\title{
\#USGS
}

science for a changing world

Prepared in cooperation with the U.S. Fish and Wildlife Service

Jaguar Surveying and Monitoring in the United States

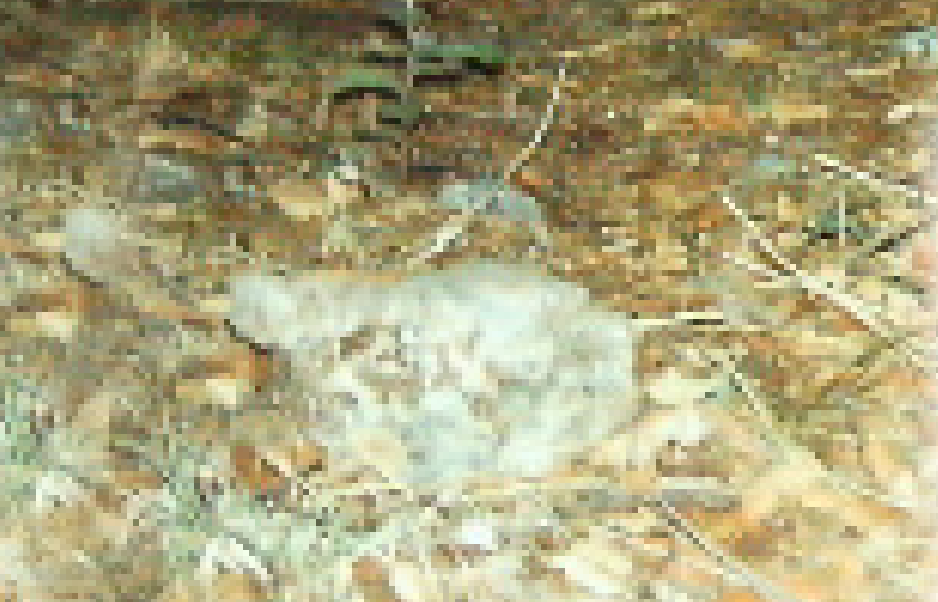

Open-File Report 2016-1095

Version 1.1, November 2016

U.S. Department of the Interior

U.S. Geological Survey
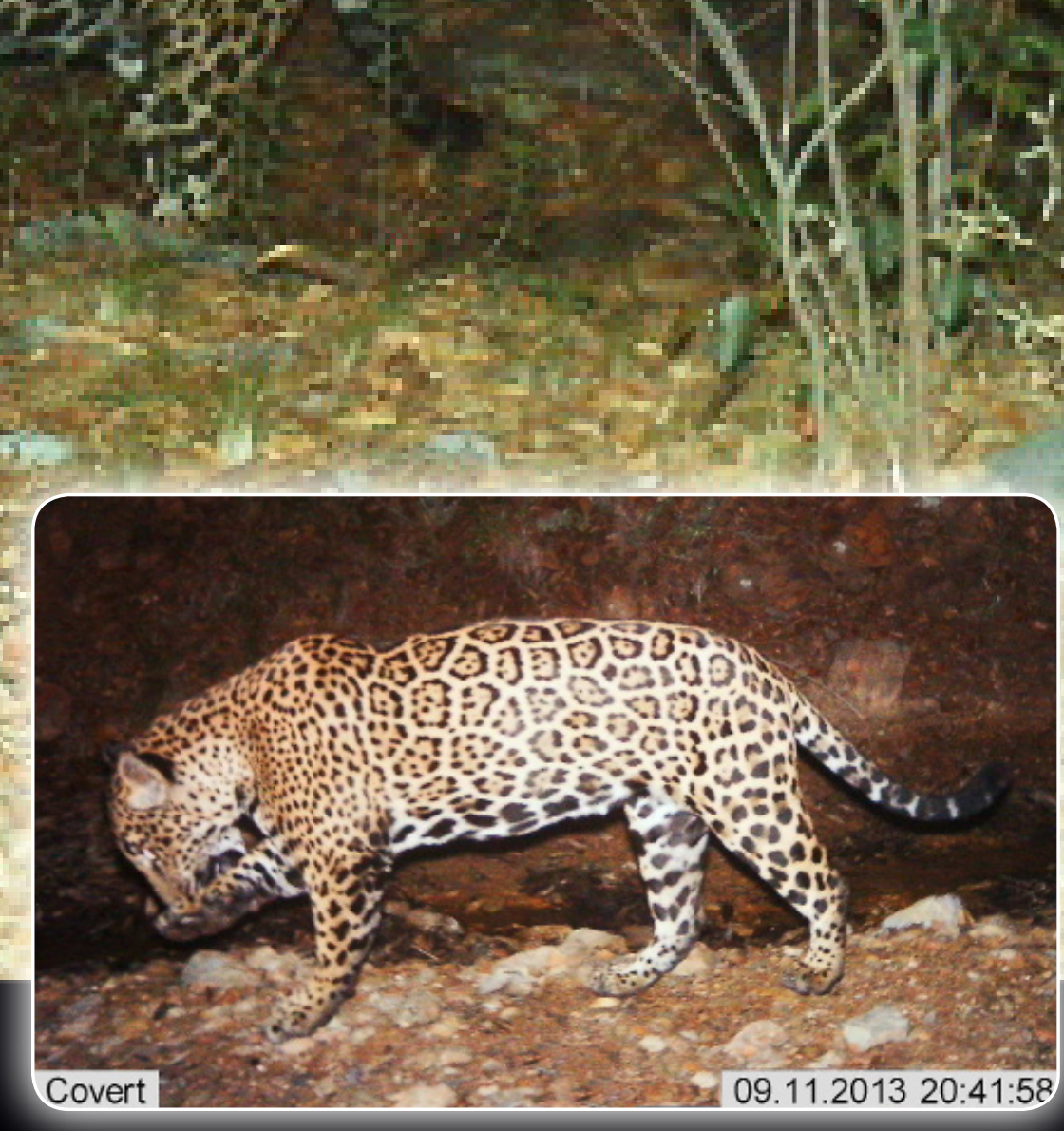
Cover: Photographs of jaguar (Panthera onca), September 11, 2013. Photographs courtesy of University of Arizona and U.S. Fish and Wildlife Service. 


\section{Jaguar Surveying and Monitoring in the United States}

By Melanie Culver

Prepared in cooperation with the U.S. Fish and Wildlife Service

Open-File Report 2016-1095

Version 1.1, November 2016

U.S. Department of the Interior

U.S. Geological Survey 


\title{
U.S. Department of the Interior SALLY JEWELL, Secretary
}

\section{U.S. Geological Survey \\ Suzette M. Kimball, Director}

\author{
U.S. Geological Survey, Reston, Virginia \\ First release: June 2016 \\ Revised: November 2016 (ver. 1.1)
}

For more information on the USGS—-the Federal source for science about the Earth, its natural and living resources, natural hazards, and the environment-visit http://www.usgs.gov/ or call 1-888-ASK-USGS (1-888-275-8747).

For an overview of USGS information products, including maps, imagery, and publications, visit http://store.usgs.gov.

Findings and conclusions in this article are those of the authors and do not necessarily represent the views of the U.S. Fish and Wildlife Service.

Any use of trade, firm, or product names is for descriptive purposes only and does not imply endorsement by the U.S. Government.

Although this information product, for the most part, is in the public domain, it also may contain copyrighted materials as noted in the text. Permission to reproduce copyrighted items must be secured from the copyright owner.

Suggested citation:

Culver, Melanie, 2016, Jaguar surveying and monitoring in the United States (ver. 1.1, November 2016): U.S. Geological Survey Open-File Report 2016-1095, 228 p., http://dx.doi.org/10.3133/ofr20161095. 


\section{Acknowledgments}

I recognize the very substantial contribution and expertise of the project manager, Susan Malusa, during all phases of this project, including report writing. I recognize the substantial contribution in field work, data analyses, and report writing of Jack Childs, the field team manager for this project. I recognize the substantial contribution of the entire jaguar field team who wrote the original proposal for funding (most of them), set and monitored cameras throughout the 17 mountain ranges, and helped with report writing -including Tim Fagan, Lisa Haynes, James G. Sanderson, Tom Skinner, Nick Smith, Kyle Thompson, and Ron W. Thompson. I appreciate Lisa Haynes’ tireless work up-front in communicating our project to the Arizona and New Mexico ranching communities; later in the project she had assistance from Jatta H. Sheehy. I appreciate James G. Sanderson's significant involvement in photo data analyses for this study. I appreciate the expert GIS analyses conducted by Patricia M. Harveson. I appreciate Kirk Emerson for guiding us through the challenges of handling media requests and any conflicts that arose, regardless of which stakeholder or individual was involved. I acknowledge Chris Bugbee in working on the first draft of this report and also his participation as a member of the jaguar field team in the latter half of the project. All jaguar team members listed above (except Chris Bugbee) are involved in some way with the continued camera effort, which is still monitoring 6 of the original 17 mountain ranges (by our team of more than 20 citizen scientists) either by monitoring cameras or providing assistance. Chris Bugbee is no longer associated with this project or with the citizen science team. I recognize the substantial support of Region 2 and the Tucson and New Mexico Ecological Services Field Offices of the USFWS for this 3-year study, which was funded by the USFWS. In particular I would like to thank biologists Susan Sferra, Erin Fernandez, Marit Alanen, Patricia Zenone, and Sarah Rinkevich at USFWS. I would like to thank biologists with extensive expertise in felids Dave Brown, Harley Shaw, Tom Waddell, and Randy Babb for many helpful discussions. I thank the Armendaris Ranch for hosting team meetings. I would like to thank Tim Snow for his help with many facets of this study, from planning to technical aspects to implementation. I would like to thank Grant Harris for his help and support of this project. I would like to thank George Ferguson for help with species identification in our photos. I would like to thank Jessica Lamberton for providing Sky Island Alliance ocelot photos to us to examine and compare to ours, and other Sky Island Alliance staff for meeting with us and providing assistance and advice. I thank Alexander Ochoa for genetic analyses of scat samples. I want to thank several members of the UA media group for their help with media relations: Daniel Stolte, Jennifer Fitzenberger, and Susan McGinley. I thank Jeff Humphrey, USFWS Public Information Officer, for his wisdom and advice. I would like to thank Joshua Estavillo and the legal team at the UA for their help. I would like to thank Andy Honoman, Jesse Schaa, and Mickey Reed for help with data servers for storage and technical aspects of GIS maps. I would like to thank our student intern Elizabeth Painter. Steve Knox was immensely helpful assisting with the permitting load at the start of the project, for which I am so grateful. I would like to thank our very enthusiastic and effective crew of Citizen Science volunteers at the UA, led by Randy Gimblett and Emily Reynolds, who have been helping with some of the camera monitoring for this project. I would like to thank all volunteers who have worked with our field team on camera checks during this project. I thank Jim Stuart, New Mexico Department of Game and Fish, who was very helpful with his advice and assistance. I thank Joe Oliver, Don Jones, and Jeff Snavely, Public Lands Liaison officers with the Border Patrol, and many others with Border Patrol who helped considerably with communications and coordination with their agency. Likewise I am very grateful for the support and assistance from Bureau of Land Management staff in both Arizona and New Mexico: Marcia Radke, Karen Simms, Jack Barnitz, Ray Lister, and Bill Childress. I thank Bill Radke, USFWS San Bernardino National Wildlife Refuge, for his very helpful advice and support throughout the project. I thank Stephen Williams for his 
assistance as liaison with the Arizona State Lands Department. I thank Mitch McClaren and Mark Heitlinger for their support from the Santa Rita Experimental Range. I thank Sheridan Stone and Dawn Rohr with Fort Huachuca for all their help facilitating our project on the Fort. I thank Peter Warren, The Nature Conservancy, for his very helpful advice and support throughout the project. I thank Don Swann and Jason Mateljak for their assistance on National Park Service lands. I would like to thank the following individuals for assistance with rancher and landowner coordination: Kristen Egen and Don Decker, with the Natural Resources Conservation Service; George Ruyle, Kim McReynolds, Dean Fish, and Mark Apel of the UA Cooperative Extension group; Kerry Baldwin and his staff members Jennifer Psillas, John Sullivan, and Doug Siegel, with Pima County Natural Resources; biologists Richard Gerhart, Marc Stamer, and John Kraft, range staff Sean Lockwood, Joe Harris, James Heitholt, Tom Lorenz, and Ed Holloway, all from Coronado National Forest. I would like to thank the following individuals for their help with permitting: Sally Gall and Juliette Gutierrez for assisting with the Buenos Aires National Wildlife Refuge permitting process; Eric Gardner for his assistance with AGFD permitting; Marty Tuegel for his assistance with USFWS permitting; Duane Bennett who assisted with USFS permitting; and Commissioner, Ray Powell, as well as his staff liaison, John Romero, for facilitating the New Mexico State Lands permitting. I am especially grateful to the 20 private landowners who gave us permission cross or work on their private lands. Finally, I would like to express my deep appreciation to many members of the ranching community who provided thoughtful conversations and advice, sometimes over coffee or meals at their kitchen tables, at times even providing our team members with a place to stay overnight. I appreciate their knowledge of the land and wildlife. Several members of the Altar Valley Conservation Alliance were instrumental in providing input for the landowner coordination protocols. I very much appreciate the Malpai Borderlands Group for their hospitality and opportunities to coordinate with their members, and also the Southern Arizona Cattlemen's Protective Association for the invitation to speak at their annual meeting. 


\section{Contents}

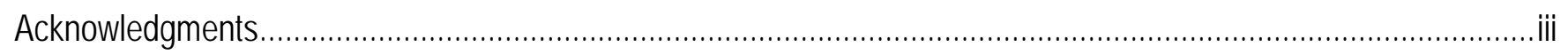

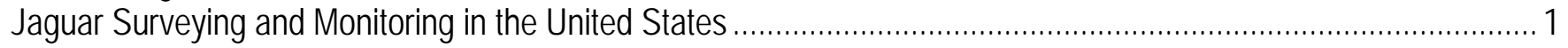

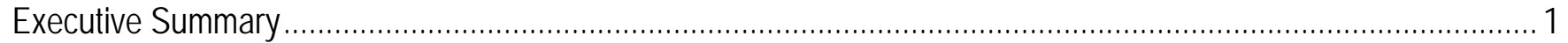

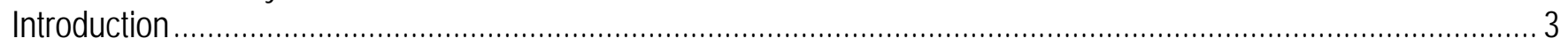

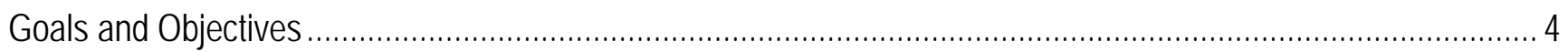

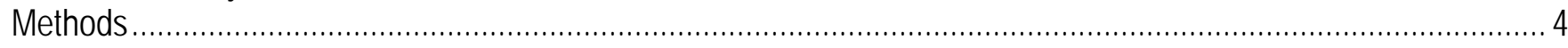

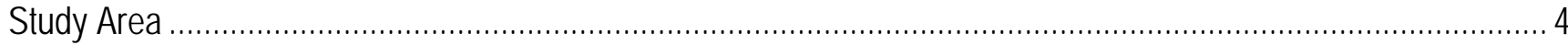

Fieldwork for Wildlife Photos/Videos and Field Safety Protocol .................................................................. 5

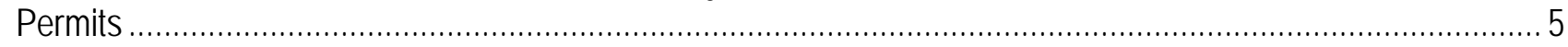

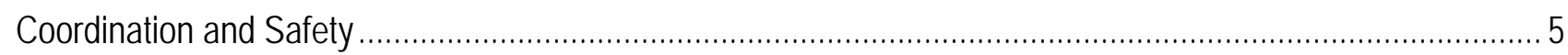

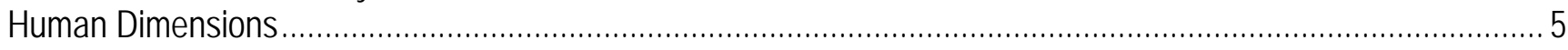

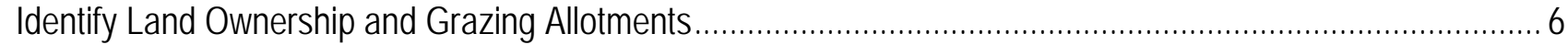

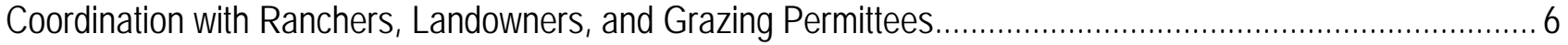

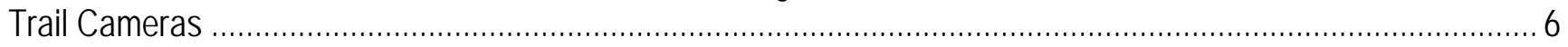

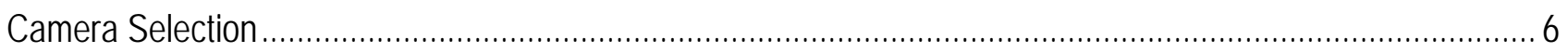

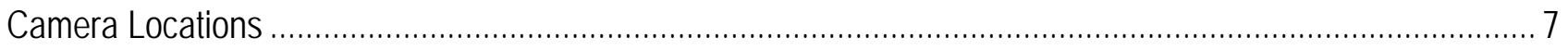

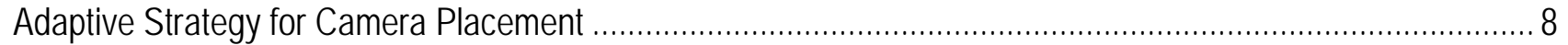

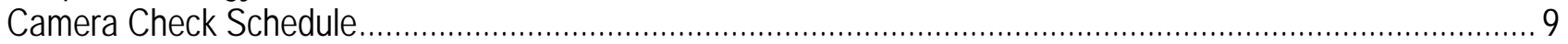

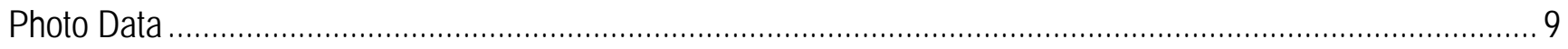

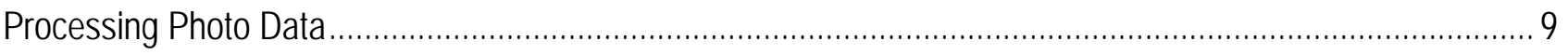

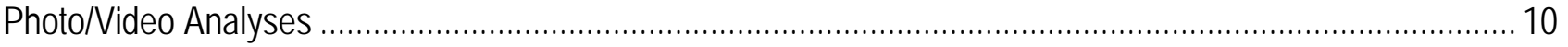

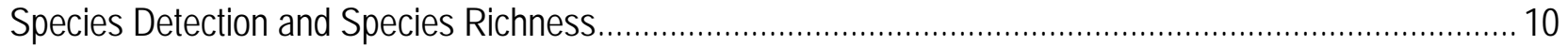

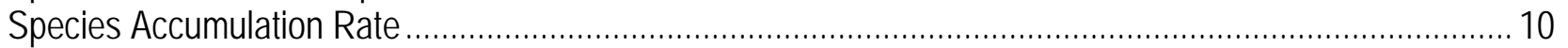

Species Total and Relative Abundance ................................................................................... 10

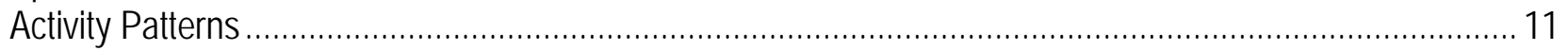

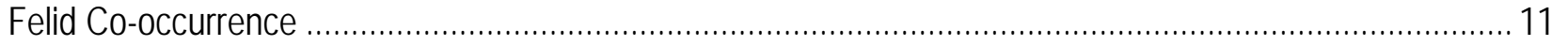

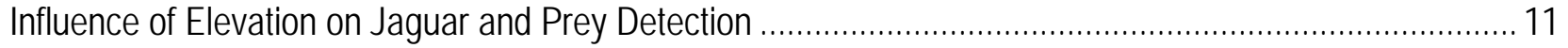

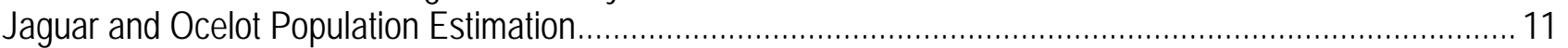

Jaguar and Ocelot Home Ranges ................................................................................................ 11

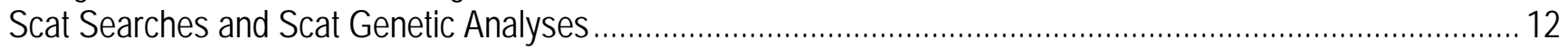

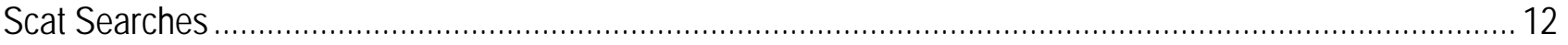

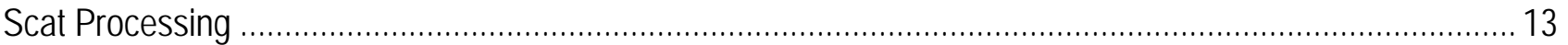

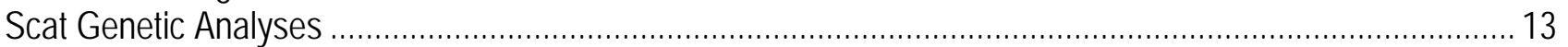

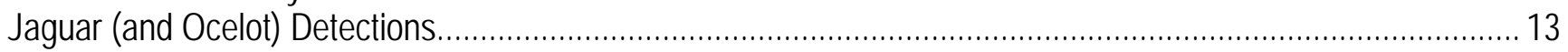

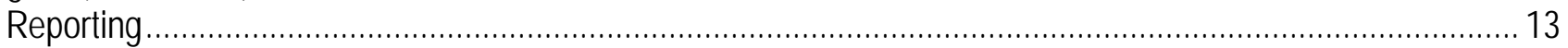

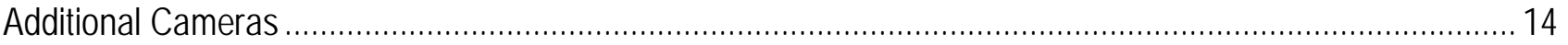

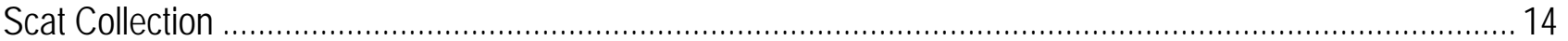

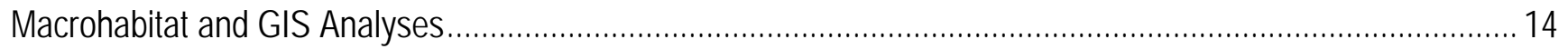

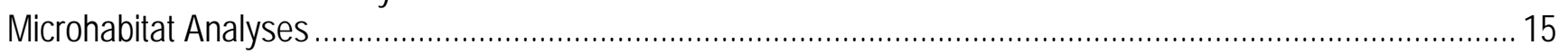

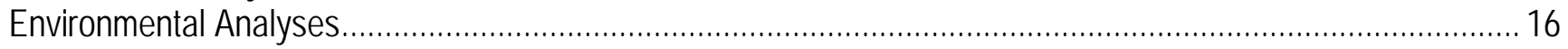

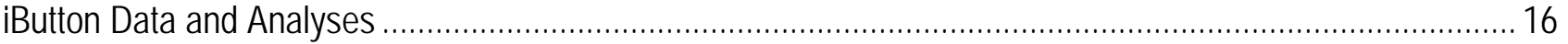

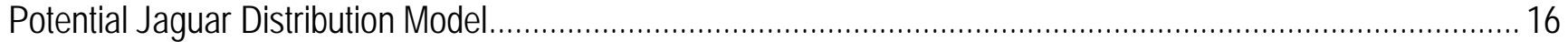




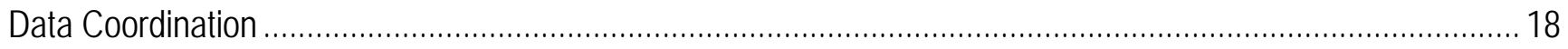

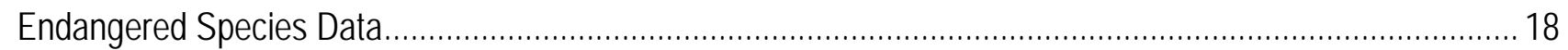

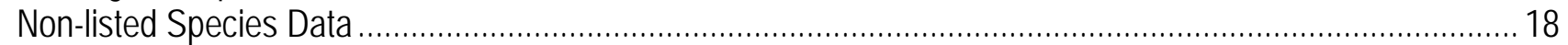

Coordination with USFWS, Jaguar Recovery Team, and Others .......................................................... 18

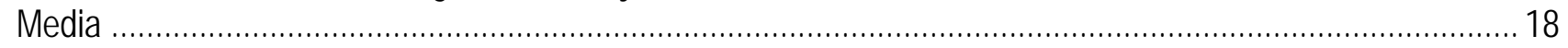

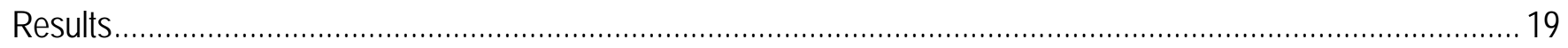

Fieldwork for Wildlife Photos/Videos and Field Safety ...................................................................... 19

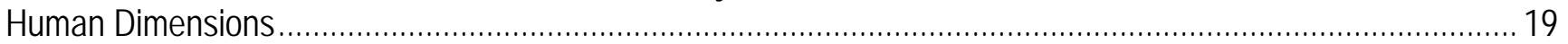

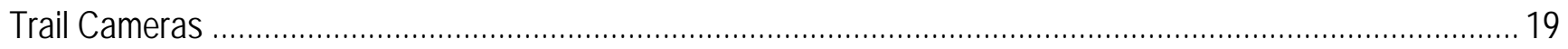

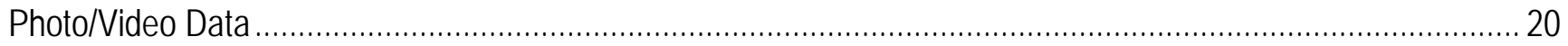

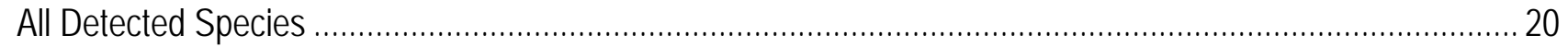

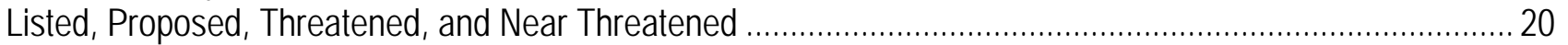

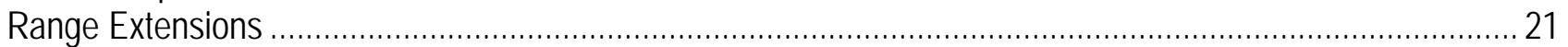

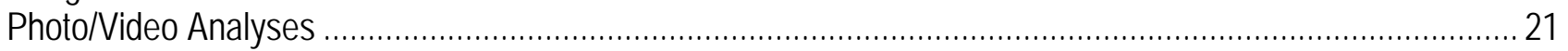

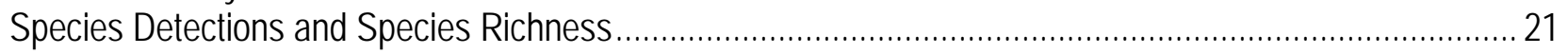

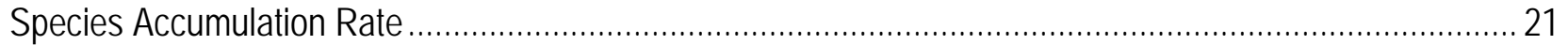

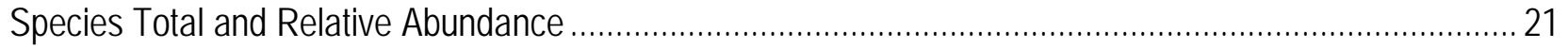

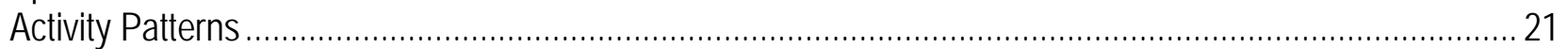

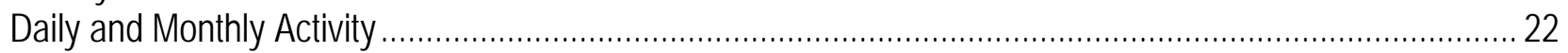

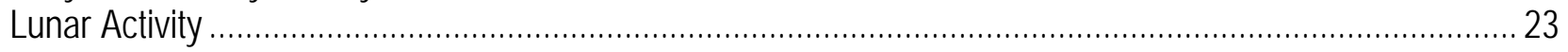

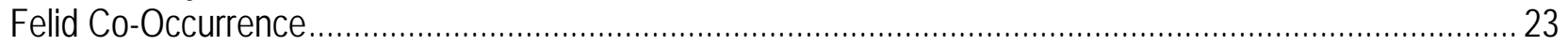

Influence of Elevation on Jaguar and Prey Detection .................................................................... 24

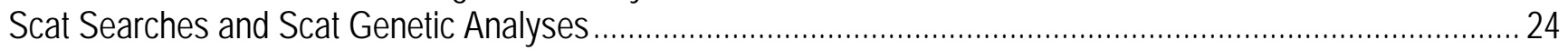

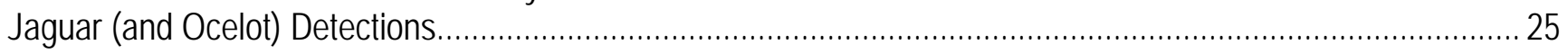

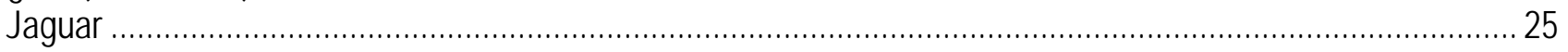

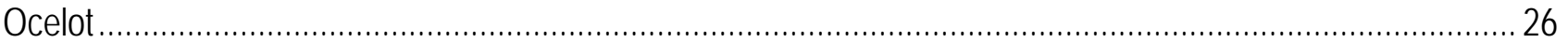

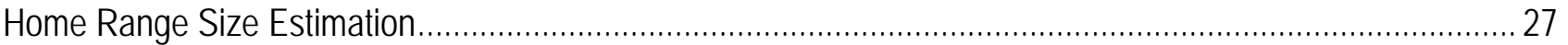

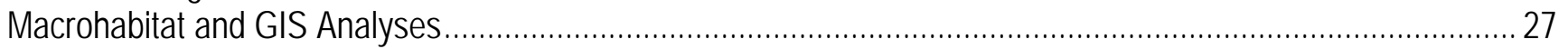

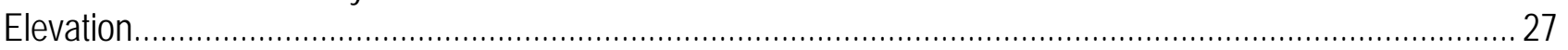

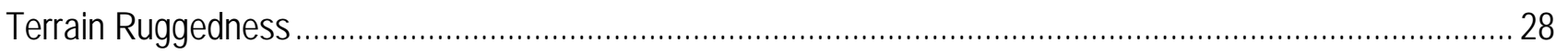

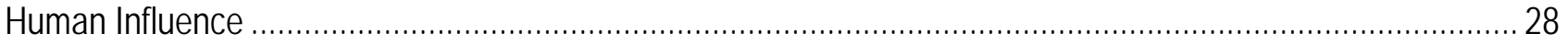

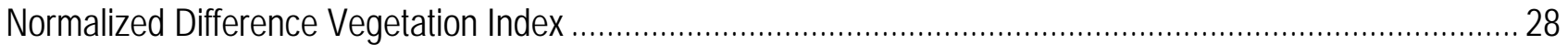

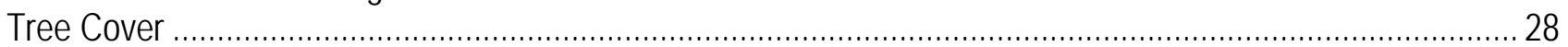

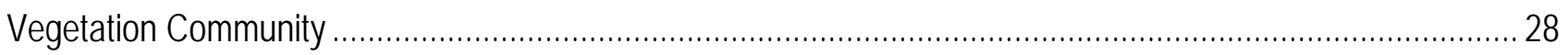

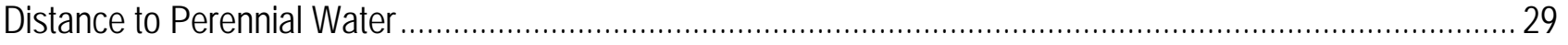

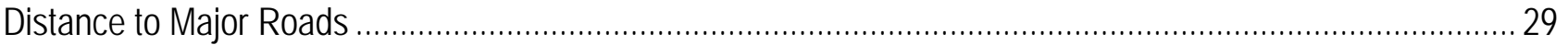

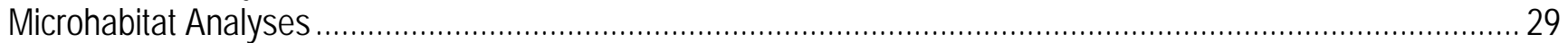

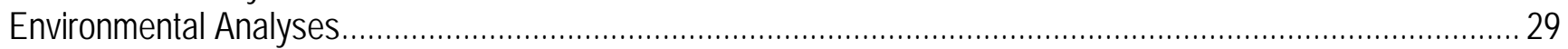

Jaguar Detection and Non-Detection Locations Across the Study Area.......................................................2 29

Ocelot Detection and Non-Detection Locations Across the Study Area ………...........................................30

Jaguar Detection and Non-Detection Locations in the Santa Rita Mountains ...............................................30

Ocelot Detection and Non-Detection Locations in the Santa Rita Mountains ................................................30

Ocelot Detection and Non-Detection Locations in the Huachuca Mountains..................................................30

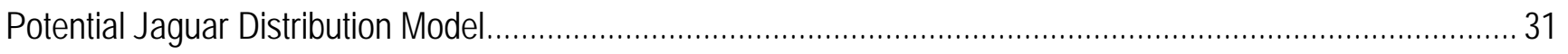




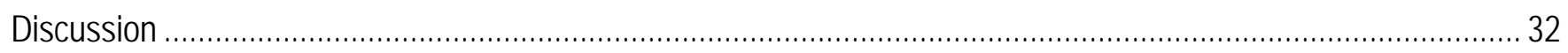

Fieldwork for Wildlife Photos/Videos and Field Safety Protocol .................................................................. 32

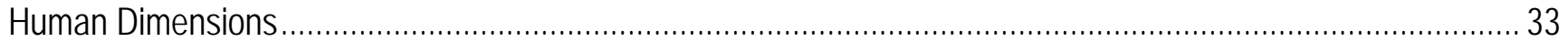

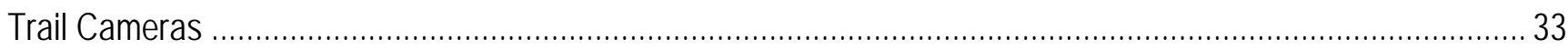

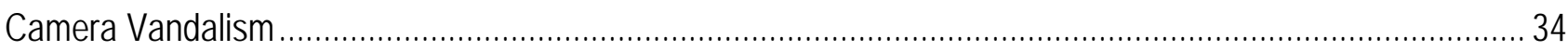

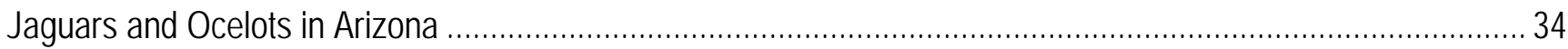

Behavioral Patterns of Arizona Felids (and Other Wildlife)......................................................................... 36

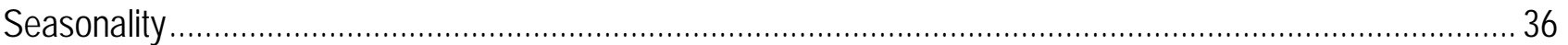

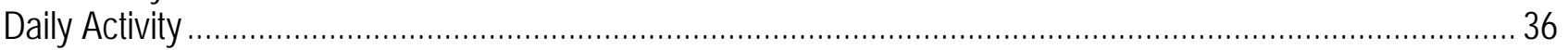

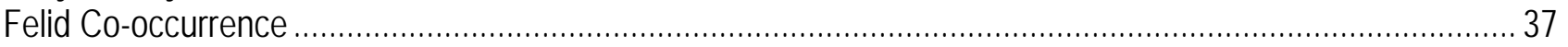

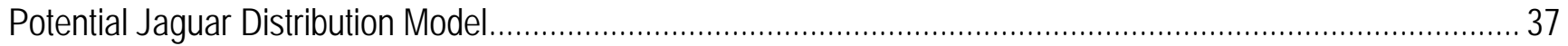

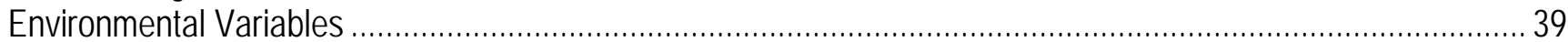

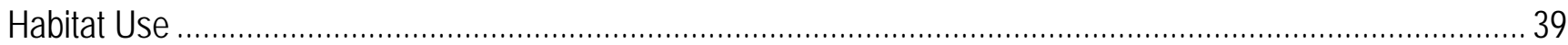

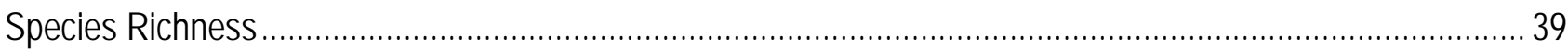

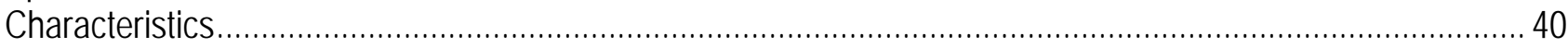

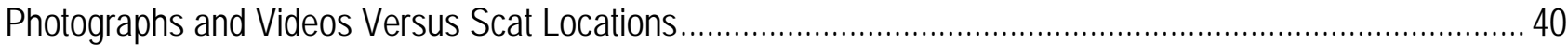

Historical Versus Current Jaguar Locations .................................................................................... 41

Jaguar and Ocelot Travel Corridors between Mountain Ranges ................................................................ 41

Potential Jaguar Habitat in Arizona Outside Our Study Area ……............................................................. 41

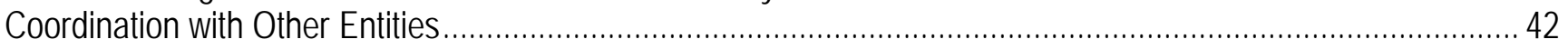

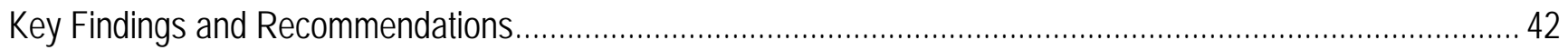

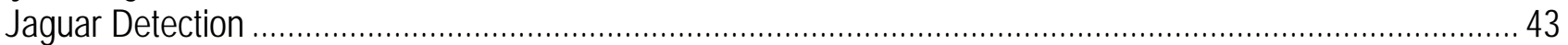

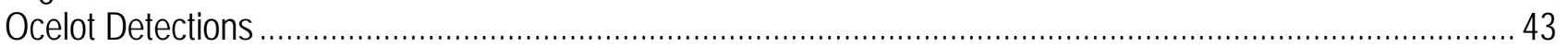

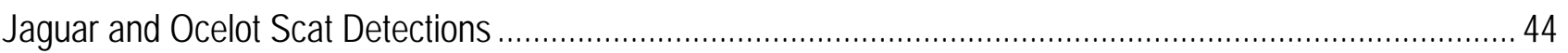

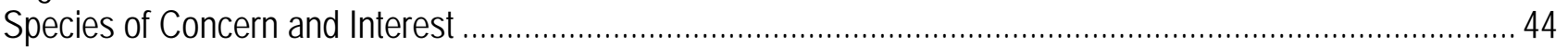

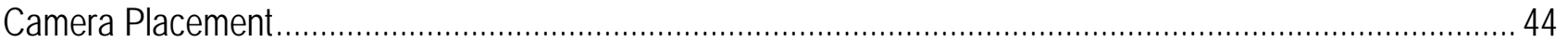

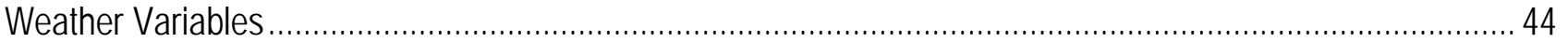

Dispersal from Mexico and International Issues ................................................................................ 45

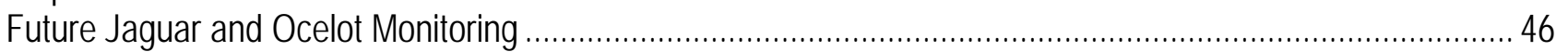

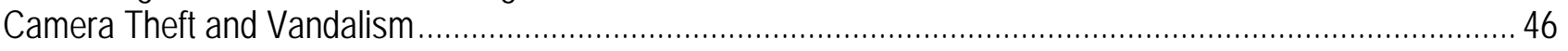

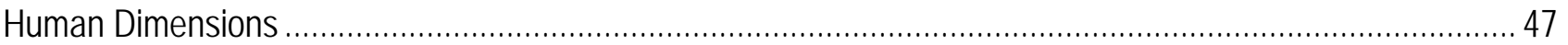

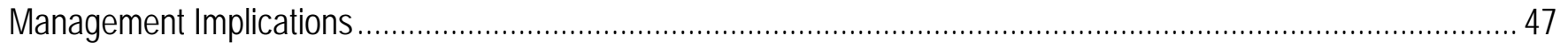

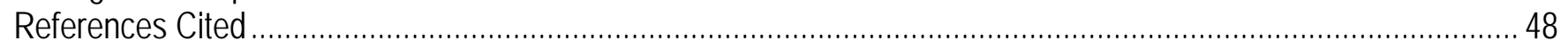

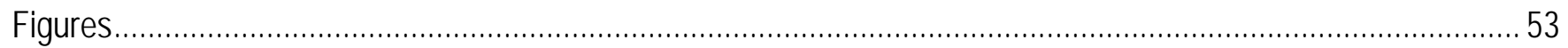

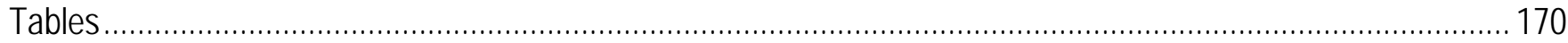




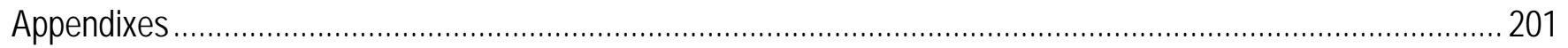

Appendix 1. Document Provided to Landowners and Grazing Permittees about the Jaguar Monitoring Project to Solicit Cooperation in Access to Land That They Own or Use .................................................... 201 Appendix 2. All Camera Sites with Rounded Coordinates (UTM 12N - NAD83) and Environmental Characteristics from Arizona and New Mexico, 2012-2015 ...................................................................... 203 Appendix 3. Survey Sites (UTM coordinates) by Mountain Range and Number of Days Camera(s) Were Active at Each Site

Appendix 4. Spatial Representation of Camera Theft and Vandalism from April 2012 to February 2015 on Eastern Side of Study Area.

Appendix 5. Spatial Representation of Camera Theft and Vandalism from April 2012 to February 2015 on Western Side of Study Area.....

Appendix 6. Quantity of Cameras Stolen/Vandalized per Quarter of Study. Data Represents April 2012 through February 2015

\section{Figures}

Figure 1a. Map showing jaguar survey and monitoring western study area (west of Interstate 19) -north of the United States-Mexico Border, south of Interstate 10.

Figure $1 \mathrm{~b}$. Map showing jaguar survey and monitoring western study area (east of Interstate 19) -north of the United States-Mexico Border, south of Interstate 10

Figure 1c. Map showing aguar survey and monitoring eastern study area-North of the United States-Mexico Border, south of Interstate 10.

Figure 2. Diagram showing layout of transects for sampling microhabitat features at jaguar and ocelot detection sites 56

Figure 3. Species composition in the Atascosa Mountain range as detected by trail cameras from April 2012 to February 2015

Figure 4. Species composition in the Baboquivari Mountain range as detected by trail cameras from April 2012 to February 2015 58

Figure 5. Species composition in the Canelo Hills as detected by trail cameras from April 2012 to February 2015

Figure 6. Species composition in the Cerro Colorado Mountain range as detected by trail cameras from April 2012 to February 2015

Figure 7. Species composition in the Chiricahua Mountain range as detected by trail cameras from April 2012 to February 2015

Figure 8. Species composition in the Coyote Mountain range as detected by trail cameras from April 2012 to February 2015.

Figure 9. Species composition in the Dos Cabezas Mountain range as detected by trail cameras from April 2012 to February 2015

Figure 10. Species composition in the Huachuca Mountain range as detected by trail cameras from April 2012 to February 2015

Figure 11. Species composition in the Pajarito (East) Mountain range as detected by trail cameras from April 2012 to February 2015

Figure 12. Species composition in the west Pajarito (West) Mountain range as detected by trail cameras from April 2012 to February 2015

Figure 13. Species composition in the Patagonia Mountain range as detected by trail cameras from April 2012 to February 2015 
Figure 14. Species composition in the Peloncillo Mountain range as detected by trail cameras from April 2012 to February 2015

Figure 15. Species composition in the San Luis (West) Mountain range as detected by trail cameras from April 2012 to February 2015.

Figure 16. Species composition in the Santa Rita Mountain range as detected by trail cameras from

April 2012 to February 2015

Figure 17. Species composition in the Sierrita Mountain range as detected by trail cameras from

April 2012 to February 2015

Figure 18. Species composition in the Tumacacori Mountain range as detected by trail cameras from

April 2012 to February 2015

Figure 19. Species composition in the Whetstone Mountain range as detected by trail cameras from

April 2012 to February 2015

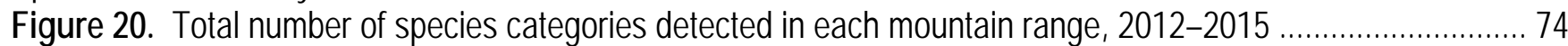

Figure 21. Percent of total detected species categories by site in the Atascosa Mountain range, 2012-2015 ..... 75

Figure 22. Percent of total detected species categories by site in the Baboquivari Mountain range, 2012-2015 .. 76

Figure 23. Percent of total detected species categories by site in the Canelo Hills, 2012-2015........................ 77

Figure 24. Percent of total detected species categories by site in the Cerro Colorado Mountain range, 2012-2015.

Figure 25. Percent of total detected species categories by site in the Chiricahua Mountain range, 2012-2015... 79

Figure 26. Percent of total detected species categories by site in the Dos Cabezas Mountain range,

2012-2015.

Figure 27. Percent of total detected species categories by site in the Huachuca Mountain range, 2012-2015 .... 81

Figure 28. Percent of total detected species categories by site in the Pajarito Mountain range, 2012-2015 ....... 82

Figure 29. Percent of total detected species categories by site in the Patagonia Mountain range, 2012-2015 .... 83

Figure 30. Percent of total detected species categories by site in the Peloncillo Mountain range, 2012-2015..... 84

Figure 31. Percent of total detected species categories by site in the Santa Rita Mountain range, 2012-2015 .... 85

Figure 32. Percent of total detected species categories by site in the Santa Rita Mountain range, 2012-2015 .... 86

Figure 33. Percent of total detected species categories by site in the Sierrita Mountain range, 2012-2015......... 87

Figure 34. Percent of total detected species categories by site in the San Luis Mountain range (Arizona),

2012-2015.

Figure 35. Percent of total detected species categories by site in the Tumacacori Mountain range, 2012-2015. . 89

Figure 36. Percent of total detected species categories by site in the Whetstone Mountain range, 2012-2015 .... 90

Figure 37. Species accumulation curve for all sites, 2012-2015 ........................................................... 91

Figure 38. Total number of each species and species categories detected from April 2012 to February 2015 ..... 92

Figure 39. Total number of species and species categories detected per 60-minute

period (independent records).

Figure 40. Total daily and monthly detections of Chiropterans (bats), 2012-2015 ........................................ 94

Figure 41. Daily and monthly detections of Ursus americanus (bears), 2012-2015 ....................................... 95

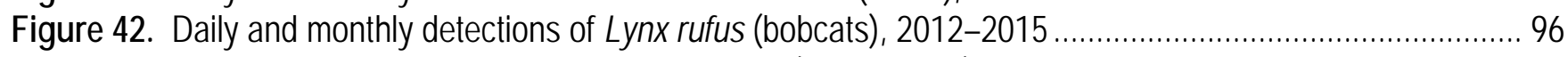

Figure 43. Daily and monthly detections of Nasua narica (coatimundis), 2012-2015 .................................... 97

Figure 44. Daily and monthly detections of Sylvilagus spp. (cottontails), 2012-2015 _..................................... 98

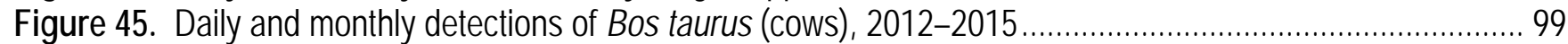

Figure 46. Daily and monthly detections of Canis latrans (coyotes), 2012-2015....................................... 100

Figure 47. Daily and monthly detections of Urocyon cinereoargenteus (gray foxes), 2012-2015................... 101

Figure 48. Daily and monthly detections of Heloderma suspectum (Gila monsters), 2012-2015 ..................... 102

Figure 49. Daily and monthly detections of Homo sapiens (humans), 2012-2015 ....................................... 103

Figure 50. Daily and monthly detections of Lepus spp. (jackrabbits), 2012-2015 .................................... 104 
Figure 51. Daily and monthly detections of Panthera onca (jaguars), 2012-2015 .......................................... 105

Figure 52. Daily and monthly detections of Pecari tejacu (javelina), 2012-2015 .......................................... 106

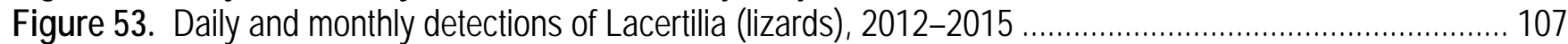

Figure 54. Daily and monthly detections of Odocoileus hemionus (mule deer), 2012-2015 .............................. 108

Figure 55. Daily and monthly detections of Leopardus pardalis (ocelots), 2012-2015 .................................... 109

Figure 56. Daily and monthly detections of Didelphis virginiana (opossums), 2012-2015 ................................ 110

Figure 57. Daily and monthly detections of Puma concolor (pumas), 2012-2015 .......................................... 111

Figure 58. Daily and monthly detections of Procyon lotor (raccoons), 2012-2015 ....................................... 112

Figure 59. Daily and monthly detections of Bassariscus astutus (ringtails), 2012-2015 ................................ 113

Figure 60. Daily and monthly detections of non-Sciurid Rodentia (rodents, not including squirrels),

2012-2015.

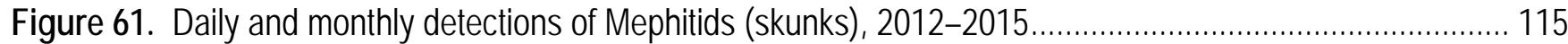

Figure 62. Daily and monthly detections of Serpentes (snakes), 2012-2015 ................................................... 116

Figure 63. Daily and monthly detections of Sciurus spp. (squirrels), 2012-2015 ........................................ 117

Figure 64. Daily and monthly detections of Gopherus morafkai (tortoises), 2012-2015 .................................. 118

Figure 65. Daily and monthly detections of Meleagris gallopavo (turkeys), 2012-2015................................. 119

Figure 66. Daily and monthly detections of Odocoileus virginiana (white-tailed deer), 2012-2015.................... 120

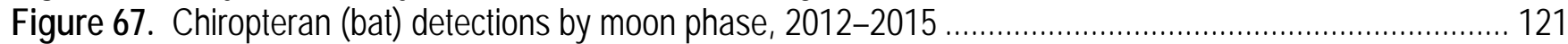

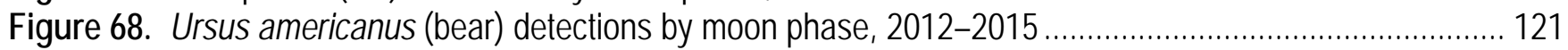

Figure 69. Lynx rufus (bobcat) detections by moon phase, 2012-2015 ..................................................... 122

Figure 70. Nasua narica (coatimundi) detections by moon phase, 2012-2015 ............................................. 122

Figure 71. Sylvilagus spp. (cottontail) detections by moon phase, 2012-2015 ............................................. 123

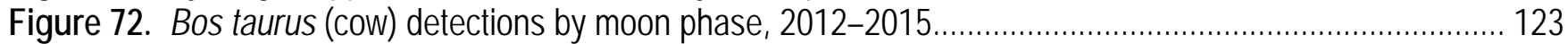

Figure 73. Canis latrans (coyote) detections by moon phase, 2012-2016 ..................................................... 124

Figure 74. Urocyon cinereoargenteus (gray fox) detections by moon phase, 2012-2016 ................................. 124

Figure 75. Ranid (frog) detections by moon phase, 2012-2016 ............................................................. 125

Figure 76. Lepus spp. (jackrabbit) detections by moon phase, 2012-2016 ….............................................. 125

Figure 77. Panthera onca (jaguar) detections by moon phase, 2012-2016 .................................................. 126

Figure 78. Pecari tejacu (javelina) detections by moon phase, 2012-2016 ................................................... 126

Figure 79. Leopardus pardalis (ocelot) detections by moon phase, 2012-2016 …......................................... 127

Figure 80. Didelphis virginiana (opossum) detections by moon phase, 2012-2016 ..................................... 127

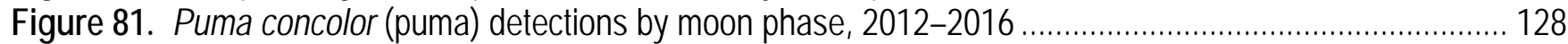

Figure 82. Procyon lotor (raccoon) detections by moon phase, 2012-2016 ................................................ 128

Figure 83. Bassariscus astutus (ringtail) detections by moon phase, 2012-2016 ......................................... 129

Figure 84. Non-Sciurid Rodentia (rodent, not including squirrel) detections by moon phase, 2012-2016 .......... 129

Figure 85. Mephitid (skunk) detections by moon phase, 2012-2016 ........................................................ 130

Figure 86. Odocoileus virginiana (white-tailed deer) detections by moon phase, 2012-2016 ......................... 130

Figure 87. Monthly detection frequencies of Arizona felids (bobcat, jaguar, puma, and ocelot), 2012-2016 ....... 131

Figure 88. Comparisons of detection rates of jaguar, white-tailed deer, and javelina by elevation and

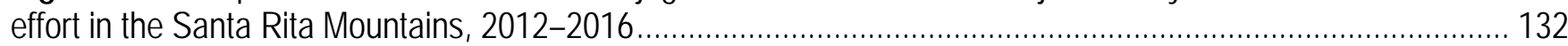

Figure 89. Elevation of camera sites, random points, current ocelot and jaguar locations, and historical

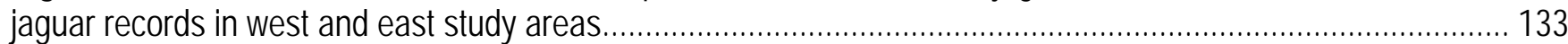

Figure 90. Terrain Ruggedness Index (TRI) of camera sites, random points, current ocelot and jaguar

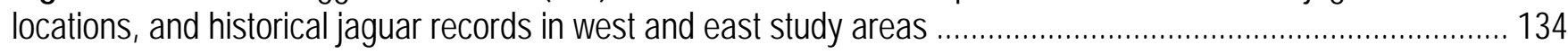

Figure 91. Human influence index of camera sites, random points, current ocelot and jaguar locations, and historical jaguar records in west and east study areas. 
Figure 92. Normalized Difference Vegetation Index (NDVI) for March 2015 (top two graphs) and August 2014 (bottom two graphs) of camera sites, random points, current ocelot and jaguar locations, and historical jaguar records in west and east study areas

Figure 93. Tree cover of camera sites, random points, current ocelot and jaguar locations, and historical jaguar records in west and east study areas......

Figure 94. Vegetation community (Brown, 1994) of camera sites, random points, current ocelot and jaguar locations, and historical jaguar records in west and east study areas

Figure 95. Distance to perennial water for camera sites, random points, current ocelot and jaguar locations, and historical jaguar records in west and east study areas.

Figure 96. Distance to major roads from camera sites, random points, current ocelot and jaguar locations, and historical jaguar records in west and east study areas

Figure 97. Habitat and species composition characteristics of jaguar detection site SAN01 in the Santa Rita Mountains

Figure 98. Habitat and species composition characteristics of jaguar detection site SAN08 in the Santa Rita Mountains

Figure 99. Habitat and species composition characteristics of jaguar detection site SAN10 in the Santa Rita Mountains....

Figure 100. Habitat and species composition characteristics of jaguar detection site SAN11 in the Santa Rita Mountains

Figure 101. Habitat and species composition characteristics of jaguar detection site SAN12 in the Santa Rita Mountains

Figure 102. Habitat and species composition characteristics of jaguar detection site SAN13 in the Santa Rita Mountains.

Figure 103. Habitat and species composition characteristics of jaguar detection site SAN14 in the Santa Rita Mountains

Figure 104. Habitat and species composition characteristics of jaguar detection site SAN15 in the Santa Rita Mountains

Figure 105. Habitat and species composition characteristics of jaguar detection site SAN17 in the Santa Rita Mountains

Figure 106. Habitat and species composition characteristics of jaguar detection site SAN19 in the Santa Rita Mountains

Figure 107. Habitat and species composition characteristics of jaguar detection site SAN22 in the Santa Rita Mountains

Figure 108. Habitat and species composition characteristics of jaguar detection site SAN23 in the Santa Rita Mountains

Figure 109. Habitat and species composition characteristics of jaguar detection site SAN24 in the Santa Rita Mountains

Figure 110. Habitat and species composition characteristics of jaguar detection site SAN25 in the Santa Rita Mountains

Figure 111. Habitat and species composition characteristics of jaguar detection site SAN27 in the Santa Rita Mountains

Figure 112. Habitat and species composition characteristics of jaguar detection site SAN28 in the Santa Rita Mountains.

Figure 113. Habitat and species composition characteristics of jaguar detection site SAN29 in the Santa Rita Mountains

Figure 114. Habitat and species composition characteristics of jaguar detection site SAN30 in the Santa Rita Mountains..... 
Figure 115. Habitat and species composition characteristics of jaguar detection site SAN31 in the Santa Rita Mountains

Figure 116. Habitat and species com position characteristics of jaguar detection site SAN32 in the Santa Rita Mountains

Figure 117. Habitat and species composition characteristics of jaguar detection site SAN37 in the Santa Rita Mountains

Figure 118. Habitat and species composition characteristics of jaguar detection site SAN39 in the Santa Rita Mountains

Figure 119. Overstory and midstory cover characteristics of jaguar detection sites in the Santa Rita

Figure 120a. A comparison of daily average relative humidity (in percent) for jaguar detection ( $n=9$, red line) and jaguar non-detection ( $n=35$, black line) locations in Julian days (days 90-120 correspond to April, 330-360 to December).

Figure 120b. A comparison of daily average temperature (in degrees Celsius) for jaguar detection $(n=9$, red line) and jaguar non-detection ( $n=35$, black line) locations in Julian days (days 90-120 correspond to April, 330-360 to December).

Figure 120c. A comparison of daily average relative humidity (in percent) for ocelot detection ( $n=6$, red line) and ocelot non-detection ( $n=38$, black line) locations in Julian days (days 90-120 correspond to April, 330-360 to December).

Figure 120d. A comparison of daily average temperature (in degrees Celsius) for ocelot detection ( $n=6$, red line) and ocelot non-detection ( $n=8$, black line) locations in Julian days (days 90-120 correspond to April, 330-360 to December).

Figure 121a. For the Santa Rita Mountains, a comparison of daily average relative humidity (in percent) for jaguar detection ( $n=9$, red line) and jaguar non-detection ( $n=6$, black line) locations in Julian day (days 90-120 correspond to April, 330-360 to December).

Figure 121b. For the Santa Rita Mountains, a comparison of daily average temperature (in degrees Celsius) for jaguar detection ( $n=9$, red line) and jaguar non-detection ( $n=6$, black line) locations in Julian days (days 90-120 correspond to April, 330-360 to December).

Figure 121c. For the Santa Rita Mountains, a comparison of daily average relative humidity (in percent) for ocelot detection ( $n=4$, red line) and ocelot non-detection ( $n=12$, black line) locations in Julian days (days 120-150 correspond to May, 330-360 to December).

Figure 121d. For the Santa Rita Mountains, a comparison of daily average temperature (in degrees Celsius) for ocelot detection ( $n=4$, red line) and ocelot non-detection ( $n=12$, black line) locations in Julian days (days 120-150 correspond to May, 330-360 to December).

Figure 122a. For the Huachuca Mountains, a comparison of daily average relative humidity (in percent) for ocelot detection ( $n=2$, red line) and ocelot non-detection ( $n=3$, black line) locations in shown versus Julian days (days 90-120 correspond to April, 330-360 to December).....

Figure 122b. For the Huachuca Mountains, a comparison of daily average temperature (in degrees Celsius) for ocelot detection ( $n=2$, red line) and ocelot non-detection ( $n=3$, black line) locations in Julian days (days 90-120 correspond to April, 330-360 to December).

Figure 123. Map showing camera survey sites buffered by a local adaptive convex-hull polygon (buffer distance $=10 \mathrm{~km}$, alpha=2) used as a bias file for background selection in MaxEnt. 163

Figure 124. Response curves for each variable in the final model showing showing changes to the logistic prediction in response to changes in each variable independently (red line, mean; blue line, \pm 1 standard deviation) 164

Figure 125. Potential distribution map for jaguars in southeastern Arizona and southwestern New Mexico focused on the west project study area. 
Figure 126. Potential distribution map for jaguars in southeastern Arizona and southwestern

New Mexico focused on the east project study area. 166

Figure 127. Map showing minimum Convex Polygon (MCP) surrounding jaguar detection sites with 24-hour Mean Maximum Distance Moved (MMDM) buffer (indicated by the green $5.90 \mathrm{~km}$ buffer) .....

Figure 128. Map showing designated jaguar critical habitat in southeastern Arizona and southwestern

New Mexico 168

Figure 129. Jaguar occurrence data from 1900 to present 169

\section{Tables}

Table 1. Permits and user agreements obtained to conduct the jaguar monitoring project, including agency, permit number, and expiration date 170

Table 2. Digital data used in geographic information system (GIS) macrohabitat analyses at sites with positive jaguar and ocelot detections.

Table 3. Established camera survey sites for jaguar monitoring project from April 25, 2012 to

February 15, 2015, including mountain range, date deployed, global positioning system (GPS) coordinates in 1983 North America Datum, Universal Transverse Marcator (NAD83, UTM), elevation, UTM zone, and total sites per mountain range

Table 4. Jaguar and ocelot detections from photographs and genetically verified scat collection from April 2012 to February 2015

Table 5a. Species $(n=50)$ and species categories $(n=32)$ used in the analysis. Common and Latin names included.

Table 5b. Species and species categories detected with trail cameras and corresponding mountain range in southern Arizona and southwestern New Mexico.

Table 6. Federally designated threatened and formerly proposed candidate species, species of concern, and species range extensions detected from April 2012 to February 2015

Table 7. Species and species categories and percent of analyzed sites $(n=233)$ at which each species or species category was detected from 2012 to 2015.

Table 8. Total number of independent photos per species/species category, average number of individuals per independent photo, and total abundance and relative abundance for species detected from April 2012 to February 2015

Table 9. Ranked differences for species activity based on full moons versus new moons for species detected from April 2012 to February 2015.

Table 10. Sample identification (ID), collection date, collection location, and analysis date for 60 scat samples collected and analyzed for species ID

Table 11. A list of mountain ranges showing 43 semi-randomly selected camera locations where iButtons were deployed.

Table 12. WorldClim bioclimatic variables evaluated for jaguar habitat suitability model performance. 199

Table 13. Evaluation statistics for top performing jaguar habitat suitability models for southeastern Arizona and southwestern New Mexico

Table 14. Bioclimatic and environmental variables included in the four top performing jaguar habitat suitability models (Models 1, 2, 3, and 4) for southeastern Arizona and southwestern New Mexico...... 200

Table 15. Jaguar habitat suitability statistics for current photo locations (2012-2015), all historical locations (1965-2009), and these locations combined 


\section{Conversion Factors}

Inch/Pound to International System of Units

\begin{tabular}{lll}
\hline & Multiply & To obtain \\
\hline & Length & \\
\hline mile (mi) & 1.609 & kilometer $(\mathrm{km})$ \\
\hline
\end{tabular}

International System of Units to Inch/Pound

\begin{tabular}{lcl}
\hline & Multiply & \multicolumn{1}{c}{ To obtain } \\
\hline & Length & \\
\hline millimeter $(\mathrm{mm})$ & 0.03937 & inch (in.) \\
meter $(\mathrm{m})$ & 3.281 & foot (ft) \\
kilometer $(\mathrm{km})$ & 0.6214 & mile (mi) \\
\hline & Area & \\
\hline square kilometer $\left(\mathrm{km}^{2}\right)$ & 247.1 & acre \\
square kilometer $\left(\mathrm{km}^{2}\right)$ & 0.3861 & square mile $\left(\mathrm{mi}^{2}\right)$ \\
\hline
\end{tabular}

Temperature in degrees Celsius $\left({ }^{\circ} \mathrm{C}\right)$ may be converted to degrees Fahrenheit $\left({ }^{\circ} \mathrm{F}\right)$ as ${ }^{\circ} \mathrm{F}=\left(1.8 \times{ }^{\circ} \mathrm{C}\right)+32$.

\section{Datums}

Vertical coordinate information is referenced to the North American Vertical Datum of 1988 (NAVD 88).

Horizontal coordinate information is referenced to the North American Datum of 1983 (NAD 83).

Elevation, as used in this report, refers to distance above the vertical datum. 


\title{
Jaguar Surveying and Monitoring in the United States
}

\author{
By Melanie Culver ${ }^{1,2,3}$
}

\section{Executive Summary}

The University of Arizona (UA) established and implemented a noninvasive system for detecting and monitoring jaguars (Panthera onca) in southeastern Arizona and southwestern New Mexico for a U.S. Fish and Wildlife Service contract F11PXO5778 awarded on September 20, 2011. UA team members working on this study had diverse expertise that included extensive field experience specifically focused on wild cats (including jaguars), facilitation/human dimensions experience, spatial analysis and modeling focused on large carnivores, and conservation genetics. Originally planned as a 3-year field study, factors such as adapting our implementation schedule to address permit/permission delays (some permits taking more than 1 year to receive), and other influences on the project timeline (such as having to remove cameras from the field sooner than expected), mean this report includes approximately 34 months of data and results (from April 2012 through February 2015).

We placed the first camera in the field on April 25, 2012, with full deployment achieved on May 31, 2013. We began removing cameras from the field on January 22, 2015, with full removal achieved on March 2, 2015. The study area incorporates most of the mountainous areas north of the United States -Mexico international border and south of Interstate 10, from the Baboquivari Mountains in Arizona to the Animas Mountains in New Mexico. In the surveying and monitoring phase of the project, there were four possible methods of confirming jaguar presence: by camera, by genetically confirmed jaguar scat, by verified tracks, or through activities outside of our project, such as other trail cameras or a jaguar being treed by puma hunters. We used two primary methods to detect exact jaguar locations: motionsensor trail cameras that passively monitored wildlife activity in the area, and genetic testing of scat collected in the field. Only noninvasive sampling methods were employed (scat and photographs) and no capturing or handling of jaguars or any other wildlife occurred during this project. Secondarily, the project focused on ocelot (Leopardus pardalis) detection and monitoring in the same manner as described for jaguars. Prior to January 22, 2015, 250 camera sites with paired or single cameras were set throughout the study area. We recorded a total of 131 jaguar detections, including 118 jaguar photographs or videos of the same individual (male) and 13 confirmed jaguar scats in one mountain range. These 131 jaguar detections represent 35 sites. We recorded 13 ocelot detections representing 3 individual males (including 1 photo/detection in which gender could not be determined) across two

\footnotetext{
${ }^{1}$ U.S. Geological Survey.

${ }^{2}$ Arizona Cooperative Fish and Wildlife Research Unit.

${ }^{3}$ University of Arizona, School of Natural Resources and the Environment.
} 
mountain ranges. These detections included 3 photographs, each of 2 ocelot males in one mountain range, and 7 photographs of 1 ocelot male in a second mountain range, representing a total of 5 sites. No confirmed ocelot scat was collected. Additional data collection included weather data (temperature and humidity) at select camera sites and detailed vegetation surveys at all camera sites. We also analyzed photograph data to examine species relationships and patterns, and used geographic information system (GIS) analyses to examine habitat suitability for jaguars and ocelots.

The key findings of this study involve the detectability, detection rates, and co-occurrence with other felids, for jaguar and ocelot. Jaguars appear to still enter Arizona/New Mexico at the rate of about 1 jaguar every 3 years. Additionally, we photographed 3 ocelots during the 3-year study period; this is notable because this study was not designed to detect ocelots. Jaguar scat detection sites $(\mathrm{n}=12)$ versus photo jaguar detection sites $(n=23)$ made up approximately one third of the total jaguar detection sites, representing a significant portion of the detections. We presume the 1 male jaguar detected in this study, whose home range size we calculated to be $90 \mathrm{~km}^{2}$ (note that our study was not designed to determine home range size, so this calculation should be used with caution), is resident in the Santa Rita Mountain range, and we presume that 1 of the ocelots (identified statewide as ocelot \#4) may be resident in the Huachuca Mountain range. In a study area with the possibility of detecting a jaguar (for example, if a jaguar is thought to be present on the landscape), the puma may be a reasonable surrogate for jaguar detection in combination with other environmental variables, and can help guide camera placement, as pumas were observed at all but 1 jaguar photo/video detection site in this study. Pumas were detected at many sites where no jaguars were detected, but the majority of those were in mountain ranges that we believe do not have jaguars present. In a study area with the possibility of detecting an ocelot (for example, if an ocelot is thought to be present on the landscape), puma and bobcat may be reasonable surrogates for ocelot detection in combination with other environmental variables, and can help guide camera placement, as both were observed at all ocelot photo detection sites in this study. Finally, continued monitoring and research for jaguars and ocelots in Arizona/New Mexico, United States, and Sonora, Mexico are needed and we recommend that a long-term monitoring system be implemented on both sides of the international border.

The methods used in this study proved valuable for detecting terrestrial mammals and a number of non-mammalian, rare species. In addition to the endangered species detected (jaguar and ocelot), this study detected species that are Endangered Species Act (ESA) threatened (Mexican spotted owl), ESA formerly proposed candidate (Sonoran desert tortoise, now withdrawn from the ESA candidate list), and International Union for Conservation of Nature (IUCN) Near Threatened (Sonora mud turtle and Gila monster). All of these species are listed as vulnerable in Arizona, and the Sonora mud turtle (photographed in New Mexico) is listed on the New Mexico Department of Game and Fish's list of Species of Greatest Conservation Need.

Because jaguars and ocelots most likely disperse into Arizona from northern Mexico, adequate conservation and monitoring of these species cannot occur without knowledge of both sides of the United States-Mexico border. The aim of this study was to monitor jaguars in core jaguar habitat on the United States side of the border. Continued monitoring and research on jaguars and ocelots in Arizona/New Mexico, United States, and Sonora, Mexico are needed, particularly in potential jaguar corridors and core habitat. Declining numbers of jaguars and ocelots suggested by current research in Sonora, Mexico may indicate increasing threats to these felids in the nearest core breeding populations for these two species-located $200 \mathrm{~km}$ and $50 \mathrm{~km}$ south of the United States-Mexico border, respectively. 
Given the complexities of land ownership, agency coordination, and the sensitive, high-profile nature of these species, it would be most desirable to have a small team of professional field biologists carry out future monitoring studies of jaguars and ocelots. Monitoring could potentially be conducted by citizen scientist volunteers, but professional personnel would still be required to train, coordinate, and oversee these volunteers. Monitoring could be expanded into Mexico in mountain ranges between the United States and the closest known populations of jaguars and ocelots in Sonora (located approximately 200 and $50 \mathrm{~km}$ south of the international border, respectively). The first priority areas for continued monitoring should be the detection areas; the next priority areas should be those that have historical jaguar/ocelot detections (such as the Baboquivari and Chiricahua Mountains in Arizona, and the San Luis Mountains in New Mexico); and the final priority areas should be mountain ranges in southeastern Arizona and southwestern New Mexico that have quality jaguar and ocelot habitat where no jaguars or ocelots have yet been detected.

\section{Introduction}

Because of the jaguar's (Panthera onca) endangered status under the Endangered Species Act (ESA) of 1973 throughout its range (from Arizona in the north to Argentina in the south), jaguar individuals and populations are monitored to varying degrees throughout their range. Knowledge gained from monitoring jaguars is helpful for wildlife managers who are responsible for conserving this species. The University of Arizona (UA) has conducted a multiyear surveying and monitoring effort for jaguars and ocelots in southern Arizona and New Mexico. The purpose of this work was to establish an effective surveying and monitoring system for jaguars along the United States-Mexico border.

Surveying and monitoring in this study focused on the United States side of the border, but the methods could also be used in Mexico. The intent was to develop and implement a surveying and monitoring system that would provide the greatest probability of recording jaguar presence in, and passage through, the border area.

This project established and implemented a noninvasive system for detecting and monitoring jaguars. The study area incorporates most of the mountainous areas north of the United States-Mexico international border and south of Interstate 10, from the Baboquivari Mountains in Arizona to the Animas Mountains in New Mexico. We used two primary methods to detect exact jaguar locations: paired motion-sensor trail cameras, and genetic testing of large carnivore scat collected in the field. We emphasize that this project used entirely noninvasive methods and no jaguars were captured, radiocollared, baited, or harassed in any way. Scat sample collection occurred during the entire field part of the study, but was intensified with the use of a trained scat detection dog following the first jaguar photo detection event (photo detection event was October 2012, scat detection dog began working January 2013). We also collected weather, vegetation, and geographic information system (GIS) data to analyze in conjunction with photo and video data. The results of this study are intended to aid and inform future management and conservation practices for jaguars and ocelots in this region. 


\section{Goals and Objectives}

The long-term goals of this project were to (1) provide new knowledge about jaguars to public land managers and the general public; (2) contribute to policy and management decisions for jaguar conservation; (3) create a useable dataset that can inform management decisions for jaguars on the ground; (4) demonstrate the value of long-term monitoring; and (5) if possible, document the occurrence of jaguars and ocelots on the United States side of the United States-Mexico border.

Project objectives were to (1) deploy and monitor approximately 120 paired wildlife camera sites (240 cameras total) in the study area to photograph and identify individual jaguars; (2) collect potential jaguar scat encountered in the field and use genetic analyses to determine species and identify individuals, if possible; (3) estimate the maximum number of wildlife species photographed at each camera site; and (4) model potential jaguar distribution and habitat use in the study area to assist with future conservation planning.

\section{Methods}

\section{Study Area}

The study area was determined by the Jaguar Recovery Team (JRT) and provided to us by the U.S. Fish and Wildlife Service (USFWS). It extended from the crest of the Baboquivari Mountains in southern Arizona, east to the "Boot-heel" of southwestern New Mexico. The southern extent of the study area was bounded by the United States-Mexico border and extended approximately 90 kilometers $(\mathrm{km})$ north (south of Interstate 10). Fieldwork was confined to mountain ranges in this area and encompassed portions of the Coronado National Forest, National Park Service lands, lands managed by the Bureau of Land Management, Arizona and New Mexico State lands, and some private ranches. The study area was predefined by the USFWS in their Request for Proposals to encompass areas where jaguars are most likely to occur in the United States (figs. 1a-1c). The study area was divided into western and eastern sections, which were not connected. The western section was divided by Interstate 19 for purposes of illustrating camera sites and detections; we include west of Interstate 19 on one map and east of Interstate 19 on a separate map.

The study area is characterized by sky island mountain ranges distributed throughout southern Arizona and southwestern New Mexico. Topography of these isolated sky islands varies from lowelevation desert flats and steep canyons to high-elevation coniferous forests and meadows. Elevation ranges from approximately 500 meters (m) to 2,900 m. The hot season ranges from April through October, with maximum temperatures reaching more than 40 degrees Celsius $\left({ }^{\circ} \mathrm{C}\right)$ at lower elevations and $30^{\circ} \mathrm{C}$ at higher elevations (Western Regional Climate Center, 2012). Winter temperatures can drop below freezing and snow is common at high elevations. Annual rainfall is approximately 400 millimeters, half of which occurs between July and September (Hass, 2002).

The study area includes ecologically diverse communities, from Chihuahuan desertscrub at the driest and lowest elevation areas, to Plains and Great Basin grassland, semidesert grassland, Madrean evergreen woodland, and Petran montane conifer forest (Brown, 1994). 


\section{Fieldwork for Wildlife Photos/Videos and Field Safety Protocol}

\section{Permits}

This broad study area encompasses an extraordinarily complex mosaic of land ownership across Arizona and New Mexico. We coordinated project details (permits, land access, safety, project updates, and so on) with a variety of Federal and State agencies and entities that manage wildlife and land resources in the study area (table 1). Most of these agencies had their own reporting requirements in terms of reports due and scheduling, camera location mapping, and field activity coordination, in addition to reporting requirements for the USFWS. Our fieldwork schedule depended on permits, especially from the U.S. Forest Service and the Arizona State Land Department, and we adapted our implementation schedule to address permit delays or other influences on the project timeline.

\section{Coordination and Safety}

We implemented a staged deployment approach (by mountain range) to ensure that adequate communication and coordination took place prior to camera placement and for subsequent camera checks. The deployment schedule depended on receipt of key permits, especially from the U.S. Forest Service and the Arizona State Land Department.

Field teams drove to the general area of camera locations using four-wheel drive vehicles and then accessed remote camera sites by hiking, all-terrain vehicle (ATV), or horseback. Prior to fieldwork by any team member, a team leader was notified of route of travel, day, time of entry, and expected return time. Teams of two or more individuals (when possible) worked together to place the cameras. SPOT messaging units (2014 Spot LLC) were used by field team members as a safety protocol. SPOT messaging units indicated to designated recipients the exact global positioning system (GPS) location and departure time of team members, the team's camera-check calendar indicated the GPS locations of each camera site visited that day, and the team leader was on alert until the field team sent a "returned safely" message. A phone tree of team members and buddy lists was maintained and distributed in the event that a team member sent a help or SOS message.

UA also provided updates to U.S. Customs and Border Protection personnel every 2-3 weeks about fieldwork locations, camping locations, vehicles, primary mode of transport (park and hike, ATV, horse/mule), and fieldwork dates.

\section{Human Dimensions}

As an endangered species, jaguars have been the subject of considerable public and Federal and State agency interest. This project has been followed closely by a number of interest groups with regard to methods used and study results. Throughout the course of this project, we addressed communities and stakeholders that encompass a full range of interests and concerns in the future of jaguars in the United States. More significantly, however, are the long-term outcomes this project seeks to achieve that depend on fostering trust between scientists, public land managers, interest groups, regulatory agencies, grazing permittees, and private landowners. 


\section{Identify Land Ownership and Grazing Allotments}

To communicate effectively with ranchers, landowners, and grazing permittees, we needed to know whom to contact. We found no one source for all information on land ownership and grazing allotment holders; therefore, we assembled these data ourselves. This data collection and analysis process required additional time and resources. We engaged GIS specialists at the Advanced Resource Technology Lab at the UA to acquire and assemble all requisite GIS and data layers from state land departments, federal land management agencies, and other sources.

\section{Coordination with Ranchers, Landowners, and Grazing Permittees}

Jaguars in the United States depend on landscapes that are primarily managed as public and private ranchlands; therefore, project members made a commitment to be proactive, communicative, and collaborative with the ranching community. We developed coordination protocols (appendix 1) to ensure that project activities were communicated and carried out in a courteous and respectful manner.

Coordination and communication with landowners and grazing permittees were crucial to project success. The Performance Work Statement from USFWS required that we obtain written permission before crossing (access routes) or conducting fieldwork on private lands. In addition, we felt it important to notify ranchers and grazing permittees on public or state lands about this project. To this effect, and in conjunction with several members of the Altar Valley Conservation Alliance, we developed a short summary of the project combined with a landowner/grazing permittee communication protocol (appendix 1). This summary was provided to those landowners and grazing permittees where we requested access. In addition, and because jaguar critical habitat was proposed by the USFWS at about the same time as this project was initiated, USFWS developed an accompanying letter explaining the implications of jaguar critical habitat designation with respect to the ranching community. The message that critical habitat designation was not expected to affect normal ranching activities or public land range management, and that the jaguar had been listed as an endangered species since 1997 and had been addressed in routine grazing-lease permitting processes on federal lands since that time, helped us develop a common understanding with the ranching community during this project.

\section{Trail Cameras}

\section{Camera Selection}

When setting large numbers of cameras over a wide geographic area, one of the first considerations is the size and weight of the camera and its mounting box. Most sites were visited on foot in rugged terrain and cameras were carried in a backpack. Firstly, we limited our camera selections to the size range of 5 by 10 by 15 centimeters. Secondly, we selected camera brands that met or exceeded high industry standards, but that were also reasonably priced (less than \$250), given the risk of damage to cameras being placed in areas associated with illegal human activity, recreational use, and severe weather.

As the objective of the project was to document jaguars, it was necessary to recognize individual jaguars by their unique spot patterns; therefore, we chose to use cameras with a white flash for nighttime photos. In our experience, cameras with infrared flash do not produce suitable clarity for spot-pattern identification. 
We conducted field tests on seven camera models and the Covert Deuce camera was selected based on these tests. We purchased 220 Covert Deuce cameras in our original camera purchase. In August 2013, additional cameras were ordered to complete camera deployment and to replace cameras that were vandalized or stolen. At this time, Covert Deuce cameras were no longer manufactured; after testing several models, the ScoutGuard SG565 was selected as the replacement camera. Eighty ScoutGuard SG565 cameras were purchased in August 2013. All cameras were secured and placed in lockable tamper-proof housing to minimize damage (vandalism and other) or theft in the field.

We maintained a spreadsheet of all cameras purchased for the project to track the status and condition of the cameras as they were set in the field, maintained, damaged, or no longer functional. Cameras and associated equipment will be returned to USFWS at the end of the project with the exception of those cameras that will remain deployed and actively used for future citizen science jaguar monitoring.

\section{Camera Locations}

Wildlife cameras were placed throughout the study area (figs. 1a-1c). Because photographic sampling is commonly used to survey for rare carnivores, we used this as our primary method to locate and identify jaguars throughout the designated study area (Karanth and others, 2004; Kays and McKelvey, 2008). We began deploying trail cameras (also known as motion-detection cameras, remote cameras, or game cameras) on April 25, 2012, and completed deployment on May 31, 2013. Cameras were eventually placed at 250 sites across the study area, but because we implemented an adaptive strategy for camera placement, cameras were relocated as needed throughout the study. Cameras were relocated if felids (jaguar and ocelot, or the much more common mountain lion and bobcat) were not detected. Cameras were paired (two opposing cameras at each location) whenever possible to capture photographs of both sides of each animal for identification (Karanth and others, 2004). Because of vandalism and theft, however, some cameras were intentionally not paired to reduce potential losses. Because of the large size of the study area, cameras were strategically placed to maximize the probability of jaguar detection (Long and others, 2008). We began removing cameras from the field on January 22, 2015, with full removal achieved on March 2, 2015.

We used the following three-tiered process to determine survey sites:

Tier 1-The first tier of analysis to determine camera sites was to categorize mountain ranges in the study area into three Priority Zones: High, Medium, and Low. These Zones were based on distance from the source population of jaguars south of the international border in Mexico, documented jaguar locations from previous surveys (analysis of 35,000 trail camera photos by Borderland Jaguar Detection Project, 2001 to 2009 [Jack Childs, written commun., August 2011), and other recent (post-1995) sightings (U.S. Fish and Wildlife Service, written commun., April 2013). Areas nearest to the international border with jaguar detections and natural land features that could serve as corridors were listed as High Priority Zones. Areas farthest north of the border with no past jaguar detections, or isolated mountain ranges, were designated as Low Priority Zones. Areas with few or no jaguar detections, with no repeat detections (indicating a jaguar merely passed through and did not linger), or that were isolated from other areas but nearer to the border, were assigned as Medium Priority Zones. These Zone designations determined the density of cameras locations and the frequency of monitoring to download photo/video data. If a jaguar (or ocelot) was photographed, that camera site became a High Priority Zone and was left in place for fixed monitoring. Figures 1a-1c show camera sites within the study area. 
Tier 2-In the second tier of analysis to determine camera sites within Zones, we used National Geographic topographic maps (TOPO! 2006 National Geographic) in conjunction with team knowledge and other published research on jaguar habitat use and movements (for example, Brown and López González, 2001; McCain and Childs, 2008). Because jaguars are rare within our study area and because variability of topography and habitat is extreme, we maximized our chances of locating a jaguar by placing cameras in areas with the highest detection probability (Long and others, 2008) and by using inferred knowledge of jaguars, pumas, and other wildlife in the region. These sites were located where (1) jaguars were recorded since 1996, and (2) they may serve as travel ways for large felids based on topographic characteristics (for example, canyons, ridgelines, saddles). We also considered the probability of potential jaguar movements from Mexico, on the basis of our knowledge of known extant populations, habitat suitability, and connectivity to Mexico. The habitat suitability model created by the JRT was also used to define suitable jaguar habitat for camera placement (Sanderson and Fisher, 2013).

Tier 3-In the third tier of analysis, the field team used its expertise and knowledge to determine cameras sites in the field by ground-truthing local field conditions. Assessment of natural microsite characteristics with respect to wildlife sign, unmapped water sources, natural trails and travel ways, and degree of human activity not quantified in available GIS layers or in the above tiers, were all used to fine-tune camera locations.

Once sites were selected, cameras were mounted on natural structures such as trees and stumps. Cameras were housed in tamper-proof boxes designed by the camera manufacturer, and were locked and secured to the supportive structure with a cable lock. No ground disturbance took place during installation. Because the cameras detect motion and heat, branches or vegetation were trimmed to avoid false triggering of cameras. All camera locations began with setting paired cameras (when possible) facing each other so that the animal would walk between them. Additionally, paired cameras (1) allow both sides of a jaguar to be photographed to identify individual rosette patterns; (2) maximize the higher detection rate related to paired cameras than a single camera (primarily because animals can walk behind one camera and still be photographed by the opposing camera); and (3) have the advantage of a second camera serving as a backup if one is nonfunctional. However, the high incidence of camera theft and vandalism necessitated the use of only single cameras at some sites to reduce the risk of losing two cameras at each theft or vandalism event. Cameras and site characteristics were photo-documented in place for the record.

\section{Adaptive Strategy for Camera Placement}

The broad geographical scale of this project made it important for our team to (1) survey as much area as possible, and (2) choose the best possible sites to detect jaguars. Therefore, we implemented an adaptive strategy for camera placement, similar to that used in Smith and others (2003). During Year 1, all areas were surveyed (that is, cameras were meant to be temporary and were moved within the pre-assigned Zones to assess such factors as prey abundance, mammalian diversity, puma abundance, and human presence) to determine probability of jaguar occurrence in an area, although in several instances, the initial site was the ideal and best site in the area, in which case the cameras were left at that site. Once the best camera locations were determined (based on the factors above or if a jaguar was detected in the area), the camera locations became fixed and became monitoring sites for the duration of the project (Years 2 and 3). An exception was locations where jaguars had previously been recorded - these were fixed monitoring sites from the outset. Some sites, including positive jaguar detection sites, experienced heavy and often repeated vandalism of camera equipment. These sites were moved, left offline, and later resurrected as single-camera sites (to prevent additional losses), or abandoned for safer locations. 


\section{Camera Check Schedule}

The study area was divided into High, Medium, and Low Priority Zones and the visitation schedule was developed on the basis of Zone classification. Because of the size and ruggedness of the study area, it was often necessary to maximize the time interval between camera checks. High Priority Zones were visited most frequently, every 4 weeks. Medium Priority Zones were visited every 7 weeks. Low Priority Zones were visited every 11 weeks. By maximizing intervals between camera checks, we were able to place more cameras into more areas and increase our chances of detecting a jaguar or ocelot.

While checking the cameras, photographs/videos were viewed in the field using the onboard viewing capabilities of the Covert Deuce or ScoutGuard 560/565 cameras. Viewing images in the field allowed us to evaluate proper camera function and to adjust the aiming point or location if needed. Batteries were checked and replaced as needed and memory cards were removed and replaced with a blank card. Memory cards were labeled with site name, which camera of the pair, date, time, and number of images, and were taken from the field to be downloaded to a computer and processed.

\section{Photo Data}

\section{Processing Photo Data}

Photograph files were processed using software developed by J. Sanderson, which included DataOrganize, DataAnalyze, MyRenamer, UpdateInput, CreateInput, and CorrectInput (Harris and others, 2010). We also used the program ReNamer (Kozlov, 2009), which automatically relabeled each file with the unique date and time the photo or video was taken. Species observed in images were identified by trained team members and images were sorted by team members into hierarchical folders labeled as follows: location / species / \#-of-individuals. A more detailed description of the process is available online at http://www.smallcats.org/CTA-executables.html.

Jaguar and ocelot photos or videos were identified to the individual, if possible, on the basis of spot pattern. Spot patterns of jaguars and ocelots are unique and laterally asymmetric, meaning the pattern is different on each side of an individual. Therefore, if both sides of an individual can be photographed with opposing cameras, subsequent photographs of either side can be used to uniquely identify the individual (Silver and others, 2004).

For the purposes of this project, non-focal species, which the study was not designed to detect, were grouped together including cottontails (Sylvilagus audubonii and S. floridanus), jackrabbits (Lepus californicus and L. alleni), squirrels (Sciurus variegatus and S. arizonensis), and skunks (Mephitis mephitis, M. macroura, Conepatus leuconotus, and Spilogale gracilis). We also grouped lizards (Lacertids), snakes (Serpentes), all birds (excluding turkeys, Meleagris gallopavo), all non-Sciurid rodents (Rodentia), and bats (Chiropterans). Depending on the analyses, some non-mammalian groups were omitted from analysis because of relative rarity and lack of species-level identification. Because of grouping and selected omissions, our total species count (as described in section, “Discussion”) is lower than the actual number of species observed. 
Once photos (and videos) were renamed and filed, they were analyzed and the information was routinely synthesized and compared among camera sites to better understand presence and absence of large carnivores and important prey species. We used a 1-hour interval between photos to determine photo independence (photos were considered pseudo-replicates if taken within 1 hour of each other; therefore, if one photo was taken of a species, all other photos of that species were ignored for analyses for the subsequent 60 minutes), as this same criteria has been used in recent studies pertaining to jaguars and ocelots (Silver and others, 2004; Di Bitetti and others, 2006; Thapa and others, 2013). The 1-hour interval is appropriate for large carnivore analyses, although we acknowledge that there is no single time interval that suits the range of species we detected in this study. All data were stored and backed up at a central location.

\section{Photo/Video Analyses}

Analyses during the survey period (Year 1) were primarily useful for determining the best locations for detecting jaguars and their prey. This enabled us to select the best, fixed monitoring sites for the duration of the study (Years 2 and 3). Data collected during a longer period from fixed sites provide a better evaluation of species habitat preference and movement patterns.

In addition to obtaining jaguar and ocelot detection data, we analyzed indices that could be monitored through time to examine the wildlife species community as a whole. Analyzed metrics are explained in the following sections.

\section{Species Detection and Species Richness}

Species detection and richness measures the total number of species in an area. This was used for ecological examinations, such as studying relations between the number of species at individual sites and within mountain ranges (Harris and others, 2010; DiBitetti and others, 2014) Detection rates were measured in detections per 100 days divided by the total number of days multiplied by 100 . Detection rates were also analyzed geographically and temporally by location, month, and year.

\section{Species Accumulation Rate}

Species accumulation rate is the rate at which new species are found and was calculated as the accumulated number of species over time (days). This was measured by the day a new species was recorded, the total number of new species recorded, and the name of the species that was recorded (Harris and others, 2010). The accumulation curve lists the order that each species was recorded and the number of calendar days into the study.

\section{Species Total and Relative Abundance}

The use of camera detection rates as an index of species abundance has been widely debated because the index needs to be calibrated with independent estimators of density. When pooling different species in these analyses, studies ideally need to control for species-specific variations in detection rates associated with factors such as body size, behavior (including territoriality, foraging behavior, gregariousness, and trail use) and daily range-all factors that affect frequency of revisits to cameras. 
Trail cameras may not provide a complete record of wildlife in an area because some species will never be detected on trail cameras, particularly those set to preferentially detect felids. However, total and relative abundance of a species among mountain ranges and camera locations can still be examined. Species total abundance is the total number of individuals of a species/species category in all independent photos (Harris and others, 2010). Species relative abundance is the total number of independent photos of the species/species category divided by the total number of independent photos of all species/species categories multiplied by 100 (Harris and others, 2010).

\section{Activity Patterns}

Activity patterns were measured as the number of times that a species was photographed during each 1-hour period throughout a 24-hour day throughout the study period.

For lunar activity pattern, a difference value was calculated to describe the activity patterns of species during full and new moons. This value was calculated from the square root of the sum of the square of the difference in detection frequencies across the two moon phases. This number was then squared and summed over 24 hours (Harris and others, 2010).

\section{Felid Co-occurrence}

Co-occurrence is estimated using the number of locations each species pair co-occurred (Harris and others, 2010).

Influence of Elevation on Jaguar and Prey Detection

Elevation was divided into 200-m zones using actual detections, and the percentage of each animal's detections was plotted within each elevation zone. Camera effort (the number of camera trap days expended in each zone) was compared with the number of detections (Harris and others, 2010).

\section{Jaguar and Ocelot Population Estimation}

To estimate the jaguar and ocelot population within our study area, we created a probability model using the detection history of each camera location in our study. This method required identifying unique jaguar and ocelot individuals within the study area, as described previously, with the understanding that additional jaguars and ocelots avoided detection, thus, the observed number of individuals was lower than the actual number present. With only 1 jaguar and 3 ocelots detected in this study, we were not able to employ a probability model for jaguar or ocelot population size.

\section{Jaguar and Ocelot Home Ranges}

To estimate home range sizes for the jaguar and ocelots detected in this study, we applied the Minimum Convex Polygon (MCP) (Trolle and Kery, 2005; Dillon and Kelly, 2008) method using detections, to which we added a surrounding band, the width of which was the 24-hour Mean Maximum Distance Moved (24-hour MMDM) (Wilson and Anderson, 1985; Trolle and Kery, 2005). The MCP is calculated by creating a polygon around the outer perimeter of all detection sites, which is by definition the minimum observed range of an individual. The actual range may be much larger. To account for this, the 24-hour MMDM method adds a buffer to this polygon that is determined by the average known distances traveled in a 24-hour period by animals within the study area (Soisalo and Cavalcanti, 2006). The photos used to determine average distances for each animal in our study were those that occurred within a 24-hour time frame for each jaguar and ocelot. Scats were not used for the 24-hour MMDM because scats do not have a time stamp. 


\section{Scat Searches and Scat Genetic Analyses}

\section{Scat Searches}

The field team searched for scat and other sign (that is, tracks, scrapes, or prey kills) during the process of setting and checking cameras. In general, cameras were set in the same types of places that felid scat and other sign are often located (for example, in canyon bottoms, natural funnel zones, along ridge lines; Childs 1998). When appropriate, we searched other areas close to cameras as time and logistics permitted, or walked out a different canyon or travel route than covered on the way in. Based on our experiences, stationary transects that would be repeatedly surveyed for tracks and scat were not useful in this study because of the short time period and large study area.

To increase our probability of scat detections, the Culver Conservation Genetics Laboratory obtained a scent detection dog from the Border Patrol Canine Program, which we were able to use to recognize jaguar and ocelot scat. One team member served as the dog handler for this project and began detector training sessions in January 2013. The dog was trained to locate jaguar and ocelot scat in the field and to vocalize upon detection of target scents for delivery of a reward; we then brought the scat to the laboratory for genetic analyses. The advantage of the detector dog is that the dog is not limited to areas where cameras are located. Detector dogs have the ability to significantly increase the number and variety of detection locations (Morrel, 2011).

In the field, the dog worked unleashed (under close supervision of the handler) and covered several times the distance of the handler. Because of rugged terrain and topography, grids were not used for scat surveys. Instead, dog and handler walked travel paths presumably used by a focal cat. Scat surveys included periodic positive reinforcement using known jaguar or ocelot scat, and, to keep the dog on point during long hikes, target scat or scented toys were placed in the field ( $0-3$ times per survey), and the dog was rewarded for finding them. After this procedure, the planted scat samples, toy, and(or) substrate upon which the scent was set (leaves or gravel) was removed so that no scent remained in the environment. Target scats were placed intermittently and unpredictably so that the dog became accustomed to prolonged walks that generated very few target hits (and sometimes none at all), thus mirroring the nature of the work.

Survey effort was more heavily weighted on localities around cameras with multiple photographs or videos, but also focused on suspected travel corridors between detection sites, especially when consecutive images occurred in a short period. Searches typically began at detection (camera) sites and were based on time and direction of jaguar travel. All surveys incorporated best-guess routes of jaguar travel.

An average of 1-3 scat searches per week were conducted throughout that timeframe and were often, but not always, coupled with camera checks. Scat searches by the dog handler typically covered 5-12 km/day, depending on terrain, with 4-6 hours on the ground. All large felid scat encountered during detector dog surveys were collected in the Santa Rita Mountains and medium-size felid scat was collected in the Huachuca Mountains until the dog was able to correctly identify jaguar and ocelot scat as verified by genetic analyses. Scat samples singled out by the dog were confirmed in the genetics lab. After the dog was shown to identify scat correctly, scat collection was more selective. In addition, opportunistic felid scat samples were collected by other field team members during routine camera checks throughout the study from all mountain ranges. Some of these opportunistically collected scat samples were tested for species identification, with the remainder available for genetic testing for species identification, if needed. In non-detection mountain ranges, opportunistic scat collection continued throughout the study. 


\section{Scat Processing}

All collected scat was stored at $-20^{\circ} \mathrm{C}$ in the Culver Conservation Genetics Lab at the UA. Scat samples were thawed and the surface was swabbed for sloughed epithelial cells so that DNA could be extracted using a stool DNA extraction kit (Rutledge and others, 2009; Wasser and others, 2011). All samples were assessed in two steps. First, samples were analyzed for species identification using polymerase chain reaction (PCR) amplification and DNA sequencing of the mitochondrial DNA cytochrome $b$ gene. The resulting sequence was compared with sequences in the GenBank database for a matching sequence at 98 percent or greater, indicating both sequences originated from the same species. Second, if the scat sample was from a jaguar, individual identification was assessed using PCR amplification of 28 felid microsatellite DNA loci shown to be polymorphic in jaguars (Eizirik and others, 2001). Following PCR amplification, the amplicons were subjected to fragment analysis to determine the exact size of each fragment, with each individual containing 2 fragments per locus. The composite genotype across all microsatellite loci were analyzed to indicate if each scat sample represented the same individual from previous jaguar scat samples, or if they were from new individuals. All jaguar samples identified from scat provided new detection events, and generally new locations.

We attempted to perform individual identification using felid microsatellite markers for all scat samples obtained and verified to be jaguar or ocelot. However, while genetic analyses are reliable, some scat samples did not have sufficient high-quality DNA to be successfully analyzed, particularly to the level of individual identification. Scats collected during searches (as many as 10 scats per detection event, in addition to opportunistically collected scats of as many as 10 per year) were analyzed in the Culver Conservation Genetics Lab.

\section{Scat Genetic Analyses}

Genetic sequence data for species identification were edited using SEQUENCHER (version 5.0, Gene Codes Corp, Ann Arbor, Michigan) and compared with an existing database (GeneBank, National Library of Medicine) of mammal sequences to determine the species of origin for each sample. This analysis was used to identify jaguar versus other carnivore scat.

Microsatellite data for individual identification were analyzed using GENOTYPER (version 1.1; Applied Biosystems Inc., Foster City, CA). Once a composite genotype was compiled for each sample of as many as ten felid microsatellite DNA loci known to be polymorphic in jaguars, pairwise genetic distances were calculated among scat samples using MICROSAT (Version 1.5d). All pairs of samples with a distance of zero (that is, complete sharing of microsatellite allelic data) were presumed to have originated from the same individual, allowing an estimate of the number of unique individuals serving as a minimum number of jaguars for this study area.

\section{Jaguar (and Ocelot) Detections}

\section{Reporting}

Specific details of each jaguar (or ocelot) detection were immediately communicated to USFWS, as well as the respective state wildlife agency, the landowner/land management agency, and Border Patrol. GPS coordinates or place-name location of jaguars and ocelots were kept confidential to outside entities. Only the date and mountain range were released to outside sources, via USFWS. This helped ensure the safety of the animals, landowners, and UA personnel. We maintained privacy of private landowners/grazing permittees who did not wish to be named. 


\section{Additional Cameras}

If a jaguar or ocelot was photographed, the camera site became a High Priority fixed site and additional cameras were placed strategically in the area. For each detection event, we purchased 20 additional cameras and set these cameras at 10 new locations in the general detection area for expanded monitoring. The goal was to obtain additional detection events of the individual and(or) other individuals in the area, as well as gather insight into home range and movement patterns (photos of the same individual at different locations), habitat use, and other variables. Topography and habitat characteristics determined the location of these additional camera sites. These cameras were given High Priority status and checked monthly (for the remainder of the project) as long as the jaguars and ocelots continued to be detected in the area. The camera locations were continually fine-tuned throughout the project.

\section{Scat Collection}

Prior to the first detection event, all field team members collected scat opportunistically. Following detection events, we increased our probability of finding jaguar scat by using the scent detection dog in detection areas.

\section{Macrohabitat and GIS Analyses}

At each camera location and sites with positive jaguar detections (confirmed photographs, videos, tracks, or scat), we collected macrohabitat or landscape-level data including geographic location, and physiographic and habitat characteristics. The project study area was separated into two distinct and noncontiguous areas, west (figs. 1a,b) and east (fig. 1c). Each study area differed for most environmental variables. For example, human influence differed considerably between study areas, with greater values (influence) in the west than in the east. Thus, camera sites in the west $(n=209)$ and east ( $\mathrm{n}=41 ; 25$ in Arizona and 16 in New Mexico) study areas were analyzed separately. Environmental variables used to characterize camera sites without, and sites with, positive jaguar and ocelot detections included elevation, terrain ruggedness, human influence, normalized difference vegetation index (NDVI), tree cover, vegetation community, distance to perennial water, and distance to major roads (table 2).

Elevation analysis was similar at 30-m (National Elevation Dataset) and 1-km (Global Land One-km Base Elevation dataset) scales; therefore, we used the 1-km analysis only because this was the resolution we used when modeling jaguar habitat. Terrain ruggedness was examined using a Terrain Ruggedness Index (TRI), which was calculated from digital elevation model maps (1-km resolution) for the study areas using ArcGIS 10 software by Esri. Map categories for TRI were assigned as described by Riley and others (1999). Human influence was assessed using the Global Human Influence Index Dataset of the Last of the Wild Project (Wildlife Conservation Society and Center for International Earth Science Information Network, Columbia University 2005). The 1-km scale raster map of human influence was based on human population density, human land use and infrastructure (built-up areas, nighttime lights, land use/land cover), and human access (coastlines, roads, railroads, navigable rivers). NDVI is a measure of "greenness" and higher values represent live green vegetation. NDVI was assessed by using two types of imagery collected during two periods. NDVI values for March 2015 were obtained from eMODIS imagery and for August 2014 from AVHRR imagery. NDVI-eMODIS images were acquired at 250-m spatial resolution and were rescaled to $1 \mathrm{~km}$ to match other environmental layers. NDVI-AVHRR images were acquired at 1-km spatial resolution. Tree cover was assigned based on the Terra MODIS Vegetation Continuous Fields image at a scale of $250 \mathrm{~m}$ and was rescaled to a 1-km resolution to match all other variables. Biotic community (or vegetation community) 
was assessed using a digital representation of Brown and Lowe's "Biotic Communities of the Southwest” map as described in Brown (1994) developed by Nature Conservancy (2006). Distance to water for camera sites was analyzed and compared with random points. In keeping with previous analyses performed by USFWS for jaguar proposed critical habitat in 2012 (metadata and GIS coverages provided by USFWS), water was limited to those of the following feature types (FType): Estuary, Ice Mass, Lake Pond, Playa, Reservoir, and Swamp Marsh. Distance to roads for camera sites was analyzed and compared with random points. Roads were limited to U.S. highways, state highways, and major roads. See table 2 for a summary of data layers, authors, and sources.

Environmental characteristics were compared between camera sites, current positive jaguar and ocelot detection sites, historical jaguar detection sites, and random points for the west and east study areas. Current jaguar and ocelot locations were obtained from camera photos or videos or positively identified scat locations from 2012 to 2015. Historical jaguar records included 130 undisputed class I records (U.S. Fish and Wildlife Service, 2012) recorded in Arizona and New Mexico from 1965 to 2009 (data provided by U.S. Fish and Wildlife Service, written commun., April 2013). We acknowledge that we run the risk of biasing our analyses based on one individual that was observed many times at several locations. Even with the historical locations, the majority of our jaguar locations were of one individual many times at several locations. Analyses were conducted using ArcGIS 10.1 software by Esri, where each location was overlaid with digital coverages to determine the environmental characteristics at each site (Appendix 2). Positive detection sites included current jaguar and ocelot photographs and videos from cameras and scat locations. They also included random points that were generated for each study area (1,000 in each) and used to compare with camera sites. These random points served as the available habitat for each study site and were the expected values if cameras were placed at random throughout the study areas. Chi-square and Mann-Whitney U tests were performed to determine if camera site characteristics differed significantly from those that were available in each study area (Zar, 2010).

\section{Microhabitat Analyses}

Microhabitat analysis included on-the-ground measurements of vegetation (as opposed to macrohabitat analysis, which used GIS coverages (digital maps) from vector data and satellite images as described in table 2). At sites with positive jaguar detections (confirmed photographs, videos, tracks, or scat), we collected detailed microhabitat data including species composition, canopy cover, and screening cover of vegetation. We characterized geomorphology, vegetation composition, and vegetation structure of each sampling site by establishing $20 \times 20 \mathrm{~m}$ square plots centered over the focal target of the two cameras and parallel to the stream course or trail (fig. 2). From the focal point, we measured distance to the nearest perennial water, nearest town, and nearest unmaintained, maintained, and paved roads. To characterize local geomorphology (the general shape of the landform near sampling sites resulting from presence of washes, cliffs, and canyons), we classified each sampling site as a wash, canyon, trail, saddle, or ridge. We then measured the bank-to-bank width of washes or canyons at heights of $1 \mathrm{~m}$ and $1.75 \mathrm{~m}$ above the ground at the focal target to characterize the shape of the stream course or trail. To characterize vegetation composition and structure, we established three 20-m transects parallel to the stream course, one in the center of the plot and two $7.5 \mathrm{~m}$ on either side of plot center (fig. 2). Along each transect, we used the line-intercept method at three heights from the ground ( $<1 \mathrm{~m}, 1-2 \mathrm{~m},>2 \mathrm{~m}$ ) to estimate percent cover of (1) bare soil, rock, litter/debris, grass, and forbs (understory), (2) subshrubs and shrubs (midstory), and (3) tree canopies (overstory) (Elzinga and others, 2009). We also recorded the species of each tree and shrub and the highest point in the canopy along each transect. If the transect could not be surveyed because of inaccessible topography, we recorded the transect length occluded by boulders or cliffs. Finally, we recorded whether seasonal or perennial water was present in the plot. 
At sites with positive jaguar detections (confirmed photographs or videos, tracks, or scat), descriptive statistics were calculated for species composition, canopy cover, and screening cover of vegetation. Species diversity measures were calculated by evaluating species diversity (using the Shannon-Wiener diversity index) and evenness (using the Camargo E-prime measurement of evenness) for each site (Krebs, 1999).

\section{Environmental Analyses}

\section{iButton Data and Analyses}

Relative humidity and temperature can alter the movement and behavior of wildlife (Hofmeister and others, 2010). Such variables can be collected hourly using an iButton (Maxim Integrated Products, Inc., San Jose, CA), a thumbnail-sized mini-weather station, installed near a camera location. Sixty iButtons were deployed throughout the study area to collect hourly temperature and humidity readings for 1 year (March 2014-February 2015). We deployed iButtons such that jaguar detection locations could be compared with non-detection locations, as well as other sites of interest (such as where jaguar prey items occur). The iButtons were placed inside plastic funnels and hung from tree limbs in hidden locations to prevent them from being taken or tampered with by humans during the course of this study.

iButton weather data were downloaded from the field onto a downloader device, and subsequently downloaded from the device into spreadsheets in preparation for analyses. All weather data were matched to photo/video data from the associated camera site.

To compute an average daily relative humidity and average daily temperature for each iButton location, hourly relative humidity and temperature data were averaged over 24 hours beginning at midnight.

Additionally, we linked weather variables (average relative humidity and average temperature) to photographic data using correlation analyses. We ranked the correlation of climate data features to features in photographs or camera sites (such as jaguar detections versus non-jaguar detections) using Correlation Feature Selection (Hall 1999).

\section{Potential Jaguar Distribution Model}

We used MaxEnt 3.3 software to generate a potential jaguar distribution map for southeastern Arizona and southwestern New Mexico. MaxEnt (http://www.cs.princeton.edu/ schapire/maxent/) is a program that allows users to generate ecological niche models using presence-only data (such as the data available from remote cameras) and to identify which environmental variables, or combinations of them, better predict jaguar occurrence (Phillips and others, 2006). A presence-only approach is needed for species such as jaguars with a reduced distribution where an absence can be the result of unsuitable habitat, low population numbers, or inadequate sampling. Rodríguez-Soto and others (2011) used similar methods to predict potential jaguar distribution in Mexico. Our model included jaguar locations from the current study and historical records in conjunction with environmental variables. Current jaguar locations were obtained from camera photos and videos or positively identified scat locations from 2012 to 2015. Historical jaguar records included 130 undisputed class I records (U.S. Fish and Wildlife Service, 2012) recorded in Arizona and New Mexico from 1965 to 2009 (data provided by U.S. Fish and Wildlilfe Service, written commun., April 2013). 
Environmental variables were evaluated for their usefulness in predicting jaguar locations and included variables used in the macrohabitat analysis (elevation, terrain ruggedness, various vegetation indices, distance to water and roads, and human influence; table 2), and climate data (temperature and precipitation). WorldClim bioclimatic data (Hijmans and others, 2005; http://www.worldclim.org) were used to represent annual mean temperature (bio1), minimum temperature during the coldest month (bio6), annual precipitation (bio12), and precipitation during the coldest month (bio19). These data resemble elevation maps in that each pixel (1- $\mathrm{km}^{2}$ cell) has a unique value representing one of these temperature or precipitation variables. Bioclimatic and environmental variables were selected on the basis of their contribution to the overall model.

All variable layers were processed using ArcGIS 10.1 to create raster maps that were projected similarly (UTM 12N Nad83) and scaled to approximately $1 \mathrm{~km}^{2}$. All layers were clipped using a mask to include all of Arizona and New Mexico and snapped to match the elevation grid so they had the exact extent and pixel size. All environmental and bioclimatic layers were analyzed as continuous data with the exception of the vegetation community layer, which was categorical. All data (jaguar locations and layers) were manipulated in ArcGIS 10.1, then exported as ASCII files for use in the program MaxEnt.

Current and historical jaguar location data were combined and used as training and testing data. Separating data into training and testing subsets allowed for model testing with data not used in its creation - training data are used to create the model and testing data are used to evaluate the model. Only nonduplicate locations were used to create the model. For example, if multiple jaguar locations occurred within one $1-\mathrm{km}^{2}$ pixel, then that pixel was only used once in analysis. A bias file was created and used to limit the extraction of background characteristics to areas that were surveyed by cameras (Phillips and others, 2009). A bias file is recommended to account for uneven sampling across the landscape. Cameras were placed in areas that were most likely to detect jaguars, which excluded some areas of the study site, such as those at lower elevations. All camera sites were buffered with a local adaptive convex-hull polygon with a buffer distance equal to $10 \mathrm{~km}$ and alpha equal to 2 (fig. 123). Models were evaluated on the basis of mean area under the receiver operating characteristic curve values on test data (AUC test) and Akaike information criterion $\left(\mathrm{AIC}_{\mathrm{c}}\right)$ scores (Warren and Seifert, 2011). Cross validation $(\mathrm{k}=5)$ was used to resample data for training and testing of models with model performance reported as $\mathrm{AUC}_{\text {test }}$. Initially, all variables were evaluated in the model and a jackknife procedure was used to determine (1) the regularized training gain with each variable independently, and (2) the decrease in the regularized training gain when each variable was excluded. Variables that contributed minimally to the training gain, either by not adding much unique information on their own or by not decreasing the training gain when removed, were not included in subsequent models. Subsequent models were evaluated similarly with low-contributing variables being eliminated while still preserving high model AUC test values.

Multiple models were created for each combination of environmental and bioclimatic variables by varying the regularization parameter (beta=1,3,5,7,9). Varying the beta coefficient was used to reduce the number of parameters in the model and to avoid fitting too complex of a model to the jaguar location data (Elith and others, 2011; Warren and Seifert, 2011; Merow and others, 2013). All models were then evaluated using $\mathrm{AIC}_{\mathrm{c}}$ scores calculated using ENMTools 1.4.3 (Warren and others, 2010). 


\section{Data Coordination}

\section{Endangered Species Data}

The following is stated in the Performance Work Statement:

"Information gathered with funding from this project is confidential and not for public release without the approval of USFWS. The contractor will maintain the confidentiality of all data collected under this project, including, but not limited to, any information relating to threatened and endangered species survey and detection locations. Data will be shared with the USFWS, the Arizona Game and Fish Department, and the New Mexico Department of Game and Fish only for the purposes of implementing the Endangered Species Act. Access to location information will be restricted unless required by the Freedom of Information Act, Public Records Law, or court order. Private property owners will have access to the information for all listed species locations on their own properties."

Accordingly, we followed the above protocol regarding any detection of listed species.

Detections of listed species were reported within 24 hours to USFWS, and through them, the respective state wildlife agency (Arizona or New Mexico), Department of Interior (DOI), Border Patrol, the land management agency (if applicable), and the private landowner or grazing lease permittee.

\section{Non-listed Species Data}

We amassed a large dataset of images of a variety of wildlife across the study area, and the majority of which were of non-listed wildlife species. These data, and our analysis of such, provide a remarkable dataset for understanding the mammalian biota of this region.

Coordination with USFWS, Jaguar Recovery Team, and Others

We coordinated with the USFWS and the JRT regarding photo/video data sharing, habitat measurements, and other analyses such as GIS modeling. Our dataset yields one of the most comprehensive region-wide analyses of the wildlife community in southern Arizona and southwestern New Mexico to date and is valuable for future collaboration and data sharing.

\section{Media}

The UA media team coordinated with the USFWS Public Information Officer, Border Mitigation Coordinator, U.S. Customs and Border Protection, DOI, U.S. Geological Survey, media, Arizona Game and Fish Department (AGFD), New Mexico Department of Game and Fish, and other USFWS staff in directing and coordinating media releases, when appropriate. When jaguars and ocelots were detected, UA and USFWS staff worked together to provide the public with appropriate and timely information, while taking into consideration the information sensitivity with respect to the animals' safety and the privacy of landowners/grazing permittees. 


\section{Results}

\section{Fieldwork for Wildlife Photos/Videos and Field Safety}

Permits were obtained from 13 Federal and State agencies prior to conducting the fieldwork portion of this study. Permits and user agreements obtained for this study are listed in table 1.

Landowner agreements were also obtained from 21 landowners prior to working on private lands during this study. Altogether, it took 2.5 years to obtain permits from all 13 agencies and 21 landowners.

Our camera-check schedule and safety protocols performed without any problems in almost 3 years of fieldwork. Only one help message was sent on a SPOT device, when one field team member had two flat tires in the same day; we were easily able to retrieve that field team member and fix the flat tires.

\section{Human Dimensions}

We believe that it was critical to the success of this project that ranchers, in particular, were notified and involved in this project. We communicated in person, by phone, and(or) by email with close to 60 members of the ranching community, plus attended many ranch meetings during the project period. Although not required, some ranchers or landowners asked to accompany us in the field when checking cameras, to which we agreed.

\section{Trail Cameras}

From April 25, 2012, to February 15, 2015, a total of 250 camera sites were set throughout the study area, including 5 in the Atascosa Mountains, 18 in the Baboquivari Mountains, 15 in the Canelo Hills, 3 in the Cerro Colorado Mountains, 15 in the Chiricahua Mountains, 5 in the Coyote Mountains, 3 in the Dos Cabezas Mountains, 23 in the Huachuca Mountains, 24 in the Pajarito Mountains, 28 in the Patagonia Mountains, 25 in the Peloncillo Mountains (18 in New Mexico and 7 in Arizona), 55 in the Santa Rita Mountains, 3 in the Sierrita Mountains, 8 in the San Luis Mountains (Arizona), 5 in the Tumacacori Mountains, and 9 in the Whetstone Mountains (figs. 1a-1c). We requested, but were not granted permission, to place cameras on private land in the Animas Mountains of New Mexico. The general location description, date deployed, Universal Transverse Mercator (UTM) coordinates and zone, and elevation for each camera survey site are listed in table 3 . We lost 78 cameras (22\% of cameras) to theft/vandalism (15 vandalized and 63 stolen) and lost 43 sites to camera malfunction (31 from mechanical malfunction and 12 from malfunction due to environmental reasons). The duration of camera site activity is provided in Appendix 3. We analyzed stolen and vandalized cameras spatially and temporally. Maps showing locations of stolen and vandalized cameras are included (Appendixes 4 and 5) to show the spatial distribution of these cameras - clearly throughout much of the study area with hot spots along the international border and in the Santa Rita Mountain range. Appendix 6 shows the number of cameras vandalized or stolen by quarter of a year, starting with the first vandalized camera in October 2012 until the most recent vandalized camera in May 2015. The highest camera loss was from April through September 2013, with two secondary peaks from January to March 2014, and from October to December 2014. 


\section{Photo/Video Data}

\section{All Detected Species}

While cameras were placed at 250 sites, if two sites were less than or equal to 300 yards apart, the associated data from both camera sites were combined. Twelve camera sites were combined with others, resulting in a total of 238 sites. Another 5 sites yielded too little data to include in the analyses and were removed, resulting in 233 sites analyzed. Table 4 includes all jaguar and ocelot photo and video detections from these 233 camera sites; however, we detected a minimum total of 50 species in our entire study area, which are grouped into 32 species/species categories (table 5). These 32 species/species categories are used throughout the analyses unless otherwise noted. Many of the mountain ranges surveyed demonstrated varying species composition, illustrated in figures 3-19.

Of the 32 species/species categories, 25 were observed in the Santa Rita Mountains, all of which represent potential jaguar prey (except the jaguar itself) (table 5b). This study also detected 23 species/species categories in the Huachuca mountains (table 5b), of which 12 could reasonably be considered potential ocelot prey items, including birds, coati (Nasua nasua), gray fox (Urocyon cinereoargenteus), cottontails (Sylvilagus spp.), opossum (Didelphis virginiana), raccoon (Procyon lotor), ringtail (Bassariscus astutus), skunks, squirrels, turkey, and snakes and lizards. It may also be possible for ocelots to prey upon small domestic dogs (Canis lupus familiaris) and the young of coyotes (Canis latrans), javelina (Pecari tecaju), and white-tailed deer (Odocoileus virginiana), which would increase the potential prey species (categories) to 16.

\section{Listed, Proposed, Threatened, and Near Threatened}

Of the species detected in this study area, 2 are federally listed as endangered under the ESA (jaguar and ocelot), 1 is federally listed as threatened (Mexican spotted owl, Strix occidentalis lucida), 1 was proposed as a candidate for federal listing (Sonoran desert tortoise, Gopherus morafkai, now removed from the candidate list), and 2 are listed as Near Threatened by the International Union for Conservation of Nature (IUCN) (Gila monster, Heloderma suspectum, and Sonora mud turtle, Kinosternon sonoriense sonoriense) (figs. 1a-1c; table 6). All of these species are listed as vulnerable in Arizona (Arizona Game and Fish Department, 2012), and the Sonora mud turtle, photographed in New Mexico, is listed on the New Mexico Department of Game and Fish's list of Species of Greatest Conservation Need (http://www.bison-m.org/booklet.aspx?id=030425).

We obtained 118 photos/videos of a jaguar, 13 photos of ocelots, 2 photos of Mexican spotted owls, 142 photos of desert tortoises, 58 photos of mud turtles, and 11 photos of Gila monsters. Tortoises were photographed in the Baboquivari, Cerro Colorado, and Coyote Mountain ranges. Gila monsters were photographed in the Baboquivari, Coyote, San Luis (AZ), and Sierrita Mountain ranges. Mud turtles were photographed in the Peloncillo Mountain range in New Mexico. A Mexican spotted owl was photographed by our cameras in the Huachuca Mountains, and by our team member with his personal camera in the Santa Rita Mountains (Chris Bugbee, written commun., November 2014) and included in this study). Sensitive species photographs are included with the digital data. 


\section{Range Extensions}

In this study, and based on photographs and scat samples, no species were detected beyond their normal and previously described ranges. Our study documented bighorn sheep (Ovis canadensis nelsoni) (13 photos; table 6) in the Coyote Mountains in May 2013 and February 2014. Bighorn sheep in this mountain range are rare, and the Coyote Mountains may have been used by bighorn sheep in recent years (John Clemmons, written commun., March 2014; Jim Heffelfinger, written commun., March 2014). However, it has been several decades since bighorn sheep have been observed in this mountain range. The two pronghorn detected in the Sierra San Luis Mountains are most likely from the non-endangered Antilocapra americanus americanus subspecies, although depending on which map you examine, the historical range of the Sonoran pronghorn (Antilocapra americanus sonoriensis) extends all the way to Interstate 19. We cannot identify subspecies in our photo detections; however, from discussion with the Sonoran pronghorn recovery team leader (Jim Atkinson, written commun., February 2015) we do not believe these two observations are likely Sonoran pronghorn subspecies, but it is possible given their location within the historical range of the subspecies.

\section{Photo/Video Analyses}

\section{Species Detections and Species Richness}

Figure 20 illustrates the total number of species/species categories (richness) detected by mountain range. The Santa Rita Mountains had the highest species richness, followed by the Huachuca and Baboquivari Mountains. However, species richness is influenced by sampling effort and sampling effort was greatest in the Santa Rita Mountains. Table 7 summarizes the 32 species/species categories and the percentage of total camera sites at which each was detected. The most ubiquitous species detected were white-tailed deer, humans, fox, puma, and skunks, all detected at more than 80 percent of the total camera sites for the project. Figures 21-36 indicate the proportion of total species/species categories detected per mountain range and per site. The top three sites with the highest proportion of total species/species categories detected for the project were in the northern Santa Rita Mountains.

\section{Species Accumulation Rate}

Figure 37 illustrates the accumulation rate of each species/species category, that is, how long it took before the first detection of a given species or species category occurred. Almost half of the species/species categories were detected in the first 48 days of the camera session; however, some species/species categories were not detected until 2 years of surveying were completed.

\section{Species Total and Relative Abundance}

The species/species categories with the overall highest total and relative abundance were whitetailed deer, gray fox, humans, birds, and skunks. Table 8 summarizes total and relative abundance for species/species categories detected in this study. Figures 38-39 illustrate species/species category abundance by total detections and by total independent photographs (per 60 minute interval).

\section{Activity Patterns}

For each species/species category, we summarized activity patterns with graphs by hour, by month, and by moon phase (figs. 40-86). 
Daily and Monthly Activity

Chiropterans were active from 1800 to 0600 , but activity peaked from 2000 to 2100 . Bats showed a marked peak of activity in the month of June (fig. 40). Bears showed a generally crepuscular activity pattern, but there were records from every hour of the day (fig. 41). Bears showed a sharp decrease in activity from December through March, and increase in activity in April. Peak bear detections occurred in June. Bobcats were detected throughout the day and night, although detections were slightly higher at night (fig. 42). Bobcat detections peaked in November through January and a secondary peak occurred in May through June (fig. 42). Cottontail detections peaked from 0500 to 0800, and detections were low by midday (fig. 43). Cottontail detections were lowest in February and rose steadily to a peak in July (fig. 43). Cattle were active throughout the day but showed a peak in the late afternoon into dusk. They were also the only species to show a peak in detections in March (fig. 44). Coyotes were active throughout the 24-hour period but showed a peak in the morning daylight hours. Coyote detection wavered starting in January but showed a high peak in June (fig. 45). Gray fox were strongly nocturnal but remained active steadily through the night (fig. 46). Gray fox detections were higher in the late fall and winter but also peaked strongly in June (fig. 47). Gila monsters were strongly diurnal and were detected from March through September with a strong peak in June (fig. 48). Humans were detected at every hour of the day but peaked strongly in the late morning with a steady decline throughout the afternoon (fig. 49). Summer detections of humans were lower than in the spring or fall (fig. 49). Jackrabbits showed a strongly crepuscular pattern of activity but were active throughout the night as well (fig. 50). Jackrabbits showed a strong peak in detections in June. The jaguar was strongly nocturnal and detections rose sharply in May (fig. 51). Javelina were active throughout the day and night but peaked around 2000-2200. A secondary peak occurred in the late morning hours and detections were lowest in the afternoon (fig. 52). Seasonally, javelina detections were relatively steady throughout the year but peaked in June and in October through November (fig. 52). Lizards were strongly diurnal, and showed a sharp rise in detection in March through April that declined steadily throughout the summer (fig. 53). Mule deer were most often detected in the morning daylight hours, and showed a strong increase in detections in June (fig. 54). All but one of the ocelot detections occurred at night. Like the jaguar, ocelots showed a sharp increase in detections in May (fig.55). Opossums were strongly nocturnal with no daytime records. They peaked in October through November with a secondary peak in June (fig. 56). Puma were active throughout the 24-hour period but showed a lull in midday and a peak in the early part of the night (fig. 57). Puma detections peaked in May through June (fig. 57). Raccoons were strongly nocturnal. Uniquely, raccoons showed a steady increase in detection from April through December (fig. 58). Ringtails were also strongly nocturnal, and showed peaked activity in the winter months (fig. 59). Rodents were primarily nocturnal with the most activity recorded in the early morning darkness (fig. 60). Rodent activity peaked in late fall to early winter (fig. 60). Skunks were strongly nocturnal and activity peaked in June, with a secondary peak recorded in the fall (fig. 61). Snakes were mostly diurnal and showed a June peak in activity (fig. 62). Squirrel detections showed a perfect bell curve during daylight hours, with a peak from 1200 to 1300. Squirrel detections peaked seasonally in June and November (fig. 63). Tortoises were strongly diurnal and detections peaked in August (fig. 64). Turkeys were highly diurnal and detections peaked in May (fig. 65). Whitetailed deer were detected throughout the 24-hour period, but activity was highest in mid- to late morning (fig. 66). Like many other species, white-tailed deer detections peaked in June (fig. 66). 
Lunar Activity

To examine relations between activity and moon phase, we first omitted all highly diurnal species, including birds, butterflies, lizards, horses, humans, mule deer, snakes, squirrels, tortoise, and turkeys. We excluded dogs because they were highly associated with diurnal humans. We excluded any species/species category (other than ocelot) with less than 10 records because our analysis required at least 10 records to determine activity patterns. Although cows and coatis are chiefly diurnal, we had some nighttime records of these species, so they were included in the lunar activity analyses.

Bats showed greater activity during the new moon (fig. 67). Bears showed almost no change in activity during different moon phases (fig. 68). Bobcats demonstrated an increase in nocturnal activity during new moons (fig. 69). Coatis had a slightly higher incidence of nighttime detections during the new moon, while cows showed slightly more nighttime activity during full moons (figs. 70, 71). Cottontails had slightly higher nocturnal detections during full moons (fig. 72). Coyotes showed mixed results (fig. 73). While detections were slightly higher from dusk until midnight during new moons, detections were higher during full moons from the early morning hours until dawn. Fox nocturnal detections were higher during new moons (fig. 74). Frogs showed higher rates of detection during full moons (fig. 75). Jackrabbits typically demonstrated a higher nocturnal detection rate during full moons (fig. 76), but during some of the crepuscular hours detection rates were higher during the new moon. The jaguar showed little influence of moon phase, but demonstrated a late night spike during full moon conditions between $2200^{1}$ and 2300, and an early morning spike during new moon conditions between 0300 and 0400 (fig. 77). Javelina were generally detected more during new moons, but the relative differences were slight (fig. 78). Six of our seven nighttime ocelot detections occurred during a full moon (fig. 79). Our one daytime detection of an ocelot occurred during a new moon cycle. Opossum showed no obvious preference for either moon phase (fig. 80). Puma, like coyotes, showed mixed results (fig. 81). Detections were slightly higher from dusk until midnight during new moons, but were higher during full moons from the early morning hours until dawn. However, overall, these differences were slight. Raccoon detections were equal during full moons and new moons (fig. 82). Raccoons showed no obvious preference for moon phase in terms of activity (fig. 82). Ringtail showed higher detections during new moons (fig. 83). Rodents showed a markedly higher nighttime activity pattern during new moons (fig. 84). However, the inverse was true in the 2100-2200 hour, when there was a higher peak of activity during full moon conditions. Skunks showed a slight increase in activity during new moons, especially between the hours of 2300 and 0400 (fig. 85). White-tailed deer showed a slightly higher activity pattern at night during full moons (fig. 86).

A species/species category list ranked (highest to lowest) by differences in activity as a function of moon phase is provided in table 9 .

\section{Felid Co-Occurrence}

The most ubiquitous felid in our study was the puma. Pumas were documented at 188 of 233 camera locations (80.7\%). Bobcats were detected at 171 of 233 camera locations (73.4\%). Most locations that detected puma also detected bobcats (89\%). The top four mountain ranges for puma detections (by percentage more than 5\%) were the Atascosa, Baboquivari, Pajarito, and Tumacacori Mountains (figs. 3-4, 11-12, 18). Of the 17 mountain ranges included in our study, only in the Coyote, Patagonia, and Sierrita Mountains (figs. 8, 13, 17) were bobcats detected more often than pumas.

\footnotetext{
${ }^{1}$ Times are indicated in a 24-hour format with noon $=1200$ hours and midnight $=2400$ hours.
} 
In the Santa Rita Mountains, 4 of the top 5 puma sites were also jaguar detection sites, and 3 of the top 4 bobcat sites were also jaguar detection sites. The jaguar was documented at 23 of 233 camera sites (9.8\%) in the Santa Rita Mountains. Only 1 jaguar detection site never documented a puma, but puma sign was observed in the immediate area on several occasions. Bobcats were not detected at 5 of the $23(21.7 \%)$ jaguar detection sites.

Ocelots were documented at 10 of 233 locations (3.8\%) representing 5 locations in the Santa Rita Mountains (based on previously defined naming conventions, ocelots in the Santa Rita Mountains were given the names Ocelot \#5 and Ocelot unknown) and 5 locations in the Huachuca Mountains (ocelots in the Huachucas were previously named by the Arizona Game and Fish Department as Ocelots \#3 and \#4). In the Santa Rita Mountains, pumas and bobcats were detected at all ocelot detection sites. Pumas were detected in all but one of the Huachuca locations (HUA23), and bobcats were also detected in all but one of the Huachuca locations (HUA09).

Two locations in the northern Santa Rita Mountains (SAN01 and SAN15) documented all four Arizona felids. All four felids showed the same general trend in detections - a peak in late spring and a much less defined secondary peak in fall (fig. 87).

Influence of Elevation on Jaguar and Prey Detection

We also examined the influence of elevation on jaguar detection rates and its presumed primary prey species - white-tailed deer and javelina (fig. 88). In the Santa Rita Mountains, camera site elevation ranged from 1,280 $\mathrm{m}$ to 2,395 m (table 3). Camera placement effort decreased as elevation increased. As effort decreased, the number of detections decreased for all three species (fig. 88). This effect is an indirect effect of our adaptive management strategy of camera placement, as all cameras that were not photographing Arizona felids were moved to other locations identified as good felid travel routes. White-tailed deer and javelina were detected below $1,400 \mathrm{~m}$ in low numbers but proportional to effort. White-tailed deer were detected proportional to effort above 2,000 m. No jaguars were photographed below the elevation of $1,400 \mathrm{~m}$, and no jaguars or javelina were photographed above 2,000 m (fig. 88).

Jaguar detection rates were higher per effort in the 1,400-1,800 m elevation range (fig. 88). White-tailed deer detections were slightly higher per effort in the 1,800-2,000 $\mathrm{m}$ elevation range, and javelina detections were higher per effort in the 1,400-1,600 m elevation range (fig. 88).

\section{Scat Searches and Scat Genetic Analyses}

Scat searches with the detector dog were conducted from November 27, 2012, through May 1, 2015. A total of 60 scat samples were tested in the lab, 46 from detector dog scat searches and 14 scat samples from other team members. All samples tested originated from the Santa Rita $(n=48)$, Huachuca $(n=4)$, Patagonia $(n=2)$, Coyote $(n=2)$, Baboquivari $(n=2)$, Chiricahua $(n=1)$, and Whetstone $(n=1)$ Mountain ranges. A total of 46 samples yielded a species-identification result, while 14 samples had too little DNA or degraded DNA and did not yield a species-identification result. Of the genetically verified species identifications, 13 were determined to be jaguar, all of which were collected by the detector dog and handler in the Santa Rita Mountains (fig. 1b; table 4). No genetically verified ocelot scats were recovered.

Approximately 23 of the 46 scats identified by the detector dog as jaguar were genetically tested before the dog was able to correctly select jaguar scat. Of those initial 23 scats tested, 1 was jaguar for a 4-percent jaguar identification rate. Following the first genetically verified jaguar scat, the jaguar identification rate for scat identified in the field by the detector dog and handler was about 50 percent (12 verified jaguars from the second set of 23 scats tested). An additional 6 scats were strongly 
suspected to be jaguar, but, because of age and degradation, no DNA was recovered for species confirmation for these samples. Following verification of species identification of scats, individual ID was performed on the 13 scats verified to be jaguar, by PCR amplification of 28 felid microsatellite loci. Of 28 loci, between 22 and 27 loci amplified for each scat sample. The composite genotype was compared for a match to each sample and to the original genotype obtained from this male jaguar from hair samples collected when he was treed and photographed by a lion hunter in the Whetstone Mountains in 2011 (Young, 2011) immediately prior to our study. The probability of identity (PID probability of this marker set to correctly identify unique individuals) of our microsatellite marker set was $9.8 \times 10^{-31}$ (Alexander Ochoa Hein, University of Arizona, School of Natural Resources and the Environment, written commun., 2013) based on the 22 markers that successfully amplified in all scat samples.

Complete results of analyzed scat samples are provided in table 10, including sample locations, PCR results, and results of GenBank search results for jaguar and non-jaguar scat. UTM coordinates for the verified jaguar scat samples are included in table 4. Scat deposit elevations ranged from 1,639 $\mathrm{m}$ to $1,778 \mathrm{~m}$ (table 4).

\section{Jaguar (and Ocelot) Detections}

Detections of jaguar or ocelot, from photograph, video, or scat, are indicated by date, time, and UTM coordinates for each of 143 detections between November 10, 2012, and February 2, 2015 (table 4). These are further broken down into 118 jaguar photographs/videos of the same individual, 13 jaguar detections of the same individual from scat, and 13 ocelot photographs of a minimum of 3 individuals. Maps of jaguar and ocelot detections are provided in figs. 1a-1c.

Jaguar

UA cameras were deployed in the Santa Rita Mountains beginning September 30, 2012, and were placed in a total of 61 locations, with 37 currently active. As of February 2015, we obtained 118 photographs/videos and 13 scats of a single male jaguar verified by genetics. All jaguar photos and videos for the project were from the Santa Rita Mountains. A total of 46 photographs were obtained from 5 locations in Pima County, and 72 photographs/videos were obtained from 18 locations in Santa Cruz County. The likelihood that 2 individuals carry the same spot pattern approaches zero, as with fingerprints; however, the accuracy of photos to identify individuals ranges between 64.2 percent (for tail), 66.7 percent (for mid-quarter) and 83.9 percent (for hind-quarter) in leopard cats where independent investigators assessed photo identification accuracy (Bashir and others, 2013). All 13 scats came from Santa Cruz County. The probability that our scat samples came from individuals not already represented in our dataset was $9.8 \times 10^{-31}$. The jaguar is a male, likely more than 6 years old, and appears in very good physical condition with no apparent injuries or ailments. Age estimates are based on the assumption that the jaguar would likely have been at least 2 years old at the time it was first treed in the Whetstones in 2011 (Young, 2011), the age at which male dispersal from natal locales typically takes place (Sunquist and Sunquist, 2002). 
Ocelot

As of February 2015, the team obtained a total of 13 photographs of a minimum of 3 individuals in Arizona. More specifically, as of February 2015, the team obtained 6 photographs of two individual male ocelots from 5 locations in the Huachuca Mountains, Cochise County. One male ocelot was photographed twice in the same location. Both animals were adults and appeared in very good physical condition with no apparent injuries or ailments. UA cameras were deployed in the Huachuca Mountains in Cochise County beginning July 31, 2012, and were placed in a total of 23 locations, with 13 currently active.

As of February 2015, the team obtained 7 photographs of at least 1 male ocelot in the Santa Rita Mountains (one photo was not adequate for individual identification), 6 in Pima County, and 1 in Santa Cruz County. The male individual was an adult and appeared in very good physical condition with no apparent injuries or ailments. UA cameras were deployed in the Santa Rita Mountains (Pima and Santa Cruz Counties) beginning September 30, 2012, and were placed in a total of 61 locations.

The numbering system used to track recent ocelot sightings in Arizona was devised by the NonGame Program Manager for Region 5 of the Arizona Game and Fish Department, and is based on the unique spot pattern of each individual. Of the 5 ocelots observed in Arizona since 2009, Ocelots \#3, \#4, and \#5 were detected in this study; each of these ocelots has also been detected by other entities.

Ocelot \#3, a male, was photographed 2 times in October 2012 and May 2013 in the Huachuca Mountains. Prior to this study, this animal was treed in the Huachuca Mountains by a dog owned by a private citizen in February 2011. The duration from the first time this animal was observed by this private citizen to the last detection in our study (May 2013) was 834 days. Another private citizen photographed this animal in the Patagonia Mountains on a trail camera in May 2012 (photo and location verified by Jack Childs). This location was $42 \mathrm{~km}$ from where he was most observed during our study. Other private citizens photographed this animal on their trail cameras; however, the locations of those photos were vague or were within the bounds of where we had photographed him, so these locations were not used in calculating the minimum observed range for this individual.

Ocelot \#4, a male, was photographed 4 times in the Huachuca Mountains by this study, from February 2013 through December 2014. Prior to this study, this animal was treed in the Huachuca Mountains by a dog owned by a private citizen in May 2012. The duration from the first time this animal was observed by this private citizen to the last detection in our study (December 2014) was 959 days. This individual was also photographed several times by private citizens in May, September, and November 2012, but the locations of those sightings were vague and were not used in calculating the minimum observed range.

Ocelot \#5, a male, was photographed 6 times by this study over 43 days from April through May 2014 in the Santa Rita Mountains. In this interim, a private citizen also captured a video of this animal in the Santa Rita Mountains (video and location verified by Jack Childs). This location was used in calculating the minimum observed range for this animal. In December 2013, we captured an ocelot photo of poor quality in the vicinity of the other photos of this male ocelot. We were not able to positively identify the individual ocelot in the December 2013 photo, but if this was in fact ocelot \#5, the duration this animal was observed would increase to 150 days. 


\section{Home Range Size Estimation}

Using the 24-hour MMDM resulted in a minimum home range size estimate of $90 \mathrm{~km}^{2}$ for the single male jaguar currently living in the Santa Rita Mountains. The 24-hour MMDM is a conservative minimum estimate of the jaguar's home range in the Santa Rita Mountains, as we know it traveled from the Whetstone Mountains to the Santa Rita Mountains, a distance of at least $35.5 \mathrm{~km}$. Our estimate should be used with caution, considering the small sample size and that our study was not designed to determine home range size.

We were unable to estimate the 24-hour MMDM home range size of the ocelots in this study because we did not have enough detections to complete these analyses. Therefore, we estimated only the minimum observed range of each ocelot using MCP. The estimated minimum observed range in the Huachuca Mountains for ocelot \#3 was $7.76 \mathrm{~km}^{2}$. The foray he took into the Patagonia Mountains increased this range to $67.46 \mathrm{~km}^{2}$. The minimum observed range of ocelot \#4 was $8.62 \mathrm{~km}^{2}$. The minimum observed range of ocelot \#5 was $19.11 \mathrm{~km}^{2}$. Combined, the average minimum observed range of the 3 ocelots detected in this study was $11.83 \mathrm{~km}^{2}$ (this used the smaller home range size for ocelot \#3).

\section{Macrohabitat and GIS Analyses}

Initially, analysis was conducted at various scales (30 m and $1 \mathrm{~km}$ ) for certain factors such as elevation and terrain ruggedness to assess characteristics at course and fine scales. In all cases, the landscape-level analysis at $1 \mathrm{~km}$ was selected as the most appropriate and meaningful for variable selection and future habitat modeling. For all environmental layers, results have been included at the 1km scale. See Appendix 2 for environmental characteristics at each camera location.

Camera site locations, among all camera sites, differed significantly from random points for most environmental variables. For the west study area, camera sites differed significantly from random points for all variables (distance to water, $\mathrm{P}=0.001$; all other variables, $\mathrm{P}<0.001$ ). For the east study area, camera sites did not differ significantly from random points for distance to water $(\mathrm{P}=0.434)$, but did differ significantly for all other variables $(\mathrm{P}<0.001)$. Statistical tests were not performed on positive detections of ocelots and jaguars (photos and scat) because of small sample sizes. However, for all location types (random, camera, current jaguar and ocelot detections, and historical jaguar records) summary statistics were calculated and graphed for each environmental variable and are summarized in the following sections.

\section{Elevation}

In the west and east study areas, the majority of camera sites were located between 1,500 $\mathrm{m}$ and 2,000 m elevations (55\% and $81 \%$, respectively; fig. 89). Average elevation for all camera sites in the west was $1,617 \mathrm{~m}$ and $1,737 \mathrm{~m}$ in the east. Ocelots were detected 13 times at 10 different camera sites with an average elevation of 1,832 $\mathrm{m}$ in the west study area. Jaguars were detected in 118 photographs/videos at 23 camera sites and 13 scats were also collected at surrounding sites in the west study area. Average elevation for all current jaguar locations was 1,702 m. Average elevation for historical jaguar records was 1,268 m. 


\section{Terrain Ruggedness}

Approximately 50 percent of all camera sites in west and east study sites were categorized as highly rugged and 50 percent as extremely rugged (fig. 90). This differed from the available habitat in that cameras were placed more often in more rugged areas than would have occurred if placed at random. This is even more exaggerated if we look at detection cameras, because 85 percent of all ocelot detections and 77 percent of all jaguar detections occurred in areas classified as extremely rugged. This finding differs from historical jaguar locations, where only 21 percent occurred in extremely rugged areas and 78 percent occurred in areas classified as highly rugged.

\section{Human Influence}

The Human Influence Index for the study area was assessed using random points and ranged from 5 to 48 in the west and from 0 to 30 in the east, with higher values indicating more human influence or impact (0-64 represents the full range). The average Human Influence Index for all camera sites was 10. Most camera sites in the west (99\%) were located in areas with an index less than 20, whereas all camera sites in the east were located in areas with an index less than 10 (fig. 91). The average Human Influence Index for ocelot locations was 15, current jaguar locations was 8, and historical jaguar records was 9.

\section{Normalized Difference Vegetation Index}

NDVI is a measure of "greenness" ranging from -1.0 to 1.0, with higher values representing live green vegetation. For March 2015, average NDVI values were 0.36 for cameras in the west and 0.33 for cameras in the east. Forty percent of cameras in the west and 49 percent of cameras in the east were located in areas ranging from 0.3 to 0.4 NDVI (fig. 92a). Ocelot locations average 0.39 NDVI, current jaguar locations averaged 0.37 NDVI, and historical jaguar records averaged 0.31 NDVI.

For August 2014, average NDVI values were 0.50 for cameras in the west and 0.47 for cameras in the east. Eighty-eight percent of cameras in the west and 71 percent of cameras in the east were located in areas with values higher than 0.40 NDVI (fig. 92b). Ocelot locations average 0.53 NDVI, current jaguar locations averaged 0.57 NDVI, and historical jaguar records averaged 0.43 NDVI.

\section{Tree Cover}

The average tree cover for camera sites was 19 percent $(\mathrm{SD}=10 \%)$ in the west and 18 percent $(\mathrm{SD}=11 \%)$ in the east study areas. This differed from random points, where the average tree cover was 9 percent $(\mathrm{SD}=8 \%)$ in the west and 7 percent $(\mathrm{SD}=9 \%)$ in the east. Thirty-nine percent of camera sites in the west and 42 percent of sites in the east were located in areas with 10-20 percent tree cover (fig. 93). Ocelot locations averaged 23 percent tree cover, current jaguar locations averaged 22 percent tree cover, and historical jaguar records averaged 14 percent tree cover.

\section{Vegetation Community}

The majority of camera sites were located in the Madrean evergreen woodland community (71\% west, 76\% east; fig. 94). The second-most surveyed community was the semidesert grassland (23\% west, $15 \%$ east). Ocelot detections from our study were located in the semidesert grassland (46\%), Madrean evergreen woodland (46\%), and Great Basin grassland (8\%) biotic communities. The majority of current jaguar detections were located in the Madrean evergreen woodland community (72\%) and the semidesert grassland community (28\%). Historical jaguar records were located in semidesert grassland (57\%) and Madrean evergreen woodland (42\%) biotic communities. 


\section{Distance to Perennial Water}

The average distance of cameras sites to water was 1,896 $\mathrm{m}$ and 1,566 $\mathrm{m}$ in west and east study areas, respectively. Eighty percent of camera sites in the west were less than $3 \mathrm{~km}$ from water and 93 percent of camera sites in the east were less than $3 \mathrm{~km}$ from water (fig. 95). On average, ocelot locations were 2,335 m from water sites. Current jaguar detections were on average 1,389 m from water sites and historical jaguar records were on average 1,743 m from water sites.

\section{Distance to Major Roads}

The average distance of camera sites to major roads was 8,254 $\mathrm{m}$ and 13,113 $\mathrm{m}$ in the west and east study areas, respectively. Distance to roads varied for camera sites in the west and east study areas, with the majority of camera sites occurring in the 5-10-km category for the west and the 10-15-km category for the east (fig. 96). Ocelot detections were on average 6,337 m from major roads. Current jaguar detections were on average 8,251 $\mathrm{m}$ from major roads, and historical jaguar records were on average $8,634 \mathrm{~m}$ from roads.

\section{Microhabitat Analyses}

Habitat and species composition characteristics are described for each jaguar detection site in figures 97-118. Overstory cover ranged from 0 to 100 percent (average 49\%) and midstory cover ranged from 8 to 53 percent (average 19\%) for all jaguar detection sites (fig. 119). We did not model microhabitat characteristics for ocelots using these analyses.

\section{Environmental Analyses}

Some iButtons lost data because of equipment failure with downloading devices or for other technical reasons. iButtons with retrievable data were deployed at jaguar and ocelot detection locations ( $n=9$ and $n=6$, respectively) and at semi-randomly selected camera locations throughout the study area ( $n=43$ in total, including jaguar and ocelot detection locations; table 11).

For all mountain ranges in the study area, average temperature and relative humidity for jaguar and ocelot detection and non-detection locations are shown in figures 120a-d. There were no significant differences in relative humidity between detection and non-detection locations throughout the study area, and all areas followed the same patterns of monthly humidity fluctuations. The same is true in our comparison of detection and non-detection sites in the Santa Rita Mountain range (figs. 121a-d), where temperature variations were a product of season, and likewise in the Huachuca Mountain range (figs. 122a-122b).

\section{Jaguar Detection and Non-Detection Locations Across the Study Area}

For each day, average relative humidity and average temperature for all jaguar detection locations with iButton data $(n=9)$ were compared with the average relative humidity and average temperature for all jaguar non-detection locations $(n=35)$ throughout the study area. Marginal, but not significant, differences can be seen in figures 120a and 120b. The average elevation of jaguar detection locations (of detection sites that had recorded, retrieved, and non-corrupted iButton data; not all jaguar detection sites are represented) was 1,638 m, and for a random sample of jaguar non-detection locations was $1,536 \mathrm{~m}$, a difference of $102 \mathrm{~m}$. 


\section{Ocelot Detection and Non-Detection Locations Across the Study Area}

For each day, average relative humidity and average temperature for all ocelot detection locations with iButton data $(n=6)$ was compared with the average relative humidity and average temperature for all ocelot detection locations $(n=38)$ throughout the study area. Marginal, but not significant, differences can be seen in figures 120c and 120d. The average elevation of ocelot detection locations (of detection sites that had recorded, retrieved, and non-corrupted iButton data; not all ocelot detection sites are represented) was $1,643 \mathrm{~m}$, and for a random sample of ocelot non-detection locations was $1,543 \mathrm{~m}$, a difference of $100 \mathrm{~m}$.

\section{Jaguar Detection and Non-Detection Locations in the Santa Rita Mountains}

For each day, average relative humidity and average temperature for all jaguar detection locations in the Santa Rita Mountains with iButton data $(n=9)$ were compared with the average relative humidity and average temperature for all jaguar non-detection locations in the Santa Rita Mountains $(n=6)$. Again, a marginal, but not significant, difference is seen in figures 121a and 121b. The average elevation of jaguar detection locations was 1,638 m, and for jaguar non-detection locations was 1,708 $\mathrm{m}$, a difference of $70 \mathrm{~m}$ (4.3\%). Table 11 lists locations in the Santa Rita Mountains where iButtons collected hourly relative humidity and temperature data, and in bold, iButton locations where jaguars were recorded.

\section{Ocelot Detection and Non-Detection Locations in the Santa Rita Mountains}

For each day, average relative humidity and average temperature for ocelot detection locations in the Santa Rita Mountains with iButton data $(n=4)$ were compared with the average relative humidity and average temperature for all ocelot non-detection locations in the Santa Rita Mountains $(n=12)$. Relative humidly and temperature differences between detection and non-detection locations throughout the year were negligible, as seen in figures 121c and 121d. The average elevation of ocelot detection locations was $1,553 \mathrm{~m}$, and for ocelot non-detection locations was $1,707 \mathrm{~m}$, a difference of $155 \mathrm{~m}$ (10\%). Table 11 lists locations in the Santa Rita Mountains where iButtons collected hourly relative humidity and temperature data, and in italics, iButton locations where ocelots were recorded.

\section{Ocelot Detection and Non-Detection Locations in the Huachuca Mountains}

For each day, average relative humidity and average temperature for ocelot detection locations in the Huachuca Mountains with iButton data $(n=2)$ were compared with the average relative humidity and average temperature for all ocelot non-detection locations in the Huachuca Mountains $(n=3)$. Relative humidly and temperature differences between detection and non-detection locations throughout the year were negligible, as seen in figures 122a and 122b. The average elevation of ocelot detection locations was $1,825 \mathrm{~m}$, and for ocelot non-detection locations was 1,880 m, a difference of $55 \mathrm{~m}$ (3\%). Table 11 lists locations in the Huachuca Mountains where iButtons collected hourly relative humidity and temperature data, and in italics, iButton locations where ocelots were recorded. 


\section{Potential Jaguar Distribution Model}

Only nonduplicate current and historical jaguar locations (at the 1-km scale) were used in the model. One historical location was removed because of its location in a high human impact area. The bias file was used to further restrict the area for jaguar locations and background points (fig. 123). This resulted in the elimination of two additional historical jaguar locations, as they fell outside the surveyed area defined by the bias file. Thus, 23 current (2012-2015) and 35 historical (1965-2009) jaguar locations were used in the model.

All environmental variables were evaluated and eliminated based on jackknife testing and model contribution. TRI, tree cover, and NDVI (March 2015) had the highest training gain when used in isolation. TRI and tree cover each resulted in the highest decrease in training gain when removed, indicating that they contributed information not available from other variables.

Low-performing variables were those that contributed minimally to training gain. Variables that were removed because of low performance included distance to water, distance to roads, and vegetation community. Bioclimatic variables were evaluated and reduced to one temperature and one precipitation variable (table 12). All other variables were evaluated in various combinations while maintaining high AUC $_{\text {test }}$ values. Additional models were created on the basis of each variable combination by varying the regularization parameter. These models were then evaluated by using $\mathrm{AIC}_{\mathrm{c}}$ scores calculated with ENMTools (table 13). The top ranking model included the following variables: Human Influence Index, NDVI (March 2015), NDVI (August 2014), tree cover, Terrain Ruggedness Index, bio1, and bio12 (table 14). Each variable's effect on the model's logistic prediction is shown in response curves (fig. 124). Response curves were created by varying the values of each variable independently while keeping all other variables at their average values.

Although the model included the Human Influence Index variable, it tended to overpredict jaguar habitat in areas of high human influence. This overprediction likely results from the limited amount of data used to create the model $(n=58)$ and over extrapolation into areas beyond the collected data. Current and historical jaguar locations occurred in areas with a Human Influence Index ranging from 0 to 18. The one exception occurred in an area with a Human Influence Index value of 34 and was not used in creating the model. The overprediction was corrected in the model by setting areas of high human influence (Human Influence Index>18) to 0, regardless of model prediction.

The final jaguar potential distribution map ranges in values from 0 (low probability) to 1 (high probability). While the map was projected to all of Arizona and New Mexico, areas outside the project study area should be viewed skeptically, as the values are extrapolated past the data used to build the model. Predicted values were evaluated for all jaguar locations and these ranges were used to classify the map. Historical locations tended to be in areas having lower values whereas current jaguar locations were in areas with higher values (table 15).

Predicted distribution maps are commonly classified as binary maps based on the 10 percent omissions threshold. We classified our map using three thresholds based on the occurrence of jaguar locations: (1) 10 percent omission of locations (red), (2) 5 percent omission (red and orange), and (3) 0 percent omission of locations (red, orange, and yellow; figs. 125-126). Tier 1 includes 90 percent of all current and historic jaguar locations. Tiers 1 and 2 (combined) include 95 percent of all current and historic jaguar locations. Tiers 1, 2, and 3 include all (100\%) of current and historic jaguar locations. The inclusion of all three thresholds may be helpful in identifying corridors that contain areas of lesser quality habitat, but that may still be important in ensuring connectivity along patchy habitat. It should be noted that size of habitat patches and distance between patches were considered in the mapping. As such, an area may be highly rated, but may also be too small and too distant from other patches to support a jaguar. When using the map and identifying areas of potential jaguar distribution, care should be taken to evaluate factors other than habitat suitability. 


\section{Discussion}

\section{Fieldwork for Wildlife Photos/Videos and Field Safety Protocol}

Prior to fieldwork, we obtained 13 permits from State and Federal agencies. In our study, we selected sites within mountain ranges, and narrow canyons and perennial water gave us the best chance to detect the maximum number of animals moving through the terrain. We chose not to monitor major river drainages because no jaguars have been documented in these riparian corridors, with the exception of a jaguar killed in the Santa Cruz river drainage in 1971. Jaguars could occur in the area between rivers and foothills, but the flatness of the terrain makes it nearly impossible to predict where jaguars would walk. These same factors also apply to ocelots.

Working on a field project close to the international border between the United States and Mexico comes with difficulties. Our team tried to maintain a high level of safety by working in pairs when possible. Illegal human traffic and other illegal activities were a source of concern for every team member, and each team member encountered some illegal activity during routine fieldwork. We strove to be prepared for these encounters as well as for the difficulties of conducting fieldwork in temperatures between -5 and $35^{\circ} \mathrm{C}$. In future studies, it would be helpful to have a GPS track of each camera site location posted on our internal team database - this would improve flexibility by allowing team members to check sites that they do not routinely check.

To assist the team in selecting the approach route to a particular camera, we posted maps of land ownership on our internal team website. This helped with planning and helped the Project Manager know what notification needed to be made in advance. A Google calendar was used to keep record of who was in the field at all times, which also helped the Principal Investigator and Project Manager keep track of safety protocols and notifications.

Safety of project personnel was considered the most important part of our project, and should be for any future borderlands project. The use of the buddy system worked well-for each camera check event, someone was aware of the camera checkers' location and route. The use of SPOT satellite GPS trackers and text communicators greatly facilitated the safety system. Check-ins and checkouts were the foundation of our safety program. When possible, camera checkers also traveled in teams of two.

Although most drug trafficking events were recorded during nighttime hours, overlap of illicit drug movement past project cameras was documented. Actual contact in the field with such illegal activity was rare, but expected and planned for. All possible scenarios were discussed prior to project implementation. Contact with injured undocumented aliens in need of emergency care was more common and Department of Homeland Security personnel were immediately contacted to assist. Contacting Homeland Security personnel prior to camera checks and provision of vehicle descriptions and license plates was not always effective as field agents often were not apprised of our activities. Project participants were prepared as first responders and assisted individuals experiencing dehydration and severe foot injuries. During those contacts, the individuals assisted were more than grateful to be given medical help.

Our safety protocols were effective in keeping our field team safe during this project, despite harsh working conditions and illegal activity across the study area. At no time were project personnel injured, stranded in the field for more than a few hours, or ever in need of post-camera-check medical treatment. 


\section{Human Dimensions}

Coordination and communication with landowners and grazing permittees was crucial to the success of this project. This took a tremendous amount of time and resources before and during the project. We believe this investment paid off and ensured a smoothly operating project, and may have developed good will for jaguar monitoring and stewardship in the future. Additional time and resources were needed to accommodate the intense media interest and scrutiny for this high-profile species, which was not expected, and which required a significant amount of staff time to handle, as well. This needs to be accounted for in the future.

Wildlife research is usually conducted to guide management practices and conservation programs. However, it is too often done without fully recognizing and accommodating the social matrix in which wildlife studies are conducted. If management and conservation efforts are to succeed, they must be accepted socially, culturally, and politically by a critical mass of the public, particularly those who may be affected by the research. Researchers are often the first point of contact with these key members of the public; therefore, it is critical that research scientists and their funders, in this case USFWS, anticipate, fund, and plan for the upfront work that needs to be done, possibly even with the assistance of social scientists and(or) professional facilitators. Otherwise, the research project may be problematic for the research entity and the funding agency, and future conservation efforts may be put at risk. In the current human environment, science is as much about relationship building as it is about data gathering.

We did not anticipate the additional effort and time it took us to address the landowners' and ranchers' concerns about critical habitat for the jaguar. This task would have normally been handled by USFWS, but because the UA team was already interacting with the landowners and ranchers, the UA team took time to address the questions and concerns. Variable factors (such as critical habitat) are important to consider when conducting a study on a high-profile species surrounded by controversial issues affecting the stakeholders. We believe that proactive communication with stakeholders is one of the most important aspects that led to the success of this project on the ground. In addition, the trust built with the public was not only important to the success of this project, but also to future projects and stakeholder relationships with USFWS.

\section{Trail Cameras}

The monitoring phase of this project, from the first camera deployed to the last camera removed, was conducted from April 25, 2012, to March 2, 2015 ( 2 years and 10 months). However, full deployment (from when the last camera was placed in the field to when the first camera was removed from the field) was conducted from May 31, 2013 to January 22, 2015 (1 year and 8 months). At the start of this project, the UA team anticipated that the entire permitting process would probably take 3-6 months at most; however, obtaining all the permits took 2.5 years, including those critical for the project in certain areas. Areas that we monitored by camera for the least amount of time were those where permits took longer to obtain. Additionally, obtaining landowner permissions took a substantial amount of time, including meetings with landowners across the study area. There are no precedents that we know of for such an intensive research project conducted in this region on such a large geographic scale across such a complex matrix of stakeholders and jurisdictional entities. These issues and workloads together functionally shortened the amount of time our cameras were monitored in some areas. To offset permitting and other delays, we adopted a strategy of setting cameras in a single mountain range as soon as all permits required for that mountain range were complete, thereby allowing us to start collecting data in parts of our study area while waiting for permits in other parts. 


\section{Camera Vandalism}

In certain areas, we had high rates of theft and vandalism of camera equipment. To reduce losses, we reduced many sites to single cameras instead of paired cameras. Many newer extended monitoring sites were set as single cameras for the same reasons, especially those in proximity to sites with previously recorded high human activity. Rather than abandon some continually vandalized sites, we shifted to infrared flash at some camera sites to avoid using bright flashes that may have revealed the cameras. This appeared to help us maintain cameras at these sites, although the tradeoff was reduced image quality. Vandalism may have impacted our results, as cameras that may have held important data were stolen or vandalized at six active jaguar detection sites.

\section{Jaguars and Ocelots in Arizona}

Based on our observations, we are confident that the jaguar in the Santa Rita Mountains is the only jaguar inhabiting that mountain range at the present time. Whether or not that individual periodically leaves the mountain range is unknown, but average time between detections since the first detection is less than two weeks. While we do not know where it roams on days when it is not photographed, it seems to use the east side of the Santa Rita Mountains with regularity.

The jaguar's minimum home range (fig. 127) was estimated using MCP with a surrounding band representing the 24-hour MMDM (Wilson and Anderson 1985); note that this estimate should be used with caution, because of small sample size and considering our study was not designed to determine home range size. The relatively small area of the current jaguar's estimated minimum home range is probably a function of high prey density and a lack of conspecific competition. It is unknown how interspecific competition between pumas and jaguars influences the way both species use the landscape and resources, but because the jaguar has been detected regularly in this area for 3 years, it may be assumed that it has adequate food, water, and cover in this relatively small area.

Based on our detections, we monitored a lone male jaguar, on average, every 7.9 days (out of 1,035 calendar days) in the Santa Rita Mountains, with a range in detection frequency of 2 hours to 101 days. Overall, our surveillance suggests he did not leave the Santa Rita Mountain Range for the duration of our study, from September 2012 through June 2015. We consider this current jaguar to be a breeding age adult, non-breeding resident male (Sweanor and others, 2000; Haines and others, 2005; Blankenship and others, 2006; Balme and others, 2009; Weingarth and others, 2012). Through most of the jaguar's natural range, resident males are usually tied to reproductive females, while resident females are tied to sufficient resources to raise young. However, male cats can survive in habitat that will not support breeding females, in many cases at the fringe of the natural range. These males may be transient, as they spend their time searching widely for a mate (Mel Sunquist, written commun., October 2014). Some male felids appear to abandon searching for females and settle into an area without females, where they remain as non-breeding residents. These situations may occur at the periphery of a breeding population in poor quality habitat, or at the edge of a species' distribution. The result is an influx of young male dispersers and a few resident males that have given up searching for mates and are now occupying the best available locales (Mel Sunquist, written commun., October 2014). This could be the case with the jaguar currently in the Santa Rita Mountains.

Based on the relative rarity of ocelot detections, we cannot make statements as to the residency of ocelots north of the international border. Too much time passed between detections to know what the ocelots were doing and where they were going. Ocelot \#3 was observed between February 2011 and May 2013. Based on detections from this project and from private parties, it was confirmed to have traveled from the Huachuca Mountains to the Patagonia Mountains and back, a one-way distance of 42 km (Jack Childs, written commun., February 2016). If ocelots regularly travel these distances, they 
could be traveling back and forth from mountains in northern Sonora during times in between detections. In south Texas, GPS-monitored adult male ocelots (some 4-6 years old that seemed to hold consistent territories over several months to years) would suddenly leave in different directions (Mitch Sternberg, written commun., October 2014). This would happen with multiple males at the same time, presumably following some unknown disturbance. With a paucity of females, resident males may have reached a tipping point where continual encounters with incoming young dispersers caused a series of wandering male ocelots (Mitch Sternberg, written commun., October 2014).

The historical records of ocelots in Arizona are scant. From 1887 to 2008, only 11 ocelots were documented with physical evidence (for example, carcass, hide, skull; note that the exact location of 4 of these records is questionable [Tim Snow, oral commun., June 2015). A 10,000-year-old fossilized skull also was found near Reddington, Arizona near the San Pedro River. Of the 11 records from 1887 to 2008, the gender is unknown for 5; 5 were males, and 1 was a lactating female detected circa 19801985 in the San Pedro River Valley. More recently (since 2009), 5 ocelots have been detected either from photographs or, in one case, a carcass, as detailed below.

Ocelot \#1, gender unknown, was photographed by a trail camera by the Sky Island Alliance in the Whetstone Mountains in 2009. Ocelot \#2, a male, was hit by a car east of Globe in Hells (or Devil's) Canyon in 2010. Ocelot \#3, a male, was first photographed in a tree by a houndsman in the Huachuca Mountains in 2011, then by our study in the same mountain range. This same ocelot was photographed by a private party in the Patagonia Mountains in 2012, then in the Huachuca Mountains later that year. Ocelot \#4, a male, first photographed by a private party in 2012, was photographed by our study and others in the Huachuca Mountains. Ocelot \#5, a male, was first photographed by our study in the Santa Rita Mountains in 2014, then subsequently in the same mountain range by a private citizen (note that we photographed an ocelot in the Santa Rita Mountains in 2013, but could not distinguish the individual in the image). If we look at individual movement based on detections of ocelot \#5 for the minimum 6 weeks that he was present in the Santa Rita Mountains, he appeared to move up and down the west side of the range at least twice.

Comparing ocelots north and south of the United States-Mexico border can be useful for determining behavioral differences between individuals in Arizona and Sonora. Since 2009, two of our team members have worked on a camera study in Sonora, Mexico, approximately $240 \mathrm{~km}$ south of the United States-Mexico border, where there is a resident breeding population of ocelots. As of March 2014, 25 individual ocelots were identified, including males, females, and kittens. Each individual was observed from 1 to 1,541 days. The average minimum observed range of 9 of these ocelots was 11.75 $\mathrm{km}^{2}$ (1.97 $\mathrm{km}^{2}$ to $31.49 \mathrm{~km}^{2}$ ). One male was documented leaving its minimum observed range and traveling $34.93 \mathrm{~km}$, was then photographed at 2 locations $10.63 \mathrm{~km}$ apart over 2 months, and then returned to his home range (Jack Childs, written commun., May 2015). This increased his minimum observed range from $11.65 \mathrm{~km}^{2}$ to $241.89 \mathrm{~km}^{2}$. This wandering behavior was also documented for a GPS-collared male jaguar in the same study area (Ron Thompson, written commun., May 2015).

While average estimated values of the area occupied, length of time in an area, and exploration of areas outside their observed range for ocelots documented by the UA study in Arizona differ somewhat from the Sonora population, sample sizes are too small for statistical comparisons. In Arizona, the minimum observed range of ocelots was $11.83 \mathrm{~km}^{2}$, and for ocelots in Sonora it was very similar at $11.75 \mathrm{~km}^{2}$. The average time the 3 ocelots spent in the area was 612 days (43 to 959 days), whereas the average time for ocelots in Sonora was 805 days (1 to 1,541 days). While statistical comparisons between the two ocelot populations (Arizona and Sonora) are not possible because of small sample size, it can be useful to observe the similarities and differences between the areas occupied by individual ocelots and the length of time they spend in an area. Additionally, the fact that our study was 
not designed to detect ocelots but still photographed 3 individuals is notable, and suggests that other ocelots could be detected if a study were designed to do so.

Although we have not detected jaguars and ocelots in other mountain ranges within the study area, we cannot say they are absent, or that they are unlikely to be detected at any given time in the future. Continued monitoring of remote mountain canyons is needed to further document these two endangered cats in Arizona and the possible presence of jaguars in New Mexico.

\section{Behavioral Patterns of Arizona Felids (and Other Wildlife)}

\section{Seasonality}

Although data for ocelots were relatively scarce, all four felids (jaguars, ocelots, pumas, and bobcats) showed the same general trend in detections: a primary peak in late spring and a much less defined secondary peak in the fall (fig. 87). Jaguar and ocelot detections, however, appeared to peak about a month earlier than puma and bobcat. Forty-five percent of our ocelot detections occurred in May, and in that month we also recorded 20 percent of our jaguar detections (compared with an expected 8.3 percent, assuming no monthly difference in detections). All felids displayed a less-defined secondary detection peak in the fall: jaguars in September, ocelots in October, and pumas and bobcats in November.

Other species such as bats, bears, coyotes, fox, jackrabbits, skunks, and white-tailed deer all showed peak detections in June. Species that exhibited a fall peak in activity also showed a secondary peak in June. June is the hottest and driest month (figs. 120-121), and summer monsoons typically begin in July. During dry periods, wildlife may spend more time in canyon bottoms searching for remaining pools of water, and therefore, would be more likely to cross our cameras. All species that showed a June peak in detections demonstrated a decline in detections through July and August with the onset of summer monsoons. For example, only 4 jaguar detections occurred in July-August (compared with an expected 14 if no seasonal difference existed) and these summer detections represented less than 5 percent of the total. Activity itself may not have declined so much as detectability. As canyon bottoms fill and water becomes more ubiquitous across the landscape, wildlife may spend less time traveling linear canyon bottoms and more time on slopes and in higher elevations, less tied to relatively scarce and scattered water resources. In general, some wildlife species were not detected until 2 years into our monitoring effort, giving justification for long-term versus short-term monitoring efforts to attain the best representation possible of biodiversity present.

\section{Daily Activity}

In terms of daily activity, bobcats appeared to be active throughout the 24-hour day. However, bobcats also demonstrated an increase in nocturnal activity during new moons (fig. 69). Pumas were more nocturnal than bobcats, but their activity peaked earlier in the night, from dusk until about 21002200 (fig. 57). Although data were sparse, ocelots, like pumas, showed a peak of activity in the earlier hours of darkness between 2100 and 2200 (fig. 55). The jaguar was highly nocturnal and its peaks of activity came between 2200 and 2300, and again between 0300 and 0400 (fig. 51). This pattern may allude to a temporal separation of activity between jaguars and pumas, but more data are needed. While the jaguar showed little response to moon phase, the two peaks of daily activity tended to occur during different lunar conditions. The jaguar demonstrated a late night spike during full moon conditions between 2200 and 2300, and an early morning spike during new moon conditions from 0300 to 0400 (fig. 77). Pumas, like coyotes, showed mixed results during the influence of lunar light (fig. 81). Detections were slightly higher from dusk until midnight during new moons, but were higher during full 
moons from the early morning hours until dawn. Although overall differences were slight, pumas showed a lunar response opposite of the jaguar, which was active later during new moons, and earlier during full moons.

Interestingly, 6 of 7 nighttime ocelot detections occurred during a full moon (fig. 79), and 1 daytime detection occurred during a new moon cycle. Emmons and others (1998) suggested that ocelots use open trails less often and move into thicker vegetation during high levels of lunar light, presumably because high light levels allow for better hunting in thick habitat patches. Our results indicate that ocelot detectability may increase overall with increasing lunar light, which conflicts with Emmons and others (1998), as our cameras were all placed on open trails and canyon bottoms. However, our data are very few, and the conclusions reached by Emmons and others (1998) were based on more robust observations of radio-collared animals. Although our data are sparse, we suggest the ideal time for ocelot detections in our study area would be in April-May during a full moon.

\section{Felid Co-occurrence}

In the Santa Rita Mountains, 4 of the 5 camera sites most often visited by pumas were also jaguar detection sites, and 3 of the 4 camera sites most often visited by bobcats were also jaguar detection sites. Two locations in the northern Santa Rita Mountains (SAN01 and SAN15) documented all four Arizona felids. In a camera study in Sonora, Mexico, pumas were detected at 34 of 38 locations, while jaguars were detected at 22 locations, overlapping with pumas at 21 of those locations (James Sanderson, written commun., 2015). In a study in Suriname, pumas and jaguars co-occurred at all 42 camera locations (James Sanderson, written commun., 2011). These studies show that jaguars and pumas often share the same locations; hence, high-value camera sites are those that also photograph pumas. The same dynamic was examined with respect to bobcats and ocelots at a study site in Sonora. Moreno and others (2013) discuss the coexistence of all four felids in the Sierra Madre Mountains of northeastern Sonora.

We suggest that very high camera detections of wild felids, particularly pumas, in areas of jaguar habitat may predict potential jaguar occurrence. Camera site selection for our study focused on sites that detected cats, particularly pumas. If cameras detected a high number of pumas, those cameras were not moved to other locations. This strategy worked in the Santa Rita Mountains in that many cameras sites eventually detected the jaguar in that range. Although sites where pumas and bobcats were detected in other mountain ranges did not also detect jaguars, we believe the strategy of using pumas as predictors for sites with a high potential of detecting jaguars is valuable and should be considered. However, the same cannot be said for ocelots. While pumas and bobcats did co-occurr with ocelots, a similar relation of very high camera detection of wild felids (pumas and bobcats) in ocelot habitat to predict potential ocelot occurrence, did not exist based on our data.

\section{Potential Jaguar Distribution Model}

Understanding jaguar occurrence and distribution is essential to their conservation and management. Ecological niche modeling allows for identification of additional areas currently unoccupied by a species; thus, it predicts (describes) a species realized niche (Phillips and others, 2006). However, ecological niche models have also been used to identify areas of potential distribution based on their fundamental niche, with the idea that barriers preventing a fundamental niche from becoming a realized niche are removed (Elith and others, 2006; Phillips and others, 2006). Niche modeling attempts to predict the realized niche by relating species occurrence and the environmental conditions that shape their distribution. For example, ecological niche modeling has been used to predict potential distribution of invasive species and species whose ranges and distributions have contracted (Peterson and Vieglais, 
2001; Raxworthy and others, 2003; Ficotela and others, 2007). Jaguars were extirpated from their former range in Arizona and New Mexico; therefore, predicting areas for potential distribution based on habitat suitability will be useful in locating and conserving this species in the future.

Several models depicting potential jaguar habitat in Arizona and New Mexico have been developed (see, for example, Menke and Hayes, 2003; Hatten and others, 2005; Robinson and others, 2006). Most recently, the JRT created a model predicting jaguar habitat within the Northwestern Recovery Unit for the jaguar (which includes southeastern Arizona and southwestern New Mexico) as part of the jaguar recovery planning process (Sanderson and Fisher, 2013), which was used by USFWS in the development of critical habitat for the jaguar (U.S. Fish and Wildlife Service, 2014; fig. 128). This habitat model included jaguar records prior to 2012, and did not incorporate the information collected during our study. Therefore, one objective of our study was to model potential jaguar distribution and habitat use in southeastern Arizona and southwestern New Mexico using the additional data obtained from our study (jaguar camera photos, videos, and scat locations collected from 2012 to 2015) to assist with future conservation planning. Our potential jaguar distribution map (figs. 125-126) identifies areas that are most likely to contain habitat suitable for jaguars, but it does not identify habitat necessary for jaguar conservation. Therefore, it should be used in conjunction with, not opposed to, the designated critical habitat map for jaguars. Additionally, our model includes areas outside of our study area, and these areas should be viewed with caution, as they likely do not adequately predict potential jaguar distribution due to extrapolation outside the geographic range of the data (current and historical jaguar locations) used to create the model. Finally, our model is based on many observations of a single animal, thus, pseudo replication may be an inherent source of error in our model (Bird and others, 2014).

While our jaguar potential distribution map is similar to the jaguar critical habitat map, they do differ slightly. Our potential distribution model predicts areas outside what was included in the designated critical habitat map. Our map does not predict distribution in some areas that are designated as critical habitat for jaguars. The reasons for these differences likely result from (1) different methods used in modeling, (2) different variables included in the model, and (3) different modeling goals. First, we used a species distribution modeling approach using MaxEnt software to evaluate the relationship between jaguar presence locations and different environmental variables to create a potential distribution model for jaguars. We used MaxEnt software because it performs well with presence-only records. A presence-only approach is needed for species such as jaguars, which have a reduced distribution, meaning an absence can be the result of unsuitable habitat, low population numbers, or inadequate sampling. In the case of jaguars in Arizona and New Mexico, there is only one known individual present. Thus, surveyed points with no detections could be a true absence due to unsuitable habitat, or a false absence due to the limited number of individuals. Our distribution, therefore, may not represent the full-potential distribution for the current jaguar in Arizona.

Second, variables selected in our potential jaguar distribution model differed from those used to create the USFWS jaguar critical habitat map. In our analysis, we evaluated all variables that were used to identify critical habitat for jaguars by USFWS, as well as additional variables such as temperature and precipitation. Fourteen environmental and bioclimatic variables (tables 2 and 12, respectively) were evaluated and seven were selected for use in the final model (table 14-model 1). Variables were included in the model on the basis of model performance, meaning low-performing variables were eliminated from our model regardless of their previous inclusion in the model used to determine jaguar critical habitat.

Third, our goal was to map the potential distribution of jaguars in southeastern Arizona and southwestern New Mexico based on habitat suitability. Our model identifies areas that are most likely to contain habitat suitable for jaguars and does not identify habitat necessary for jaguar conservation. 
While the two goals may seem similar, they do differ. In our map, we have rated areas in a three-tier system on the basis of current and historical jaguar locations. The upper-tier (shown in red, figs. 125126) indicates the highest probability (or suitability) that includes 90 percent of all current and historical jaguar locations. The second tier (shown in orange) includes 90-95 percent of all current and historical jaguar locations. The third tier (shown in yellow) includes 95-100 percent of all current and historical jaguar locations. All other areas on the map (not colored) either contained no jaguar locations or were removed because of high human influence. In using this approach, our model can also be considered a habitat suitability model defined by three gradients of suitability, where suitability of habitat for jaguars declines from high suitability (red) to low suitability (yellow). We used this approach to account for the uncertainty associated with historical jaguar locations. While only undisputed class I jaguar records (U.S. Fish and Wildlife Service, 2012) were used in this analysis, some uncertainty may remain as to exact jaguar location (for example, somewhere within a mountain range), or whether that location represented suitable jaguar habitat as opposed to lower quality habitat used only for travel between areas of high suitability. For instance, we removed one historical jaguar record from analysis because of its occurrence in an area with high human influence. By classifying the map into three tiers, we can choose to take a more conservative or generous approach to assessing suitability and potential jaguar distribution with regard to available data. The most conservative approach would be to use the upper tier (10\% omission threshold containing $90 \%$ of all current and historical jaguar locations, red), whereas a moderate approach would be to include the second tier (5\% omission threshold containing 95\% of all current and historical jaguar locations, orange) and the most generous approach would be to include the third tier ( $0 \%$ omission threshold containing $100 \%$ of all current and historical jaguar locations, yellow). The inclusion of all three tiers may help to identify corridors that contain areas of lesser quality habitat that still serve as important connectors between areas of higher suitability.

Unlike the critical habitat model, we made no attempt to analyze the potential of an area to support a jaguar based on size or connectivity. The jaguar critical habitat map identifies three areas of lower quality habitat that link directly or indirectly to Mexico, whereas our map only identifies areas of most likely jaguar occurrence (or highest habitat suitability).

\section{Environmental Variables}

A comparison of the average daily relative humidity and average daily temperature of locations at which the jaguar was recorded, with the average daily relative humidity and average daily temperature of locations at which the jaguar was not recorded, showed only slight differences. These differences can be explained by the $102 \mathrm{~m}$ difference in average elevation between the groups of locations. The same was found for ocelots (100.4 m difference). Within the Santa Rita Mountains (for the jaguar) and Santa Rita and Huachuca Mountains (for the ocelot), these same comparisons for jaguar and ocelot also showed no significant differences. Relative humidity and temperature do not appear to exert a direct influence on habitat selection for these predators; apparently, they are able to tolerate temperatures ranging from $29^{\circ} \mathrm{C}$ to $-3^{\circ} \mathrm{C}$.

\section{Habitat Use}

\section{Species Richness}

In our study, the Santa Rita Mountains had the highest species/species category richness, followed by the Baboquivari Mountains. Both mountain ranges were documented to have supported jaguars and(or) ocelots (this study; McCain and Childs, 2008). The top three sites for greatest proportion of total species/species categories detected were located in the northern Santa Rita Mountains, in the 
same area where we documented all four Arizona felids, and the only place in the United States known to have had jaguar and ocelot detections in the same mountain range. Mountain ranges with high species richness may be more likely to support a neotropical felid on the fringe of its range by supporting a wide range of prey. According to our study, species/species category richness may be a predictor of where jaguars and ocelots occur in Arizona and New Mexico. However, jaguars are known to have occurred in the Patagonia (most recently in 1965), Chiricahua (most recently between 1926 and 1930), and Dos Cabezas (most recently in 1986) Mountain ranges (Brown and López González, 2001), as well as the Peloncillo (most recently 1996; Warner Glenn, written commun., 1996), Atascosa (most recently 2009; Jack Childs, written commun., 2007), and San Luis Mountain (New Mexico) ranges (most recently 2006; U.S. Fish and Wildlife, 2014). With the exception of the San Luis Mountains (New Mexico), which we did not monitor, each of these mountain ranges had a lower species/species category count in our study than the Santa Rita and Baboquivari Mountain ranges, with the Dos Cabezas Mountains having the lowest overall (although we only had 3 sites in this mountain range). White-tailed deer were the most common species detected overall in 10 of the 16 individual mountain ranges, including both mountain ranges where we detected the jaguar and ocelots. White-tailed deer are probably an important food source for jaguars in Arizona.

The information above is useful for documenting species/species category richness in each of the 16 mountain ranges in our study area; however, we acknowledge that species richness may be affected by effort. The Santa Rita Mountains, which had the highest species/species category richness, also had the greatest effort (57 sites total); in contrast, the Dos Cabezas Mountains, which had the lowest species/species category richness, also had one of the lowest efforts (3 sites total). Therefore, the level of effort in each mountain range could contribute to the level of species/species category richness documented in that mountain range. However, the Coyote Mountains, with only three camera sites total, tied for having the third highest species/species category richness (the other two ranges were the Huachuca and San Luis Mountains, Arizona). Thus, number of camera sites does not necessarily correlate with species/species category richness.

\section{Characteristics}

The majority (72\%) of current jaguar detections (photo, video, and scat) and 46 percent of ocelot detections were located in the Madrean evergreen woodland community. The remaining jaguar detections (28\%) and 46 percent of the ocelot detections were in semidesert grassland (Brown, 1994; the remaining 8 percent of ocelot detections were in Great Basin grassland). At a coarse scale, these vegetation communities may not represent higher canopy cover and complex vegetative characteristics (such as those found near springs and narrow canyons) of detection sites that jaguars and ocelots use, particularly within the semidesert grassland type. In fact, most of our jaguar and ocelot detections that occurred in grassland were actually in rocky canyons with mature oaks, sycamores, mesquite, and(or) acacia. Because of their coarse scale, these habitat classifications may be misleading. At a finer scale, microhabitat characteristics of detection sites were highly variable and did not appear to be a driving factor in predicting jaguar or ocelot detections.

\section{Photographs and Videos Versus Scat Locations}

Confirmed jaguar scat generally had a higher average elevation than jaguar photographs and videos (table 4). The lowest confirmed scat was collected at 1,650 m, and 9 of 12 were collected at elevations above 1,700 m. Most cameras were located in canyon bottoms, but based on scat locations, the jaguar also traveled on slopes and ridges, and spent more time on slopes and ridges than in canyon bottoms. Scat searches in the Huachuca Mountains did not yield positive ocelot samples, but low 
detection rates of scat for cryptic predators are typical results of such surveys, especially in arid climates (Rinkevich, 2012). Habitat use by ocelots in Arizona is still largely unknown.

\section{Historical Versus Current Jaguar Locations}

Jaguars were found in more rugged terrain in our study than in historical accounts (fig. 90). Two factors that complicated our analyses and mapping efforts included vague historical site descriptions, and some occurrence records that reported locations where jaguars were taken (killed), which were not necessarily locations used by the jaguar. With these complications in mind, Hatten and others (2003) found that 92 percent of jaguar sightings used in their study occurred in intermediately rugged to extremely rugged terrain. They went on to suggest that the association of jaguars and terrain ruggedness might result from the biomes typically found in mountainous habitats of Arizona. Our analyses, using current and historical jaguar locations, provided a result similar to Hatten and others (2003), although it appears that historically jaguars were found in less rugged terrain than currently. It is notable, however, that in our analyses of historical locations, most records (122 out of 129) were from 2000 to 2009, which are older than our current jaguar locations but still from a time when GPS units could accurately place the location to within several meters. Thus, this result may represent variation of habitat choice among individuals rather than a temporal difference among Arizona jaguars.

\section{Jaguar and Ocelot Travel Corridors between Mountain Ranges}

It is apparent that jaguars use the same travel corridors as those used by ocelots, based on mapping of historical jaguar and ocelot sightings (Dave Brown, written commun., May 2015). Jaguars have recently, since 2001, been documented entering the United States through the Atascosa Mountains west of Interstate 19. Two individuals were photographed in the region by the Borderlands Jaguar Detection project from 2001 to 2009 (McCain and Childs, 2008). They also appeared travel along the Arizona-New Mexico border from the Cajon Bonita area of Sonora, north to the Peloncillo Mountain range and San Luis Mountain range (New Mexico), as there are verified records from both of these mountain ranges from 1996 and 2006, respectively (Warner Glenn, written commun., 1996, oral commun., 2006). The areas south of Interstate 10 with the most sightings correspond closely to the geographical areas we studied. These areas also correspond to our jaguar habitat modeling, as well as to areas south of Interstate 10 identified in 2003 by the Arizona/New Mexico Jaguar Conservation Team as potential jaguar habitat. It is reasonable to assume that our habitat modeling criteria could also be applied to areas with historical jaguar sightings north of Interstate 10.

Continuation of monitoring the habitat condition and the presence of jaguars and ocelots in the areas previously discussed, supports conservation efforts related to these animals. The spotted cats documented in Arizona and New Mexico are generally transient animals from known breeding populations south of the United States-Mexico international border, a connection supported by recent DNA evidence (Alexander Ochoa Hein, University of Arizona, School of Natural Resources and the Environment, written commun., 2013). Data suggests that the numbers of jaguars and ocelots living in Sonora, the Tres Rios area near the junction of the Bavispe, Aros, and Yaqui Rivers, are rapidly declining. Measures to protect this population would allow for continued dispersal into the Southwestern United States.

\section{Potential Jaguar Habitat in Arizona Outside Our Study Area}

Historically, jaguars have been documented over a wide geographical range in Arizona and New Mexico. The vast majority of these sightings occurred from the United States-Mexico international boundary north to the Mogollon Rim area in the central portions of both states. It is not known if these 
animals were resident or transient individuals, as all animals documented prior to 1996 were killed. It is likely that these animals were not born here, as the majority (77\%) were males. These animals likely immigrated to the United States from Sonora, Mexico. It was not discovered until after the year 2000, when wildlife camera studies for jaguars were first initiated, that some individual jaguars chose to inhabit certain mountain ranges for extended periods. A male, known as Macho A, was documented in Arizona for 3 years from December 2001 to December 2004. A second animal, known as Macho B, was documented by remote camera for 4.5 years from August 2004 to February 2009. He was also treed by a houndsman and photographed 8 years prior to being detected by trail cameras. The current male jaguar was first documented in November 2011, when he was treed by a houndsman in the Huachuca Mountains. The latest photo of this animal by this study was in June 2015. He has been residing in a relatively small area within the study area for 3.5 years. All three of these animals were photographed within the boundaries of this study. No jaguars have been documented north of Interstate 10 since 1964, but it is hypothesized that habitat suitable to sustain jaguars could exist outside the bounds of this study in Arizona and New Mexico. As these animals are nocturnal and sightings are often unreliable and hard to verify, we will not know the full extent of the range of jaguars in the United States until further camera studies have been completed. This could take several years to accomplish.

\section{Coordination with Other Entities}

A number of independent entities, such as the Northern Jaguar Project (NJP), Naturalia, Sky Island Alliance, and Primero Conservation (Primero Conservation has members who are also part of the UA team), are working to detect, monitor, and conserve jaguars in northern Mexico. It is our goal to collaborate and comprehensively share and compare data such that population status and distribution of northern jaguars can be assessed on a region-wide scale, to aid in the ultimate goal of jaguar conservation. For example, we have had one coordination meeting with Sky Island Alliance with respect to their tracking program (largely conducted in potential wildlife corridors in the valleys between mountain ranges in the United States and Mexico). We fully recognize that the data gathered through this project are confidential, cannot be shared without USFWS permission, and stand alone for the purpose of this contract. However, data from Sonora has been shared with us and we continue to make data and photos from other projects available to facilitate this collaborative process. It is our hope that the USFWS and the JRT will take the lead in structuring and ensuring that this collaborative process moves forward, which is essential to holistic, region-wide jaguar conservation goals in the United States and Mexico.

We have amassed a large dataset of photos of a variety of wildlife across the study area. These data, and our analysis of such, are providing a remarkable understanding of the mammalian biota of this region. We continue to coordinate with the USFWS and the JRT regarding photo data sharing, habitat measurements, and other analyses such as GIS modeling. Our dataset yields one of the most comprehensive, region-wide analyses of a wildlife community to date. We will coordinate data sharing from this and other projects with the USFWS and the JRT for the purpose of a region-wide analysis, if so desired.

\section{Key Findings and Recommendations}

One male jaguar and three male ocelots were detected and identified in 3 of the 16 mountain ranges surveyed between April 25, 2012 and March 2, 2015. 


\section{Jaguar Detection}

One jaguar was consistently detected in the Santa Rita Mountains for the duration of the project, which is a relatively small minimum home range. This jaguar was detected in 118 photographs/videos and 13 scats. The minimum home range was estimated to be $90 \mathrm{~km}^{2}$, although our sample size was small and this study was not designed to determine home range size, so this calculation should be used with caution. We presume the single male jaguar detected in this study is a resident because he was photographed by our cameras every month of the year from November 2012 to February 2015.

Figure 129 represents all recorded jaguar sightings in Arizona and New Mexico from 1900 to 2015. From 1900 to 1941, 52 jaguar individuals were sighted in Arizona/New Mexico at a rate of 1 every 0.8 years. From 1942 to 1972, when jaguars were granted legal protection under the Endangered Species Conservation Act of 1969, 10 jaguars individuals were sighted in Arizona/New Mexico at a rate of 1 every 3.1 years. From 1973 to 2000, only 3 jaguar individuals were sighted in Arizona/New Mexico at a rate of 1 every 9.3 years during this period. From 2001 to 2015, when researchers engaged in actively monitoring for jaguars, 4 individual jaguars were sighted, bringing the detection rate close to the pre-1973 level of 1 every 3.8 years.

The steady decline in jaguar numbers in the 20th century probably results from depredation by man. After 1972, when it became punishable by law to kill a jaguar, the numbers of reported jaguars dropped drastically. One jaguar was killed during the period from 1972 to 2000, but it is possible that if any other jaguars were killed during this period, they may not have been reported for fear of prosecution. Trail cameras deployed to monitor for jaguars from 2001 to 2015 (including those deployed during our study from 2012 to 2015) confirm the rate has returned to close to the pre-1973 level of 1 individual detected every 3.8 years. The presence of researchers and remote cameras actively monitoring jaguar habitat, as well as public education and outreach bringing attention to the plight of the jaguar, may be deterrents to poaching. These positive results justify continued long-term surveillance south of Interstate 10 and expansion northward to encompass areas of past jaguar sightings.

\section{Ocelot Detections}

The high number of ocelot detections in this study was unexpected. Three ocelots were detected in the Huachuca Mountains, Santa Rita Mountains, and Patagonia Mountains (this last detection was from a private citizen). These ocelots appeared to be transient, with the average of their minimum observed ranges calculated at $11.83 \mathrm{~km}^{2}$. However, we cannot rule out the possibility that one of these ocelots, ocelot \#4, is a resident, based on 15 photo detections collected over 3 years by AGFD, private citizens, and this study. The fact that this ocelot was photographed by a number of different entities emphasizes the importance of sharing data among different studies, agencies, and other organizations and persons.

According to AGFD records, 11 ocelots were documented in Arizona from 1887 to 2010, or 1 every 11.3 years. From 2011 to 2015, 3 ocelots were photographed (including by this study) for a rate of 1 every 1.7 years. Prior to 2011, only 1 ocelot was documented by a trail camera in Arizona. While the level of effort and methods used to incidentally detect ocelots over the years have been inconsistent, it is notable that our study, which was not designed to detect ocelots, detected 3 individuals. Therefore, a study specifically designed to detect and monitor ocelots in the United States by using the information in this study to focus camera placement within ocelot habitat, has potential to detect more ocelot individuals than this study at a higher frequency. 


\section{Jaguar and Ocelot Scat Detections}

We used a detector dog to increase jaguar and ocelot detections, and the dog was a valuable asset to this study. Scats detected by this dog accounted for one third of jaguar detection locations, for a total of 13 jaguar scats from 1 jaguar. No ocelot scats were detected by the detector dog. As this study was primarily designed to detect jaguars, more time was spent searching for jaguars than for ocelots, which likely contributed to the lack of ocelot scats detected by the dog. Our results indicate that the trained scat detector dog increased jaguar detections and has potential to increase ocelot detections.

Species of Concern and Interest

A wide variety of species were detected and monitored during this study, including ESA endangered, ESA threatened, ESA formerly proposed candidate, and IUCN near threatened. Photo detections for this study include the Mexican spotted owl (ESA threatened), Sonoran desert tortoise (ESA formerly proposed candidate), Sonora mud turtle (Arizona and New Mexico state vulnerable and IUCN Near Threatened), and Gila monster (IUCN Near Threatened). Additionally, in the spring of 2013 and 2014, bighorn sheep were photographed in the Coyote Mountains. One ram and three ewes were photographed, indicating that a breeding population of bighorn sheep is located in this mountain range.

This method has proven valuable in detecting terrestrial mammals on the landscape, and, increased numbers of rare species were detected over time. Our results indicate that camera placements that are focused on known movement/behavior patterns of the species group of interest should maximize detections of all species of interest.

\section{Camera Placement}

The fact that white-tailed deer and pumas were in the top four species detected is indicative of camera placement success for detecting large felids and their prey. The top four species also included humans, which indicates large human influence on the landscape and a large presence associated with illegal activity. We did not quantify the proportion of human presence associated with illegal activity. The high human presence at our camera sites is expected to affect the jaguar, as our habitat analyses showed that habitat suitability is inversely associated with human presence.

In a study area with the possibility of detecting a jaguar (for example, if a jaguar is thought to be present on the landscape), the puma may be a reasonable surrogate for jaguar detection and can help guide camera placement, as pumas were observed at all but one jaguar photo detection site in this study. Pumas were detected at many sites where no jaguars were detected, but the majority of those were in mountain ranges that we believe do not have jaguars present. In a study area with the possibility of detecting an ocelot (for example, if an ocelot is thought to be present on the landscape), puma and bobcat may be reasonable surrogates for ocelot detection and can help guide camera placement, as both were observed at all ocelot photo detection sites in this study.

\section{Weather Variables}

Our analyses of temperature and humidity for detection sites versus non-detection sites, even within a single mountain range, did not show a significant weather-related trend. The temperature and humidity data would be useful to describe microlevel differences in habitat, which may be more useful for species with low mobility. For a wide-ranging large carnivore, microclimate variables may not be as valuable. 


\section{Dispersal from Mexico and International Issues}

In April 2009, a camera study was implemented east of the junction of the Bavispe, Aros, and Yaqui Rivers in Sonora, Mexico, approximately $200 \mathrm{~km}$ south of the United States-Mexico international border. This area is a possible source population of the jaguars (and potentially the ocelots) photographed in southern Arizona, as supported by DNA evidence on jaguars (Alexander Ochoa Hein, University of Arizona, School of Natural Resources and the Environment, written commun., 2013). This study is being sponsored by Primero Conservation, a nonprofit organization based in Portal, Arizona. Twenty-three individual jaguars have been identified, including 11 males, 12 females, and 2 kittens. Twenty-five individual ocelots have also been identified, including 16 males, 8 females, and 1 kitten.

The number of individuals present by month was plotted for both species in the Primero Conservation study. The trend line shows that the number of individuals present in any given month for both species is declining. Jaguar numbers decreased from a high of 8 to 10 jaguars present until mid2011 to a low of 2 individuals at the end of 2013. Six jaguars were verified to have been killed. Ocelots show a similar reduction, dropping from a high of 7 to 9 individuals until mid-2012 to a low of 2 individuals at the end of 2013.

In 2003, the NJP established a jaguar reserve in the area of the Bavispe, Aros, and Yaqui Rivers in Sonora, Mexico. Livestock were removed and various studies are underway to examine the response in the jaguar population. The Primero Conservation study, just across the Aros River from the NJP reserve, provides incentives not to kill jaguars by selling white-tailed deer hunts and by donating the proceeds to ranchers who pledge not to kill jaguars. However, even with both programs, 6 jaguars have been intentionally killed in this area since 2009. All these studies occur in a rugged and thinly populated corner of northern Sonora, making law enforcement difficult or nonexistent.

Several jaguars photographed by NJP have also been photographed by Primero Conservation on opposite sides of the Aros River. This is not surprising, considering home ranges of jaguars can range from $10 \mathrm{~km}^{2}$ in Belize (Rabinowitz and Nottingham, 1986) to $959 \mathrm{~km}^{2}$ in Paraguay (Hernandez-Santin, 2007), and nightly forays out of their core areas as far as $20 \mathrm{~km}$ have been documented (Nuñez Pérez, 2006). These large home ranges also help explain the presence of jaguars in Arizona.

Because jaguars and ocelots most likely disperse into Arizona from northern Mexico, adequate conservation and monitoring of these species cannot occur without knowledge of both sides of the United States-Mexico border. Continued monitoring of and research on jaguars and ocelots in Arizona/New Mexico, United States, and Sonora, Mexico are needed, particularly in potential jaguar corridors and core habitat. Declining numbers of ocelots and jaguars suggested by current research in Sonora, Mexico may indicate increasing threats to these felids in the nearest core breeding populations for these two species-located 200 and $50 \mathrm{~km}$ south of the United States-Mexico border, respectively. There are benefits to jaguar conservation in both countries, if U.S. government agencies join with Mexican officials and agencies, to explore ways to better enforce wildlife laws and improve conservation efforts beneficial to jaguars and ocelots in this binational region. Examples of jaguar and ocelot conservation and research efforts that involve both countries include the following: surveys of potential travel corridors between the core jaguar population in Sonora and the Southwestern United States, researching the cause of decline for, and threats to, jaguars and ocelots populations south of the United States-Mexico international border. 


\section{Future Jaguar and Ocelot Monitoring}

Given that a new jaguar is detected in the United States approximately every 3-5 years, results of this study indicate a long-term monitoring system implemented for jaguars, in jaguar habitat, in the United States has the potential to detect a large proportion of these individuals. A long-term system is necessary because, as we saw in our study, individuals or species may not be detected until 2 years into the study in some areas. Because jaguars have large home ranges, and some may become residents, it is likely that new cats could be detected with 3 to 12 high-detection-probability cameras per mountain range (depending on the size of the mountain range; for example, 3 cameras in the Dos Cabeza Mountain Range and 12 cameras in the Santa Rita Mountain Range) which could be monitored every several years. Based on our intensive project, we can now identify a minimal number of highprobability camera sites for each mountain range, which could be monitored on a periodic or rotating basis. For example, every 5 years these high-priority sites throughout the study area could be monitored intensively for 1 year. Alternatively, a more logistically feasible option could be to monitor each mountain range in sequence, moving from west to east (or vice versa). The latter option could be accomplished by a team of two highly-qualified field biologists, moving from one mountain range to the next.

The high number of ocelot detections in this study is an important finding, especially given that our cameras were set to detect large felids (jaguar and puma). We feel that if a future study were initiated to specifically to detect ocelots, one which focused on camera placements in ocelot habitat in Arizona, the ocelot detection rate could markedly increase (that is, ocelots would be detected with greater frequency).

Given the complexities of land ownership, agency coordination, and the sensitive, high-profile nature of these species, it would be most desirable to have a small team of professional field biologists carry out future monitoring of jaguars and ocelots. Monitoring could potentially be conducted by volunteer citizen scientists, but professional personnel would still be required to train, coordinate, and oversee these citizen science volunteers. Monitoring could be expanded into Mexico in mountain ranges between the United States and the only known jaguar population in Sonora (located approximately 50$200 \mathrm{~km}$ south of the International Border). The first priority areas for continued monitoring should be the detection areas; the next priority areas should be those that have historical jaguar/ocelot detections (such as the Baboquivari and Chiricahua Mountains in Arizona, and the San Luis Mountains in New Mexico); and the final priority areas should be mountain ranges in southeastern Arizona and southwestern New Mexico where no jaguars or ocelots have been detected. Finally, resources and efforts to monitor jaguar habitat are needed on both sides of the international border.

\section{Camera Theft and Vandalism}

Camera theft/vandalism and resulting loss of data will always be a problem in United StatesMexico border areas, so researchers need to design an optimal study plan and be prepared with a large number of replacement cameras. Our strategy of using infrared cameras in high vandalism areas was effective in reducing theft/vandalism. The rate of theft/vandalism for this study was about 22 percent over 3 years, so one would need to be prepared to replace about 7 percent of total cameras per year. 


\section{Human Dimensions}

The data collection process required effective upfront communication with landowners and grazing permittees, which necessitated additional time and resources. All upfront preparation to conduct fieldwork for this project—such as coordination and communication with landowners and grazing permittees, and obtaining permits from State, County, and Federal agencies_- took a tremendous amount of time, but paid off with good will and cooperation from stakeholders. Pre-field project planning, agency permitting and coordination, strategic media outreach, and focused, intensive communication with key stakeholders, especially the people living and working on the land require considerable time and resources. All involved with conducting this project grossly underestimated the time and frontloading of effort that the project required before fieldwork could even begin. Allowing more than ample time to receive all permits and permissions is a practical consideration for a large-scale project like this.

Any project this high profile in nature involving a complex matrix of stakeholders and land ownership needs to accommodate and fund this extra time and effort prior to and during fieldwork and other research activities. We also recommend that future research projects follow the landowner/grazing permittee coordination protocol developed herein, with the proviso that once initial coordination is established and project vehicle descriptions are known, that, with agreement by both parties, ongoing notifications of field activities are not always necessary, particularly on public lands. However, if ongoing notifications are requested, then accommodating that request to continue to generate good will and conservation relationships has clear, long-term, good-will benefits.

\section{Management Implications}

The management implications provided here are based on our knowledge and experiences studying the jaguar in Mexico, and surveying and monitoring jaguars and ocelots in the United States. The following list of potential actions not only apply to the single known male jaguar in the United States, but also apply to species recovery which is important to a properly functioning ecosystem and to maintaining conservation and biodiversity goals in the United States and Mexico:

- All parties (nonprofits, governmental agencies) involved in jaguar and ocelot research and monitoring on both sides of the international border will benefit from sharing and incorporating their findings into a single report to examine the closest source population of jaguars (in Sonora and northern Sinaloa) to the United States.

- Science-based management will benefit from initiation of a survey protocol and long-term monitoring of travel corridors between the core source jaguar and ocelot populations in Sonora and the Southwestern United States.

- Initiation of future ocelot studies is warranted, and studies can be designed to detect and monitor ocelots in their habitat in the United States, and in predicted ocelot corridors between habitat in the United States and known breeding populations in Mexico. Studies would benefit from using information in this study to focus on camera placements that maximize felid detections within ocelot habitat.

- Public and private livestock management and research programs that promote and include synchronized breeding and calving periods timed to be sympatric with native prey birth pulses are beneficial to livestock-predator co-existence, and other depredation-intervention techniques, such as shifting to cattle breeds more resistant to predation, should also be encouraged.

- Research is needed on successful translocations of both sexes of ocelots and jaguars.

- Research is needed on prey selection/diet of ocelots and jaguars. 
- Native prey population (if below carrying capacity) improvements would benefit ocelots and jaguars in areas of detection and known core areas.

- Existing programs that pay landowners who provide useful jaguar and ocelot photo data, benefit wildlife conservation. We encourage the continuation or expansion of these programs on both sides of the border.

- Examples of conservation easement programs have provided tax savings for landowners and habitat protection for wildlife. Conservation easement programs that would establish new refugia with quality habitat for jaguars or ocelots can benefit conservation efforts for jaguar and ocelot.

- Landscape-scale conservation incentive programs in working landscapes (for example, active ranchlands) are effective because they provide financial support to landowners in return for best land-management practices (for example, onsite water-retention, grass-banking, reducing overgrazing).

- Continued jaguar monitoring in the survey area is warranted and is described in section, "Key Findings and Future Recommendations.”

- The use of large, comprehensive photo datasets of wildlife to examine the effects of land use, human impacts, climate change, and other variables on wildlife has positive impacts; this use is encouraged.

\section{References Cited}

Arizona Game and Fish Department, 2012, Arizona’s State Wildlife Action Plan-2012-2022: Arizona Game and Fish Department, 233 p.

Balme, G.A., Slotow, R., and Hunter, L.T.B., 2009, Impact of conservation interventions on the dynamics and persistence of a persecuted leopard (Panthera pardus) population: Biological Conservation, v. 142, no. 11, p. 2681-2690.

Bashir, T., Bhattacharya, T., Poudyal, K., Sathyakumar, S., and Qureshi, Q., 2013, Estimating leopard cat Prionailurus bengalensis densities using photographic captures and recaptures: Wildlife Biology, v. 19, p. 462-472.

Bird, T.J., Bates, A.E., Lefcheck, J.S., Hill, N.A., Thomson, R.J., Edgar, G.J., Stuart-Smith, R.D., Wotherspoon, S., Krkosek, M., Stuart-Smith, J.F., Pecl, G.T., Barrett, N., and Frusher, S., 2014, Statistical solutions for error and bias in global citizen science datasets: Biological Conservation v. 173, p. 144-154.

Blankenship, T.L., Haines, A.M., Tewes, M.E., and Silvy, N.J., 2006, Comparing survival and causespecific mortality between resident and transient bobcats Lynx rufus: Wildlife Biology, v. 12, no. 3, p. 297-303.

Brown, D.E., ed., 1994, Biotic communities_-southwestern United States and northwestern Mexico: Salt Lake City, University of Utah Press, 346 p.

Brown, D.E., and López González, C.A., 2001, Borderland jaguars—tigres de la frontera: Salt Lake City, University of Utah Press, 184 p.

Childs, J.L., 1998, Tracking the felids of the borderlands: El Paso, Tex., Printing Corner Press, 77 p.

Di Bitetti, M.S., Paviolo, A., and De Angelo, C., 2006, Density, habitat use and activity patterns of ocelots (Leopardus pardalis) in the Atlantic Forest of Misiones, Argentina: Journal of Zoology, v. 270, no. 1, p. 153-163.

Di Bitetti, M.S., Paviolo, A., and De Angelo, C., 2014, Camera trap photographic rates on roads vs. off roads-location does matter: Mastozoologia Neotropical, v. 21, p. 37-46. 
Dillon, A., and Kelly, M.J., 2008, Ocelot home range, overlap and density-comparing radio telemetry with camera trapping: Journal of Zoology, v. 275, no. 4, p. 391-398.

Eizirik, E., Kim, J-H., Menotti-Raymond, M., Crawshaw, P.G., Jr., O’Brien, S.J., and Johnson, W.E., 2001, Phylogeography, population history and conservation genetics of jaguars (Panthera onca, Mammalia, Felidae): Molecular Ecology, v. 10, no. 1, p. 65-79.

Elith, J., Graham, C.H., Anderson, R.P., Dudík, M., Ferrier, S., Guisan, A., Hijmans, R.J., Huettmann, F., Leathwick, J.R., Lehmann, A., Li, J., Lohmann, L.G., Loiselle, B.A., Manion, G., Moritz, C., Nakamura, M., Nakazawa, Y., Overton, J.McC.M., Peterson, A.T., Phillips, S.J., Richardson, K., Scachetti-Pereira, R., Schapire, R.E., Soberón, J., Williams, S., Wisz, M.S., and Zimmermann, N.E., 2006, Novel methods improve prediction of species' distributions from occurrence data: Ecography, v. 29, no. 2, p. 129-151.

Elith, J., Phillips, S.J., Hastie, T., Dudík, M., Chee, Y.E., and Yates, C.J., 2011, A statistical explanation of MaxEnt for ecologists: Diversity and Distributions, v. 17, no. 1, p. 43-57.

Elzinga, C.L., Salzer, D.W., and Willoughby, J.W., 2009, Monitoring plant and animal populations-a handbook for field biologists: Hoboken, N.J., Wiley, 368 p.

Emmons, L.H., Sherman, P., Bolster, D., Goldizen, A., and Terborgh, J., 1998, Ocelot behavior in moonlight, in Redford, K.H., Eisenberg, J.F., eds., Advances in neotropical mammalogy: Gainesville, Fla., Sandhill Crane Press, Inc., p. 233-242.

Ficetola, G.F., Thuiller, W., and Miaud, C., 2007, Prediction and validation of the potential global distribution of a problematic alien invasive species - the American bullfrog: Diversity and Distributions, v. 13, no. 4, p. 476-485.

Haines, A.M., Tewes, M.E., and Laack, L.L., 2005, Survival and sources of mortality in ocelots: Journal of Wildlife Management, v. 69, no.1, p. 255-263.

Hall, M.A., 1999, Correlation-based feature selection for machine learning: Hamilton, New Zealand, University of Waikato, Ph.D. dissertation, 178 p.

Harris, G., Thompson, R., Childs, J.L., and Sanderson, J.G., 2010, Automatic storage and analysis of camera trap data: Bulletin of the Ecological Society of America, v. 91, no. 3, p. 352, accessed June 2013 at http://www.esajournals.org/doi/abs/10.1890/0012-9623-91.3.352.

Hass, C.C., 2002, Home range dynamics of white-nosed coatis in southeastern Arizona: Journal of Mammalogy, v. 83, no. 4, p. 934-946.

Hatten, J.R., Averill-Murray, A., and Van Pelt, W.E., 2005, A spatial model of potential jaguar habitat in Arizona: Journal of Wildlife Management, v. 69, no. 3, p. 1024-1033.

Hernandez-Santin, L., 2007, Movements and range sizes of jaguars in Paraguay based on GPStelemetry: Alpine, Tex., Sul Ross State University, M.S. thesis, 109 p.

Hijmans, R.J., Cameron, S.E., Parra, J.L., Jones, P.G., and Jarvis, A., 2005, Very high resolution interpolated climate surfaces for global land areas: International Journal of Climatology, v. 25, no. 15, p. 1965-1978.

Hofmeister, Erik, Rogall, G.M., Wesenberg, K., Abbott, R., Work, T., Schuler, K., Sleeman, J., and Winton, J., 2010, Climate change and wildlife health—direct and indirect effects: U.S. Geological Survey Fact Sheet 2010-3017, 4 p.

Karanth, K.U., Nicholos, J.D., and Kumar, N.S., 2004, Photographic sampling of elusive mammals in tropical forests, in Thompson, W.L., ed., Sampling rare or elusive species: Washington, D.C., Island Press, p. 229-247. 
Kays, R.C., and McKelvey, K.S., 2008, Remote cameras, in Long, R.A., MacKay, P., Zielinski, W.J., and Ray, J.C., eds., Noninvasive survey methods for carnivores: Washington, D.C., Island Press, p. 110-140.

Kozlov, D., 2009, ReNAmer’s user manual: SnapFiles Web site, accessed June 2015 at http://www.snapfiles.com/downloads/denReNamer/dldenReNamer.html.

Krebs, C.J., 1999, Ecological methodology (2d ed.): Menlo Park, Calif., Addison-Wesley Educational Publishers, Inc., 620 p.

Long, R.A., MacKay, P., Zielinski, W.J., and Ray, J.C., 2008, Noninvasive survey methods for carnivores: Washington, D.C., Island Press, 400 p.

McCain, E.B., and Childs, J.L., 2008, Evidence of resident jaguars (Panthera onca) in the southwestern United States and the implications for conservation: Journal of Mammalogy, v. 89, p. 1-10.

Menke, K.A., and Hayes, C.L., 2003, Evaluation of the relative suitability of potential jaguar habitat in New Mexico: New Mexico Department of Game and Fish, 31 p.

Merow, C., Smith, M.J., and Silander, J.A., Jr., 2013, A practical guide to MaxEnt for modeling species' distributions—what it does, and why inputs and settings matter: Ecography, v. 36, p. 10581069.

Moreno, J., Medellín, R.A., Cassaigne, I., Valdez, R., Alcumbrac, O., Galaz, M., Thompson, R., Childs, J.L., Smith, N., Dieterich, S., Shallcross, K., Culver, M., and Sanderson, J. G., 2013, Coexistence of four felids in the Sierra Madre Mountains of northeastern Sonora, Mexico: Wild Felid Monitor, v. 6, no. 1, p. 19-20.

Morell, V., 2011, Scat-sniffing dogs nose out clues to caribou decline: ScienceMag, June 22, 2011, accessed June 2013 at http://news.sciencemag.org/2011/06/scat-sniffing-dogs-nose-out-clues-cariboudecline.

Nature Conservancy, 2006, Biotic communities of the southwest GIS layer: Nature Conservancy’s Center for Science and Public Policy Web site, accessed May 2016 at http://azconservation.org/downloads/biotic_communities_of_the_southwest_gis_data.

Nuñez Pérez, R., 2006, Área de actividad, patrones de actividad y movimiento del jaguar (Panthera onca) y del puma (Puma concolor), en la Reserva de la Biosfera “Chamela-Cuixmala,” Jalisco: México, D.F., Universidad Nacional Autónoma de México, M.S. thesis.

Peterson, A.T., and Vieglais, D.A., 2001, Predicting species invasions using ecological niche modeling - new approaches form bioinformatics attack a pressing problem: BioScience, v. 51, no. 5, p. 363-371.

Phillips, S.J., Anderson, R.P., and Schapire, R.E., 2006, Maximum entropy modeling of species geographic distributions: Ecological Modelling, v. 190, nos. 3-4, p. 231-259.

Phillips, S.J., Dudík, M., Elith, J., Graham, C.H., Lehmann, A., Leatherwick, J., and Ferrier, S., 2009, Sample selection bias and presence-only distribution models-implications for background and pseudo-absence data: Ecological Applications, v. 19, no. 1, p. 181-197.

Rabinowitz, A., and Nottingham, B.G., Jr., 1986, Ecology and behavior of the jaguar (Panthera onca) in Belize, Central America: Journal of Zoology, v. 210, no. 1, p. 149-159.

Raxworthy, C.J., Martinez-Meyer, E., Horning, N., Nusssbaum, R.A., Schneidere, G.E., Ortega-Huerta, M.A., and Peterson, A.T., 2003, Predicting distributions of known and unknown reptile species in Madagascar: Nature, v. 426, p. 837-841.

Riley, S.J., DeGloria, S.D., and Elliot, R., 1999, A terrain ruggedness index that quantifies topographic heterogeneity: Intermountain Journal of Sciences, v. 5, p. 23-27. 
Rinkevich, S.E., 2012, Population size, diet, and cultural significance of Mexican gray wolves in Arizona: Tucson, Ariz., University of Arizona, Ph.D. dissertation, 290 p.

Robinson, M.J., Bradley, C., and Boyd, J., 2006, Potential habitat for jaguars in New Mexico: Report to Arizona Game and Fish Department from the Center for Biological Diversity, 26 p.

Rodríguez-Soto, C., Monroy-Vilchis, O., Maiorano, L., Boitani, L., Faller, J.C., Briones, M.A., Núñez, R., Rosas-Rosas, O., Ceballos, G., and Falcucci, A., 2011, Predicting potential distribution of the jaguar in Mexico—identification of priority areas for conservation: Diversity and Distributions, v. 17, no. 2, p. 350-361.

Rutledge, L., Holloway, J.J., Patterson, B.R., and White, B.N., 2009, An improved field method to obtain DNA for individual identification from wolf scat: Journal of Wildlife Management, v. 73, no. 8, p. $1430-1435$.

Sanderson, E.W., and Fisher, K., 2013, Jaguar habitat modeling and database update: U.S. Fish and Wildlife Service and Wildlife Conservation Society, 42 p.

Silver, S.C., Ostro, L.E.T., Marsh, L.K., Maffei, L., Noss, A.J., Kelly, M.J., Wallace, R.B., Gómez, H., and Ayala, G., 2004, The use of camera traps for estimating jaguar (Panthera onca) abundance and density using capture/recapture analysis: Oryx, v. 38, no. 2, p. 148-154.

Smith, D.R., Villella, R.F., and Lemarie, D.P., 2003, Application of adaptive cluster sampling to lowdensity populations of freshwater mussels: Environmental and Ecological Statistics, v. 10, p. 7-15.

Soisalo, M.K., and Cavalcanti, S.M.C., 2006, Estimating the density of a jaguar population in the Brazilian Pantanal using camera-traps and capture-recapture sampling in combination with GPS radio-telemetry: Biological Conservation, v. 129, no. 4, p. 487-496.

Sunquist, M.E., and Sunquist. F.C., 2002, Wild cats of the world: Chicago, University of Chicago Press, $416 \mathrm{p}$.

Sweanor, L.L., Logan, K.A., and Hornocker, M.G., 2000, Cougar dispersal patterns, metapopulation dynamics, and conservation: Conservation Biology, v. 14, no. 3, p. 798-808.

Thapa, K., Kelly, M.J., Karki, J.B., and Subedi, N., 2013, First camera trap record of pack hunting dholes in Chitwan National Park, Nepal [distribution update]: Canid Biology and Conservation, v. 16, p. 4-7.

Trolle, M., and Kery, M., 2005, Camera-trap study of ocelot and other secretive mammals in the northern Pantanal: Mammalia, v. 69, nos. 3-4, p. 405-412.

U.S. Fish and Wildlife Service, 2012, Endangered and threatened wildlife and plants; designation of critical habitat for jaguar; proposed rule: Federal Register, v. 77, no. 161, p. 50214-50242.

U.S. Fish and Wildlife Service, 2014, Endangered and threatened wildlife and plants; designation of critical habitat for jaguar; final rule: Federal Register, v. 79, no. 43, p. 12572-12654.

Warren, D.L., Glor, R.E., and Turelli, M., 2010, ENMTools-a toolbox for comparative studies of environmental niche models: Ecography, v. 33, no. 3, p. 607-611.

Warren, D.L., and Seifert, S.N., 2011, Ecological niche modeling in Maxent-the importance of model complexity and the performance of model selection criteria: Ecological Applications, v. 21, no. 2, p. 335-342.

Wasser, S.K., Keim, J.L., Taper, M.L., and Lele, S.R., 2011, The influences of wolf predation, habitat loss, and human activity on caribou and moose in the Alberta oil sands: Frontiers in Ecology and the Environment, v. 9, no. 10, p. 546-551. 
Weingarth, K., Heibl, C., Knauer, F., Zimmermann, F., Bufka, L., and Heurich, M., 2012, First estimation of Eurasian lynx (Lynx lynx) abundance and density using digital cameras and capturerecapture techniques in a German national park: Animal Biodiversity and Conservation, v. 35, no. 2, p. 197-207.

Western Regional Climate Center, 2012, Arizona climate summaries: Western Regional Climate Center Web site, accessed April 2012 at http://www.wrcc.dri.edu/summary/azF.html.

Wildlife Conservation Society and Center for International Earth Science Information Network, 2005, Last of the Wild Project, version 2, 2005 (LWP-2): Global Human Influence Index (HII) Dataset (Geographic), NASA Socioeconomic Data and Applications Center (SEDAC), accessed June 2013 at http://sedac.ciesin.columbia.edu/data/set/wildareas-v2-human-influence-index-geographic.

Wilson, K.R., and Anderson, D.R., 1985, Evaluation of two density estimators of small mammal population size: Journal of Mammalogy, v. 66, p. 13-21.

Young, G.C., 2011, Rare jaguar treed by hunter in Arizona: Outdoor Life, November 29, 2011, accessed June 2013 at http://www.outdoorlife.com/blogs/newshound/2011/11/jaguar-spotted-first-time-twoyears-arizona.

Zar, J.H., 2010, Biostatistical Analysis (5th ed.): Upper Saddle River, N.J., Pearson Prentice-Hall, $944 \mathrm{p}$. 


\section{Figures}

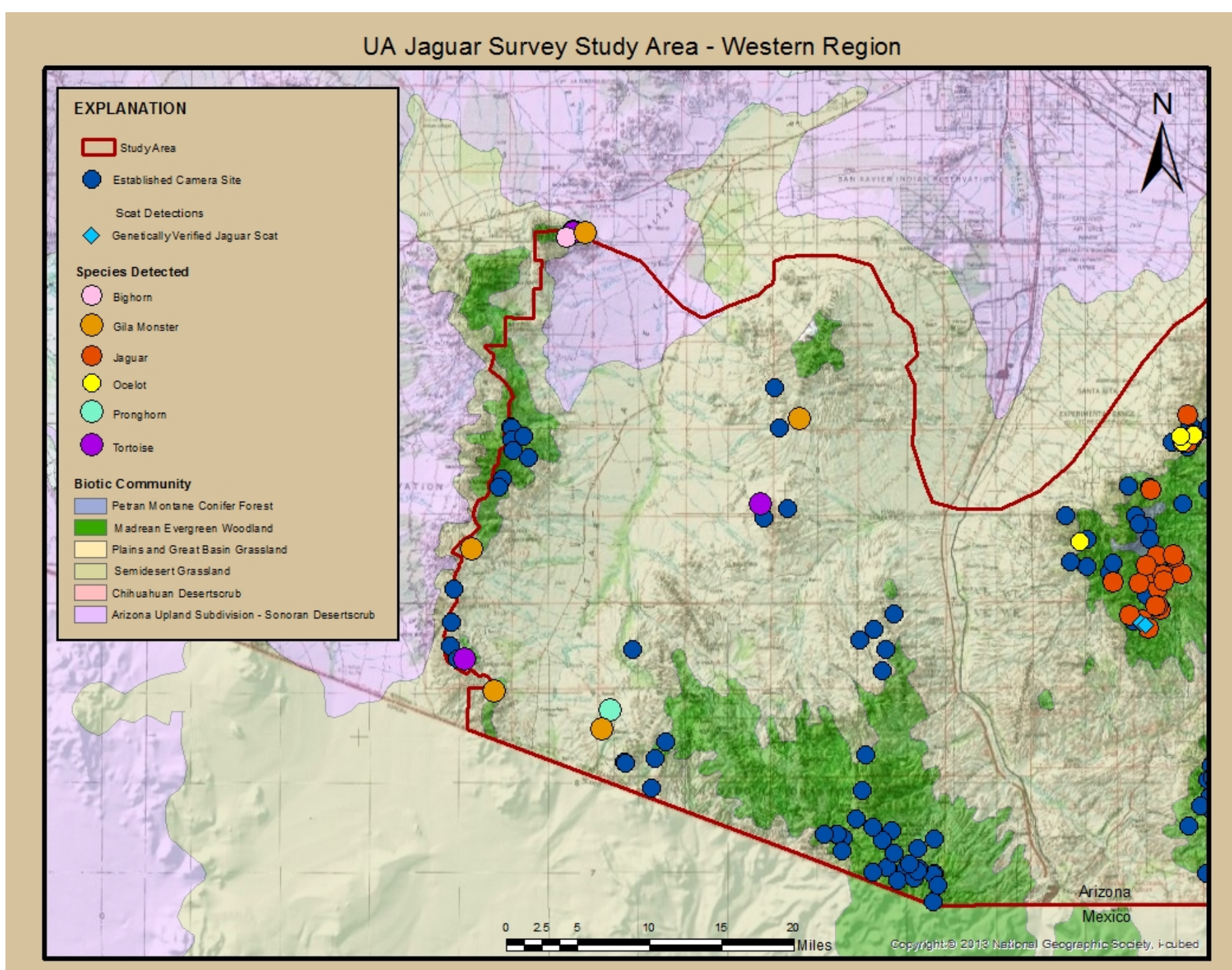

Figure 1a. Map showing jaguar survey and monitoring western study area (west of Interstate 19)—north of the United States-Mexico Border, south of Interstate 10. Map image from National Geographic Society used under Esri Master License Agreement \#102282. 


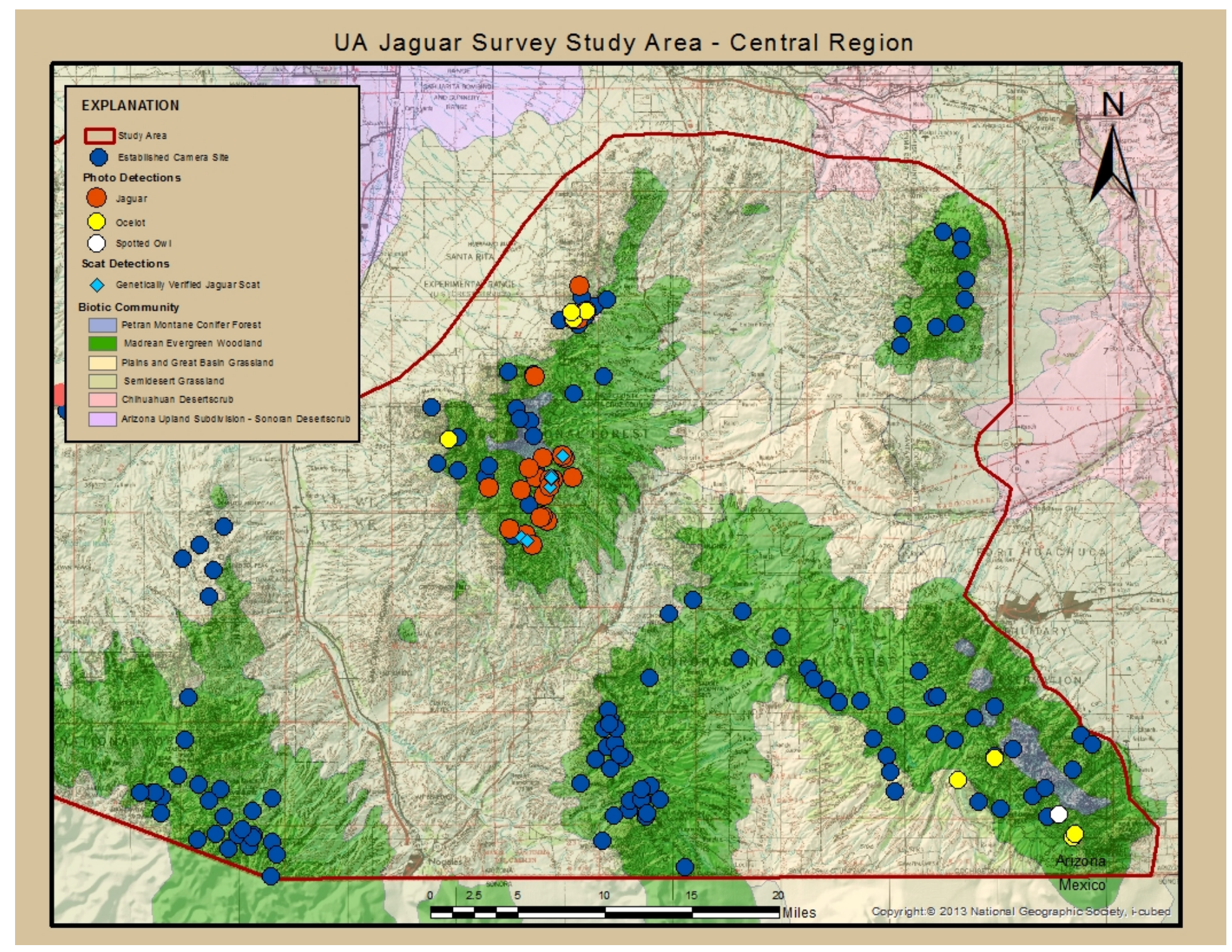

Figure 1b. Map showing jaguar survey and monitoring western study area (east of Interstate 19)-north of the United States-Mexico Border, south of Interstate 10. Map image from National Geographic Society used under Esri Master License Agreement \#102282. 


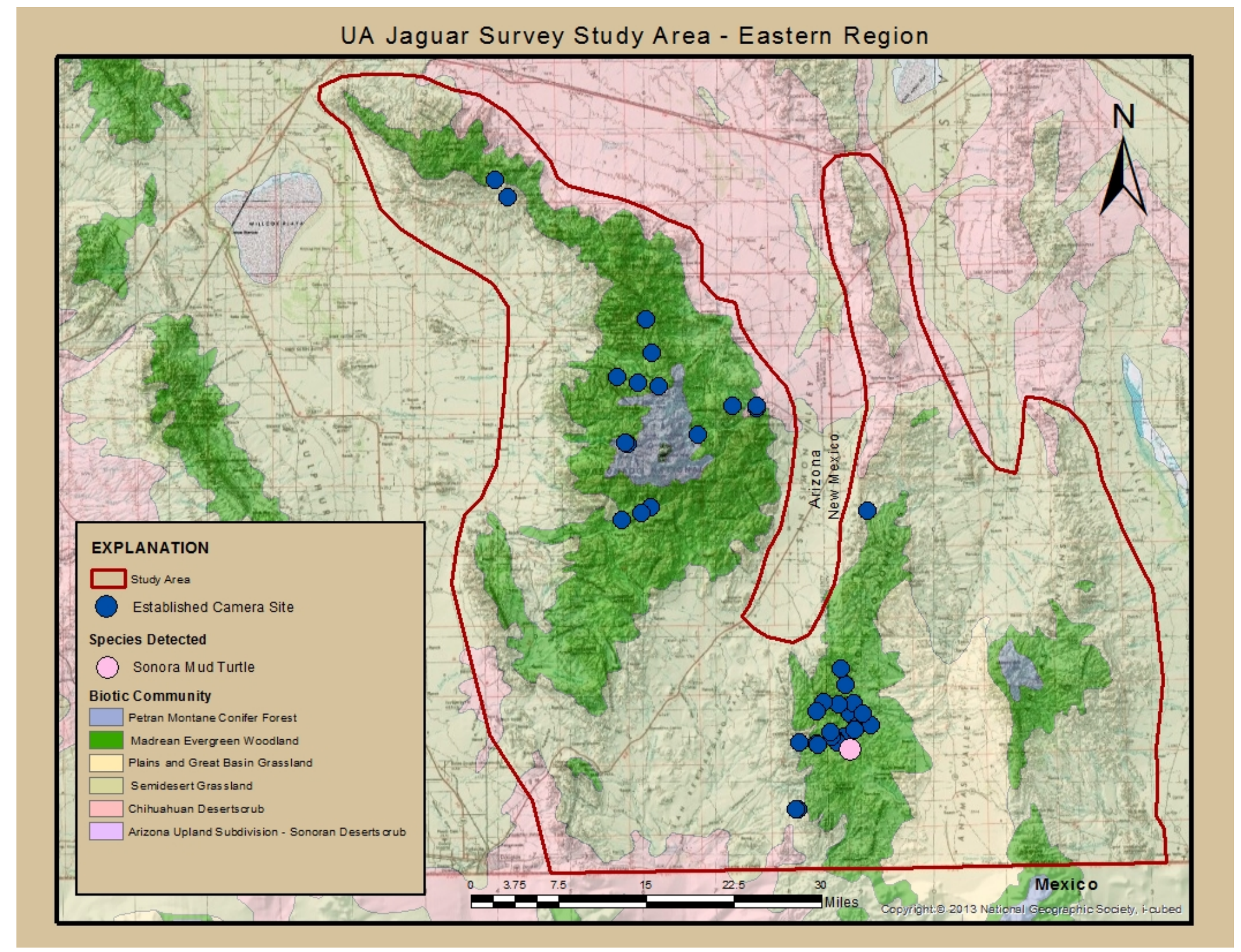

Figure 1c. Map showing aguar survey and monitoring eastern study area-North of the United States-Mexico Border, south of Interstate 10. Map image from National Geographic Society used under Esri Master License Agreement \#102282. 


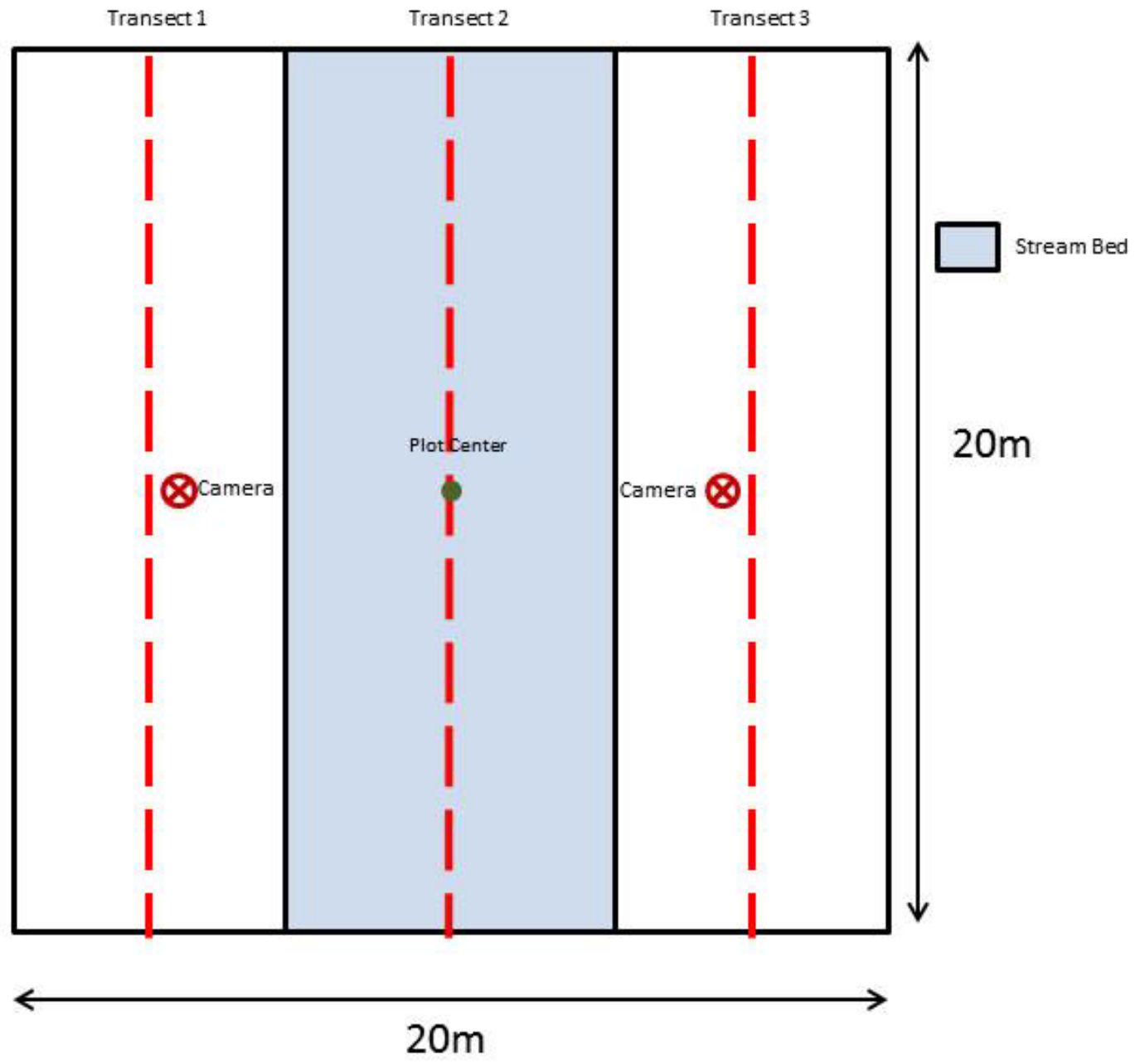

Figure 2. Diagram showing layout of transects for sampling microhabitat features at jaguar and ocelot detection sites. ( $m$, meters.) 


\section{Atascosa Mountains \\ Effort $=\mathbf{5}$ sites for $\mathbf{3 7 5 1}$ camera days}

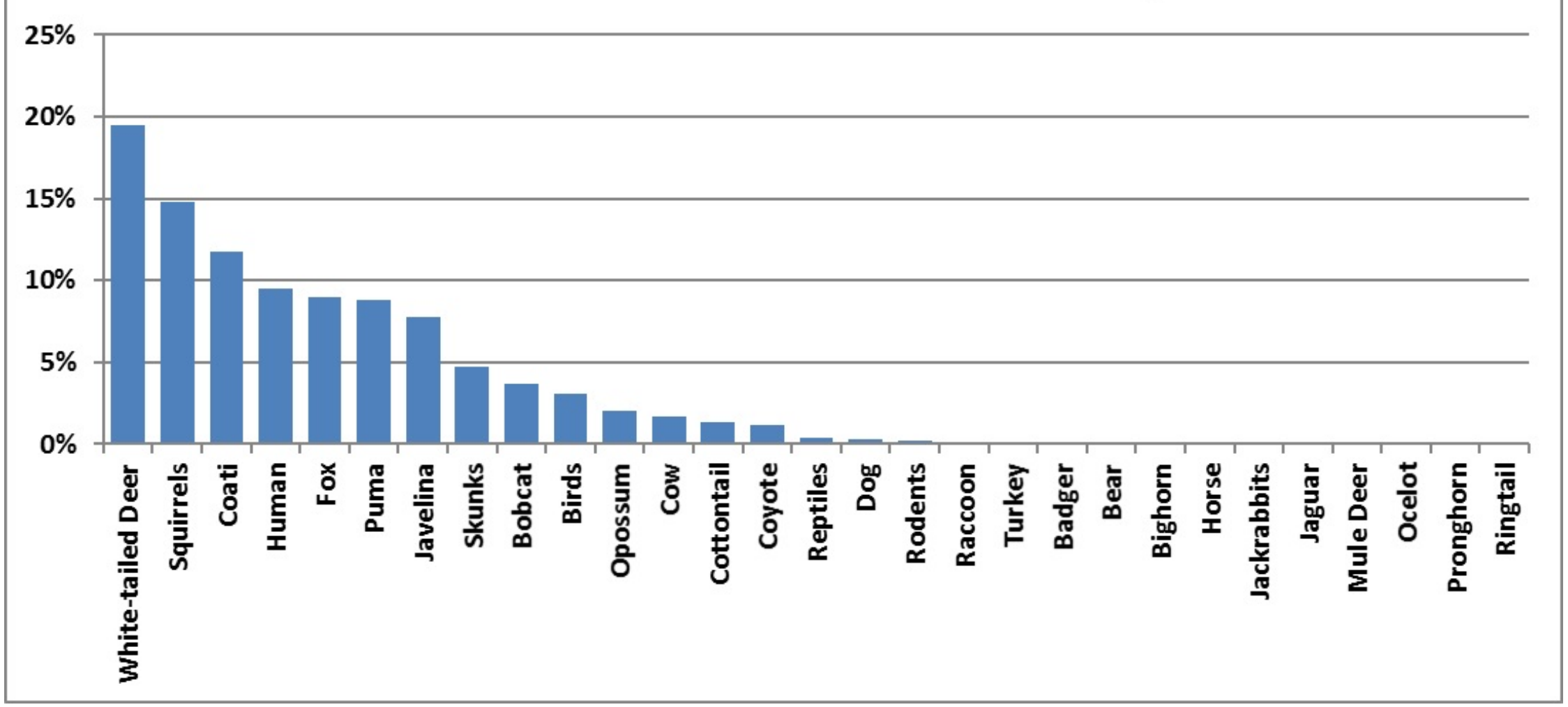

Figure 3. Species composition in the Atascosa Mountain range as detected by trail cameras from April 2012 to February 2015. Squirrels are separated from the rodent species category, and turkeys are separated from the bird species category. 


\section{Baboquivari Mountains \\ Effort $=17$ sites for 6,761 camera days}

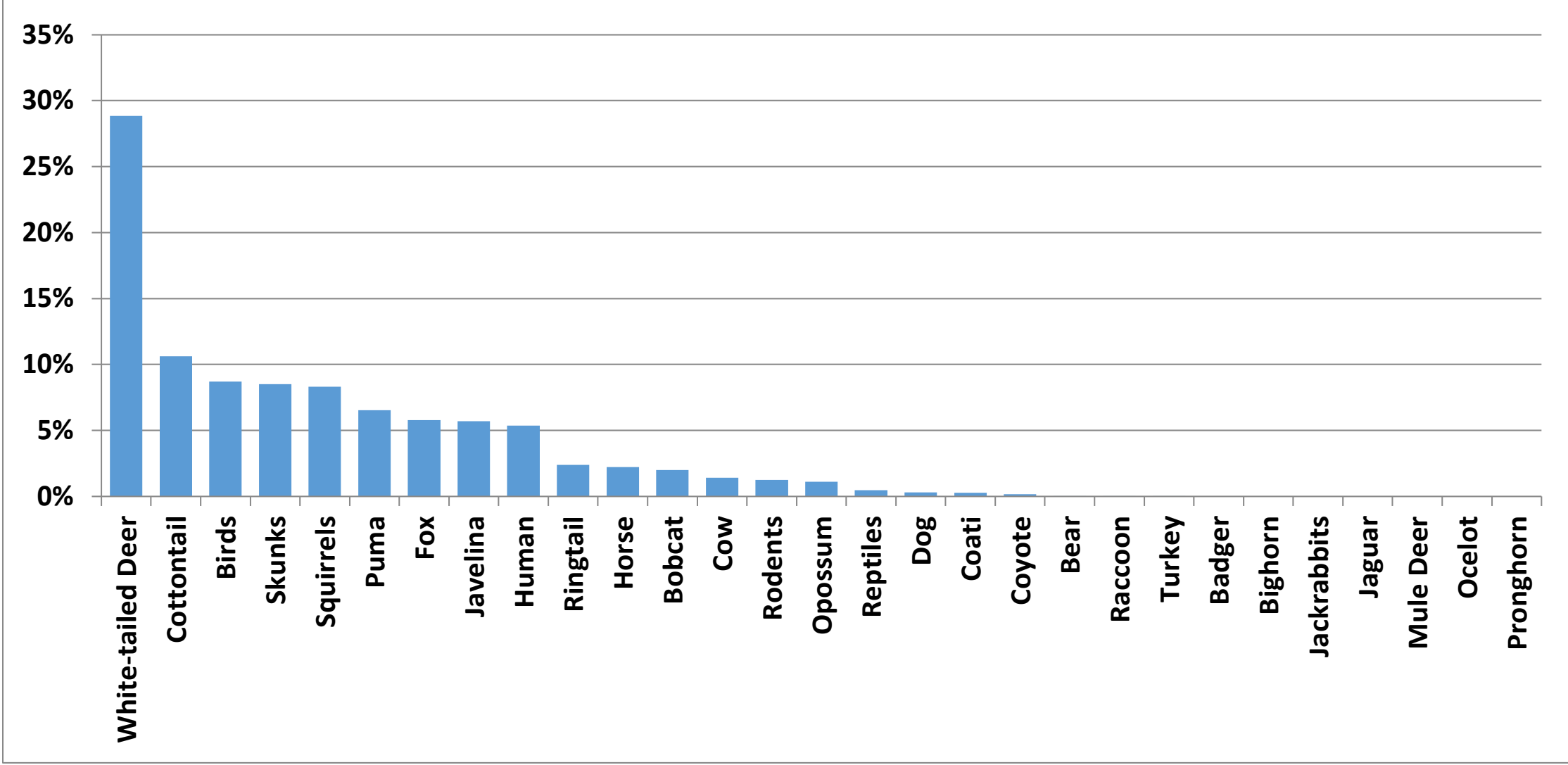

Figure 4. Species composition in the Baboquivari Mountain range as detected by trail cameras from April 2012 to February 2015. Squirrels are separated from the rodent species category, and turkeys are separated from the bird species category. 


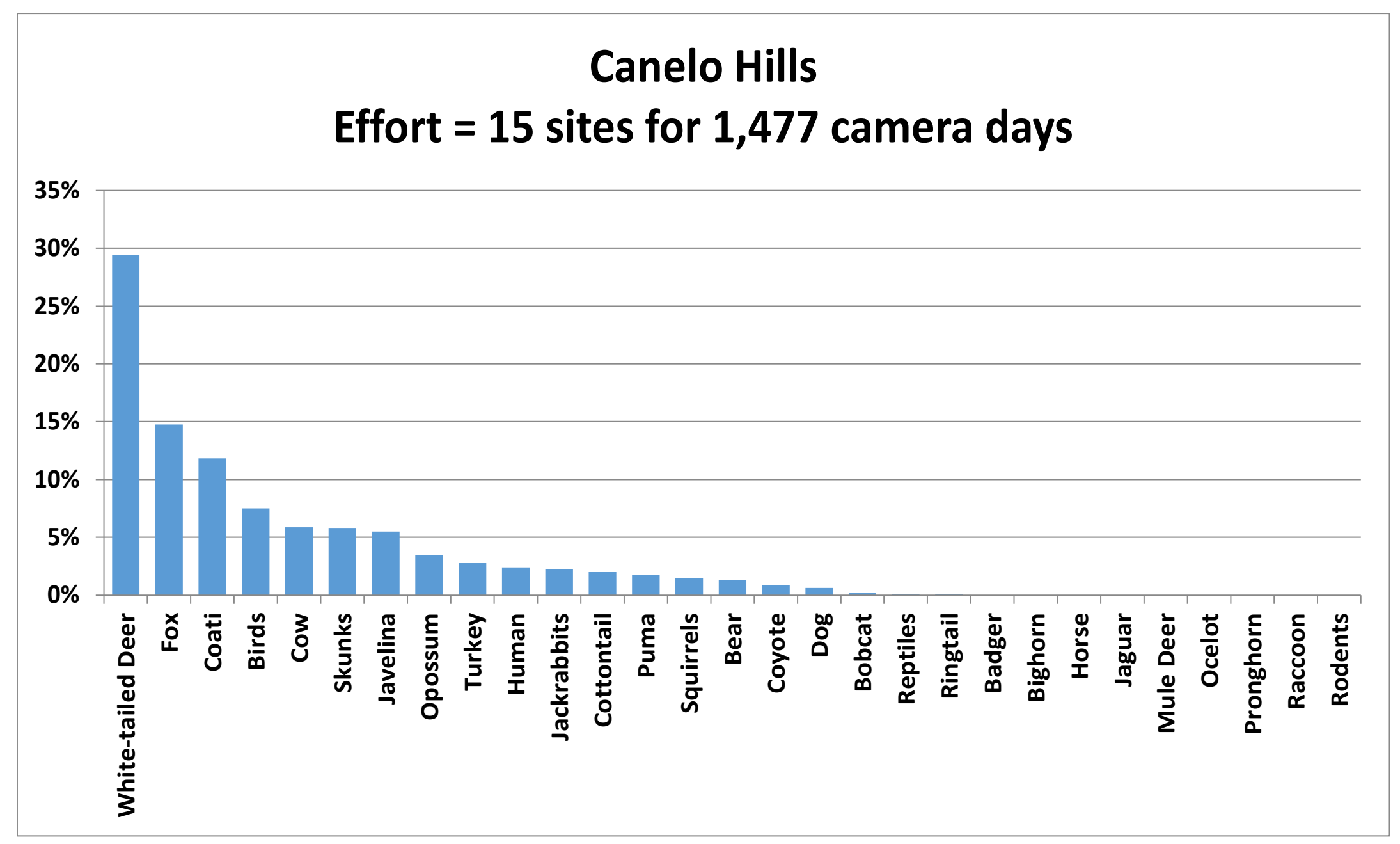

Figure 5. Species composition in the Canelo Hills as detected by trail cameras from April 2012 to February 2015. Squirrels are separated from the rodent species category, and turkeys are separated from the bird species category. 


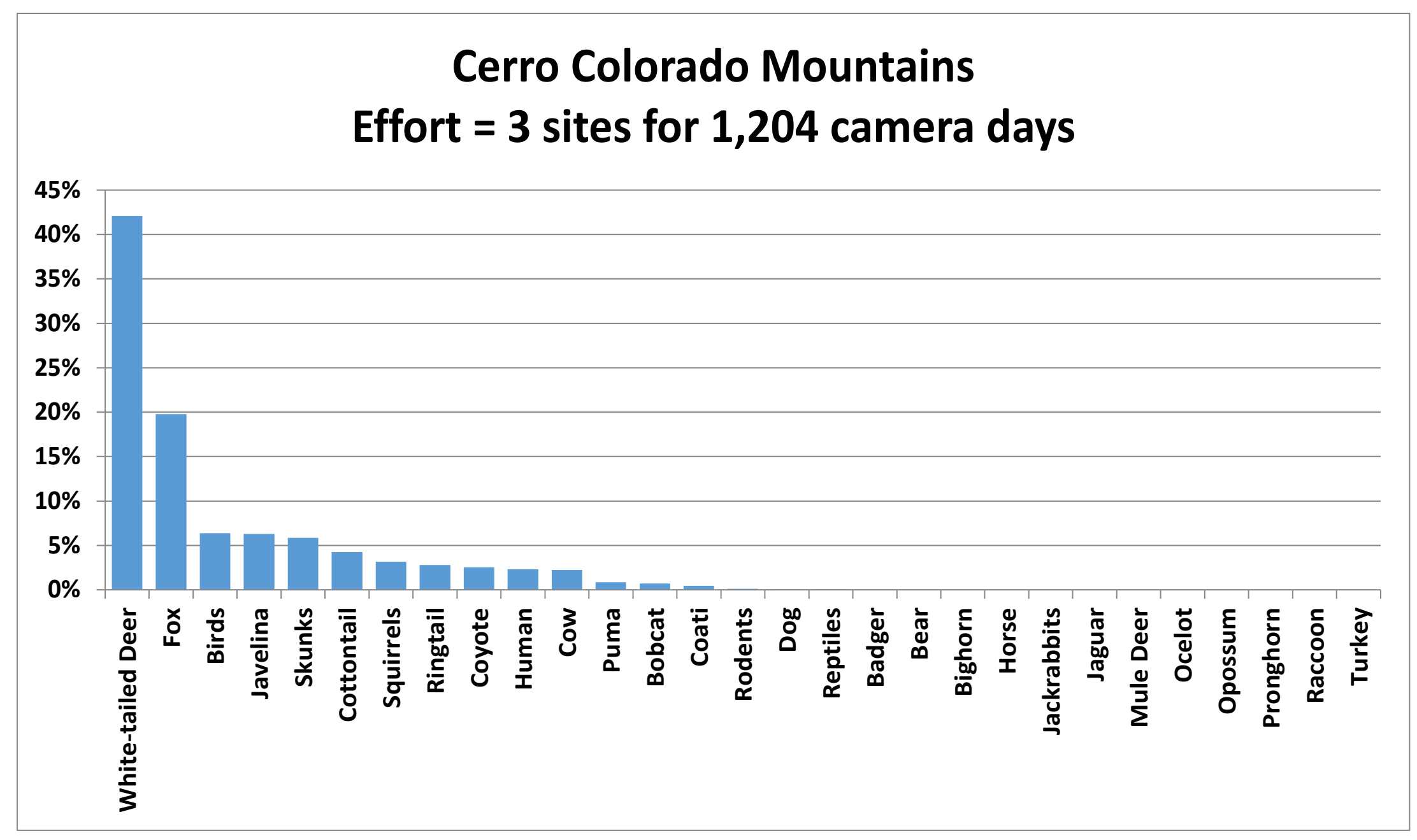

Figure 6. Species composition in the Cerro Colorado Mountain range as detected by trail cameras from April 2012 to February 2015. Squirrels are separated from the rodent species category, and turkeys are separated from the bird species category. 


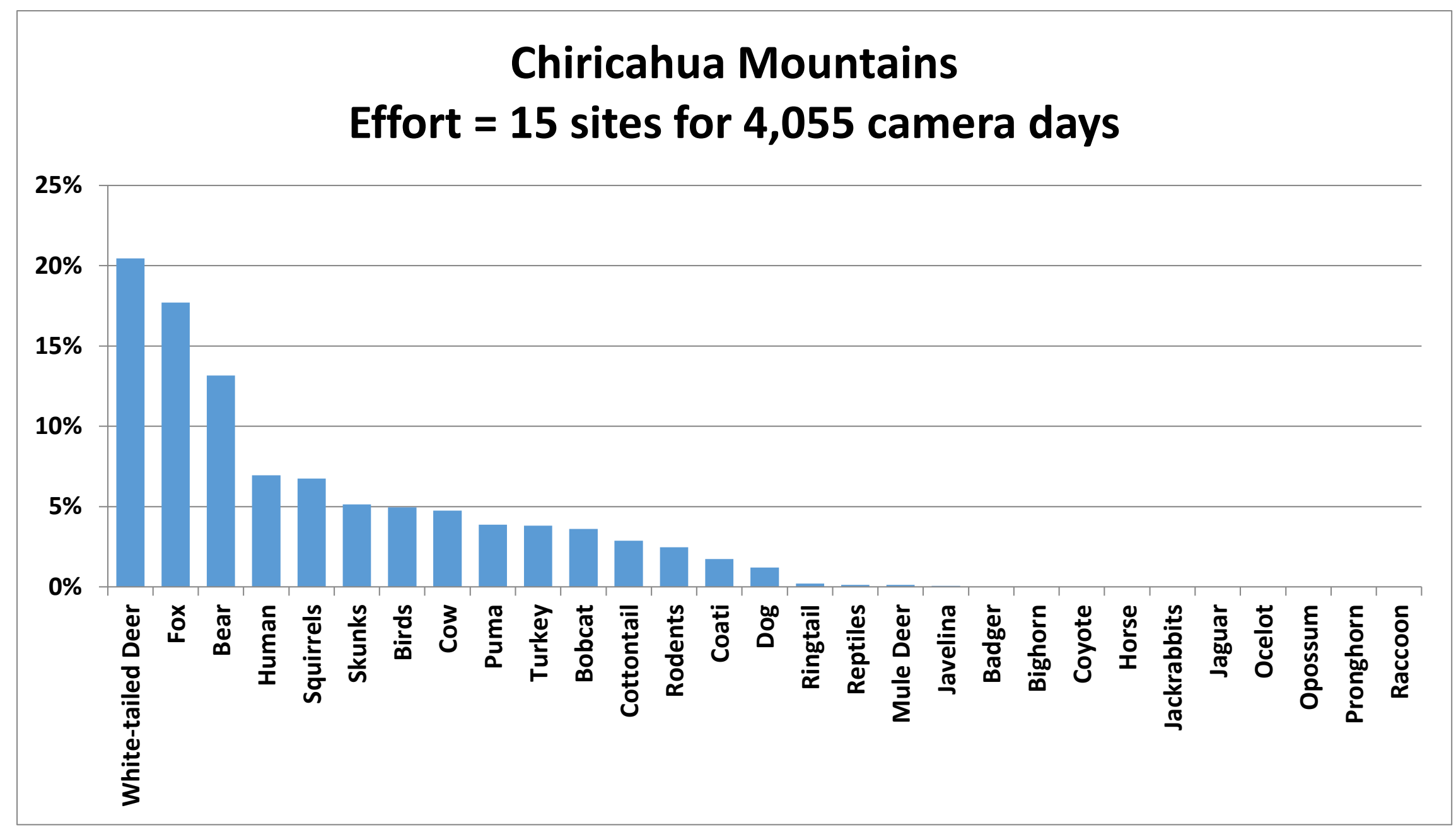

Figure 7. Species composition in the Chiricahua Mountain range as detected by trail cameras from April 2012 to February 2015. Squirrels are separated from the rodent species category, and turkeys are separated from the bird species category. 


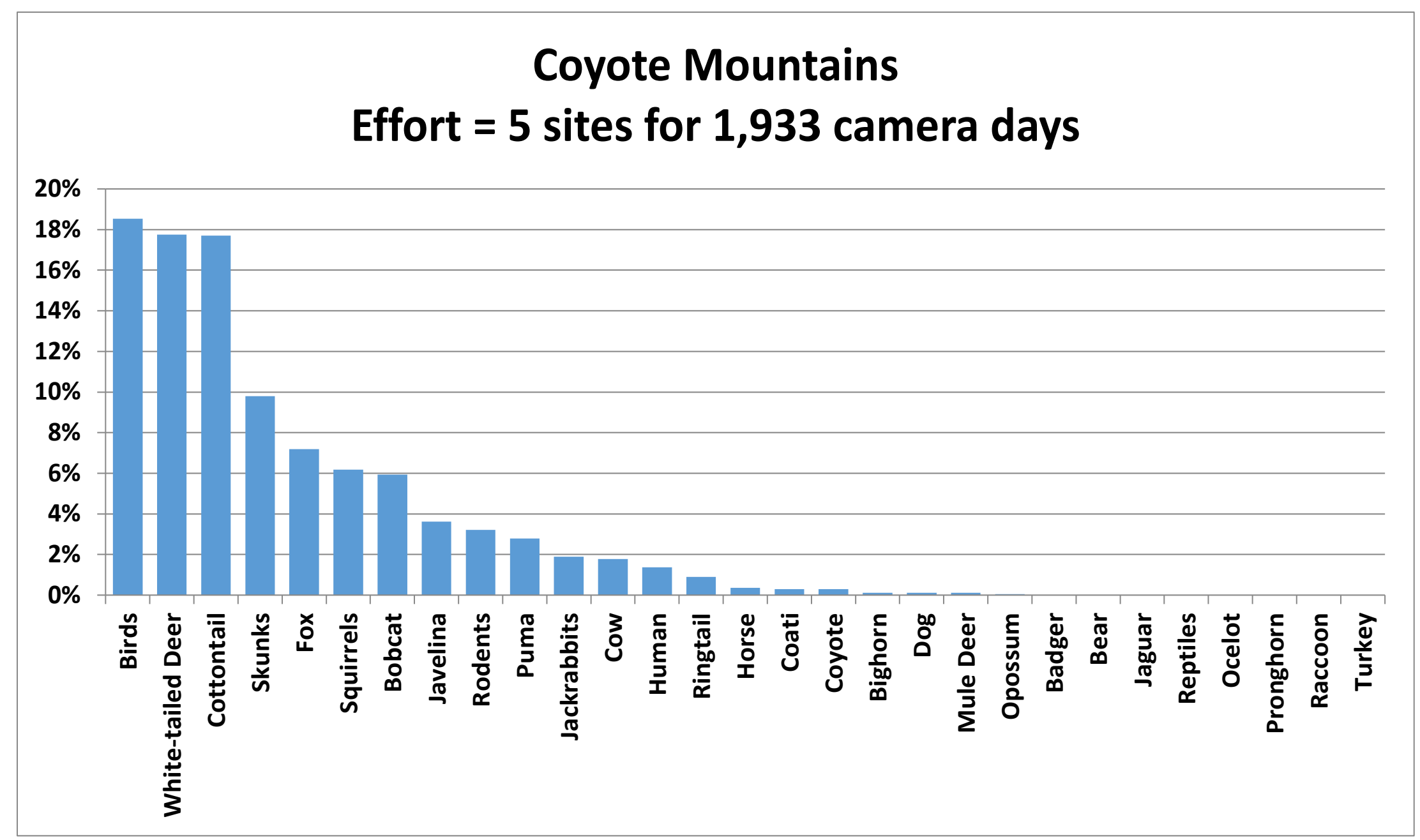

Figure 8. Species composition in the Coyote Mountain range as detected by trail cameras from April 2012 to February 2015. Squirrels are separated from the rodent species category, and turkeys are separated from the bird species category. 


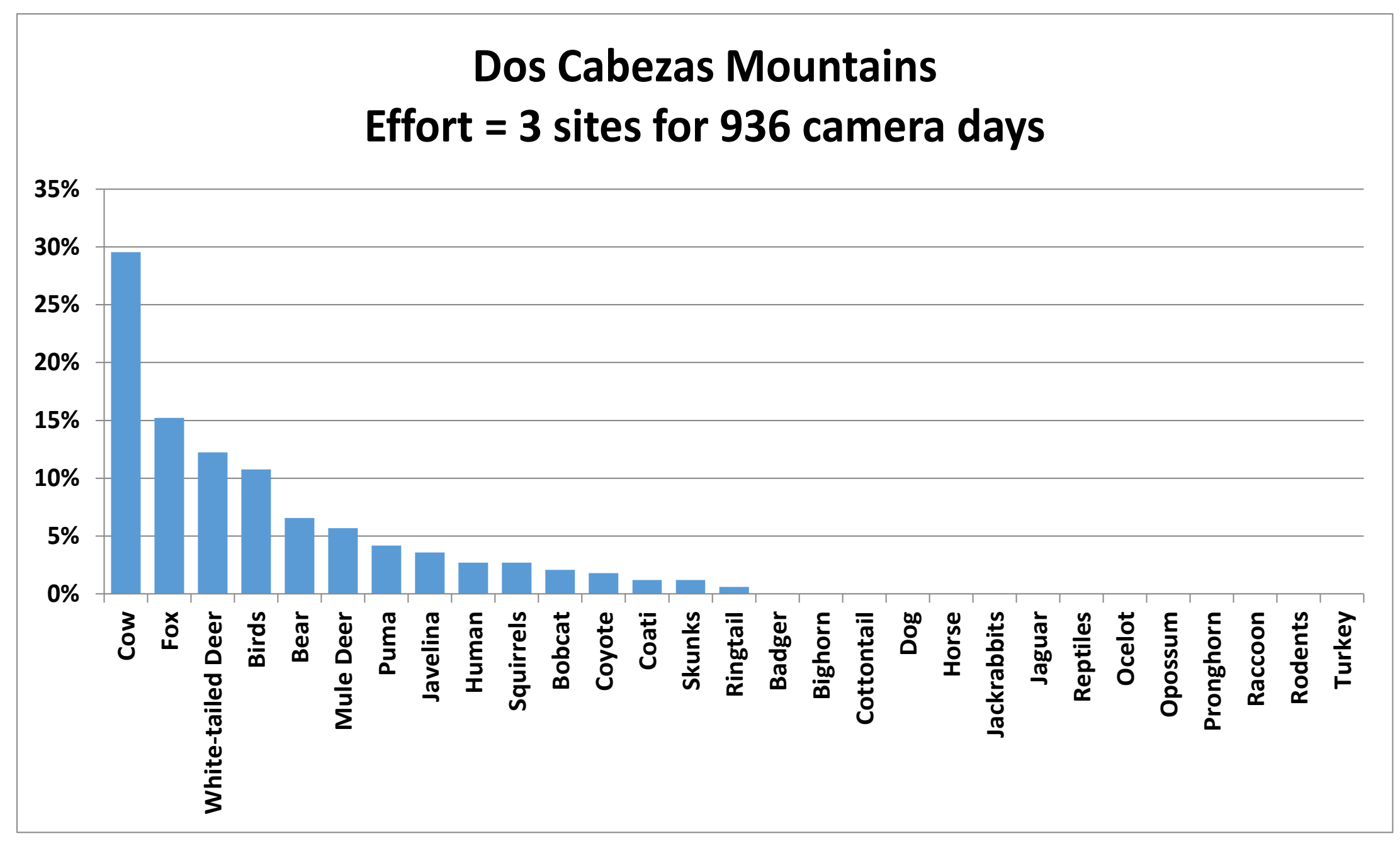

Figure 9. Species composition in the Dos Cabezas Mountain range as detected by trail cameras from April 2012 to February 2015. Squirrels are separated from the rodent species category, and turkeys are separated from the bird species category. 


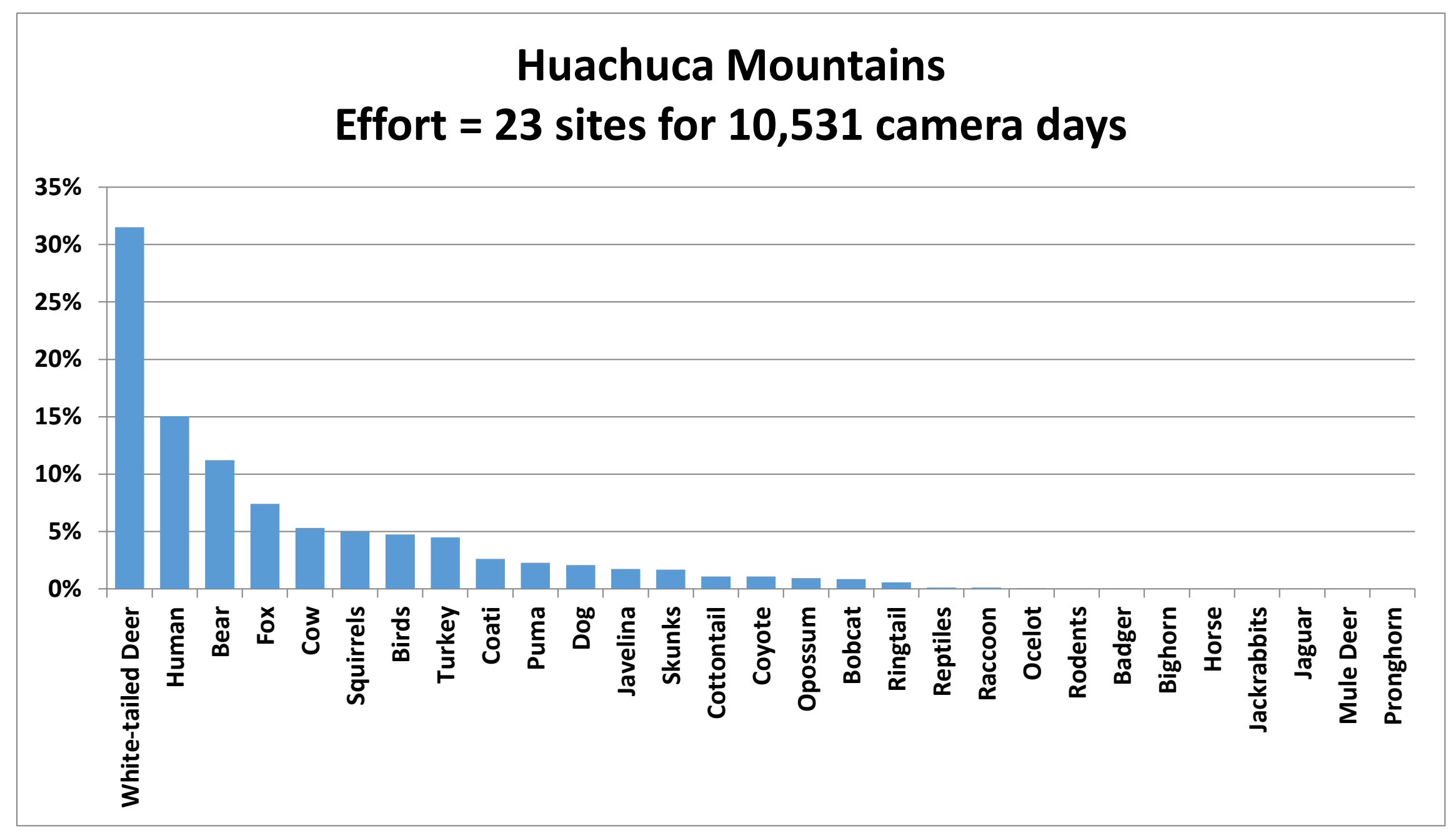

Figure 10. Species composition in the Huachuca Mountain range as detected by trail cameras from April 2012 to February 2015. Squirrels are separated from the rodent species category, and turkeys are separated from the bird species category. 


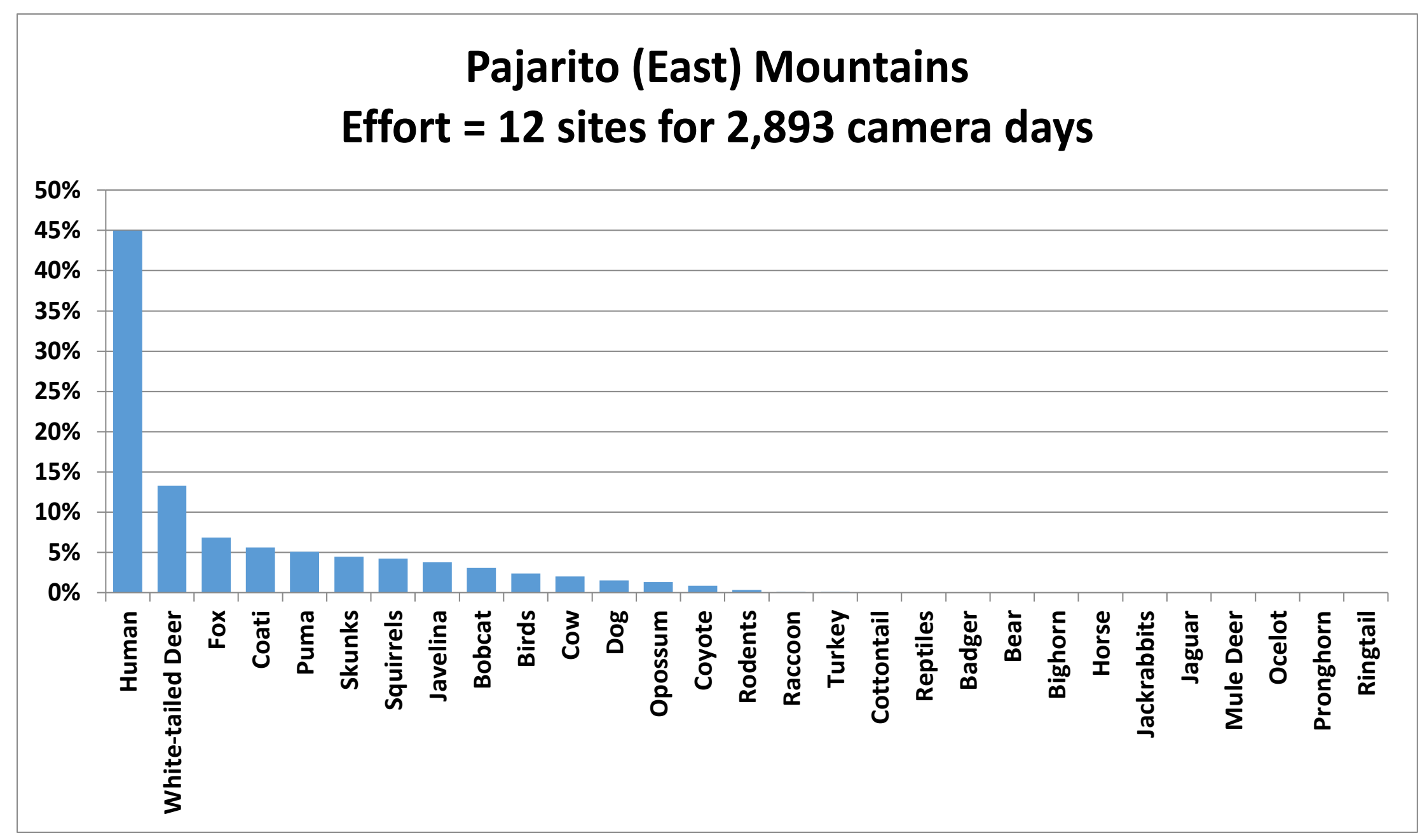

Figure 11. Species composition in the Pajarito (East) Mountain range as detected by trail cameras from April 2012 to February 2015 . Squirrels are separated from the rodent species category, and turkeys are separated from the bird species category. 


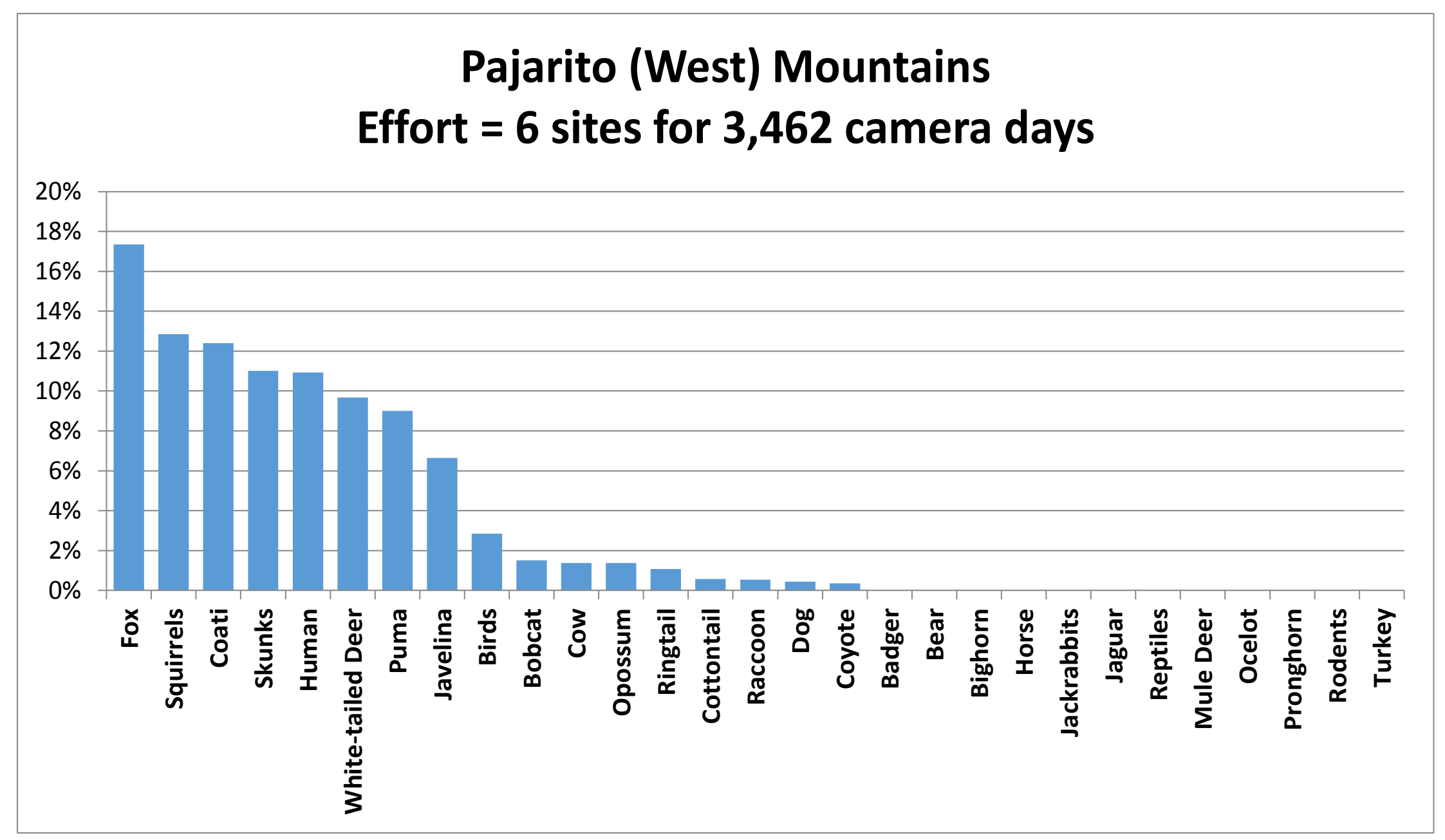

Figure 12. Species composition in the west Pajarito (West) Mountain range as detected by trail cameras from April 2012 to February 2015. Squirrels are separated from the rodent species category, and turkeys are separated from the bird species category. 


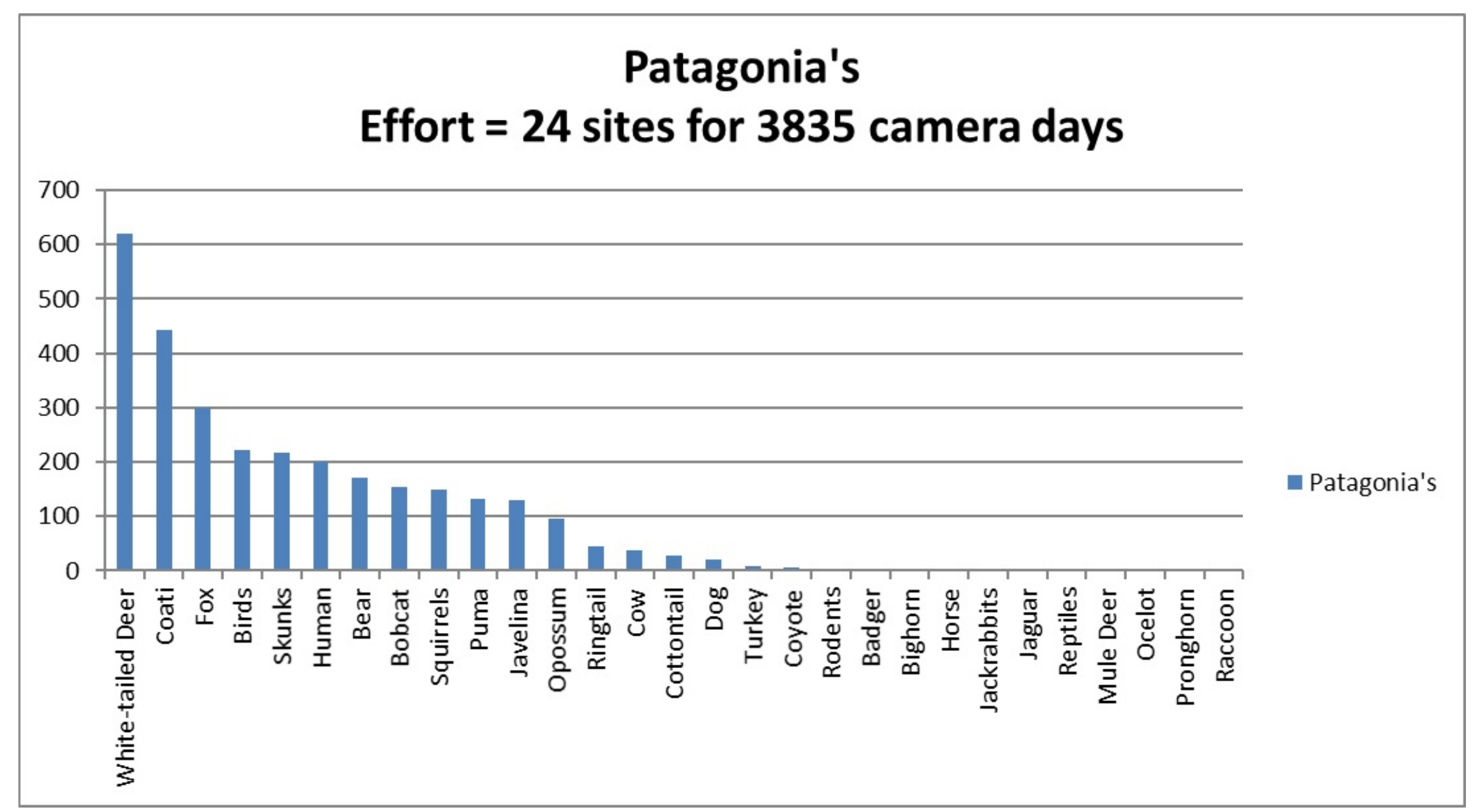

Figure 13. Species composition in the Patagonia Mountain range as detected by trail cameras from April 2012 to February 2015. Squirrels are separated from the rodent species category, and turkeys are separated from the bird species category. 


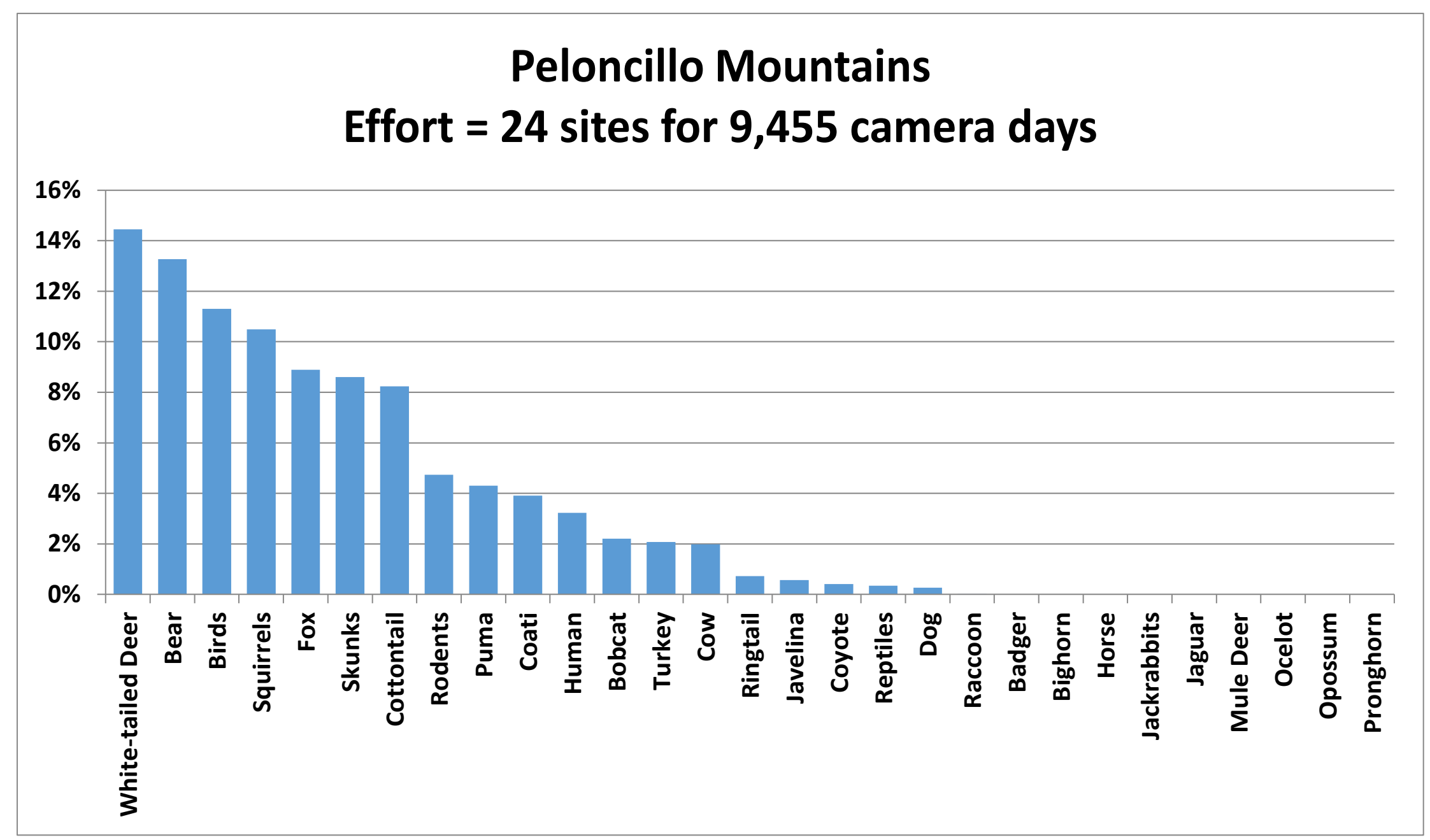

Figure 14. Species composition in the Peloncillo Mountain range as detected by trail cameras from April 2012 to February 2015. Squirrels are separated from the rodent species category, and turkeys are separated from the bird species category. 


\section{San Luis (West) Mountains Effort $=7$ sites for 2,963 camera days}

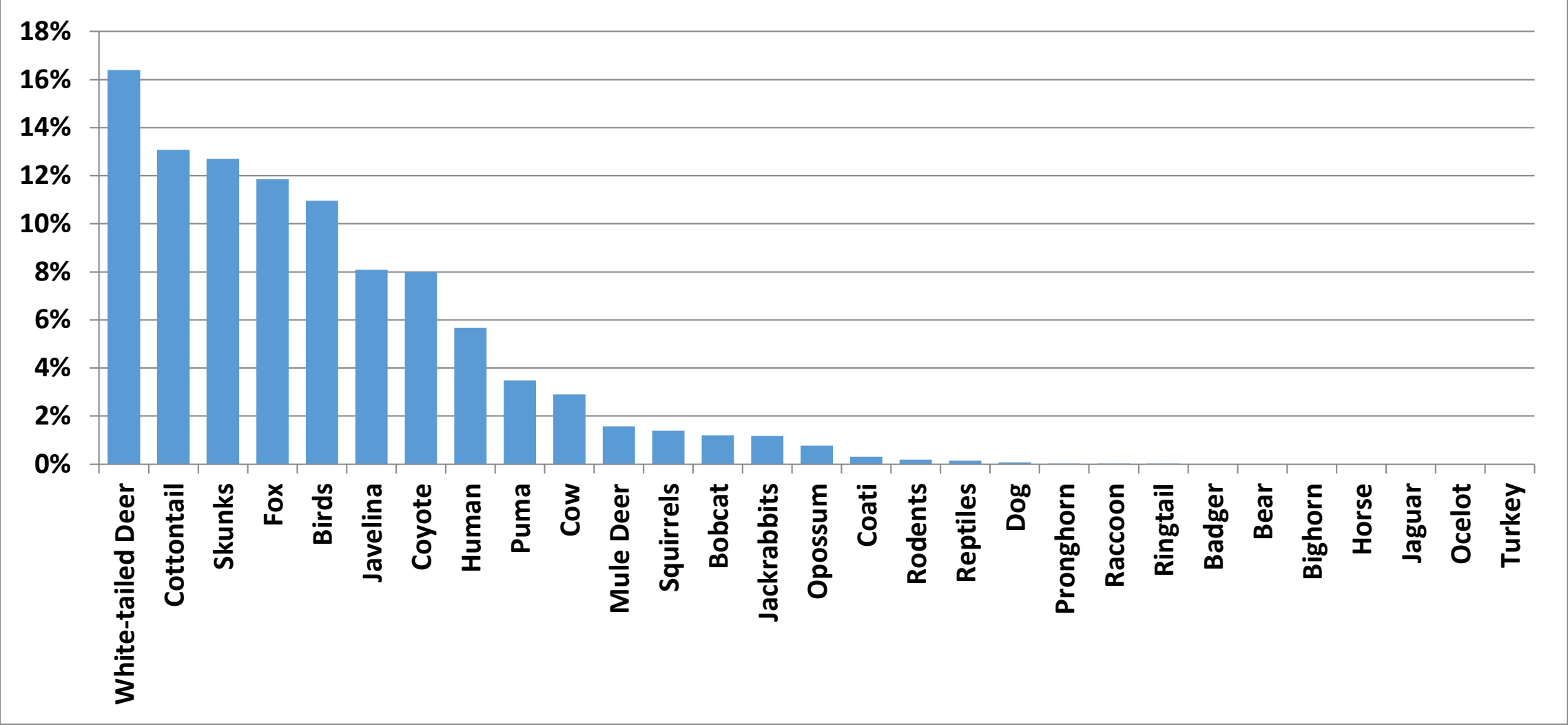

Figure 15. Species composition in the San Luis (West) Mountain range as detected by trail cameras from April 2012 to February 2015. Squirrels are separated from the rodent species category, and turkeys are separated from the bird species category. 


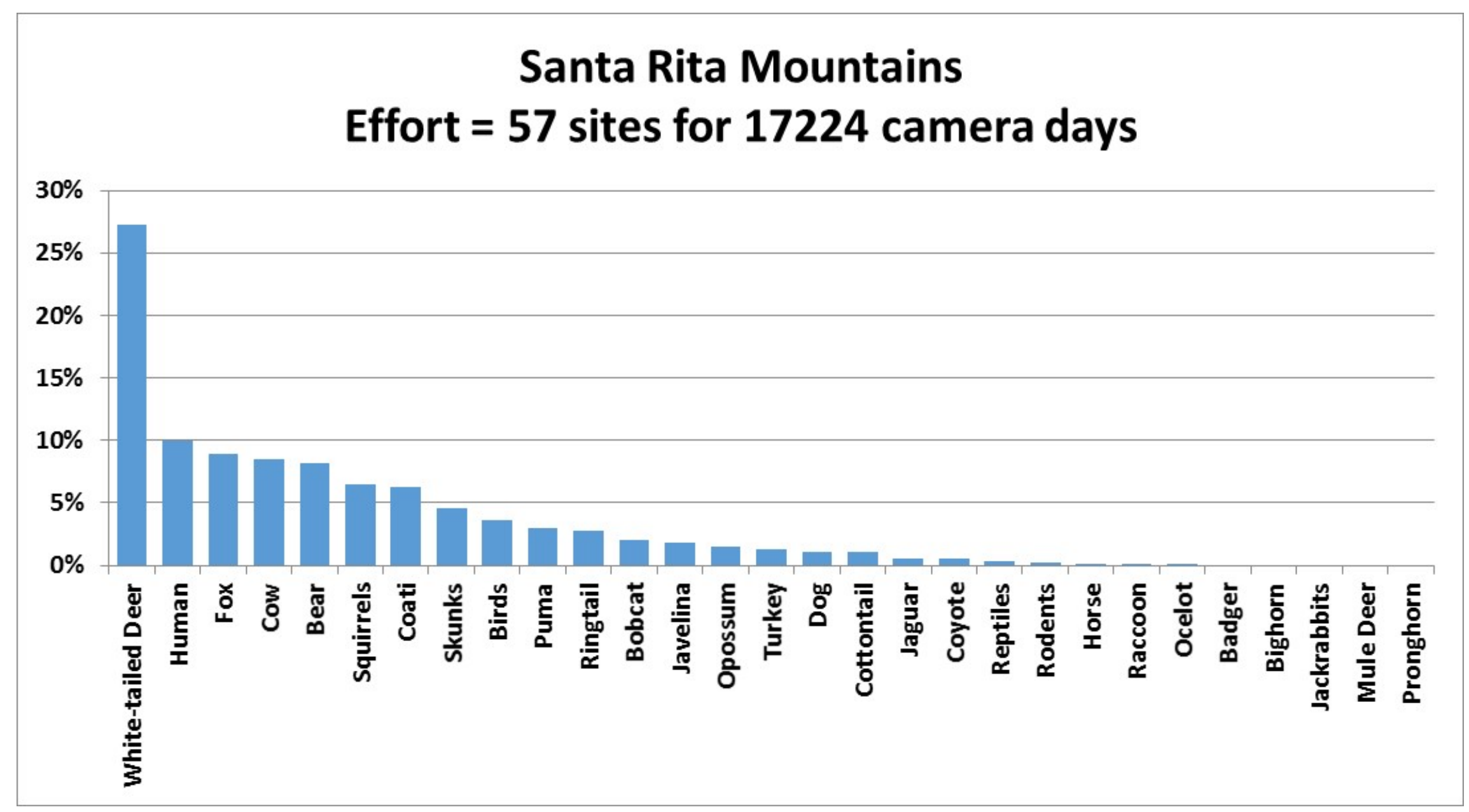

Figure 16. Species composition in the Santa Rita Mountain range as detected by trail cameras from April 2012 to February 2015. Squirrels are separated from the rodent species category, and turkeys are separated from the bird species category. 


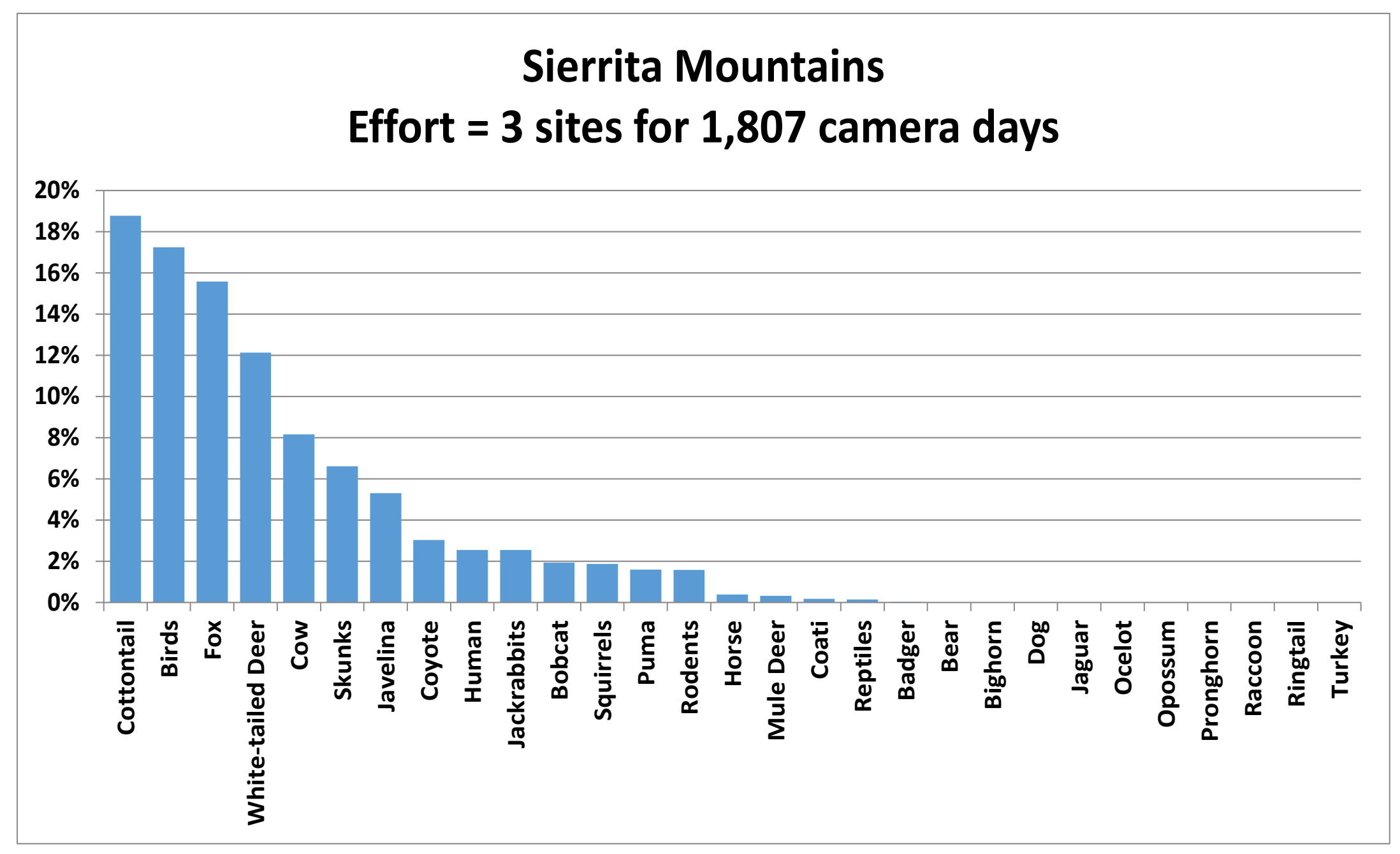

Figure 17. Species composition in the Sierrita Mountain range as detected by trail cameras from April 2012 to February 2015. Squirrels are separated from the rodent species category, and turkeys are separated from the bird species category. 


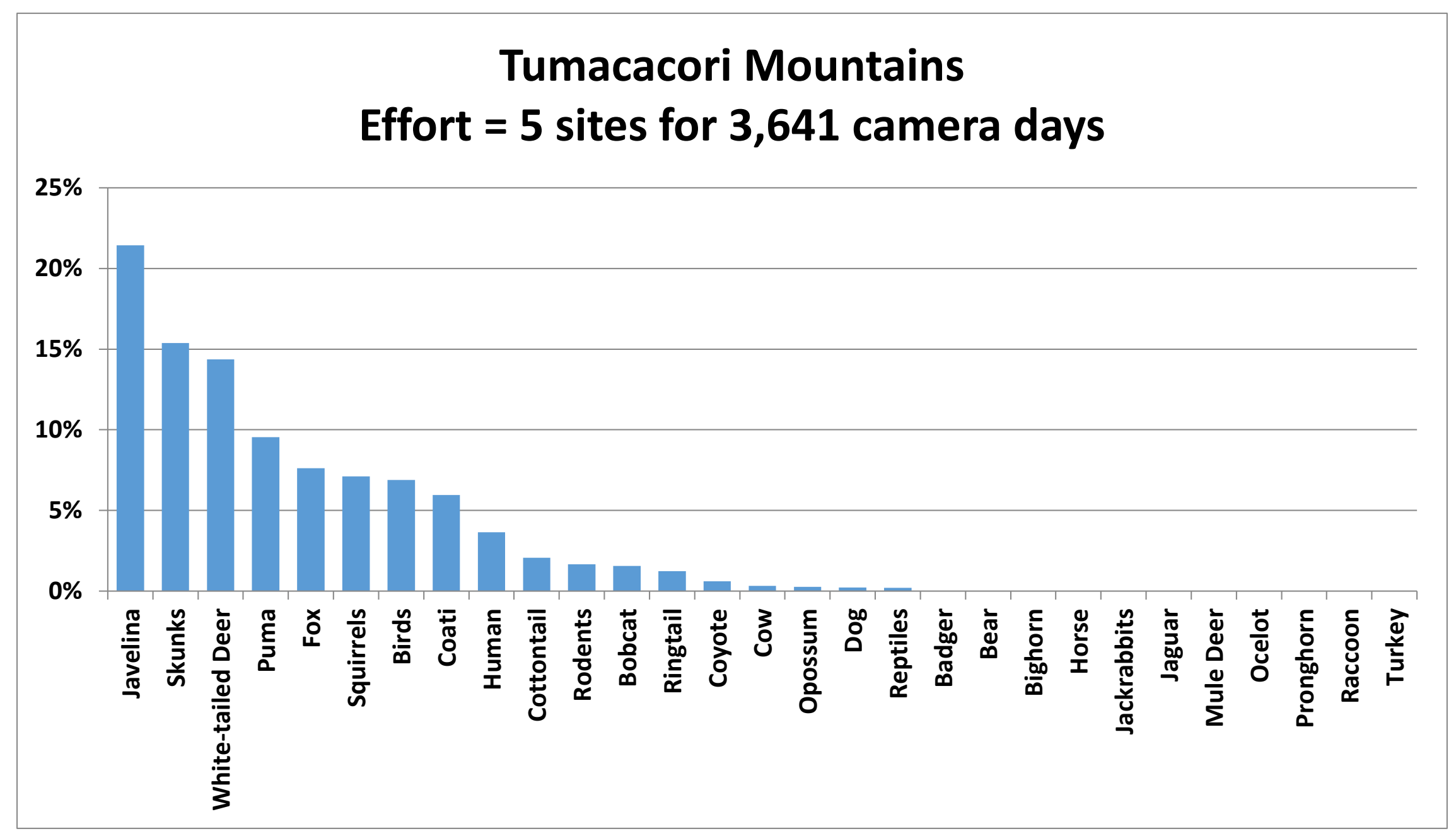

Figure 18. Species composition in the Tumacacori Mountain range as detected by trail cameras from April 2012 to February 2015 . Squirrels are separated from the rodent species category, and turkeys are separated from the bird species category. 


\section{Whetstone Mountains \\ Effort $=9$ sites for 5,393 camera days}

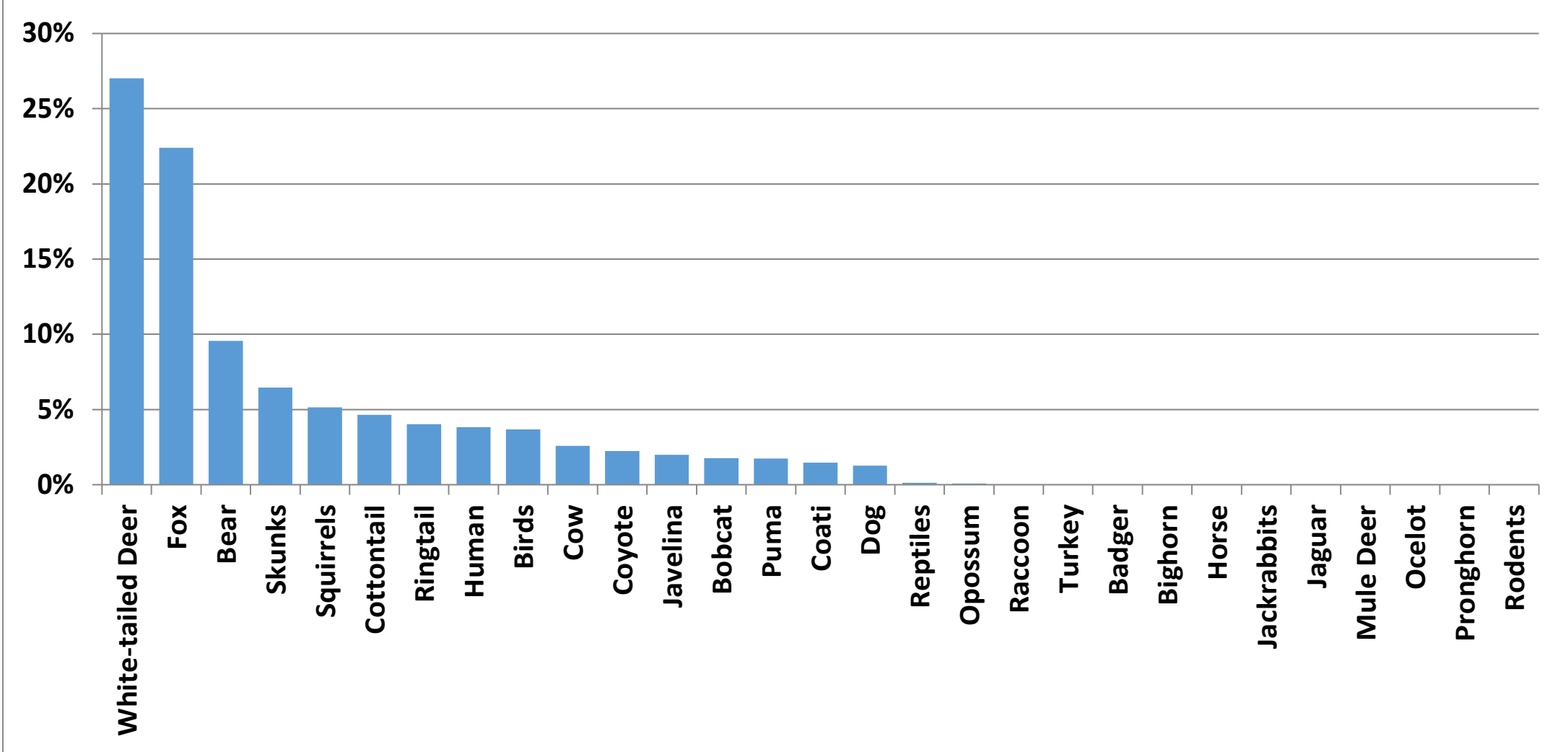

Figure 19. Species composition in the Whetstone Mountain range as detected by trail cameras from April 2012 to February 2015. Squirrels are separated from the rodent species category, and turkeys are separated from the bird species category. 


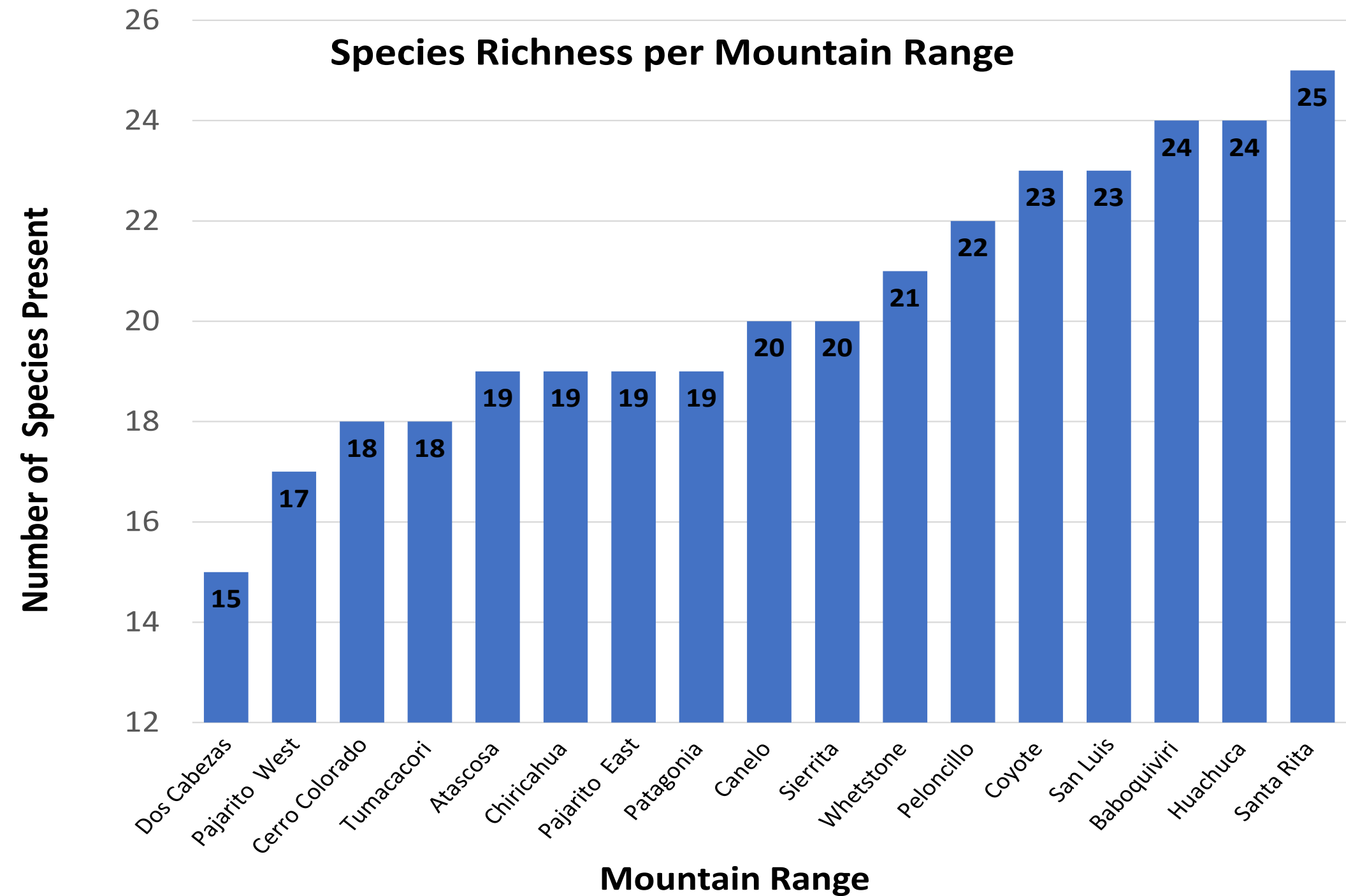

Figure 20. Total number of species categories detected in each mountain range, 2012-2015. 


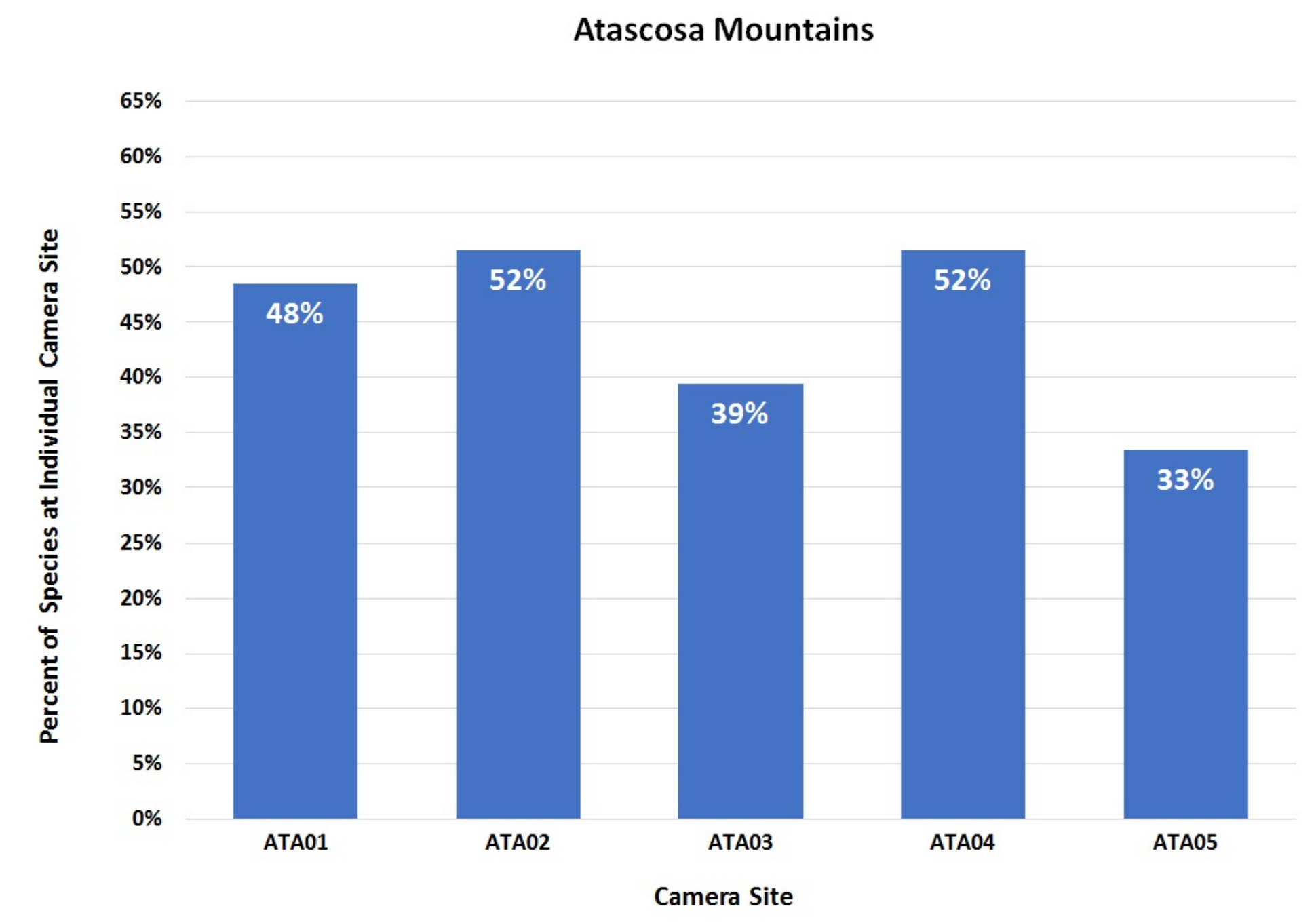

Figure 21. Percent of total detected species categories by site in the Atascosa Mountain range, 2012-2015. 


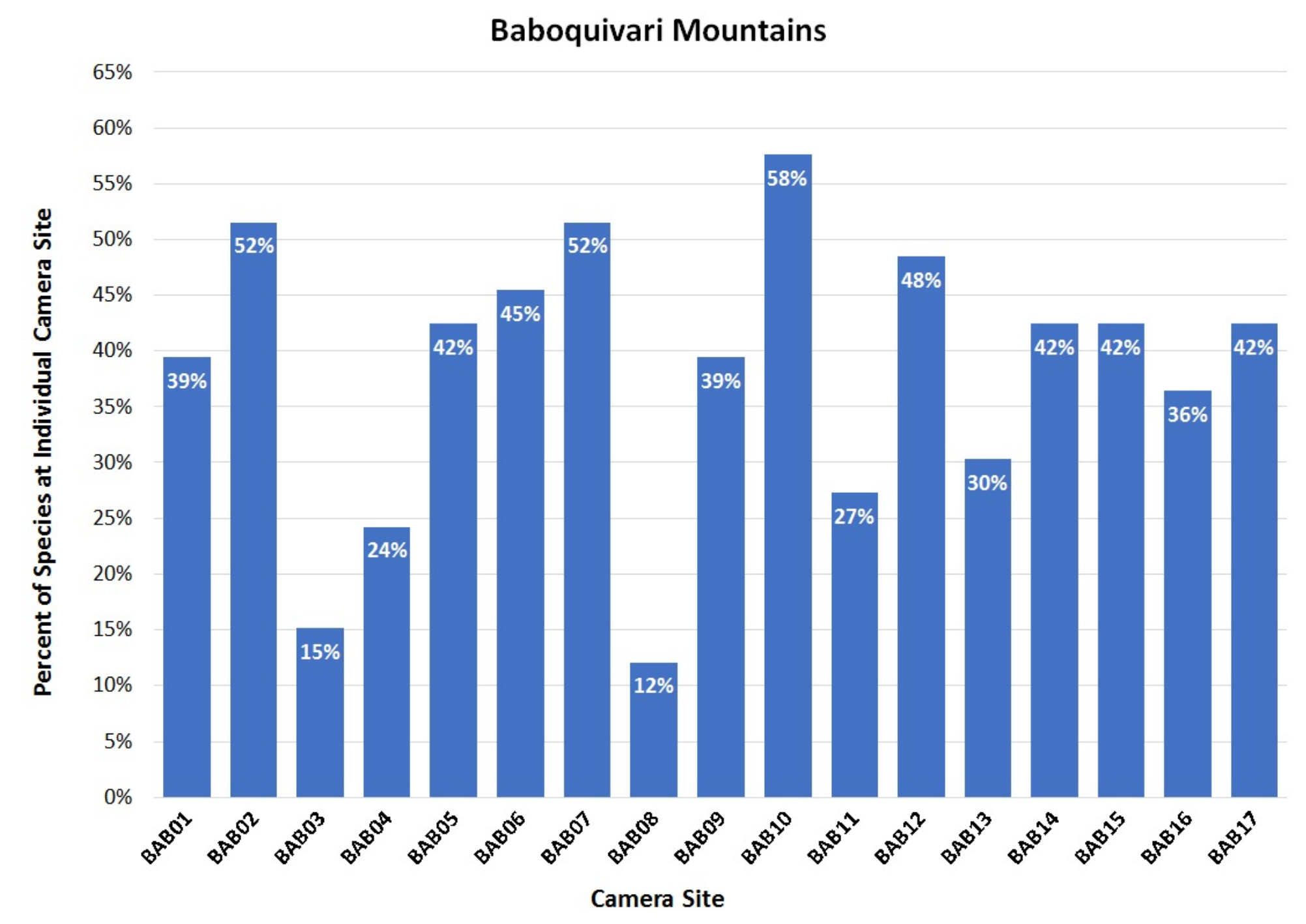

Figure 22. Percent of total detected species categories by site in the Baboquivari Mountain range, 2012-2015. 


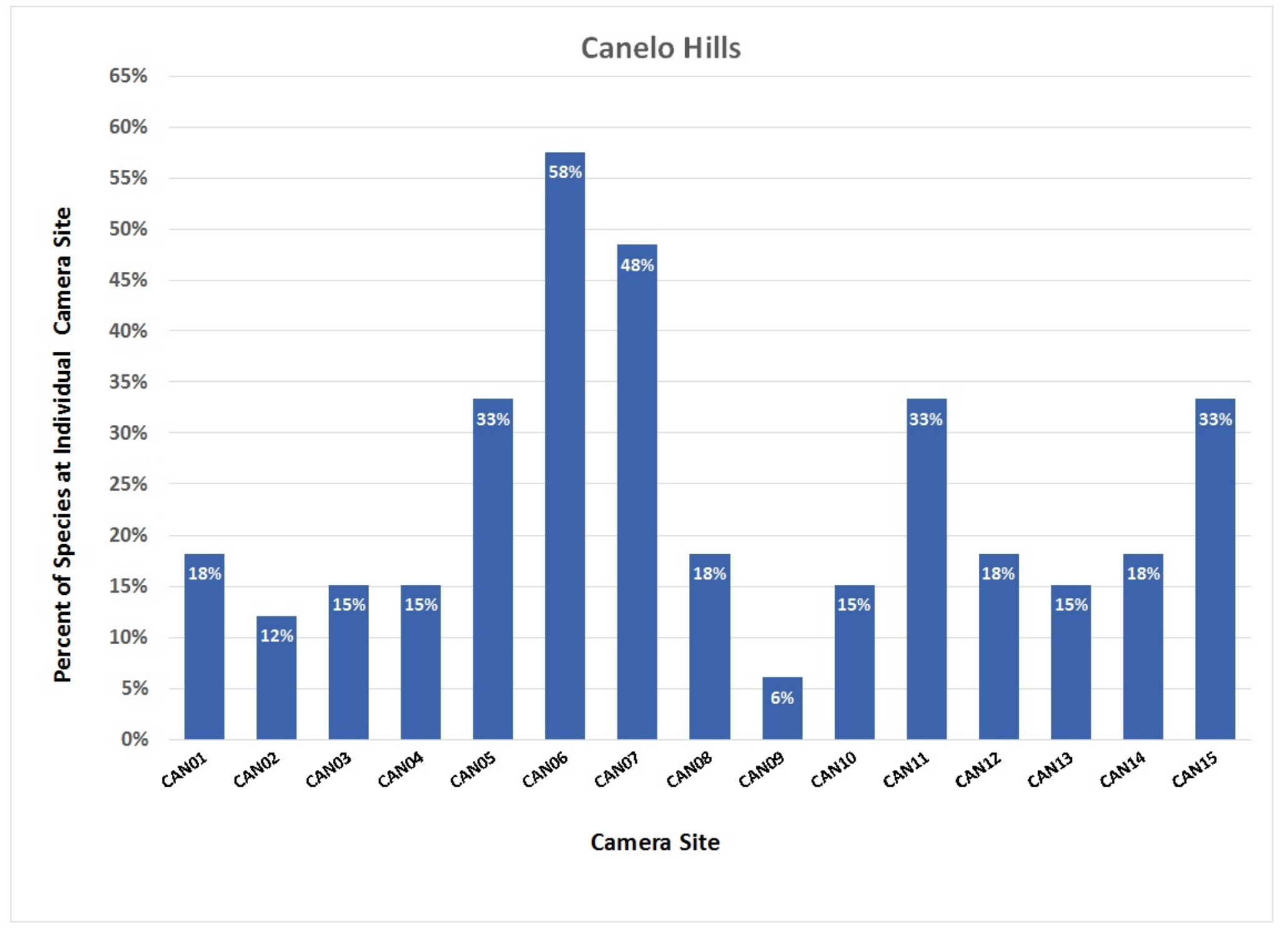

Figure 23. Percent of total detected species categories by site in the Canelo Hills, 2012-2015. 


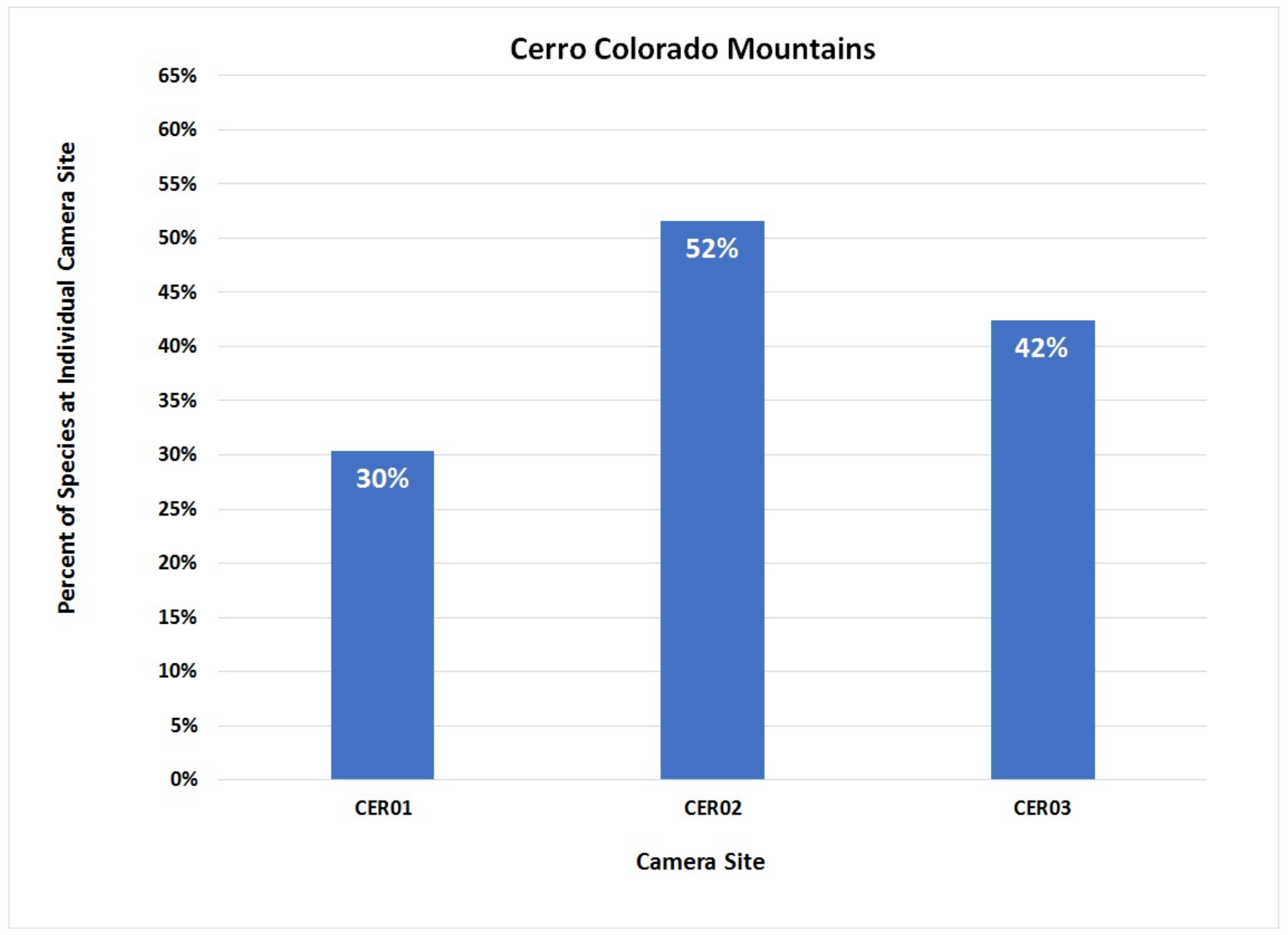

Figure 24. Percent of total detected species categories by site in the Cerro Colorado Mountain range, 2012-2015. 


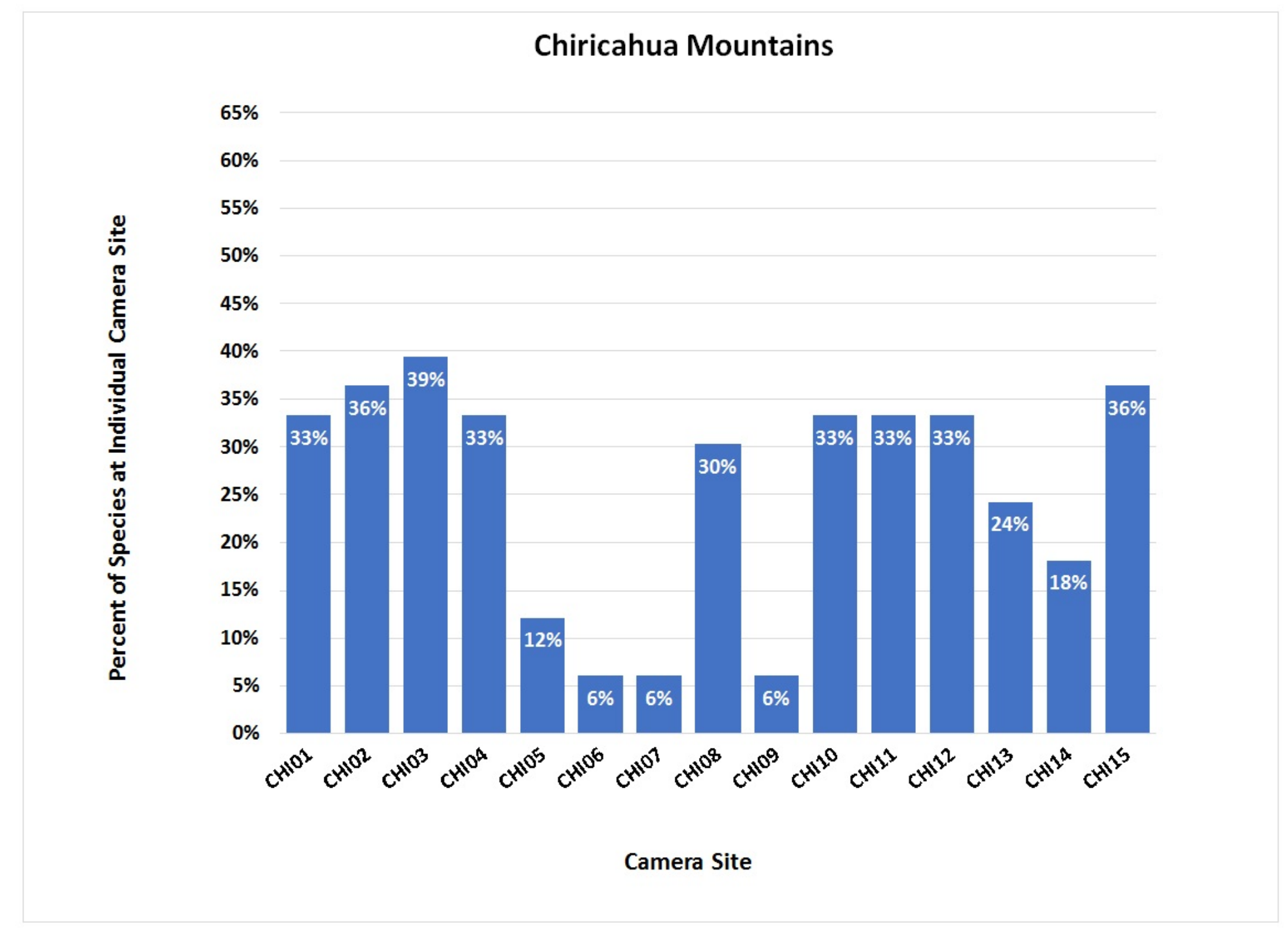

Figure 25. Percent of total detected species categories by site in the Chiricahua Mountain range, 2012-2015. 


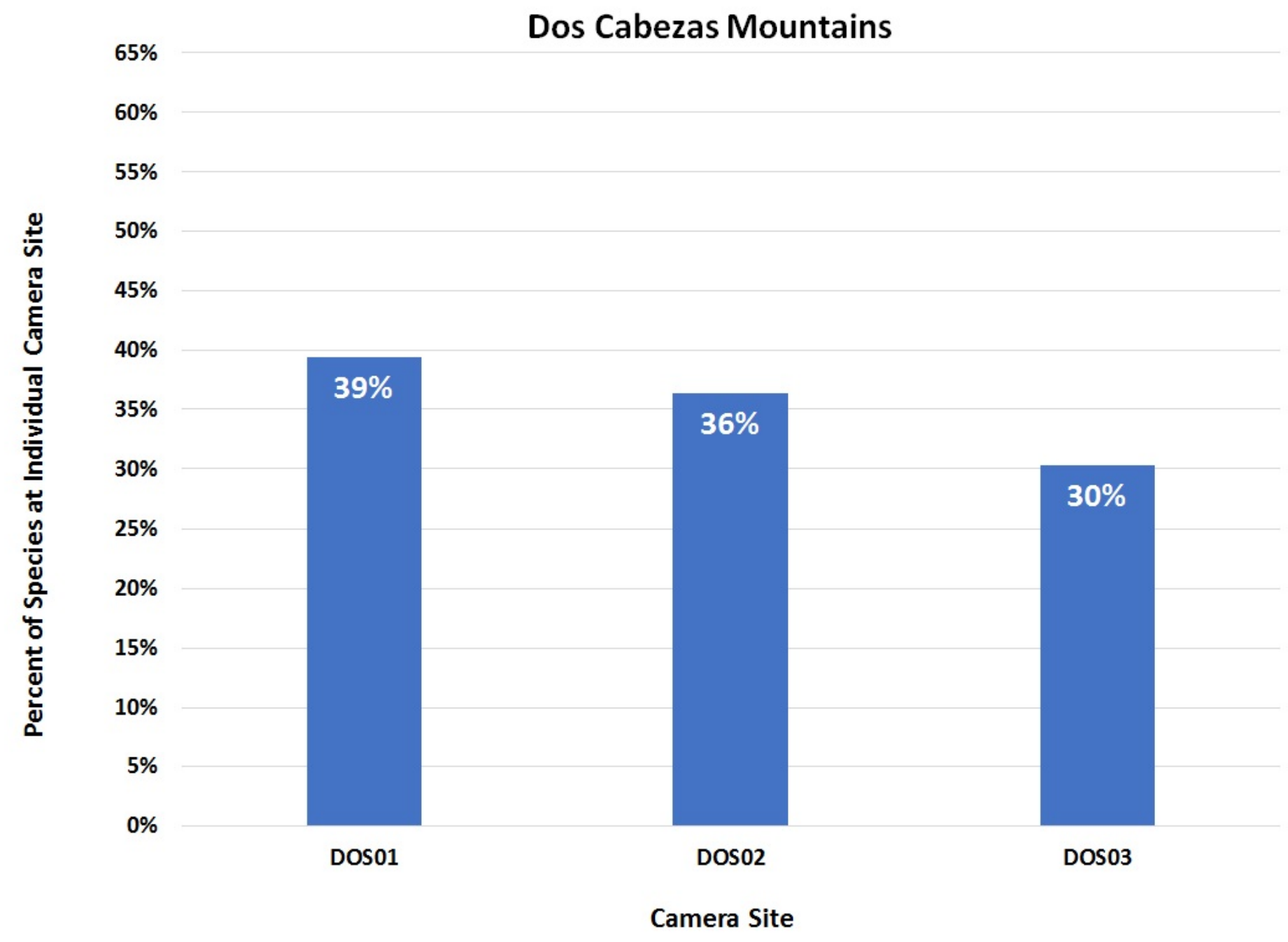

Figure 26. Percent of total detected species categories by site in the Dos Cabezas Mountain range, 2012-2015. 


\section{Huachuca Mountains}

$65 \%$

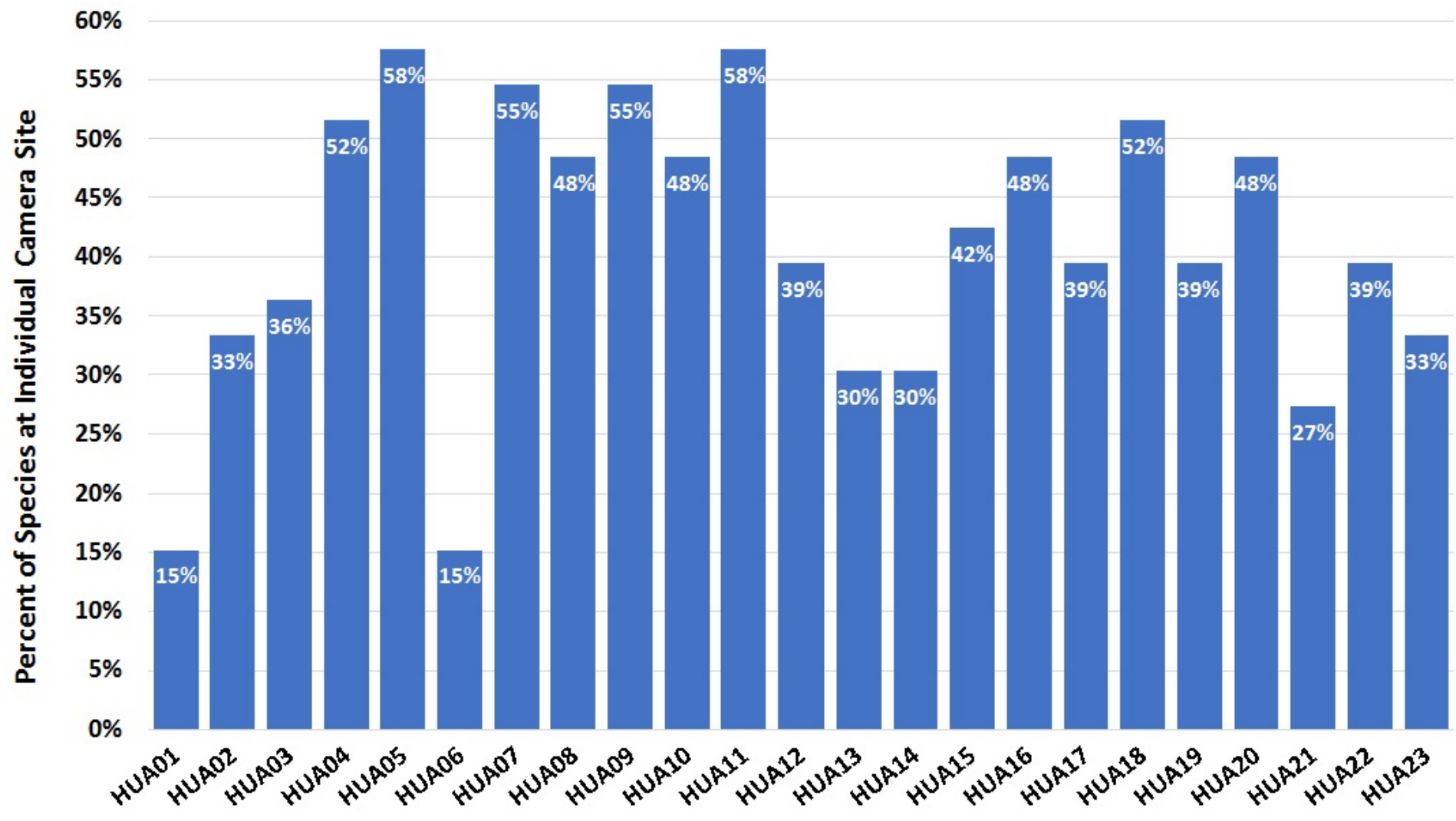

Camera Site

Figure 27. Percent of total detected species categories by site in the Huachuca Mountain range, 2012-2015. 


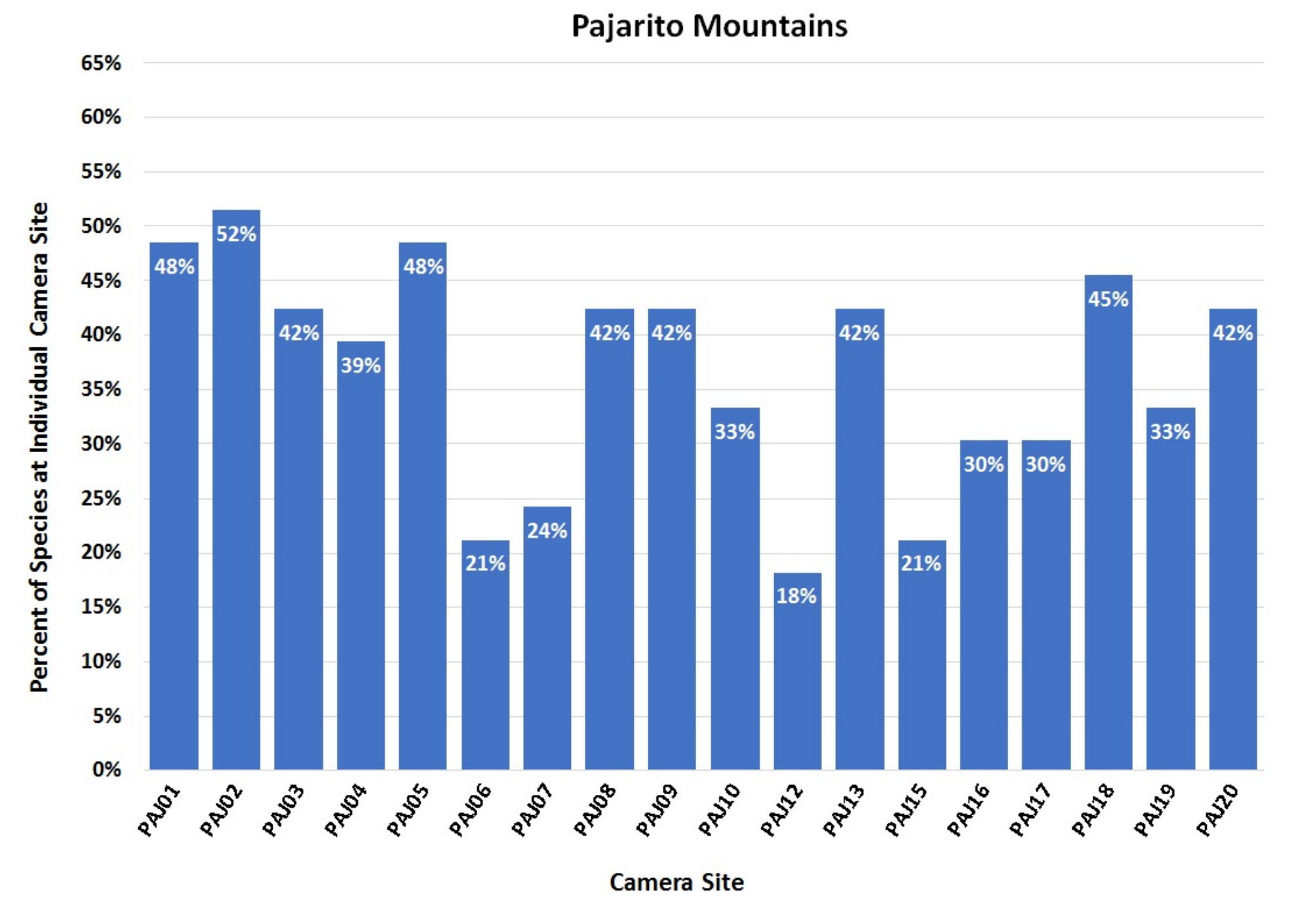

Figure 28. Percent of total detected species categories by site in the Pajarito Mountain range, 2012-2015. 


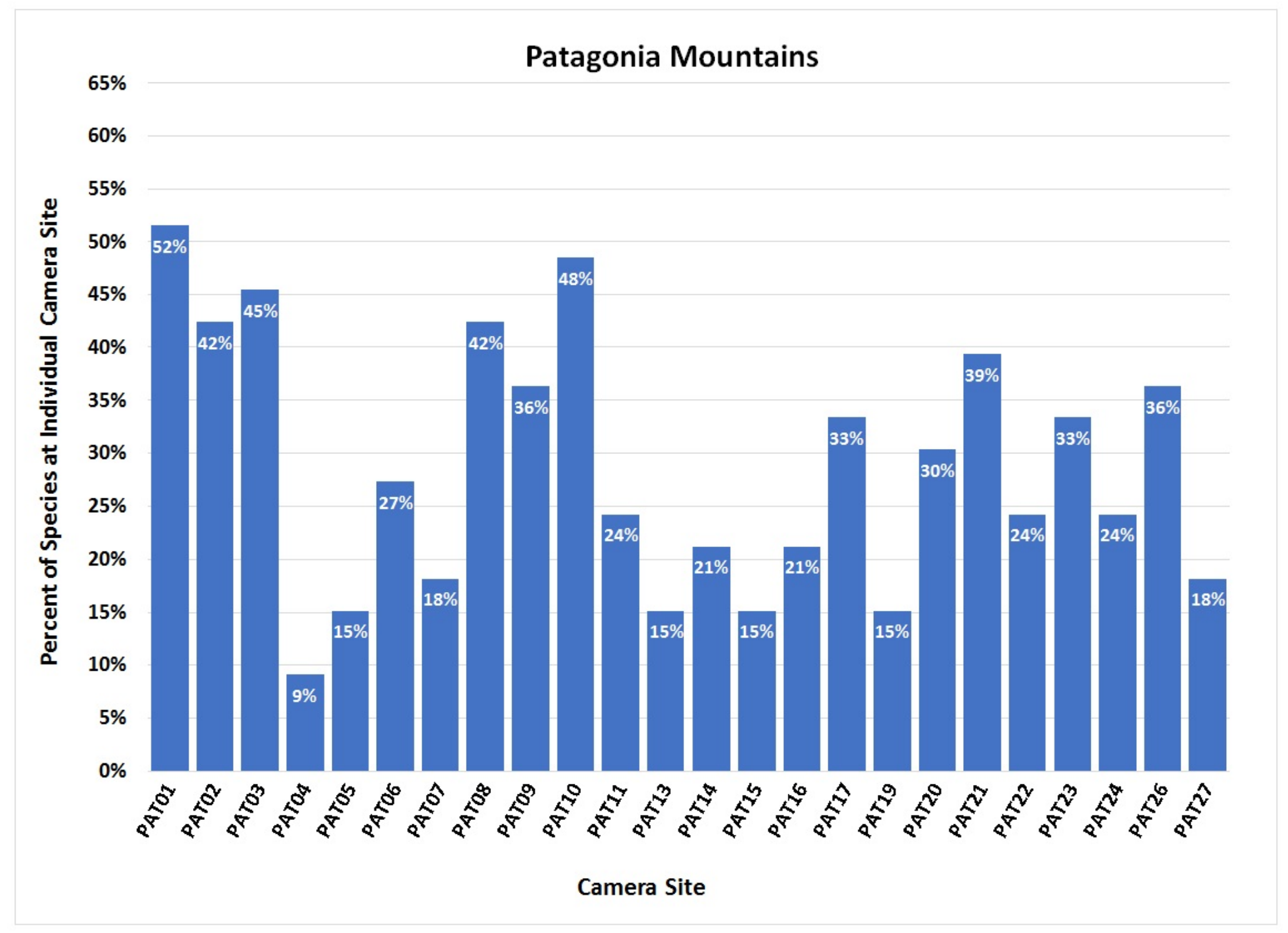

Figure 29. Percent of total detected species categories by site in the Patagonia Mountain range, 2012-2015. 


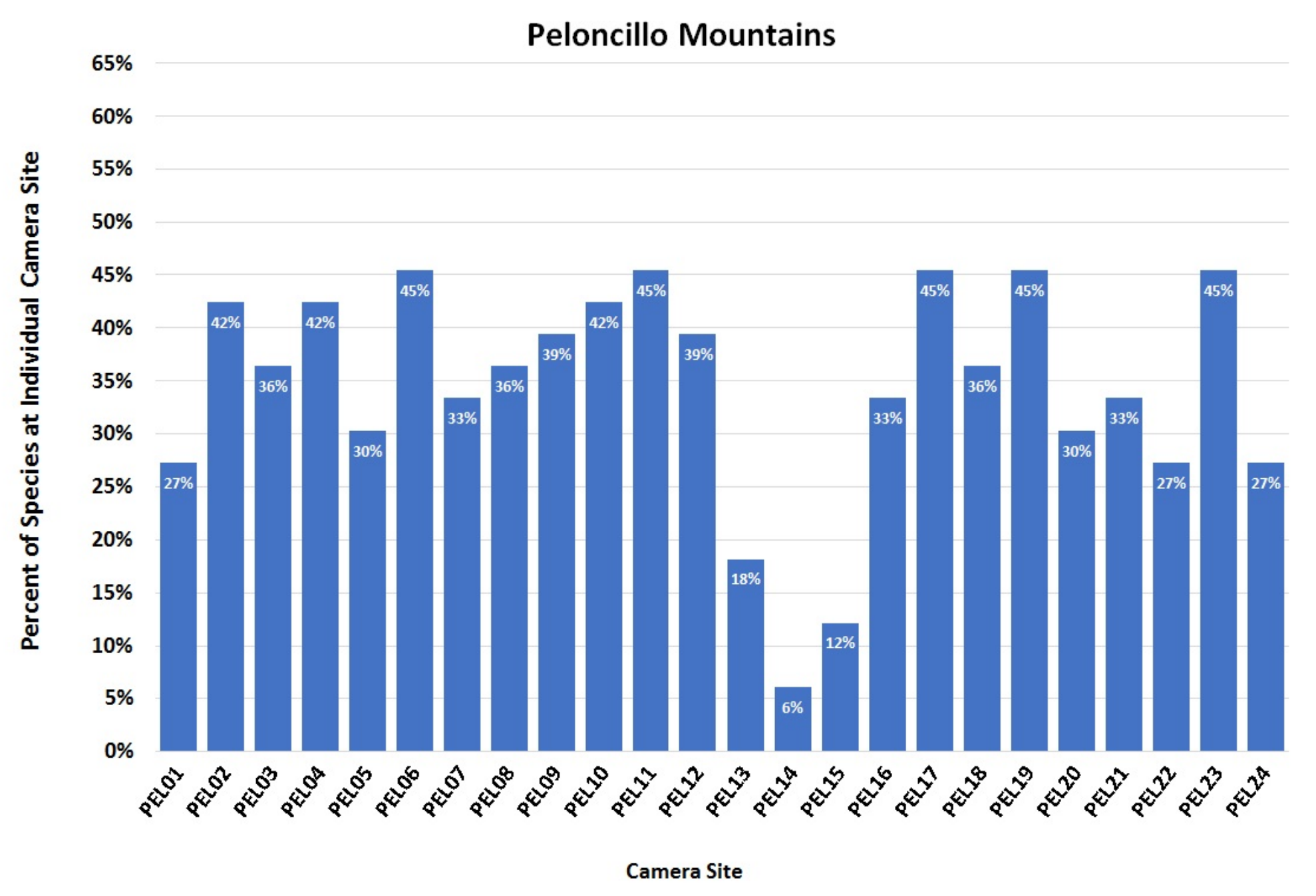

Figure 30. Percent of total detected species categories by site in the Peloncillo Mountain range, 2012-2015. 


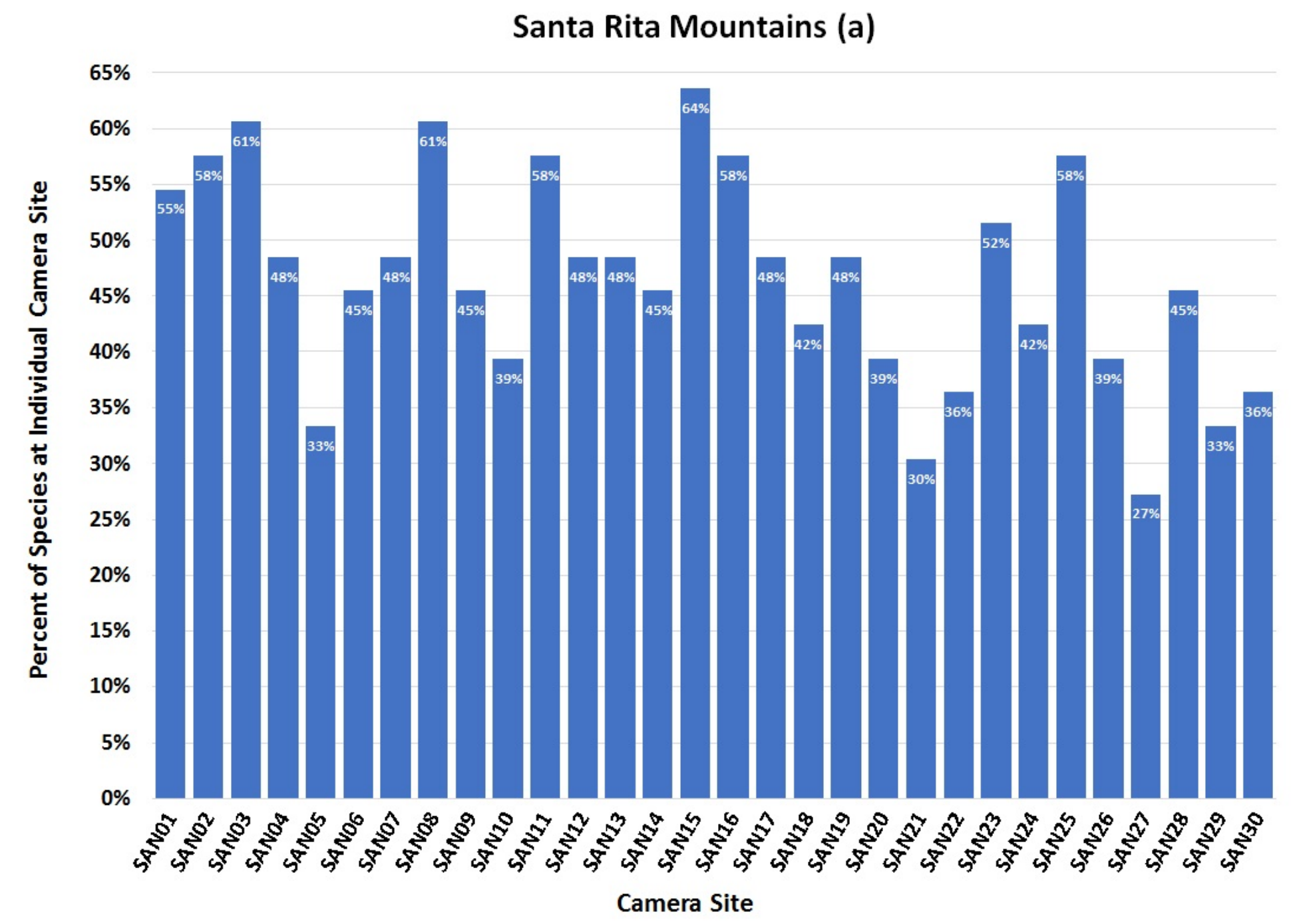

Figure 31. Percent of total detected species categories by site in the Santa Rita Mountain range, 2012-2015. 


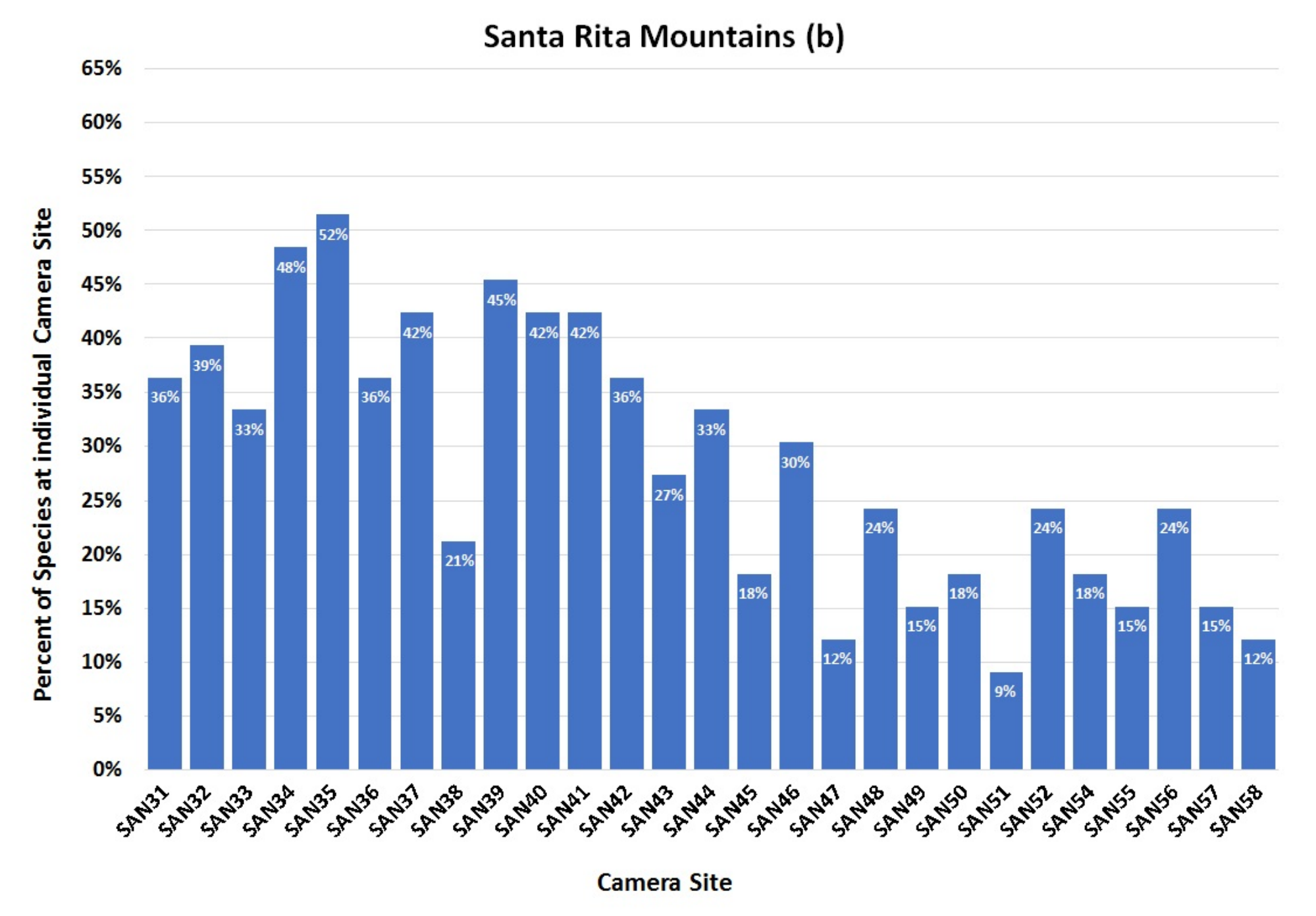

Figure 32. Percent of total detected species categories by site in the Santa Rita Mountain range, 2012-2015. 


\section{Sierrita Mountains}

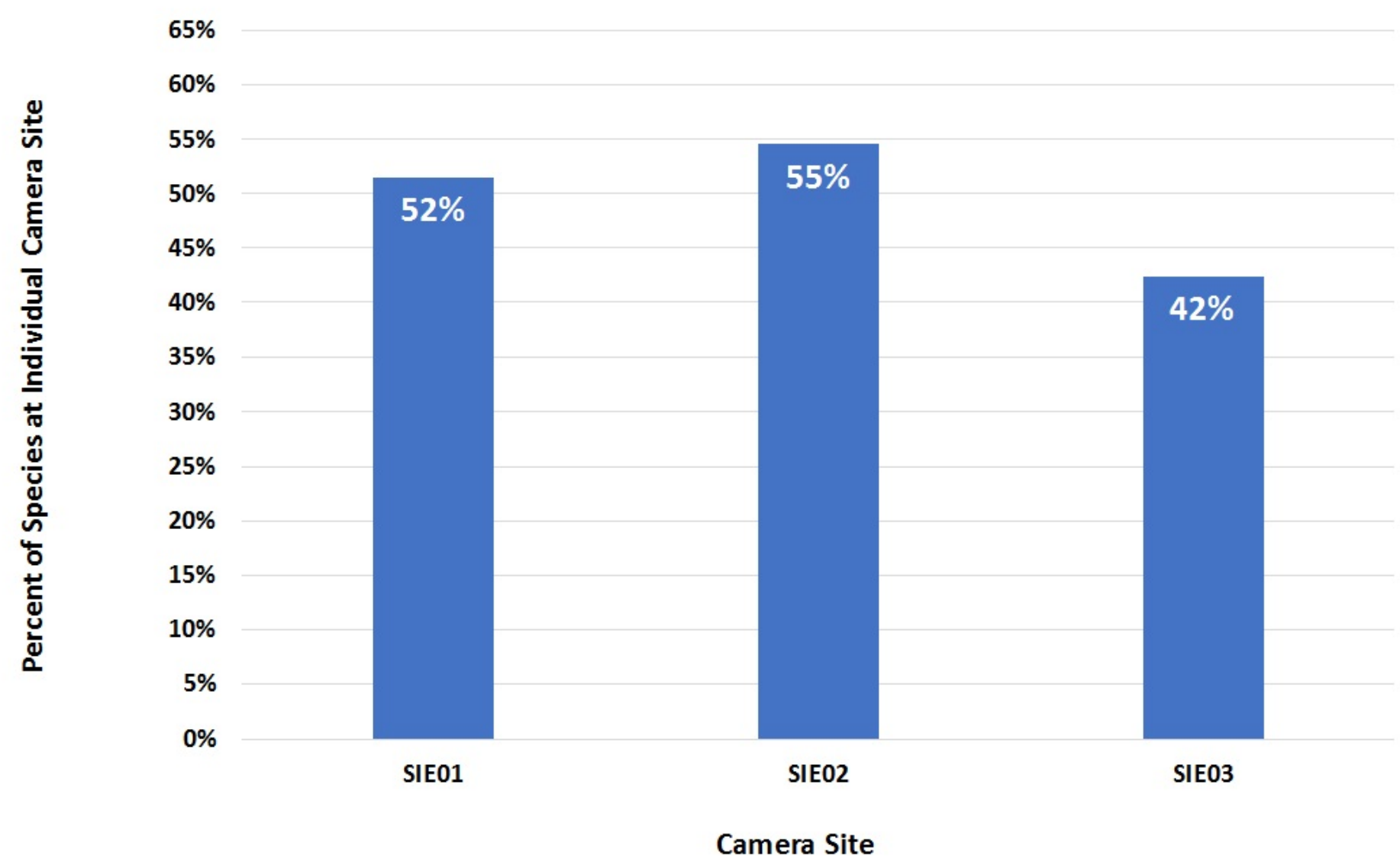

Figure 33. Percent of total detected species categories by site in the Sierrita Mountain range, 2012-2015. 


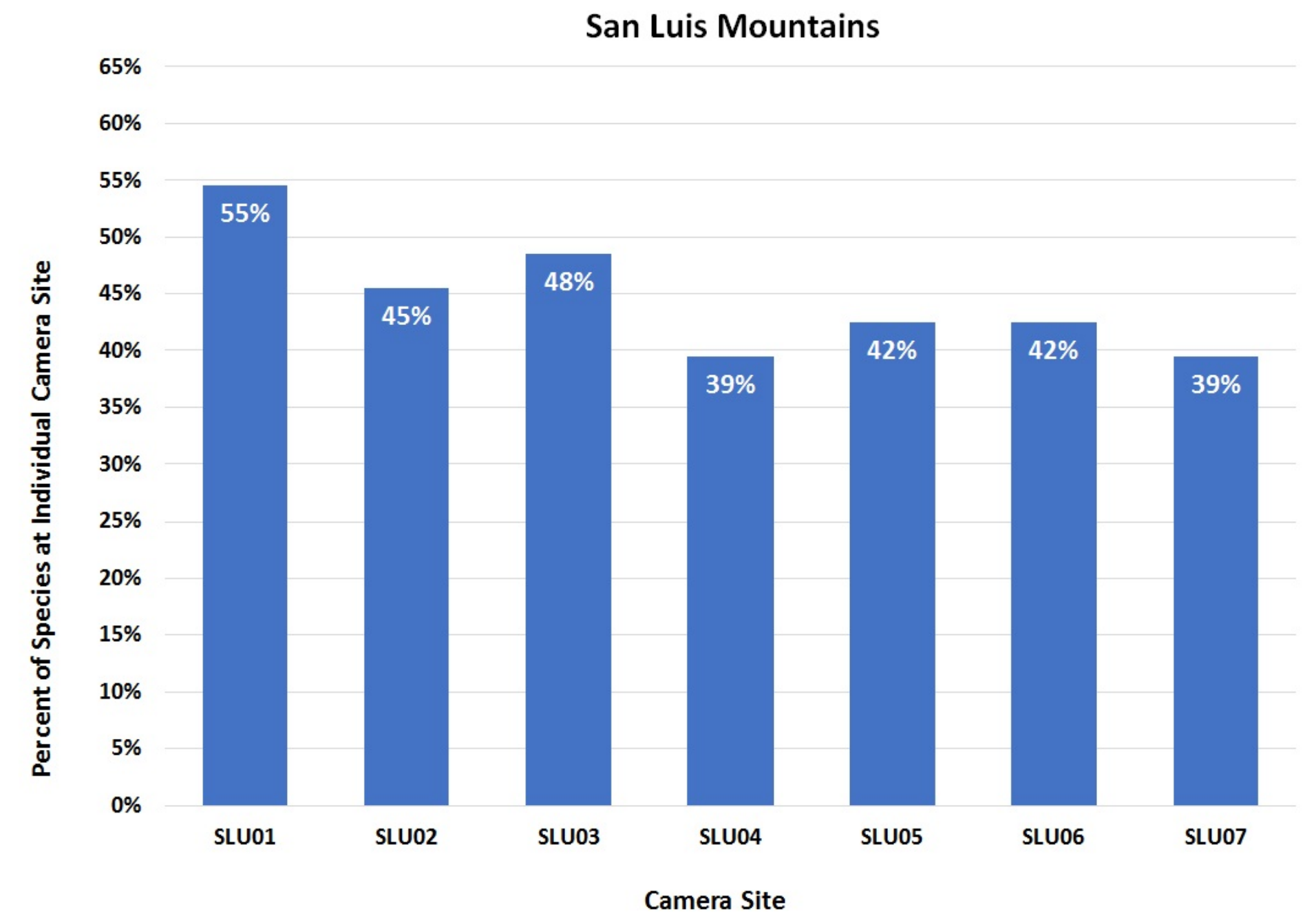

Figure 34. Percent of total detected species categories by site in the San Luis Mountain range (Arizona), 2012-2015. 


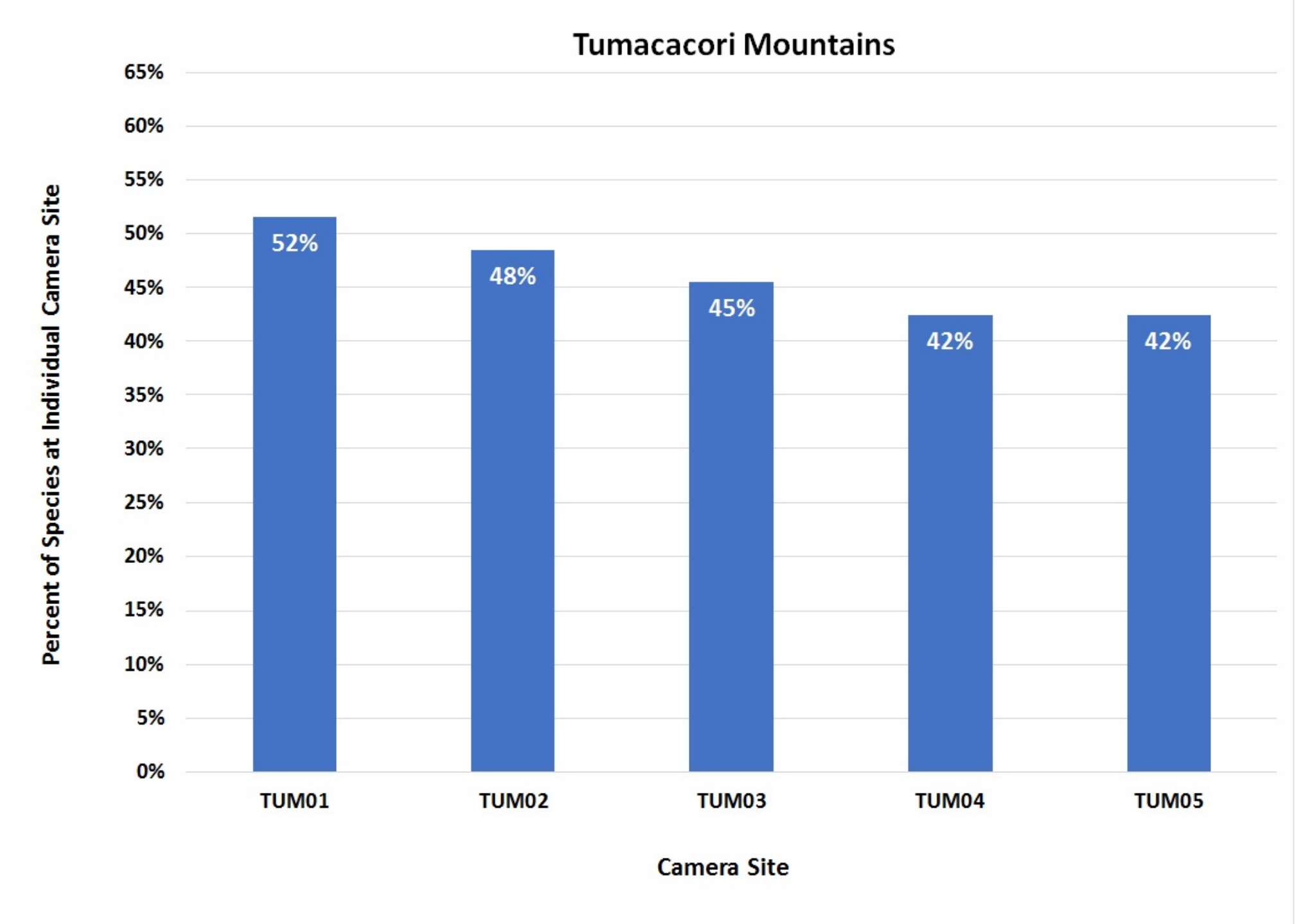

Figure 35. Percent of total detected species categories by site in the Tumacacori Mountain range, 2012-2015. 


\section{Whetstone Mountains}

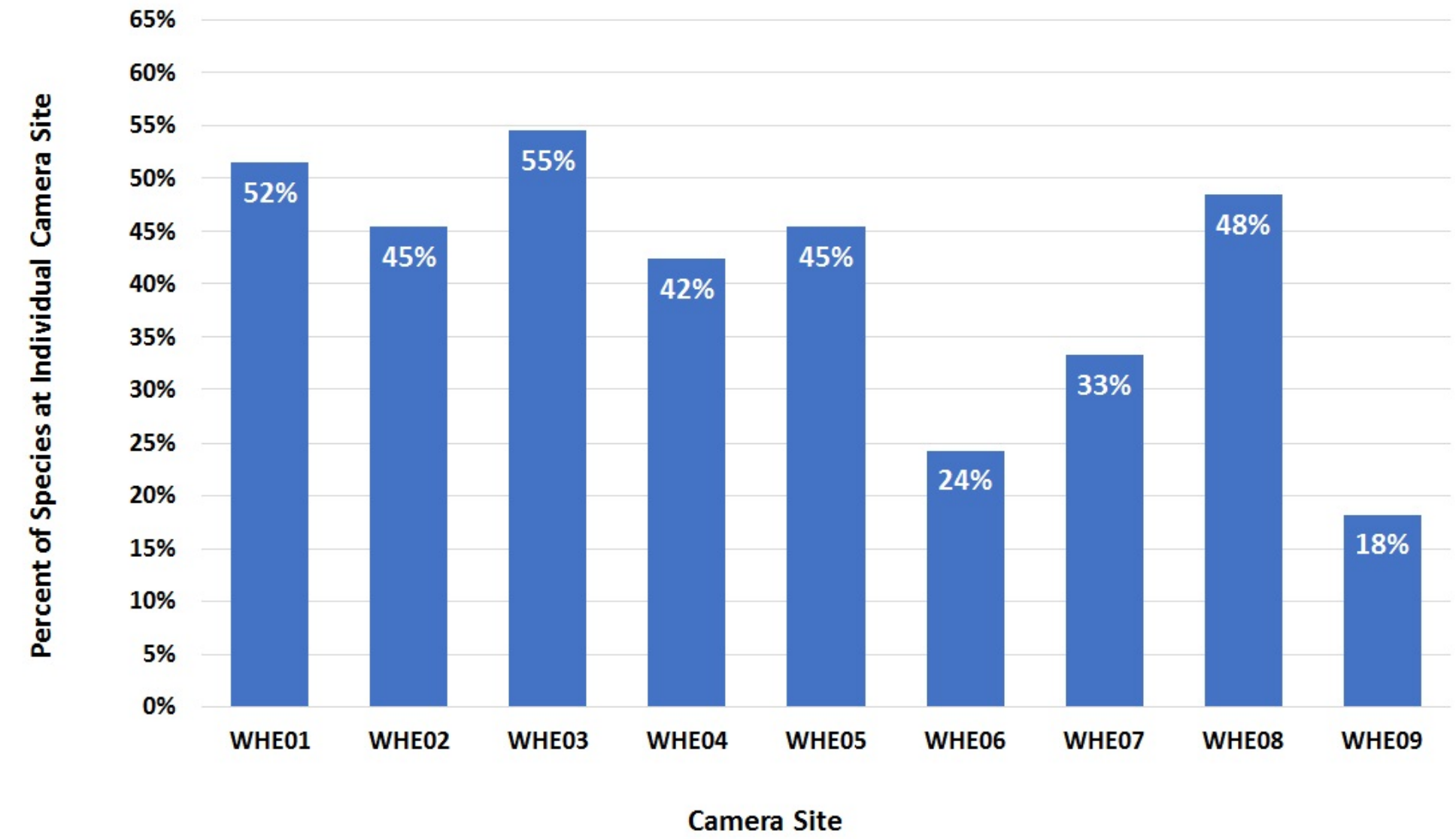

Figure 36. Percent of total detected species categories by site in the Whetstone Mountain range, 2012-2015. 


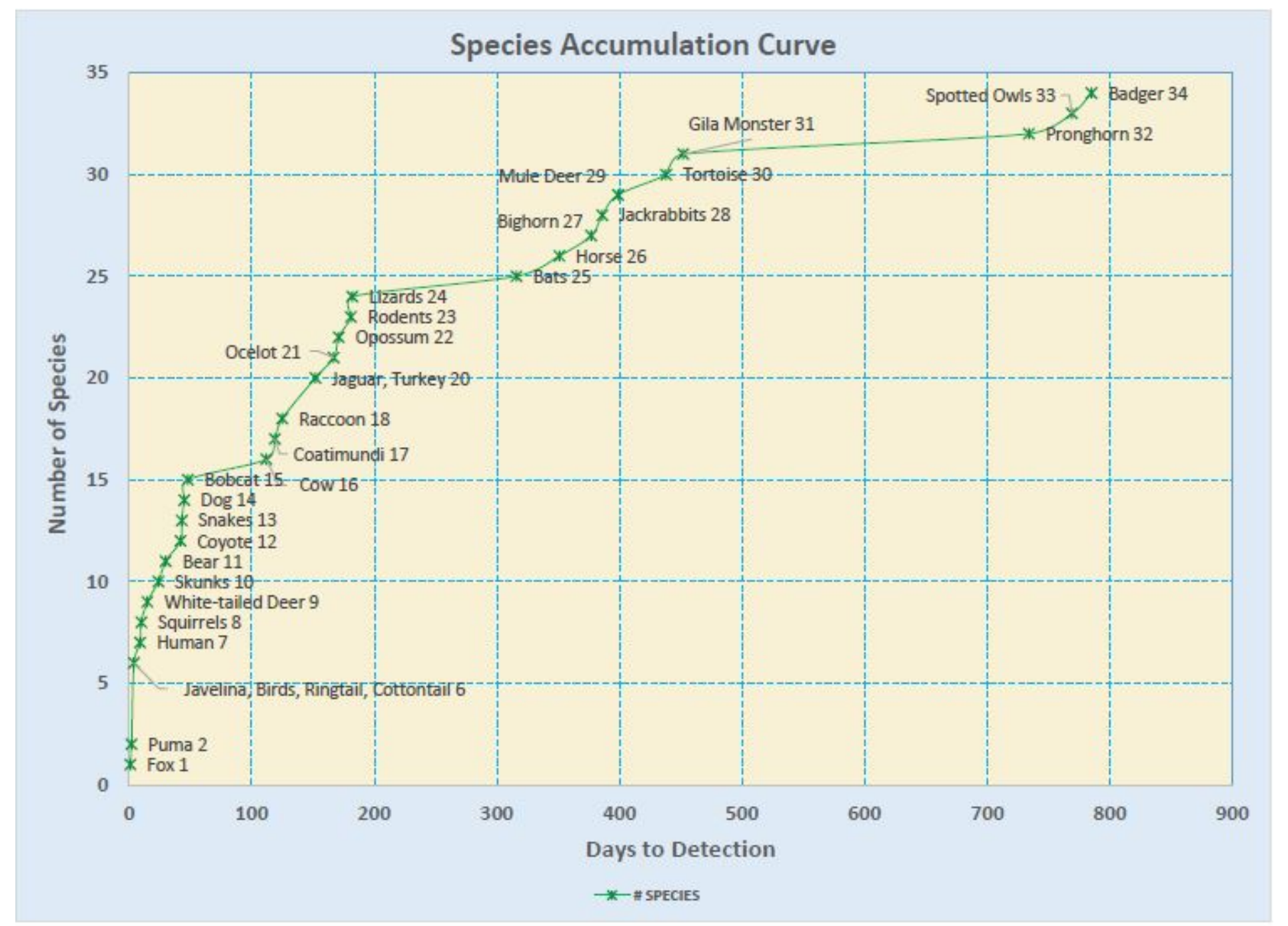

Figure 37. Species accumulation curve for all sites, 2012-2015. Squirrels are separated from the rodent species category, and turkeys are separated from the bird species category. 


\section{Total Photo Detections}

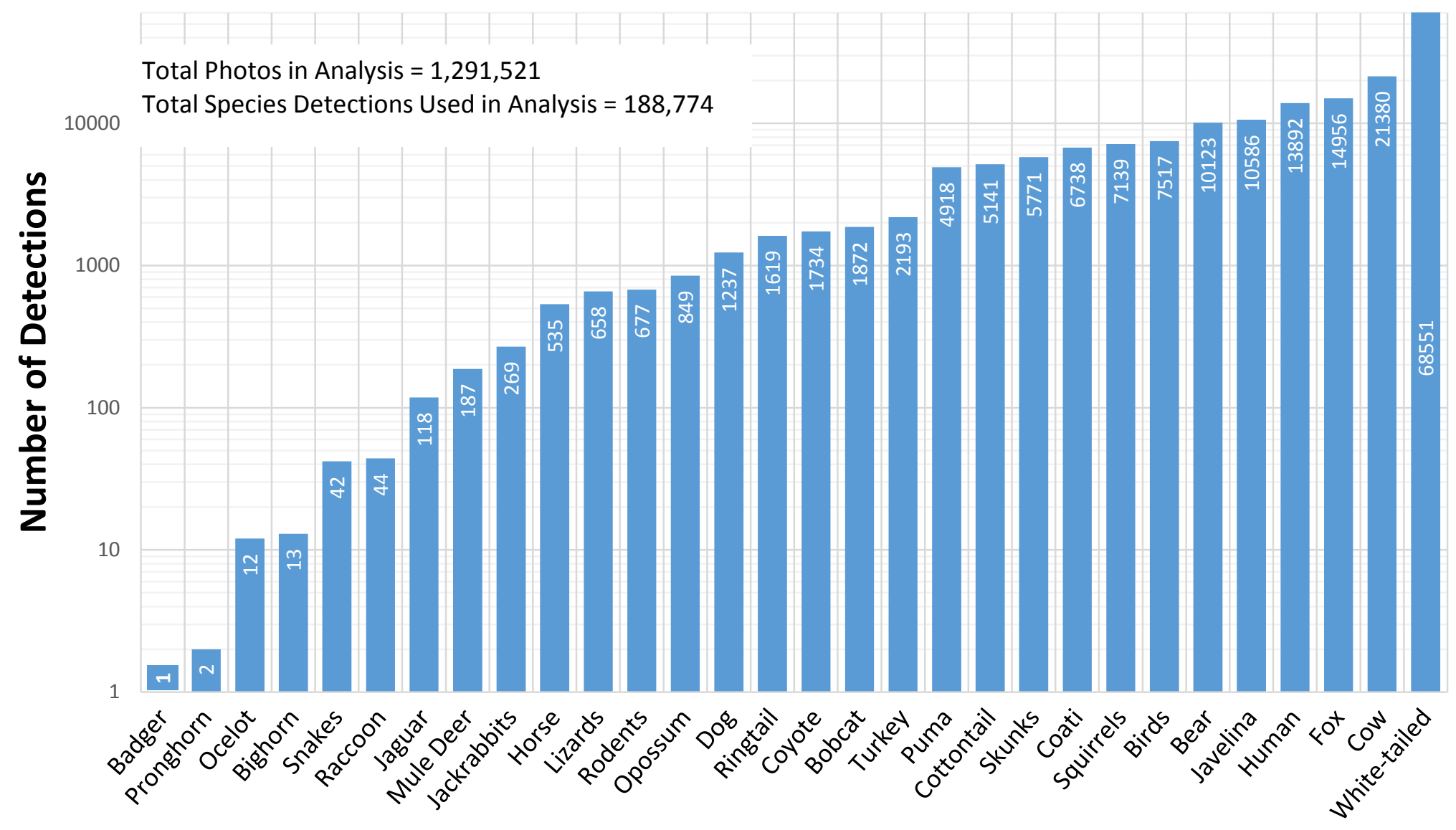

Species

Figure 38. Total number of each species and species categories detected from April 2012 to February 2015. Squirrels are separated from the rodent species category, and turkeys are separated from the bird species category. 


\section{Total Number of Species Detected/*Period}

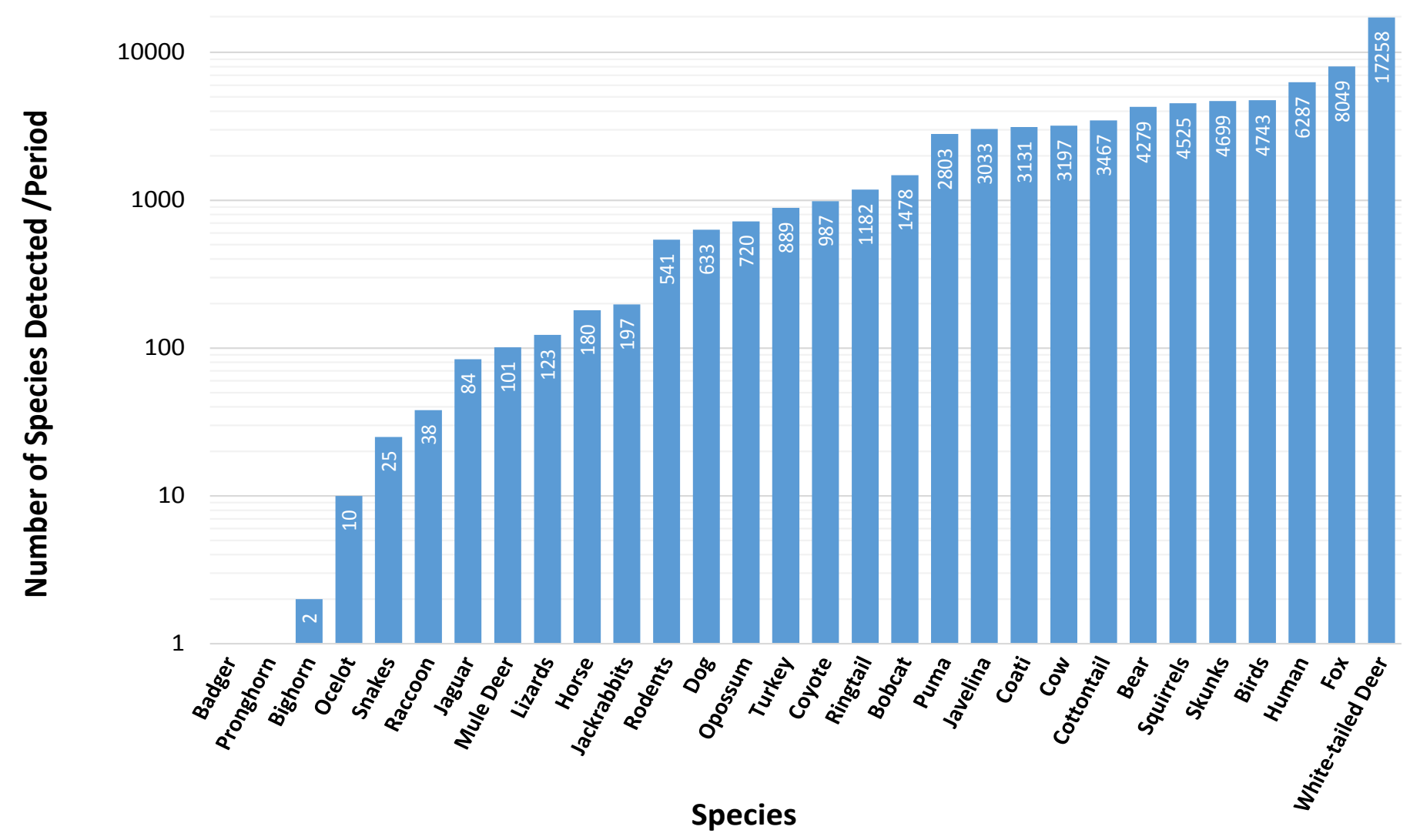

* Period equals 60 minute interval

Figure 39. Total number of species and species categories detected per 60-minute period (independent records). Squirrels are separated from the rodent species category, and turkeys are separated from the bird species category. 


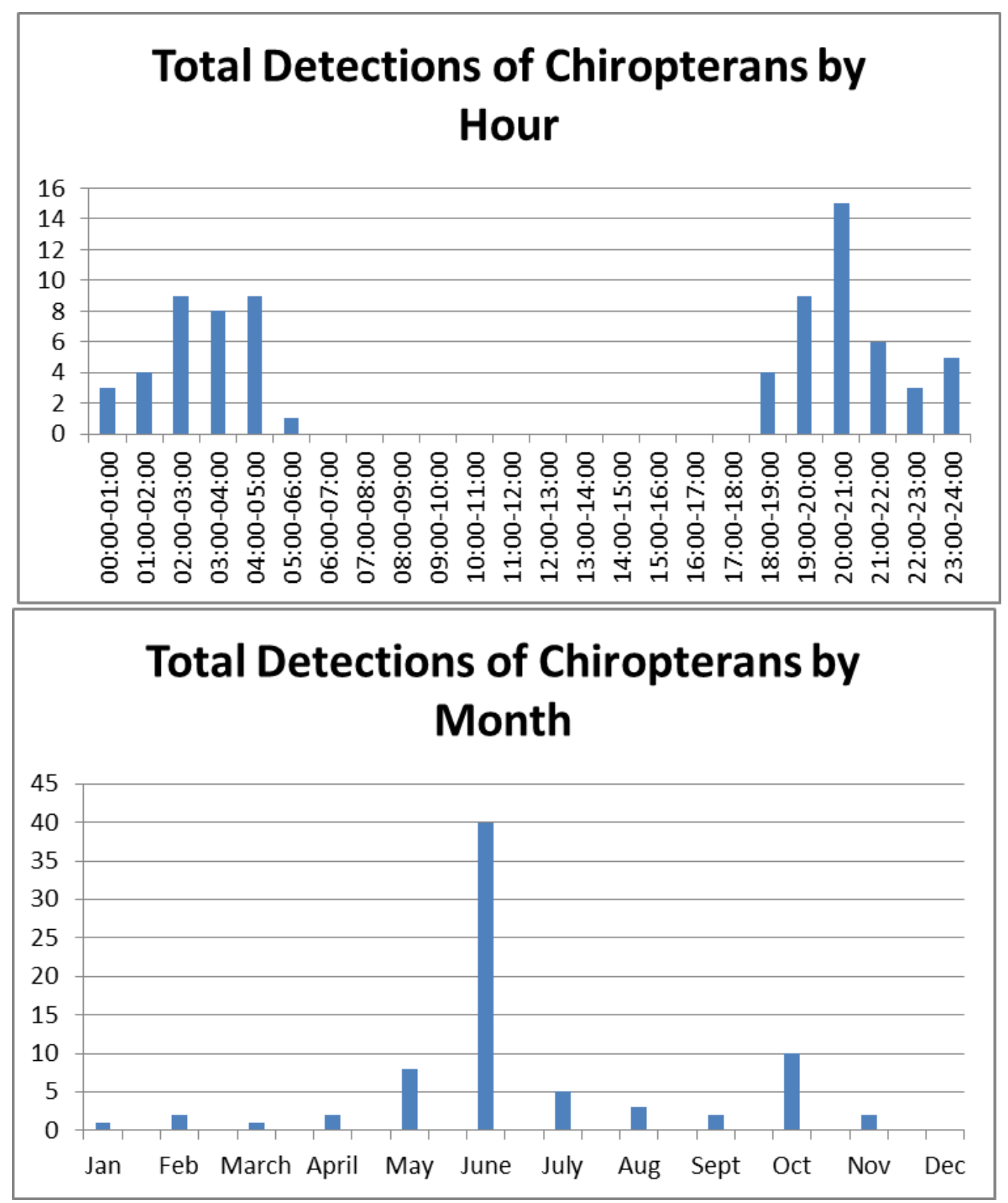

Figure 40. Total daily and monthly detections of Chiropterans (bats), 2012-2015. 


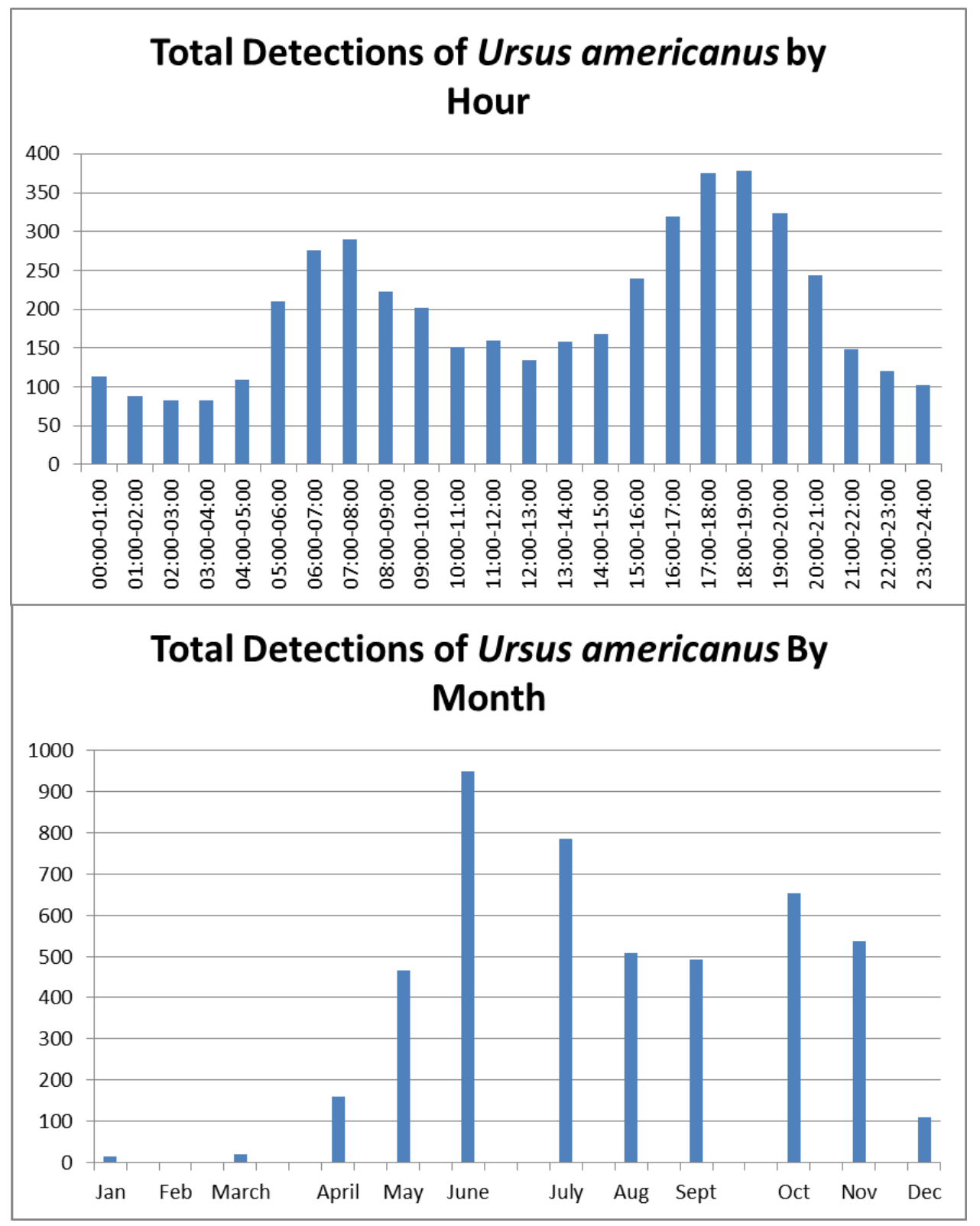

Figure 41. Daily and monthly detections of Ursus americanus (bears), 2012-2015. 


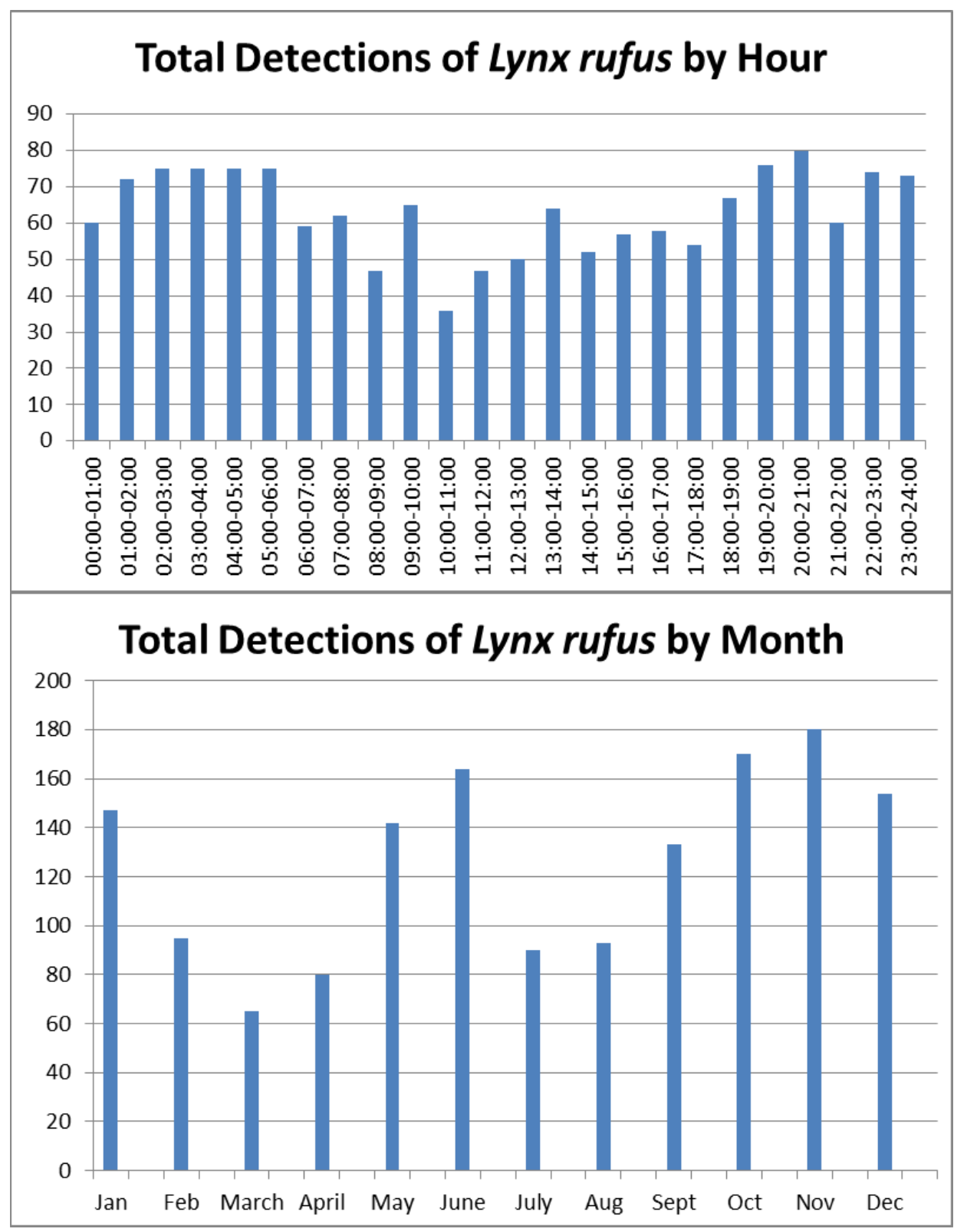

Figure 42. Daily and monthly detections of Lynx rufus (bobcats), 2012-2015. 


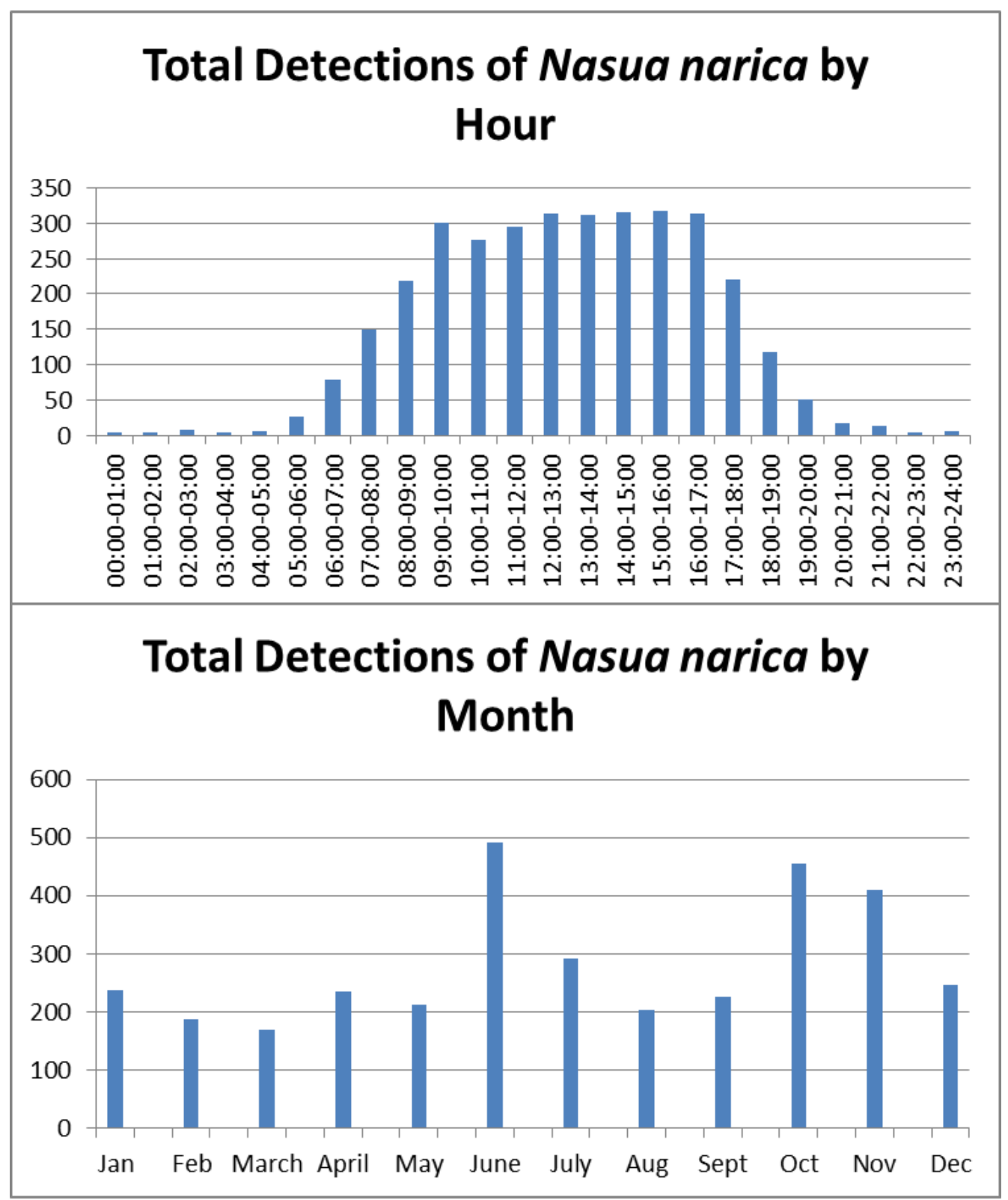

Figure 43. Daily and monthly detections of Nasua narica (coatimundis), 2012-2015. 


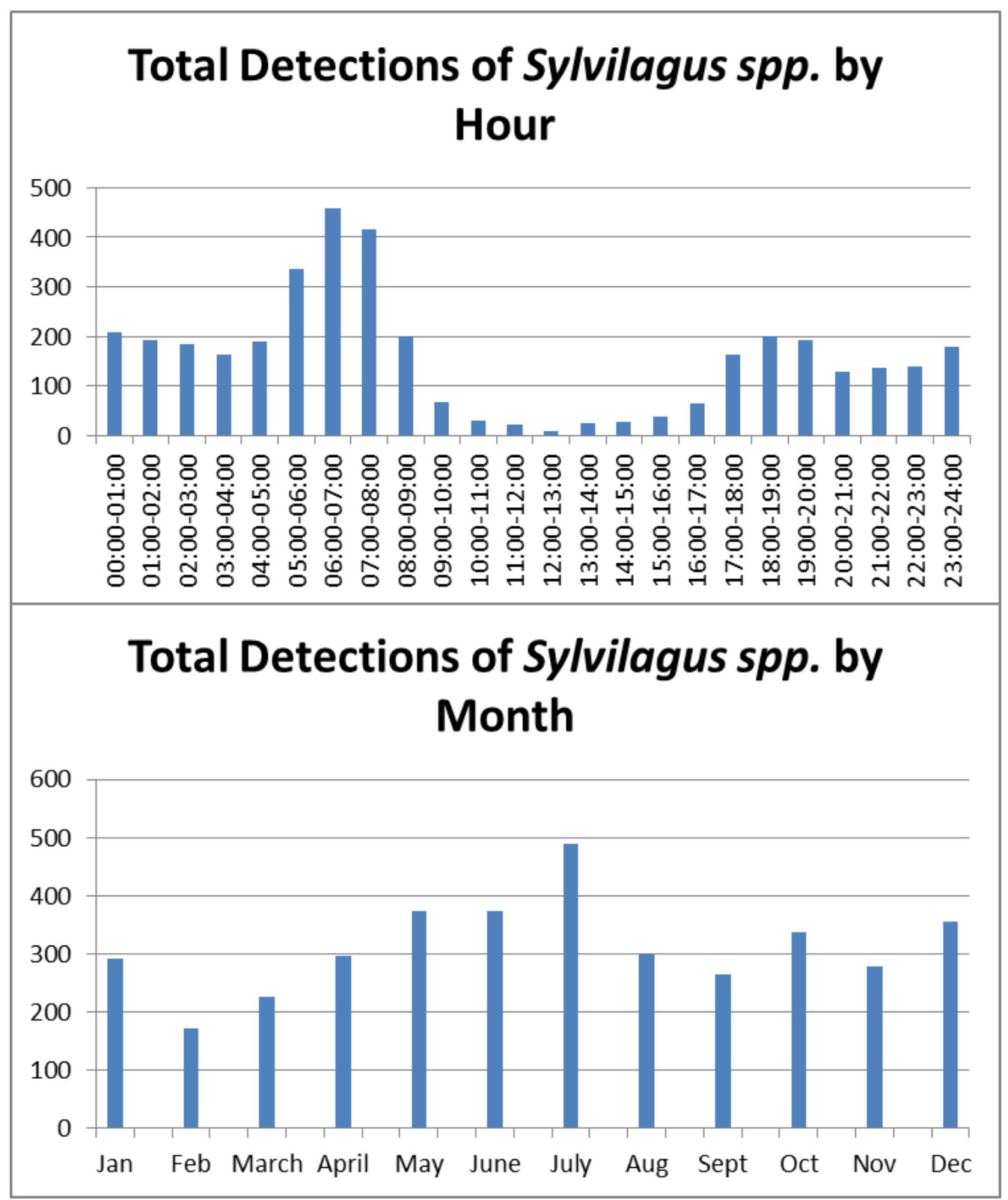

Figure 44. Daily and monthly detections of Sylvilagus spp. (cottontails), 2012-2015. 


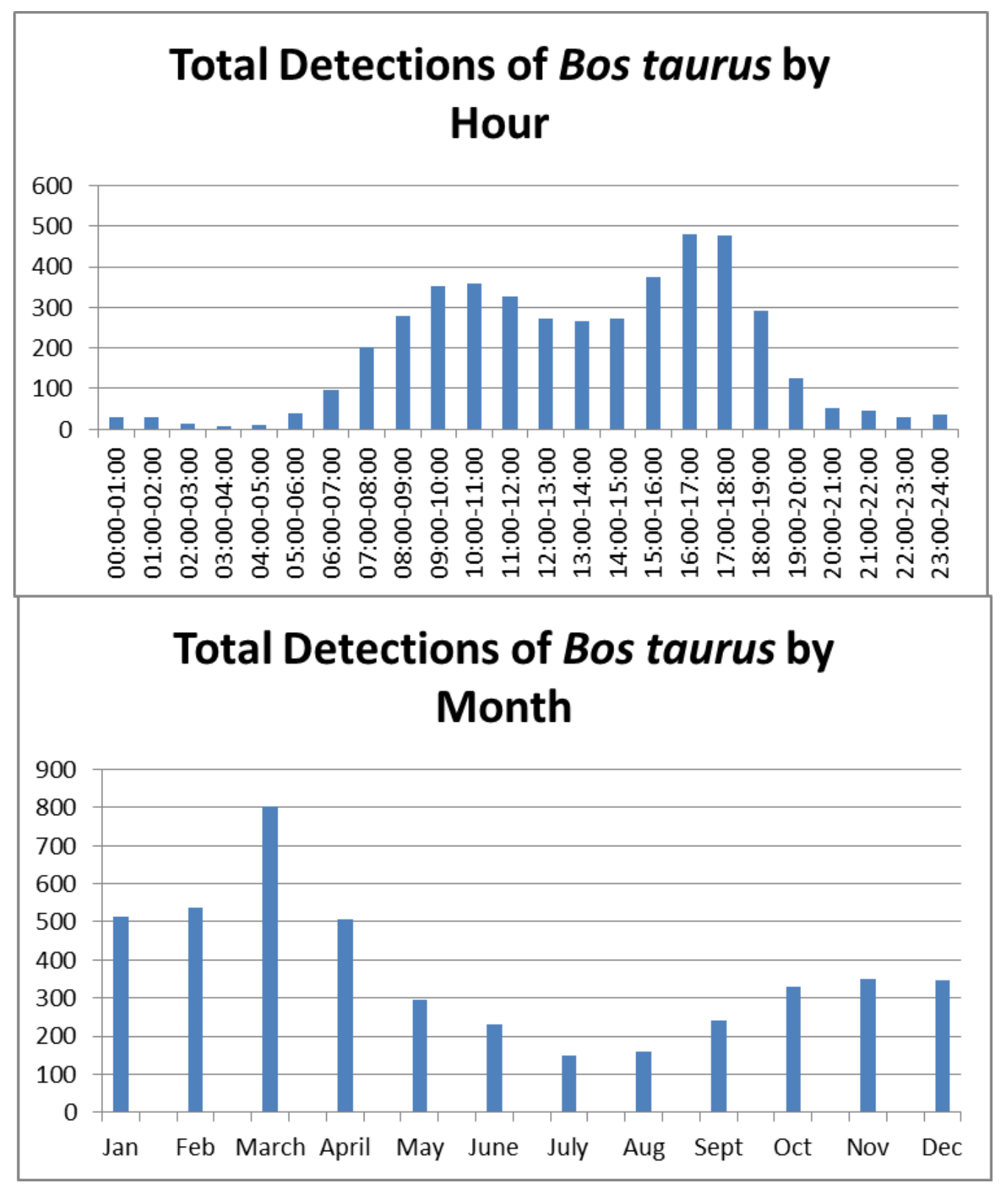

Figure 45. Daily and monthly detections of Bos taurus (cows), 2012-2015. 


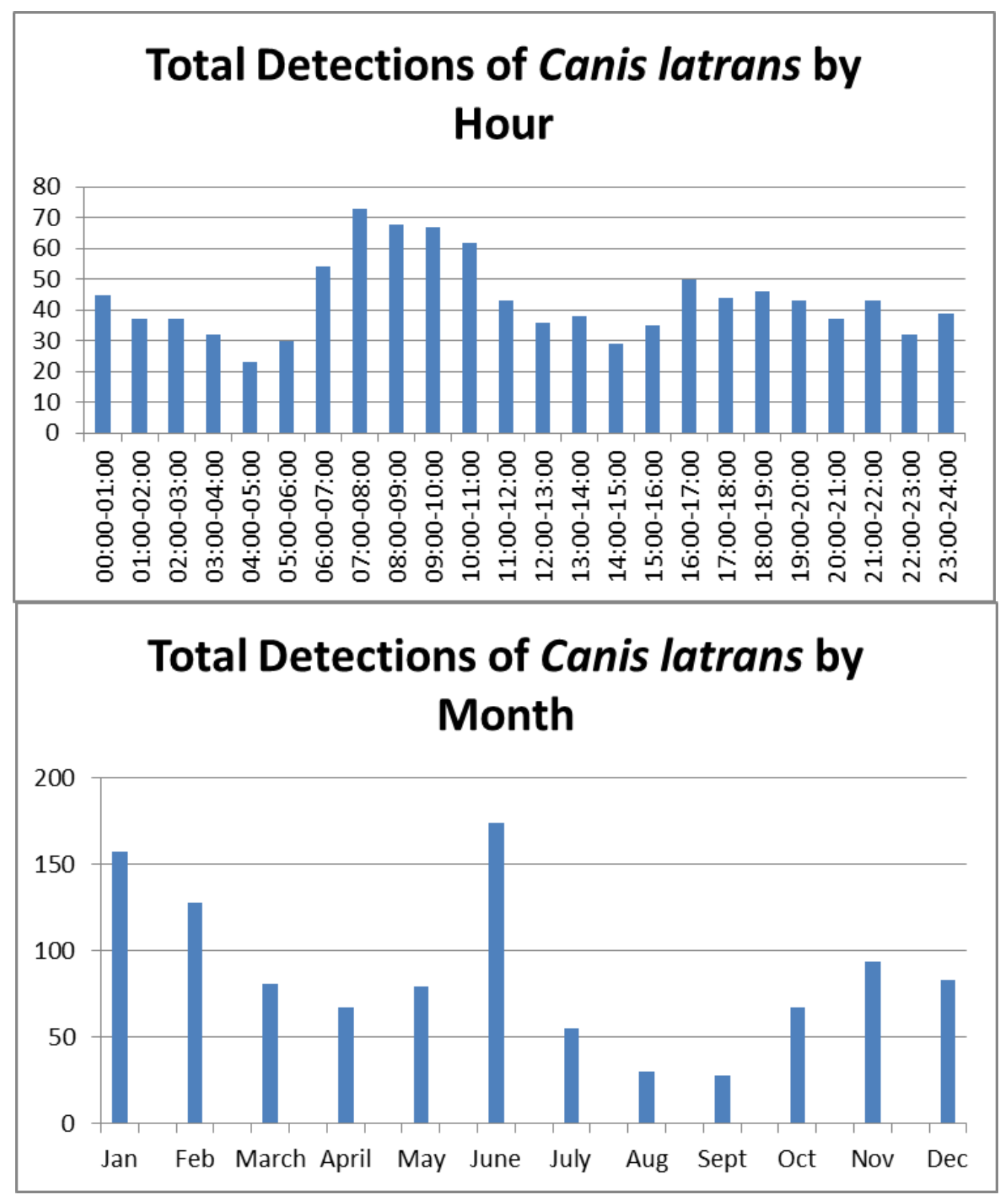

Figure 46. Daily and monthly detections of Canis latrans (coyotes), 2012-2015. 


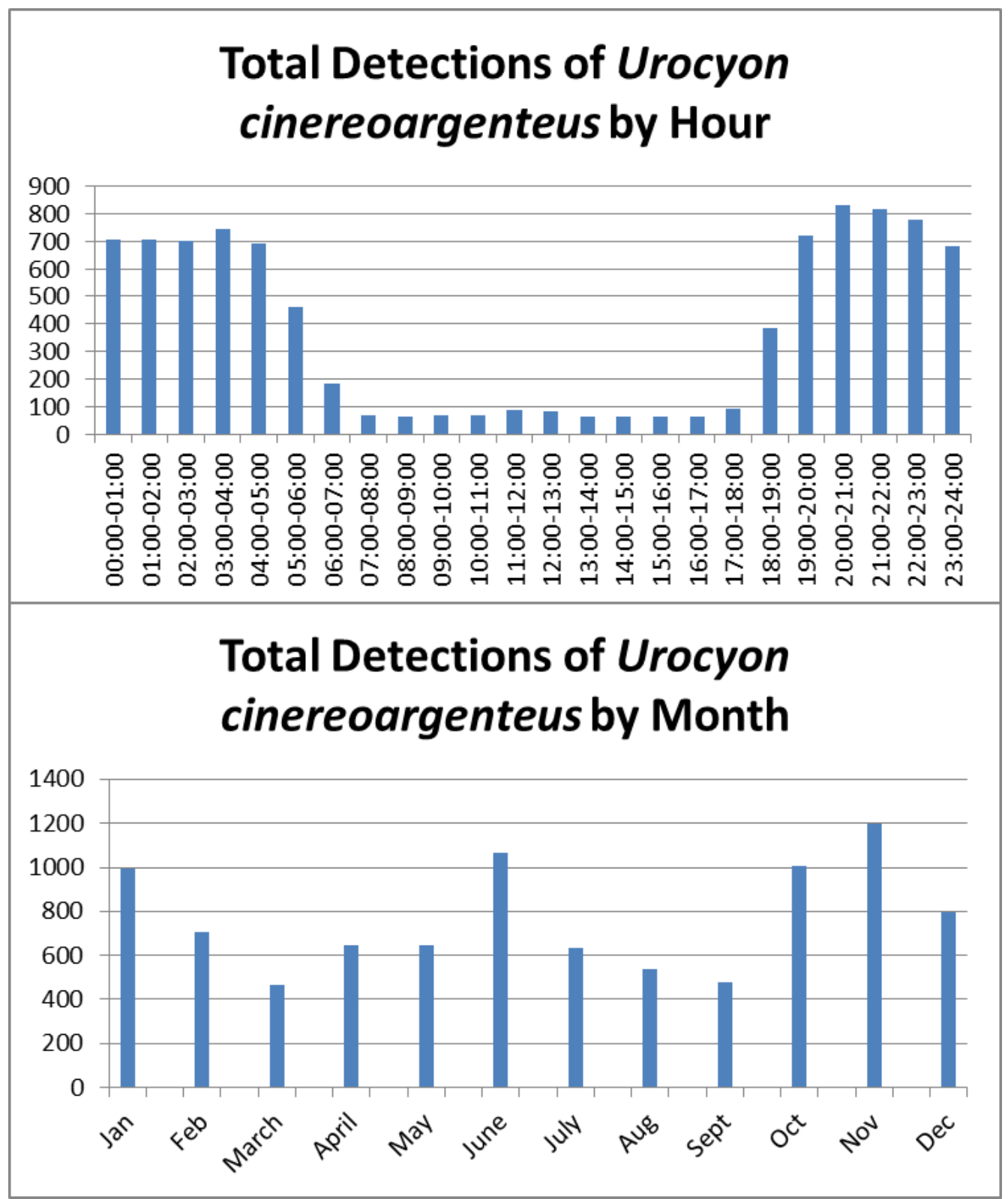

Figure 47. Daily and monthly detections of Urocyon cinereoargenteus (gray foxes), 2012-2015. 


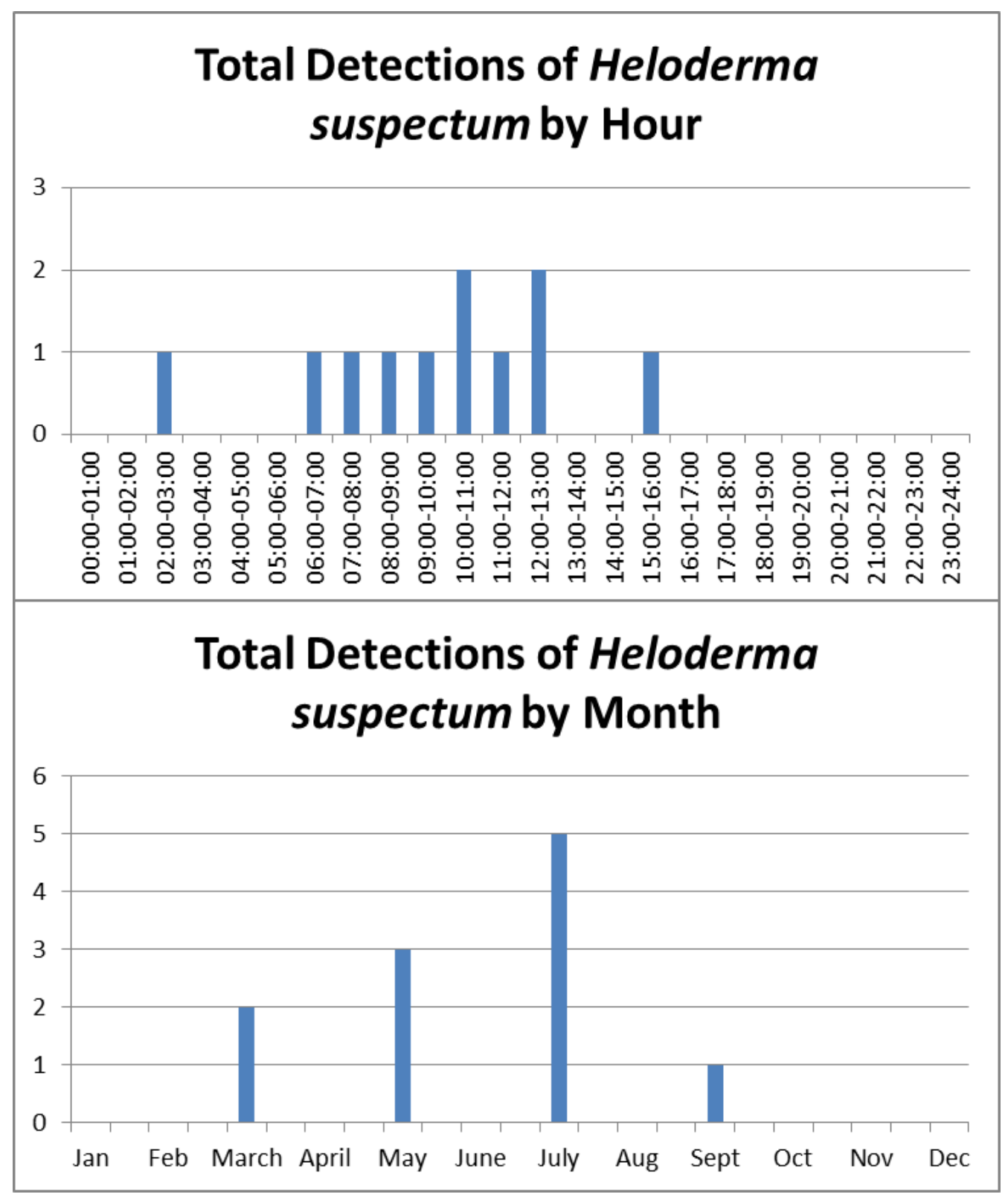

Figure 48. Daily and monthly detections of Heloderma suspectum (Gila monsters), 2012-2015. 


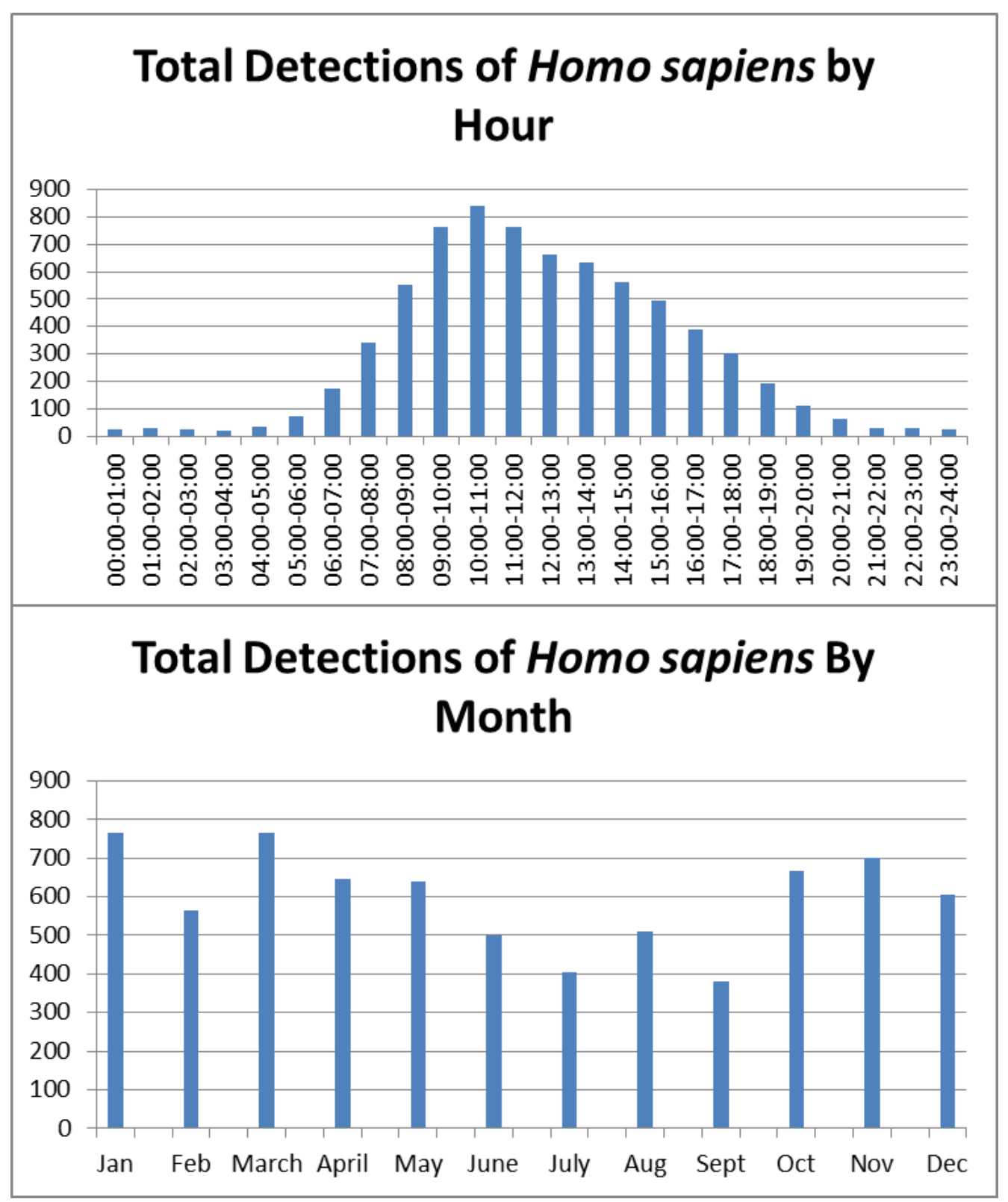

Figure 49. Daily and monthly detections of Homo sapiens (humans), 2012-2015. 


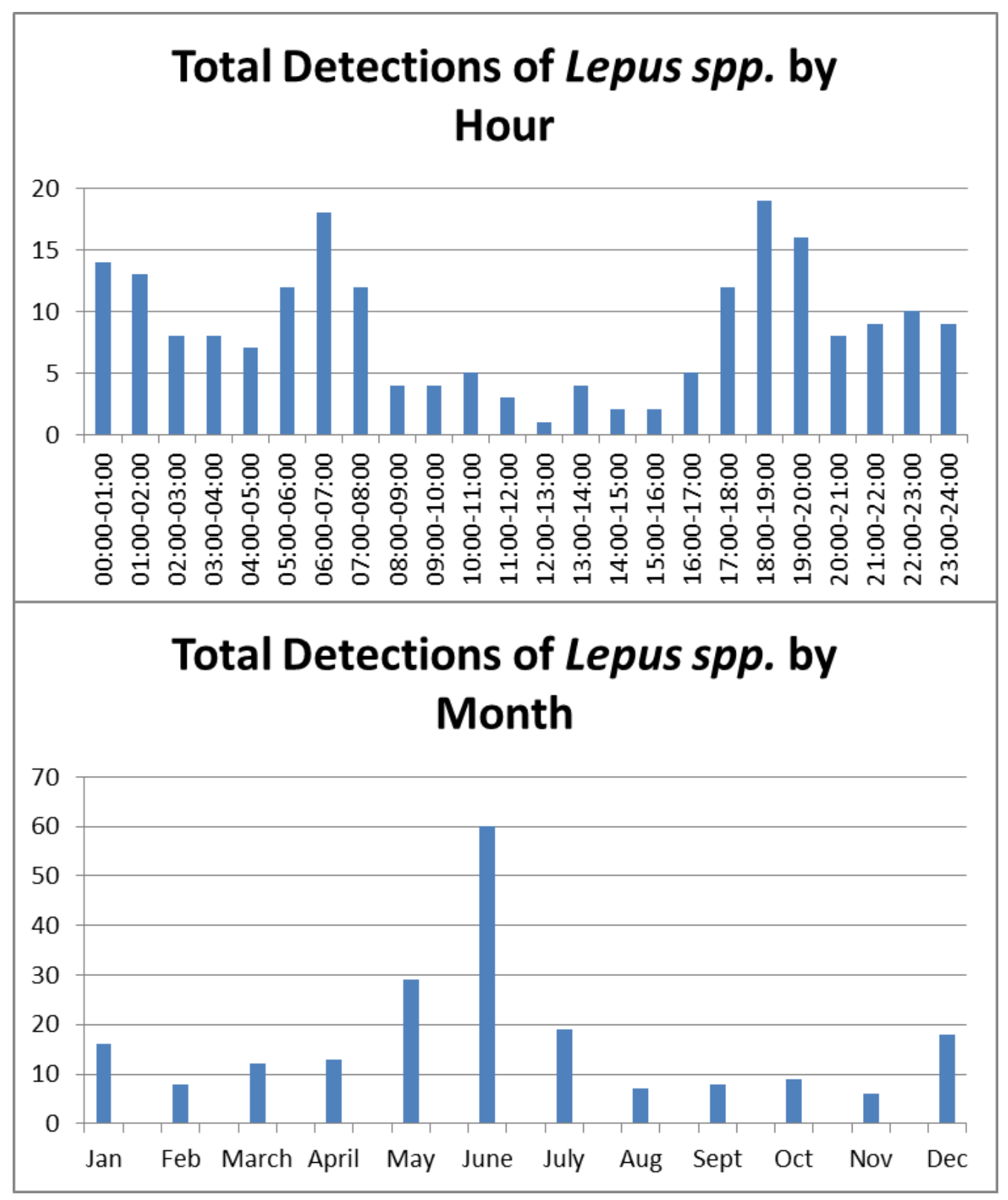

Figure 50. Daily and monthly detections of Lepus spp. (jackrabbits), 2012-2015. 


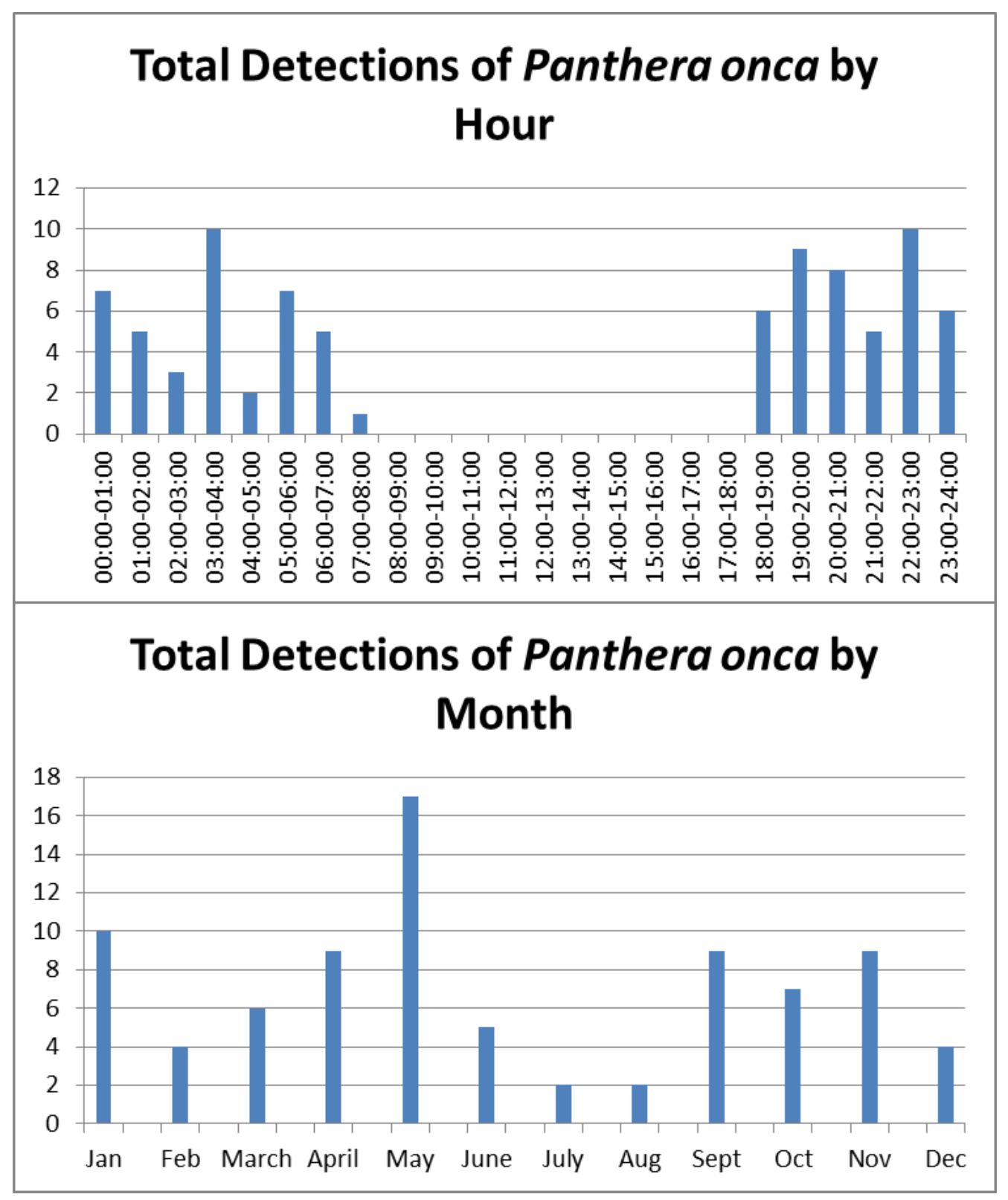

Figure 51. Daily and monthly detections of Panthera onca (jaguars), 2012-2015. 


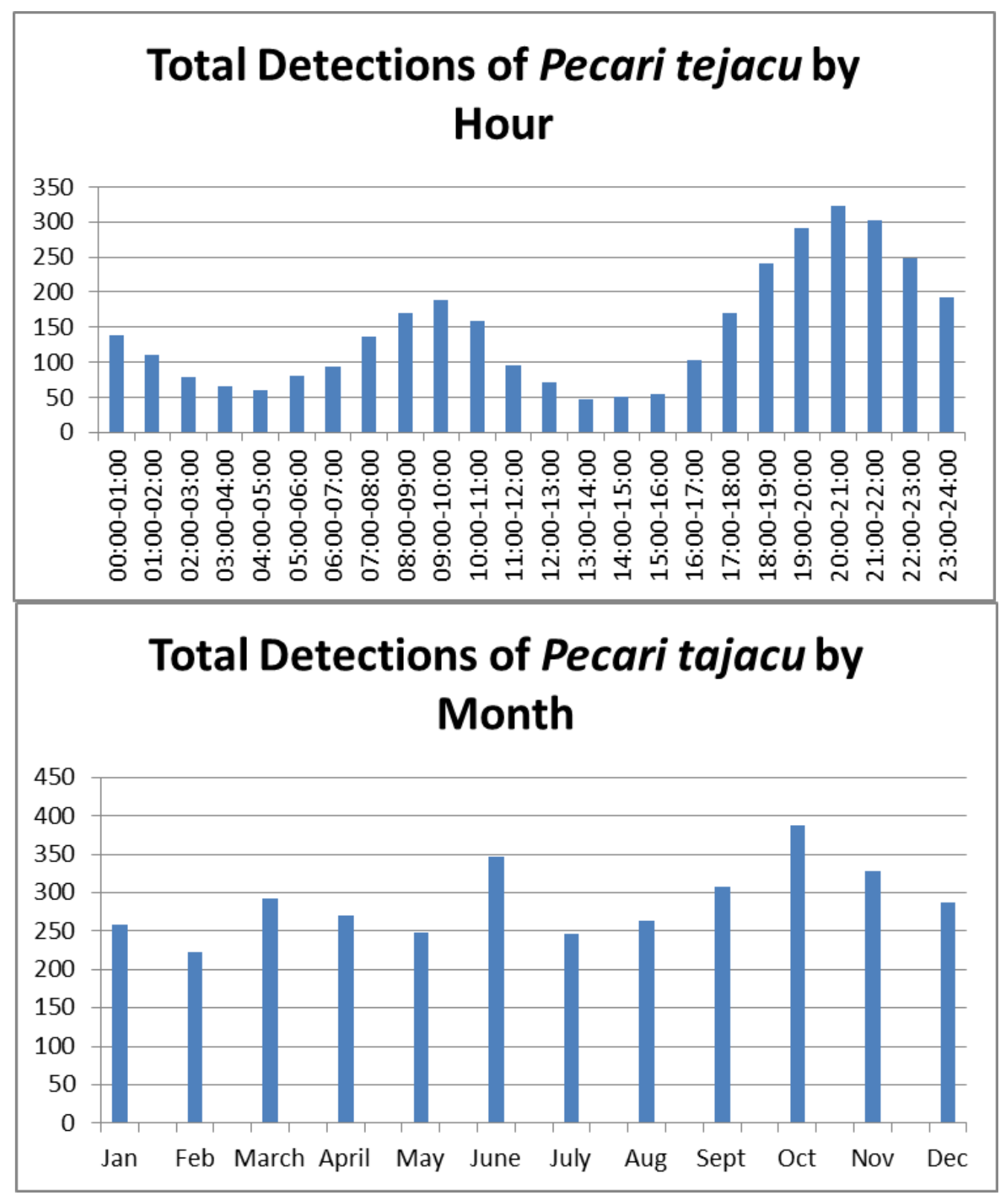

Figure 52. Daily and monthly detections of Pecari tejacu (javelina), 2012-2015. 


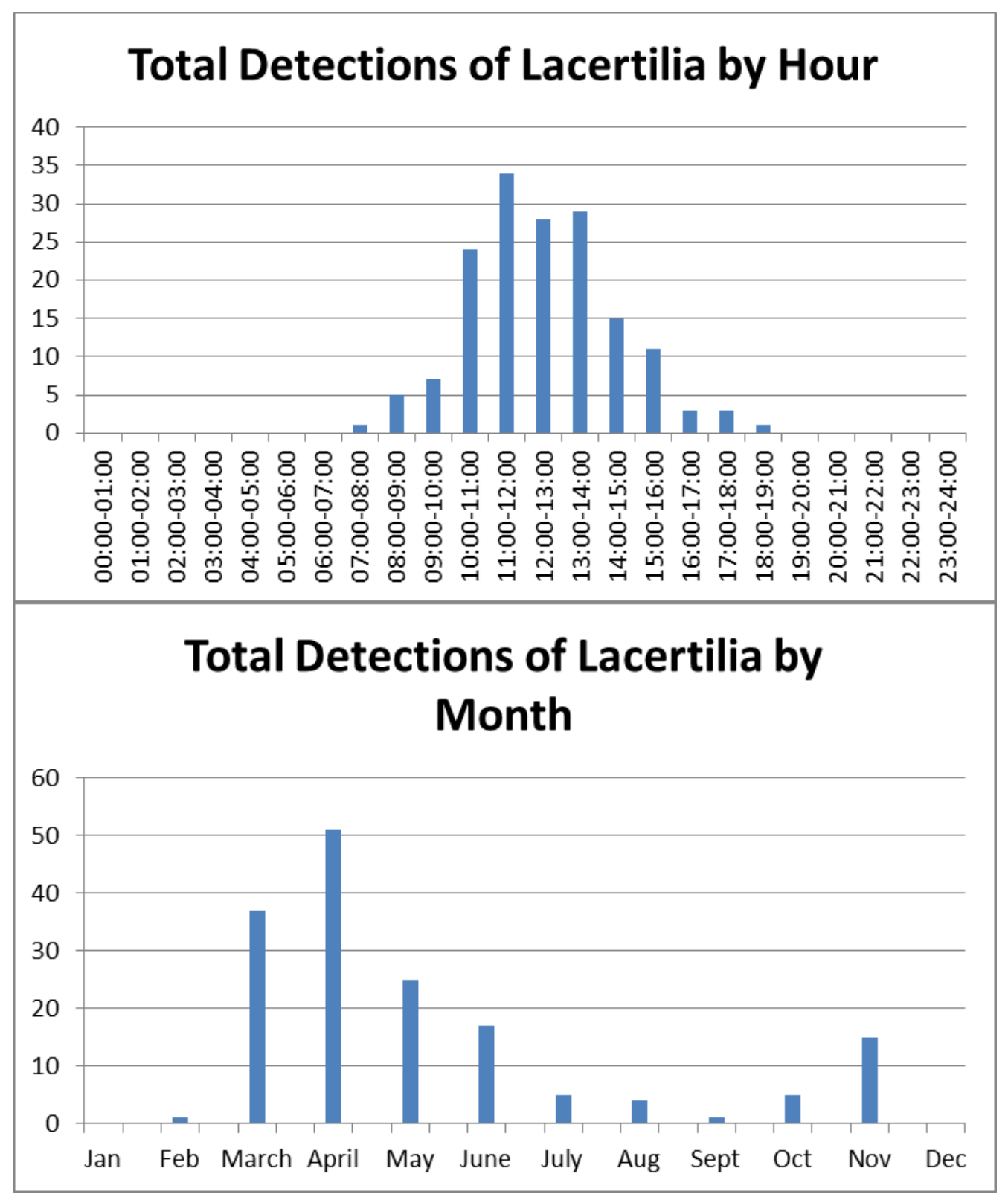

Figure 53. Daily and monthly detections of Lacertilia (lizards), 2012-2015. 


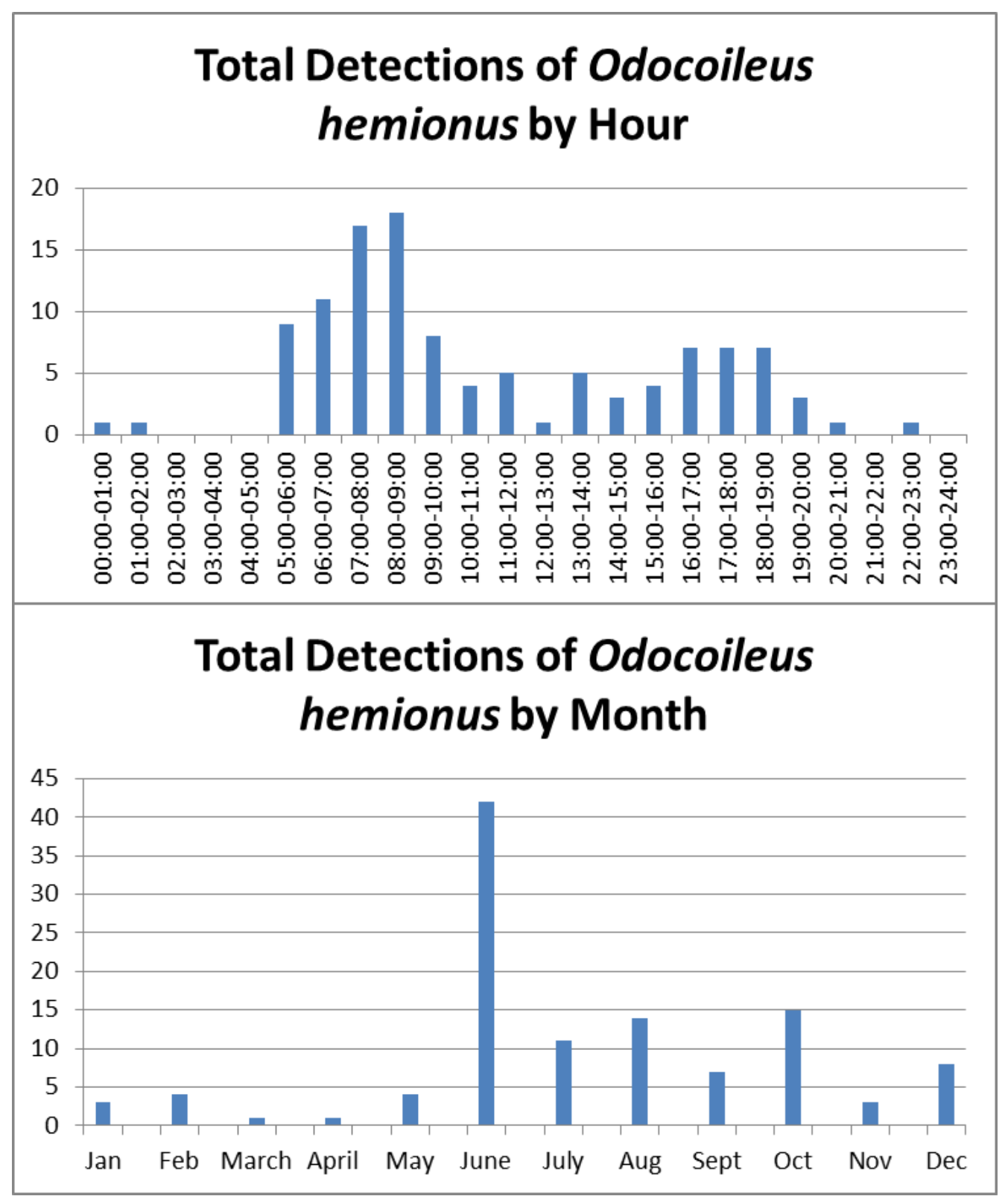

Figure 54. Daily and monthly detections of Odocoileus hemionus (mule deer), 2012-2015. 


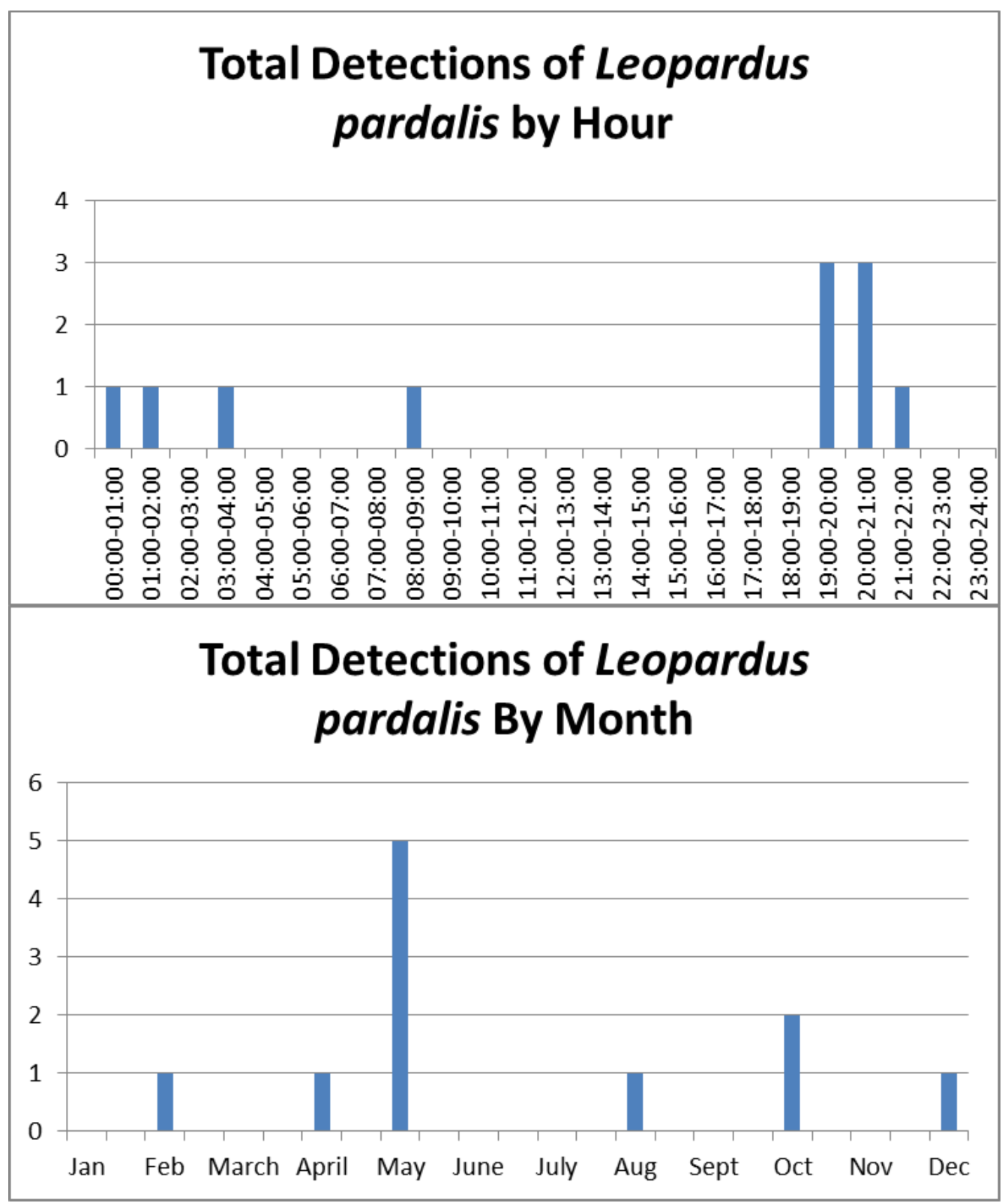

Figure 55. Daily and monthly detections of Leopardus pardalis (ocelots), 2012-2015. 


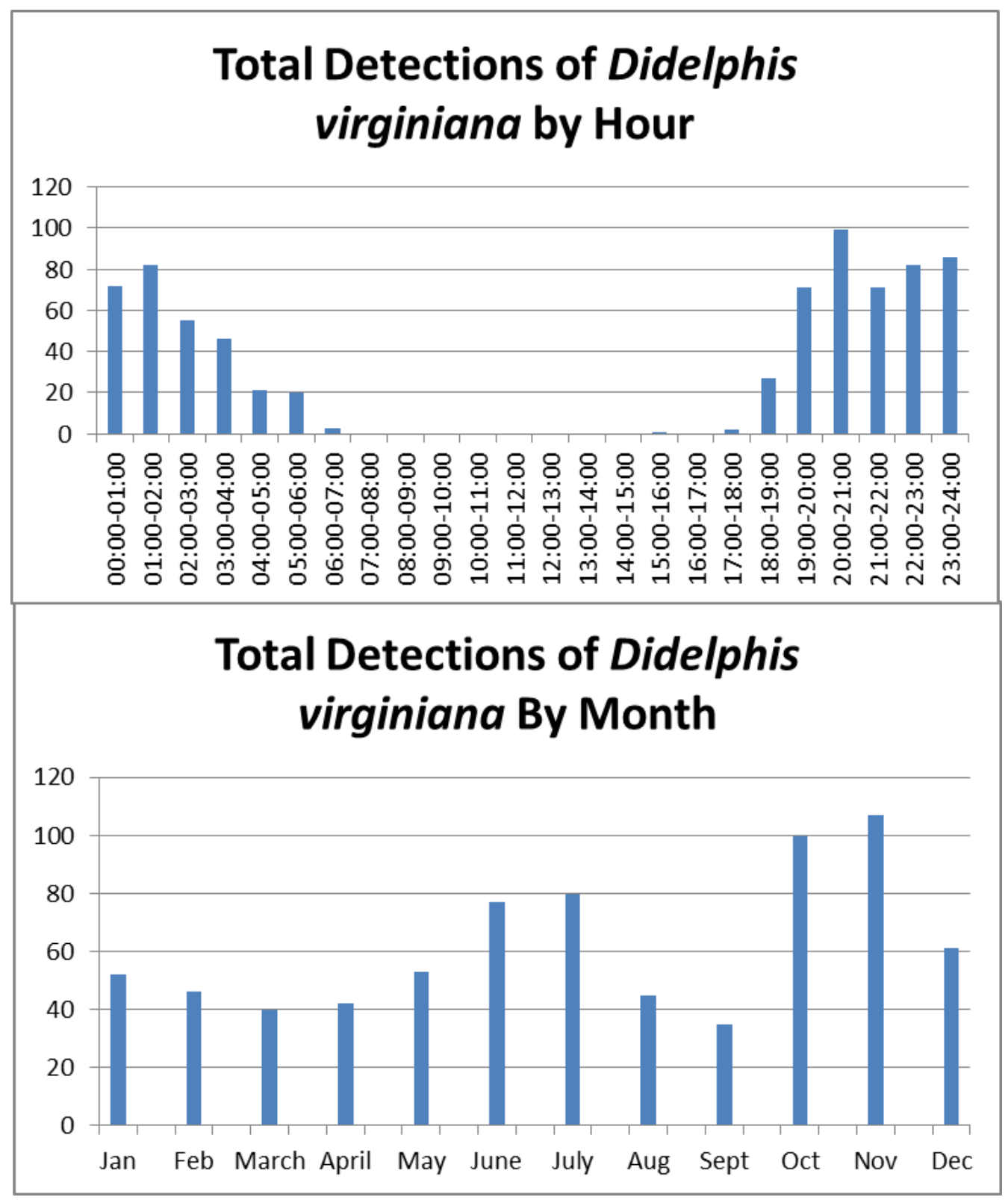

Figure 56. Daily and monthly detections of Didelphis virginiana (opossums), 2012-2015. 


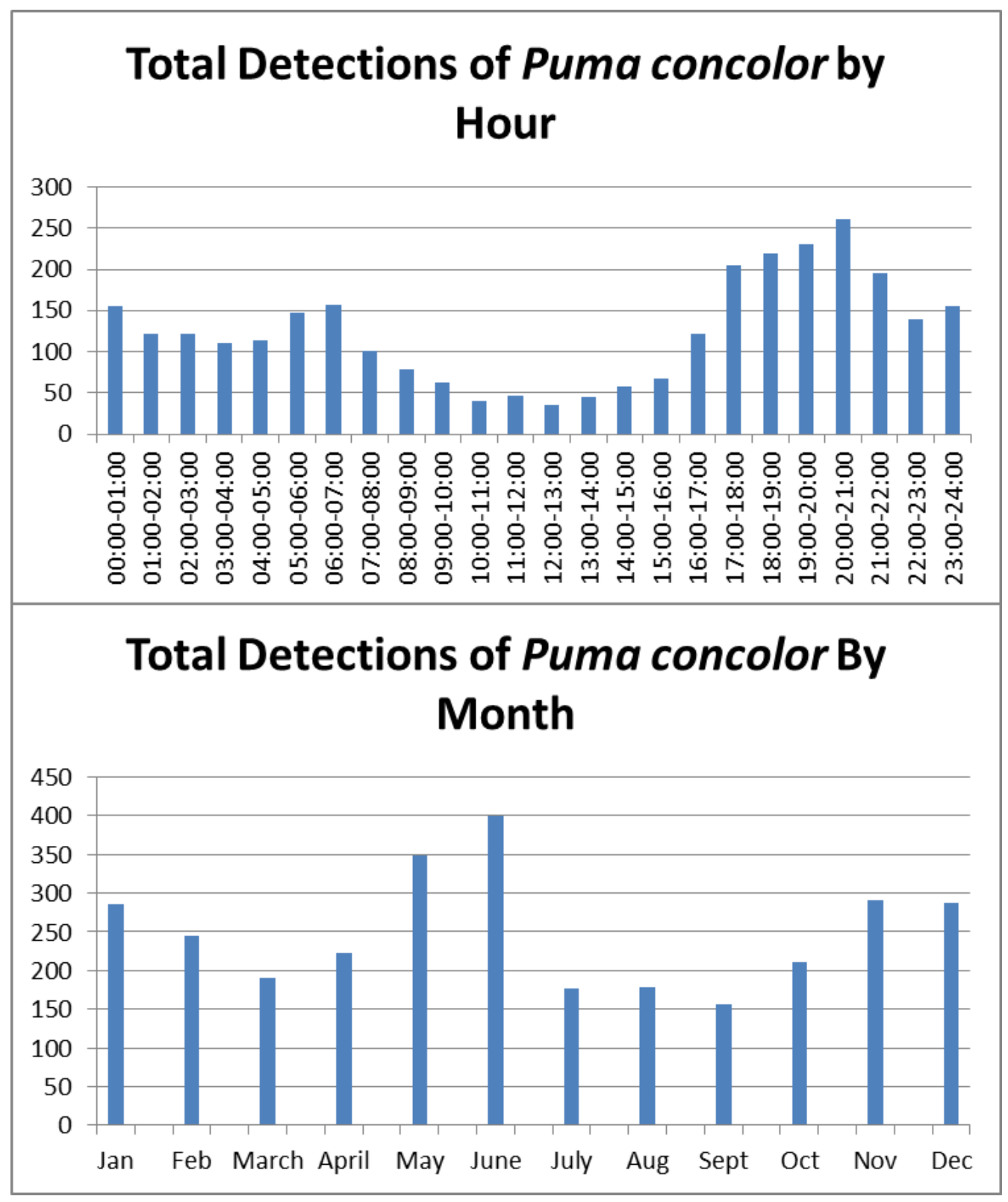

Figure 57. Daily and monthly detections of Puma concolor (pumas), 2012-2015. 


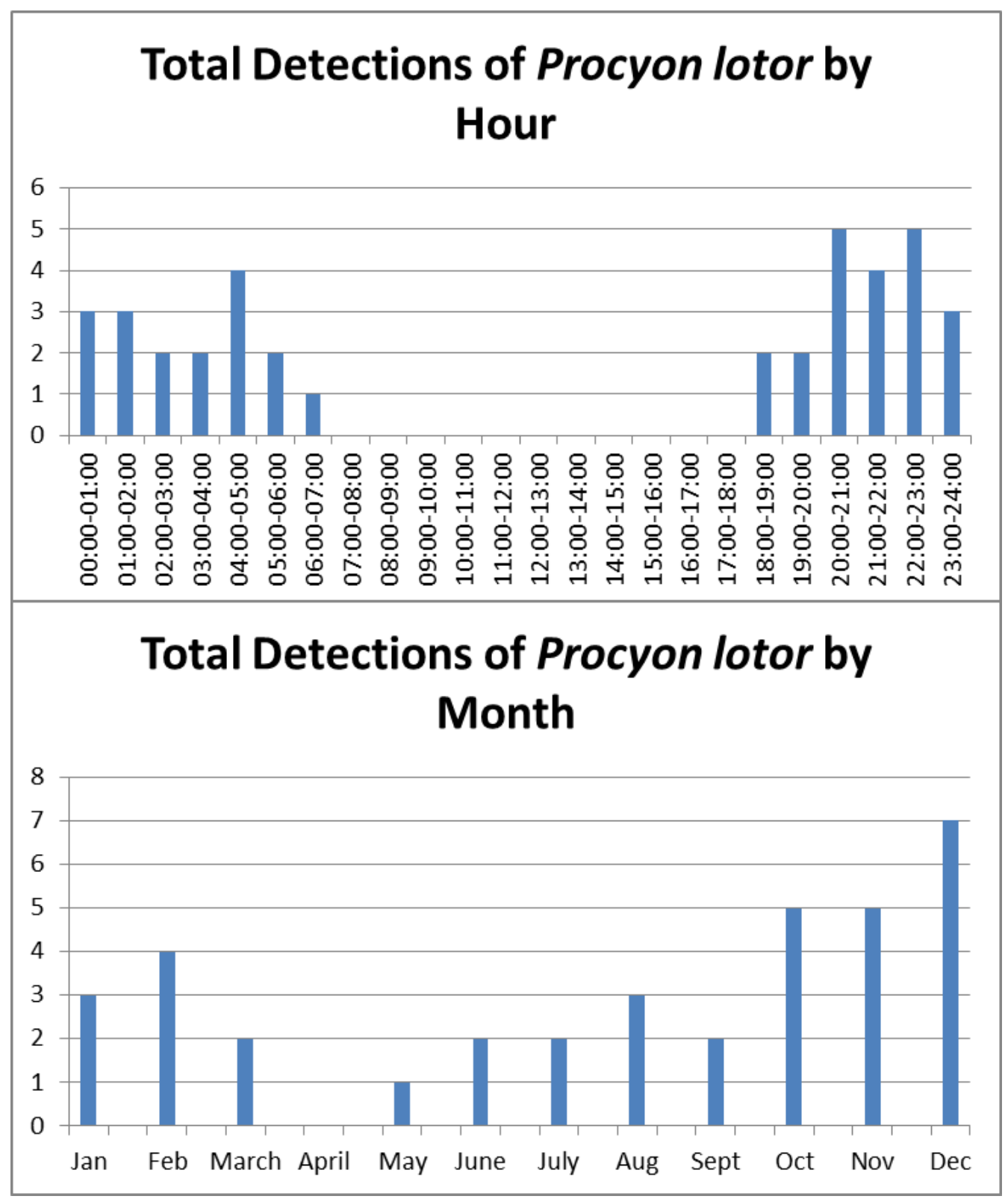

Figure 58. Daily and monthly detections of Procyon lotor (raccoons), 2012-2015. 


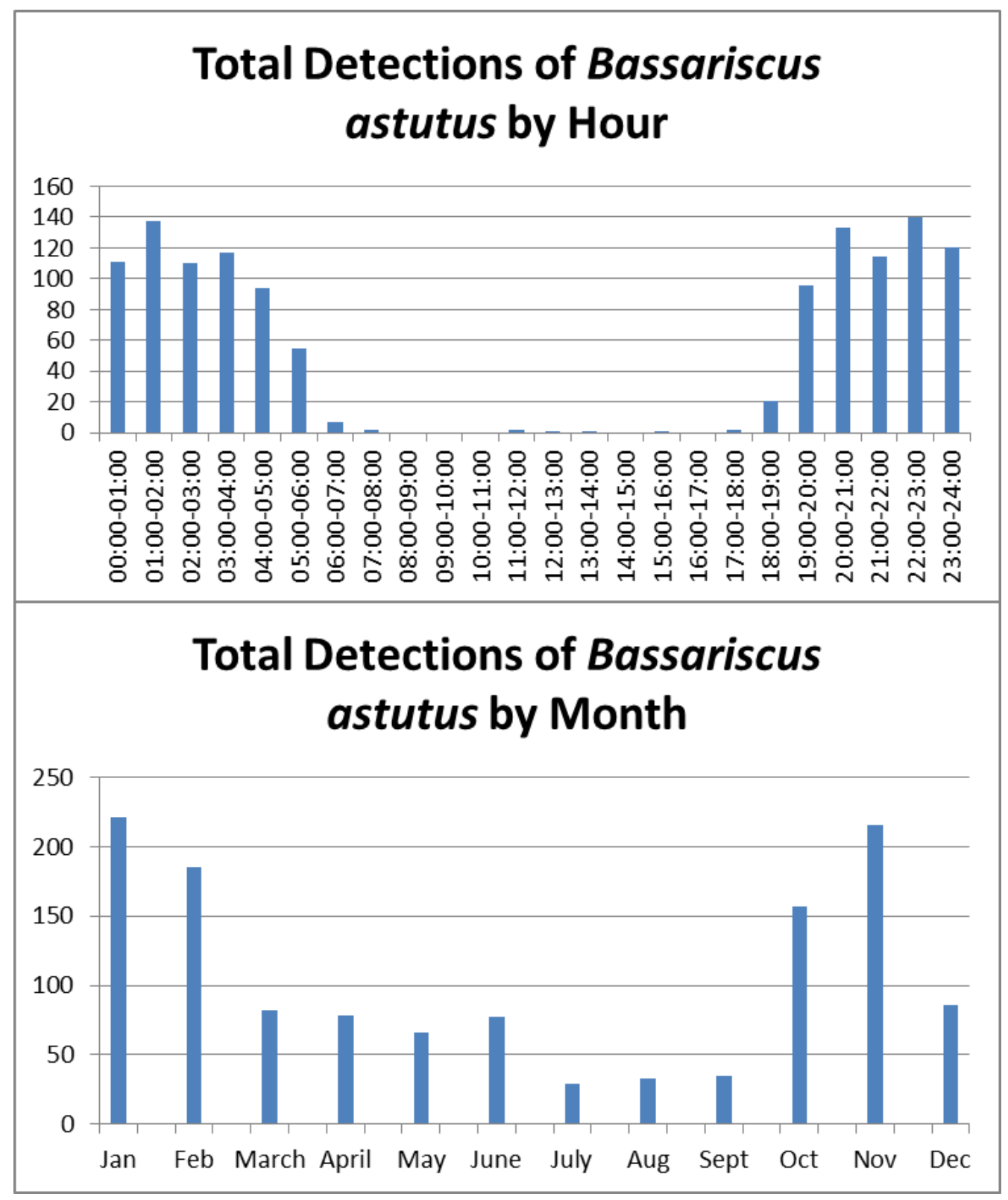

Figure 59. Daily and monthly detections of Bassariscus astutus (ringtails), 2012-2015. 


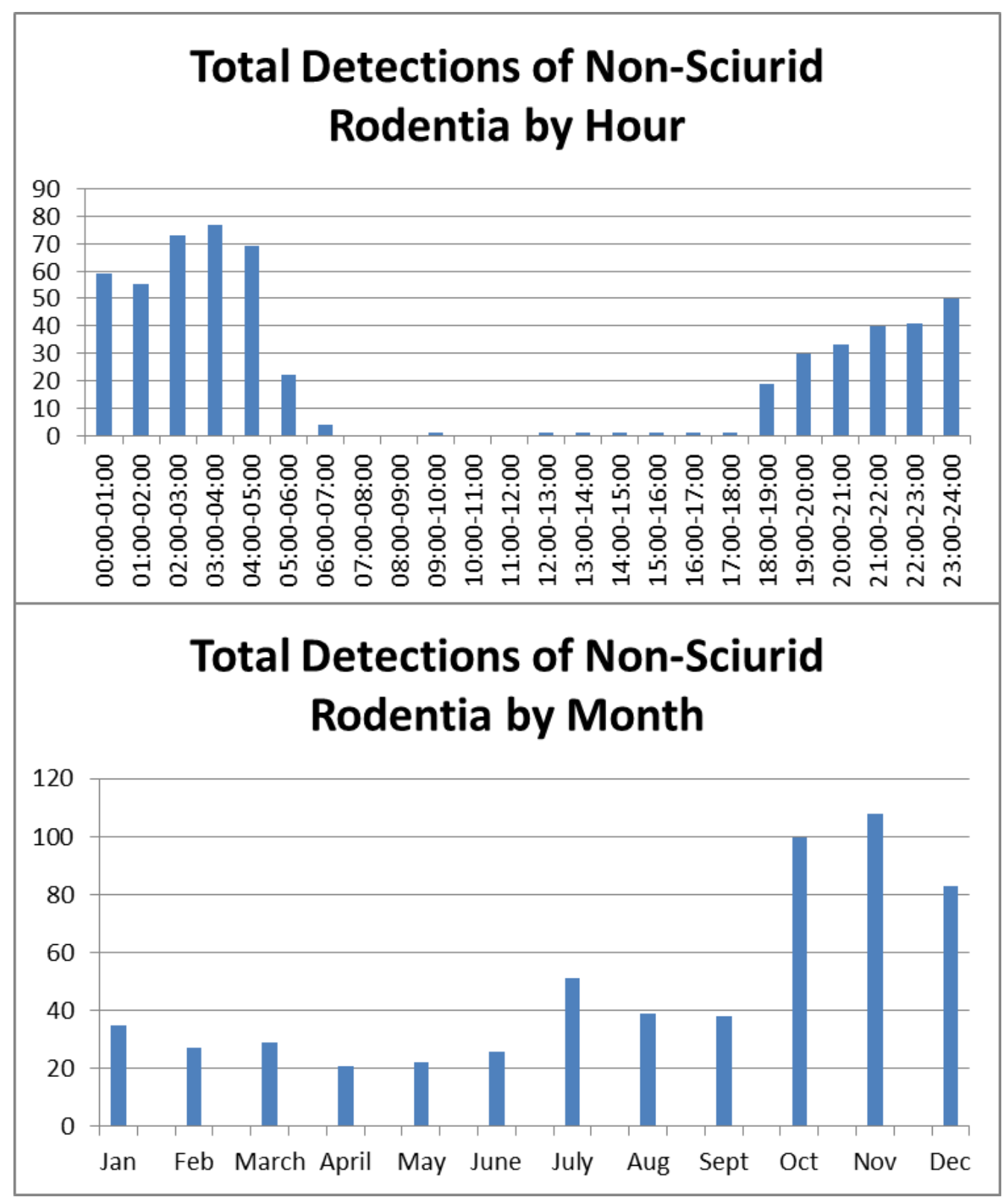

Figure 60. Daily and monthly detections of non-Sciurid Rodentia (rodents, not including squirrels), 20122015. 


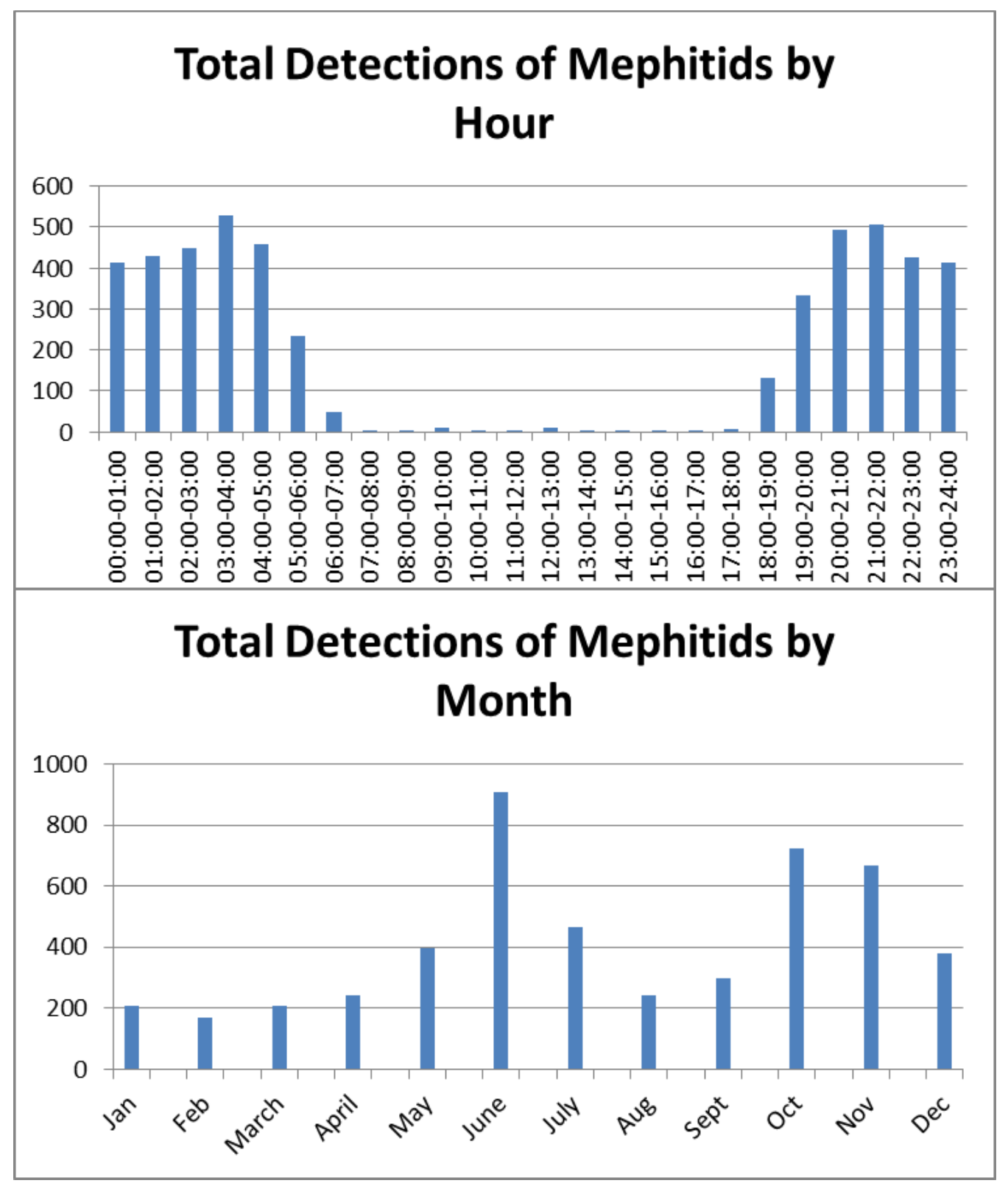

Figure 61. Daily and monthly detections of Mephitids (skunks), 2012-2015. 


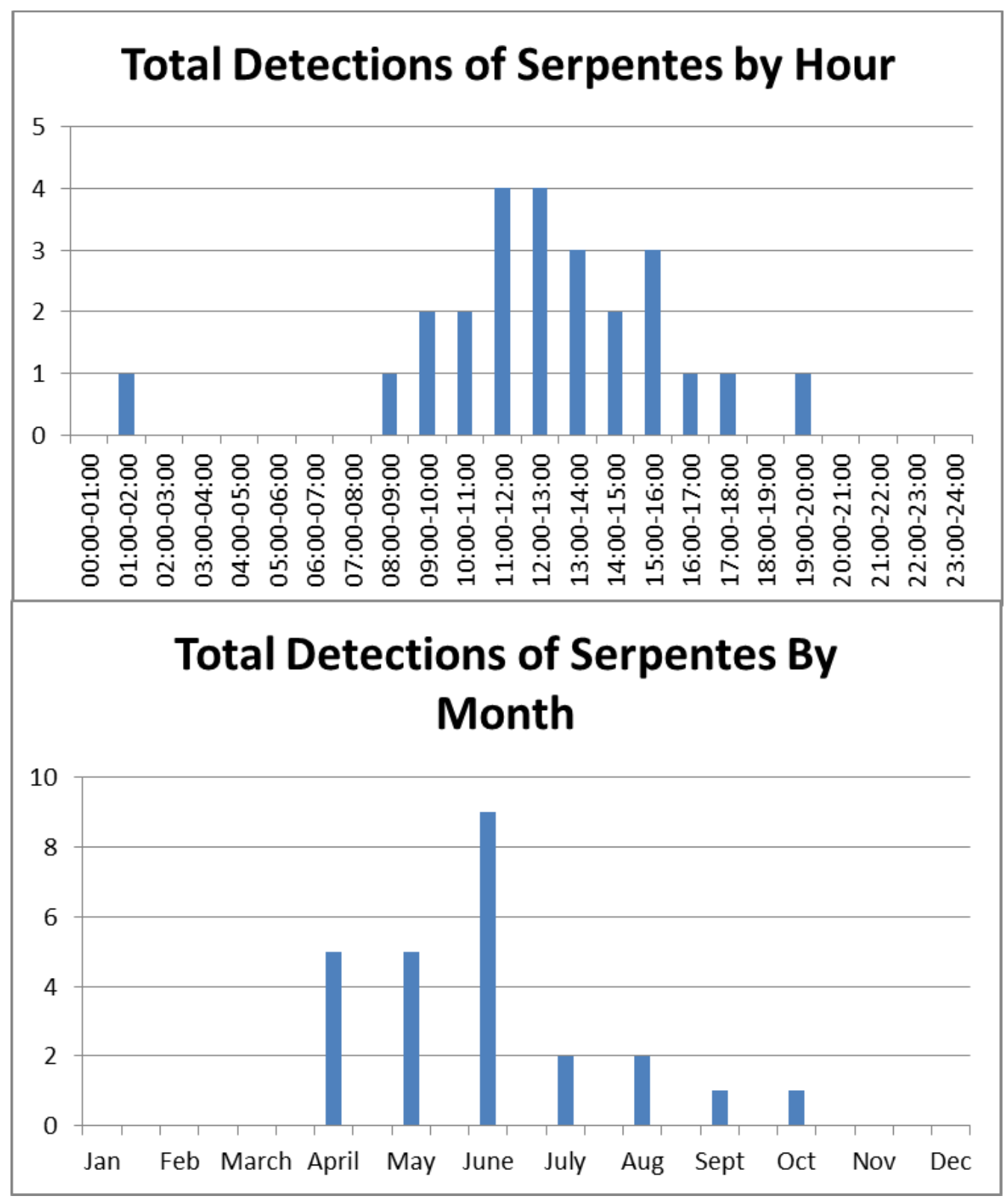

Figure 62. Daily and monthly detections of Serpentes (snakes), 2012-2015. 


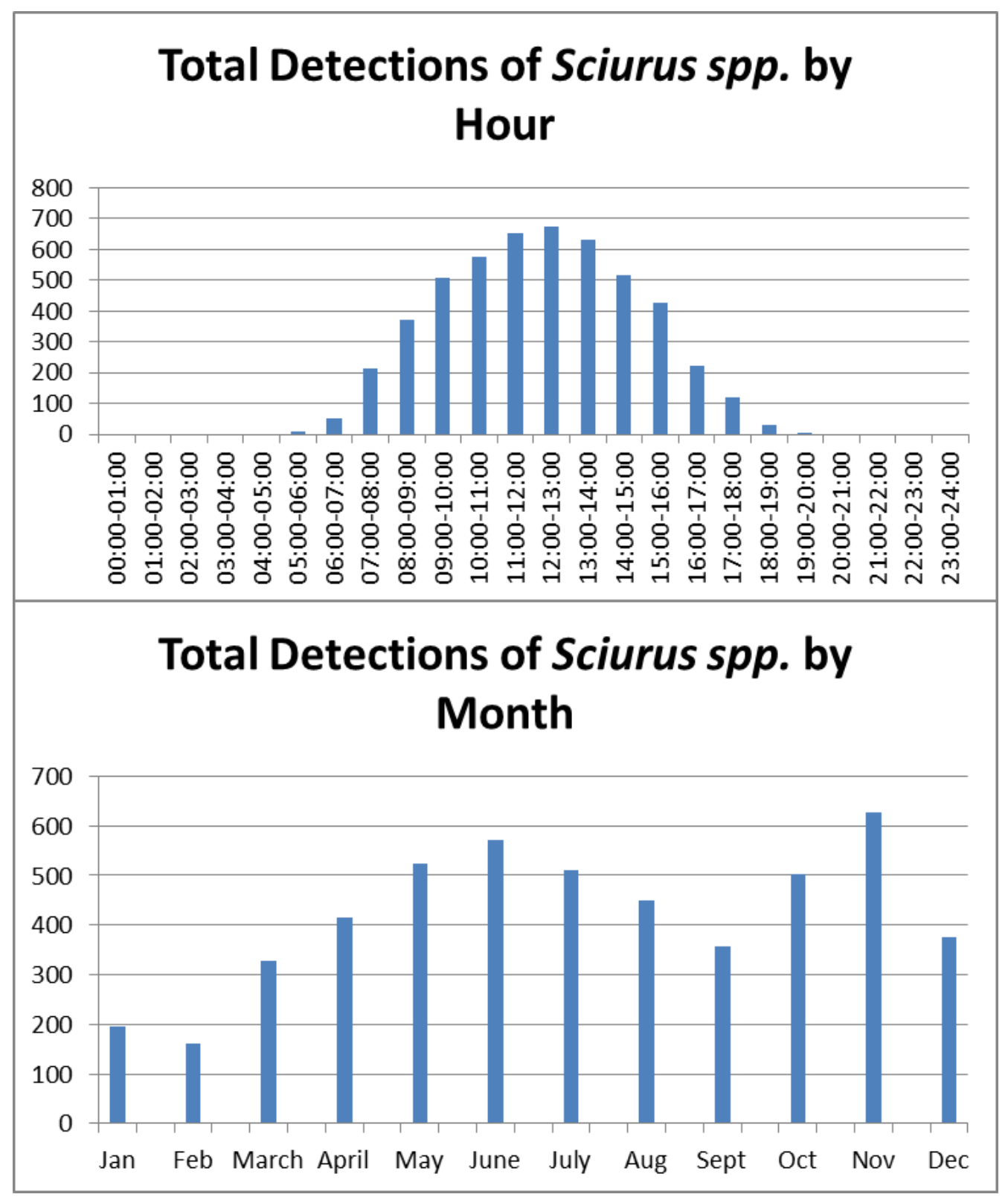

Figure 63. Daily and monthly detections of Sciurus spp. (squirrels), 2012-2015. 


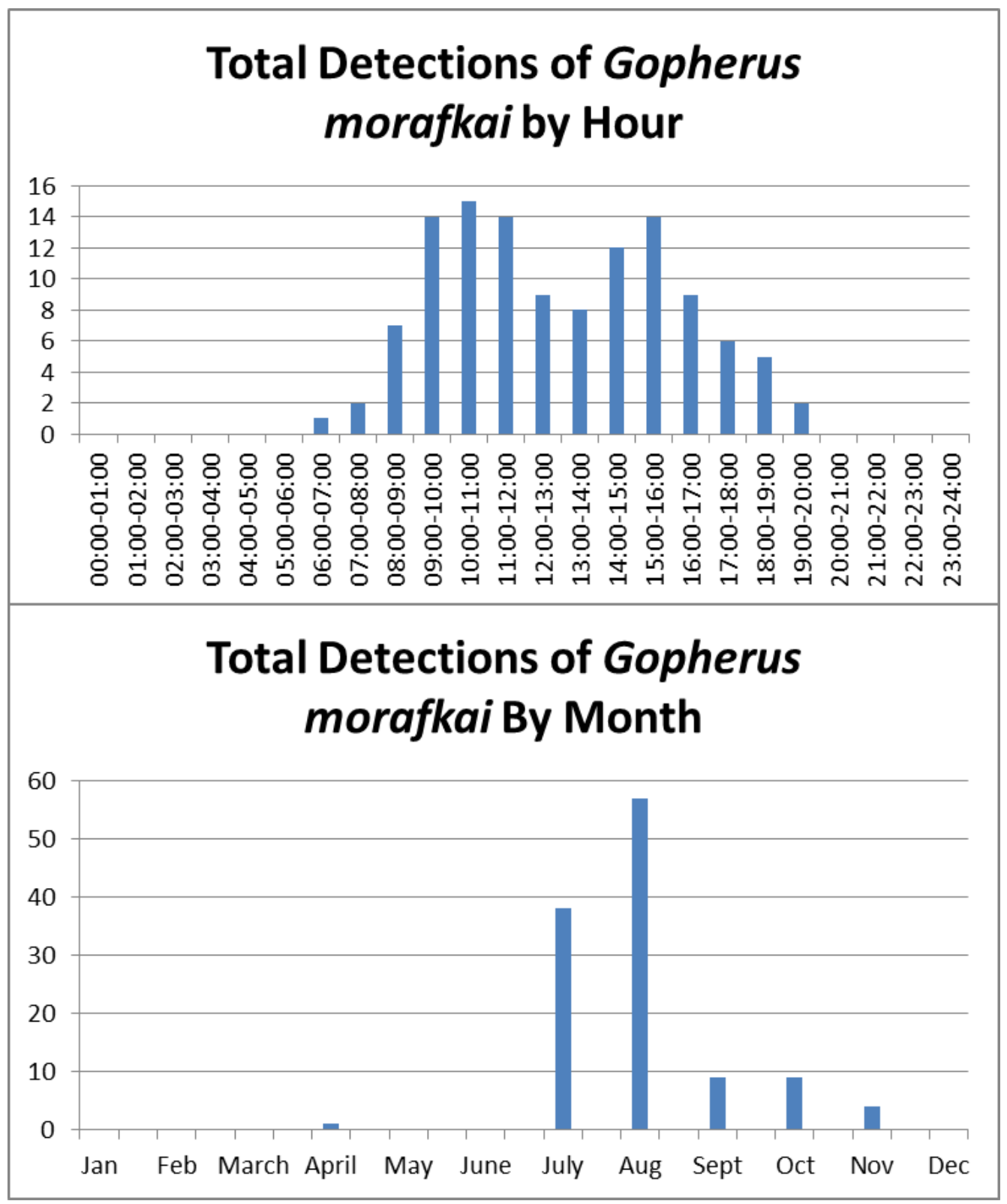

Figure 64. Daily and monthly detections of Gopherus morafkai (tortoises), 2012-2015. 


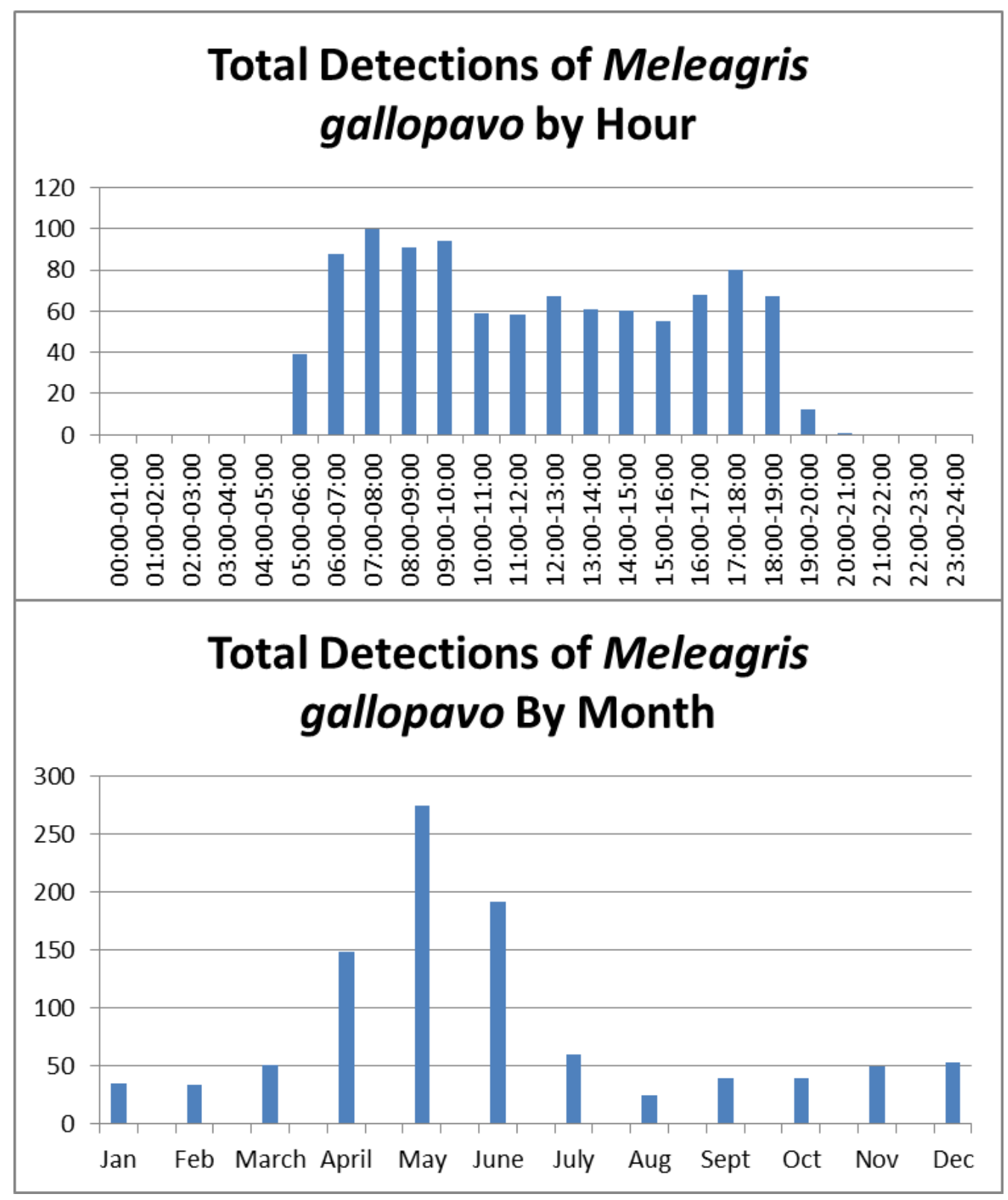

Figure 65. Daily and monthly detections of Meleagris gallopavo (turkeys), 2012-2015. 


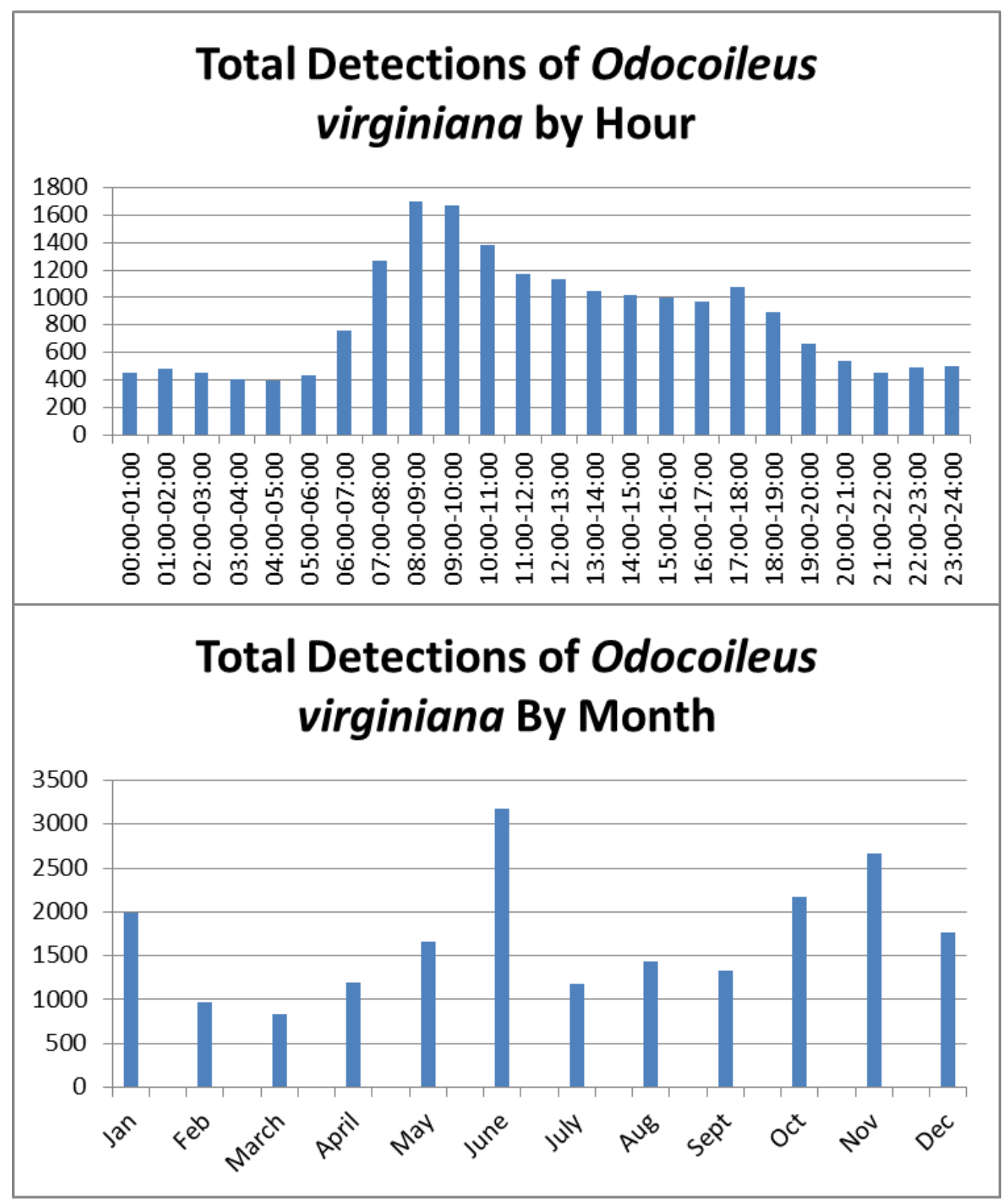

Figure 66. Daily and monthly detections of Odocoileus virginiana (white-tailed deer), 2012-2015. 


\section{Bat Detections by Moon Phase}

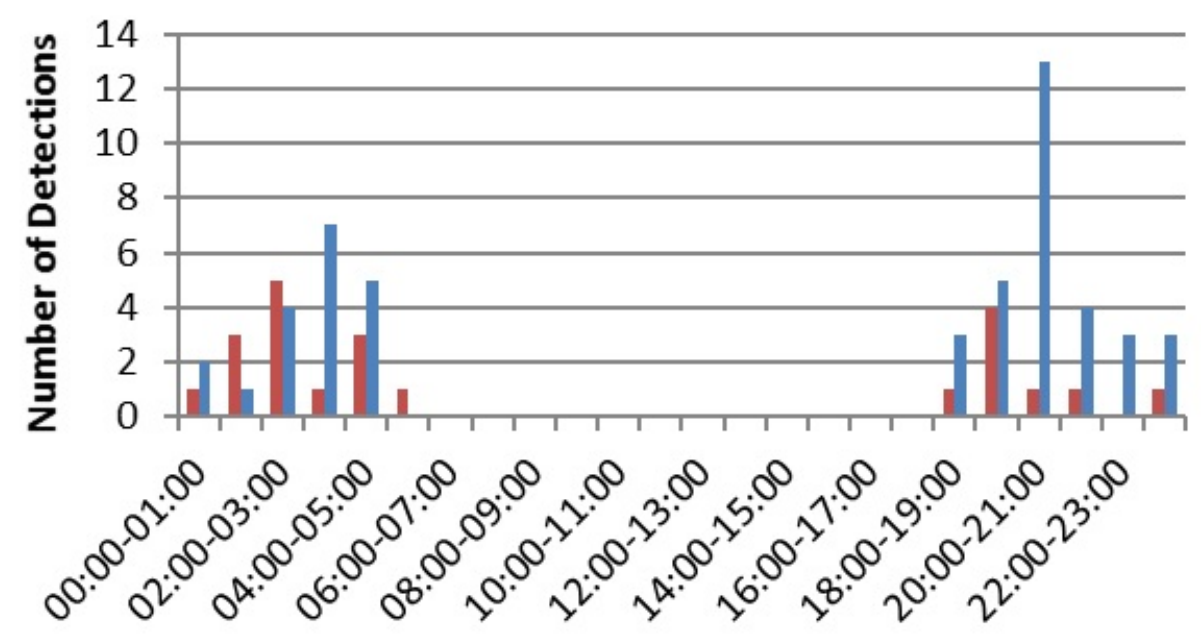

- Full Moon

new Moon

Time

Figure 67. Chiropteran (bat) detections by moon phase, 2012-2015.

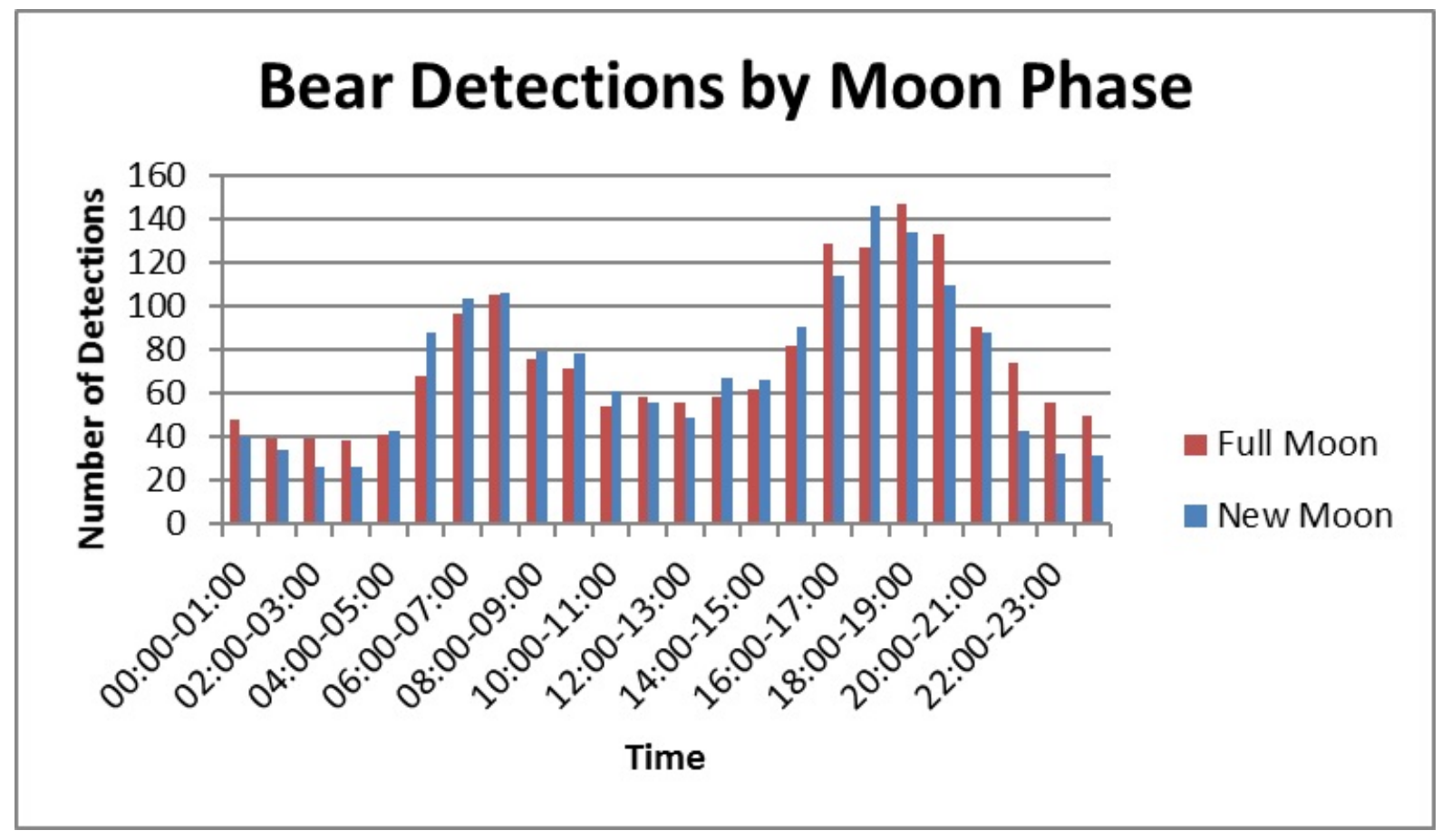

Figure 68. Ursus americanus (bear) detections by moon phase, 2012-2015. 


\section{Bobcat Detections by Moon Phase}

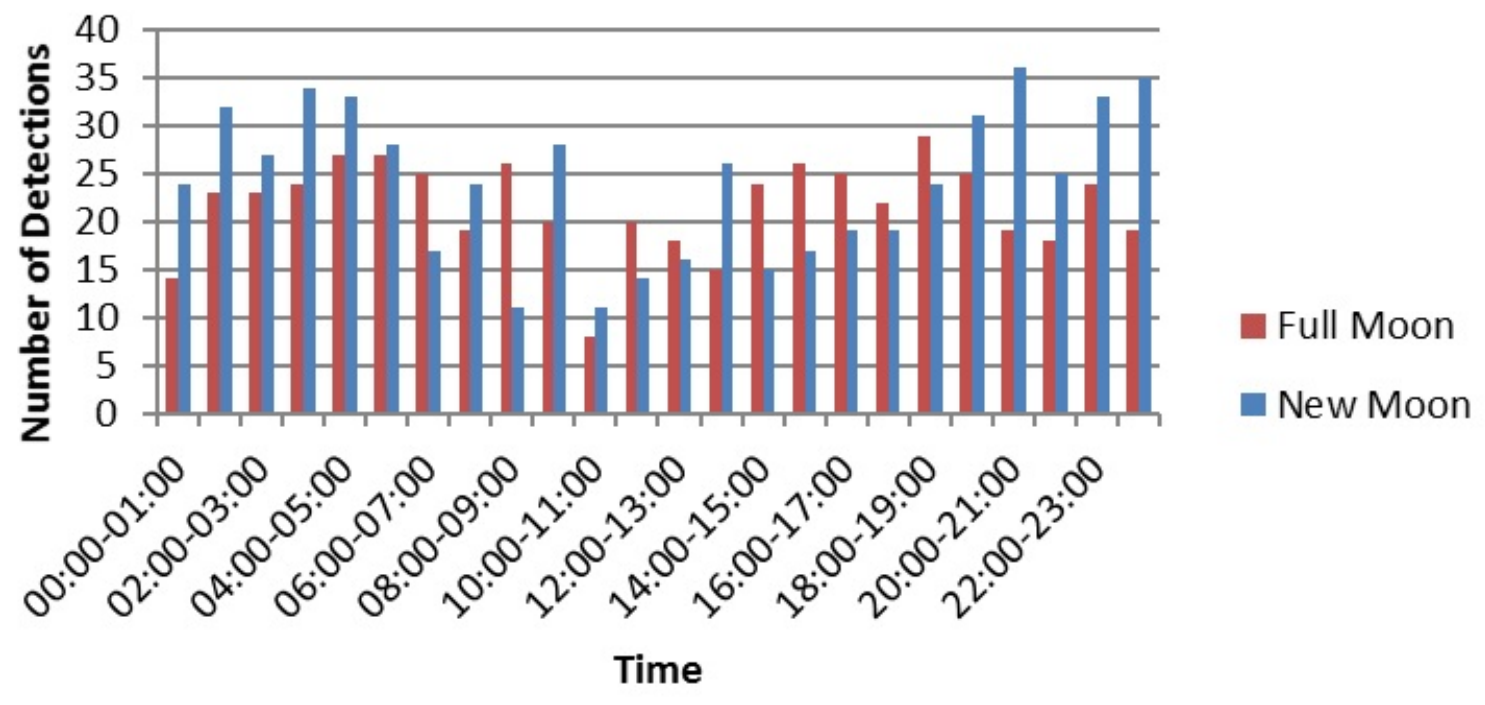

Figure 69. Lynx rufus (bobcat) detections by moon phase, 2012-2015.

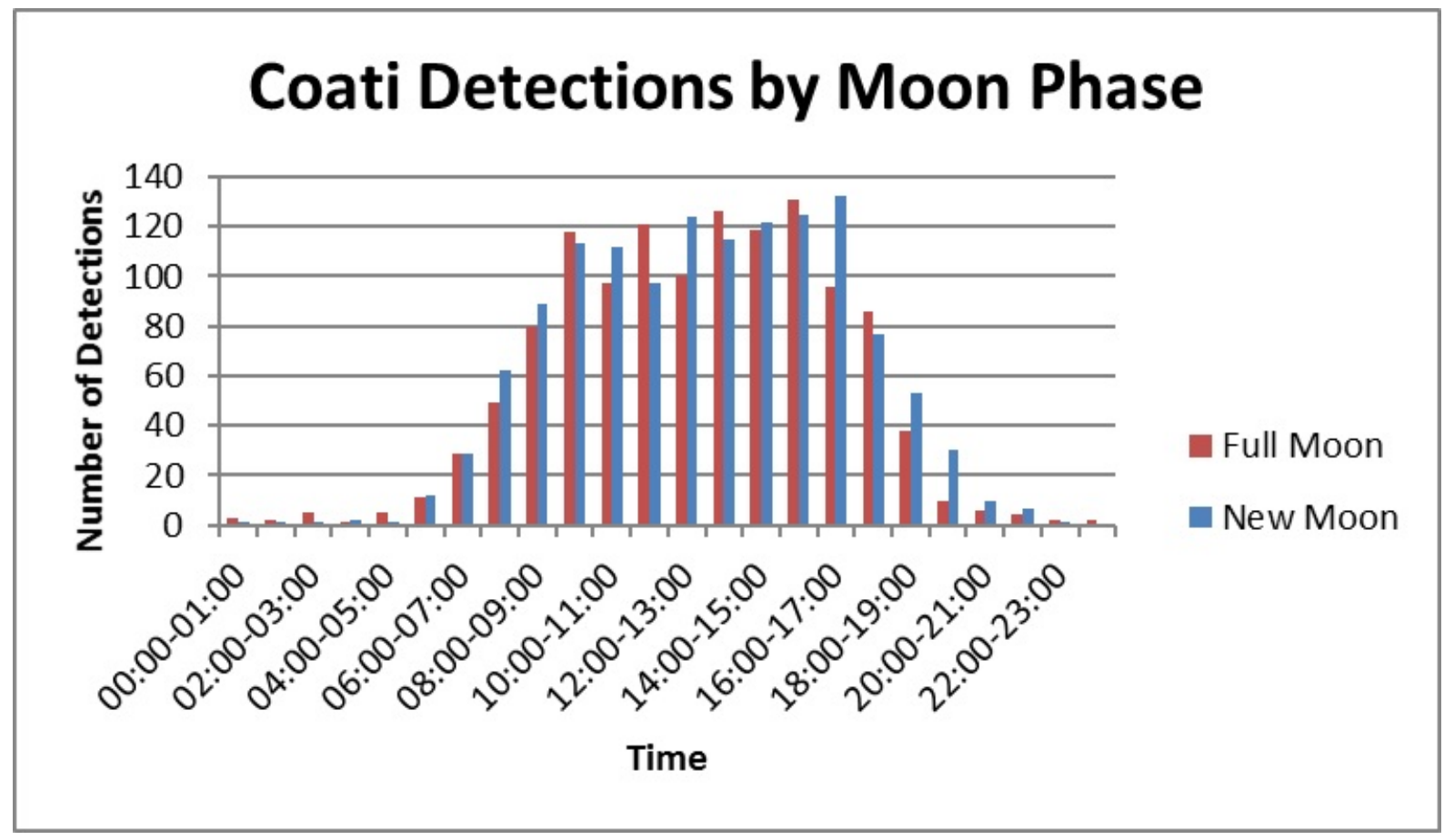

Figure 70. Nasua narica (coatimundi) detections by moon phase, 2012-2015. 


\section{Cottontail Detections by Moon Phase}

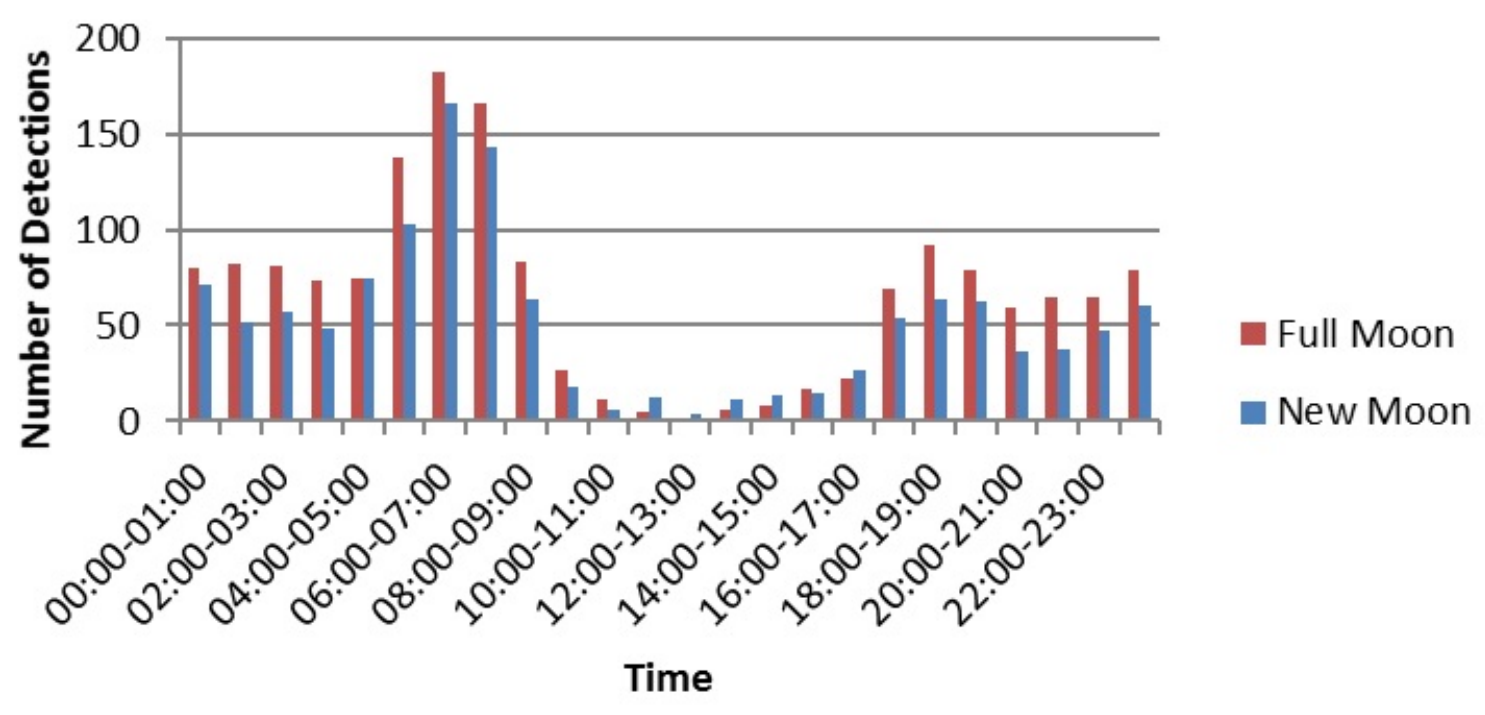

Figure 71. Sylvilagus spp. (cottontail) detections by moon phase, 2012-2015.

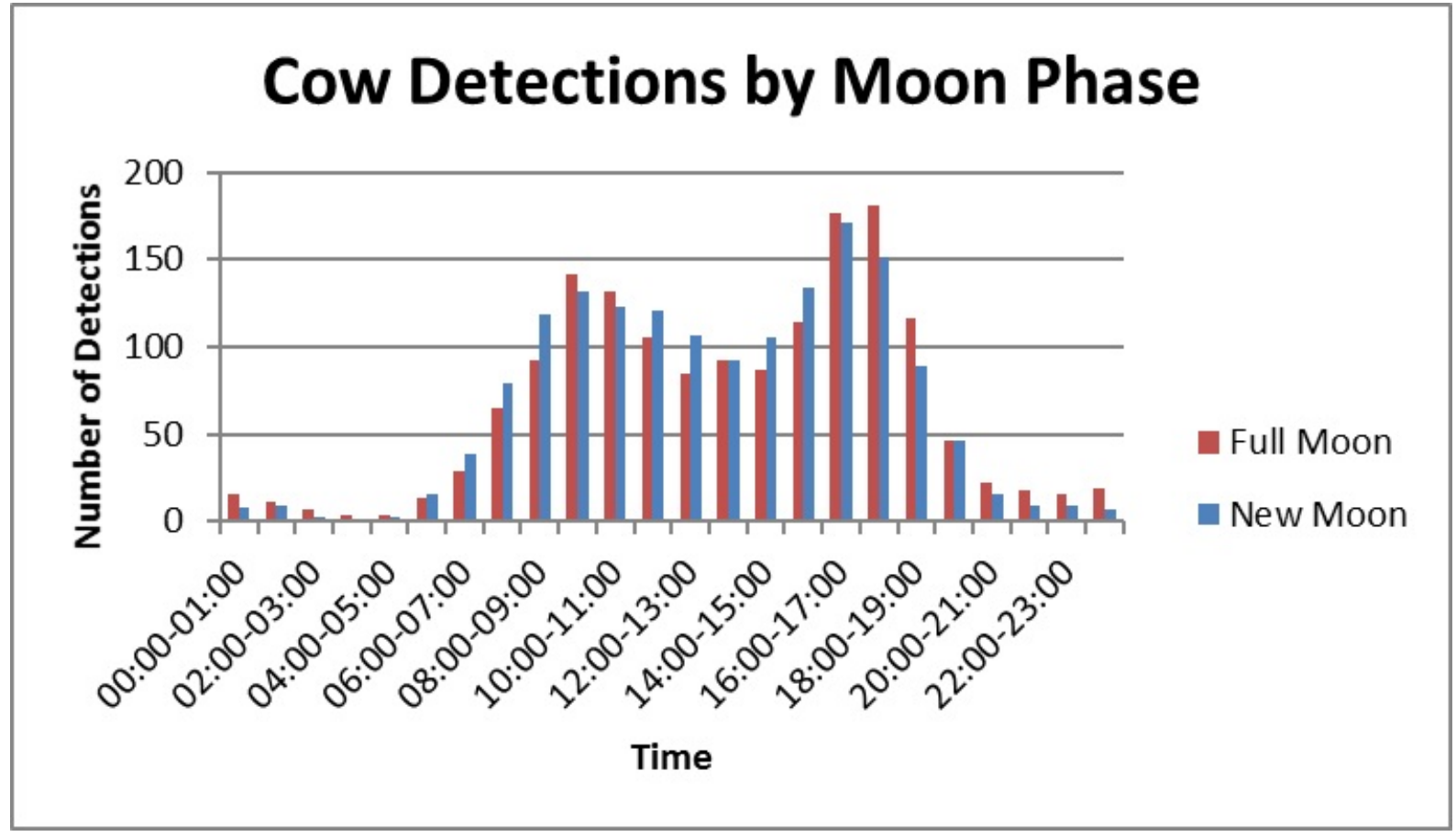

Figure 72. Bos taurus (cow) detections by moon phase, 2012-2015. 


\section{Coyote Detections by Moon Phase}

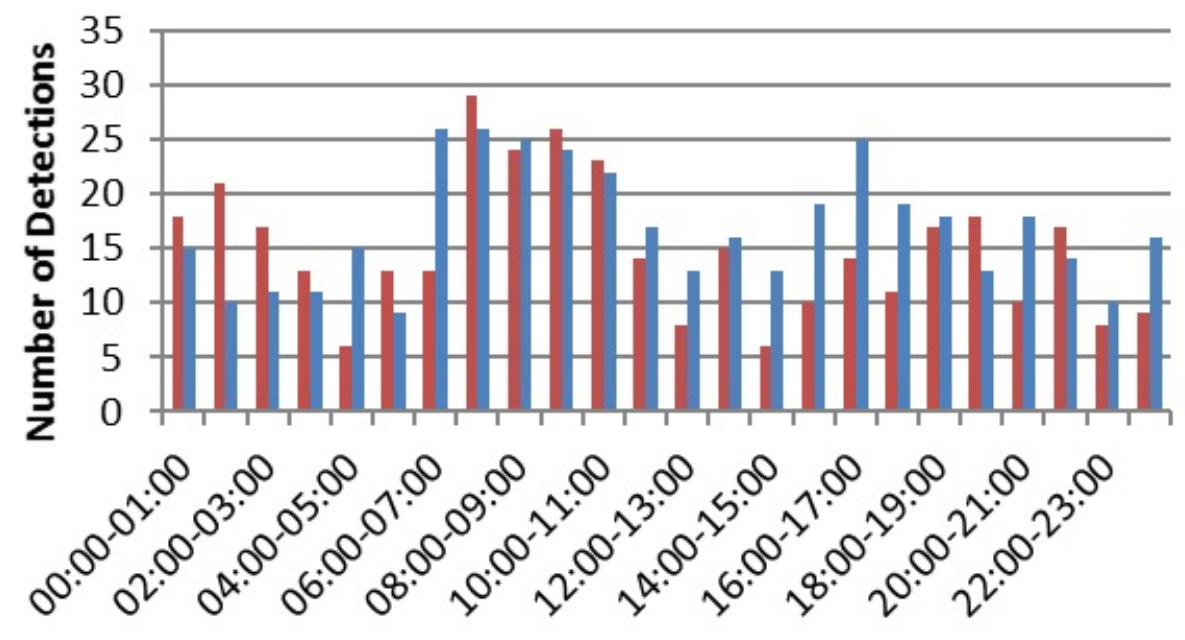

- Full Moon

New Moon

Time

Figure 73. Canis latrans (coyote) detections by moon phase, 2012-2016.

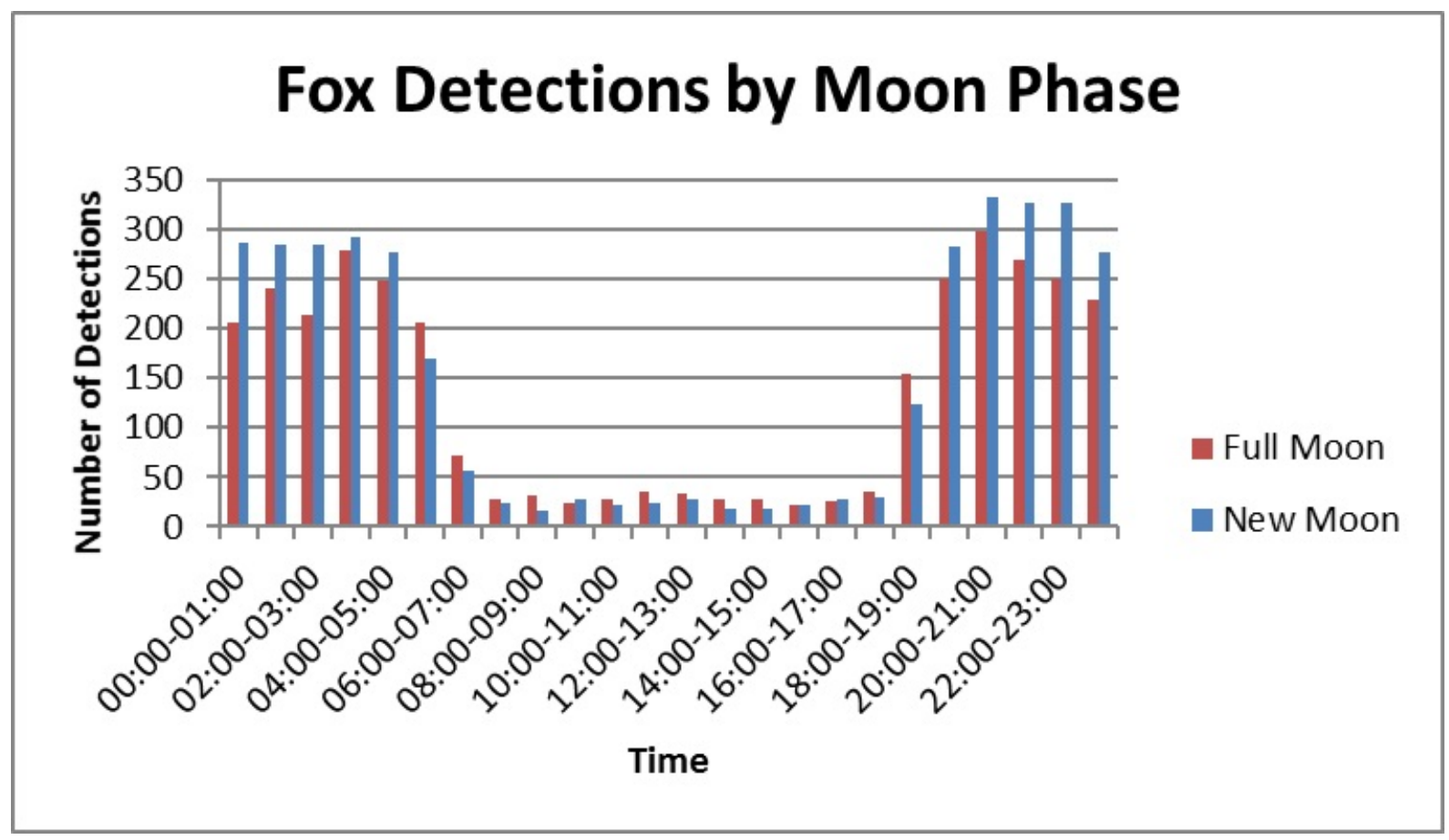

Figure 74. Urocyon cinereoargenteus (gray fox) detections by moon phase, 2012-2016. 


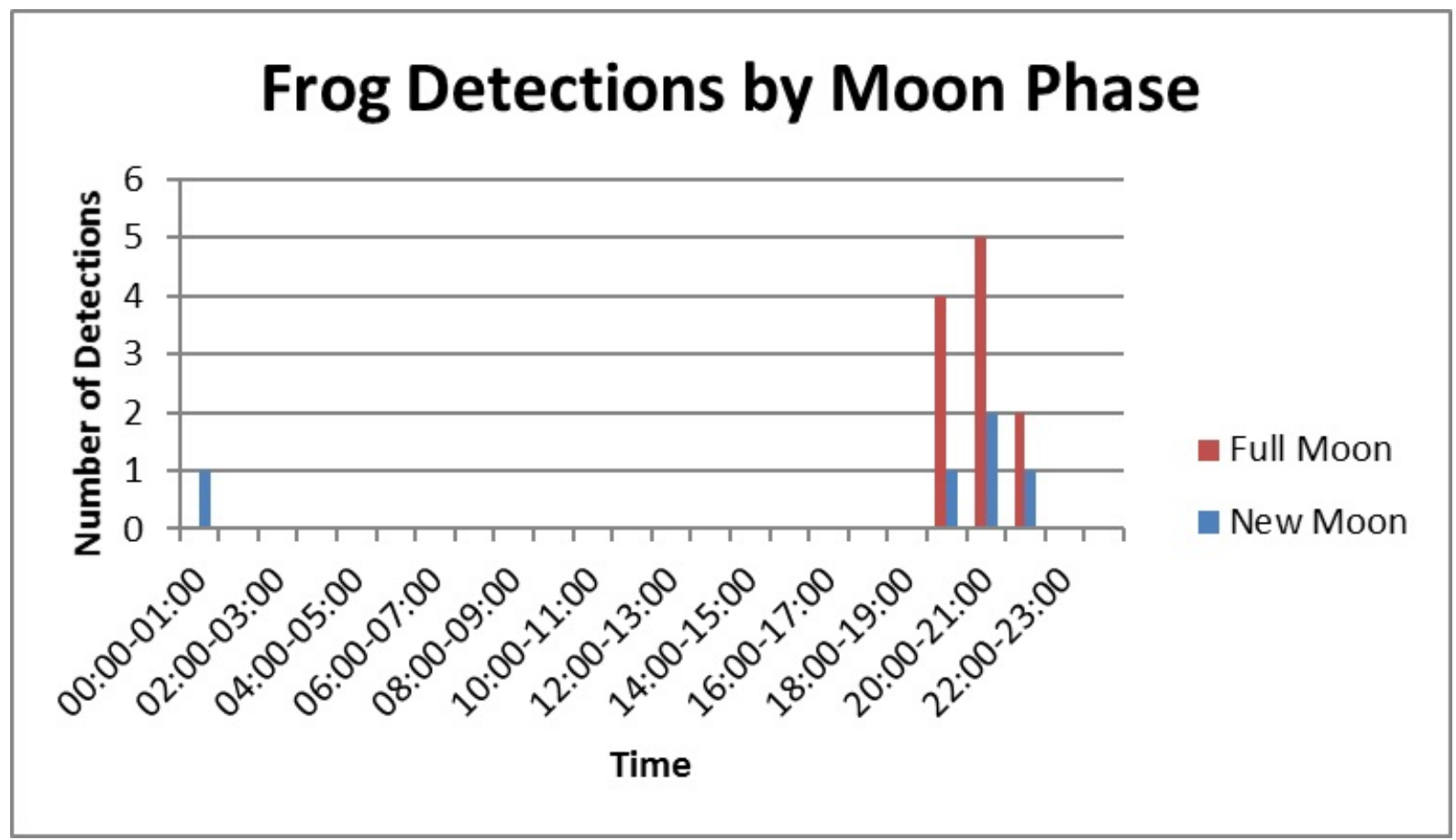

Figure 75. Ranid (frog) detections by moon phase, 2012-2016.

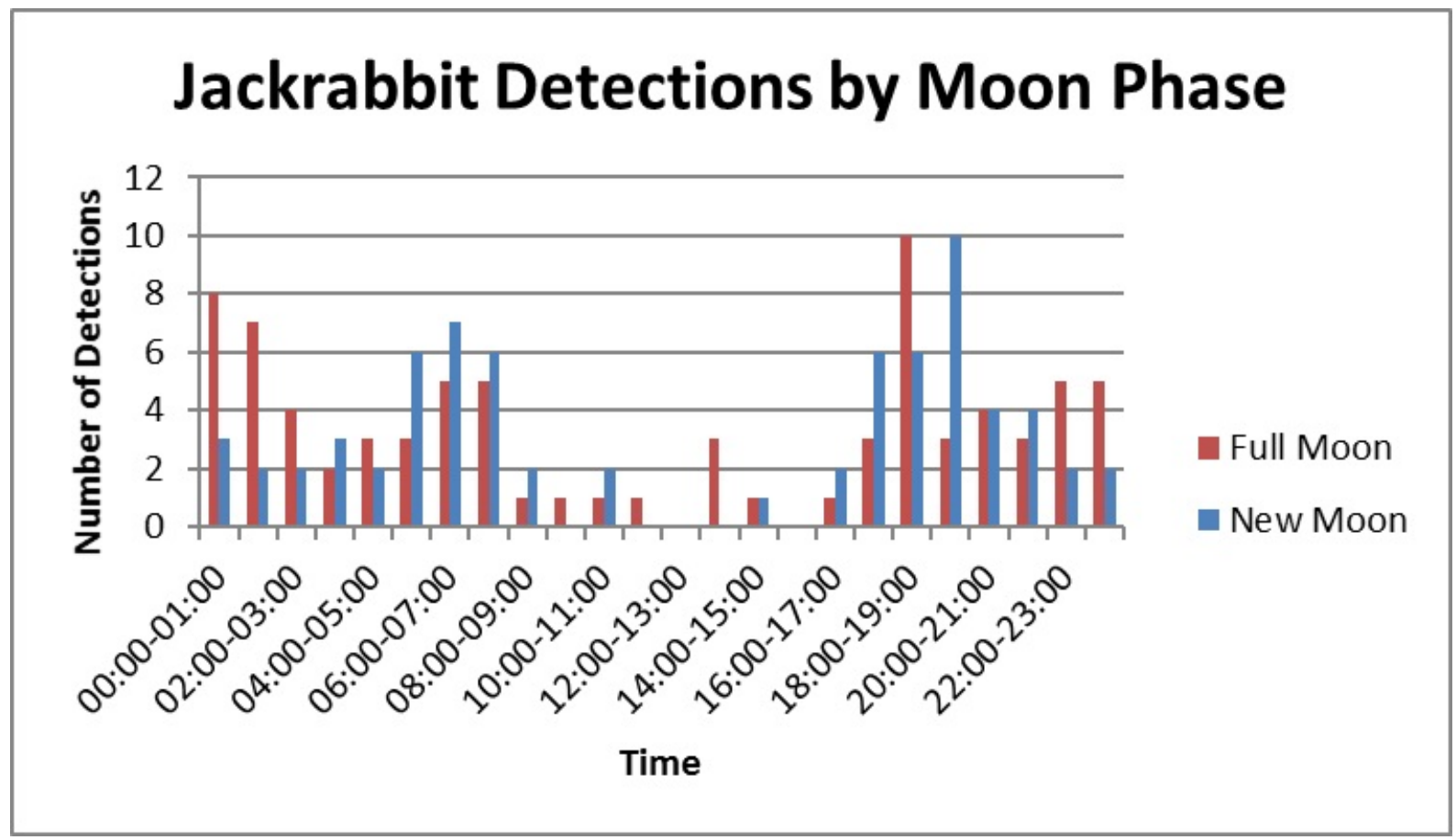

Figure 76. Lepus spp. (jackrabbit) detections by moon phase, 2012-2016. 


\section{Jaguar Detections by Moon Phase}

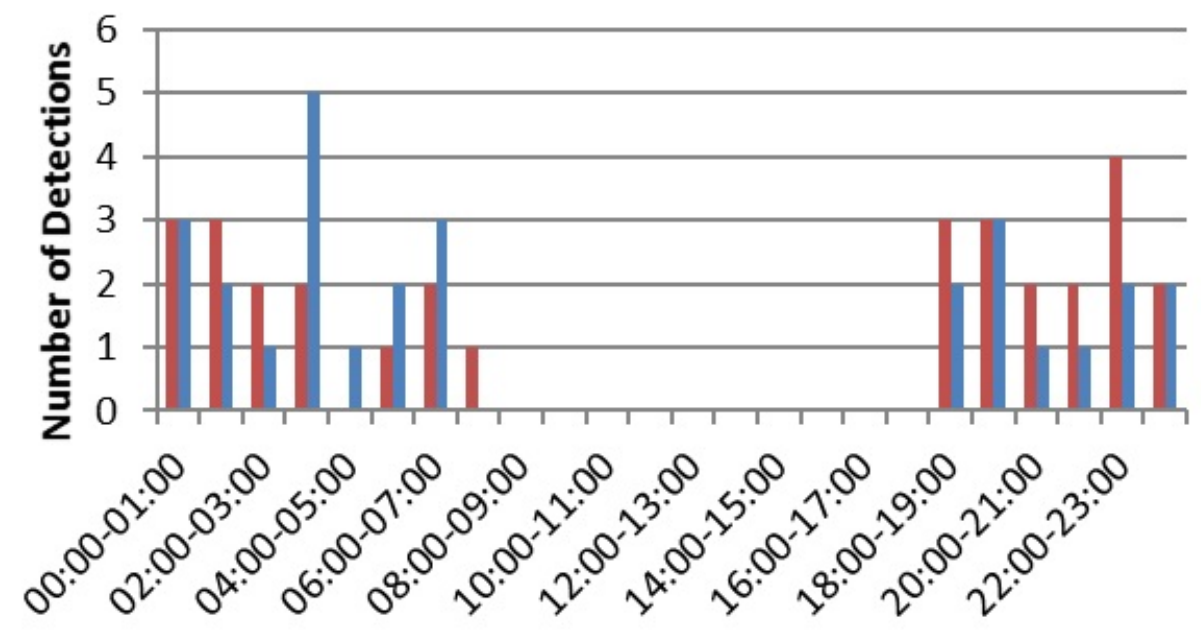

- Full Moon

new Moon

Time

Figure 77. Panthera onca (jaguar) detections by moon phase, 2012-2016.

\section{Javelina Detections by Moon Phase}

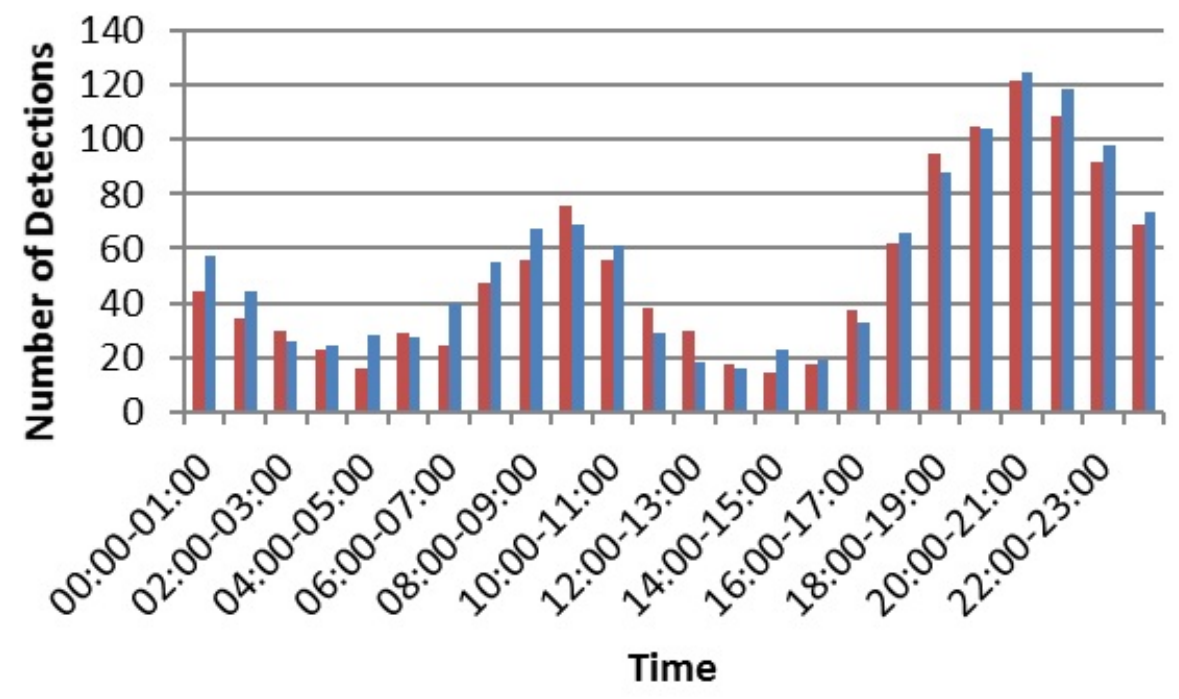

Figure 78. Pecari tejacu (javelina) detections by moon phase, 2012-2016. 


\section{Ocelot Detections by Moon Phase}

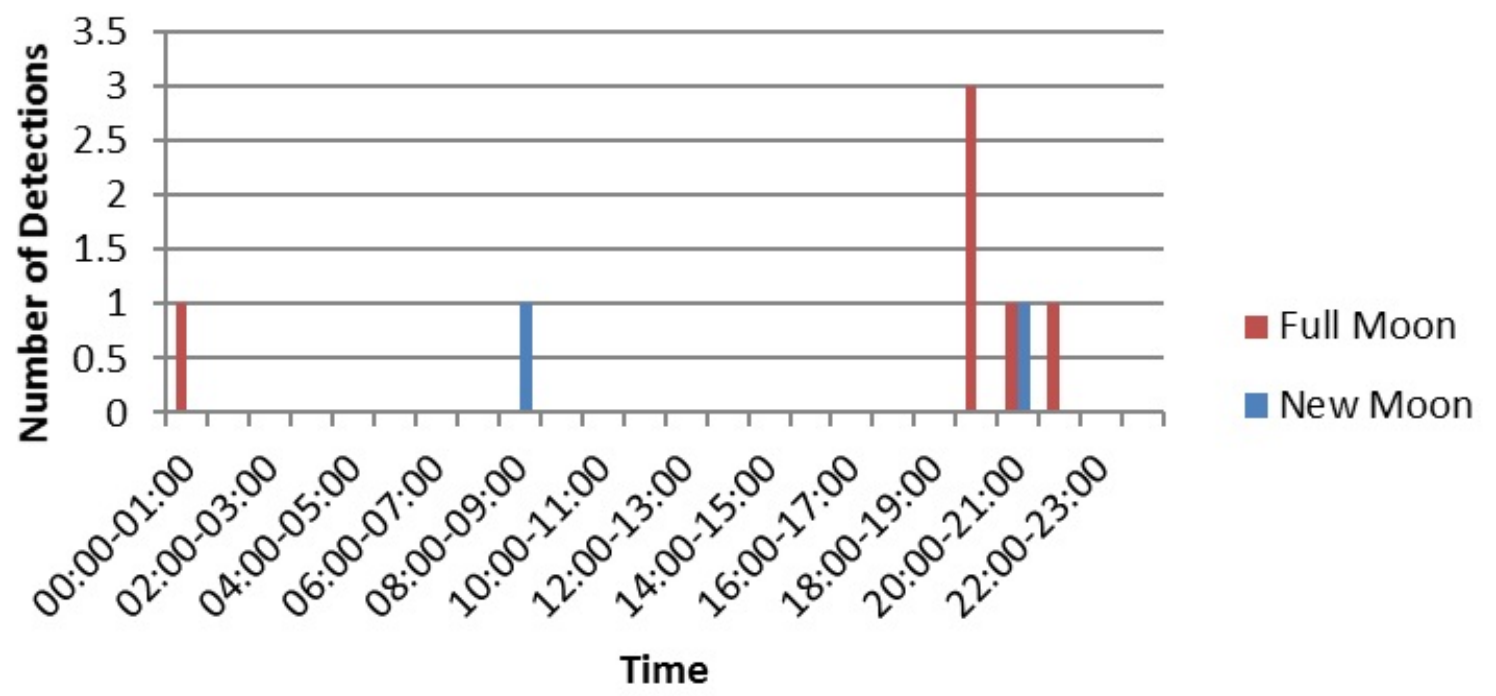

Figure 79. Leopardus pardalis (ocelot) detections by moon phase, 2012-2016.

\section{Opossum Detections by Moon Phase}

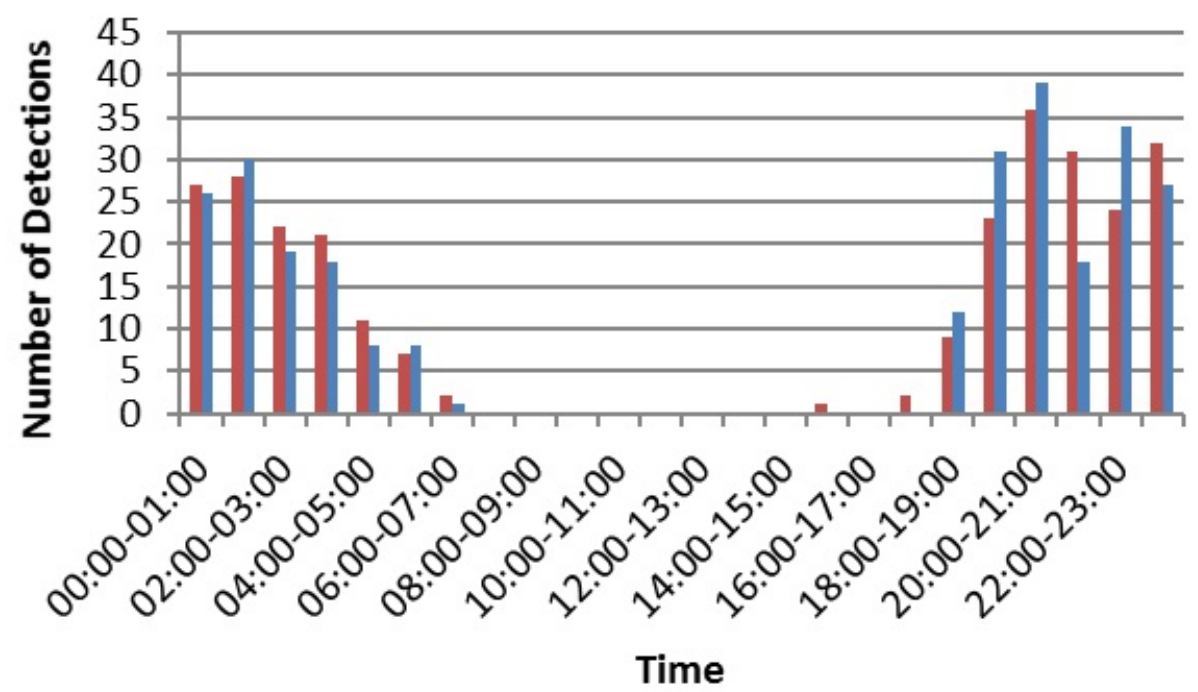

Full Moon

New Moon

Figure 80. Didelphis virginiana (opossum) detections by moon phase, 2012-2016. 


\section{Puma Detections by Moon Phase}

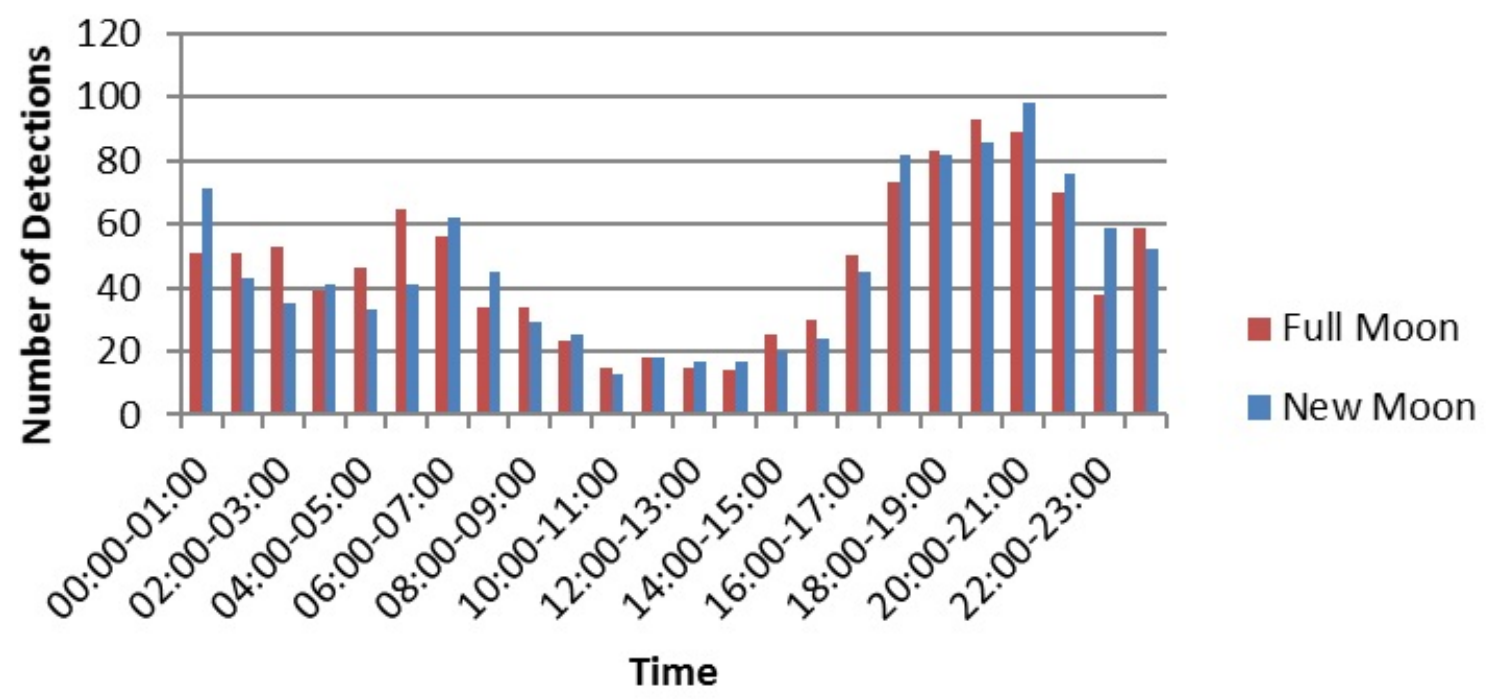

Figure 81. Puma concolor (puma) detections by moon phase, 2012-2016.

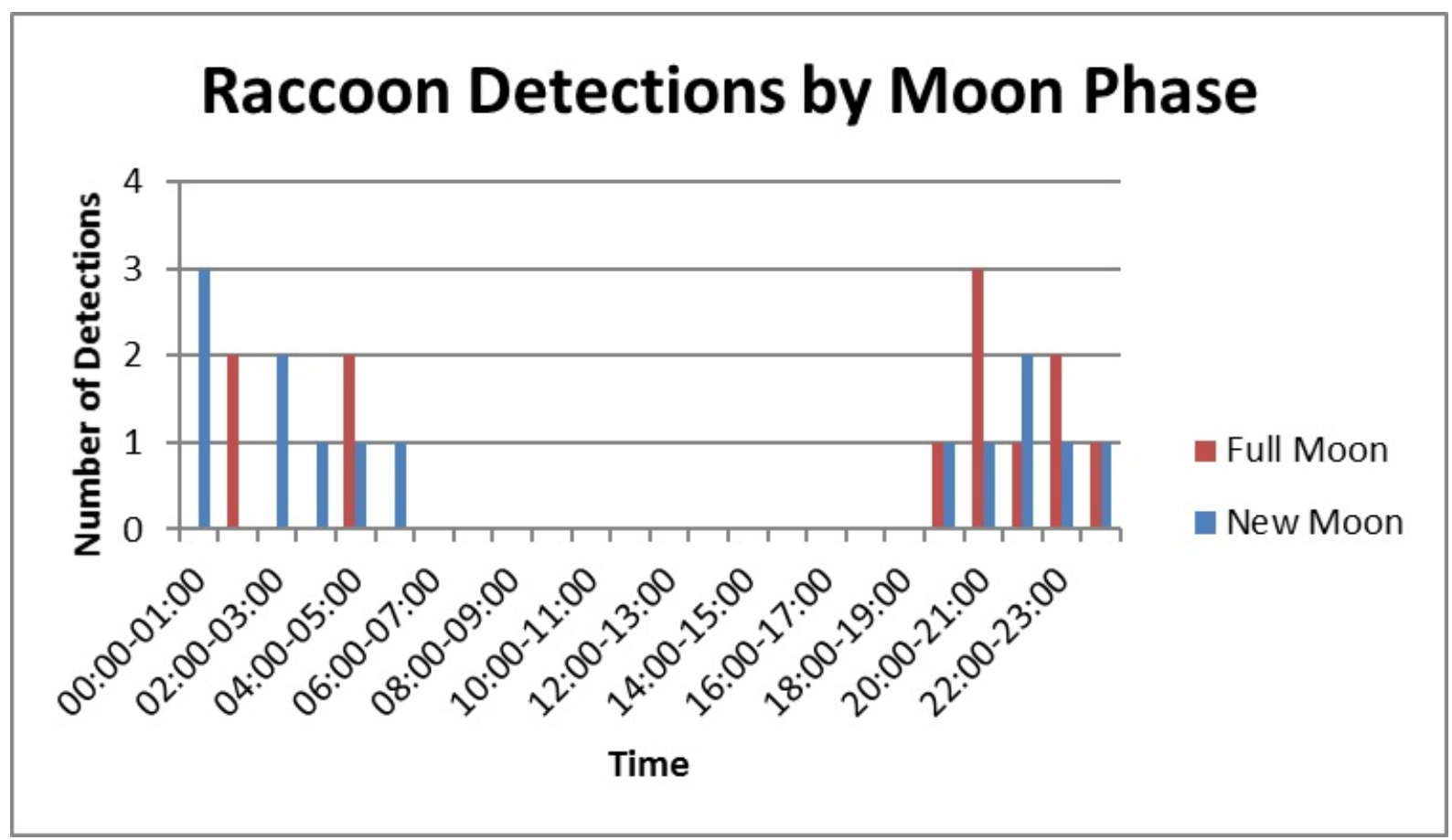

Figure 82. Procyon lotor (raccoon) detections by moon phase, 2012-2016. 


\section{Ringtail Detections by Moon Phase}

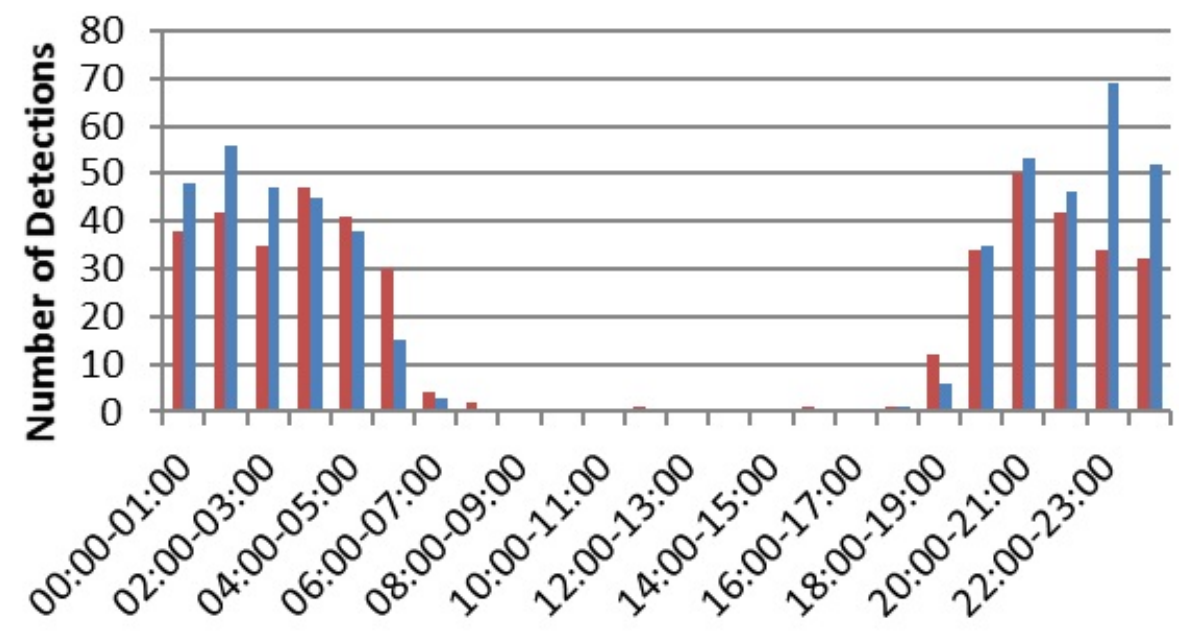

- Full Moon

New Moon

Time

Figure 83. Bassariscus astutus (ringtail) detections by moon phase, 2012-2016.

\section{Rodent Detections by Moon Phase}

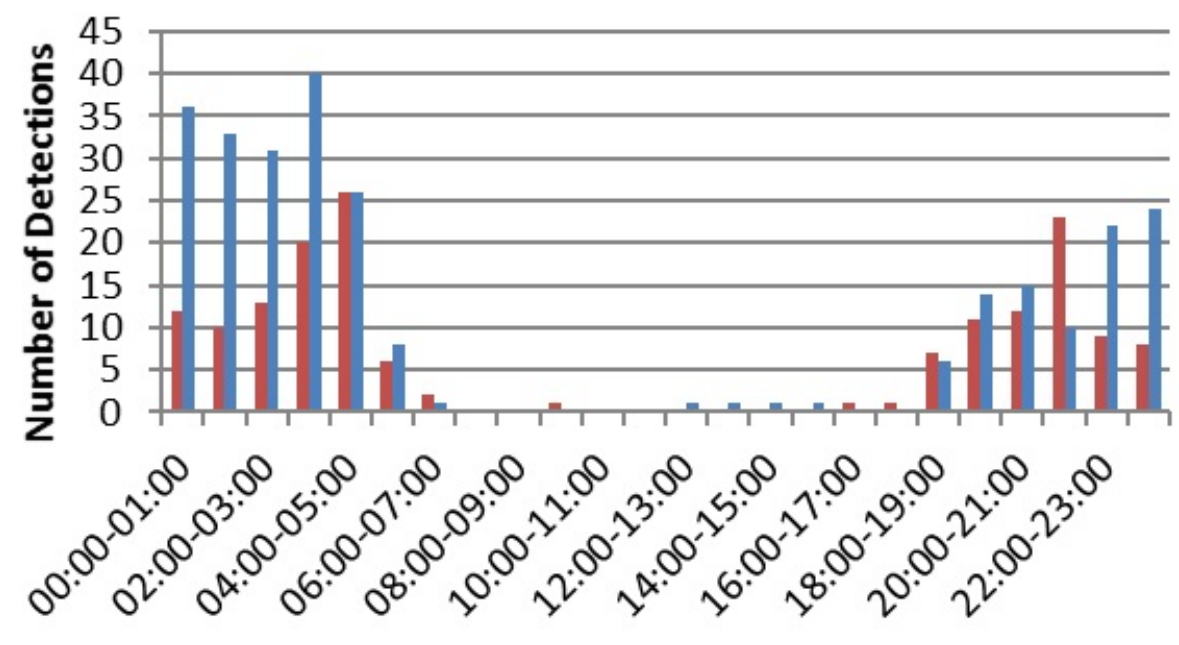

- Full Moon

New Moon

Time

Figure 84. Non-Sciurid Rodentia (rodent, not including squirrel) detections by moon phase, 2012-2016. 


\section{Skunk Detections by Moon Phase}

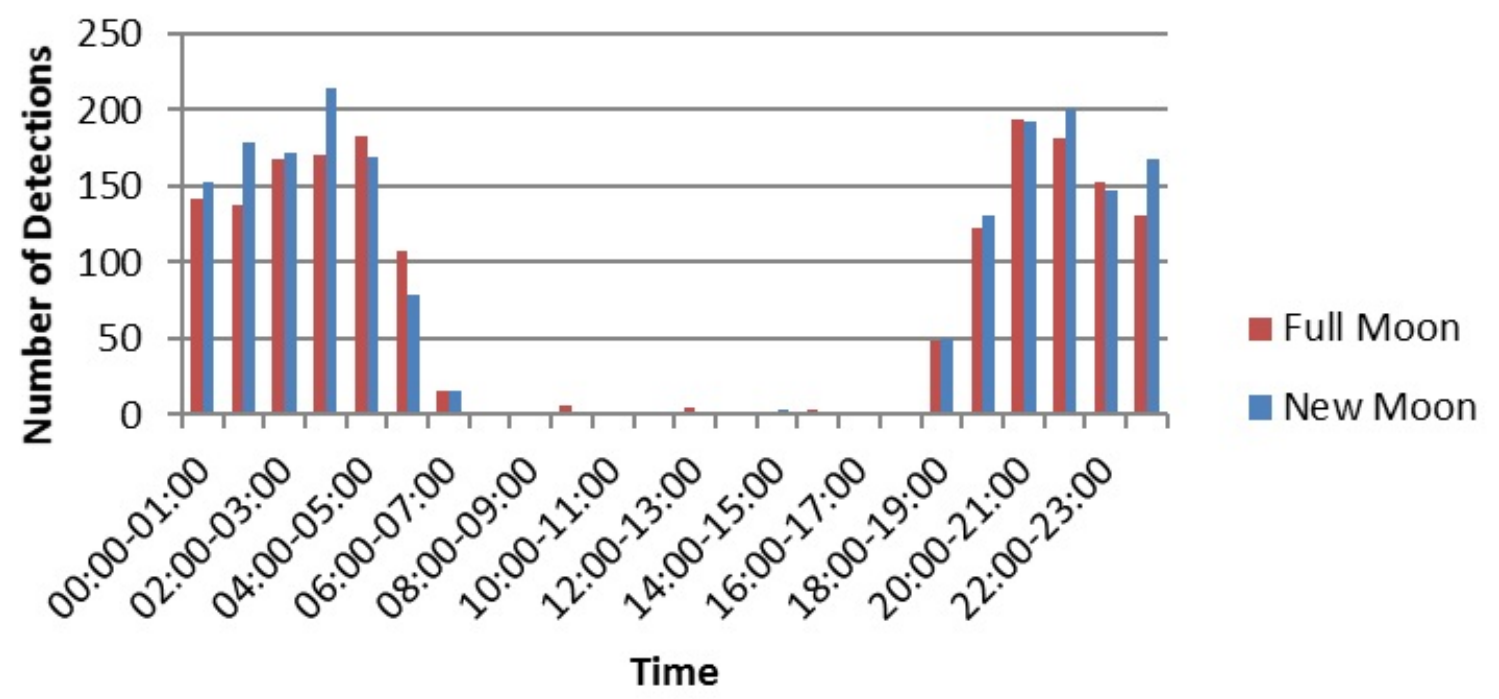

Figure 85. Mephitid (skunk) detections by moon phase, 2012-2016.

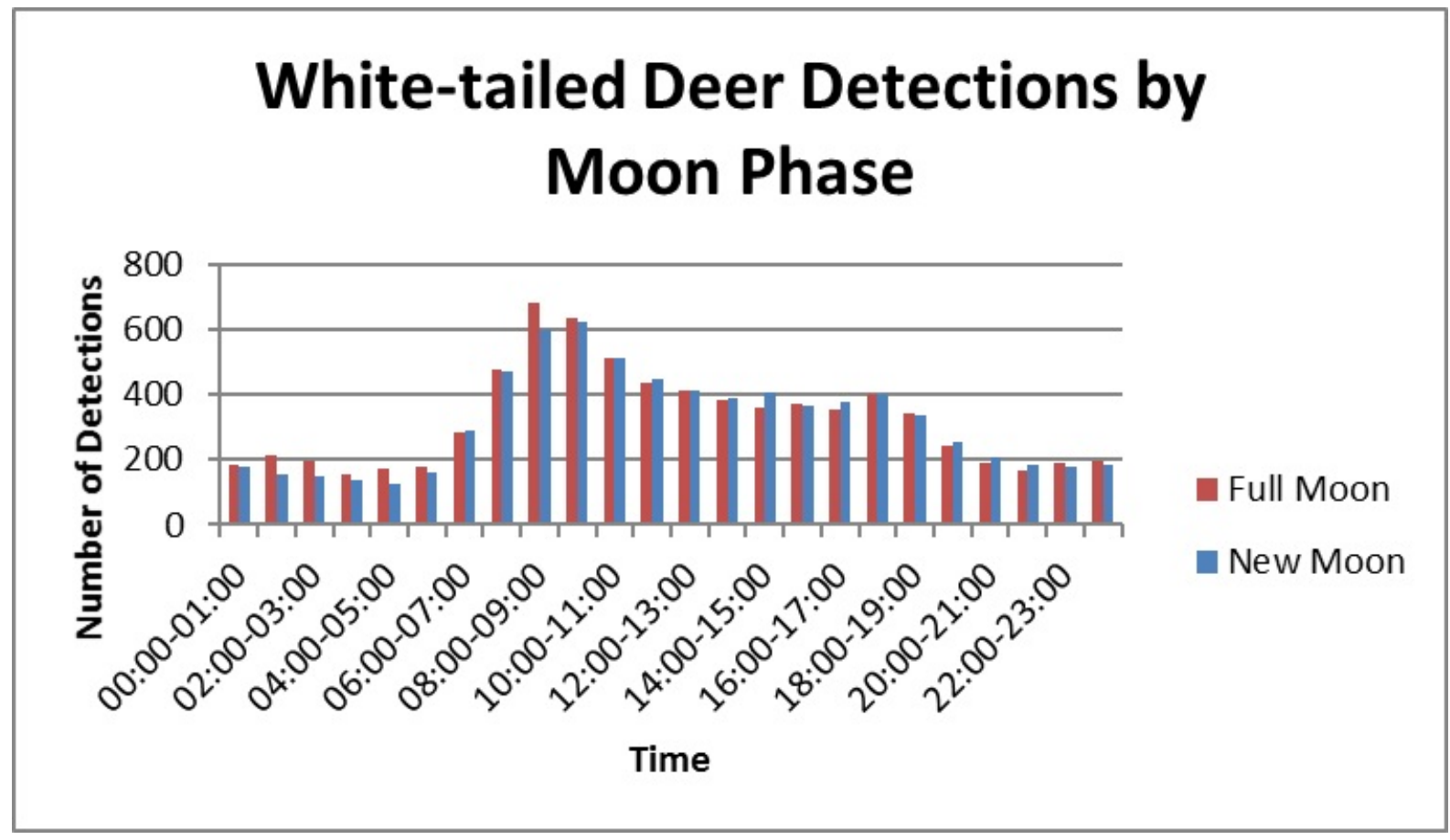

Figure 86. Odocoileus virginiana (white-tailed deer) detections by moon phase, 2012-2016. 


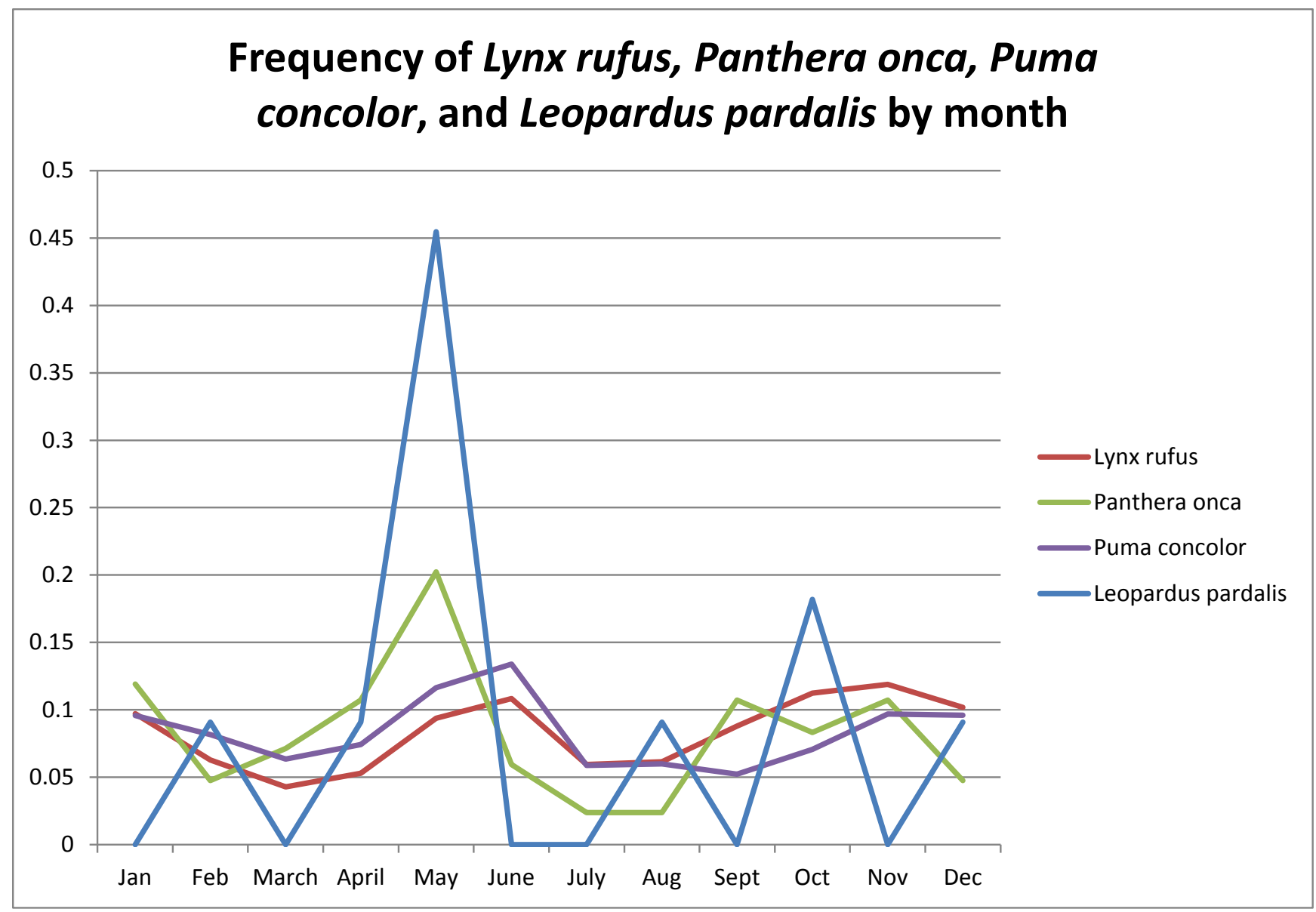

Figure 87. Monthly detection frequencies of Arizona felids (bobcat, jaguar, puma, and ocelot), 2012-2016. 


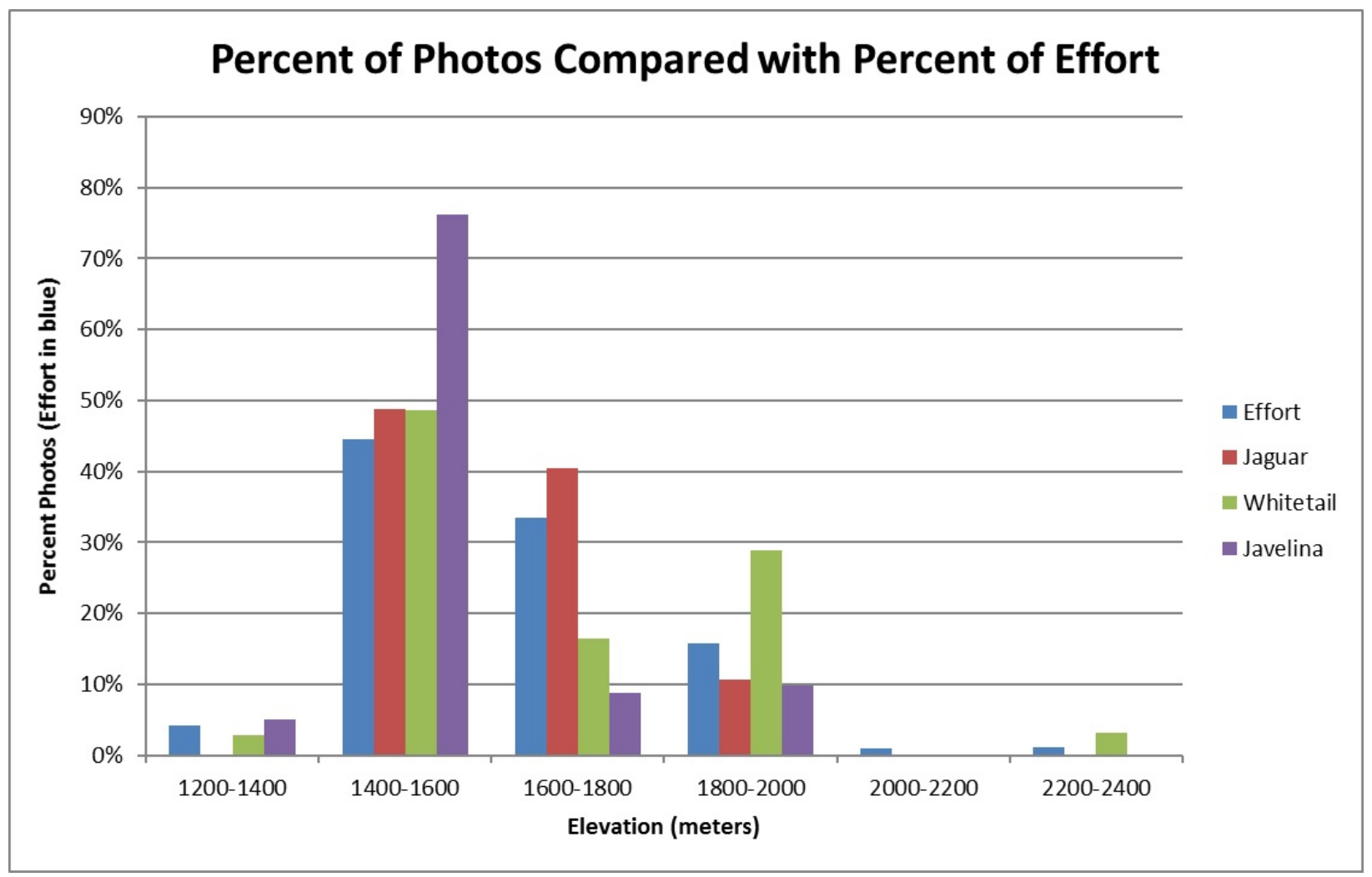

Figure 88. Comparisons of detection rates of jaguar, white-tailed deer, and javelina by elevation and effort in the Santa Rita Mountains, 2012-2016. 


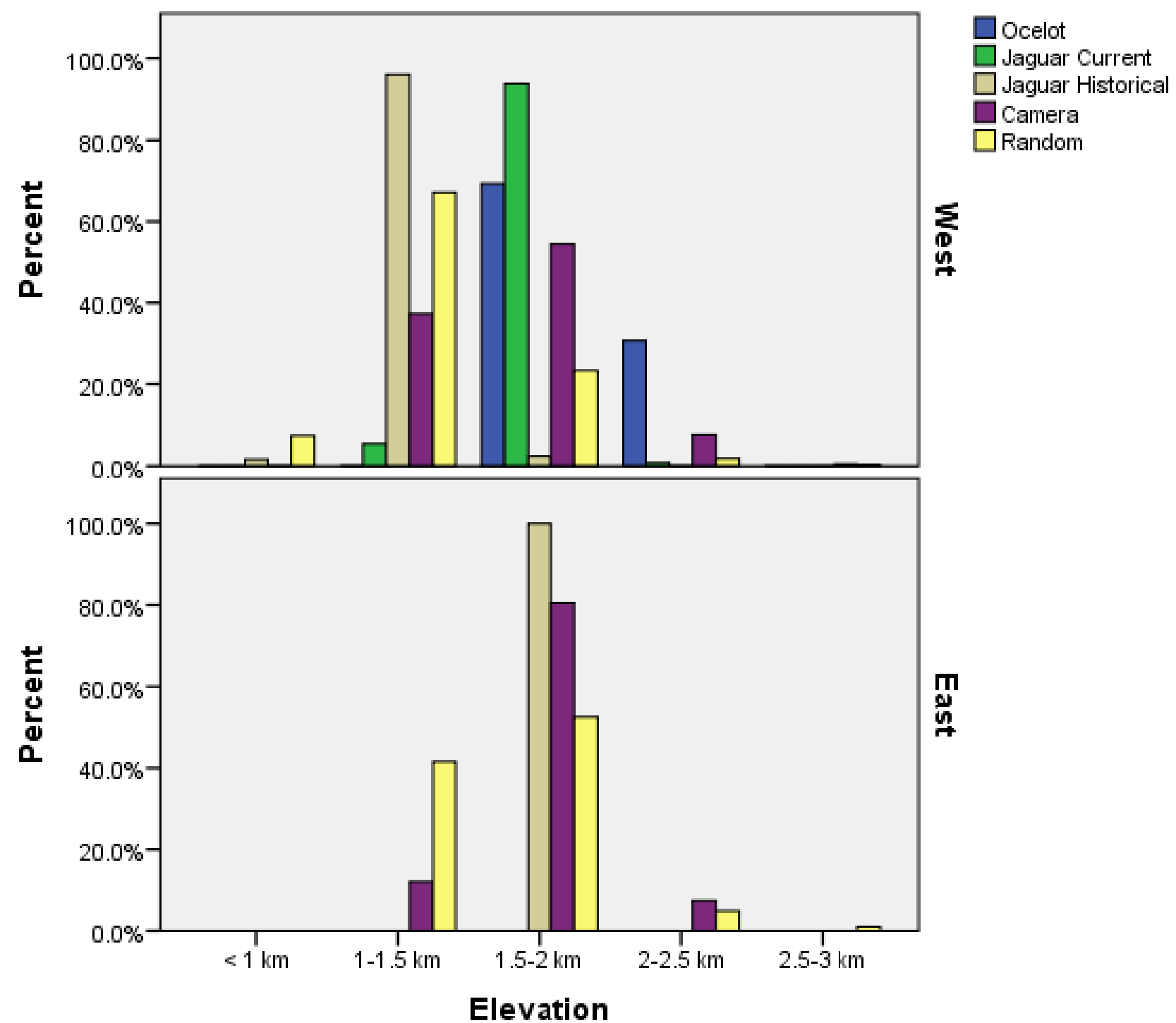

Figure 89. Elevation of camera sites, random points, current ocelot and jaguar locations, and historical jaguar records in west and east study areas. 


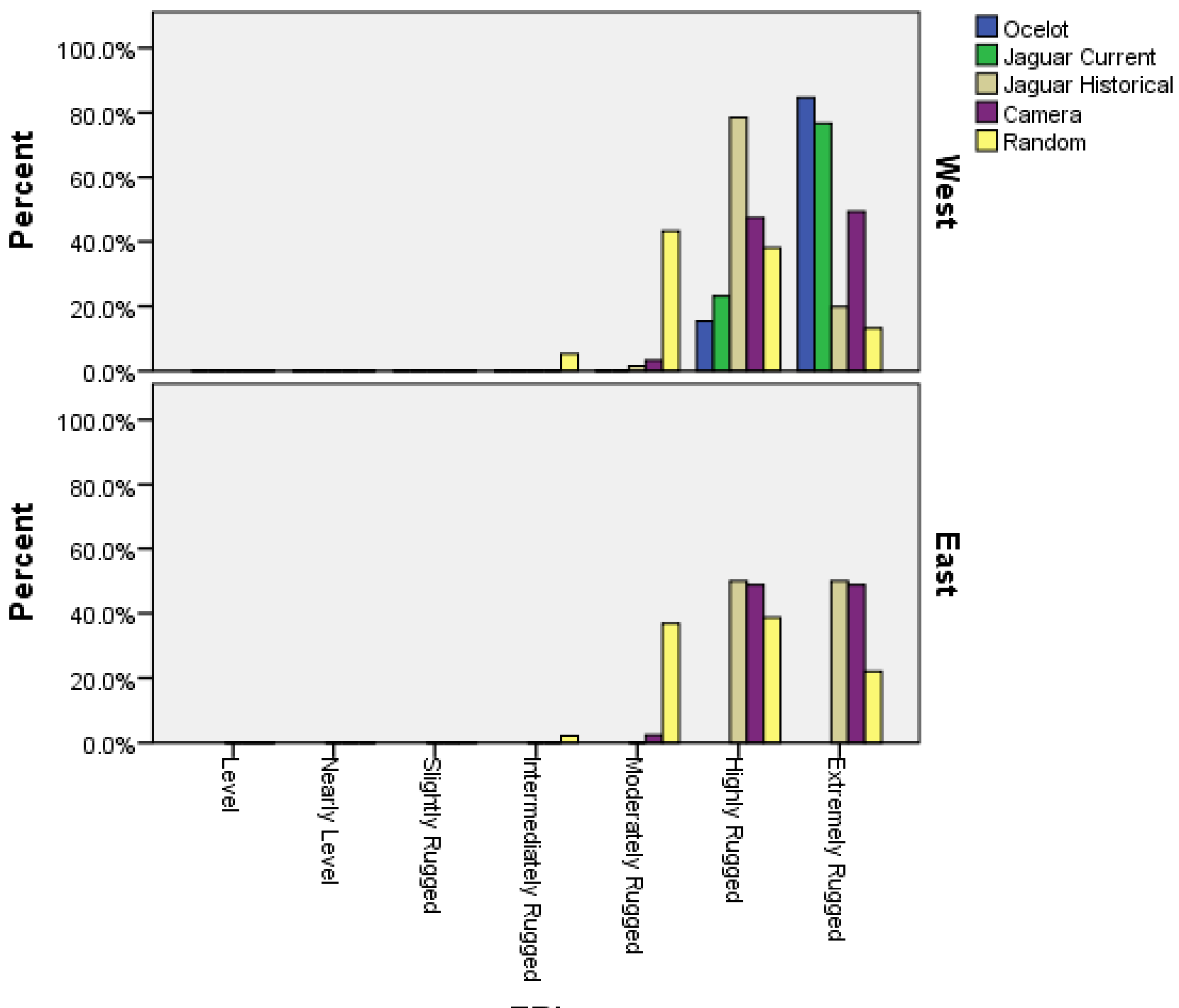

TRI

Figure 90. Terrain Ruggedness Index (TRI) of camera sites, random points, current ocelot and jaguar locations, and historical jaguar records in west and east study areas. 


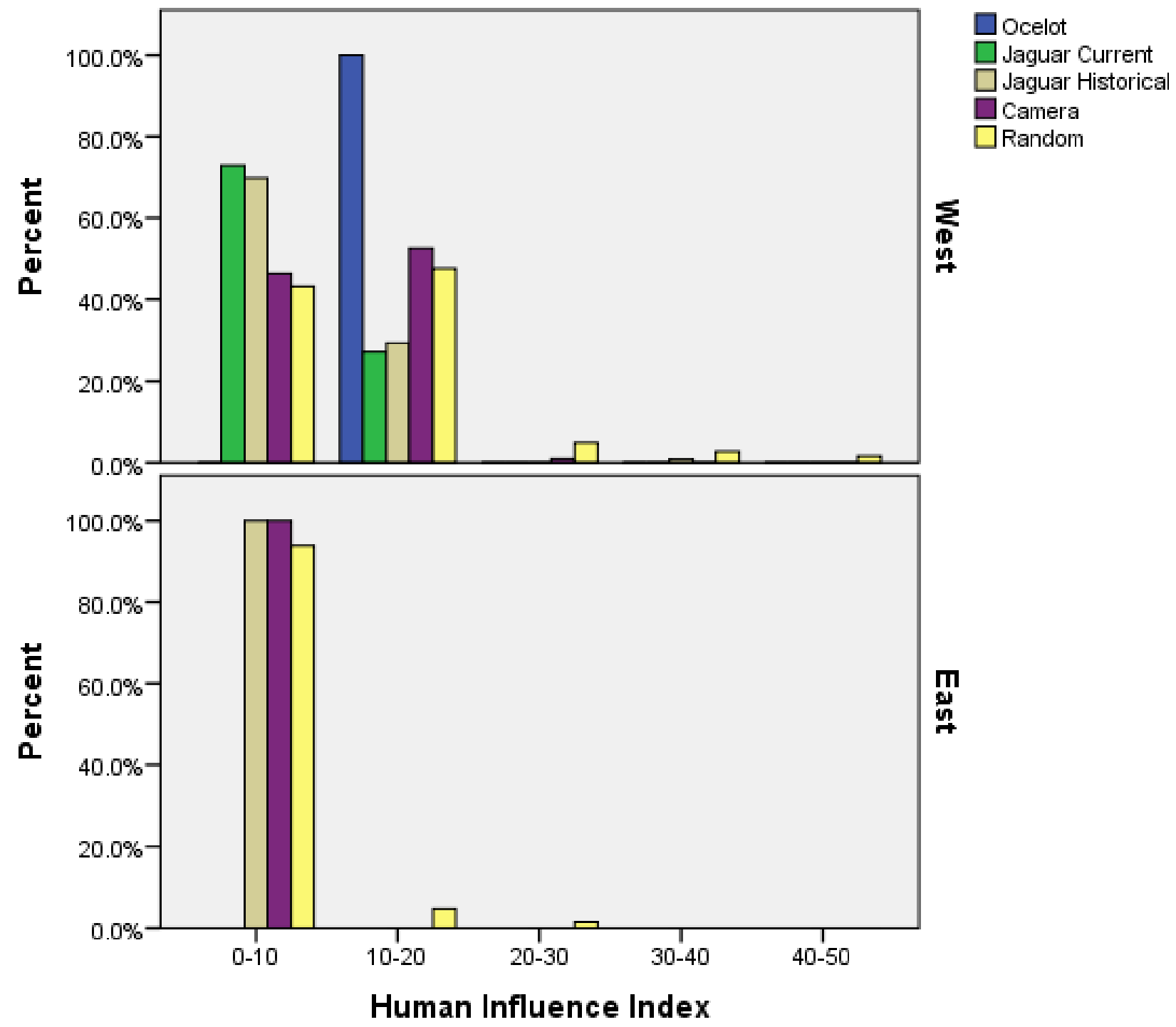

Figure 91. Human influence index of camera sites, random points, current ocelot and jaguar locations, and historical jaguar records in west and east study areas. 

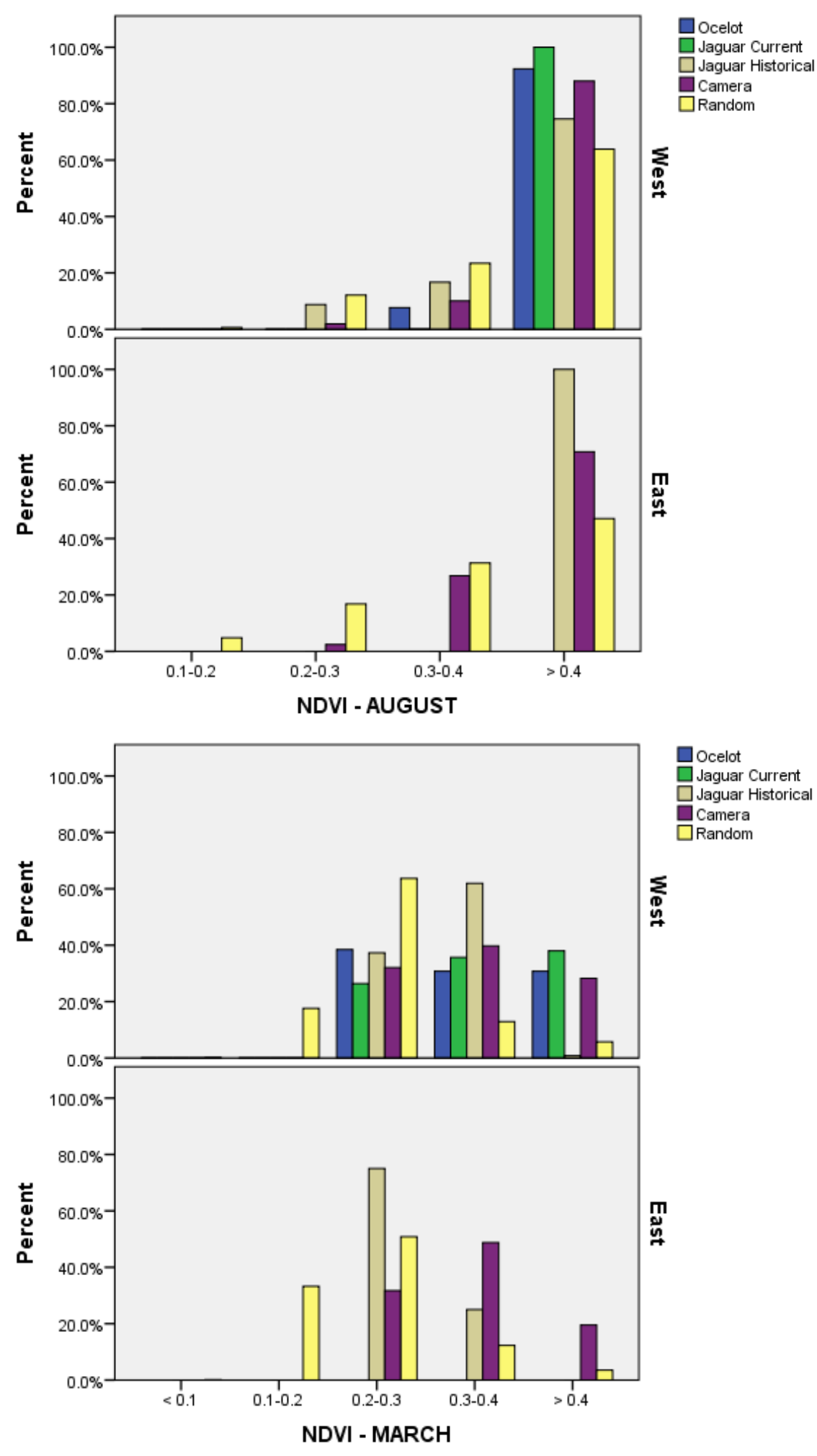

Figure 92. Normalized Difference Vegetation Index (NDVI) for March 2015 (top two graphs) and August 2014 (bottom two graphs) of camera sites, random points, current ocelot and jaguar locations, and historical jaguar records in west and east study areas. 


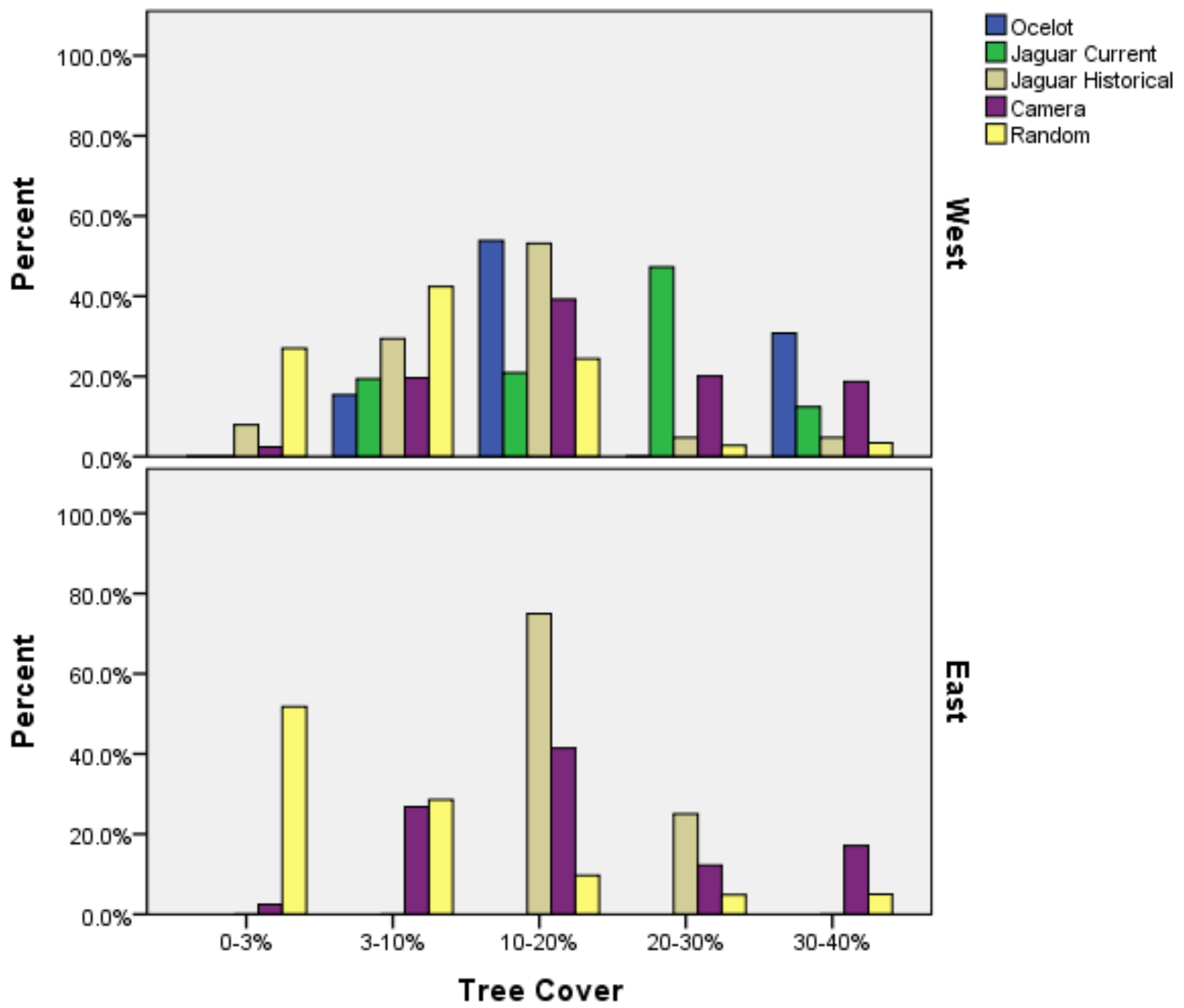

Figure 93. Tree cover of camera sites, random points, current ocelot and jaguar locations, and historical jaguar records in west and east study areas. 


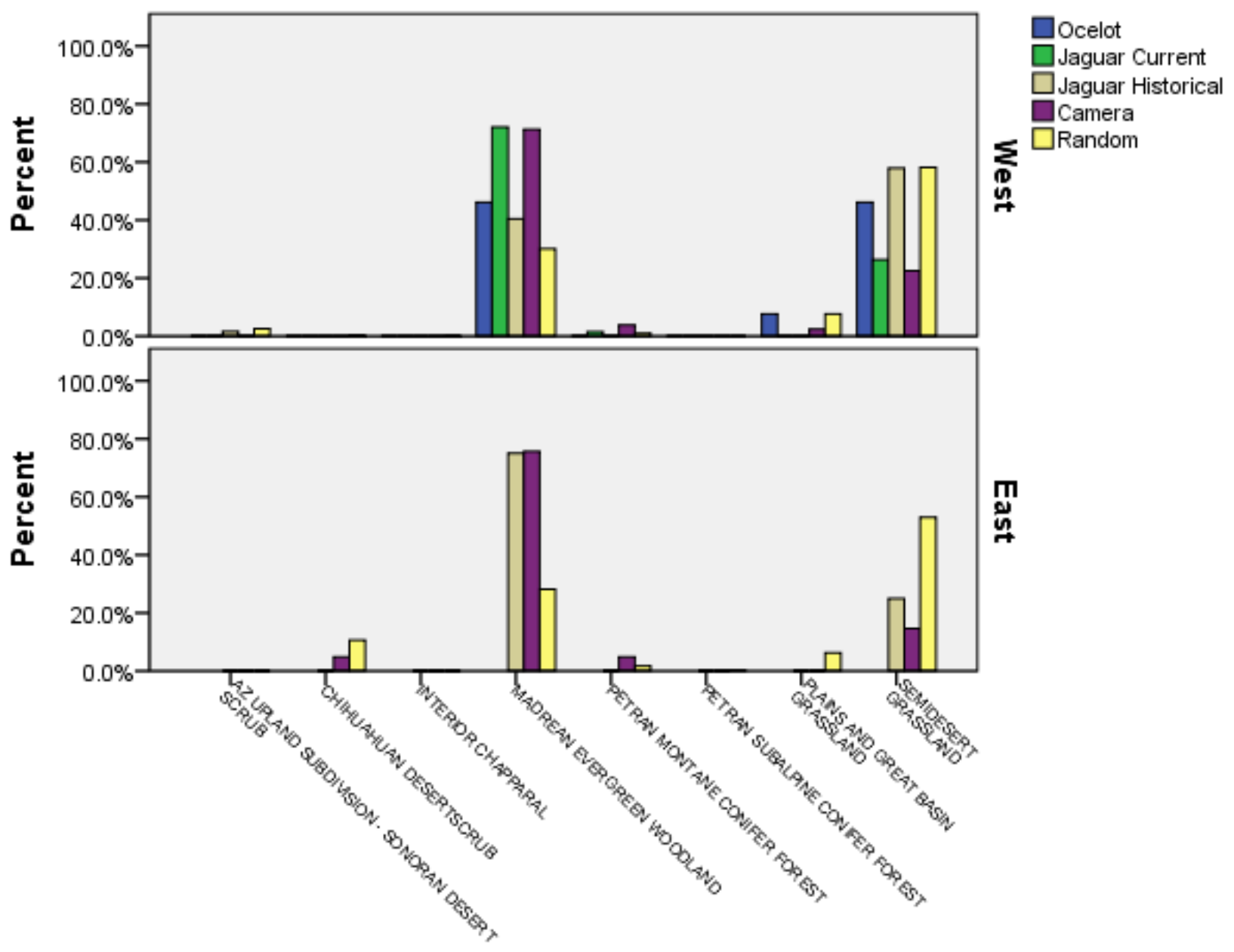

\section{Vegetation Community}

Figure 94. Vegetation community (Brown, 1994) of camera sites, random points, current ocelot and jaguar locations, and historical jaguar records in west and east study areas. 


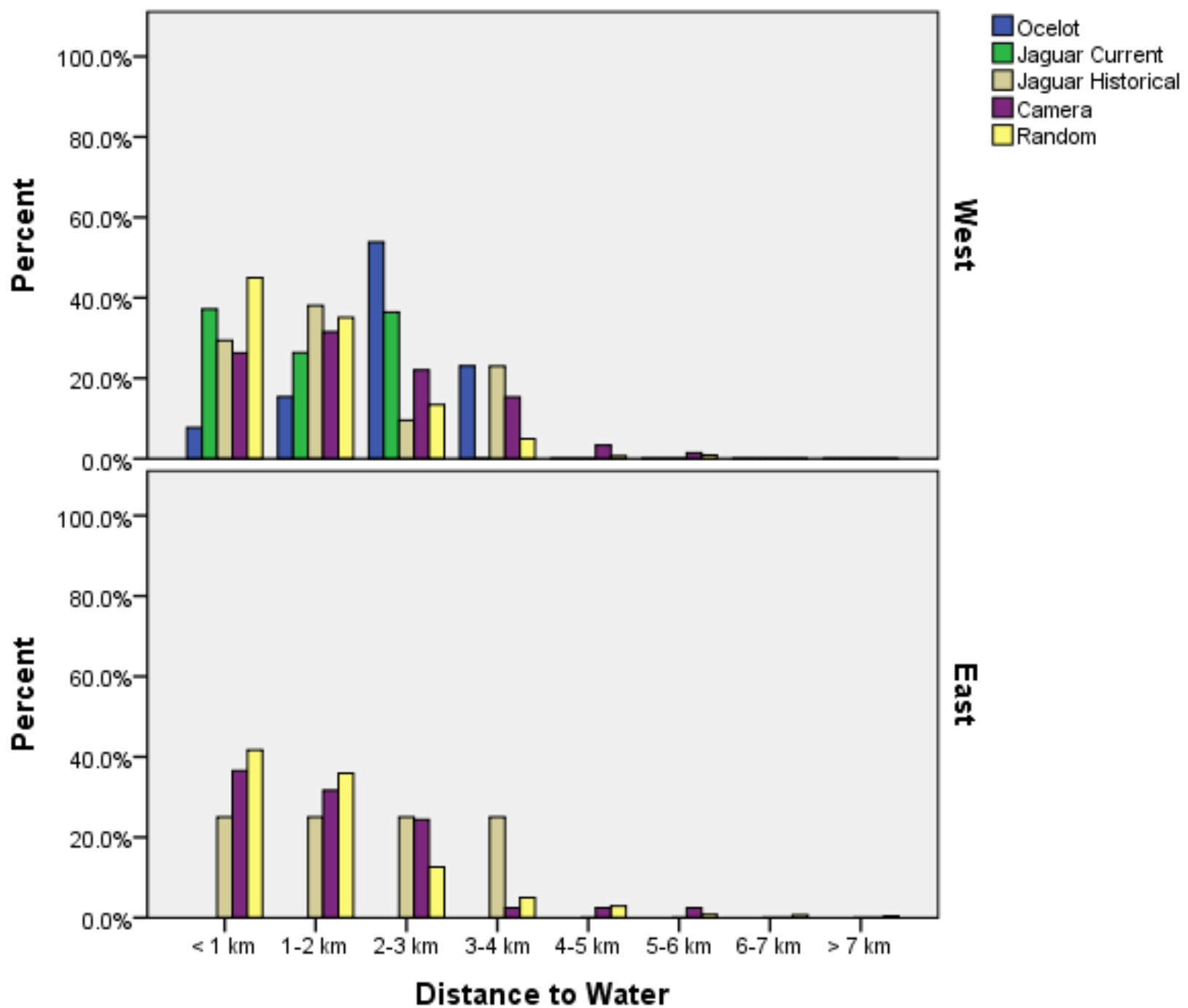

Figure 95. Distance to perennial water for camera sites, random points, current ocelot and jaguar locations, and historical jaguar records in west and east study areas. 


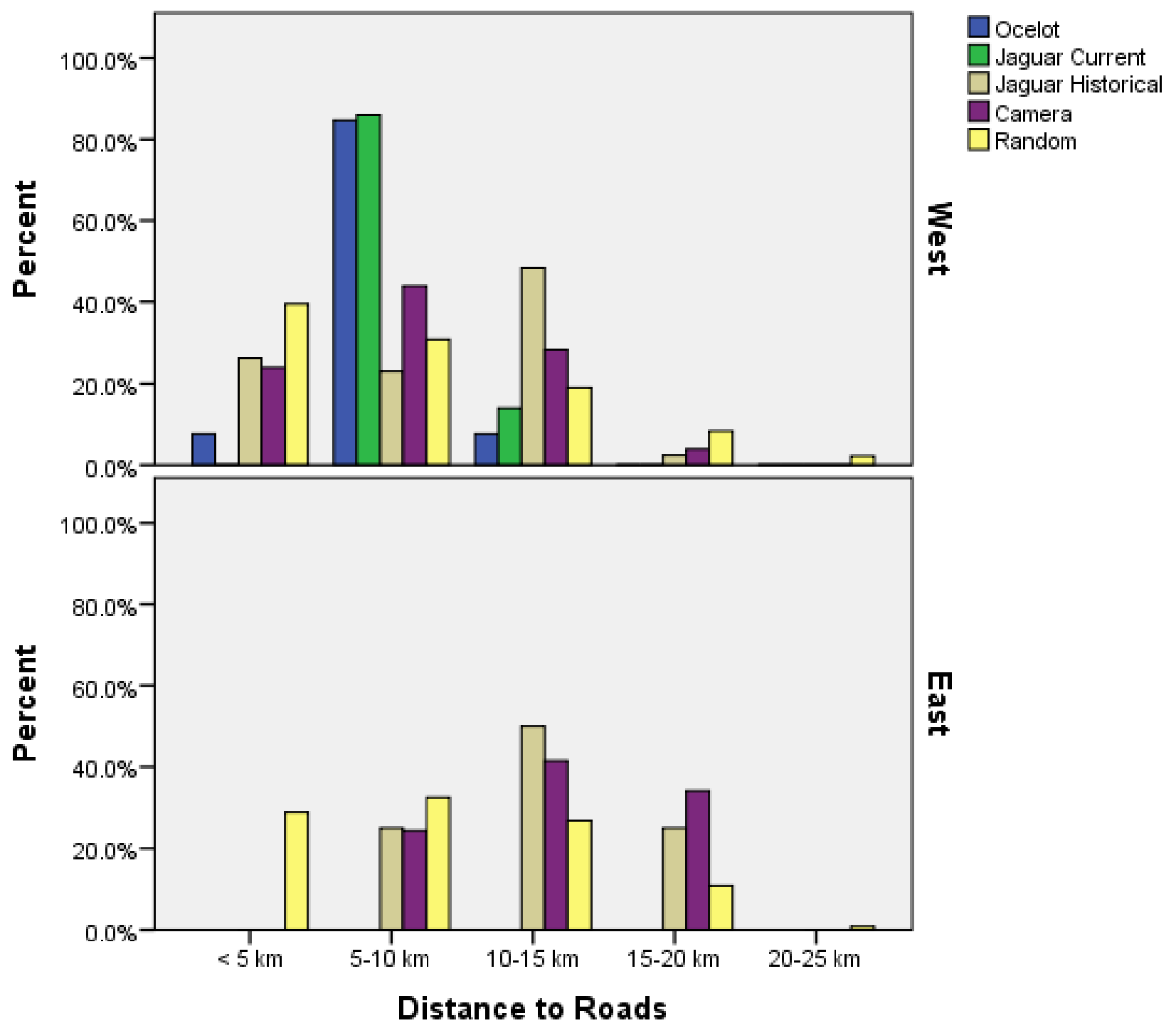

Figure 96. Distance to major roads from camera sites, random points, current ocelot and jaguar locations, and historical jaguar records in west and east study areas. 


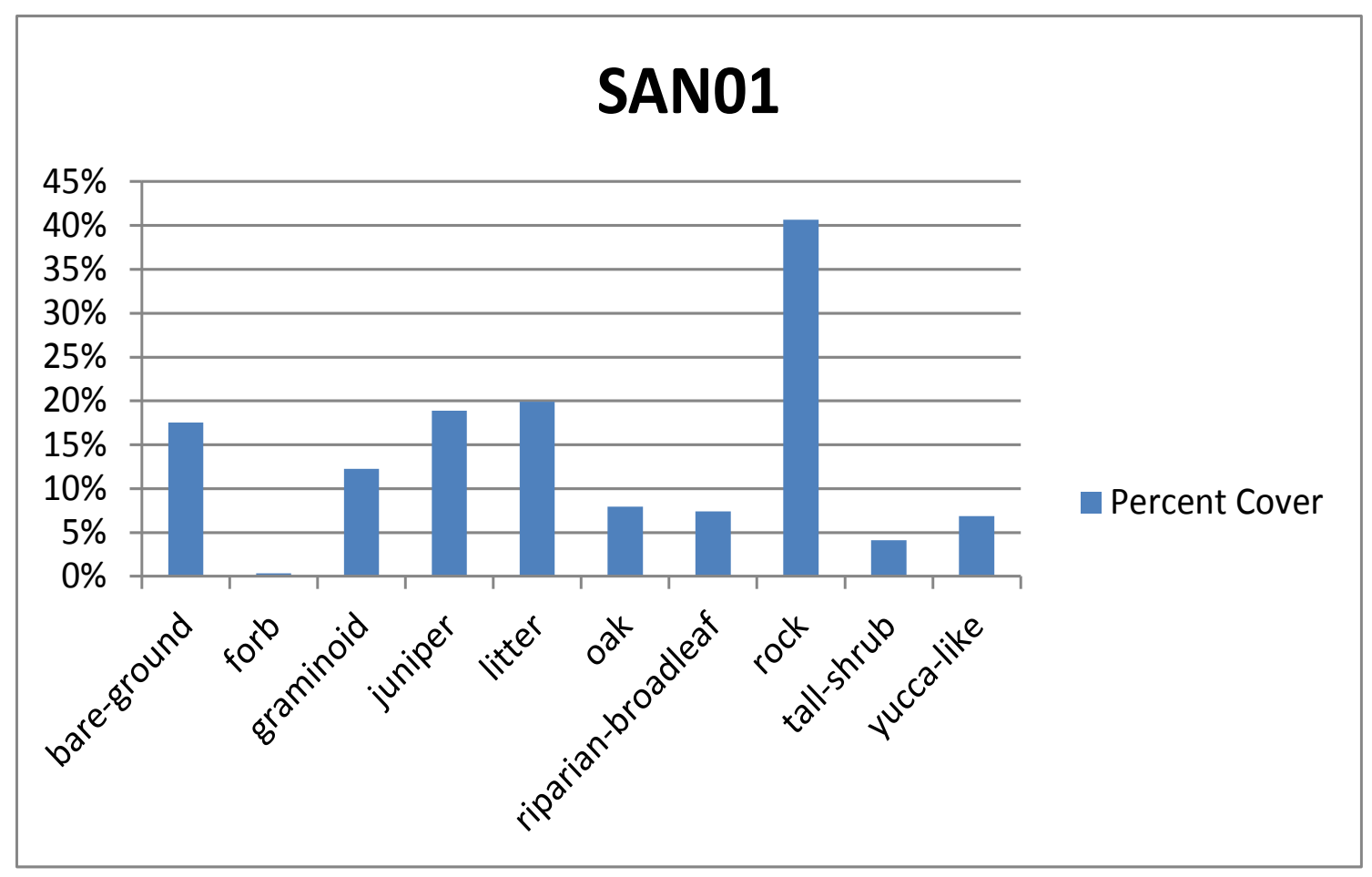

Figure 97. Habitat and species composition characteristics of jaguar detection site SAN01 in the Santa Rita Mountains.

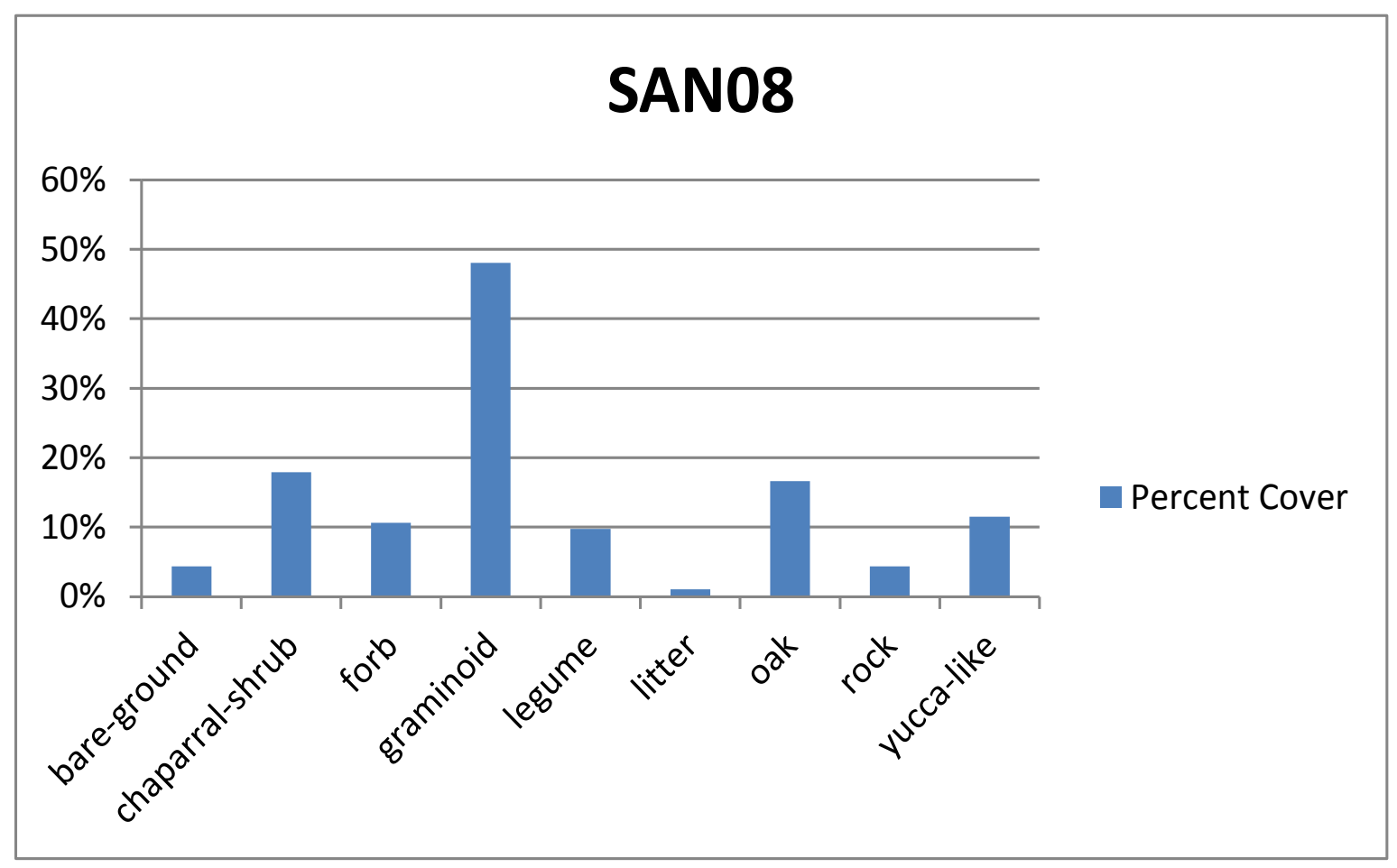

Figure 98. Habitat and species composition characteristics of jaguar detection site SAN08 in the Santa Rita Mountains. 


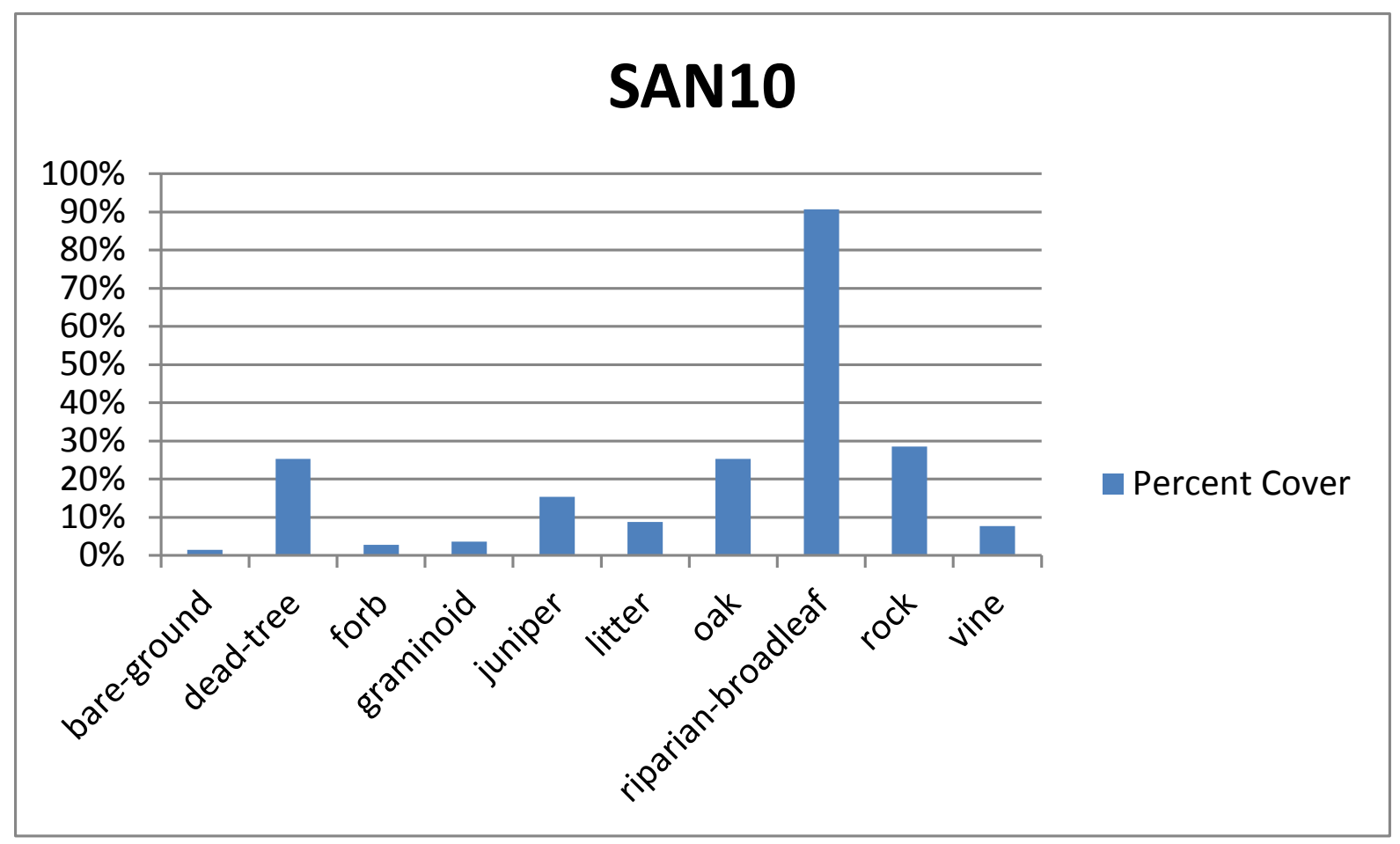

Figure 99. Habitat and species composition characteristics of jaguar detection site SAN10 in the Santa Rita Mountains.

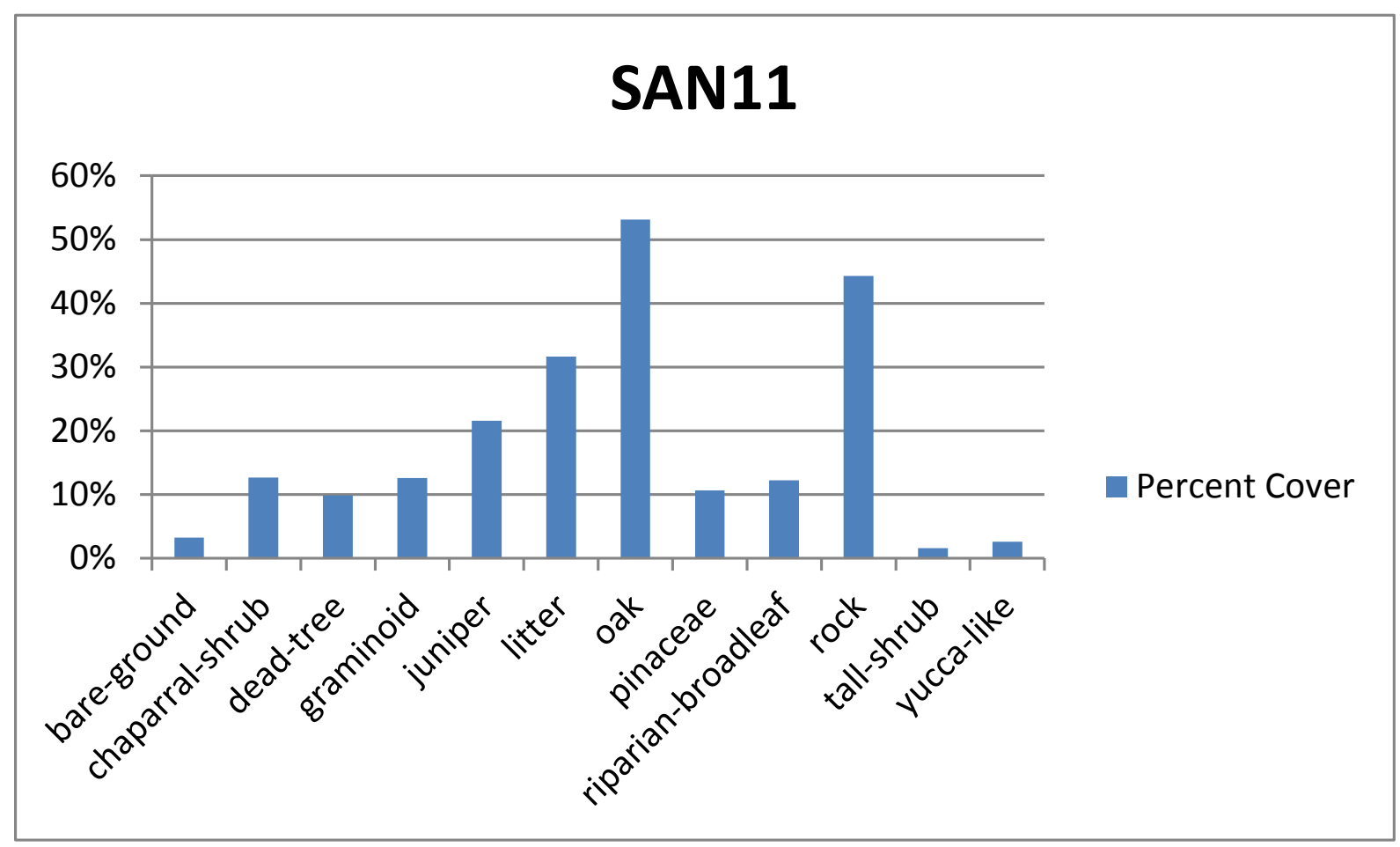

Figure 100. Habitat and species composition characteristics of jaguar detection site SAN11 in the Santa Rita Mountains. 


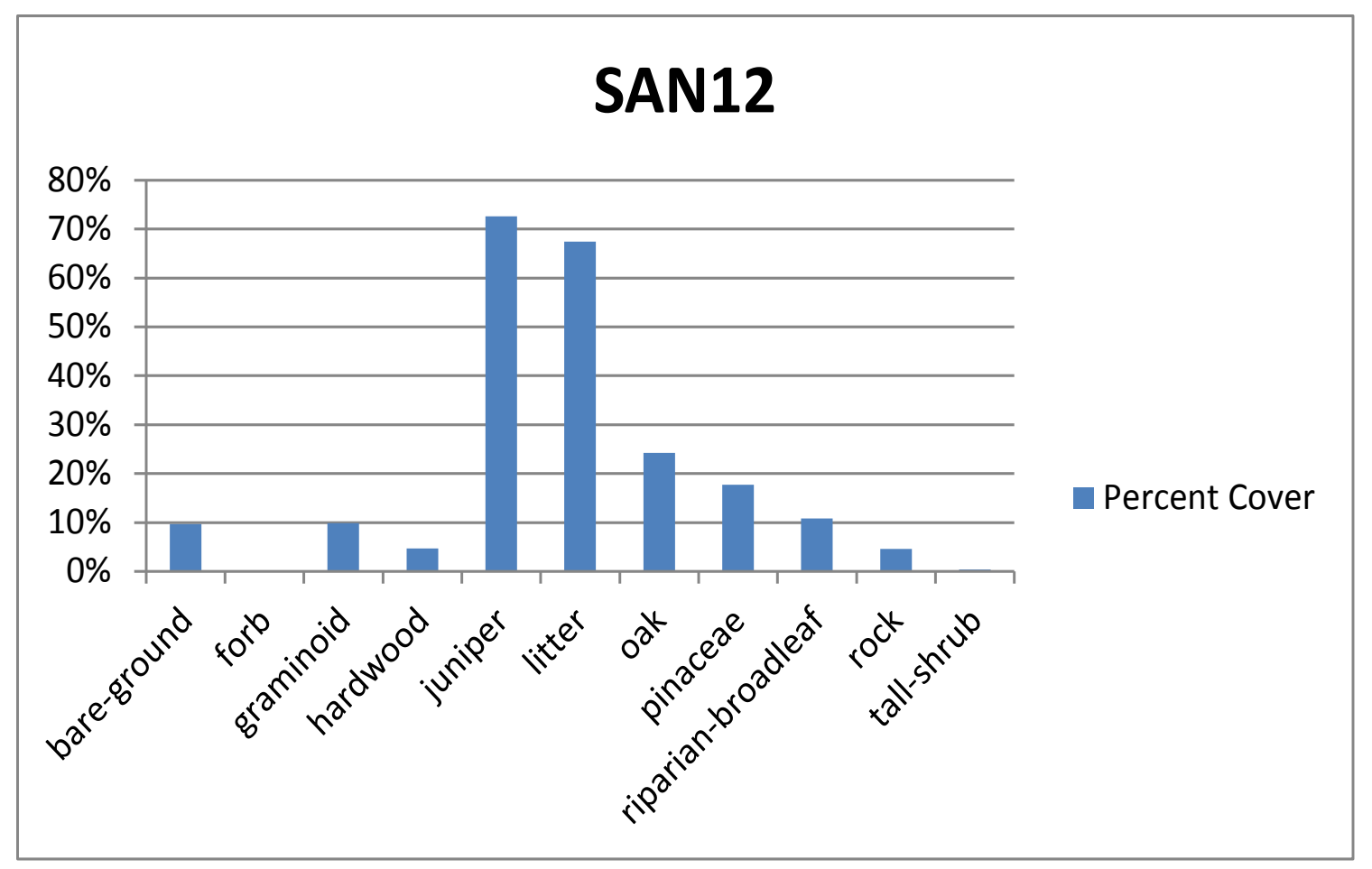

Figure 101. Habitat and species composition characteristics of jaguar detection site SAN12 in the Santa Rita Mountains.

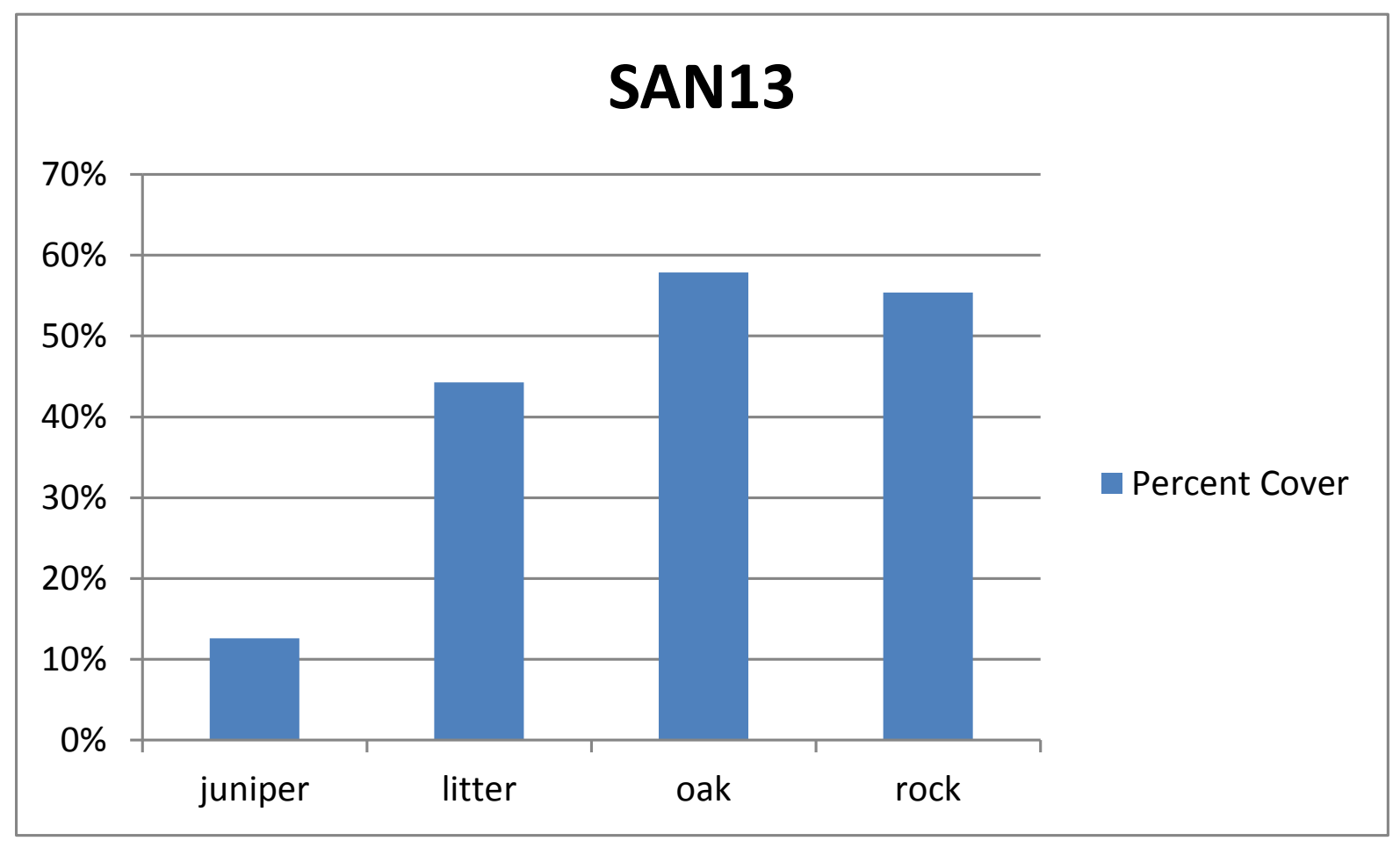

Figure 102. Habitat and species composition characteristics of jaguar detection site SAN13 in the Santa Rita Mountains. 


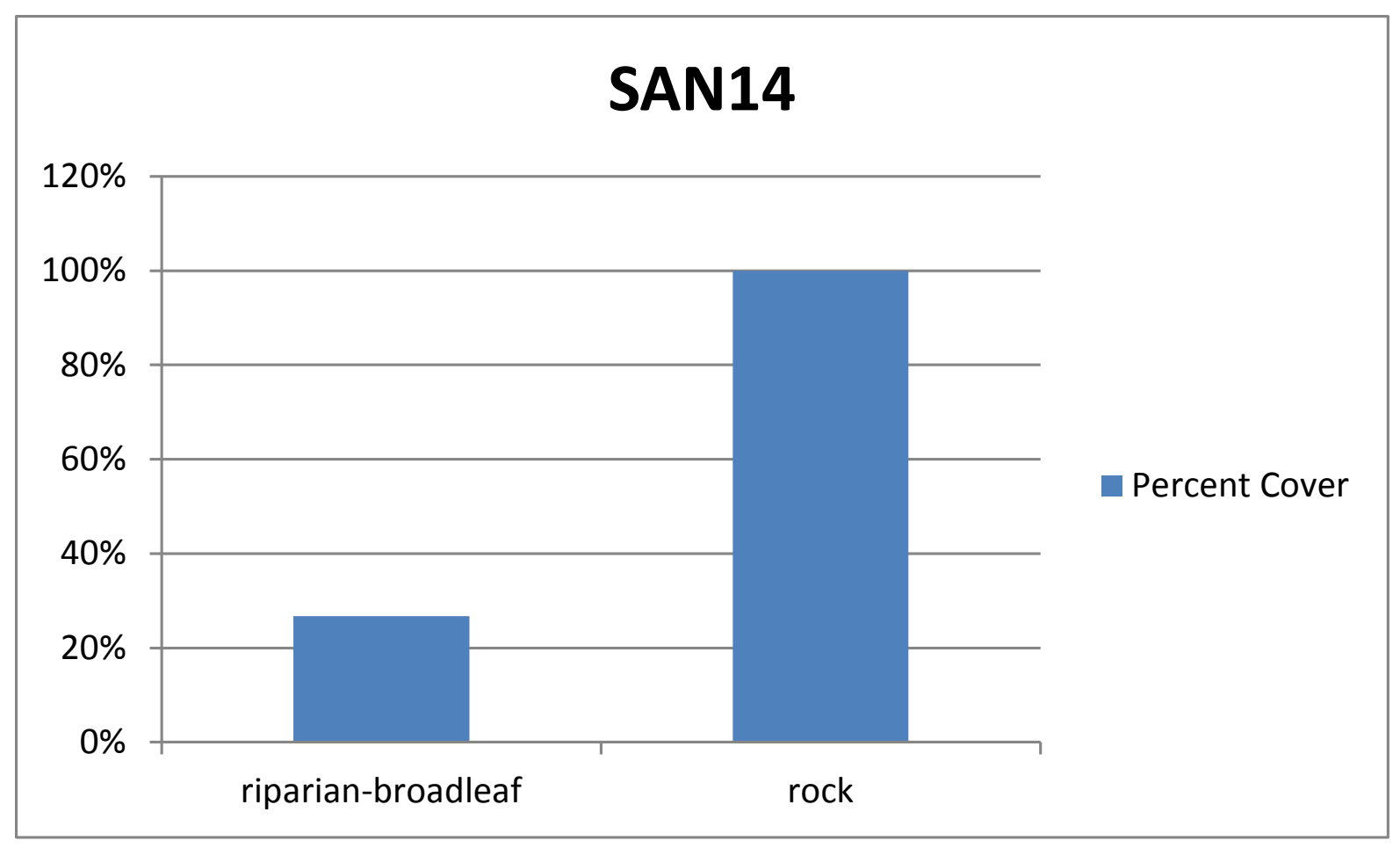

Figure 103. Habitat and species composition characteristics of jaguar detection site SAN14 in the Santa Rita Mountains.

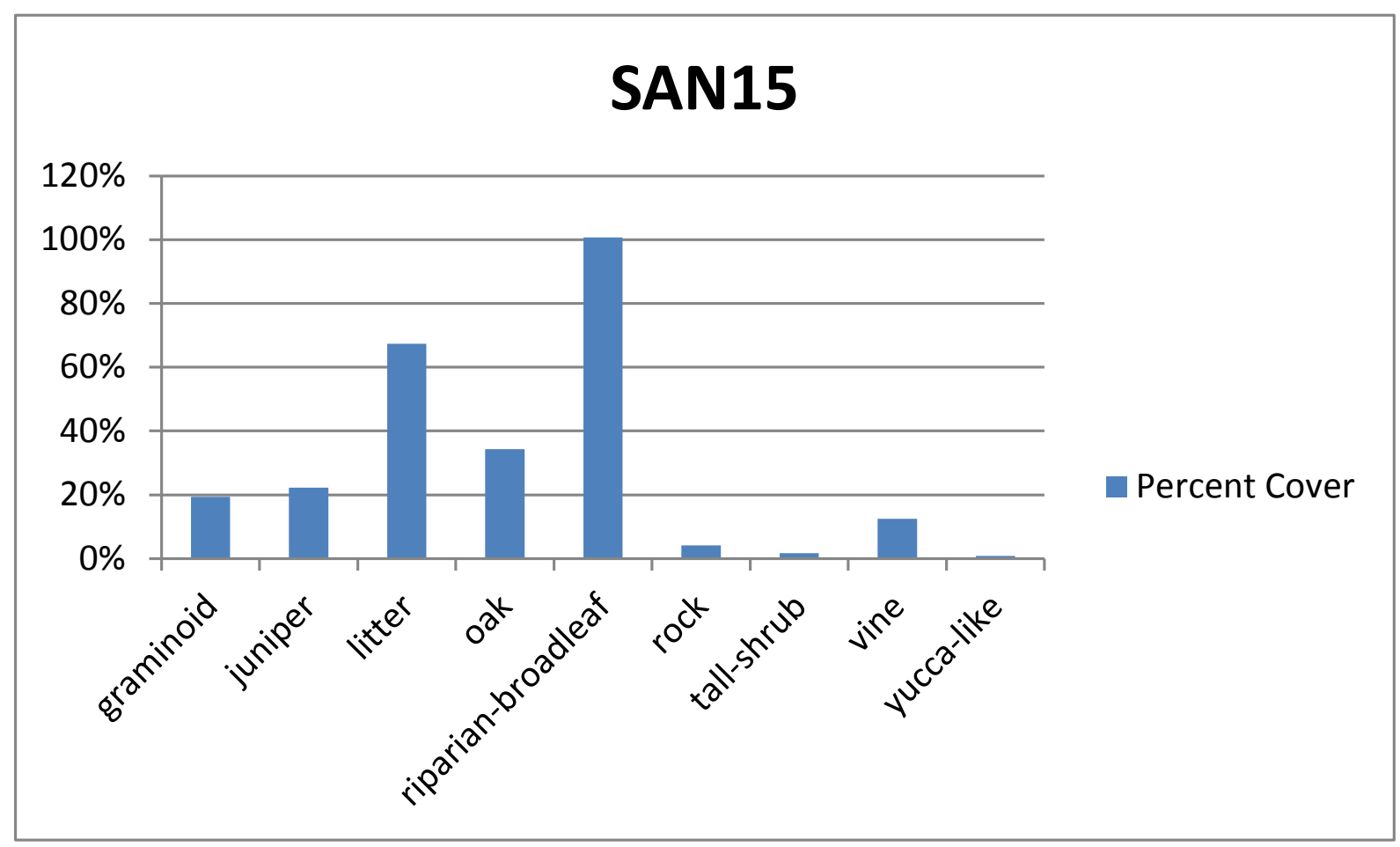

Figure 104. Habitat and species composition characteristics of jaguar detection site SAN15 in the Santa Rita Mountains. 


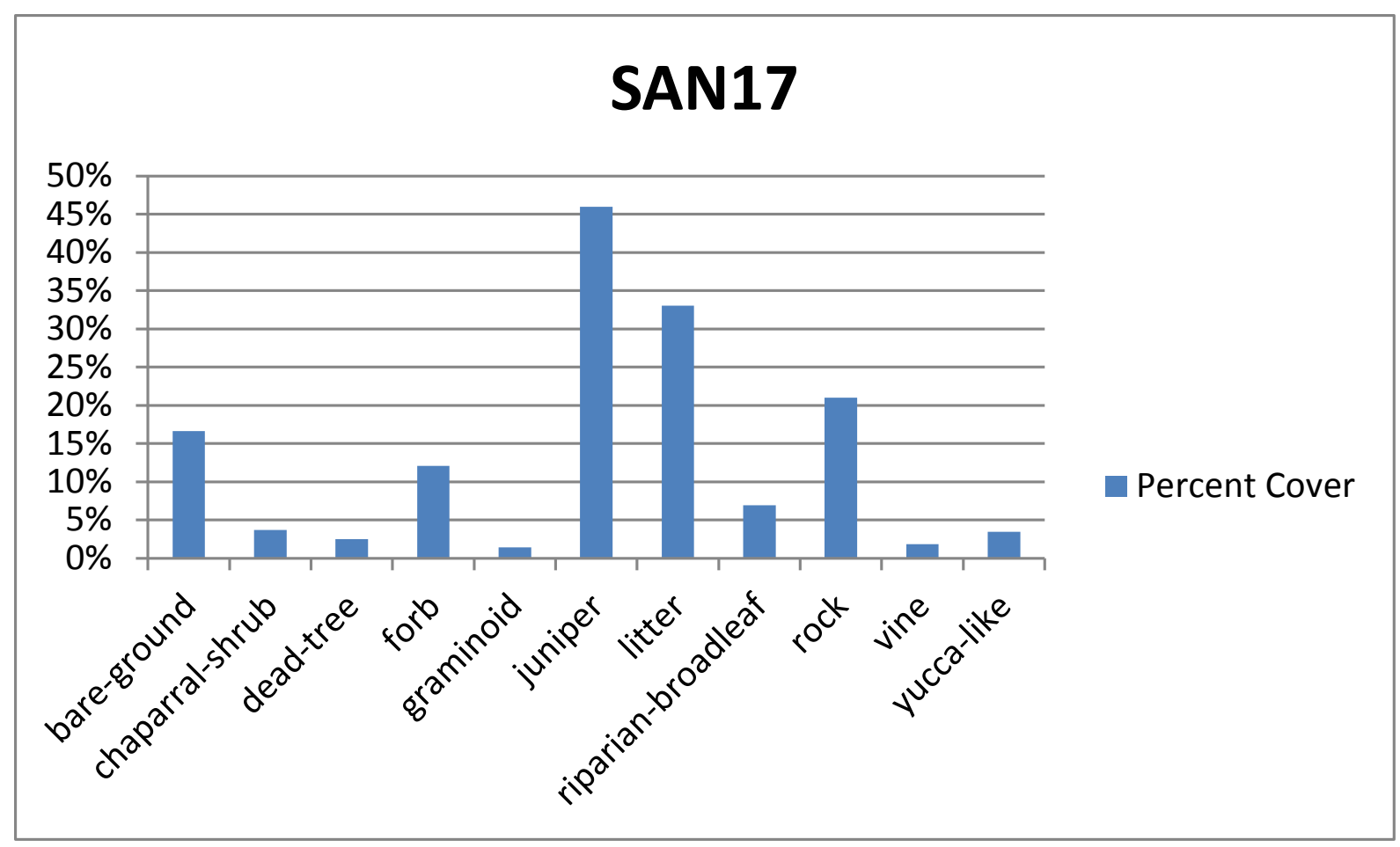

Figure 105. Habitat and species composition characteristics of jaguar detection site SAN17 in the Santa Rita Mountains.

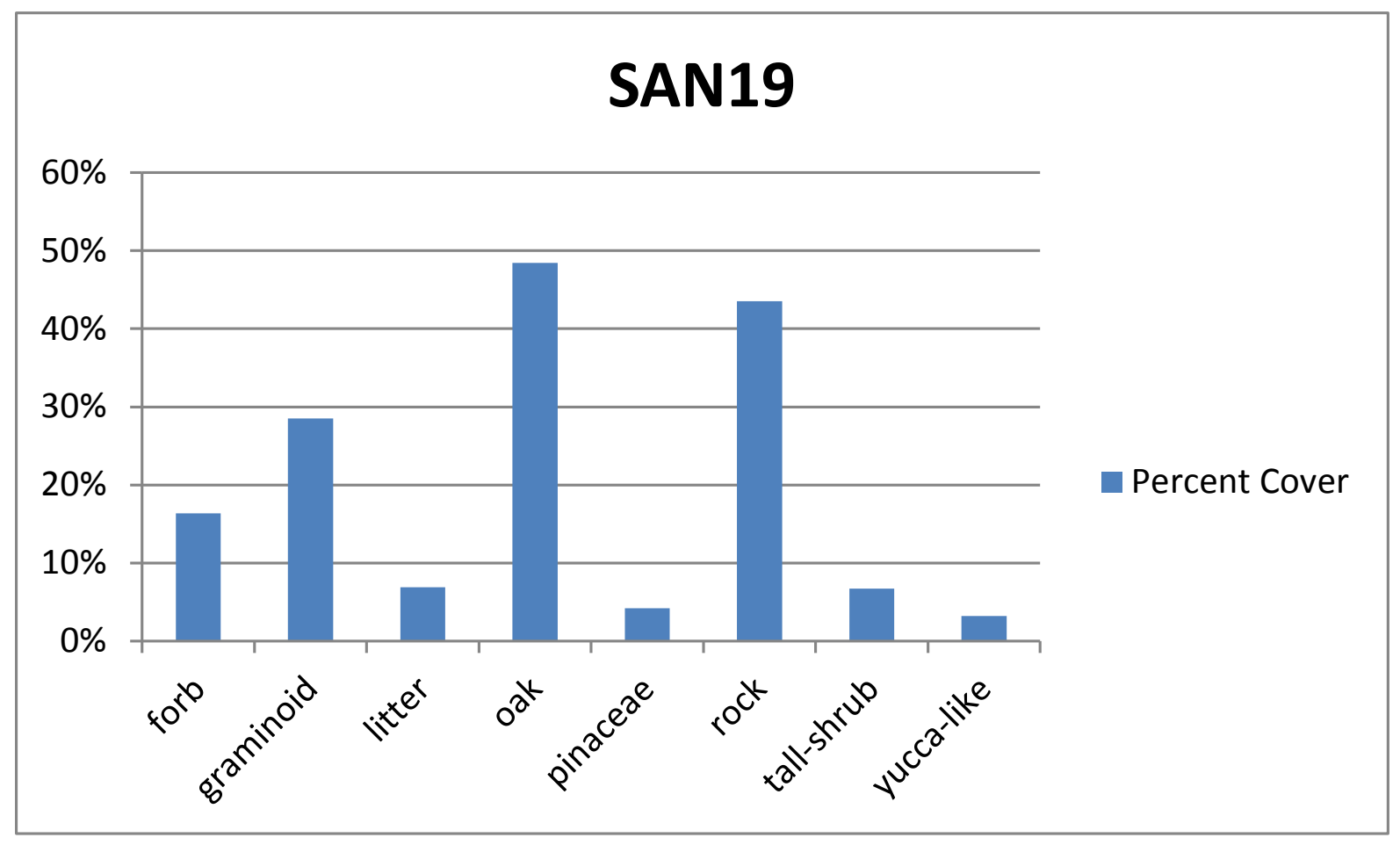

Figure 106. Habitat and species composition characteristics of jaguar detection site SAN19 in the Santa Rita Mountains. 


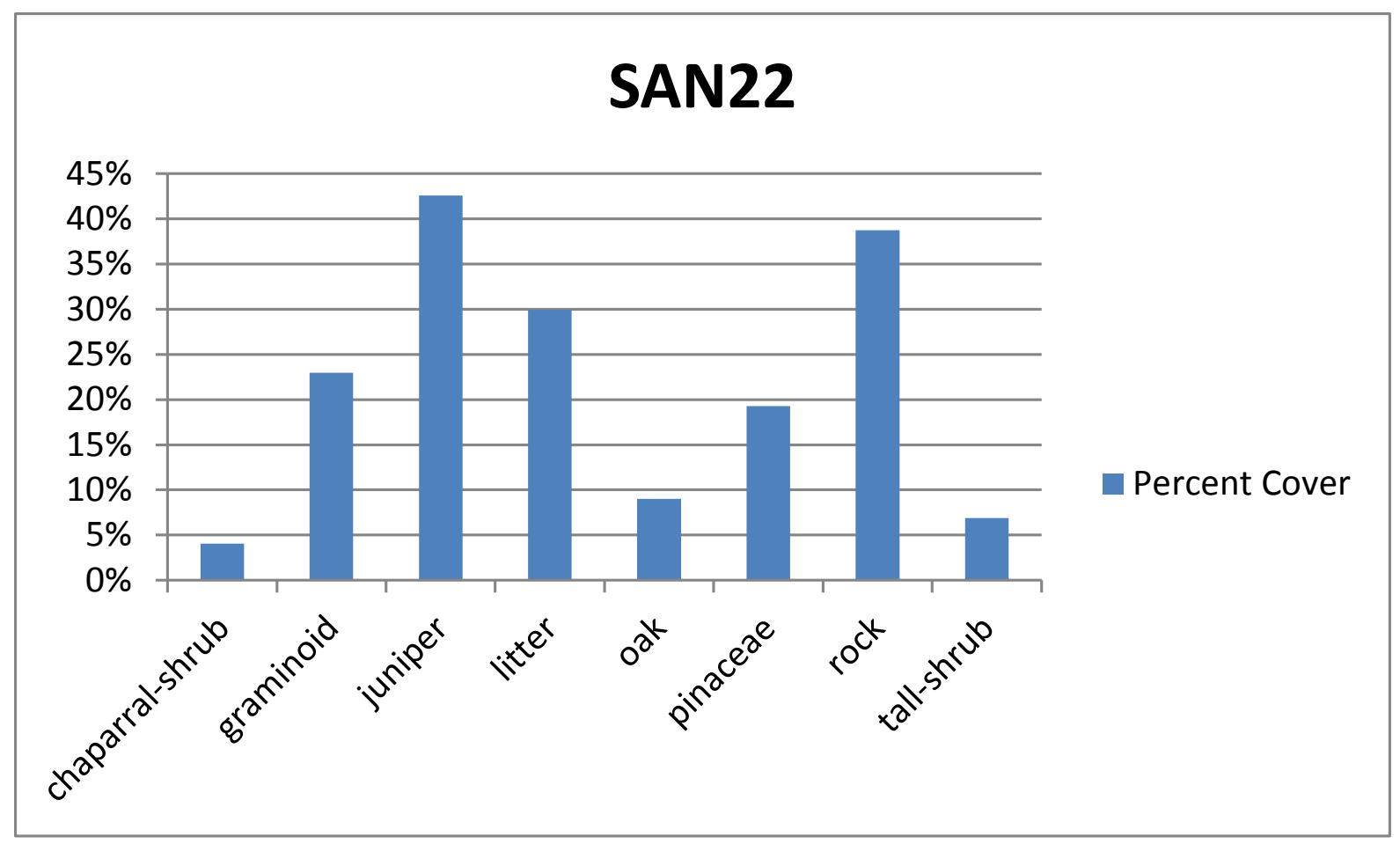

Figure 107. Habitat and species composition characteristics of jaguar detection site SAN22 in the Santa Rita Mountains.

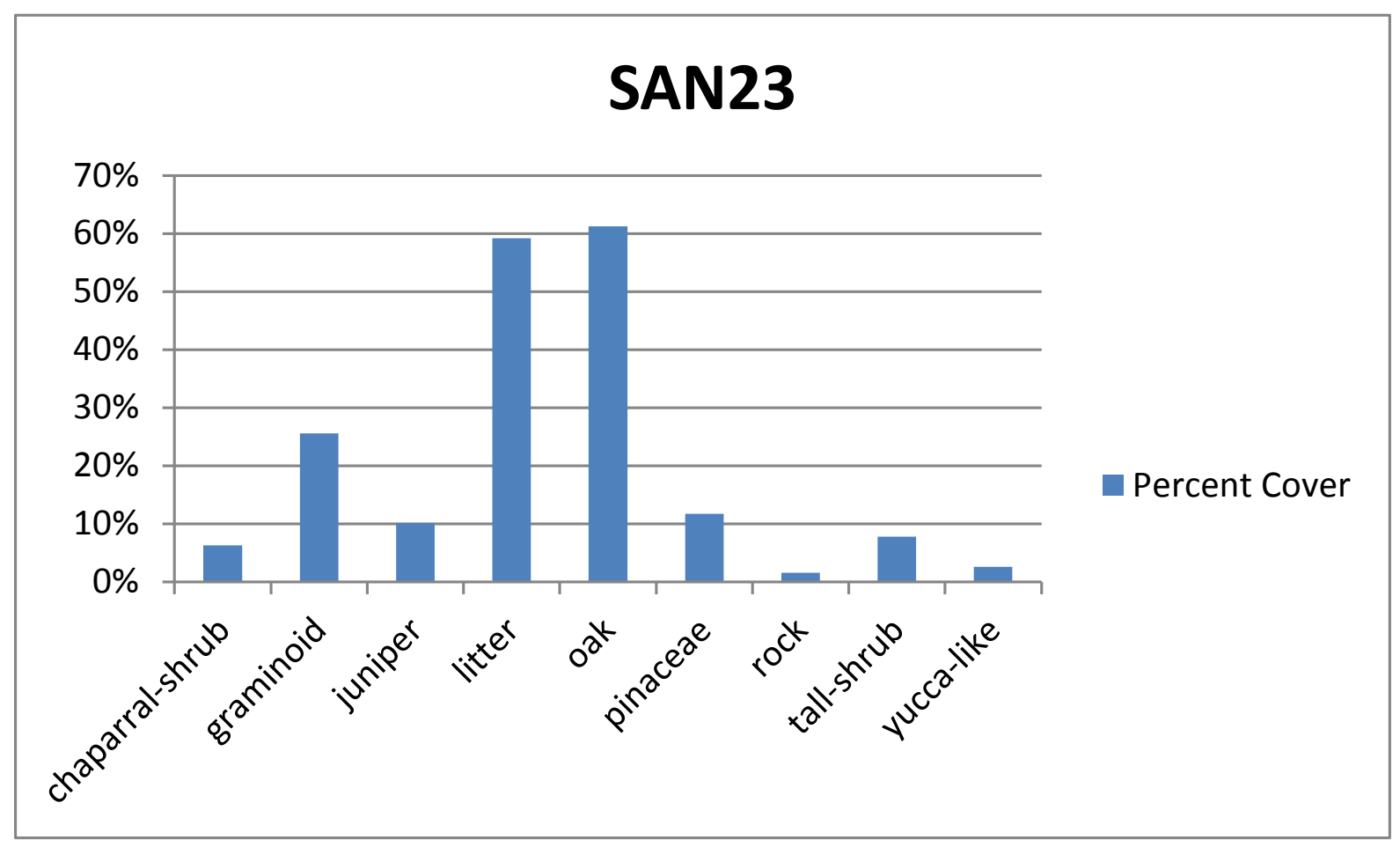

Figure 108. Habitat and species composition characteristics of jaguar detection site SAN23 in the Santa Rita Mountains. 


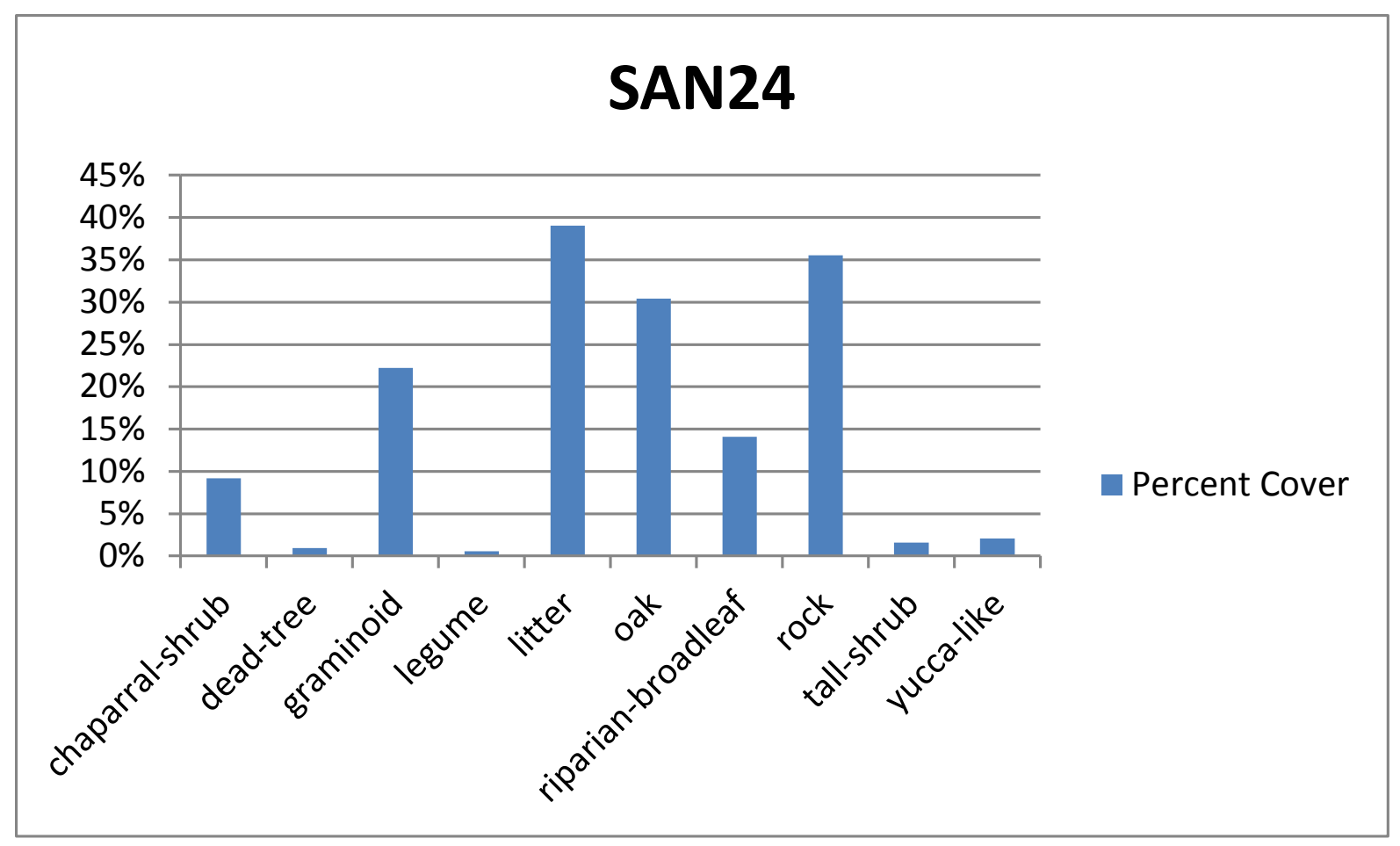

Figure 109. Habitat and species composition characteristics of jaguar detection site SAN24 in the Santa Rita Mountains.

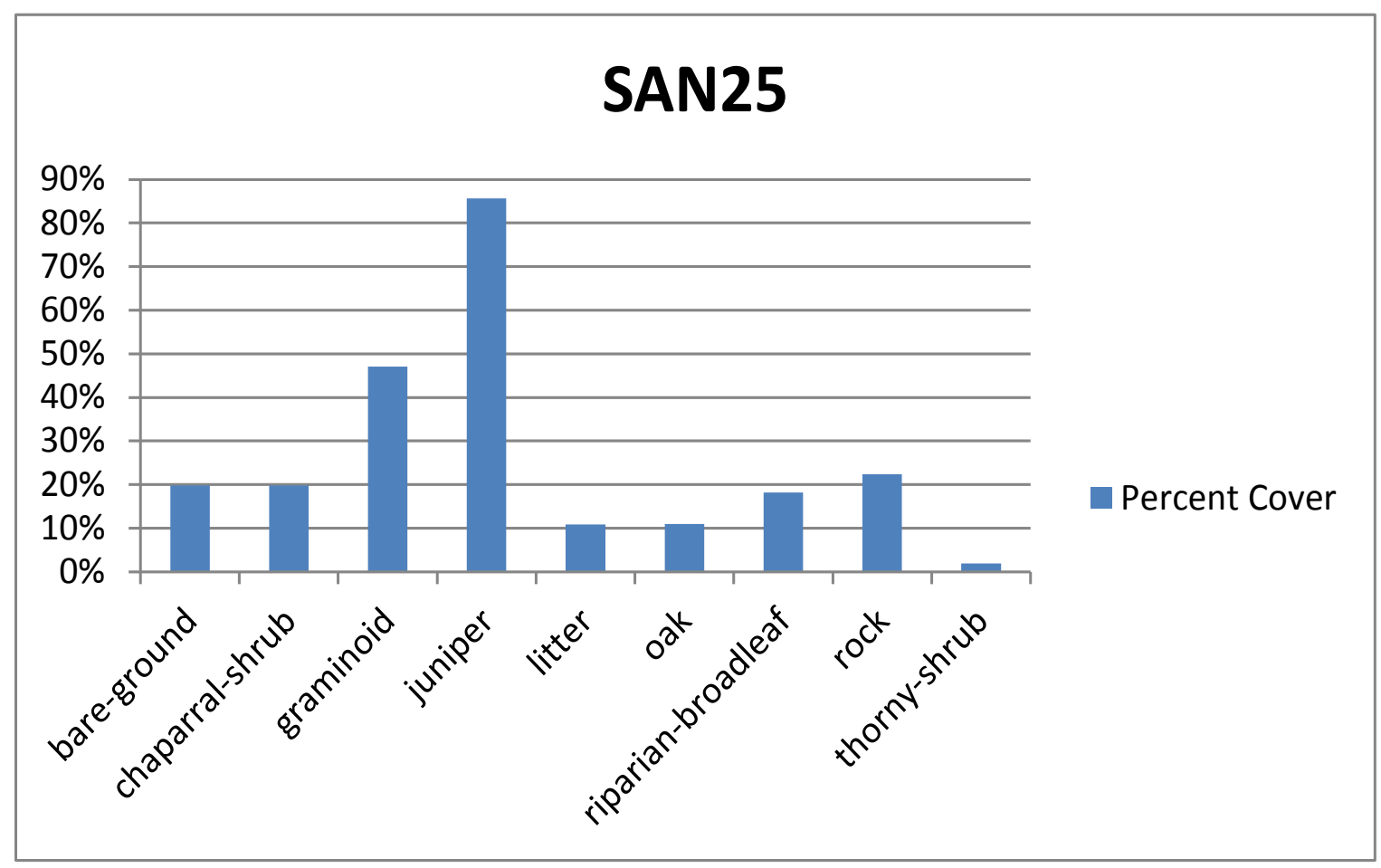

Figure 110. Habitat and species composition characteristics of jaguar detection site SAN25 in the Santa Rita Mountains. 


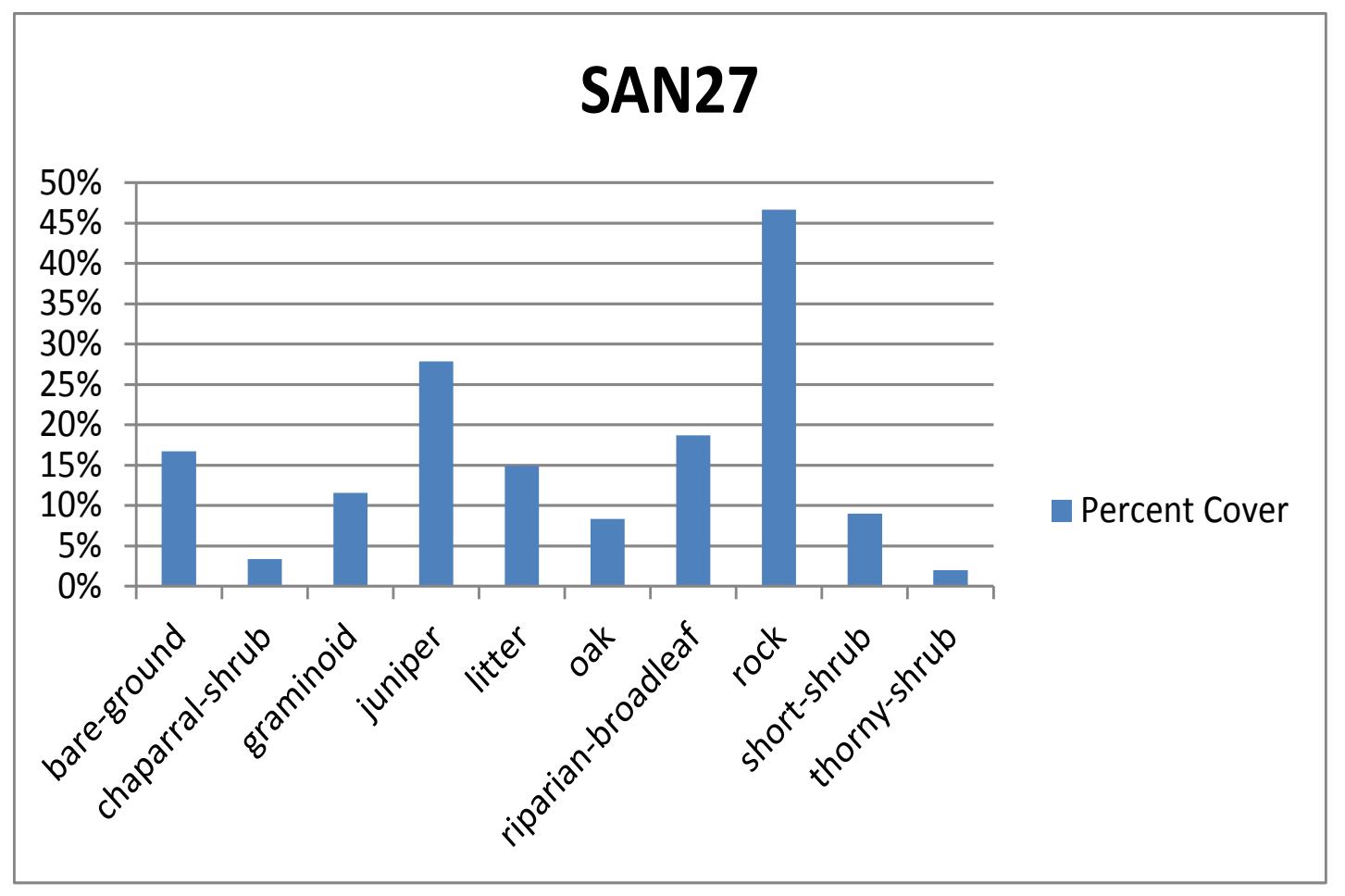

Figure 111. Habitat and species composition characteristics of jaguar detection site SAN27 in the Santa Rita Mountains.

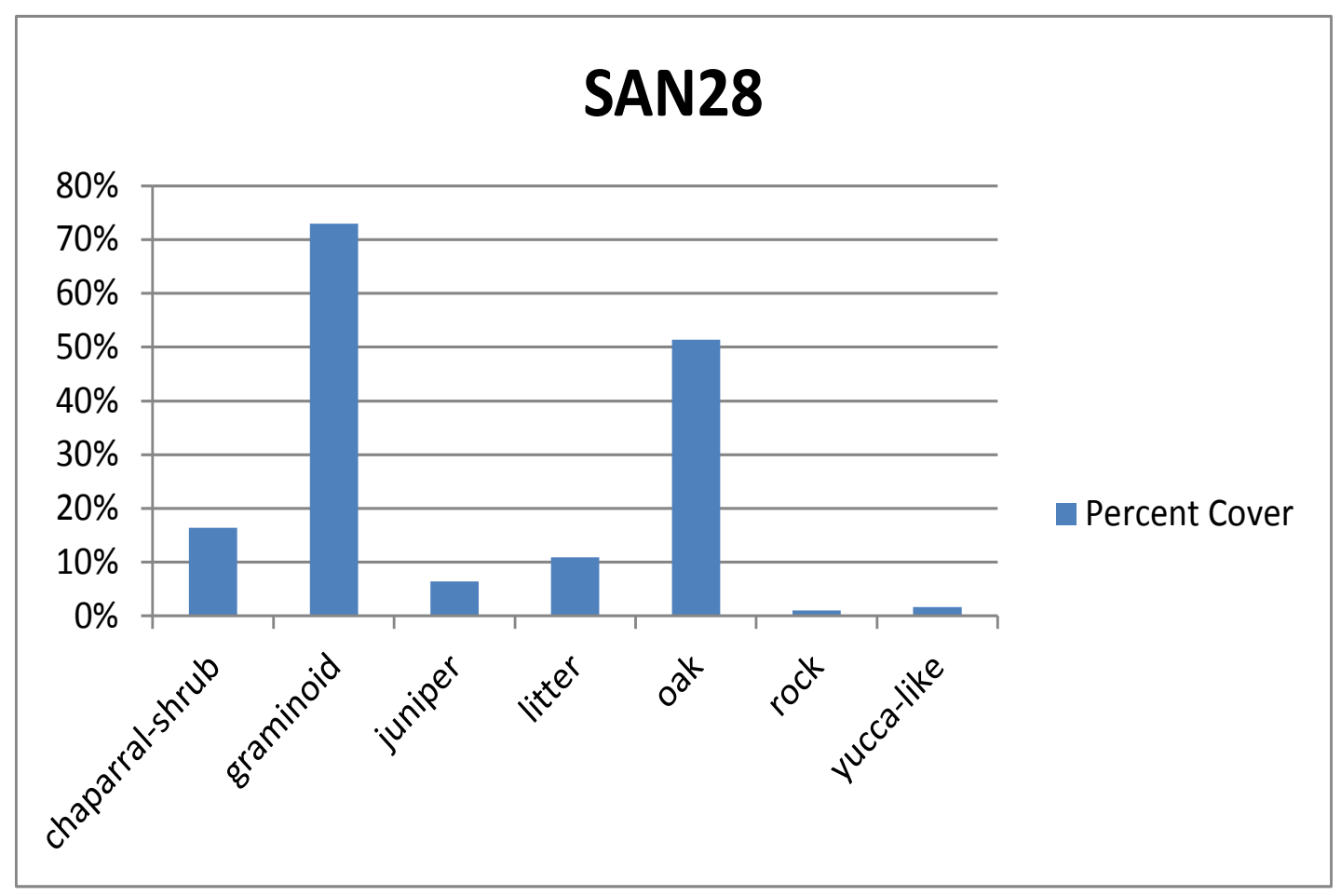

Figure 112. Habitat and species composition characteristics of jaguar detection site SAN28 in the Santa Rita Mountains. 


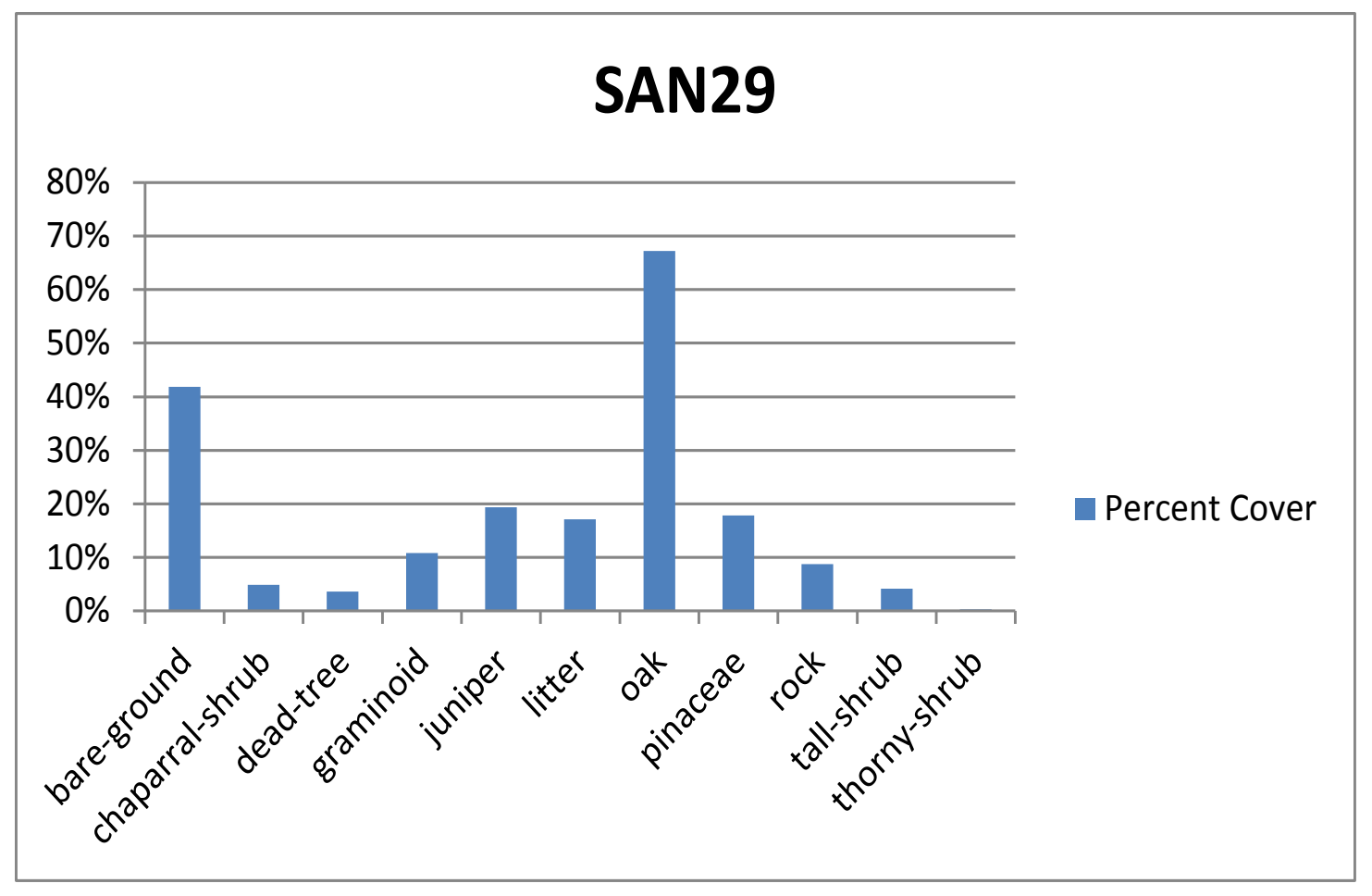

Figure 113. Habitat and species composition characteristics of jaguar detection site SAN29 in the Santa Rita Mountains.

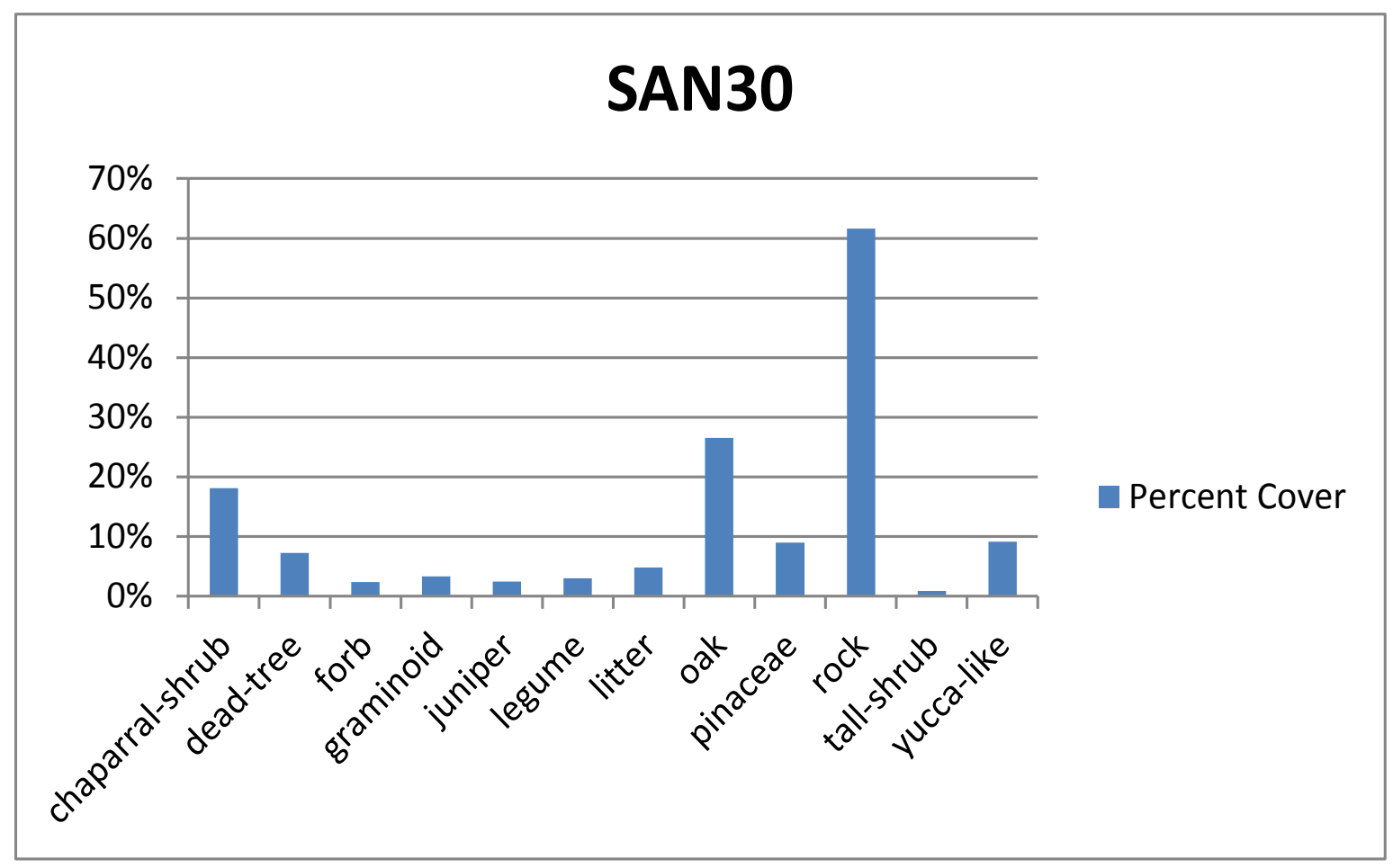

Figure 114. Habitat and species composition characteristics of jaguar detection site SAN30 in the Santa Rita Mountains. 


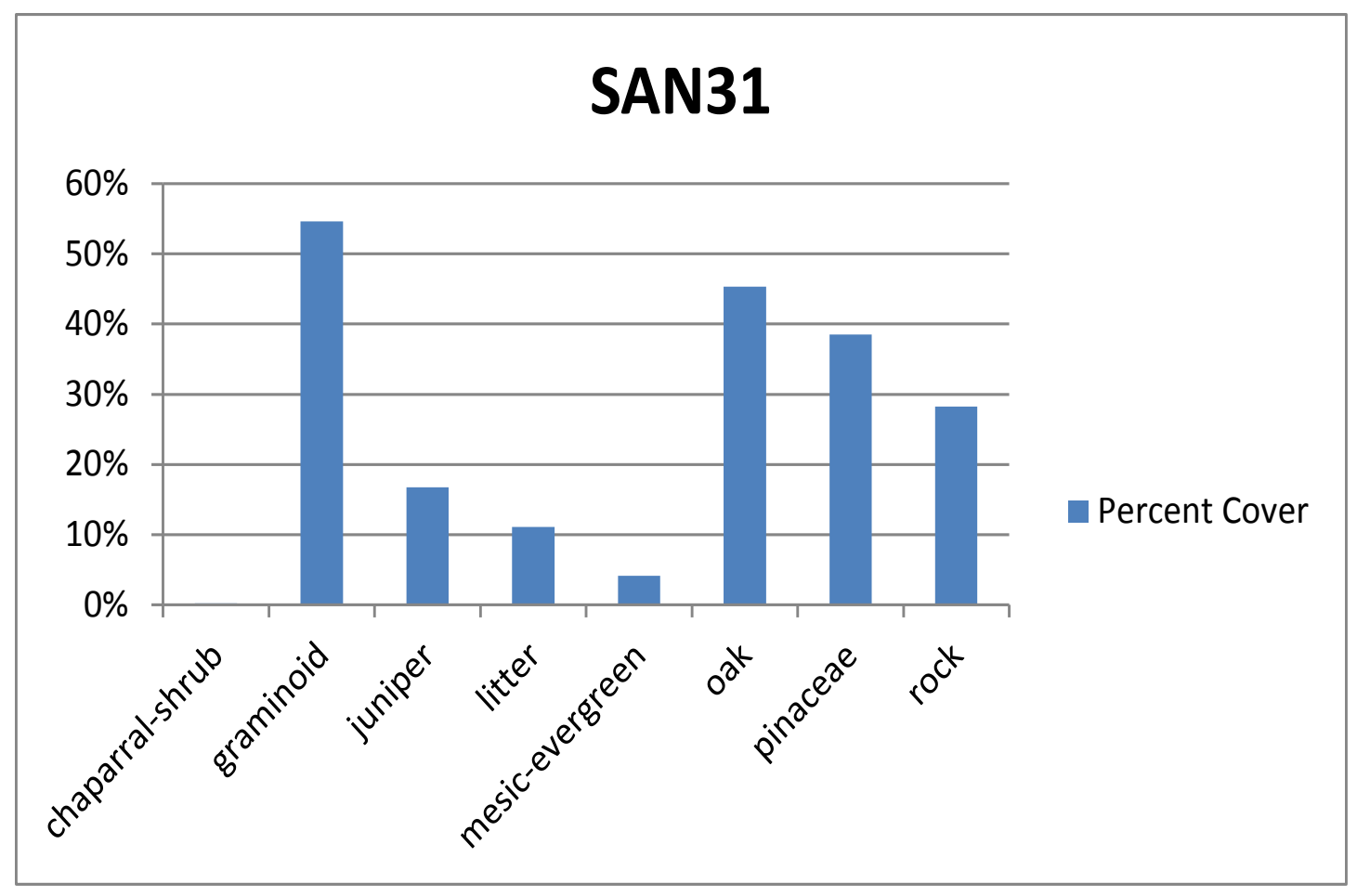

Figure 115. Habitat and species composition characteristics of jaguar detection site SAN31 in the Santa Rita Mountains.

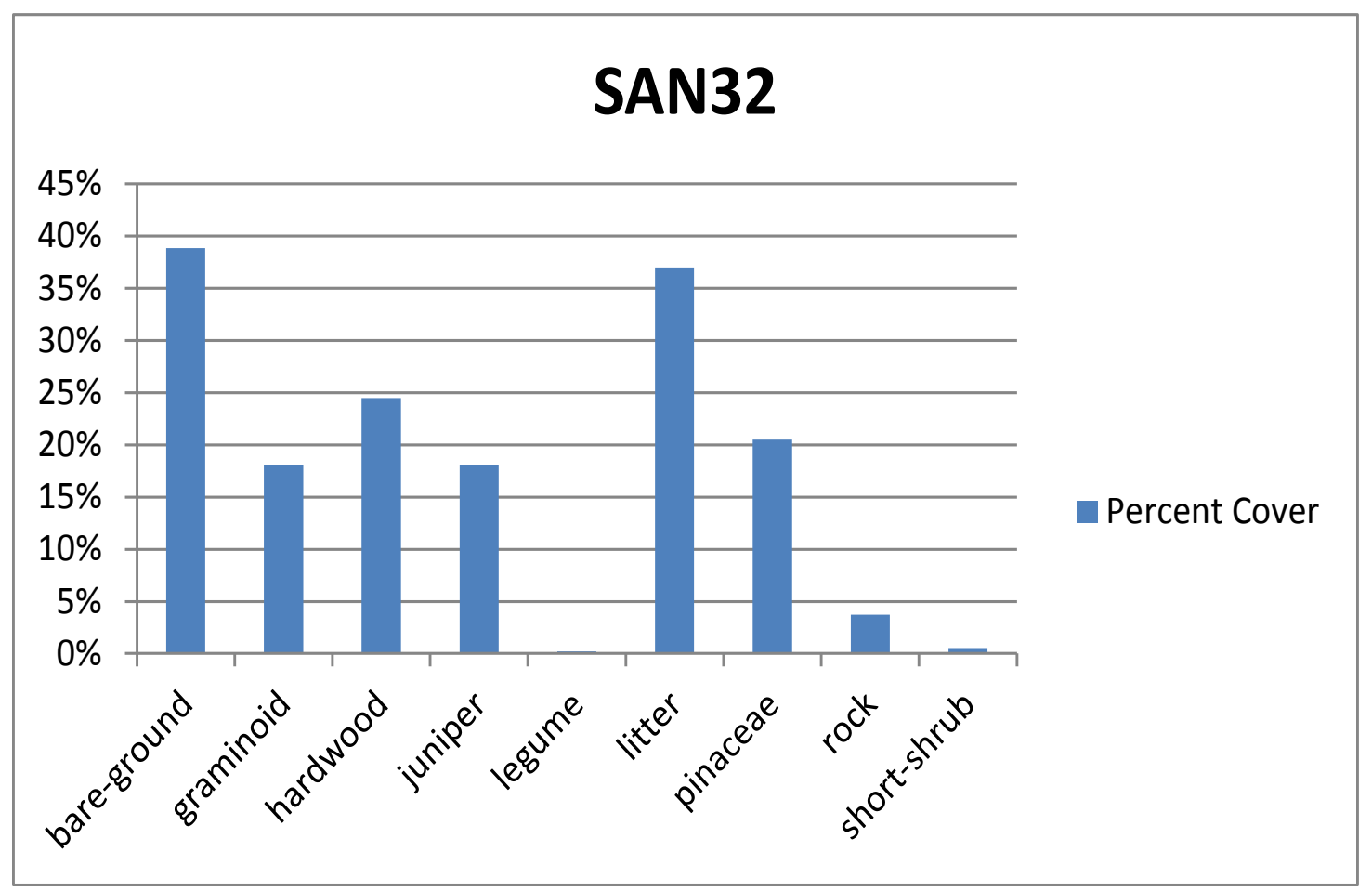

Figure 116. Habitat and species com position characteristics of jaguar detection site SAN32 in the Santa Rita Mountains. 


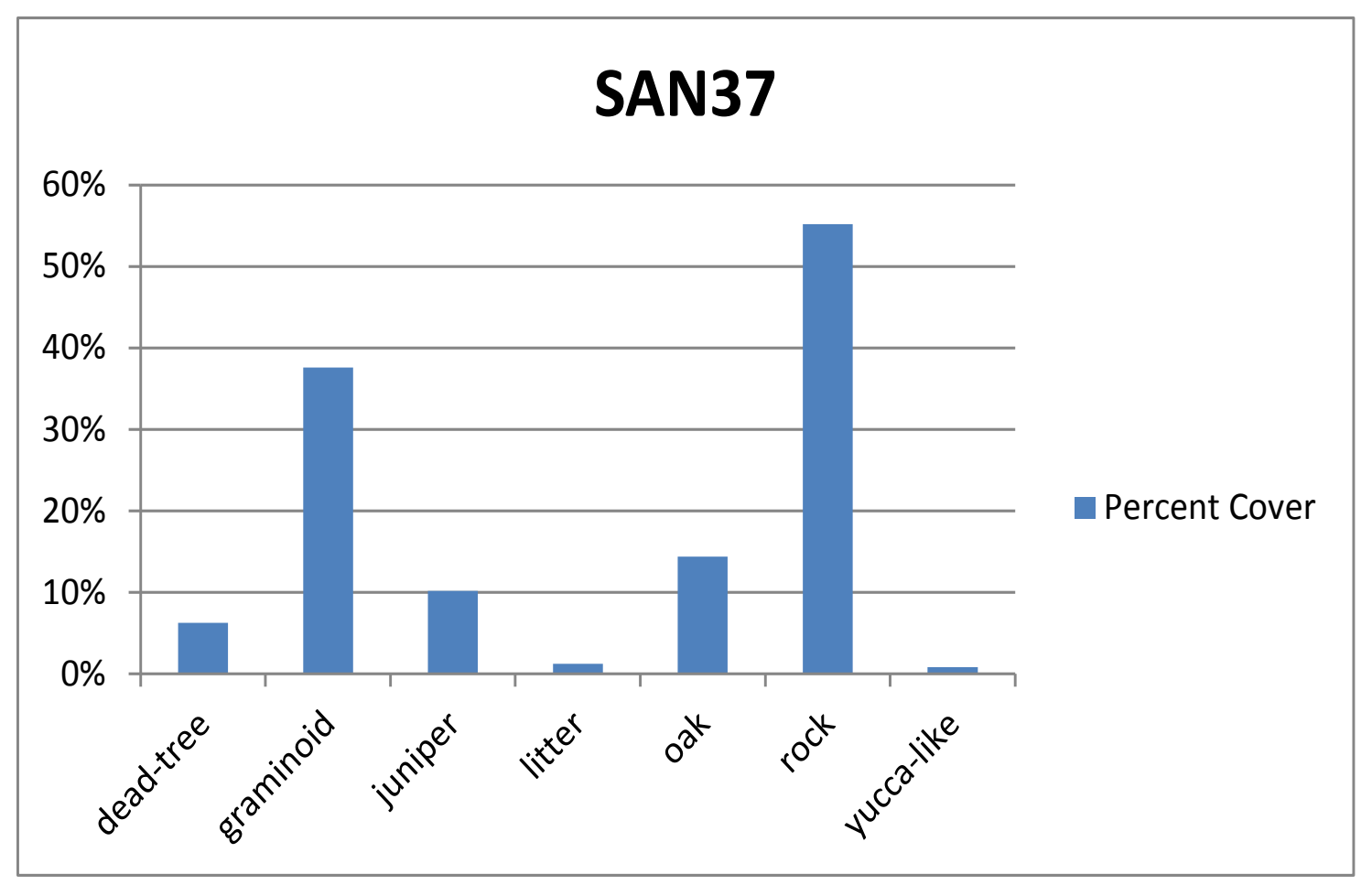

Figure 117. Habitat and species composition characteristics of jaguar detection site SAN37 in the Santa Rita Mountains.

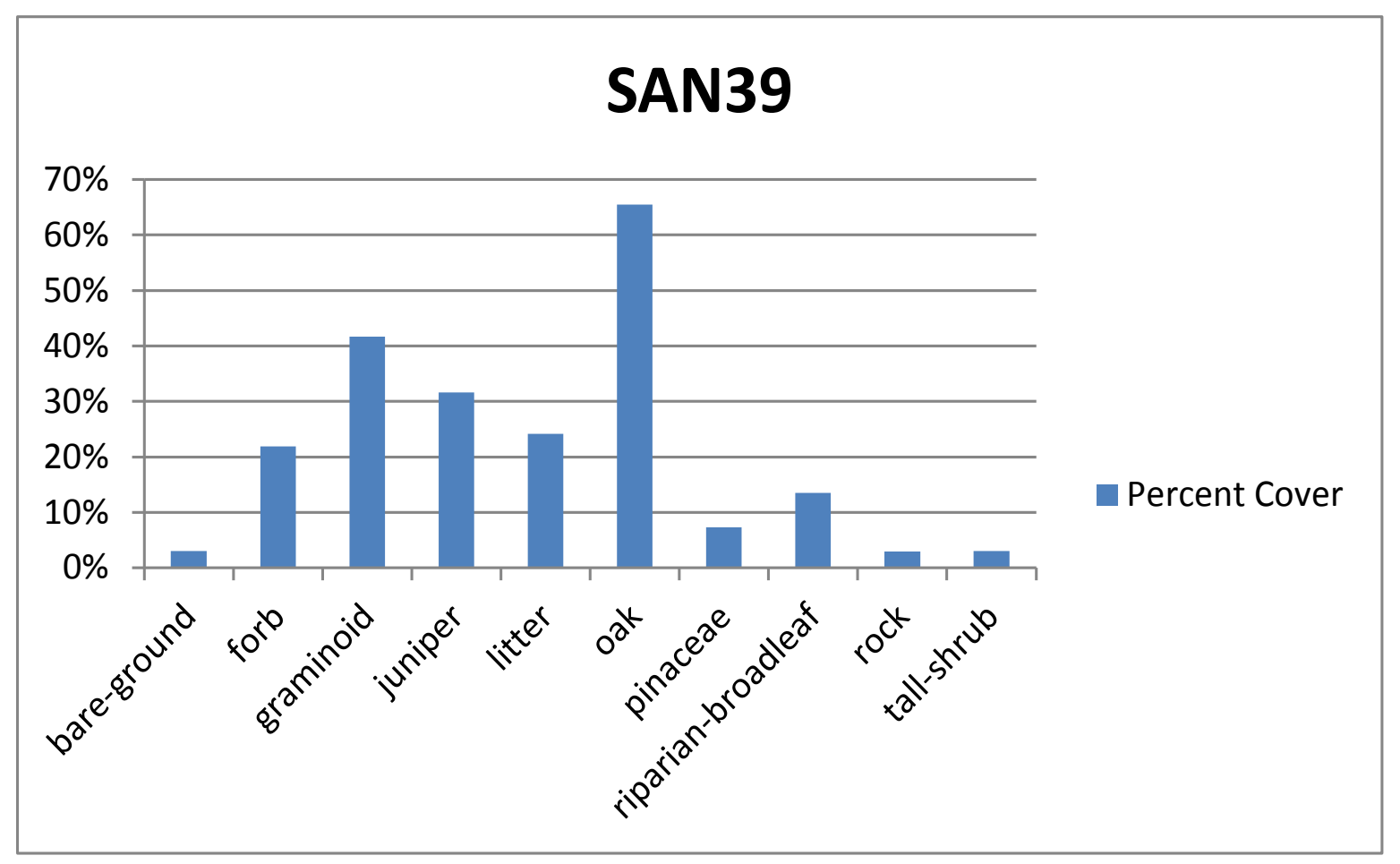

Figure 118. Habitat and species composition characteristics of jaguar detection site SAN39 in the Santa Rita Mountains. 


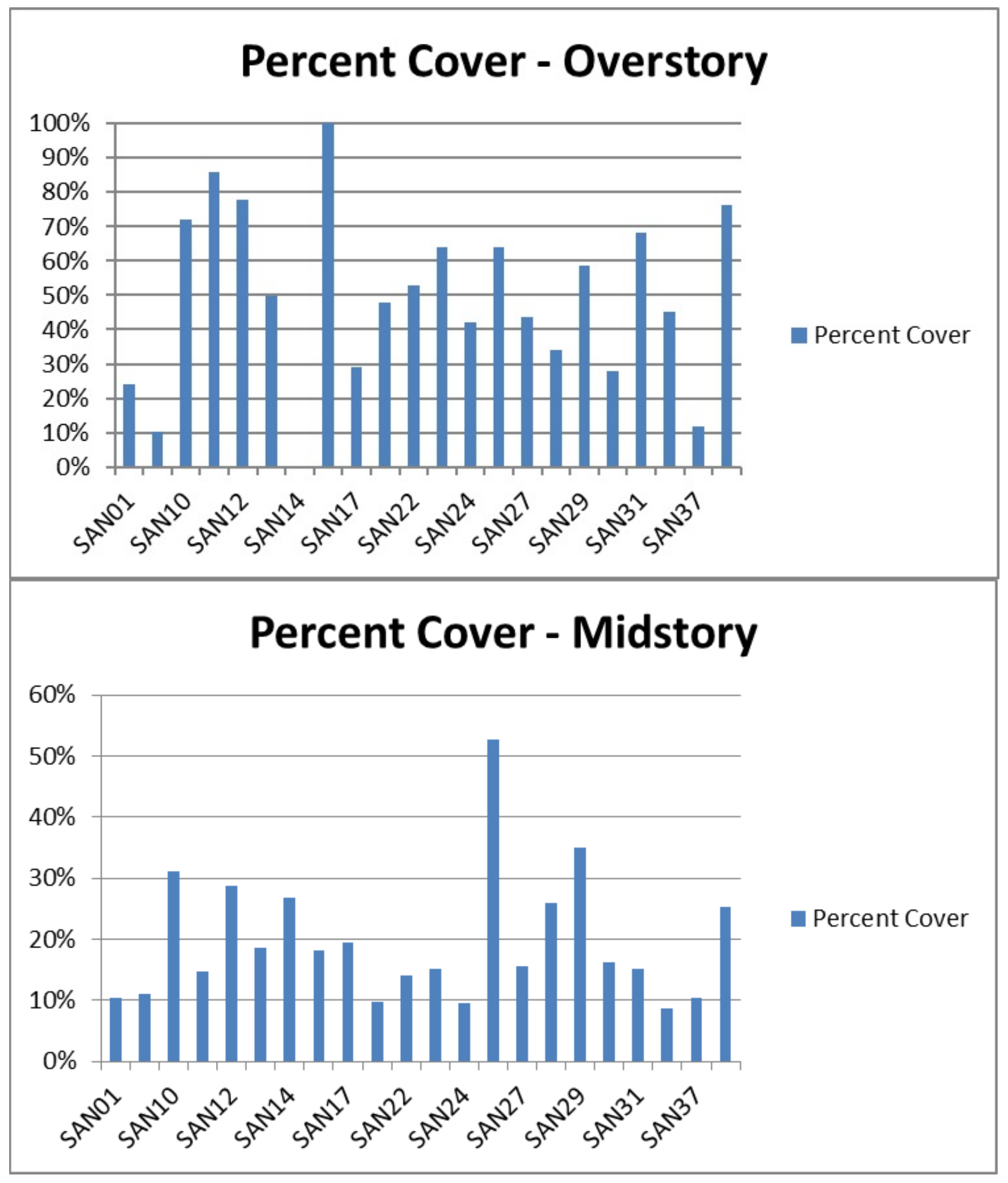

Figure 119. Overstory and midstory cover characteristics of jaguar detection sites in the Santa Rita Mountains. 


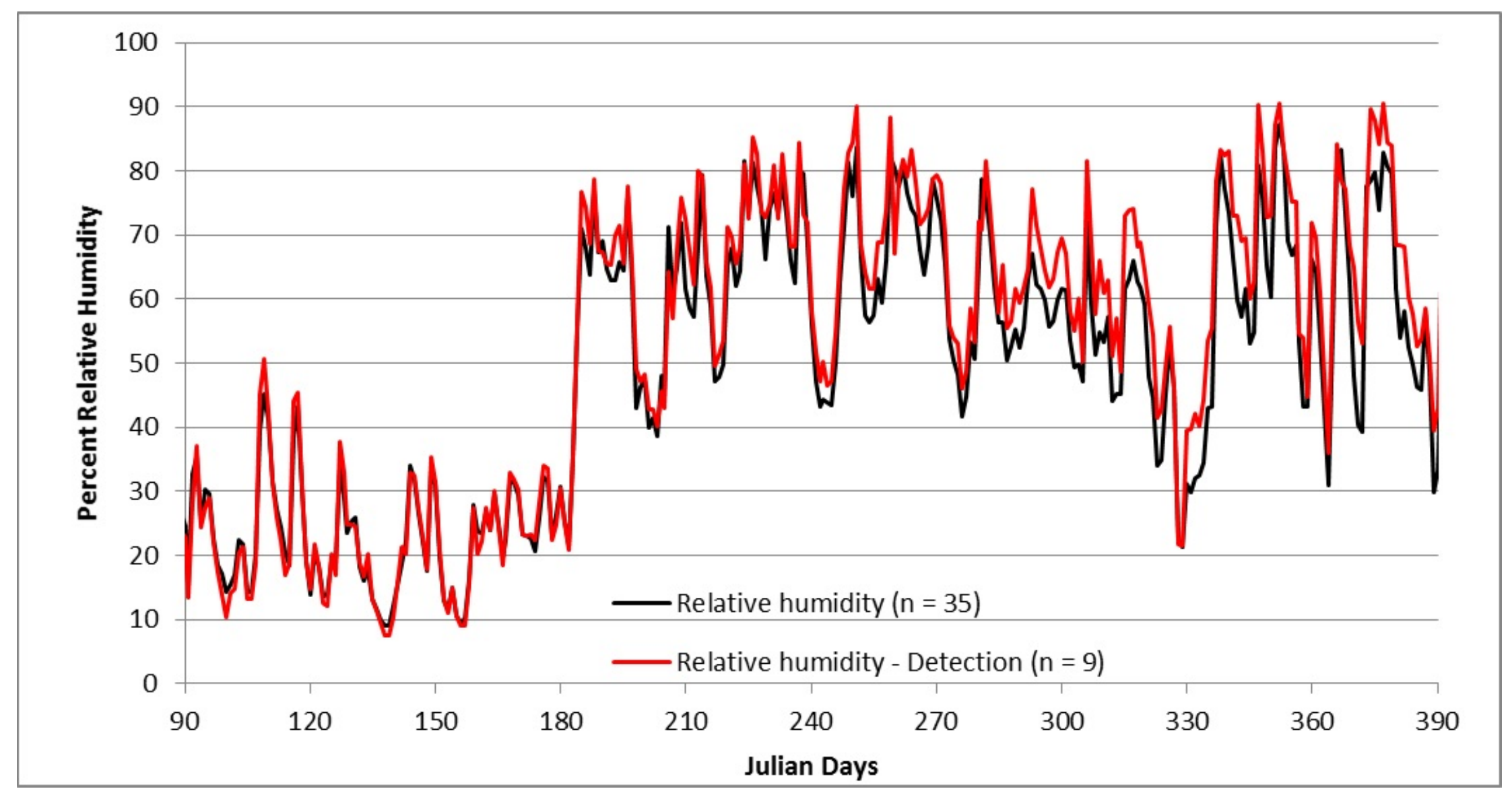

Figure 120a. A comparison of daily average relative humidity (in percent) for jaguar detection ( $n=9$, red line) and jaguar non-detection ( $n=35$, black line) locations in Julian days (days 90-120 correspond to April, 330-360 to December). 


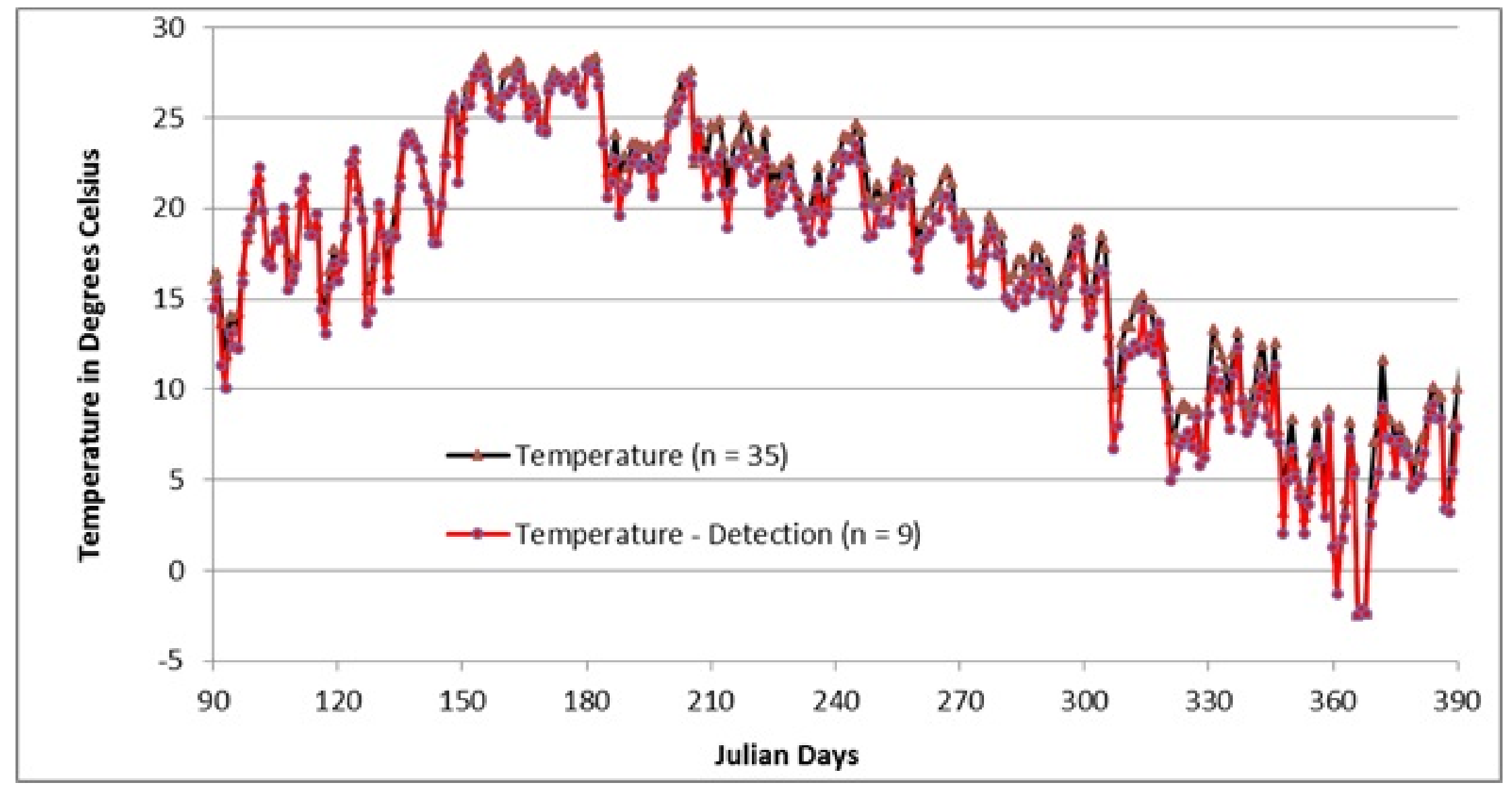

Figure 120b. A comparison of daily average temperature (in degrees Celsius) for jaguar detection ( $n=9$, red line) and jaguar non-detection ( $n=35$, black line) locations in Julian days (days 90-120 correspond to April, 330-360 to December). 


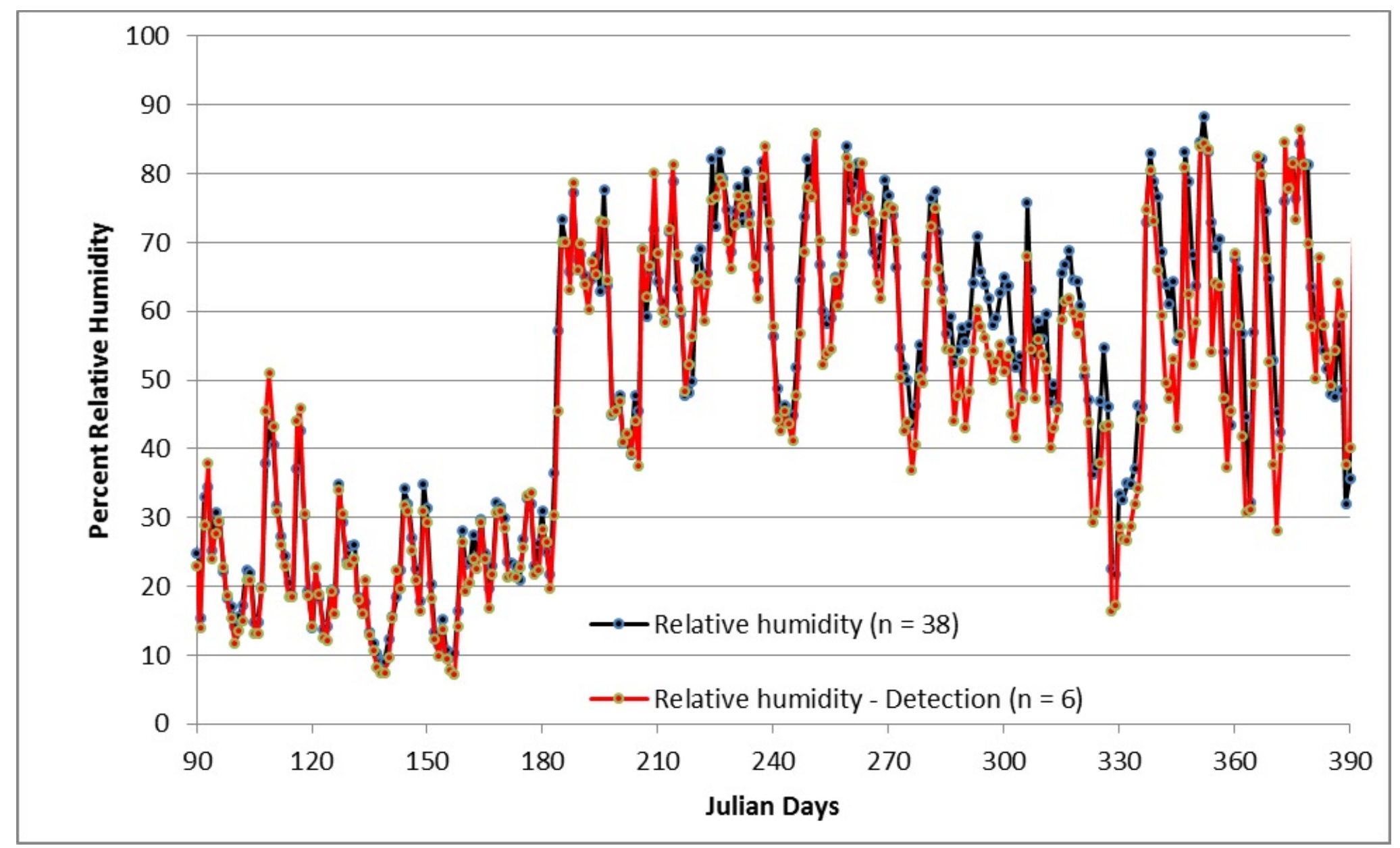

Figure 120c. A comparison of daily average relative humidity (in percent) for ocelot detection ( $n=6$, red line) and ocelot non-detection ( $n=38$, black line) locations in Julian days (days 90-120 correspond to April, 330-360 to December). 


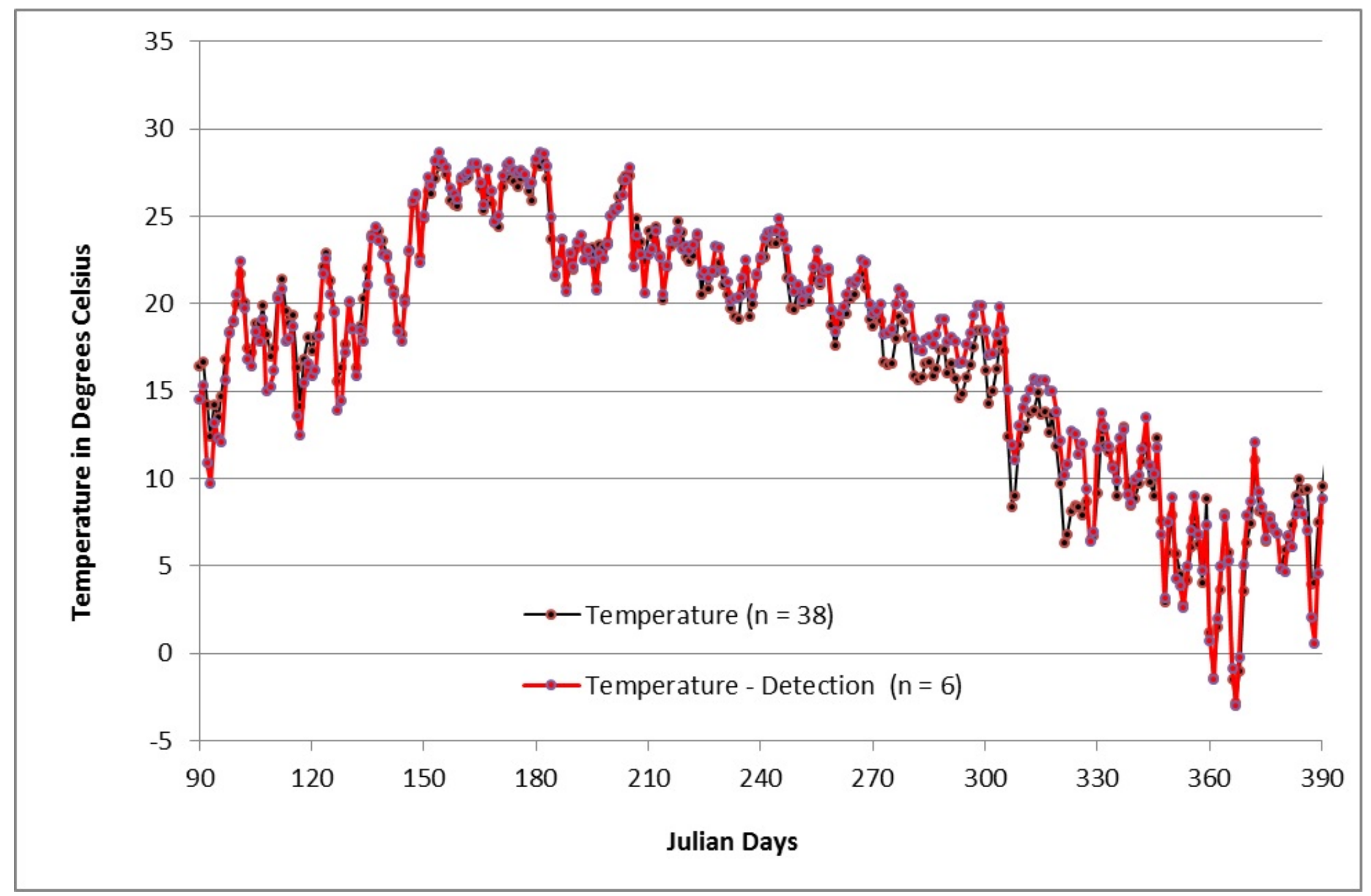

Figure 120d. A comparison of daily average temperature (in degrees Celsius) for ocelot detection ( $n=6$, red line) and ocelot non-detection ( $n=8$, black line) locations in Julian days (days 90-120 correspond to April, 330-360 to December). 


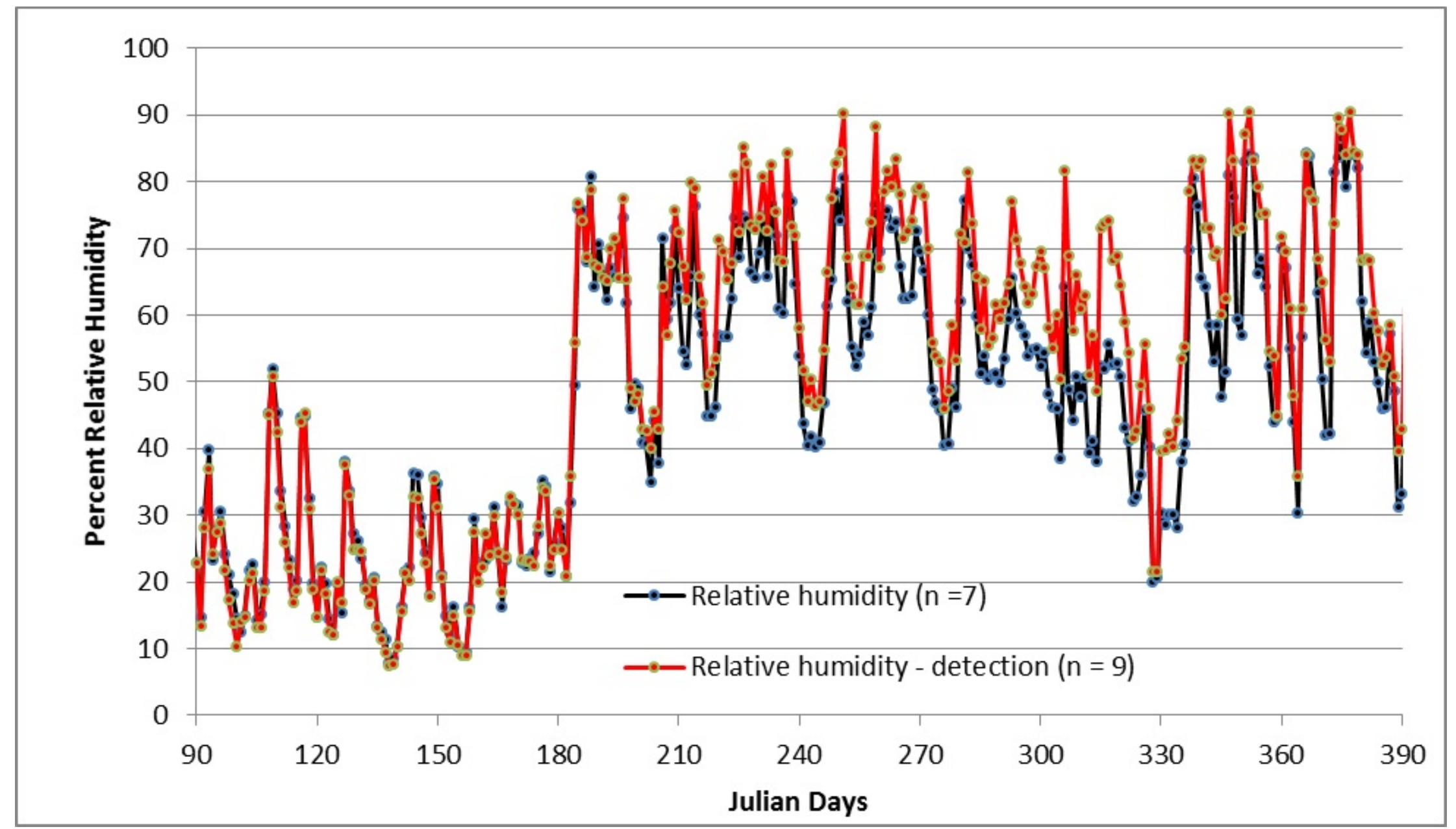

Figure 121a. For the Santa Rita Mountains, a comparison of daily average relative humidity (in percent) for jaguar detection ( $n=9$, red line) and jaguar non-detection ( $n=6$, black line) locations in Julian day (days 90-120 correspond to April, 330-360 to December). 


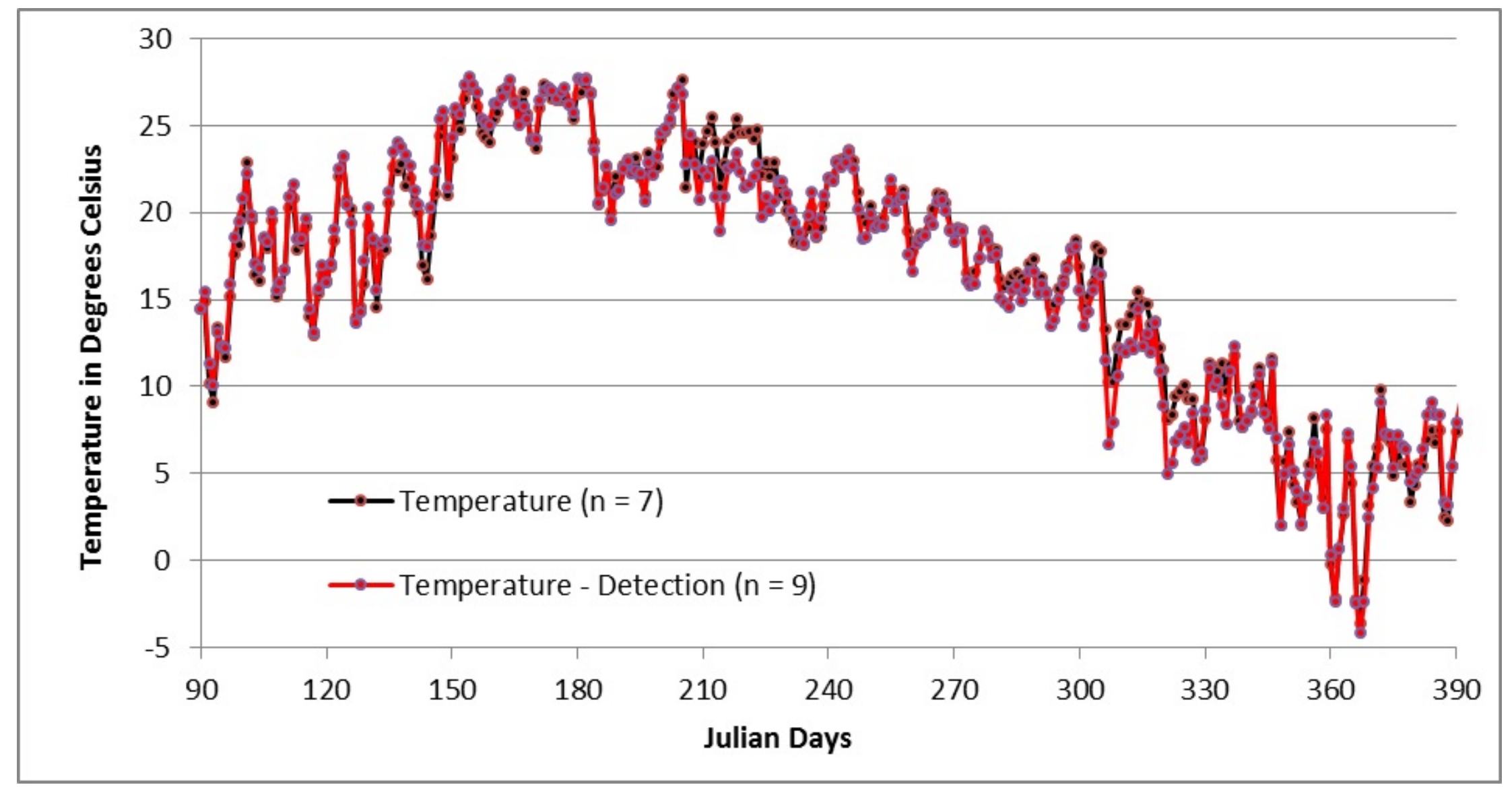

Figure 121b. For the Santa Rita Mountains, a comparison of daily average temperature (in degrees Celsius) for jaguar detection ( $n=9$, red line) and jaguar non-detection ( $n=6$, black line) locations in Julian days (days 90-120 correspond to April, 330-360 to December). 


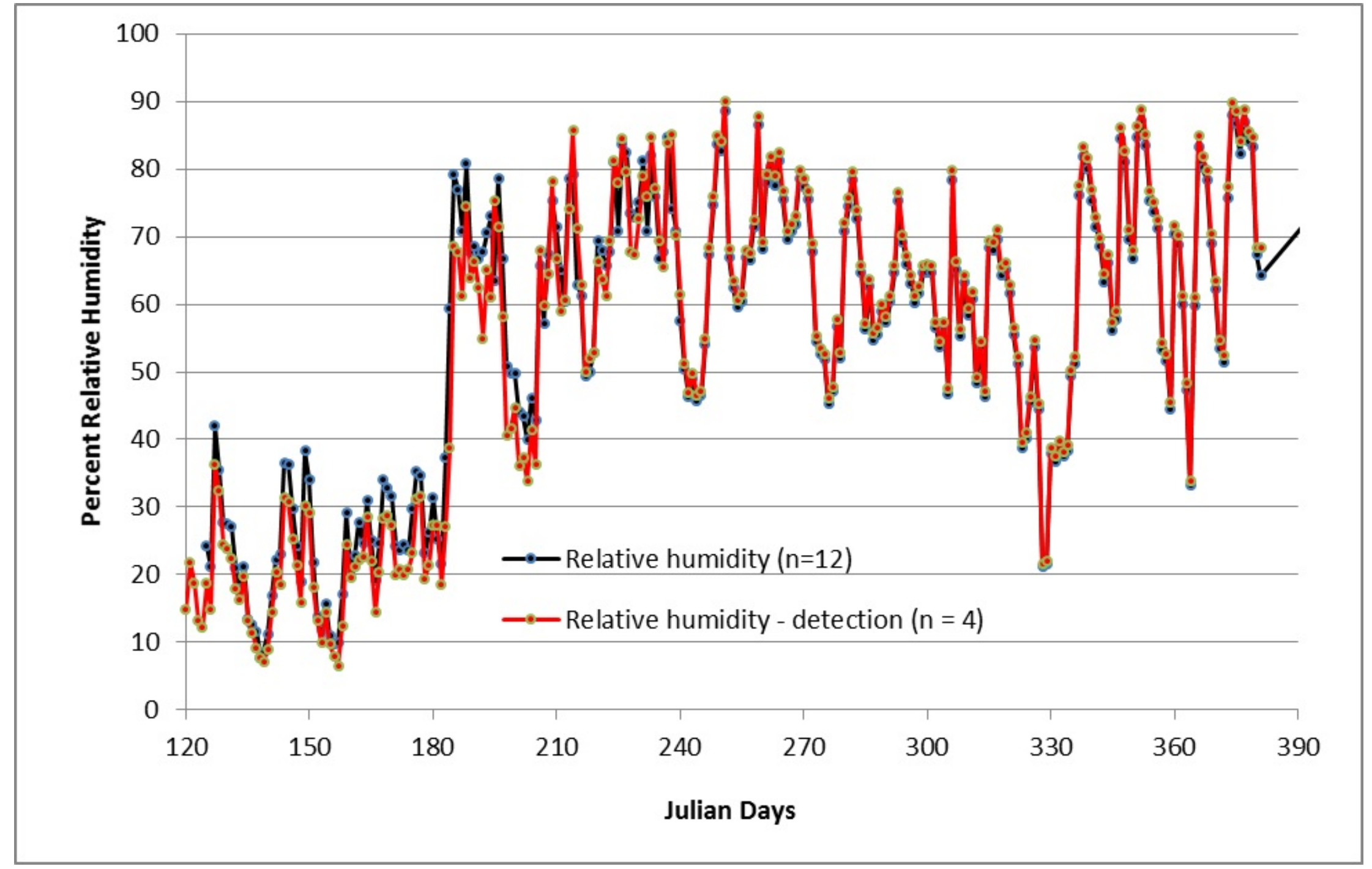

Figure 121c. For the Santa Rita Mountains, a comparison of daily average relative humidity (in percent) for ocelot detection ( $\mathrm{n}=4$, red line) and ocelot non-detection ( $n=12$, black line) locations in Julian days (days 120-150 correspond to May, 330-360 to December). 


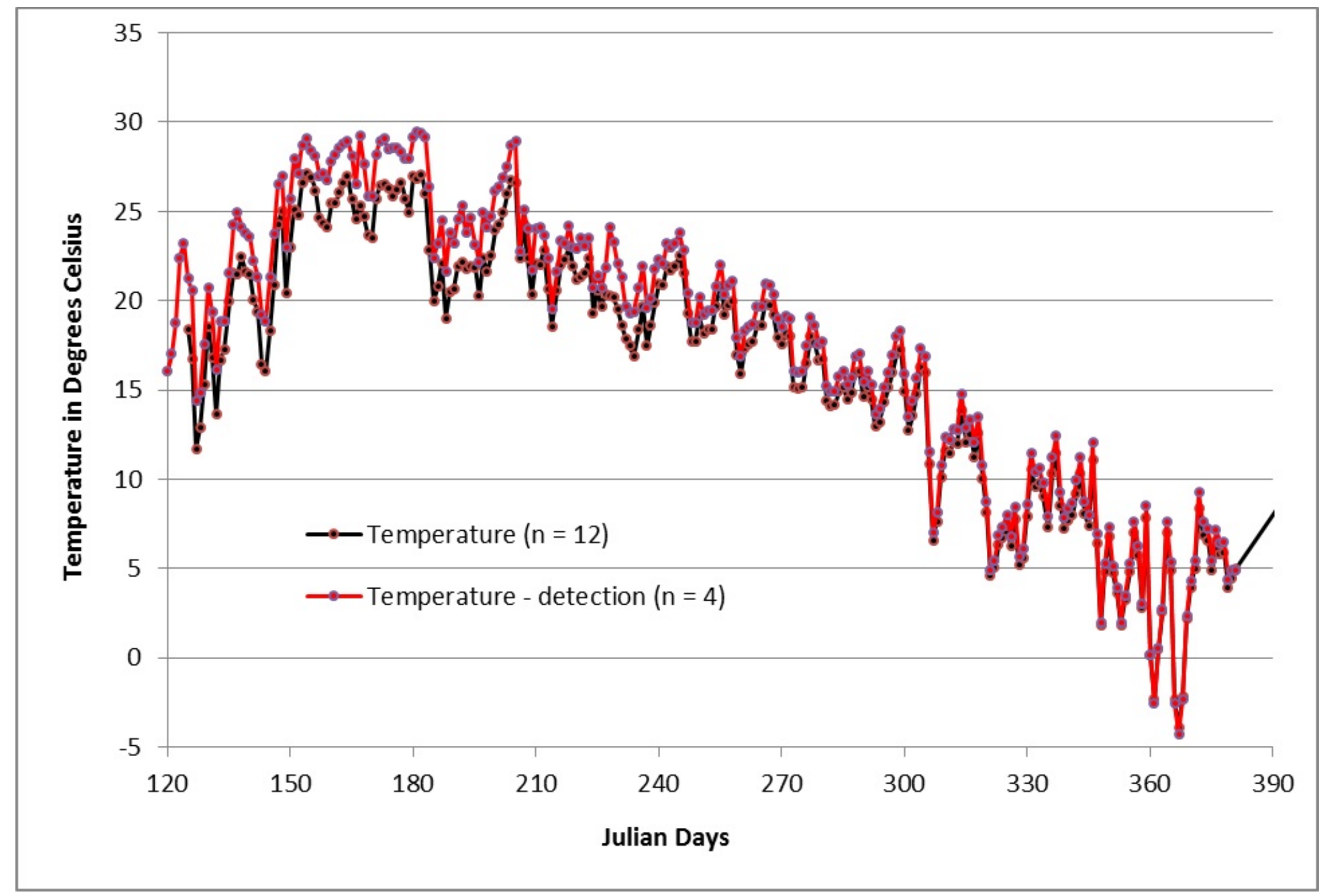

Figure 121d. For the Santa Rita Mountains, a comparison of daily average temperature (in degrees Celsius) for ocelot detection ( $n=4$, red line) and ocelot non-detection ( $n=12$, black line) locations in Julian days (days 120-150 correspond to May, 330-360 to December). 


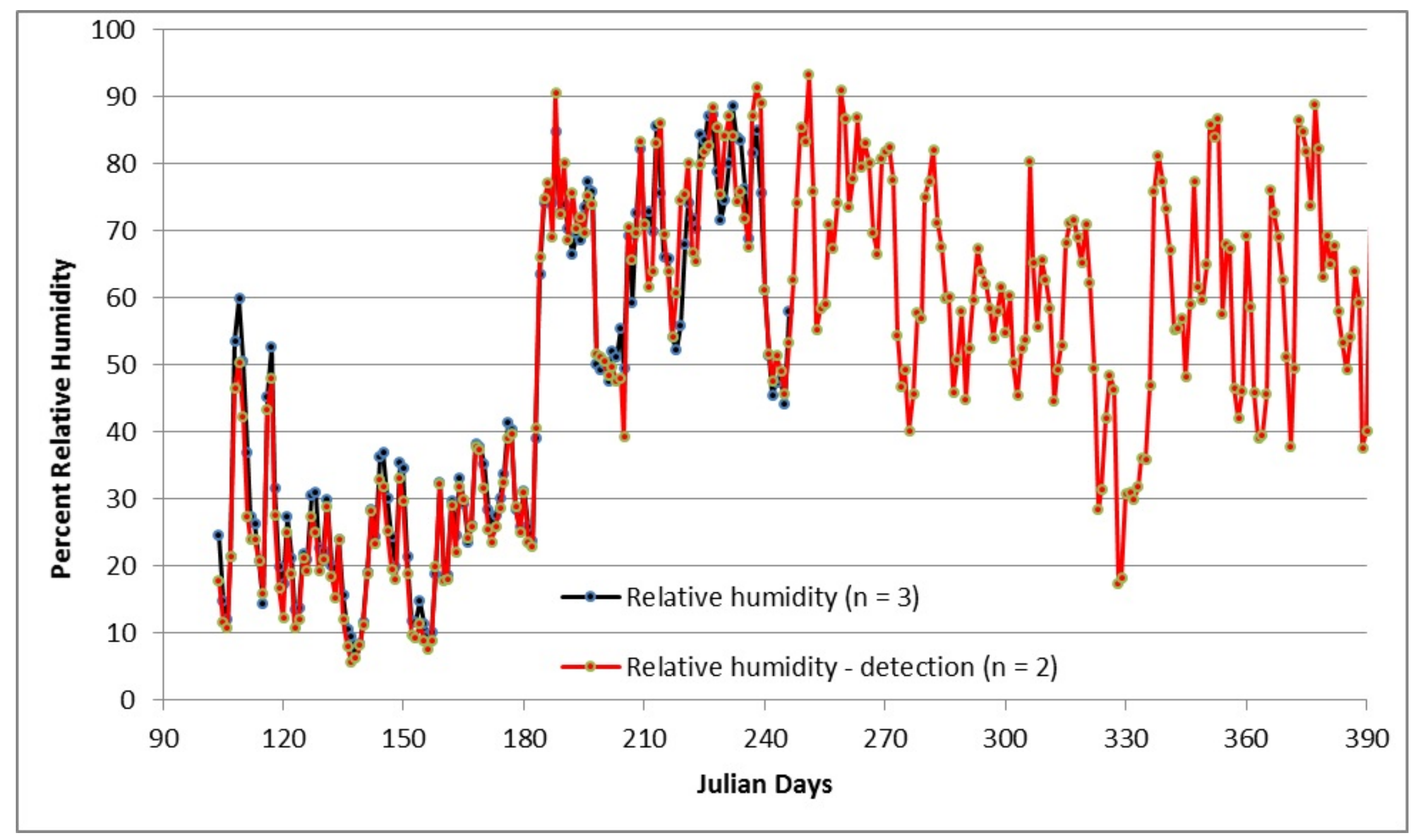

Figure 122a. For the Huachuca Mountains, a comparison of daily average relative humidity (in percent) for ocelot detection ( $\mathrm{n}=2$, red line) and ocelot non-detection ( $n=3$, black line) locations in shown versus Julian days (days 90-120 correspond to April, 330-360 to December). 


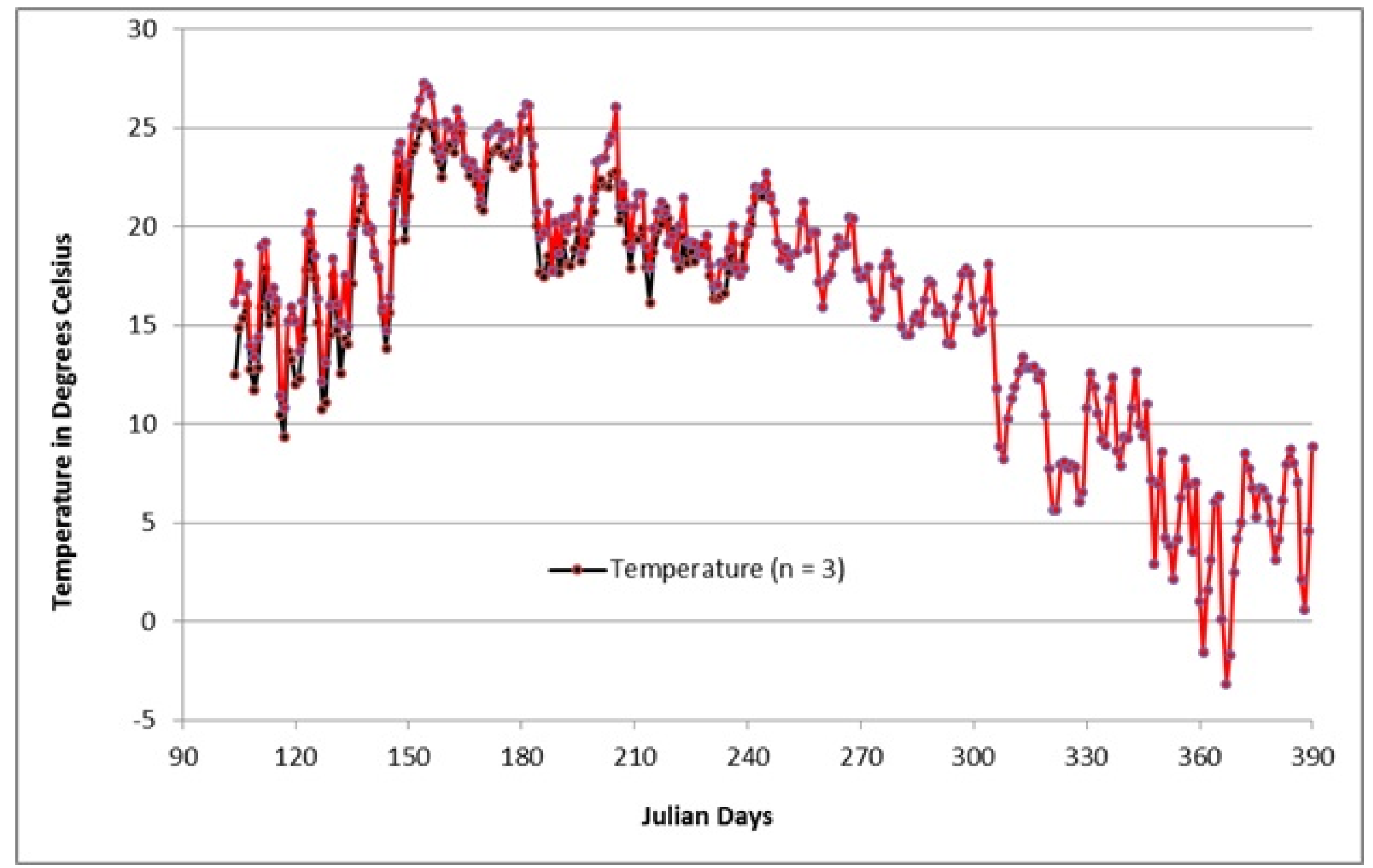

Figure 122b. For the Huachuca Mountains, a comparison of daily average temperature (in degrees Celsius) for ocelot detection ( $n=2$, red line) and ocelot non-detection ( $n=3$, black line) locations in Julian days (days 90-120 correspond to April, 330-360 to December). 


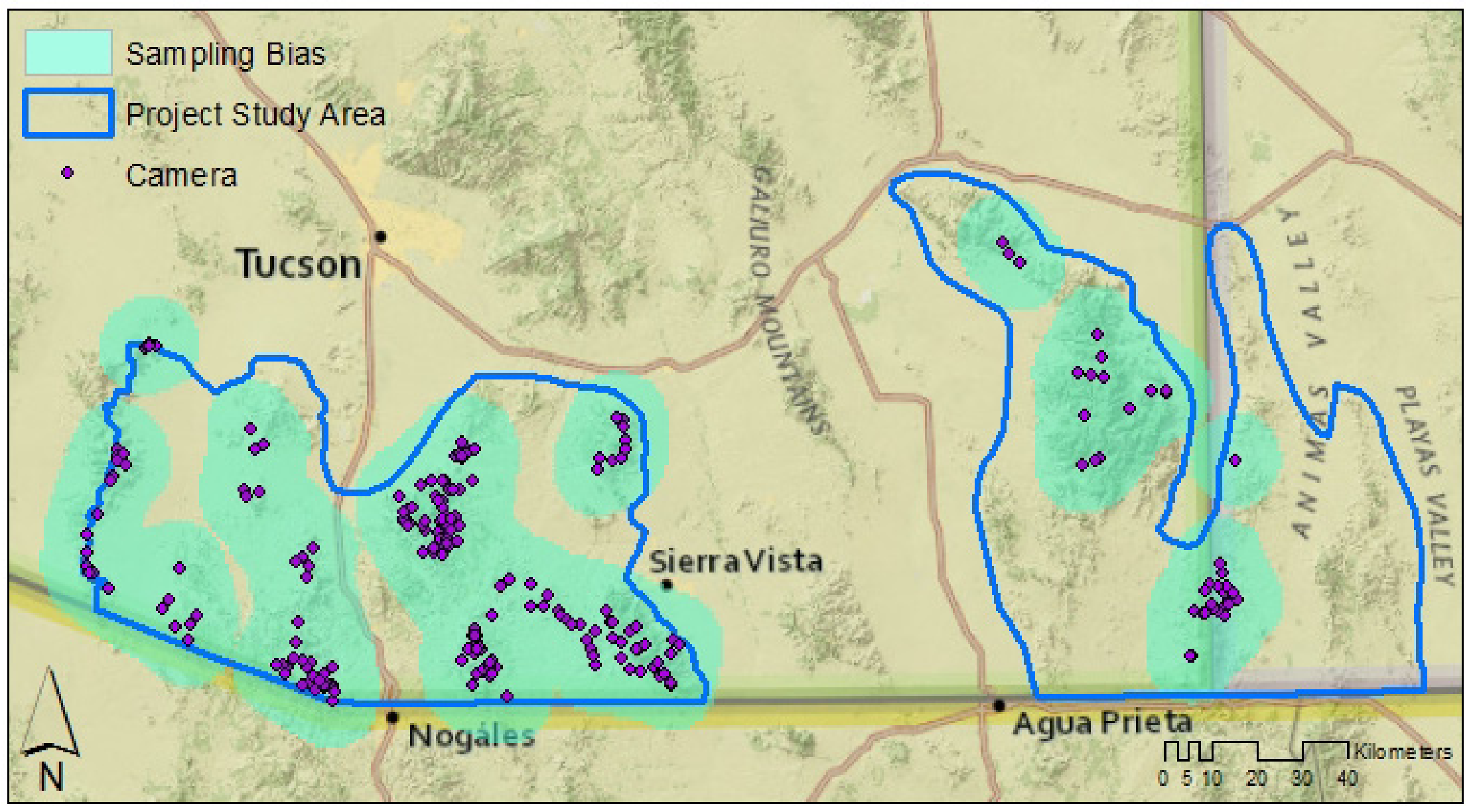

Figure 123. Map showing camera survey sites buffered by a local adaptive convex-hull polygon (buffer distance=10 km, alpha=2) used as a bias file for background selection in MaxEnt. 
bio 1

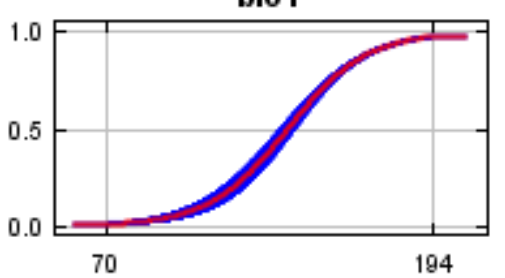

nckiavhrr

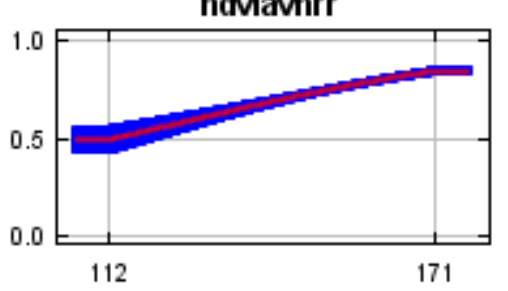

bio12

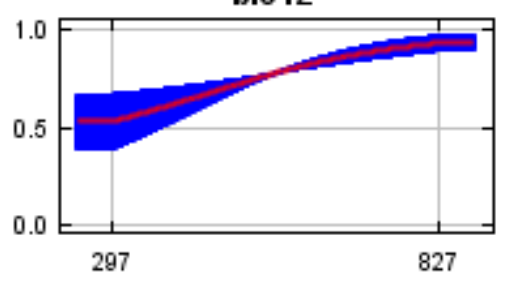

treecover

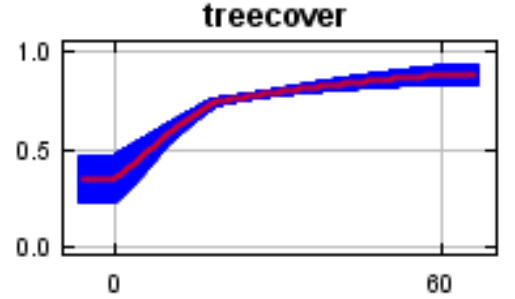

hii
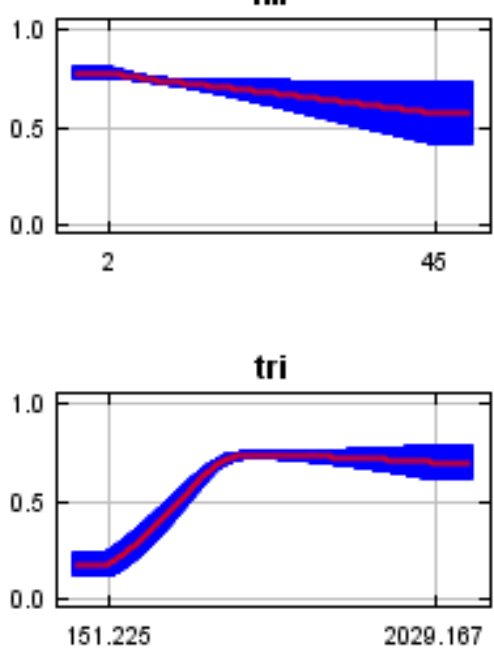

novi

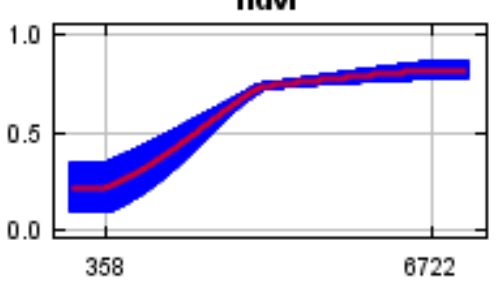

Figure 124. Response curves for each variable in the final model showing showing changes to the logistic prediction in response to changes in each variable independently (red line, mean; blue line, \pm 1 standard deviation). Variable definitions are bio1, annual mean temperature in degrees centigrade ${ }^{\circ} \mathrm{C}$; bio12, annual precipitation in millimeters $(\mathrm{mm})$; hii, human influence index (gradient from low to high); ndvi, normalized difference vegetation index for March (MODIS, rescaled as ndvi times 10,000); ndviavhrr, normalized dfference vegetation index for August 2014 (AVHRR, rescaled as (ndvi times 100) plus 100); tree cover, percent tree cover; tri, terrain ruggedness index (gradient from level to extremely rugged). 


\section{West Study Area}
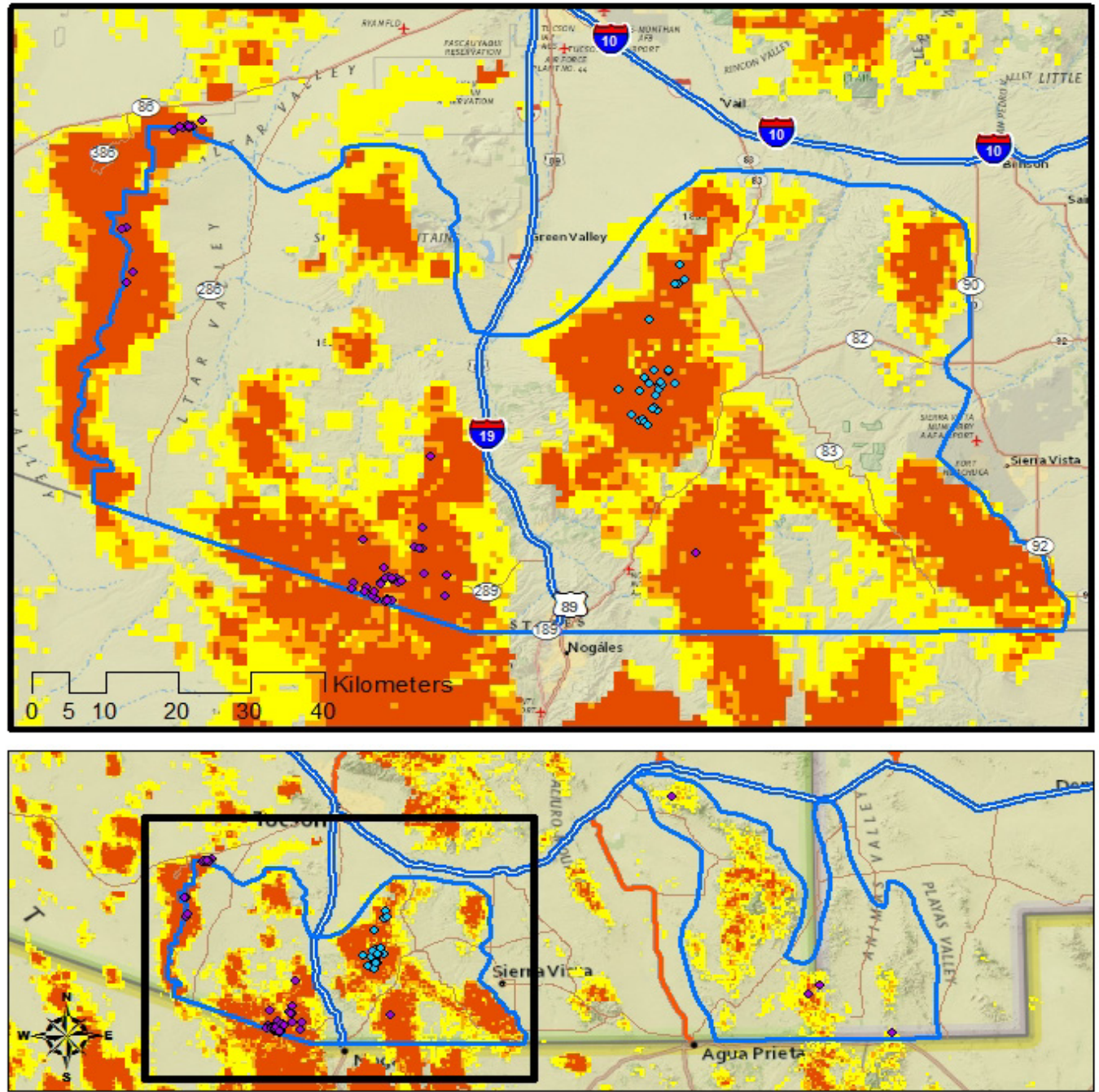

\section{Jaguar Habitat Suitability}

Tier $1-10 \%$ omission threshold

Tier $2-5 \%$ omission threshold

Tier $3-0 \%$ omission threshold

\section{Jaguar Locations}

- Current (2012-2015)

- Historical (1965-2009)

Project Study Area

Figure 125. Potential distribution map for jaguars in southeastern Arizona and southwestern New Mexico focused on the west project study area. Tier 1 includes $90 \%$ of all current and historical jaguar locations; tiers 1 and 2 include 95\% of all locations; and tiers 1, 2, and 3 include 100\% of all locations. 


\section{East Study Area}
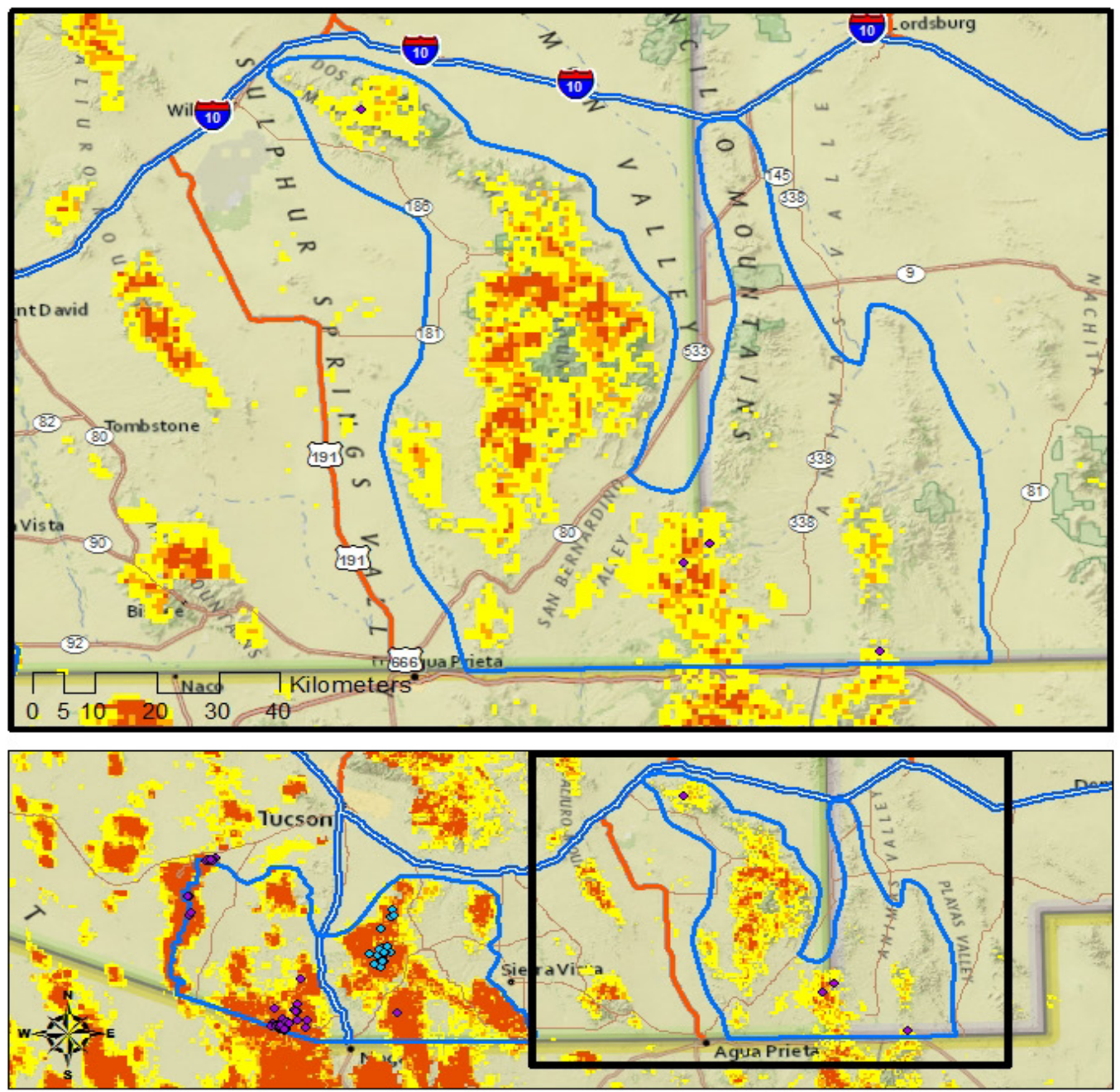

\section{Jaguar Habitat Suitability}

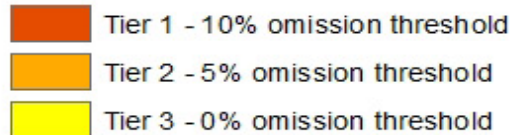

Jaguar Locations

- Current (2012-2015)

- Historical (1965-2009)

Project Study Area

Figure 126. Potential distribution map for jaguars in southeastern Arizona and southwestern New Mexico focused on the east project study area. Tier 1 includes $90 \%$ of all current and historical jaguar locations; tiers 1 and 2 include 95\% of all locations; and tiers 1, 2, and 3 include 100\% of all locations. 

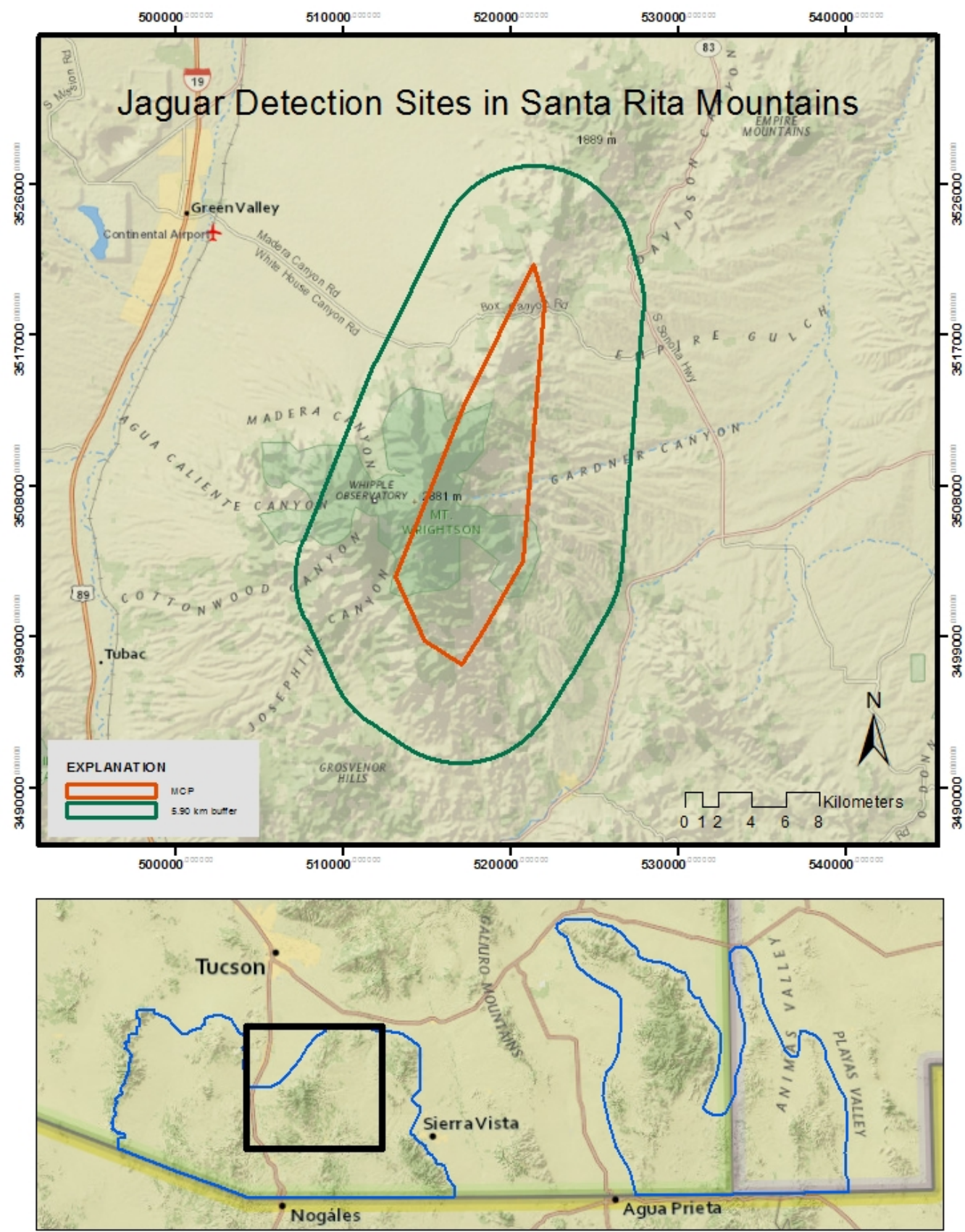

Figure 127. Map showing minimum Convex Polygon (MCP) surrounding jaguar detection sites with 24hour Mean Maximum Distance Moved (MMDM) buffer (indicated by the green $5.90 \mathrm{~km}$ buffer). Total area within the 24-hour MMDM buffer (including MCP) is $90 \mathrm{~km}^{2}$. Note that this calculation should be used with caution, as this study was not designed to determine home range size. Map image from National Geographic Society used under Esri Master License Agreement \#102282. 


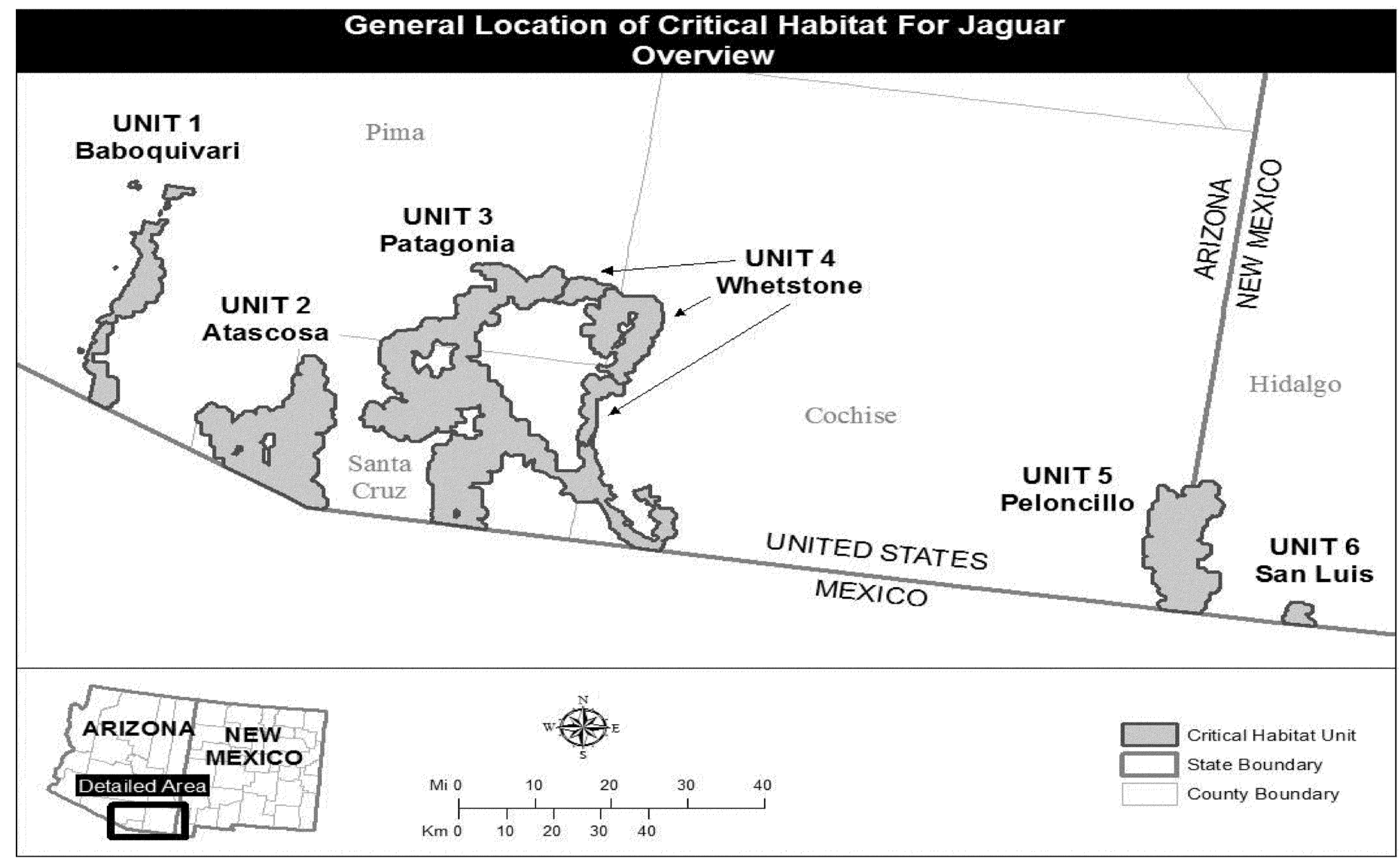

Figure 128. Map showing designated jaguar critical habitat in southeastern Arizona and southwestern New Mexico (U.S. Fish and Wildlife Service, 2014). 
All Jaguars, AZ-NM

80 Jaguar Occurances Representing 70 Individual Animals 31 Identified as to Gender-24 Males(77\%)-7 Females(23\%)-3 Kittens)

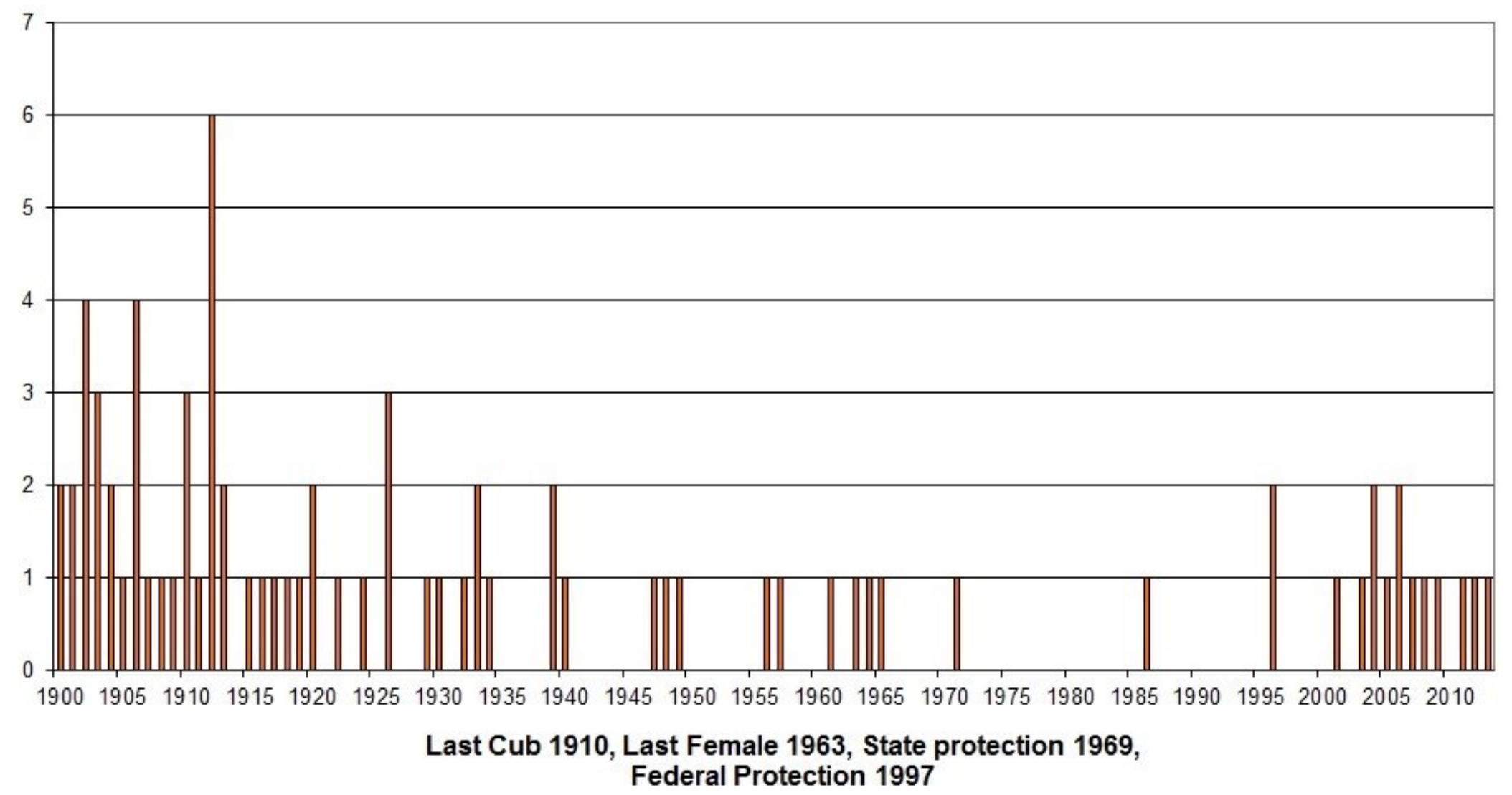

Figure 129. Jaguar occurrence data from 1900 to present. 


\section{Tables}

Table 1. Permits and user agreements obtained to conduct the jaguar monitoring project, including agency, permit number, and expiration date.

\begin{tabular}{|lll|}
\hline \multicolumn{1}{|c|}{ Agency } & \multicolumn{1}{c|}{ Permit No. } & \multicolumn{1}{c|}{ Expiration date } \\
\hline Arizona State Land Department & Permit No. 23116637-29 & 9/19/2017 \\
\hline $\begin{array}{l}\text { Arizona State Land Right of Way (ROW) } \\
\text { Permit }\end{array}$ & $30-116615-00-000$ & $\begin{array}{l}\text { Renewed 12/2014; pending } \\
\text { hardcopy with expiration date }\end{array}$ \\
\hline New Mexico State Land Department & Memorandum of Understanding & Duration of project \\
\hline $\begin{array}{l}\text { U.S. Forest Service - Coronado National } \\
\text { Forest-SO-Sierra Vista Road; Nogales Road; } \\
\text { Douglas Road }\end{array}$ & $\begin{array}{l}\text { Authorization Identification No. } \\
\text { SUP0118 }\end{array}$ & $12 / 31 / 2015$ \\
\hline Bureau of Land Management-Gila District & Re: 4180 (AZ421) & Duration of project \\
\hline $\begin{array}{l}\text { National Park Service-Coronado, National } \\
\text { Monument; Chiricahua, National Monument; } \\
\text { Fort Bowie, National Historic Site }\end{array}$ & CORO-2013-SCI-0002 CHIR- & 2013-SCI-0004 FOBO-2013- \\
\hline Buenos Aires National Wildlife Refuge & SCI-0002 & No. 2013-011 Station 22530 \\
\hline $\begin{array}{l}\text { Arizona Game and Fish Department } \\
\text { (scat dog) }\end{array}$ & $\begin{array}{l}\text { Scientific Collections Permit\# } \\
\text { SP799917 CLS }\end{array}$ & $\begin{array}{l}\text { Renewed annually/due } \\
1 / 30 / 2016\end{array}$ \\
\hline Fort Huachuca Department of Defense & IMHU-PWB (200-1) & Renewed annually/due 12/31/15 \\
\hline U.S. Border Patrol & Coordinate biweekly & Duration of project \\
\hline Santa Rita Research Station & Permit No. 12-107 & $12 / 31 / 2015$ \\
\hline U.S. Fish and Wildlife Service & Permit No. TE58781A-1 & 5/31/2020 \\
\hline Landowner Agreements & 20 confidential agreements & Duration of project \\
\hline
\end{tabular}


Table 2. Digital data used in geographic information system (GIS) macrohabitat analyses at sites with positive jaguar and ocelot detections.

[km, kilometer; m, meter]

\begin{tabular}{|c|c|c|}
\hline Variable & Author & Source \\
\hline $\begin{array}{l}\text { Elevation-National Elevation Dataset } \\
\text { (NED, } 30 \mathrm{~m} \text { ) }\end{array}$ & U.S. Geological Survey & http://ned.usgs.gov/ \\
\hline $\begin{array}{l}\text { Elevation-Global Land One-km } \\
\text { Based Elevation } \\
\text { (GLOBE, } 1 \mathrm{~km})\end{array}$ & $\begin{array}{l}\text { National Oceanic and Atmospheric } \\
\text { Administration }\end{array}$ & $\begin{array}{l}\text { http://www.ngdc.noaa.gov/mgg/topo/glti } \\
\text { les.html }\end{array}$ \\
\hline Terrain Ruggedness Index (TRI) & $\begin{array}{l}\text { Calculated according to Riley and } \\
\text { others (1999) }\end{array}$ & $\begin{array}{l}\text { TRI calculated using GLOBE elevation } \\
\text { data }\end{array}$ \\
\hline $\begin{array}{l}\text { Global Human Influence Index } \\
\text { (geographic), version } 2\end{array}$ & $\begin{array}{l}\text { Wildlife Conservation Society and } \\
\text { Center for International Earth } \\
\text { Science Information Network, } \\
\text { Columbia University }\end{array}$ & $\begin{array}{l}\text { http://sedac.ciesin.columbia.edu/data/set/ } \\
\text { wildareas-v2-human-influence-index- } \\
\text { geographic }\end{array}$ \\
\hline $\begin{array}{l}\text { Normalized Difference } \\
\text { Vegetation Index (NDVI) for March } \\
2015\end{array}$ & $\begin{array}{l}\text { U.S. Geological Survey-Earth } \\
\text { Resources Observation and Science } \\
\text { Center (EROS) Moderate Resolution } \\
\text { Imaging Spectroradiometer } \\
\text { (eMODIS) }\end{array}$ & https://lta.cr.usgs.gov/emodis \\
\hline $\begin{array}{l}\text { Normalized Difference } \\
\text { Vegetation Index (NDVI) for August } \\
2014\end{array}$ & $\begin{array}{l}\text { U.S. Geological Survey-Advanced } \\
\text { Very High Resolution Radiometer } \\
\text { (AVHRR) }\end{array}$ & https://lta.cr.usgs.gov/NDVI \\
\hline $\begin{array}{l}\text { Tree cover-- Vegetation Continuous } \\
\text { Fields Yearly L3 Global } 250 \mathrm{~m} \\
\text { (MOD44B) }\end{array}$ & U.S. Geological Survey & $\begin{array}{l}\text { https://pdaac.usgs.gov/products/modis_ } \\
\text { products_table/mod44b }\end{array}$ \\
\hline Vegetation Community & $\begin{array}{l}\text { Nature Conservancy based on Brown } \\
\text { and Lowe's "Biotic Communities of } \\
\text { the Southwest" map-1980 }\end{array}$ & $\begin{array}{l}\text { http://azconservation.org/downloads/biot } \\
\text { ic_communities_of_the_southwest_gis_ } \\
\text { data }\end{array}$ \\
\hline Water & $\begin{array}{l}\text { U.S. Geological Survey-National } \\
\text { Hydrography Dataset (Arizona and } \\
\text { New Mexico) }\end{array}$ & $\begin{array}{l}\text { ftp://nhdftp.usgs.gov/DataSets/Staged/St } \\
\text { ates/FileGDB/HighResolution/ }\end{array}$ \\
\hline U.S. Major Highways & $\begin{array}{l}\text { Environmental Systems Research } \\
\text { Institute (ESRI) }\end{array}$ & $\begin{array}{l}\text { http://www.arcgis.com/home/item.html?i } \\
\text { d=fc870766a3994111bce4a083413988e4 }\end{array}$ \\
\hline
\end{tabular}


Table 3. Established camera survey sites for jaguar monitoring project from April 25, 2012 to February 15, 2015, including mountain range, date deployed, global positioning system (GPS) coordinates in 1983 North America Datum, Universal Transverse Marcator (NAD83, UTM), elevation, UTM zone, and total sites per mountain range.

[All 250 camera sites are included, regardless of whether or not they were eventually moved. At the time of this report, 57 sites continue to be monitored in mountain ranges with positive jaguar or ocelot detections]

\begin{tabular}{|c|c|c|c|c|c|c|}
\hline Mountain range & Date deployed & $\begin{array}{l}\text { UTM E } \\
\text { (rounded) }\end{array}$ & $\begin{array}{l}\text { UTM N } \\
\text { (rounded) }\end{array}$ & $\begin{array}{c}\text { Elevation } \\
\text { (meters) }\end{array}$ & $\begin{array}{l}\text { UTM } \\
\text { zone }\end{array}$ & $\begin{array}{l}\text { Total } \\
\text { sites/ } \\
\text { range }\end{array}$ \\
\hline Atascosa & $10 / 1 / 2012$ & 488000 & 3475000 & 1,310 & $12 \mathrm{R}$ & \\
\hline Atascosa & $10 / 1 / 2012$ & 487000 & 3474000 & 1,350 & $12 \mathrm{R}$ & \\
\hline Atascosa & 10/1/2012 & 486000 & 3475000 & 1,450 & $12 \mathrm{R}$ & \\
\hline Atascosa & $10 / 5 / 2012$ & 485000 & 3479000 & 1,370 & $12 \mathrm{R}$ & \\
\hline Atascosa & 10/5/2012 & 485000 & 3483000 & 1,210 & $12 \mathrm{R}$ & \\
\hline Atascosa Mountain range & & & & & & 5 \\
\hline Baboquivari & $5 / 7 / 2013$ & 445000 & 3514000 & 1,476 & $12 \mathrm{R}$ & \\
\hline Baboquivari & $5 / 7 / 2013$ & 444000 & 3513000 & 1,377 & $12 \mathrm{R}$ & \\
\hline Baboquivari & $5 / 16 / 2013$ & 446000 & 3520000 & 1,314 & $12 \mathrm{R}$ & \\
\hline Baboquivari & $5 / 16 / 2013$ & 446000 & 3518000 & 1,447 & $12 \mathrm{R}$ & \\
\hline Baboquivari & $5 / 23 / 2013$ & 446000 & 3517000 & 1,560 & $12 \mathrm{R}$ & \\
\hline Baboquivari & 8/29/2013 & 446000 & 3517000 & 1,560 & $12 \mathrm{R}$ & \\
\hline Baboquivari & $5 / 23 / 2013$ & 448000 & 3516000 & 1,440 & $12 \mathrm{R}$ & \\
\hline Baboquivari & $5 / 19 / 2013$ & 441000 & 3506000 & 1,353 & $12 \mathrm{R}$ & \\
\hline Baboquivari & $5 / 19 / 2013$ & 439000 & 3502000 & 1,292 & $12 \mathrm{R}$ & \\
\hline Baboquivari & $5 / 19 / 2013$ & 439000 & 3498000 & 1,258 & $12 \mathrm{R}$ & \\
\hline Baboquivari & $5 / 20 / 2013$ & 444000 & 3490000 & 1,237 & $12 \mathrm{R}$ & \\
\hline Baboquivari & $5 / 20 / 2013$ & 439000 & 3495000 & 1,238 & $12 \mathrm{R}$ & \\
\hline Baboquivari & $5 / 20 / 2013$ & 441000 & 3494000 & 1,237 & $12 \mathrm{R}$ & \\
\hline Baboquivari & 6/13/2013 & 446000 & 3520000 & 1,314 & $12 \mathrm{R}$ & \\
\hline Baboquivari & 8/19/2013 & 446000 & 3518000 & 1,501 & $12 \mathrm{R}$ & \\
\hline Baboquivari & 8/29/2013 & 446000 & 3517000 & 1,560 & $12 \mathrm{R}$ & \\
\hline Baboquivari & $5 / 13 / 2014$ & 440000 & 3494000 & 1,260 & $12 \mathrm{R}$ & \\
\hline Baboquivari & $7 / 14 / 2014$ & 447000 & 3519000 & 1,340 & $12 \mathrm{R}$ & \\
\hline Baboquivari Mountain range & & & & & & 18 \\
\hline Canelo Hills & $10 / 25 / 2012$ & 550000 & 3474000 & 1,680 & $12 \mathrm{R}$ & \\
\hline Canelo Hills & $11 / 1 / 2012$ & 547000 & 3483000 & 1,620 & $12 \mathrm{R}$ & \\
\hline Canelo Hills & $3 / 4 / 2013$ & 539000 & 3487000 & 1,600 & $12 \mathrm{R}$ & \\
\hline Canelo Hills & 3/6/2013 & 536000 & 3491000 & 1,545 & $12 \mathrm{R}$ & \\
\hline Canelo Hills & $3 / 6 / 2013$ & 540000 & 3489000 & 1,680 & $12 \mathrm{R}$ & \\
\hline Canelo Hills & $6 / 11 / 2014$ & 530000 & 3491000 & 1,270 & $12 \mathrm{R}$ & \\
\hline Canelo Hills & 6/11/2014 & 532000 & 3492000 & 1,380 & $12 \mathrm{R}$ & \\
\hline Canelo Hills & 5/31/2013 & 536000 & 3487000 & 1,480 & $12 \mathrm{R}$ & \\
\hline Canelo Hills & 9/20/2013 & 542000 & 3486000 & 1,660 & $12 \mathrm{R}$ & \\
\hline Canelo Hills & 9/20/2013 & 543000 & 3485000 & 1,790 & $12 \mathrm{R}$ & \\
\hline Canelo Hills & 9/20/2013 & 544000 & 3484000 & 1,630 & $12 \mathrm{R}$ & \\
\hline
\end{tabular}




\begin{tabular}{|c|c|c|c|c|c|c|}
\hline Mountain range & Date deployed & $\begin{array}{c}\text { UTM E } \\
\text { (rounded) }\end{array}$ & $\begin{array}{c}\text { UTM N } \\
\text { (rounded) }\end{array}$ & $\begin{array}{c}\text { Elevation } \\
\text { (meters) }\end{array}$ & $\begin{array}{l}\text { UTM } \\
\text { zone }\end{array}$ & $\begin{array}{l}\text { Total } \\
\text { sites/ } \\
\text { range }\end{array}$ \\
\hline Canelo Hills & 9/30/2013 & 545000 & 3483000 & 1,670 & $12 \mathrm{R}$ & \\
\hline Canelo Hills & $1 / 16 / 2014$ & 548000 & 3479000 & 1,700 & $12 \mathrm{R}$ & \\
\hline Canelo Hills & $1 / 16 / 2014$ & 550000 & 3478000 & 1,630 & $12 \mathrm{R}$ & \\
\hline Canelo Hills & $1 / 16 / 2014$ & 550000 & 3476000 & 1,590 & $12 \mathrm{R}$ & \\
\hline Canelo Hills range & & & & & & 15 \\
\hline Cerro Colorado & $5 / 13 / 2013$ & 477000 & 3511000 & 1,205 & $12 \mathrm{R}$ & \\
\hline Cerro Colorado & 5/6/2013 & 474000 & 3511000 & 1,140 & $12 \mathrm{R}$ & \\
\hline Cerro Colorado & $5 / 13 / 2013$ & 474000 & 3510000 & 1,190 & $12 \mathrm{R}$ & \\
\hline Cerro Colorado Mountain range & & & & & & 3 \\
\hline Chiricahua & $5 / 23 / 2013$ & 660000 & 3544000 & 1,740 & $12 \mathrm{R}$ & \\
\hline Chiricahua & $5 / 23 / 2013$ & 659000 & 3535000 & 1,910 & $12 \mathrm{R}$ & \\
\hline Chiricahua & $5 / 23 / 2013$ & 667000 & 3528000 & 1,750 & $12 \mathrm{R}$ & \\
\hline Chiricahua & $5 / 23 / 2013$ & 658000 & 3527000 & 1,927 & $12 \mathrm{R}$ & \\
\hline Chiricahua & $5 / 23 / 2013$ & 661000 & 3518000 & 1,880 & $12 \mathrm{R}$ & \\
\hline Chiricahua & $5 / 23 / 2013$ & 657000 & 3516000 & 1,790 & $12 \mathrm{R}$ & \\
\hline Chiricahua & $5 / 23 / 2013$ & 660000 & 3517000 & 1,840 & $12 \mathrm{R}$ & \\
\hline Chiricahua & $5 / 23 / 2013$ & 675000 & 3532000 & 1,545 & $12 \mathrm{R}$ & \\
\hline Chiricahua & $5 / 23 / 2013$ & 675000 & 3532000 & 1,520 & $12 \mathrm{R}$ & \\
\hline Chiricahua & $5 / 23 / 2013$ & 661000 & 3539000 & 1,996 & $12 \mathrm{R}$ & \\
\hline Chiricahua & $5 / 23 / 2013$ & 657000 & 3527000 & 1,854 & $12 \mathrm{R}$ & \\
\hline Chiricahua & $5 / 23 / 2013$ & 656000 & 3536000 & 1,775 & $12 \mathrm{R}$ & \\
\hline Chiricahua & $5 / 24 / 2013$ & 662000 & 3535000 & 1,996 & $12 \mathrm{R}$ & \\
\hline Chiricahua & $10 / 4 / 2013$ & 672000 & 3532000 & 1,825 & $12 \mathrm{R}$ & \\
\hline Chiricahua & 10/6/2013 & 644000 & 3559000 & 1,527 & $12 \mathrm{R}$ & \\
\hline Chiricahua Mountain range & & & & & & 15 \\
\hline Coyote & $5 / 2 / 2013$ & 454000 & 3542000 & 935 & $12 \mathrm{R}$ & \\
\hline Coyote & $5 / 2 / 2013$ & 453000 & 3542000 & 1,058 & $12 \mathrm{R}$ & \\
\hline Coyote & $5 / 2 / 2013$ & 452000 & 3541000 & 1,470 & $12 \mathrm{R}$ & \\
\hline Coyote & 9/23/2013 & 452000 & 3541000 & 1,165 & $12 \mathrm{R}$ & \\
\hline Coyote & 3/19/2014 & 453000 & 3542000 & 1,105 & $12 \mathrm{R}$ & \\
\hline Coyote Mountain range & & & & & & 5 \\
\hline Dos Cabezas & $5 / 24 / 2013$ & 640000 & 3563000 & 1,838 & $12 \mathrm{R}$ & \\
\hline Dos Cabezas & $5 / 24 / 2013$ & 641000 & 3561000 & 1,684 & $12 \mathrm{R}$ & \\
\hline Dos Cabezas & $3 / 2 / 2014$ & 644000 & 3559000 & 1,527 & $12 \mathrm{R}$ & \\
\hline Dos Cabezas Mountain range & & & & & & 3 \\
\hline Huachuca & $8 / 8 / 2012$ & 553000 & 3486000 & 1,690 & $12 \mathrm{R}$ & \\
\hline Huachuca & 8/8/2012 & 554000 & 3480000 & 1,730 & $12 \mathrm{R}$ & \\
\hline Huachuca & $1 / 5 / 2013$ & 551000 & 3482000 & 1,680 & $12 \mathrm{R}$ & \\
\hline Huachuca & 7/31/2012 & 567000 & 3470000 & 1,910 & $12 \mathrm{R}$ & \\
\hline Huachuca & 7/31/2012 & 563000 & 3474000 & 1,980 & $12 \mathrm{R}$ & \\
\hline Huachuca & 7/31/2012 & 558000 & 3481000 & 1,930 & $12 \mathrm{R}$ & \\
\hline Huachuca & 8/2/2012 & 558000 & 3474000 & 1,690 & $12 \mathrm{R}$ & \\
\hline
\end{tabular}




\begin{tabular}{|c|c|c|c|c|c|c|}
\hline Mountain range & Date deployed & $\begin{array}{l}\text { UTM E } \\
\text { (rounded) }\end{array}$ & $\begin{array}{c}\text { UTM N } \\
\text { (rounded) }\end{array}$ & $\begin{array}{c}\text { Elevation } \\
\text { (meters) }\end{array}$ & $\begin{array}{l}\text { UTM } \\
\text { zone }\end{array}$ & $\begin{array}{l}\text { Total } \\
\text { sites/ } \\
\text { range }\end{array}$ \\
\hline Huachuca & $9 / 20 / 2012$ & 564000 & 3472000 & 1,920 & $12 \mathrm{R}$ & \\
\hline Huachuca & 9/21/2012 & 556000 & 3476000 & 1,740 & $12 \mathrm{R}$ & \\
\hline Huachuca & 9/21/2012 & 556000 & 3479000 & 1,780 & $12 \mathrm{R}$ & \\
\hline Huachuca & $2 / 14 / 2014$ & 560000 & 3478000 & 2,020 & $12 \mathrm{R}$ & \\
\hline Huachuca & 8/8/2012 & 554000 & 3483000 & 1,750 & $12 \mathrm{R}$ & \\
\hline Huachuca & 1/8/2013 & 568000 & 3480000 & 1,605 & $12 \mathrm{R}$ & \\
\hline Huachuca & 1/8/2013 & 569000 & 3479000 & 1,750 & $12 \mathrm{R}$ & \\
\hline Fort Huachuca & 4/8/2013 & 561000 & 3478000 & 2,160 & $12 \mathrm{R}$ & \\
\hline Fort Huachuca & 4/8/2013 & 560000 & 3482000 & 1,800 & $12 \mathrm{R}$ & \\
\hline Huachuca & $5 / 20 / 2013$ & 567000 & 3477000 & 2,290 & $12 \mathrm{R}$ & \\
\hline Huachuca & $11 / 6 / 2013$ & 554000 & 3483000 & 1,790 & $12 \mathrm{R}$ & \\
\hline Huachuca & $1 / 21 / 2014$ & 560000 & 3478000 & 1,997 & $12 \mathrm{R}$ & \\
\hline Huachuca & $3 / 14 / 2014$ & 560000 & 3473000 & 1,695 & $12 \mathrm{R}$ & \\
\hline Huachuca & $3 / 14 / 2014$ & 564000 & 3475000 & 2,320 & $12 \mathrm{R}$ & \\
\hline Huachuca & 4/9/2014 & 566000 & 3472000 & 2,108 & $12 \mathrm{R}$ & \\
\hline Huachuca & 4/9/2014 & 567000 & 3471000 & 2,000 & $12 \mathrm{R}$ & \\
\hline Huachuca Mountain range & & & & & & 23 \\
\hline Pajarito & $10 / 1 / 2012$ & 483000 & 3474000 & 1,210 & $12 \mathrm{R}$ & \\
\hline Pajarito & $10 / 6 / 2012$ & 484000 & 3476000 & 1,300 & $12 \mathrm{R}$ & \\
\hline Pajarito & $10 / 31 / 2012$ & 482000 & 3474000 & 1,165 & $12 \mathrm{R}$ & \\
\hline Pajarito & $4 / 22 / 2014$ & 482000 & 3474000 & 1,306 & $12 \mathrm{R}$ & \\
\hline Pajarito & $10 / 31 / 2012$ & 481000 & 3474000 & 1,145 & $12 \mathrm{R}$ & \\
\hline Pajarito & 10/30/2012 & 483000 & 3472000 & 1,290 & $12 \mathrm{R}$ & \\
\hline Pajarito & $10 / 6 / 2012$ & 490000 & 3471000 & 1,255 & $12 \mathrm{R}$ & \\
\hline Pajarito & $10 / 6 / 2012$ & 488000 & 3471000 & 1,300 & $12 \mathrm{R}$ & \\
\hline Pajarito & $10 / 30 / 2012$ & 493000 & 3470000 & 1,300 & $12 \mathrm{R}$ & \\
\hline Pajarito & $12 / 28 / 2013$ & 493000 & 3470000 & 1,300 & $12 \mathrm{R}$ & \\
\hline Pajarito $^{1}$ & 10/31/2012 & 491000 & 3470000 & 1,270 & $12 \mathrm{R}$ & \\
\hline Pajarito & $12 / 28 / 2013$ & 491000 & 3470000 & 1,300 & $12 \mathrm{R}$ & \\
\hline Pajarito & $12 / 17 / 2012$ & 489000 & 3469000 & 1,325 & $12 \mathrm{R}$ & \\
\hline Pajarito $^{1}$ & $12 / 17 / 2012$ & 491000 & 3469000 & 1,270 & $12 \mathrm{R}$ & \\
\hline Pajarito & $12 / 18 / 2012$ & 489000 & 3472000 & 1,220 & $12 \mathrm{R}$ & \\
\hline Pajarito & 8/22/2013 & 491000 & 3473000 & 1,190 & $12 \mathrm{R}$ & \\
\hline Pajarito & 2/7/2013 & 493000 & 3467000 & 1,520 & $12 \mathrm{R}$ & \\
\hline Pajarito & 2/7/2013 & 493000 & 3469000 & 1,350 & $12 \mathrm{R}$ & \\
\hline Pajarito & $12 / 28 / 2013$ & 486000 & 3470000 & 1,385 & $12 \mathrm{R}$ & \\
\hline Pajarito & $12 / 28 / 2013$ & 486000 & 3470000 & 1,385 & $12 \mathrm{R}$ & \\
\hline Pajarito & $4 / 16 / 2013$ & 493000 & 3474000 & 1,180 & $12 \mathrm{R}$ & \\
\hline Pajarito & $12 / 28 / 2013$ & 490000 & 3471000 & 1,250 & $12 \mathrm{R}$ & \\
\hline Pajarito & $12 / 28 / 2013$ & 491000 & 3470000 & 1,300 & $12 \mathrm{R}$ & \\
\hline Pajarito & $12 / 28 / 2013$ & 490000 & 3471000 & 1,250 & $12 \mathrm{R}$ & \\
\hline Pajarito Mountain range & & & & & & 24 \\
\hline
\end{tabular}




\begin{tabular}{|c|c|c|c|c|c|c|}
\hline Mountain range & Date deployed & $\begin{array}{l}\text { UTM E } \\
\text { (rounded) }\end{array}$ & $\begin{array}{c}\text { UTM N } \\
\text { (rounded) }\end{array}$ & $\begin{array}{c}\text { Elevation } \\
\text { (meters) }\end{array}$ & $\begin{array}{l}\text { UTM } \\
\text { zone }\end{array}$ & $\begin{array}{l}\text { Total } \\
\text { sites/ } \\
\text { range }\end{array}$ \\
\hline Patagonia & $8 / 24 / 2012$ & 526000 & 3473000 & 1,560 & $12 \mathrm{R}$ & \\
\hline Patagonia & 8/24/2012 & 521000 & 3475000 & 1,430 & $12 \mathrm{R}$ & \\
\hline Patagonia & $8 / 25 / 2012$ & 524000 & 3481000 & 1,680 & $12 \mathrm{R}$ & \\
\hline Patagonia & $12 / 18 / 2012$ & 524000 & 3481000 & 1,580 & $12 \mathrm{R}$ & \\
\hline Patagonia & $11 / 11 / 2012$ & 524000 & 3479000 & 1,840 & $12 \mathrm{R}$ & \\
\hline Patagonia & 8/25/2012 & 531000 & 3468000 & 1,495 & $12 \mathrm{R}$ & \\
\hline Patagonia & 10/23/2012 & 525000 & 3472000 & 1,490 & $12 \mathrm{R}$ & \\
\hline Patagonia & $12 / 18 / 2012$ & 524000 & 3477000 & 1,570 & $12 \mathrm{R}$ & \\
\hline Patagonia & $12 / 18 / 2012$ & 525000 & 3478000 & 1,570 & $12 \mathrm{R}$ & \\
\hline Patagonia & $12 / 9 / 2014$ & 525000 & 3478000 & 1,690 & $12 \mathrm{R}$ & \\
\hline Patagonia & $11 / 1 / 2012$ & 528000 & 3472000 & 1,830 & $12 \mathrm{R}$ & \\
\hline Patagonia $^{1}$ & $12 / 21 / 2012$ & 529000 & 3474000 & 1,670 & $12 \mathrm{R}$ & \\
\hline Patagonia & $12 / 9 / 2014$ & 529000 & 3474000 & 1,670 & $12 \mathrm{R}$ & \\
\hline Patagonia & 2/10/2013 & 525000 & 3479000 & 1,700 & $12 \mathrm{R}$ & \\
\hline Patagonia & 2/10/2013 & 525000 & 3478000 & 1,740 & $12 \mathrm{R}$ & \\
\hline Patagonia & 2/10/2013 & 526000 & 3474000 & 1,570 & $12 \mathrm{R}$ & \\
\hline Patagonia & 2/11/2013 & 528000 & 3475000 & 1,670 & $12 \mathrm{R}$ & \\
\hline Patagonia $^{1}$ & 4/6/2013 & 524000 & 3470000 & 1,480 & $12 \mathrm{R}$ & \\
\hline Patagonia & 4/6/2013 & 523000 & 3477000 & 1,590 & $12 \mathrm{R}$ & \\
\hline Patagonia & 4/7/2013 & 527000 & 3474000 & 1,814 & $12 \mathrm{R}$ & \\
\hline Patagonia & 4/7/2013 & 524000 & 3482000 & 1,526 & $12 \mathrm{R}$ & \\
\hline Patagonia & $6 / 21 / 2013$ & 528000 & 3472000 & 1,764 & $12 \mathrm{R}$ & \\
\hline Patagonia & $6 / 20 / 2013$ & 527000 & 3475000 & 1,710 & $12 \mathrm{R}$ & \\
\hline Patagonia & 6/28/2013 & 524000 & 3480000 & 1,726 & $12 \mathrm{R}$ & \\
\hline Patagonia & 7/3/2013 & 525000 & 3480000 & 1,674 & $12 \mathrm{R}$ & \\
\hline Patagonia & 8/26/2013 & 524000 & 3481000 & 1,669 & $12 \mathrm{R}$ & \\
\hline Patagonia & $6 / 11 / 2014$ & 528000 & 3485000 & 1,370 & $12 \mathrm{R}$ & \\
\hline Patagonia & $12 / 9 / 2014$ & 525000 & 3477000 & 1,780 & $12 \mathrm{R}$ & \\
\hline Patagonia Mountain range & & & & & & 28 \\
\hline Peloncillo & $5 / 27 / 2013$ & 688000 & 3490000 & 1,700 & $12 \mathrm{R}$ & \\
\hline Peloncillo & $5 / 27 / 2013$ & 689000 & 3491000 & 1,690 & $12 \mathrm{R}$ & \\
\hline Peloncillo & $5 / 27 / 2013$ & 688000 & 3493000 & 1,705 & $12 \mathrm{R}$ & \\
\hline Peloncillo & $5 / 27 / 2013$ & 687000 & 3496000 & 1,525 & $12 \mathrm{R}$ & \\
\hline Peloncillo & $5 / 27 / 2013$ & 687000 & 3491000 & 1,740 & $12 \mathrm{R}$ & \\
\hline Peloncillo & $5 / 27 / 2013$ & 685000 & 3491000 & 1,830 & $12 \mathrm{R}$ & \\
\hline Peloncillo & $5 / 27 / 2013$ & 684000 & 3490000 & 1,620 & $12 \mathrm{R}$ & \\
\hline Peloncillo & $5 / 27 / 2013$ & 689000 & 3488000 & 1,685 & $12 \mathrm{R}$ & \\
\hline Peloncillo & $5 / 27 / 2013$ & 688000 & 3486000 & 1,750 & $12 \mathrm{R}$ & \\
\hline Peloncillo & $5 / 27 / 2013$ & 688000 & 3485000 & 1,730 & $12 \mathrm{R}$ & \\
\hline Peloncillo & $5 / 27 / 2013$ & 691000 & 3488000 & 1,670 & $12 \mathrm{R}$ & \\
\hline Peloncillo & $5 / 29 / 2013$ & 681000 & 3476000 & 1,380 & $12 \mathrm{R}$ & \\
\hline Peloncillo & 5/29/2013 & 686000 & 3486000 & 1,690 & $12 \mathrm{R}$ & \\
\hline
\end{tabular}




\begin{tabular}{|c|c|c|c|c|c|c|}
\hline Mountain range & Date deployed & $\begin{array}{l}\text { UTM E } \\
\text { (rounded) }\end{array}$ & $\begin{array}{c}\text { UTM N } \\
\text { (rounded) }\end{array}$ & $\begin{array}{c}\text { Elevation } \\
\text { (meters) }\end{array}$ & $\begin{array}{l}\text { UTM } \\
\text { zone }\end{array}$ & $\begin{array}{l}\text { Total } \\
\text { sites/ } \\
\text { range }\end{array}$ \\
\hline Peloncillo & $5 / 30 / 2013$ & 687000 & 3486000 & 1,870 & $12 \mathrm{R}$ & \\
\hline Peloncillo & 5/30/2013 & 690000 & 3490000 & 1,700 & $12 \mathrm{R}$ & \\
\hline Peloncillo & 5/31/2013 & 681000 & 3486000 & 1,490 & $12 \mathrm{R}$ & \\
\hline Peloncillo & $11 / 12 / 2013$ & 691000 & 3518000 & 1,460 & $12 \mathrm{R}$ & \\
\hline Peloncillo & $11 / 14 / 2013$ & 686000 & 3486000 & 1,821 & $12 \mathrm{R}$ & \\
\hline Peloncillo & $11 / 12 / 2013$ & 686000 & 3487000 & 1,700 & $12 \mathrm{R}$ & \\
\hline Peloncillo & $11 / 14 / 2013$ & 681000 & 3476000 & 1,720 & $12 \mathrm{R}$ & \\
\hline Peloncillo & 9/26/2013 & 689000 & 3487000 & 1,736 & $12 \mathrm{R}$ & \\
\hline Peloncillo & $1 / 29 / 2014$ & 681000 & 3476000 & 1,370 & $12 \mathrm{R}$ & \\
\hline Peloncillo & $1 / 29 / 2014$ & 684000 & 3485000 & 1,515 & $12 \mathrm{R}$ & \\
\hline Peloncillo & $1 / 29 / 2014$ & 684000 & 3485000 & 1,515 & $12 \mathrm{R}$ & \\
\hline Peloncillo & 6/19/2014 & 688000 & 3485000 & 1,789 & $12 \mathrm{R}$ & \\
\hline Peloncillo Mountain range & & & & & & 25 \\
\hline Santa Rita & 9/30/2012 & 521000 & 3518000 & 1,440 & $12 \mathrm{R}$ & \\
\hline Santa Rita & $10 / 10 / 2012$ & 522000 & 3518000 & 1,490 & $12 \mathrm{R}$ & \\
\hline Santa Rita & $10 / 10 / 2012$ & 523000 & 3519000 & 1,580 & $12 \mathrm{R}$ & \\
\hline Santa Rita & 10/10/2012 & 522000 & 3520000 & 1,680 & $12 \mathrm{R}$ & \\
\hline Santa Rita & $10 / 10 / 2012$ & 524000 & 3520000 & 1,550 & $12 \mathrm{R}$ & \\
\hline Santa Rita & 10/16/2012 & 520000 & 3518000 & 1,340 & $12 \mathrm{R}$ & \\
\hline Santa Rita & $10 / 16 / 2012$ & 521000 & 3518000 & 1,480 & $12 \mathrm{R}$ & \\
\hline Santa Rita & $10 / 23 / 2012$ & 521000 & 3521000 & 1,810 & $12 \mathrm{R}$ & \\
\hline Santa Rita & $10 / 22 / 2012$ & 515000 & 3513000 & 1,420 & $12 \mathrm{R}$ & \\
\hline Santa Rita & 10/22/2012 & 517000 & 3513000 & 1,470 & $12 \mathrm{R}$ & \\
\hline Santa Rita & $12 / 20 / 2012$ & 519000 & 3503000 & 1,585 & $12 \mathrm{R}$ & \\
\hline Santa Rita & $12 / 20 / 2012$ & 518000 & 3502000 & 1,570 & $12 \mathrm{R}$ & \\
\hline Santa Rita & $12 / 20 / 2012$ & 516000 & 3498000 & 1,555 & $12 \mathrm{R}$ & \\
\hline Santa Rita & $12 / 20 / 2012$ & 518000 & 3499000 & 1,470 & $12 \mathrm{R}$ & \\
\hline Santa Rita & $1 / 16 / 2013$ & 522000 & 3519000 & 1,540 & $12 \mathrm{R}$ & \\
\hline Santa Rita & $1 / 16 / 2013$ & 521000 & 3519000 & 1,520 & $12 \mathrm{R}$ & \\
\hline Santa Rita & $1 / 21 / 2013$ & 521000 & 3518000 & 1,470 & $12 \mathrm{R}$ & \\
\hline Santa Rita & 4/16/2013 & 515000 & 3498000 & 1,775 & $12 \mathrm{R}$ & \\
\hline Santa Rita & $4 / 16 / 2013$ & 515000 & 3499000 & 1,770 & $12 \mathrm{R}$ & \\
\hline Santa Rita & 7/22/2013 & 517000 & 3509000 & 1,885 & $12 \mathrm{R}$ & \\
\hline Santa Rita & $7 / 22 / 2013$ & 517000 & 3507000 & 1,875 & $12 \mathrm{R}$ & \\
\hline Santa Rita & 7/24/2013 & 518000 & 3505000 & 1,755 & $12 \mathrm{R}$ & \\
\hline Santa Rita & 8/31/2013 & 517000 & 3504000 & 1,720 & $12 \mathrm{R}$ & \\
\hline Santa Rita & 8/31/2013 & 519000 & 3503000 & 1,625 & $12 \mathrm{R}$ & \\
\hline Santa Rita & 9/1/2013 & 521000 & 3503000 & 1,470 & $12 \mathrm{R}$ & \\
\hline Santa Rita & 9/4/2013 & 521000 & 3511000 & 1,825 & $12 \mathrm{R}$ & \\
\hline Santa Rita & 9/10/2013 & 520000 & 3505000 & 1,615 & $12 \mathrm{R}$ & \\
\hline Santa Rita & $10 / 30 / 2013$ & 520000 & 3506000 & 1,690 & $12 \mathrm{R}$ & \\
\hline Santa Rita & $12 / 12 / 2013$ & 516000 & 3502000 & 1,795 & $12 \mathrm{R}$ & \\
\hline
\end{tabular}




\begin{tabular}{|c|c|c|c|c|c|c|}
\hline Mountain range & Date deployed & $\begin{array}{l}\text { UTM E } \\
\text { (rounded) }\end{array}$ & $\begin{array}{c}\text { UTM N } \\
\text { (rounded) }\end{array}$ & $\begin{array}{c}\text { Elevation } \\
\text { (meters) }\end{array}$ & $\begin{array}{l}\text { UTM } \\
\text { zone }\end{array}$ & $\begin{array}{l}\text { Total } \\
\text { sites/ } \\
\text { range }\end{array}$ \\
\hline Santa Rita & $12 / 13 / 2013$ & 518000 & 3500000 & 1,495 & $12 \mathrm{R}$ & \\
\hline Santa Rita & 2/13/2014 & 517000 & 3504000 & 1,875 & $12 \mathrm{R}$ & \\
\hline Santa Rita & 2/13/2014 & 519000 & 3503000 & 1,650 & $12 \mathrm{R}$ & \\
\hline Santa Rita & $2 / 14 / 2014$ & 517000 & 3501000 & 1,745 & $12 \mathrm{R}$ & \\
\hline Santa Rita & $2 / 27 / 2014$ & 509000 & 3507000 & 1,710 & $12 \mathrm{R}$ & \\
\hline Santa Rita & $2 / 27 / 2014$ & 510000 & 3507000 & 1,970 & $12 \mathrm{R}$ & \\
\hline Santa Rita & $3 / 2 / 2014$ & 515000 & 3510000 & 2,395 & $12 \mathrm{R}$ & \\
\hline Santa Rita & $3 / 4 / 2014$ & 517000 & 3497000 & 1,585 & $12 \mathrm{R}$ & \\
\hline Santa Rita & 3/7/2014 & 510000 & 3504000 & 1,840 & $12 \mathrm{R}$ & \\
\hline Santa Rita & 3/12/2014 & 513000 & 3503000 & 1,700 & $12 \mathrm{R}$ & \\
\hline Santa Rita & 3/12/2014 & 513000 & 3504000 & 1,730 & $12 \mathrm{R}$ & \\
\hline Santa Rita & $3 / 12 / 2014$ & 513000 & 3505000 & 1,830 & $12 \mathrm{R}$ & \\
\hline Santa Rita & 6/2/2014 & 508000 & 3510000 & 1,380 & $12 \mathrm{R}$ & \\
\hline Santa Rita & 6/16/2014 & 508000 & 3505000 & 1,575 & $12 \mathrm{R}$ & \\
\hline Santa Rita & $8 / 15 / 2014$ & 508000 & 3505000 & 1,560 & $12 \mathrm{R}$ & \\
\hline Santa Rita & $5 / 7 / 2014$ & 517000 & 3504000 & 1,755 & $12 \mathrm{R}$ & \\
\hline Santa Rita & 8/13/2014 & 516000 & 3509000 & 2,117 & $12 \mathrm{R}$ & \\
\hline Santa Rita & 9/9/2014 & 524000 & 3513000 & 1,280 & $12 \mathrm{R}$ & \\
\hline Santa Rita & 9/9/2014 & 517000 & 3513000 & 1,445 & $12 \mathrm{R}$ & \\
\hline Santa Rita & 8/27/2014 & 518000 & 3501000 & 1,620 & $12 \mathrm{R}$ & \\
\hline Santa Rita & $11 / 12 / 2014$ & 517000 & 3505000 & 1,965 & $12 \mathrm{R}$ & \\
\hline Santa Rita & $11 / 14 / 2014$ & 509000 & 3505000 & 1,680 & $12 \mathrm{R}$ & \\
\hline Santa Rita & $11 / 14 / 2014$ & 509000 & 3505000 & 1,670 & $12 \mathrm{R}$ & \\
\hline Santa Rita & $11 / 14 / 2014$ & 509000 & 3505000 & 1,620 & $12 \mathrm{R}$ & \\
\hline Santa Rita & $11 / 18 / 2014$ & 512000 & 3512000 & 1,410 & $12 \mathrm{R}$ & \\
\hline Santa Rita & $12 / 1 / 2014$ & 512000 & 3512000 & 1,470 & $12 \mathrm{R}$ & \\
\hline Santa Rita ${ }^{1}$ & date pending & & & & $12 \mathrm{R}$ & \\
\hline Santa Rita & $11 / 19 / 2014$ & 517784 & 3499822 & 1,555 & $12 \mathrm{R}$ & \\
\hline Santa Rita & $12 / 1 / 2015$ & 512728 & 3497922 & 1,505 & $12 \mathrm{R}$ & \\
\hline Santa Rita & $12 / 3 / 2014$ & 518420 & 3511262 & 1,795 & $12 \mathrm{R}$ & \\
\hline Santa Rita & $12 / 3 / 2014$ & 519535 & 3511115 & 1,705 & $12 \mathrm{R}$ & \\
\hline Santa Rita & $12 / 16 / 2014$ & 518705 & 3505063 & 1,700 & $12 \mathrm{R}$ & \\
\hline Santa Rita Mountain range & & & & & & 61 \\
\hline Sierrita & $5 / 6 / 2013$ & 478000 & 3521000 & 1,210 & $12 \mathrm{R}$ & \\
\hline Sierrita & 5/6/2013 & 476000 & 3520000 & 1,200 & $12 \mathrm{R}$ & \\
\hline Sierrita & $5 / 6 / 2013$ & 475000 & 3524000 & 1,200 & $12 \mathrm{R}$ & \\
\hline Sierrita Mountain range & & & & & & 3 \\
\hline San Luis & $5 / 27 / 2013$ & 457000 & 3488000 & 1,160 & $12 \mathrm{R}$ & \\
\hline San Luis & $5 / 27 / 2013$ & 456000 & 3486000 & 1,125 & $12 \mathrm{R}$ & \\
\hline San Luis & $5 / 27 / 2013$ & 459000 & 3495000 & 1,125 & $12 \mathrm{R}$ & \\
\hline San Luis & 3/31/2014 & 458000 & 3482000 & 1,210 & $12 \mathrm{R}$ & \\
\hline San Luis & $4 / 29 / 2014$ & 458000 & 3482000 & 1,230 & $12 \mathrm{R}$ & \\
\hline
\end{tabular}




\begin{tabular}{|c|c|c|c|c|c|c|}
\hline Mountain range & Date deployed & $\begin{array}{l}\text { UTM E } \\
\text { (rounded) }\end{array}$ & $\begin{array}{c}\text { UTM N } \\
\text { (rounded) }\end{array}$ & $\begin{array}{l}\text { Elevation } \\
\text { (meters) }\end{array}$ & $\begin{array}{l}\text { UTM } \\
\text { zone }\end{array}$ & $\begin{array}{l}\text { Total } \\
\text { sites/ } \\
\text { range }\end{array}$ \\
\hline San Luis & $3 / 31 / 2014$ & 461000 & 3480000 & 1,180 & $12 \mathrm{R}$ & \\
\hline San Luis & $3 / 31 / 2014$ & 462000 & 3483000 & 1,190 & $12 \mathrm{R}$ & \\
\hline San Luis & 3/31/2014 & 463000 & 3485000 & 1,250 & $12 \mathrm{R}$ & \\
\hline San Luis Mountain range & & & & & & 8 \\
\hline Tumacacori & $2 / 5 / 2013$ & 489000 & 3499000 & 1,100 & $12 \mathrm{R}$ & \\
\hline Tumacacori & 2/5/2013 & 486000 & 3497000 & 1,200 & $12 \mathrm{R}$ & \\
\hline Tumacacori & 2/5/2013 & 488000 & 3495000 & 1,330 & $12 \mathrm{R}$ & \\
\hline Tumacacori & $2 / 6 / 2013$ & 485000 & 3496000 & 1,170 & $12 \mathrm{R}$ & \\
\hline Tumacacori & 2/6/2013 & 487000 & 3493000 & 1,300 & $12 \mathrm{R}$ & \\
\hline Tumacacori Mountain range & & & & & & 5 \\
\hline Whetstones & $6 / 1 / 2012$ & 557000 & 3526000 & 1,787 & $12 \mathrm{R}$ & \\
\hline Whetstones & $6 / 1 / 2012$ & 555000 & 3526000 & 1,668 & $12 \mathrm{R}$ & \\
\hline Whetstones & $6 / 4 / 2012$ & 557000 & 3524000 & 1,817 & $12 \mathrm{R}$ & \\
\hline Whetstones & $6 / 4 / 2012$ & 557000 & 3522000 & 1,690 & $12 \mathrm{R}$ & \\
\hline Whetstones & $6 / 2 / 2012$ & 557000 & 3520000 & 1,617 & $12 \mathrm{R}$ & \\
\hline Whetstones & 7/3/2012 & 554000 & 3517000 & 2,137 & $12 \mathrm{R}$ & \\
\hline Whetstones & 6/21/2012 & 556000 & 3518000 & 1,670 & $12 \mathrm{R}$ & \\
\hline Whetstones & $4 / 25 / 2012$ & 551000 & 3516000 & 1,729 & $12 \mathrm{R}$ & \\
\hline Whetstones & $2 / 15 / 2013$ & 551000 & 3518000 & 1,630 & $12 \mathrm{R}$ & \\
\hline Whetstone Mountain range & & & & & & 9 \\
\hline Total Sites Deployed & & & & & & 250 \\
\hline
\end{tabular}

${ }^{1}$ No data collected at site due to theft or other circumstances, therefore, this site is not included in the analyses. 
Table 4. Jaguar and ocelot detections from photographs and genetically verified scat collection from April 2012 to February 2015.

[Ocelots were named based on a historical numbering system; therefore, the numbers in their names do not reflect the total number of ocelots detected in this study. Mountain range/priority level ${ }^{1}$, elevation, Universal Transverse Mercator (UTM) zone, and Global Positioning System (GPS) coordinates in 1983 North American Datum (NAD83) are included. Photo and video detection date denotes year, month, day, hour, minute, and seconds; scat detection date denotes year, month and day]

\begin{tabular}{|c|c|c|c|c|c|c|c|c|c|}
\hline ID \# & $\begin{array}{l}\text { Mountain range } \\
\text { and priority } 1\end{array}$ & Detection date & $\begin{array}{l}\text { UTM } \\
\text { zone }\end{array}$ & $\begin{array}{c}\text { UTM E } \\
\text { (rounded) }\end{array}$ & $\begin{array}{c}\text { UTM N } \\
\text { (rounded) }\end{array}$ & $\begin{array}{l}\text { Elev. } \\
(\mathrm{m})\end{array}$ & Key & County & $\begin{array}{l}\text { Detection } \\
\text { type }\end{array}$ \\
\hline 1 & $\begin{array}{l}\text { Huachuca } \\
\text { Mountains/High }\end{array}$ & 20121008200213 & $12 \mathrm{R}$ & 560000 & 3480000 & 2,020 & Ocelot_3 & Cochise & Photo \\
\hline 2 & $\begin{array}{l}\text { Santa Rita } \\
\text { Mountains/Extended }\end{array}$ & 20121025225116 & $12 \mathrm{R}$ & 520000 & 3520000 & 1,810 & Jaguar & Pima & Photo \\
\hline 3 & $\begin{array}{l}\text { Santa Rita } \\
\text { Mountains/Extended }\end{array}$ & 20121025233659 & $12 \mathrm{R}$ & 520000 & 3520000 & 1,810 & Jaguar & Pima & Photo \\
\hline 4 & $\begin{array}{l}\text { Santa Rita } \\
\text { Mountains/Extended }\end{array}$ & 20121025233704 & $12 \mathrm{R}$ & 520000 & 3520000 & 1,810 & Jaguar & Pima & Photo \\
\hline 5 & $\begin{array}{l}\text { Santa Rita } \\
\text { Mountains/Extended }\end{array}$ & 20121025233709 & $12 \mathrm{R}$ & 520000 & 3520000 & 1,810 & Jaguar & Pima & Photo \\
\hline 6 & $\begin{array}{l}\text { Santa Rita } \\
\text { Mountains/Extended }\end{array}$ & 20121025233835 & $12 \mathrm{R}$ & 520000 & 3520000 & 1,810 & Jaguar & Pima & Photo \\
\hline 7 & $\begin{array}{l}\text { Santa Rita } \\
\text { Mountains/Extended }\end{array}$ & 20121025233840 & $12 \mathrm{R}$ & 520000 & 3520000 & 1,810 & Jaguar & Pima & Photo \\
\hline 8 & $\begin{array}{l}\text { Santa Rita } \\
\text { Mountains/Extended }\end{array}$ & 20121025233845 & $12 \mathrm{R}$ & 520000 & 3520000 & 1,810 & Jaguar & Pima & Photo \\
\hline 9 & $\begin{array}{l}\text { Santa Rita } \\
\text { Mountains/Extended }\end{array}$ & 20121110192319 & $12 \mathrm{R}$ & 520000 & 3510000 & 1,470 & Jaguar & Pima & Photo \\
\hline 10 & $\begin{array}{l}\text { Santa Rita } \\
\text { Mountains/Extended }\end{array}$ & 20121110224358 & $12 \mathrm{R}$ & 520000 & 3520000 & 1,440 & Jaguar & Pima & Photo \\
\hline 11 & $\begin{array}{l}\text { Santa Rita } \\
\text { Mountains/Extended }\end{array}$ & 20121111002636 & $12 \mathrm{R}$ & 520000 & 3520000 & 1,810 & Jaguar & Pima & Photo \\
\hline 12 & $\begin{array}{l}\text { Santa Rita } \\
\text { Mountains/Extended }\end{array}$ & 20121231001221 & $12 \mathrm{R}$ & 520000 & 3520000 & 1,440 & Jaguar & Pima & Photo \\
\hline 13 & $\begin{array}{l}\text { Santa Rita } \\
\text { Mountains/Extended }\end{array}$ & 20121231001419 & $12 \mathrm{R}$ & 520000 & 3520000 & 1,440 & Jaguar & Pima & Photo \\
\hline 14 & $\begin{array}{l}\text { Santa Rita } \\
\text { Mountains/Extended }\end{array}$ & 20121231015051 & $12 \mathrm{R}$ & 520000 & 3520000 & 1,810 & Jaguar & Pima & Photo \\
\hline 15 & $\begin{array}{l}\text { Santa Rita } \\
\text { Mountains/Extended }\end{array}$ & 20121231025011 & $12 \mathrm{R}$ & 520000 & 3520000 & 1,810 & Jaguar & Pima & Photo \\
\hline 16 & $\begin{array}{l}\text { Santa Rita } \\
\text { Mountains/Extended }\end{array}$ & 20121231025016 & $12 \mathrm{R}$ & 520000 & 3520000 & 1,810 & Jaguar & Pima & Photo \\
\hline 17 & $\begin{array}{l}\text { Santa Rita } \\
\text { Mountains/Extended }\end{array}$ & 20121231025021 & $12 \mathrm{R}$ & 520000 & 3520000 & 1,810 & Jaguar & Pima & Photo \\
\hline 18 & $\begin{array}{l}\text { Santa Rita } \\
\text { Mountains/Extended }\end{array}$ & 20130116032327 & $12 \mathrm{R}$ & 520000 & 3500000 & 1,555 & Jaguar & $\begin{array}{l}\text { Santa } \\
\text { Cruz }\end{array}$ & Photo \\
\hline 19 & $\begin{array}{l}\text { Huachuca } \\
\text { Mountains/High }\end{array}$ & 20130205201537 & $12 \mathrm{R}$ & 560000 & 3480000 & 1,740 & Ocelot_4 & Cochise & Photo \\
\hline 20 & $\begin{array}{l}\text { Santa Rita } \\
\text { Mountains/Extended }\end{array}$ & 20130426 & $12 \mathrm{R}$ & 520000 & 3500000 & 1,669 & Jaguar & $\begin{array}{l}\text { Santa } \\
\text { Cruz }\end{array}$ & Scat \\
\hline 21 & $\begin{array}{l}\text { Santa Rita } \\
\text { Mountains/Extended }\end{array}$ & 20130427213426 & $12 \mathrm{R}$ & 520000 & 3500000 & 1,689 & Jaguar & $\begin{array}{l}\text { Santa } \\
\text { Cruz }\end{array}$ & Photo \\
\hline 22 & $\begin{array}{l}\text { Santa Rita } \\
\text { Mountains/Extended }\end{array}$ & 20130427214657 & $12 \mathrm{R}$ & 520000 & 3500000 & 1,747 & Jaguar & $\begin{array}{l}\text { Santa } \\
\text { Cruz }\end{array}$ & Photo \\
\hline 23 & $\begin{array}{l}\text { Santa Rita } \\
\text { Mountains/Extended }\end{array}$ & 20130511035452 & $12 \mathrm{R}$ & 520000 & 3500000 & 1,778 & Jaguar & $\begin{array}{l}\text { Santa } \\
\text { Cruz }\end{array}$ & Photo \\
\hline 24 & $\begin{array}{l}\text { Santa Rita } \\
\text { Mountains/Extended }\end{array}$ & 20130517193715 & $12 \mathrm{R}$ & 520000 & 3500000 & 1,744 & Jaguar & $\begin{array}{l}\text { Santa } \\
\text { Cruz }\end{array}$ & Photo \\
\hline 25 & $\begin{array}{l}\text { Huachuca } \\
\text { Mountains/High }\end{array}$ & 20130522005937 & $12 \mathrm{R}$ & 560000 & 3480000 & 1,639 & Ocelot_3 & Cochise & Photo \\
\hline
\end{tabular}




\begin{tabular}{|c|c|c|c|c|c|c|c|c|c|}
\hline ID \# & $\begin{array}{l}\text { Mountain range } \\
\text { and priority1 }\end{array}$ & Detection date & $\begin{array}{l}\text { UTM } \\
\text { zone }\end{array}$ & $\begin{array}{c}\text { UTM E } \\
\text { (rounded) }\end{array}$ & $\begin{array}{c}\text { UTM N } \\
\text { (rounded) }\end{array}$ & $\begin{array}{c}\text { Elev. } \\
\text { (m) }\end{array}$ & Key & County & $\begin{array}{l}\text { Detection } \\
\text { type }\end{array}$ \\
\hline 26 & $\begin{array}{l}\text { Santa Rita } \\
\text { Mountains/Extended }\end{array}$ & 20130529001954 & $12 \mathrm{R}$ & 520000 & 3520000 & 1,744 & Jaguar & Pima & Photo \\
\hline 27 & $\begin{array}{l}\text { Santa Rita } \\
\text { Mountains/Extended }\end{array}$ & 20130529002739 & $12 \mathrm{R}$ & 520000 & 3520000 & 1,744 & Jaguar & Pima & Photo \\
\hline 28 & $\begin{array}{l}\text { Santa Rita } \\
\text { Mountains/Extended }\end{array}$ & 20130529014236 & $12 \mathrm{R}$ & 520000 & 3520000 & 1,703 & Jaguar & Pima & Photo \\
\hline 29 & $\begin{array}{l}\text { Santa Rita } \\
\text { Mountains/Extended }\end{array}$ & 20130529014446 & $12 \mathrm{R}$ & 520000 & 3520000 & 1,703 & Jaguar & Pima & Photo \\
\hline 30 & $\begin{array}{l}\text { Santa Rita } \\
\text { Mountains/Extended }\end{array}$ & 20130531204406 & $12 \mathrm{R}$ & 520000 & 3520000 & 1,743 & Jaguar & Pima & Photo \\
\hline 31 & $\begin{array}{l}\text { Santa Rita } \\
\text { Mountains/Extended }\end{array}$ & 20130531204616 & $12 \mathrm{R}$ & 520000 & 3520000 & 1,774 & Jaguar & Pima & Photo \\
\hline 32 & $\begin{array}{l}\text { Santa Rita } \\
\text { Mountains/Extended }\end{array}$ & 20130531224505 & $12 \mathrm{R}$ & 520000 & 3500000 & 1,721 & Jaguar & Pima & Photo \\
\hline 33 & $\begin{array}{l}\text { Santa Rita } \\
\text { Mountains/Extended }\end{array}$ & 20130617211427 & $12 \mathrm{R}$ & 520000 & 3500000 & 1,570 & Jaguar & $\begin{array}{l}\text { Santa } \\
\text { Cruz }\end{array}$ & Photo \\
\hline 34 & $\begin{array}{l}\text { Santa Rita } \\
\text { Mountains/Extended }\end{array}$ & 20130617211827 & $12 \mathrm{R}$ & 520000 & 3500000 & 1,570 & Jaguar & $\begin{array}{l}\text { Santa } \\
\text { Cruz }\end{array}$ & Photo \\
\hline 35 & $\begin{array}{l}\text { Santa Rita } \\
\text { Mountains/Extended }\end{array}$ & 20130801041004 & $12 \mathrm{R}$ & 520000 & 3510000 & 1,755 & Jaguar & $\begin{array}{l}\text { Santa } \\
\text { Cruz }\end{array}$ & Photo \\
\hline 36 & $\begin{array}{l}\text { Santa Rita } \\
\text { Mountains/Extended }\end{array}$ & 20130903001103 & $12 \mathrm{R}$ & 510000 & 3500000 & 1,720 & Jaguar & $\begin{array}{l}\text { Santa } \\
\text { Cruz }\end{array}$ & Photo \\
\hline 37 & $\begin{array}{l}\text { Santa Rita } \\
\text { Mountains/Extended }\end{array}$ & 20130911204158 & $12 \mathrm{R}$ & 510000 & 3500000 & 1,770 & Jaguar & $\begin{array}{l}\text { Santa } \\
\text { Cruz }\end{array}$ & Photo \\
\hline 38 & $\begin{array}{l}\text { Santa Rita } \\
\text { Mountains/Extended }\end{array}$ & 20130924053934 & $12 \mathrm{R}$ & 520000 & 3500000 & 1,720 & Jaguar & $\begin{array}{l}\text { Santa } \\
\text { Cruz }\end{array}$ & Photo \\
\hline 39 & $\begin{array}{l}\text { Santa Rita } \\
\text { Mountains/Extended }\end{array}$ & 20130924221326 & $12 \mathrm{R}$ & 520000 & 3500000 & 1,470 & Jaguar & $\begin{array}{l}\text { Santa } \\
\text { Cruz }\end{array}$ & Photo \\
\hline 40 & $\begin{array}{l}\text { Santa Rita } \\
\text { Mountains/Extended }\end{array}$ & 20130928202643 & $12 \mathrm{R}$ & 510000 & 3500000 & 1,770 & Jaguar & $\begin{array}{l}\text { Santa } \\
\text { Cruz }\end{array}$ & Photo \\
\hline 41 & $\begin{array}{l}\text { Santa Rita } \\
\text { Mountains/Extended }\end{array}$ & 20131002 & $12 \mathrm{R}$ & 520000 & 3510000 & 1,689 & Jaguar & $\begin{array}{l}\text { Santa } \\
\text { Cruz }\end{array}$ & Scat \\
\hline 42 & $\begin{array}{l}\text { Santa Rita } \\
\text { Mountains/Extended }\end{array}$ & 20131006190059 & $12 \mathrm{R}$ & 510000 & 3500000 & 1,770 & Jaguar & $\begin{array}{l}\text { Santa } \\
\text { Cruz }\end{array}$ & Photo \\
\hline 43 & $\begin{array}{l}\text { Huachuca } \\
\text { Mountains/High }\end{array}$ & 201310151931 & $12 \mathrm{R}$ & 570000 & 3470000 & 1,910 & Ocelot_4 & Cochise & Photo \\
\hline 44 & $\begin{array}{l}\text { Santa Rita } \\
\text { Mountains/Extended }\end{array}$ & 20131019184519 & $12 \mathrm{R}$ & 520000 & 3520000 & 1,810 & Jaguar & Pima & Photo \\
\hline 45 & $\begin{array}{l}\text { Santa Rita } \\
\text { Mountains/Extended }\end{array}$ & 20131019184618 & $12 \mathrm{R}$ & 520000 & 3520000 & 1,810 & Jaguar & Pima & Photo \\
\hline 46 & $\begin{array}{l}\text { Santa Rita } \\
\text { Mountains/Extended }\end{array}$ & 20131019185030 & $12 \mathrm{R}$ & 520000 & 3520000 & 1,810 & Jaguar & Pima & Photo \\
\hline 47 & $\begin{array}{l}\text { Santa Rita } \\
\text { Mountains/Extended }\end{array}$ & 20131022200928 & $12 \mathrm{R}$ & 520000 & 3500000 & 1,470 & Jaguar & $\begin{array}{l}\text { Santa } \\
\text { Cruz }\end{array}$ & Photo \\
\hline 48 & $\begin{array}{l}\text { Santa Rita } \\
\text { Mountains/Extended }\end{array}$ & 20131023 & $12 \mathrm{R}$ & 520000 & 3500000 & 1,747 & Jaguar & $\begin{array}{l}\text { Santa } \\
\text { Cruz }\end{array}$ & Scat \\
\hline 49 & $\begin{array}{l}\text { Santa Rita } \\
\text { Mountains/Extended }\end{array}$ & 20131029024001 & $12 \mathrm{R}$ & 520000 & 3500000 & 1,470 & Jaguar & $\begin{array}{l}\text { Santa } \\
\text { Cruz }\end{array}$ & Photo \\
\hline 50 & $\begin{array}{l}\text { Santa Rita } \\
\text { Mountains/Extended }\end{array}$ & 20131102005951 & $12 \mathrm{R}$ & 520000 & 3510000 & 1,615 & Jaguar & $\begin{array}{l}\text { Santa } \\
\text { Cruz }\end{array}$ & Photo \\
\hline 51 & $\begin{array}{l}\text { Santa Rita } \\
\text { Mountains/Extended }\end{array}$ & 20131102223627 & $12 \mathrm{R}$ & 520000 & 3510000 & 1,615 & Jaguar & $\begin{array}{l}\text { Santa } \\
\text { Cruz }\end{array}$ & Photo \\
\hline 52 & $\begin{array}{l}\text { Santa Rita } \\
\text { Mountains/Extended }\end{array}$ & 20131102225237 & $12 \mathrm{R}$ & 520000 & 3510000 & 1,615 & Jaguar & $\begin{array}{l}\text { Santa } \\
\text { Cruz }\end{array}$ & Photo \\
\hline 53 & $\begin{array}{l}\text { Santa Rita } \\
\text { Mountains/Extended }\end{array}$ & 20131111004221 & $12 \mathrm{R}$ & 520000 & 3500000 & 1,720 & Jaguar & $\begin{array}{l}\text { Santa } \\
\text { Cruz }\end{array}$ & Photo \\
\hline 54 & $\begin{array}{l}\text { Santa Rita } \\
\text { Mountains/Extended }\end{array}$ & 20131111004222 & $12 \mathrm{R}$ & 520000 & 3500000 & 1,720 & Jaguar & $\begin{array}{l}\text { Santa } \\
\text { Cruz }\end{array}$ & Video \\
\hline 55 & $\begin{array}{l}\text { Santa Rita } \\
\text { Mountains/Extended }\end{array}$ & 20131113223310 & $12 \mathrm{R}$ & 520000 & 3500000 & 1,625 & Jaguar & $\begin{array}{l}\text { Santa } \\
\text { Cruz }\end{array}$ & Photo \\
\hline
\end{tabular}




\begin{tabular}{|c|c|c|c|c|c|c|c|c|c|}
\hline ID \# & $\begin{array}{l}\text { Mountain range } \\
\text { and priority1 }\end{array}$ & Detection date & $\begin{array}{l}\text { UTM } \\
\text { zone }\end{array}$ & $\begin{array}{c}\text { UTM E } \\
\text { (rounded) }\end{array}$ & $\begin{array}{c}\text { UTM N } \\
\text { (rounded) }\end{array}$ & $\begin{array}{c}\text { Elev. } \\
(\mathrm{m})\end{array}$ & Key & County & $\begin{array}{c}\text { Detection } \\
\text { type }\end{array}$ \\
\hline 56 & $\begin{array}{l}\text { Santa Rita } \\
\text { Mountains/Extended }\end{array}$ & 20131213232350 & $12 \mathrm{R}$ & 520000 & 3500000 & 1,720 & Jaguar & $\begin{array}{l}\text { Santa } \\
\text { Cruz }\end{array}$ & Video \\
\hline 57 & $\begin{array}{l}\text { Santa Rita } \\
\text { Mountains/Extended }\end{array}$ & 20131222175347 & $12 \mathrm{R}$ & 520000 & 3520000 & 1,680 & $\begin{array}{l}\text { Ocelot \# } \\
\text { unknown }\end{array}$ & Pima & Photo \\
\hline 58 & $\begin{array}{l}\text { Santa Rita } \\
\text { Mountains/Extended }\end{array}$ & 201405 08a & $12 \mathrm{R}$ & 520000 & 3500000 & 1,778 & Jaguar & $\begin{array}{l}\text { Santa } \\
\text { Cruz }\end{array}$ & Scat \\
\hline 59 & $\begin{array}{l}\text { Santa Rita } \\
\text { Mountains/Extended }\end{array}$ & 201405 08b & $12 \mathrm{R}$ & 520000 & 3500000 & 1,744 & Jaguar & $\begin{array}{l}\text { Santa } \\
\text { Cruz }\end{array}$ & Scat \\
\hline 60 & $\begin{array}{l}\text { Santa Rita } \\
\text { Mountains/Extended }\end{array}$ & 20140107031736 & $12 \mathrm{R}$ & 520000 & 3500000 & 1,495 & Jaguar & $\begin{array}{l}\text { Santa } \\
\text { Cruz }\end{array}$ & Photo \\
\hline 61 & $\begin{array}{l}\text { Santa Rita } \\
\text { Mountains/Extended }\end{array}$ & 20140107032039 & $12 \mathrm{R}$ & 520000 & 3500000 & 1,495 & Jaguar & $\begin{array}{l}\text { Santa } \\
\text { Cruz }\end{array}$ & Photo \\
\hline 62 & $\begin{array}{l}\text { Santa Rita } \\
\text { Mountains/Extended }\end{array}$ & 20140116180750 & $12 \mathrm{R}$ & 520000 & 3500000 & 1,585 & Jaguar & $\begin{array}{l}\text { Santa } \\
\text { Cruz }\end{array}$ & Photo \\
\hline 63 & $\begin{array}{l}\text { Santa Rita } \\
\text { Mountains/Extended }\end{array}$ & 20140203062832 & $12 \mathrm{R}$ & 520000 & 3500000 & 1,720 & Jaguar & $\begin{array}{l}\text { Santa } \\
\text { Cruz }\end{array}$ & Video \\
\hline 64 & $\begin{array}{l}\text { Santa Rita } \\
\text { Mountains/Extended }\end{array}$ & 20140203065245 & $12 \mathrm{R}$ & 520000 & 3500000 & 1,625 & Jaguar & $\begin{array}{l}\text { Santa } \\
\text { Cruz }\end{array}$ & Photo \\
\hline 65 & $\begin{array}{l}\text { Santa Rita } \\
\text { Mountains/Extended }\end{array}$ & 20140210182258 & $12 \mathrm{R}$ & 520000 & 3500000 & 1,585 & Jaguar & $\begin{array}{l}\text { Santa } \\
\text { Cruz }\end{array}$ & Photo \\
\hline 66 & $\begin{array}{l}\text { Santa Rita } \\
\text { Mountains/Extended }\end{array}$ & 20140301230621 & $12 \mathrm{R}$ & 520000 & 3500000 & 1,585 & Jaguar & $\begin{array}{l}\text { Santa } \\
\text { Cruz }\end{array}$ & Photo \\
\hline 67 & $\begin{array}{l}\text { Santa Rita } \\
\text { Mountains/Extended }\end{array}$ & 20140305033554 & $12 \mathrm{R}$ & 520000 & 3500000 & 1,585 & Jaguar & $\begin{array}{l}\text { Santa } \\
\text { Cruz }\end{array}$ & Photo \\
\hline 68 & $\begin{array}{l}\text { Santa Rita } \\
\text { Mountains/Extended }\end{array}$ & 20140305054005 & $12 \mathrm{R}$ & 520000 & 3500000 & 1,495 & Jaguar & $\begin{array}{l}\text { Santa } \\
\text { Cruz }\end{array}$ & Photo \\
\hline 69 & $\begin{array}{l}\text { Santa Rita } \\
\text { Mountains/Extended }\end{array}$ & 20140318014240 & $12 \mathrm{R}$ & 520000 & 3500000 & 1,650 & Jaguar & $\begin{array}{l}\text { Santa } \\
\text { Cruz }\end{array}$ & Photo \\
\hline 70 & $\begin{array}{l}\text { Santa Rita } \\
\text { Mountains/Extended }\end{array}$ & 20140322222002 & $12 \mathrm{R}$ & 510000 & 3500000 & 1,770 & Jaguar & $\begin{array}{l}\text { Santa } \\
\text { Cruz }\end{array}$ & Photo \\
\hline 71 & $\begin{array}{l}\text { Santa Rita } \\
\text { Mountains/Extended }\end{array}$ & 20140323213843 & $12 \mathrm{R}$ & 520000 & 3500000 & 1,470 & Jaguar & $\begin{array}{l}\text { Santa } \\
\text { Cruz }\end{array}$ & Photo \\
\hline 72 & $\begin{array}{l}\text { Santa Rita } \\
\text { Mountains/Extended }\end{array}$ & 20140406204001 & $12 \mathrm{R}$ & 520000 & 3500000 & 1,470 & Jaguar & $\begin{array}{l}\text { Santa } \\
\text { Cruz }\end{array}$ & Photo \\
\hline 73 & $\begin{array}{l}\text { Santa Rita } \\
\text { Mountains/Extended }\end{array}$ & 20140408032529 & $12 \mathrm{R}$ & 520000 & 3520000 & 1,520 & Ocelot_5 & Pima & Photo \\
\hline 74 & $\begin{array}{l}\text { Santa Rita } \\
\text { Mountains/Extended }\end{array}$ & 20140409050827 & $12 \mathrm{R}$ & 520000 & 3500000 & 1,585 & Jaguar & $\begin{array}{l}\text { Santa } \\
\text { Cruz }\end{array}$ & Photo \\
\hline 75 & $\begin{array}{l}\text { Santa Rita } \\
\text { Mountains/Extended }\end{array}$ & 20140410073707 & $12 \mathrm{R}$ & 520000 & 3500000 & 1,495 & Jaguar & $\begin{array}{l}\text { Santa } \\
\text { Cruz }\end{array}$ & Photo \\
\hline 76 & $\begin{array}{l}\text { Santa Rita } \\
\text { Mountains/Extended }\end{array}$ & 20140410073858 & $12 \mathrm{R}$ & 520000 & 3500000 & 1,495 & Jaguar & $\begin{array}{l}\text { Santa } \\
\text { Cruz }\end{array}$ & Photo \\
\hline 77 & $\begin{array}{l}\text { Santa Rita } \\
\text { Mountains/Extended }\end{array}$ & 20140410074226 & $12 \mathrm{R}$ & 520000 & 3500000 & 1,495 & Jaguar & $\begin{array}{l}\text { Santa } \\
\text { Cruz }\end{array}$ & Photo \\
\hline 78 & $\begin{array}{l}\text { Santa Rita } \\
\text { Mountains/Extended }\end{array}$ & 20140410190319 & $12 \mathrm{R}$ & 520000 & 3500000 & 1,495 & Jaguar & $\begin{array}{l}\text { Santa } \\
\text { Cruz }\end{array}$ & Photo \\
\hline 79 & $\begin{array}{l}\text { Santa Rita } \\
\text { Mountains/Extended }\end{array}$ & 20140410190648 & $12 \mathrm{R}$ & 520000 & 3500000 & 1,495 & Jaguar & $\begin{array}{l}\text { Santa } \\
\text { Cruz }\end{array}$ & Photo \\
\hline 80 & $\begin{array}{l}\text { Santa Rita } \\
\text { Mountains/Extended }\end{array}$ & 20140411 & $12 \mathrm{R}$ & 520000 & 3500000 & 1,639 & Jaguar & $\begin{array}{l}\text { Santa } \\
\text { Cruz }\end{array}$ & Scat \\
\hline 81 & $\begin{array}{l}\text { Santa Rita } \\
\text { Mountains/Extended }\end{array}$ & 20140417064807 & $12 \mathrm{R}$ & 520000 & 3500000 & 1,625 & Jaguar & $\begin{array}{l}\text { Santa } \\
\text { Cruz }\end{array}$ & Photo \\
\hline 83 & $\begin{array}{l}\text { Santa Rita } \\
\text { Mountains/Extended }\end{array}$ & 20140428050840 & $12 \mathrm{R}$ & 520000 & 3500000 & 1,650 & Jaguar & $\begin{array}{l}\text { Santa } \\
\text { Cruz }\end{array}$ & Photo \\
\hline 84 & $\begin{array}{l}\text { Santa Rita } \\
\text { Mountains/Extended }\end{array}$ & 20140430061114 & $12 \mathrm{R}$ & 520000 & 3500000 & 1,495 & Jaguar & $\begin{array}{l}\text { Santa } \\
\text { Cruz }\end{array}$ & Photo \\
\hline 85 & $\begin{array}{l}\text { Santa Rita } \\
\text { Mountains/Extended }\end{array}$ & 20140430061418 & $12 \mathrm{R}$ & 520000 & 3500000 & 1,495 & Jaguar & $\begin{array}{l}\text { Santa } \\
\text { Cruz }\end{array}$ & Photo \\
\hline 86 & $\begin{array}{l}\text { Santa Rita } \\
\text { Mountains/Extended }\end{array}$ & 20140430061427 & $12 \mathrm{R}$ & 520000 & 3500000 & 1,495 & Jaguar & $\begin{array}{l}\text { Santa } \\
\text { Cruz }\end{array}$ & Photo \\
\hline
\end{tabular}




\begin{tabular}{|c|c|c|c|c|c|c|c|c|c|}
\hline ID \# & $\begin{array}{l}\text { Mountain range } \\
\text { and priority1 }\end{array}$ & Detection date & $\begin{array}{l}\text { UTM } \\
\text { zone }\end{array}$ & $\begin{array}{c}\text { UTM E } \\
\text { (rounded) }\end{array}$ & $\begin{array}{c}\text { UTM N } \\
\text { (rounded) }\end{array}$ & $\begin{array}{c}\text { Elev. } \\
\text { (m) }\end{array}$ & Key & County & $\begin{array}{l}\text { Detection } \\
\text { type }\end{array}$ \\
\hline 87 & $\begin{array}{l}\text { Santa Rita } \\
\text { Mountains/Extended }\end{array}$ & 20140502183935 & $12 \mathrm{R}$ & 520000 & 3500000 & 1,625 & Jaguar & $\begin{array}{l}\text { Santa } \\
\text { Cruz }\end{array}$ & Photo \\
\hline 88 & $\begin{array}{l}\text { Santa Rita } \\
\text { Mountains/Extended }\end{array}$ & 20140502191256 & $12 \mathrm{R}$ & 520000 & 3500000 & 1,720 & Jaguar & $\begin{array}{l}\text { Santa } \\
\text { Cruz }\end{array}$ & Photo \\
\hline 89 & $\begin{array}{l}\text { Santa Rita } \\
\text { Mountains/Extended }\end{array}$ & 20140502214610 & $12 \mathrm{R}$ & 510000 & 3500000 & 1,700 & Jaguar & $\begin{array}{l}\text { Santa } \\
\text { Cruz }\end{array}$ & Photo \\
\hline 90 & $\begin{array}{l}\text { Santa Rita } \\
\text { Mountains/Extended }\end{array}$ & $20140508 c$ & $12 \mathrm{R}$ & 520000 & 3500000 & 1,744 & Jaguar & $\begin{array}{l}\text { Santa } \\
\text { Cruz }\end{array}$ & Scat \\
\hline 91 & $\begin{array}{l}\text { Santa Rita } \\
\text { Mountains/Extended }\end{array}$ & 20140514192024 & $12 \mathrm{R}$ & 520000 & 3500000 & 1,650 & Jaguar & $\begin{array}{l}\text { Santa } \\
\text { Cruz }\end{array}$ & Photo \\
\hline 92 & $\begin{array}{l}\text { Santa Rita } \\
\text { Mountains/Extended }\end{array}$ & 20140514194224 & $12 \mathrm{R}$ & 510000 & 3510000 & 1,710 & Ocelot_5 & $\begin{array}{l}\text { Santa } \\
\text { Cruz }\end{array}$ & Photo \\
\hline 93 & $\begin{array}{l}\text { Santa Rita } \\
\text { Mountains/Extended }\end{array}$ & 20140515201534 & $12 \mathrm{R}$ & 520000 & 3500000 & 1,795 & Jaguar & $\begin{array}{l}\text { Santa } \\
\text { Cruz }\end{array}$ & Photo \\
\hline 94 & $\begin{array}{l}\text { Santa Rita } \\
\text { Mountains/Extended }\end{array}$ & 20140516022236 & $12 \mathrm{R}$ & 520000 & 3500000 & 1,650 & Jaguar & $\begin{array}{l}\text { Santa } \\
\text { Cruz }\end{array}$ & Photo \\
\hline 95 & $\begin{array}{l}\text { Santa Rita } \\
\text { Mountains/Extended }\end{array}$ & 20140518231042 & $12 \mathrm{R}$ & 520000 & 3500000 & 1,795 & Jaguar & $\begin{array}{l}\text { Santa } \\
\text { Cruz }\end{array}$ & Photo \\
\hline 96 & $\begin{array}{l}\text { Santa Rita } \\
\text { Mountains/Extended }\end{array}$ & 20140518231641 & $12 \mathrm{R}$ & 520000 & 3500000 & 1,795 & Jaguar & $\begin{array}{l}\text { Santa } \\
\text { Cruz }\end{array}$ & Photo \\
\hline 97 & $\begin{array}{l}\text { Santa Rita } \\
\text { Mountains/Extended }\end{array}$ & 20140519002942 & $12 \mathrm{R}$ & 510000 & 3500000 & 1,700 & Jaguar & $\begin{array}{l}\text { Santa } \\
\text { Cruz }\end{array}$ & Photo \\
\hline 98 & $\begin{array}{l}\text { Santa Rita } \\
\text { Mountains/Extended }\end{array}$ & 20140519205632 & $12 \mathrm{R}$ & 520000 & 3520000 & 1,440 & Ocelot_5 & Pima & Photo \\
\hline 99 & $\begin{array}{l}\text { Santa Rita } \\
\text { Mountains/Extended }\end{array}$ & 20140519210137 & $12 \mathrm{R}$ & 520000 & 3520000 & 1,440 & Ocelot_5 & Pima & Photo \\
\hline 100 & $\begin{array}{l}\text { Santa Rita } \\
\text { Mountains/Extended }\end{array}$ & 20140521011353 & $12 \mathrm{R}$ & 520000 & 3520000 & 1,540 & Ocelot_5 & Pima & Photo \\
\hline 101 & $\begin{array}{l}\text { Santa Rita } \\
\text { Mountains/Extended }\end{array}$ & 20140521011917 & $12 \mathrm{R}$ & 520000 & 3520000 & 1,540 & Ocelot_5 & Pima & Photo \\
\hline 102 & $\begin{array}{l}\text { Santa Rita } \\
\text { Mountains/Extended }\end{array}$ & 20140521193424 & $12 \mathrm{R}$ & 520000 & 3510000 & 1,755 & Jaguar & $\begin{array}{l}\text { Santa } \\
\text { Cruz }\end{array}$ & Photo \\
\hline 103 & $\begin{array}{l}\text { Santa Rita } \\
\text { Mountains/Extended }\end{array}$ & 20140522042519 & $12 \mathrm{R}$ & 520000 & 3520000 & 1,440 & Jaguar & Pima & Photo \\
\hline 104 & $\begin{array}{l}\text { Santa Rita } \\
\text { Mountains/Extended }\end{array}$ & 20140522043037 & $12 \mathrm{R}$ & 520000 & 3520000 & 1,440 & Jaguar & Pima & Photo \\
\hline 105 & $\begin{array}{l}\text { Santa Rita } \\
\text { Mountains/Extended }\end{array}$ & 20140522055117 & $12 \mathrm{R}$ & 520000 & 3520000 & 1,810 & Jaguar & Pima & Photo \\
\hline 106 & $\begin{array}{l}\text { Santa Rita } \\
\text { Mountains/Extended }\end{array}$ & 20140522055631 & $12 \mathrm{R}$ & 520000 & 3520000 & 1,810 & Jaguar & Pima & Photo \\
\hline 107 & $\begin{array}{l}\text { Santa Rita } \\
\text { Mountains/Extended }\end{array}$ & 20140522055632 & $12 \mathrm{R}$ & 520000 & 3520000 & 1,810 & Jaguar & Pima & Photo \\
\hline 108 & $\begin{array}{l}\text { Santa Rita } \\
\text { Mountains/Extended }\end{array}$ & 20140522055711 & $12 \mathrm{R}$ & 520000 & 3520000 & 1,810 & Jaguar & Pima & Photo \\
\hline 109 & $\begin{array}{l}\text { Santa Rita } \\
\text { Mountains/Extended }\end{array}$ & 20140522055712 & $12 \mathrm{R}$ & 520000 & 3520000 & 1,810 & Jaguar & Pima & Photo \\
\hline 110 & $\begin{array}{l}\text { Santa Rita } \\
\text { Mountains/Extended }\end{array}$ & 20140530230346 & $12 \mathrm{R}$ & 520000 & 3500000 & 1,585 & Jaguar & $\begin{array}{l}\text { Santa } \\
\text { Cruz }\end{array}$ & Photo \\
\hline 111 & $\begin{array}{l}\text { Santa Rita } \\
\text { Mountains/Extended }\end{array}$ & 20140610063642 & $12 \mathrm{R}$ & 520000 & 3500000 & 1,495 & Jaguar & $\begin{array}{l}\text { Santa } \\
\text { Cruz }\end{array}$ & Photo \\
\hline 112 & $\begin{array}{l}\text { Santa Rita } \\
\text { Mountains/Extended }\end{array}$ & 20140610063729 & $12 \mathrm{R}$ & 520000 & 3500000 & 1,495 & Jaguar & $\begin{array}{l}\text { Santa } \\
\text { Cruz }\end{array}$ & Photo \\
\hline 113 & $\begin{array}{l}\text { Santa Rita } \\
\text { Mountains/Extended }\end{array}$ & 20140615024827 & $12 \mathrm{R}$ & 520000 & 3500000 & 1,625 & Jaguar & $\begin{array}{l}\text { Santa } \\
\text { Cruz }\end{array}$ & Photo \\
\hline 114 & $\begin{array}{l}\text { Santa Rita } \\
\text { Mountains/Extended }\end{array}$ & 20140620222602 & $12 \mathrm{R}$ & 520000 & 3500000 & 1,650 & Jaguar & $\begin{array}{l}\text { Santa } \\
\text { Cruz }\end{array}$ & Photo \\
\hline 115 & $\begin{array}{l}\text { Santa Rita } \\
\text { Mountains/Extended }\end{array}$ & 20140629030631 & $12 \mathrm{R}$ & 520000 & 3500000 & 1,650 & Jaguar & $\begin{array}{l}\text { Santa } \\
\text { Cruz }\end{array}$ & Photo \\
\hline 116 & $\begin{array}{l}\text { Santa Rita } \\
\text { Mountains/Extended }\end{array}$ & 20140704032816 & $12 \mathrm{R}$ & 520000 & 3500000 & 1,495 & Jaguar & $\begin{array}{l}\text { Santa } \\
\text { Cruz }\end{array}$ & Photo \\
\hline
\end{tabular}




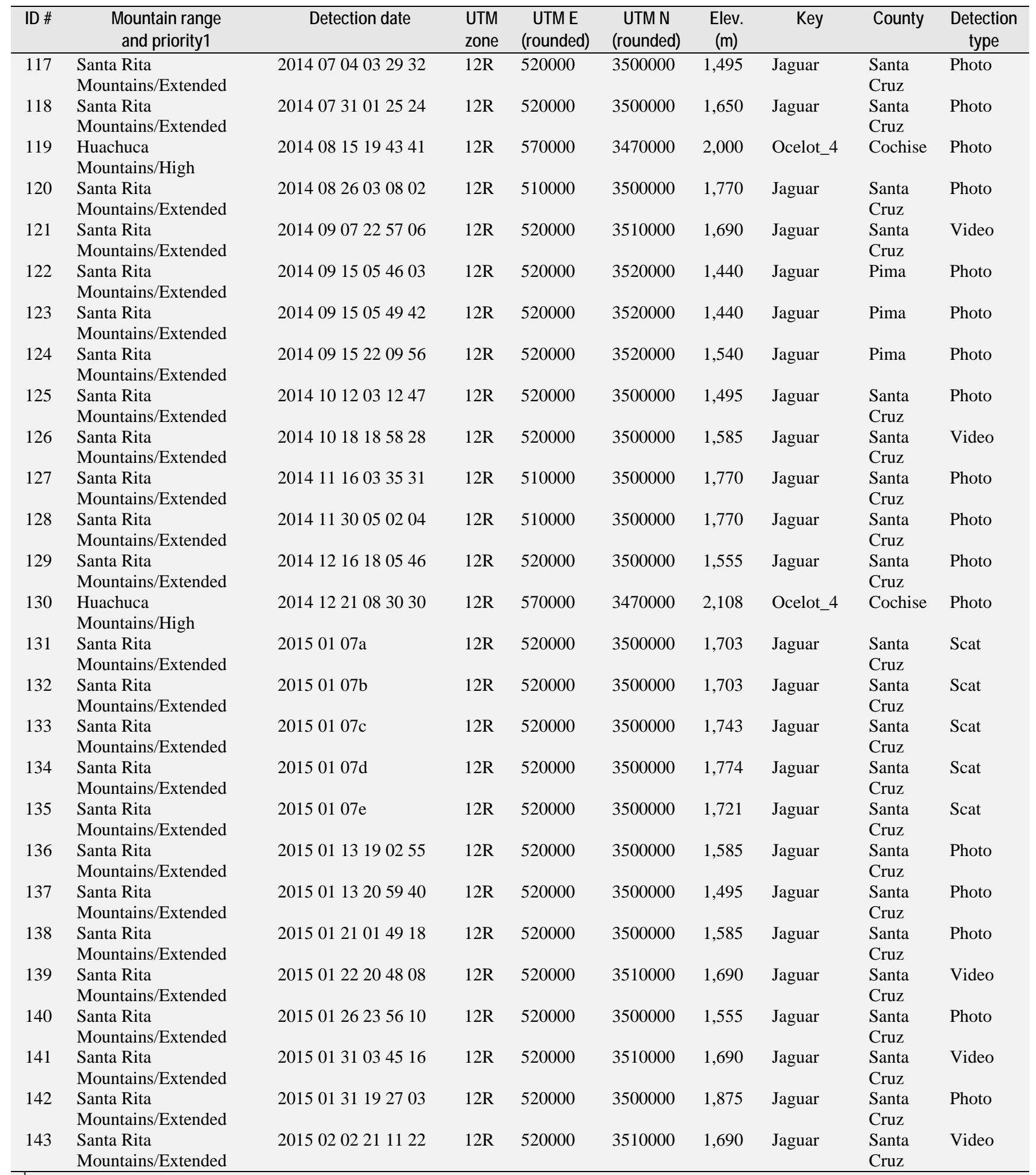

${ }^{1}$ Priority refers to the likelihood of a detection based on historical and recent records. High Priority cameras were checked every 30 days. Extended priority indicates extra cameras that were set in an area after a jaguar or ocelot was detected. Priority levels were modified as an adaptive management strategy throughout the study. 
Table 5a. Species $(n=50)$ and species categories $(n=32)$ used in the analysis. Common and Latin names included. Squirrels are separated from the rodent species category, and turkeys are separated from the bird species category.

[NA, not applicable]

\begin{tabular}{|c|c|c|}
\hline Common name & Species/species categories & Latin name \\
\hline Badger & Badger & Taxidea taxus \\
\hline Bats & Bats (in some analyses/not considered a prey item) & Many ssp. \\
\hline Bear & Bear & Ursus americanus \\
\hline Bighorn & Bighorn & Ovis Canadensis nelson \\
\hline Birds & Birds & Many ssp. \\
\hline Bobcat & Bobcat & Lynx rufus \\
\hline Butterflies & Not included, not major prey item & Many ssp. \\
\hline Coatimundi & Coatimundi & Nasua narica \\
\hline Cottontail & Cottontail & Sylvilagus auduboni \\
\hline Cow & Cow & Bos tarus \\
\hline Coyote & Coyote & Canis latrans \\
\hline Domestic Dog & Dog & Canis familiaris \\
\hline Domestic Cat & Not included, too few records & Felis catus \\
\hline Gray fox & Fox & Urocyon cinereoargenteus \\
\hline Frogs & Not included, not major prey item & Many ssp. \\
\hline Ghost & Not included, not a record & NA \\
\hline Gila Monster & Gila monster & Heloderma suspectum \\
\hline Goat & not included, too few records & Capra aegagrus \\
\hline Horse & Horse & Equus caballus \\
\hline Human & Human & Homo sapiens \\
\hline Antelope jackrabbit & Jackrabbit & Lepus alleni \\
\hline Blacktail jackrabbit & (Jackrabbit) & Lepus californicus \\
\hline Jaguar & Jaguar & Panthera onca \\
\hline Javelina & Javelina & Pecari tajacu \\
\hline Lizards & Snakes/Lizards & many ssp. \\
\hline Mule deer & Mule deer & Odocoileus hemionus \\
\hline Ocelot & Ocelot & Leopardus pardalis \\
\hline Mexican opossum & Opossum & Didelphis marsupialis \\
\hline Virginia opossum & (Opossum) & Didelphis virginiana \\
\hline Pronghorn & Pronghorn & Antilocapra Americana \\
\hline Puma & Puma & Puma concolor \\
\hline Raccoon & Raccoon & Procyon lotor \\
\hline Ringtail & Ringtail & Bassariscus astutus \\
\hline Packrat & Rodent & Neotoma albigula \\
\hline Other rodents & (Rodents) & Many ssp. \\
\hline Hooded skunk & Skunks & Mephitis macroura \\
\hline Striped skunk & (Skunks) & Mephitis mephitis \\
\hline Hog-nosed skunk & (Skunks) & Conepatus leuconotus \\
\hline Spotted skunk & (Skunks) & Spilogale gragilis \\
\hline
\end{tabular}




\begin{tabular}{lll}
\hline \multicolumn{1}{c}{ Common name } & \multicolumn{1}{c}{ Species/species categories } & \multicolumn{1}{c}{ Latin name } \\
\hline Snakes & (Snakes/Lizards) & Many ssp. \\
Mexican spotted owl & Spotted Owl & Strix occidentalis \\
Arizona gray squirrel & Squirrels & Sciurus arizonensis \\
Rock squirrel & (Squirrels) & Spermophilus varegatus \\
Other squirrels & (Squirrels) & Many ssp. \\
Test & Not included, not a record & NA \\
Sonoran desert tortoise & Tortoise & Gopherus morafki \\
Sonora mud turtle & (Tortoise) & Kinosternon sonoriense \\
Turkey & Turkey & Meleagris gallopavo \\
Unknown & Not included, not analyzable data & NA \\
White-tailed deer & White-tailed deer & Odocoileus virginianus \\
\hline
\end{tabular}


Table $5 \mathrm{~b}$. Species and species categories detected with trail cameras and corresponding mountain range in southern Arizona and southwestern New Mexico.

[Squirrels are separated from the rodent species category, and turkeys are separated from the bird species category]

\begin{tabular}{|c|c|c|c|c|c|c|c|c|c|c|c|c|c|c|c|c|c|}
\hline Species (32) & 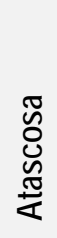 & 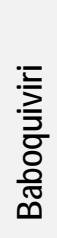 & 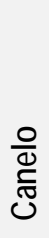 & $\begin{array}{l}\text { 융 } \\
\frac{\pi}{0} \\
\text { 응 } \\
\text { U } \\
\text { 옳 } \\
\text { U. }\end{array}$ & 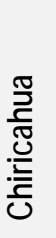 & 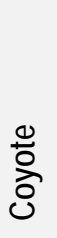 & 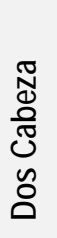 & 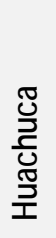 & 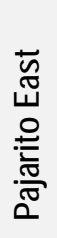 & 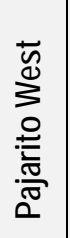 & 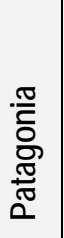 & 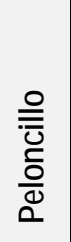 & 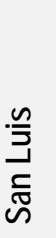 & 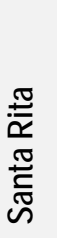 & 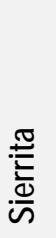 & 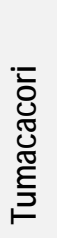 & 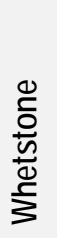 \\
\hline \multicolumn{18}{|l|}{ Badger } \\
\hline \multicolumn{18}{|l|}{ Bear } \\
\hline \multicolumn{18}{|l|}{ Bighorn } \\
\hline \multicolumn{18}{|l|}{ Birds } \\
\hline \multicolumn{18}{|l|}{ Bobcat } \\
\hline \multicolumn{18}{|l|}{ Coatimundi } \\
\hline \multicolumn{18}{|l|}{ Cottontail } \\
\hline \multicolumn{18}{|l|}{ Cow } \\
\hline \multicolumn{18}{|l|}{ Coyote } \\
\hline \multicolumn{18}{|l|}{ Dog } \\
\hline \multicolumn{18}{|l|}{ Fox } \\
\hline \multicolumn{18}{|l|}{ Gila Monster } \\
\hline \multicolumn{18}{|l|}{ Horse } \\
\hline \multicolumn{18}{|l|}{ Humans } \\
\hline \multicolumn{18}{|l|}{ Jackrabbits } \\
\hline \multicolumn{18}{|l|}{ Jaguar } \\
\hline \multicolumn{18}{|l|}{ Javelina } \\
\hline \multicolumn{18}{|l|}{ Mule Deer } \\
\hline \multicolumn{18}{|l|}{ Ocelot } \\
\hline \multicolumn{18}{|l|}{ Opossum } \\
\hline \multicolumn{18}{|l|}{ Pronghorn } \\
\hline \multicolumn{18}{|l|}{ Puma } \\
\hline \multicolumn{18}{|l|}{ Raccoon } \\
\hline Ringtail & & & & & & & & & & & & & & & & & \\
\hline Rodents & & & & & & & & & & & & & & & & & \\
\hline Skunks & & & & & & & & & & & & & & & & & \\
\hline Snakes and Lizards & & & & & & & & & & & & & & & & & \\
\hline Spotted Owl & & & & & & & & & & & & & & & & & \\
\hline Squirrels & & & & & & & & & & & & & & & & & \\
\hline Tortoise & & & & & & & & & & & & & & & & & \\
\hline Turkey & & & & & & & & & & & & & & & & & \\
\hline White-tailed Deer & & & & & & & & & & & & & & & & & \\
\hline Total Species detected & 19 & 24 & 20 & 18 & 19 & 23 & 15 & 23 & 19 & 17 & 19 & 21 & 23 & 25 & 20 & 18 & 20 \\
\hline & & & & & & & & & & & detec & & & & & & \\
\hline
\end{tabular}


Table 6. Federally designated threatened and formerly proposed candidate species, species of concern, and species range extensions detected from April 2012 to February 2015.

[Mountain range, species, detection date and time, UTM zone, UTM coordinates, and elevation in meters are included. GPS coordinates are in NAD83. Detection date denotes year, month, day, hour, minute, and seconds. Abbreviations are UTM, Universal Transverse Mercatur; GPS, Global Positioning System; NAD83, 1983 North American Datum]

\begin{tabular}{|c|c|c|c|c|c|c|}
\hline Mountain range & Species & Detection date and time & $\begin{array}{l}\text { UTM } \\
\text { zone }\end{array}$ & $\begin{array}{l}\text { UTM E } \\
\text { (rounded) }\end{array}$ & $\begin{array}{l}\text { UTM N } \\
\text { (rounded) }\end{array}$ & $\begin{array}{c}\text { Elevation } \\
\text { (meters) }\end{array}$ \\
\hline Coyote & Bighorn & 2013050619232101 & $12 \mathrm{R}$ & 450000 & 3540000 & 1,470 \\
\hline Coyote & Bighorn & 2014021417242602 & $12 \mathrm{R}$ & 450000 & 3540000 & 1,470 \\
\hline Coyote & Bighorn & 2014021417260601 & $12 \mathrm{R}$ & 450000 & 3540000 & 1,470 \\
\hline Coyote & Bighorn & 2014021417271001 & $12 \mathrm{R}$ & 450000 & 3540000 & 1,470 \\
\hline Coyote & Bighorn & 2014021417281202 & $12 \mathrm{R}$ & 450000 & 3540000 & 1,470 \\
\hline Coyote & Bighorn & 2014021417291402 & $12 \mathrm{R}$ & 450000 & 3540000 & 1,470 \\
\hline Coyote & Bighorn & 2014021417333902 & $12 \mathrm{R}$ & 450000 & 3540000 & 1,470 \\
\hline Coyote & Bighorn & 2014021417344101 & $12 \mathrm{R}$ & 450000 & 3540000 & 1,470 \\
\hline Coyote & Bighorn & 2014021417374002 & $12 \mathrm{R}$ & 450000 & 3540000 & 1,470 \\
\hline Coyote & Bighorn & 2014021417414801 & $12 \mathrm{R}$ & 450000 & 3540000 & 1,470 \\
\hline Coyote & Bighorn & 2014021417424801 & $12 \mathrm{R}$ & 450000 & 3540000 & 1,470 \\
\hline Coyote & Bighorn & 2014021417435502 & $12 \mathrm{R}$ & 450000 & 3540000 & 1,470 \\
\hline Coyote & Bighorn & 2014021417453001 & $12 \mathrm{R}$ & 450000 & 3540000 & 1,470 \\
\hline Baboquivari & Gila Monster & 2014032312182701 & $12 \mathrm{R}$ & 440000 & 3510000 & 1,353 \\
\hline Baboquivari & Gila Monster & 2014071010321101 & $12 \mathrm{R}$ & 440000 & 3510000 & 1,353 \\
\hline Baboquivari & Gila Monster & 2014071615524801 & $12 \mathrm{R}$ & 440000 & 3510000 & 1,353 \\
\hline Baboquivari & Gila Monster & 2014050307571701 & $12 \mathrm{R}$ & 440000 & 3490000 & 1,237 \\
\hline Coyote & Gila Monster & 2013072006380701 & $12 \mathrm{R}$ & 450000 & 3540000 & 935 \\
\hline Coyote & Gila Monster & 2013091112170201 & $12 \mathrm{R}$ & 450000 & 3540000 & 935 \\
\hline Coyote & Gila Monster & 2014031108482101 & $12 \mathrm{R}$ & 450000 & 3540000 & 935 \\
\hline Coyote & Gila Monster & 2014072102565201 & $12 \mathrm{R}$ & 450000 & 3540000 & 935 \\
\hline Coyote & Gila Monster & 2014072610180801 & $12 \mathrm{R}$ & 450000 & 3540000 & 935 \\
\hline Sierrita & Gila Monster & 2014051011320001 & $12 \mathrm{R}$ & 480000 & 3520000 & 1,210 \\
\hline San Luis & Gila Monster & 2014050609410301 & $12 \mathrm{R}$ & 460000 & 3490000 & 1,160 \\
\hline San Luis & Pronghorn & 2014042809205101 & $12 \mathrm{R}$ & 460000 & 3490000 & 1,160 \\
\hline San Luis & Pronghorn & 2014042809210701 & $12 \mathrm{R}$ & 460000 & 3490000 & 1,160 \\
\hline Santa Rita & Spotted Owl & 2014110411000000 & $12 \mathrm{R}$ & 520000 & 3520000 & 1,680 \\
\hline Huachuca & Spotted Owl & 2014060220490201 & $12 \mathrm{R}$ & 570000 & 3470000 & 2,108 \\
\hline Huachuca & Spotted Owl & 2014060821030601 & $12 \mathrm{R}$ & 570000 & 3470000 & 2,108 \\
\hline $\begin{array}{l}\text { Baboquivari } \\
\text { Cerro }\end{array}$ & Tortoise & 2014072915335001 & $12 \mathrm{R}$ & 440000 & 3490000 & 1,237 \\
\hline $\begin{array}{l}\text { Colorado } \\
\text { Cerro }\end{array}$ & Tortoise & 2013080410202201 & $12 \mathrm{R}$ & 470000 & 3510000 & 1,140 \\
\hline $\begin{array}{l}\text { Colorado } \\
\text { Cerro }\end{array}$ & Tortoise & 2013080819182101 & $12 \mathrm{R}$ & 470000 & 3510000 & 1,140 \\
\hline Colorado & Tortoise & 2013081414253101 & $12 \mathrm{R}$ & 470000 & 3510000 & 1,140 \\
\hline Coyote & Tortoise & 2013070608064401 & $12 \mathrm{R}$ & 450000 & 3540000 & 935 \\
\hline
\end{tabular}




\begin{tabular}{|c|c|c|c|c|c|c|}
\hline Mountain range & Species & Detection date and time & $\begin{array}{l}\text { UTM } \\
\text { zone }\end{array}$ & $\begin{array}{l}\text { UTM E } \\
\text { (rounded) }\end{array}$ & $\begin{array}{c}\text { UTM N } \\
\text { (rounded) }\end{array}$ & $\begin{array}{c}\text { Elevation } \\
\text { (meters) }\end{array}$ \\
\hline Coyote & Tortoise & 2013070815374401 & $12 \mathrm{R}$ & 450000 & 3540000 & 935 \\
\hline Coyote & Tortoise & 2013071110223401 & $12 \mathrm{R}$ & 450000 & 3540000 & 935 \\
\hline Coyote & Tortoise & 2013071112442401 & $12 \mathrm{R}$ & 450000 & 3540000 & 935 \\
\hline Coyote & Tortoise & 2013071308114601 & $12 \mathrm{R}$ & 450000 & 3540000 & 935 \\
\hline Coyote & Tortoise & 2013071415170501 & $12 \mathrm{R}$ & 450000 & 3540000 & 935 \\
\hline Coyote & Tortoise & 2013071515352101 & $12 \mathrm{R}$ & 450000 & 3540000 & 935 \\
\hline Coyote & Tortoise & 2013071518080001 & $12 \mathrm{R}$ & 450000 & 3540000 & 935 \\
\hline Coyote & Tortoise & 2013071816494301 & $12 \mathrm{R}$ & 450000 & 3540000 & 935 \\
\hline Coyote & Tortoise & 2013071915202101 & $12 \mathrm{R}$ & 450000 & 3540000 & 935 \\
\hline Coyote & Tortoise & 2013080511274301 & $12 \mathrm{R}$ & 450000 & 3540000 & 935 \\
\hline Coyote & Tortoise & 2013080713582901 & $12 \mathrm{R}$ & 450000 & 3540000 & 935 \\
\hline Coyote & Tortoise & 2013081017303701 & $12 \mathrm{R}$ & 450000 & 3540000 & 935 \\
\hline Coyote & Tortoise & 2013081316555601 & $12 \mathrm{R}$ & 450000 & 3540000 & 935 \\
\hline Coyote & Tortoise & 2013081508503401 & $12 \mathrm{R}$ & 450000 & 3540000 & 935 \\
\hline Coyote & Tortoise & 2013081512471001 & $12 \mathrm{R}$ & 450000 & 3540000 & 935 \\
\hline Coyote & Tortoise & 2013081516033701 & $12 \mathrm{R}$ & 450000 & 3540000 & 935 \\
\hline Coyote & Tortoise & 2013081614594601 & $12 \mathrm{R}$ & 450000 & 3540000 & 935 \\
\hline Coyote & Tortoise & 2013081615143201 & $12 \mathrm{R}$ & 450000 & 3540000 & 935 \\
\hline Coyote & Tortoise & 2013081814190001 & $12 \mathrm{R}$ & 450000 & 3540000 & 935 \\
\hline Coyote & Tortoise & 2013082412143901 & $12 \mathrm{R}$ & 450000 & 3540000 & 935 \\
\hline Coyote & Tortoise & 2013082715041701 & $12 \mathrm{R}$ & 450000 & 3540000 & 935 \\
\hline Coyote & Tortoise & 2013082911132701 & $12 \mathrm{R}$ & 450000 & 3540000 & 935 \\
\hline Coyote & Tortoise & 2013082911524301 & $12 \mathrm{R}$ & 450000 & 3540000 & 935 \\
\hline Coyote & Tortoise & 2013082912443801 & $12 \mathrm{R}$ & 450000 & 3540000 & 935 \\
\hline Coyote & Tortoise & 2013083016103201 & $12 \mathrm{R}$ & 450000 & 3540000 & 935 \\
\hline Coyote & Tortoise & 2013083016410801 & $12 \mathrm{R}$ & 450000 & 3540000 & 935 \\
\hline Coyote & Tortoise & 2013083016504701 & $12 \mathrm{R}$ & 450000 & 3540000 & 935 \\
\hline Coyote & Tortoise & 2013083111212401 & $12 \mathrm{R}$ & 450000 & 3540000 & 935 \\
\hline Coyote & Tortoise & 2013083114063701 & $12 \mathrm{R}$ & 450000 & 3540000 & 935 \\
\hline Coyote & Tortoise & 2013090217102701 & $12 \mathrm{R}$ & 450000 & 3540000 & 935 \\
\hline Coyote & Tortoise & 2013090414383701 & $12 \mathrm{R}$ & 450000 & 3540000 & 935 \\
\hline Coyote & Tortoise & 2013090516294001 & $12 \mathrm{R}$ & 450000 & 3540000 & 935 \\
\hline Coyote & Tortoise & 2013090711114501 & $12 \mathrm{R}$ & 450000 & 3540000 & 935 \\
\hline Coyote & Tortoise & 2013090713545901 & $12 \mathrm{R}$ & 450000 & 3540000 & 935 \\
\hline Coyote & Tortoise & 2013091517420801 & $12 \mathrm{R}$ & 450000 & 3540000 & 935 \\
\hline Coyote & Tortoise & 2013092610121701 & $12 \mathrm{R}$ & 450000 & 3540000 & 935 \\
\hline Coyote & Tortoise & 2013111210495501 & $12 \mathrm{R}$ & 450000 & 3540000 & 935 \\
\hline Coyote & Tortoise & 2014041009540901 & $12 \mathrm{R}$ & 450000 & 3540000 & 935 \\
\hline Coyote & Tortoise & 2014070713044501 & $12 \mathrm{R}$ & 450000 & 3540000 & 935 \\
\hline Coyote & Tortoise & 2014070715101301 & $12 \mathrm{R}$ & 450000 & 3540000 & 935 \\
\hline Coyote & Tortoise & 2014071412301201 & $12 \mathrm{R}$ & 450000 & 3540000 & 935 \\
\hline
\end{tabular}




\begin{tabular}{|c|c|c|c|c|c|c|}
\hline Mountain range & Species & Detection date and time & $\begin{array}{l}\text { UTM } \\
\text { zone }\end{array}$ & $\begin{array}{l}\text { UTM E } \\
\text { (rounded) }\end{array}$ & $\begin{array}{c}\text { UTM N } \\
\text { (rounded) }\end{array}$ & $\begin{array}{c}\text { Elevation } \\
\text { (meters) }\end{array}$ \\
\hline Coyote & Tortoise & 2014071415230801 & $12 \mathrm{R}$ & 450000 & 3540000 & 935 \\
\hline Coyote & Tortoise & 2014071415255501 & $12 \mathrm{R}$ & 450000 & 3540000 & 935 \\
\hline Coyote & Tortoise & 2014071415272801 & $12 \mathrm{R}$ & 450000 & 3540000 & 935 \\
\hline Coyote & Tortoise & 2014071415284001 & $12 \mathrm{R}$ & 450000 & 3540000 & 935 \\
\hline Coyote & Tortoise & 2014071415300201 & $12 \mathrm{R}$ & 450000 & 3540000 & 935 \\
\hline Coyote & Tortoise & 2014071415311501 & $12 \mathrm{R}$ & 450000 & 3540000 & 935 \\
\hline Coyote & Tortoise & 2014071415325601 & $12 \mathrm{R}$ & 450000 & 3540000 & 935 \\
\hline Coyote & Tortoise & 2014071415342301 & $12 \mathrm{R}$ & 450000 & 3540000 & 935 \\
\hline Coyote & Tortoise & 2014071415352701 & $12 \mathrm{R}$ & 450000 & 3540000 & 935 \\
\hline Coyote & Tortoise & 2014071608333401 & $12 \mathrm{R}$ & 450000 & 3540000 & 935 \\
\hline Coyote & Tortoise & 2014071609485601 & $12 \mathrm{R}$ & 450000 & 3540000 & 935 \\
\hline Coyote & Tortoise & 2014071610101202 & $12 \mathrm{R}$ & 450000 & 3540000 & 935 \\
\hline Coyote & Tortoise & 2014071610114102 & $12 \mathrm{R}$ & 450000 & 3540000 & 935 \\
\hline Coyote & Tortoise & 2014071610150202 & $12 \mathrm{R}$ & 450000 & 3540000 & 935 \\
\hline Coyote & Tortoise & 2014071610172002 & $12 \mathrm{R}$ & 450000 & 3540000 & 935 \\
\hline Coyote & Tortoise & 2014071610183902 & $12 \mathrm{R}$ & 450000 & 3540000 & 935 \\
\hline Coyote & Tortoise & 2014071614184001 & $12 \mathrm{R}$ & 450000 & 3540000 & 935 \\
\hline Coyote & Tortoise & 2014071614204501 & $12 \mathrm{R}$ & 450000 & 3540000 & 935 \\
\hline Coyote & Tortoise & 2014071709512301 & $12 \mathrm{R}$ & 450000 & 3540000 & 935 \\
\hline Coyote & Tortoise & 2014071711503901 & $12 \mathrm{R}$ & 450000 & 3540000 & 935 \\
\hline Coyote & Tortoise & 2014071713301201 & $12 \mathrm{R}$ & 450000 & 3540000 & 935 \\
\hline Coyote & Tortoise & 2014071809195901 & $12 \mathrm{R}$ & 450000 & 3540000 & 935 \\
\hline Coyote & Tortoise & 2014071815335601 & $12 \mathrm{R}$ & 450000 & 3540000 & 935 \\
\hline Coyote & Tortoise & 2014071914431701 & $12 \mathrm{R}$ & 450000 & 3540000 & 935 \\
\hline Coyote & Tortoise & 2014071915241401 & $12 \mathrm{R}$ & 450000 & 3540000 & 935 \\
\hline Coyote & Tortoise & 2014072507592701 & $12 \mathrm{R}$ & 450000 & 3540000 & 935 \\
\hline Coyote & Tortoise & 2014072611395601 & $12 \mathrm{R}$ & 450000 & 3540000 & 935 \\
\hline Coyote & Tortoise & 2014072809144001 & $12 \mathrm{R}$ & 450000 & 3540000 & 935 \\
\hline Coyote & Tortoise & 2014072809244301 & $12 \mathrm{R}$ & 450000 & 3540000 & 935 \\
\hline Coyote & Tortoise & 2014072810045801 & $12 \mathrm{R}$ & 450000 & 3540000 & 935 \\
\hline Coyote & Tortoise & 2014072814083401 & $12 \mathrm{R}$ & 450000 & 3540000 & 935 \\
\hline Coyote & Tortoise & 2014072814571301 & $12 \mathrm{R}$ & 450000 & 3540000 & 935 \\
\hline Coyote & Tortoise & 2014072908430801 & $12 \mathrm{R}$ & 450000 & 3540000 & 935 \\
\hline Coyote & Tortoise & 2014072913335701 & $12 \mathrm{R}$ & 450000 & 3540000 & 935 \\
\hline Coyote & Tortoise & 2014072914024101 & $12 \mathrm{R}$ & 450000 & 3540000 & 935 \\
\hline Coyote & Tortoise & 2014073112451501 & $12 \mathrm{R}$ & 450000 & 3540000 & 935 \\
\hline Coyote & Tortoise & 2014073113220301 & $12 \mathrm{R}$ & 450000 & 3540000 & 935 \\
\hline Coyote & Tortoise & 2014073117563501 & $12 \mathrm{R}$ & 450000 & 3540000 & 935 \\
\hline Coyote & Tortoise & 2014080118051001 & $12 \mathrm{R}$ & 450000 & 3540000 & 935 \\
\hline Coyote & Tortoise & 2014080308200401 & $12 \mathrm{R}$ & 450000 & 3540000 & 935 \\
\hline Coyote & Tortoise & 2014080309270301 & $12 \mathrm{R}$ & 450000 & 3540000 & 935 \\
\hline
\end{tabular}




\begin{tabular}{|c|c|c|c|c|c|c|}
\hline Mountain range & Species & Detection date and time & $\begin{array}{l}\text { UTM } \\
\text { zone }\end{array}$ & $\begin{array}{l}\text { UTM E } \\
\text { (rounded) }\end{array}$ & $\begin{array}{c}\text { UTM N } \\
\text { (rounded) }\end{array}$ & $\begin{array}{c}\text { Elevation } \\
\text { (meters) }\end{array}$ \\
\hline Coyote & Tortoise & 2014080310071001 & $12 \mathrm{R}$ & 450000 & 3540000 & 935 \\
\hline Coyote & Tortoise & 2014080409071501 & $12 \mathrm{R}$ & 450000 & 3540000 & 935 \\
\hline Coyote & Tortoise & 2014080409195701 & $12 \mathrm{R}$ & 450000 & 3540000 & 935 \\
\hline Coyote & Tortoise & 2014080415483301 & $12 \mathrm{R}$ & 450000 & 3540000 & 935 \\
\hline Coyote & Tortoise & 2014080609315001 & $12 \mathrm{R}$ & 450000 & 3540000 & 935 \\
\hline Coyote & Tortoise & 2014080616192801 & $12 \mathrm{R}$ & 450000 & 3540000 & 935 \\
\hline Coyote & Tortoise & 2014080709374601 & $12 \mathrm{R}$ & 450000 & 3540000 & 935 \\
\hline Coyote & Tortoise & 2014080807585801 & $12 \mathrm{R}$ & 450000 & 3540000 & 935 \\
\hline Coyote & Tortoise & 2014080813061002 & $12 \mathrm{R}$ & 450000 & 3540000 & 935 \\
\hline Coyote & Tortoise & 2014080813255901 & $12 \mathrm{R}$ & 450000 & 3540000 & 935 \\
\hline Coyote & Tortoise & 2014080816412001 & $12 \mathrm{R}$ & 450000 & 3540000 & 935 \\
\hline Coyote & Tortoise & 2014081114574101 & $12 \mathrm{R}$ & 450000 & 3540000 & 935 \\
\hline Coyote & Tortoise & 2014081206131601 & $12 \mathrm{R}$ & 450000 & 3540000 & 935 \\
\hline Coyote & Tortoise & 2014081211561001 & $12 \mathrm{R}$ & 450000 & 3540000 & 935 \\
\hline Coyote & Tortoise & 2014081309564801 & $12 \mathrm{R}$ & 450000 & 3540000 & 935 \\
\hline Coyote & Tortoise & 2014081408582901 & $12 \mathrm{R}$ & 450000 & 3540000 & 935 \\
\hline Coyote & Tortoise & 2014081412402801 & $12 \mathrm{R}$ & 450000 & 3540000 & 935 \\
\hline Coyote & Tortoise & 2014081414562301 & $12 \mathrm{R}$ & 450000 & 3540000 & 935 \\
\hline Coyote & Tortoise & 2014081415142001 & $12 \mathrm{R}$ & 450000 & 3540000 & 935 \\
\hline Coyote & Tortoise & 2014081509240901 & $12 \mathrm{R}$ & 450000 & 3540000 & 935 \\
\hline Coyote & Tortoise & 2014081610201101 & $12 \mathrm{R}$ & 450000 & 3540000 & 935 \\
\hline Coyote & Tortoise & 2014081619064801 & $12 \mathrm{R}$ & 450000 & 3540000 & 935 \\
\hline Coyote & Tortoise & 2014081810220401 & $12 \mathrm{R}$ & 450000 & 3540000 & 935 \\
\hline Coyote & Tortoise & 2014081916061302 & $12 \mathrm{R}$ & 450000 & 3540000 & 935 \\
\hline Coyote & Tortoise & 2014081916071902 & $12 \mathrm{R}$ & 450000 & 3540000 & 935 \\
\hline Coyote & Tortoise & 2014081916100102 & $12 \mathrm{R}$ & 450000 & 3540000 & 935 \\
\hline Coyote & Tortoise & 2014082112164201 & $12 \mathrm{R}$ & 450000 & 3540000 & 935 \\
\hline Coyote & Tortoise & 2014082112244601 & $12 \mathrm{R}$ & 450000 & 3540000 & 935 \\
\hline Coyote & Tortoise & 2014082112570101 & $12 \mathrm{R}$ & 450000 & 3540000 & 935 \\
\hline Coyote & Tortoise & 2014082112590501 & $12 \mathrm{R}$ & 450000 & 3540000 & 935 \\
\hline Coyote & Tortoise & 2014082113000801 & $12 \mathrm{R}$ & 450000 & 3540000 & 935 \\
\hline Coyote & Tortoise & 2014082113133501 & $12 \mathrm{R}$ & 450000 & 3540000 & 935 \\
\hline Coyote & Tortoise & 2014082113242401 & $12 \mathrm{R}$ & 450000 & 3540000 & 935 \\
\hline Coyote & Tortoise & 2014082209040601 & $12 \mathrm{R}$ & 450000 & 3540000 & 935 \\
\hline Coyote & Tortoise & 2014082209053301 & $12 \mathrm{R}$ & 450000 & 3540000 & 935 \\
\hline Coyote & Tortoise & 2014082209070801 & $12 \mathrm{R}$ & 450000 & 3540000 & 935 \\
\hline Coyote & Tortoise & 2014082218172701 & $12 \mathrm{R}$ & 450000 & 3540000 & 935 \\
\hline Coyote & Tortoise & 2014082218183001 & $12 \mathrm{R}$ & 450000 & 3540000 & 935 \\
\hline Coyote & Tortoise & 2014082218230601 & $12 \mathrm{R}$ & 450000 & 3540000 & 935 \\
\hline Coyote & Tortoise & 2014082315181901 & $12 \mathrm{R}$ & 450000 & 3540000 & 935 \\
\hline Coyote & Tortoise & 2014082315251301 & $12 \mathrm{R}$ & 450000 & 3540000 & 935 \\
\hline
\end{tabular}




\begin{tabular}{|c|c|c|c|c|c|c|}
\hline Mountain range & Species & Detection date and time & $\begin{array}{l}\text { UTM } \\
\text { zone }\end{array}$ & $\begin{array}{l}\text { UTM E } \\
\text { (rounded) }\end{array}$ & $\begin{array}{c}\text { UTM N } \\
\text { (rounded) }\end{array}$ & $\begin{array}{c}\text { Elevation } \\
\text { (meters) }\end{array}$ \\
\hline Coyote & Tortoise & 2014082318330801 & $12 \mathrm{R}$ & 450000 & 3540000 & 935 \\
\hline Coyote & Tortoise & 2014082410273802 & $12 \mathrm{R}$ & 450000 & 3540000 & 935 \\
\hline Coyote & Tortoise & 2014083109121501 & $12 \mathrm{R}$ & 450000 & 3540000 & 935 \\
\hline Coyote & Tortoise & 2014083109124201 & $12 \mathrm{R}$ & 450000 & 3540000 & 935 \\
\hline Coyote & Tortoise & 2014083109141601 & $12 \mathrm{R}$ & 450000 & 3540000 & 935 \\
\hline Coyote & Tortoise & 2014092217302201 & $12 \mathrm{R}$ & 450000 & 3540000 & 935 \\
\hline Coyote & Tortoise & 2014092217390101 & $12 \mathrm{R}$ & 450000 & 3540000 & 935 \\
\hline Coyote & Tortoise & 2014102611392201 & $12 \mathrm{R}$ & 450000 & 3540000 & 935 \\
\hline Coyote & Tortoise & 2013082016134001 & $12 \mathrm{R}$ & 450000 & 3540000 & 1,058 \\
\hline Coyote & Tortoise & 2013090514181401 & $12 \mathrm{R}$ & 450000 & 3540000 & 1,058 \\
\hline Coyote & Tortoise & 2014071610004501 & $12 \mathrm{R}$ & 450000 & 3540000 & 1,105 \\
\hline Coyote & Tortoise & 2014080217142801 & $12 \mathrm{R}$ & 450000 & 3540000 & 1,105 \\
\hline Coyote & Tortoise & 2014080318004901 & $12 \mathrm{R}$ & 450000 & 3540000 & 1,105 \\
\hline Coyote & Tortoise & 2014082612365301 & $12 \mathrm{R}$ & 450000 & 3540000 & 1,105 \\
\hline Peloncillo & Mud Turtle & 2013100711523801 & $12 \mathrm{R}$ & 690000 & 3480000 & 1,758 \\
\hline Peloncillo & Mud Turtle & 2013100711543401 & $12 \mathrm{R}$ & 690000 & 3480000 & 1,758 \\
\hline Peloncillo & Mud Turtle & 2013100711592801 & $12 \mathrm{R}$ & 690000 & 3480000 & 1,758 \\
\hline Peloncillo & Mud Turtle & 2013100811130901 & $12 \mathrm{R}$ & 690000 & 3480000 & 1,758 \\
\hline Peloncillo & Mud Turtle & 2013100811183301 & $12 \mathrm{R}$ & 690000 & 3480000 & 1,758 \\
\hline Peloncillo & Mud Turtle & 2013100811213401 & $12 \mathrm{R}$ & 690000 & 3480000 & 1,758 \\
\hline Peloncillo & Mud Turtle & 2013100811224001 & $12 \mathrm{R}$ & 690000 & 3480000 & 1,758 \\
\hline Peloncillo & Mud Turtle & 2013100811283301 & $12 \mathrm{R}$ & 690000 & 3480000 & 1,758 \\
\hline Peloncillo & Mud Turtle & 2013100811313501 & $12 \mathrm{R}$ & 690000 & 3480000 & 1,758 \\
\hline Peloncillo & Mud Turtle & 2013100811324701 & $12 \mathrm{R}$ & 690000 & 3480000 & 1,758 \\
\hline Peloncillo & Mud Turtle & 2013100811362701 & $12 \mathrm{R}$ & 690000 & 3480000 & 1,758 \\
\hline Peloncillo & Mud Turtle & 2013100811425301 & $12 \mathrm{R}$ & 690000 & 3480000 & 1,758 \\
\hline Peloncillo & Mud Turtle & 2013100811443901 & $12 \mathrm{R}$ & 690000 & 3480000 & 1,758 \\
\hline Peloncillo & Mud Turtle & 2013100811462301 & $12 \mathrm{R}$ & 690000 & 3480000 & 1,758 \\
\hline Peloncillo & Mud Turtle & 2013100811502401 & $12 \mathrm{R}$ & 690000 & 3480000 & 1,758 \\
\hline Peloncillo & Mud Turtle & 2013100811522601 & $12 \mathrm{R}$ & 690000 & 3480000 & 1,758 \\
\hline Peloncillo & Mud Turtle & 2013100909294901 & $12 \mathrm{R}$ & 690000 & 3480000 & 1,758 \\
\hline Peloncillo & Mud Turtle & 2013100909323501 & $12 \mathrm{R}$ & 690000 & 3480000 & 1,758 \\
\hline Peloncillo & Mud Turtle & 2013100909364501 & $12 \mathrm{R}$ & 690000 & 3480000 & 1,758 \\
\hline Peloncillo & Mud Turtle & 2013100909390101 & $12 \mathrm{R}$ & 690000 & 3480000 & 1,758 \\
\hline Peloncillo & Mud Turtle & 2013100909413501 & $12 \mathrm{R}$ & 690000 & 3480000 & 1,758 \\
\hline Peloncillo & Mud Turtle & 2013100909541002 & $12 \mathrm{R}$ & 690000 & 3480000 & 1,758 \\
\hline Peloncillo & Mud Turtle & 2013100910011402 & $12 \mathrm{R}$ & 690000 & 3480000 & 1,758 \\
\hline Peloncillo & Mud Turtle & 2013100910063302 & $12 \mathrm{R}$ & 690000 & 3480000 & 1,758 \\
\hline Peloncillo & Mud Turtle & 2013100910081502 & $12 \mathrm{R}$ & 690000 & 3480000 & 1,758 \\
\hline Peloncillo & Mud Turtle & 2013100910121402 & $12 \mathrm{R}$ & 690000 & 3480000 & 1,758 \\
\hline Peloncillo & Mud Turtle & 2013100910142202 & $12 \mathrm{R}$ & 690000 & 3480000 & 1,758 \\
\hline
\end{tabular}




\begin{tabular}{|c|c|c|c|c|c|c|}
\hline Mountain range & Species & Detection date and time & $\begin{array}{l}\text { UTM } \\
\text { zone }\end{array}$ & $\begin{array}{l}\text { UTM E } \\
\text { (rounded) }\end{array}$ & $\begin{array}{l}\text { UTM N } \\
\text { (rounded) }\end{array}$ & $\begin{array}{l}\text { Elevation } \\
\text { (meters) }\end{array}$ \\
\hline Peloncillo & Mud Turtle & 2013100910160202 & $12 \mathrm{R}$ & 690000 & 3480000 & 1,758 \\
\hline Peloncillo & Mud Turtle & 2013100910200402 & $12 \mathrm{R}$ & 690000 & 3480000 & 1,758 \\
\hline Peloncillo & Mud Turtle & 2013100910323202 & $12 \mathrm{R}$ & 690000 & 3480000 & 1,758 \\
\hline Peloncillo & Mud Turtle & 2013100910383202 & $12 \mathrm{R}$ & 690000 & 3480000 & 1,758 \\
\hline Peloncillo & Mud Turtle & 2013100910411001 & $12 \mathrm{R}$ & 690000 & 3480000 & 1,758 \\
\hline Peloncillo & Mud Turtle & 2013100910434801 & $12 \mathrm{R}$ & 690000 & 3480000 & 1,758 \\
\hline Peloncillo & Mud Turtle & 2013100910455801 & $12 \mathrm{R}$ & 690000 & 3480000 & 1,758 \\
\hline Peloncillo & Mud Turtle & 2013100910511201 & $12 \mathrm{R}$ & 690000 & 3480000 & 1,758 \\
\hline Peloncillo & Mud Turtle & 2013100910524801 & $12 \mathrm{R}$ & 690000 & 3480000 & 1,758 \\
\hline Peloncillo & Mud Turtle & 2013100910550201 & $12 \mathrm{R}$ & 690000 & 3480000 & 1,758 \\
\hline Peloncillo & Mud Turtle & 2013100910572401 & $12 \mathrm{R}$ & 690000 & 3480000 & 1,758 \\
\hline Peloncillo & Mud Turtle & 2013100910590301 & $12 \mathrm{R}$ & 690000 & 3480000 & 1,758 \\
\hline Peloncillo & Mud Turtle & 2013101011332401 & $12 \mathrm{R}$ & 690000 & 3480000 & 1,758 \\
\hline Peloncillo & Mud Turtle & 2013101011344201 & $12 \mathrm{R}$ & 690000 & 3480000 & 1,758 \\
\hline Peloncillo & Mud Turtle & 2013101011372901 & $12 \mathrm{R}$ & 690000 & 3480000 & 1,758 \\
\hline Peloncillo & Mud Turtle & 2013101110221301 & $12 \mathrm{R}$ & 690000 & 3480000 & 1,758 \\
\hline Peloncillo & Mud Turtle & 2013101110482801 & $12 \mathrm{R}$ & 690000 & 3480000 & 1,758 \\
\hline Peloncillo & Mud Turtle & 2013101110505001 & $12 \mathrm{R}$ & 690000 & 3480000 & 1,758 \\
\hline Peloncillo & Mud Turtle & 2013101110525601 & $12 \mathrm{R}$ & 690000 & 3480000 & 1,758 \\
\hline Peloncillo & Mud Turtle & 2013101111080901 & $12 \mathrm{R}$ & 690000 & 3480000 & 1,758 \\
\hline Peloncillo & Mud Turtle & 2013101111131501 & $12 \mathrm{R}$ & 690000 & 3480000 & 1,758 \\
\hline Peloncillo & Mud Turtle & 2013101210492001 & $12 \mathrm{R}$ & 690000 & 3480000 & 1,758 \\
\hline Peloncillo & Mud Turtle & 2013101210550701 & $12 \mathrm{R}$ & 690000 & 3480000 & 1,758 \\
\hline Peloncillo & Mud Turtle & 2013101210593801 & $12 \mathrm{R}$ & 690000 & 3480000 & 1,758 \\
\hline Peloncillo & Mud Turtle & 2013110311184201 & $12 \mathrm{R}$ & 690000 & 3480000 & 1,758 \\
\hline Peloncillo & Mud Turtle & 2013111010433401 & $12 \mathrm{R}$ & 690000 & 3480000 & 1,758 \\
\hline Peloncillo & Mud Turtle & 2013111010474101 & $12 \mathrm{R}$ & 690000 & 3480000 & 1,758 \\
\hline Peloncillo & Mud Turtle & 2013111010560801 & $12 \mathrm{R}$ & 690000 & 3480000 & 1,758 \\
\hline Peloncillo & Mud Turtle & 2013111010595701 & $12 \mathrm{R}$ & 690000 & 3480000 & 1,758 \\
\hline Peloncillo & Mud Turtle & 2013111011081301 & $12 \mathrm{R}$ & 690000 & 3480000 & 1,758 \\
\hline Peloncillo & Mud Turtle & 2013111011144001 & $12 \mathrm{R}$ & 690000 & 3480000 & 1,758 \\
\hline
\end{tabular}


Table 7. Species and species categories and percent of analyzed sites $(n=233)$ at which each species or species category was detected from 2012 to 2015.

[Squirrels are separated from the rodent species category, and turkeys are separated from the bird species category]

\begin{tabular}{|c|c|c|}
\hline Species & $\begin{array}{l}\text { Percent of sites } \\
\text { occupied (percent) }\end{array}$ & $\begin{array}{l}\text { Total sites } \\
\text { occupied }\end{array}$ \\
\hline White-tailed Deer & 91 & 211 \\
\hline Human & 89 & 207 \\
\hline Fox & 88 & 205 \\
\hline Puma & 81 & 188 \\
\hline Skunks & 80 & 186 \\
\hline Squirrels & 78 & 181 \\
\hline Birds & 77 & 180 \\
\hline Bobcat & 73 & 171 \\
\hline Coati & 71 & 166 \\
\hline Javelina & 58 & 134 \\
\hline Bear & 55 & 127 \\
\hline Cottontail & 49 & 114 \\
\hline Dog & 44 & 103 \\
\hline Cow & 39 & 91 \\
\hline Ringtail & 34 & 79 \\
\hline Coyote & 33 & 76 \\
\hline Opossum & 31 & 73 \\
\hline Turkey & 29 & 68 \\
\hline Rodents & 20 & 46 \\
\hline Lizards & 16 & 38 \\
\hline Raccoon & 11 & 25 \\
\hline Jaguar & 10 & 23 \\
\hline Snakes & 6 & 14 \\
\hline Horse & 6 & 14 \\
\hline Mule Deer & 4 & 9 \\
\hline Ocelot & 4 & 9 \\
\hline Jackrabbits & 4 & 9 \\
\hline Bighorn & 0 & 1 \\
\hline Badger & 0 & 1 \\
\hline Pronghorn & 0 & 1 \\
\hline
\end{tabular}


Table 8. Total number of independent photos per species/species category, average number of individuals per independent photo, and total abundance and relative abundance for species detected from April 2012 to February 2015.

[Squirrels are separated from the rodent species category, and turkeys are separated from the bird species category]

\begin{tabular}{|c|c|c|c|c|}
\hline Species & $\begin{array}{l}\text { Total number of } \\
\text { photos }\end{array}$ & $\begin{array}{c}\text { Average number of } \\
\text { individuals per } \\
\text { photo }\end{array}$ & Abundance & $\begin{array}{c}\text { Relative } \\
\text { abundance }\end{array}$ \\
\hline Badger & 1 & 1.00 & 1 & 0.00 \\
\hline Goat & 1 & 2.00 & 2 & 0.00 \\
\hline Pronghorn & 1 & 1.00 & 1 & 0.00 \\
\hline Bighorn & 2 & 1.50 & 3 & 0.00 \\
\hline Domestic Cat & 2 & 1.00 & 2 & 0.00 \\
\hline Ocelot & 10 & 1.00 & 10 & 0.01 \\
\hline Frogs & 25 & 1.04 & 26 & 0.02 \\
\hline Snakes & 25 & 1.00 & 25 & 0.02 \\
\hline Raccoon & 38 & 1.00 & 38 & 0.02 \\
\hline Bats & 60 & 1.05 & 63 & 0.04 \\
\hline Jaguar & 84 & 1.00 & 84 & 0.06 \\
\hline Mule Deer & 101 & 1.23 & 124 & 0.07 \\
\hline Tortoise & 105 & 1.05 & 110 & 0.07 \\
\hline Lizards & 134 & 1.00 & 134 & 0.09 \\
\hline Horse & 180 & 1.19 & 214 & 0.12 \\
\hline Jackrabbits & 197 & 1.02 & 200 & 0.13 \\
\hline Rodents & 541 & 1.00 & 541 & 0.35 \\
\hline Dog & 633 & 1.25 & 789 & 0.42 \\
\hline Opossum & 720 & 1.00 & 721 & 0.47 \\
\hline Turkey & 889 & 1.55 & 1,378 & 0.58 \\
\hline Coyote & 987 & 1.09 & 1,080 & 0.65 \\
\hline Ringtail & 1,182 & 1.01 & 1,197 & 0.78 \\
\hline Bobcat & 1,478 & 1.01 & 1,488 & 0.97 \\
\hline Puma & 2,803 & 1.02 & 2,873 & 1.84 \\
\hline Javelina & 3,033 & 1.74 & 5,281 & 1.99 \\
\hline Coati & 3,131 & 1.66 & 5,211 & 2.05 \\
\hline Cow & 3,197 & 1.40 & 4,475 & 2.10 \\
\hline Cottontail & 3,467 & 1.01 & 3,515 & 2.27 \\
\hline Bear & 4,279 & 1.06 & 4,537 & 2.81 \\
\hline Squirrels & 4,525 & 1.01 & 4,588 & 2.97 \\
\hline Skunks & 4,699 & 1.01 & 4,737 & 3.08 \\
\hline Birds & 4,743 & 1.18 & 5,590 & 3.11 \\
\hline Human & 6,287 & 1.31 & 8,224 & 4.12 \\
\hline Fox & 8,049 & 1.03 & 8,305 & 5.28 \\
\hline White-tailed deer & 17,258 & 1.24 & 21,383 & 11.32 \\
\hline
\end{tabular}


Table 9. Ranked differences for species activity based on full moons versus new moons for species detected from April 2012 to February 2015.

[Higher values indicate a greater statistical difference in activity. Number of records used to estimate differences is included.

Squirrels are separated from the rodent species category]

\begin{tabular}{|c|c|c|}
\hline Species & Difference & $\begin{array}{l}\text { Number of } \\
\text { records }\end{array}$ \\
\hline Ocelot & 0.82 & 8 \\
\hline Snakes & 0.49 & 17 \\
\hline Raccoon & 0.40 & 26 \\
\hline Bats & 0.32 & 72 \\
\hline Frogs & 0.26 & 16 \\
\hline Jackrabbits & 0.18 & 151 \\
\hline Rodents & 0.17 & 432 \\
\hline Jaguar & 0.16 & 58 \\
\hline Ringtail & 0.08 & 960 \\
\hline Coyote & 0.08 & 765 \\
\hline Opossum & 0.07 & 547 \\
\hline Bobcat & 0.07 & 1,099 \\
\hline Coati & 0.05 & 2,557 \\
\hline Puma & 0.05 & 2,238 \\
\hline Cow & 0.04 & 3,174 \\
\hline Skunks & 0.04 & 3,663 \\
\hline Bear & 0.04 & 3,511 \\
\hline Fox & 0.04 & 6,803 \\
\hline Cottontail & 0.04 & 2,809 \\
\hline Squirrels & 0.03 & 3,739 \\
\hline Javelina & 0.03 & 2,551 \\
\hline White-tailed deer & 0.02 & 15,260 \\
\hline
\end{tabular}


Table 10. Sample identification (ID), collection date, collection location, and analysis date for 60 scat samples collected and analyzed for species ID.

[Jaguar samples are highlighted in red. Species IDs were obtained by searching the GenBank online nucleotide database. ID, identification; AZ, Arizona; Mtns, mountains; NA, not available]

\begin{tabular}{|c|c|c|c|c|c|}
\hline Sample No. & Sample ID & $\begin{array}{l}\text { Species ID from } \\
\text { GenBank database }\end{array}$ & Mountain range & Collection date & Analysis date \\
\hline 1 & 64341 & Puma concolor & Chiricahua Mtns, AZ & $4 / 15 / 2013$ & 7/17/2013 \\
\hline 2 & 48298 & NA & Baboquivari Mtns, AZ & $6 / 23 / 2013$ & 7/17/2013 \\
\hline 3 & 64079 & Puma concolor & Patagonia Mtns, AZ & $7 / 3 / 2013$ & 7/17/2013 \\
\hline 4 & 33829 & Puma concolor & Baboquivari Mtns, AZ & 8/4/2012 & 7/17/2013 \\
\hline 5 & 63336 & NA & Huachuca Mtns, AZ & $12 / 3 / 2012$ & 7/17/2013 \\
\hline 6 & 0521 & NA & Coyote Mtns, AZ & $5 / 21 / 2013$ & 7/17/2013 \\
\hline 7 & 0702 & Puma concolor & Coyote Mtns, AZ & $7 / 2 / 2013$ & 7/17/2013 \\
\hline 8 & 1 & Odocoileus virginianus & Whetstone Mtns, AZ & $3 / 1 / 2012$ & 7/17/2013 \\
\hline 9 & 16121201 & Puma concolor & Santa Rita Mtns, AZ & $12 / 16 / 2012$ & 7/3/2013 \\
\hline 10 & 12011301 & Puma concolor & Santa Rita Mtns, AZ & $1 / 12 / 2013$ & 7/3/2013 \\
\hline 11 & 17011301 & Puma concolor & Santa Rita Mtns, AZ & $1 / 17 / 2013$ & 7/3/2013 \\
\hline 12 & 17011302 & Puma concolor & Santa Rita Mtns, AZ & $1 / 17 / 2013$ & 7/3/2013 \\
\hline 13 & 17011303 & Puma concolor & Santa Rita Mtns, AZ & $1 / 17 / 2013$ & 7/3/2013 \\
\hline 14 & 17011304 & Panthera onca & Santa Rita Mtns, AZ & $1 / 17 / 2013$ & 7/3/2013 \\
\hline 15 & 15021303 & Puma concolor & Santa Rita Mtns, AZ & $2 / 15 / 2013$ & 7/3/2013 \\
\hline 16 & 15021304 & NA & Santa Rita Mtns, AZ & $2 / 15 / 2013$ & 7/3/2013 \\
\hline 17 & 19021301 & Puma concolor & Santa Rita Mtns, AZ & $2 / 19 / 2013$ & 7/3/2013 \\
\hline 18 & 26021301 & NA & Santa Rita Mtns, AZ & $2 / 26 / 2013$ & 7/3/2013 \\
\hline 19 & 26021302 & Puma concolor & Santa Rita Mtns, AZ & $2 / 26 / 2013$ & 7/3/2013 \\
\hline 20 & 26021303 & Canis latrans & Santa Rita Mtns, AZ & $2 / 26 / 2013$ & 7/3/2013 \\
\hline 21 & 26021304 & Canis latrans & Santa Rita Mtns, AZ & $2 / 26 / 2013$ & 7/3/2013 \\
\hline 22 & 20031301 & Pecari tajacu & Huachuca Mtns, AZ & $3 / 20 / 2013$ & 7/3/2013 \\
\hline 23 & 20031301 & Odocoileus virginianus & Huachuca Mtns, AZ & $3 / 20 / 2013$ & 6/28/2014 \\
\hline 24 & 20031301 & Antilocapra americana & Huachuca Mtns, AZ & $3 / 20 / 2013$ & $1 / 15 / 2015$ \\
\hline 25 & 02041302 & NA & Santa Rita Mtns, AZ & $4 / 2 / 2013$ & 7/3/2013 \\
\hline 26 & 03041301 & Lynx rufus & Santa Rita Mtns, AZ & $4 / 3 / 2013$ & 7/3/2013 \\
\hline 27 & 17041301 & Puma concolor & Santa Rita Mtns, AZ & $4 / 17 / 2013$ & 7/3/2013 \\
\hline 28 & 26041301 & Panthera onca & Santa Rita Mtns, AZ & $4 / 26 / 2013$ & 7/3/2013 \\
\hline 29 & 20051301 & Puma concolor & Santa Rita Mtns, AZ & $5 / 20 / 2013$ & 7/3/2013 \\
\hline 30 & 20051309 & Puma concolor & Santa Rita Mtns, AZ & $5 / 20 / 2013$ & 7/3/2013 \\
\hline 31 & 09071301 & Ursus americanus & Santa Rita Mtns, AZ & 7/9/2013 & 9/22/2013 \\
\hline 32 & 24071301 & Puma concolor & Santa Rita Mtns, AZ & $7 / 24 / 2013$ & 9/22/2013 \\
\hline 33 & 13091301 & NA & Santa Rita Mtns, AZ & 9/13/2013 & 9/22/2013 \\
\hline 34 & 13091301 & NA & Santa Rita Mtns, AZ & 9/13/2013 & $1 / 16 / 2014$ \\
\hline 35 & 18091301 & Puma concolor & Santa Rita Mtns, AZ & 9/18/2013 & 9/22/2013 \\
\hline 36 & 02101301 & Puma concolor & Santa Rita Mtns, AZ & $10 / 2 / 2013$ & $1 / 16 / 2014$ \\
\hline 37 & 02101302 & Puma concolor & Santa Rita Mtns, AZ & $10 / 2 / 2013$ & $1 / 16 / 2014$ \\
\hline 38 & 02101303 & NA & Santa Rita Mtns, AZ & $10 / 2 / 2013$ & $1 / 16 / 2014$ \\
\hline 39 & 02101303 & Panthera onca & Santa Rita Mtns, AZ & $10 / 2 / 2013$ & $1 / 16 / 2014$ \\
\hline 40 & 23101301 & Lynx rufus & Santa Rita Mtns, AZ & $10 / 23 / 2013$ & $1 / 16 / 2014$ \\
\hline 41 & 23101302 & Panthera onca & Santa Rita Mtns, AZ & $10 / 23 / 2013$ & $1 / 16 / 2014$ \\
\hline 42 & 10121301 & NA & Santa Rita Mtns, AZ & $12 / 10 / 2013$ & $1 / 16 / 2014$ \\
\hline
\end{tabular}




\begin{tabular}{|c|c|c|c|c|c|}
\hline Sample No. & Sample ID & $\begin{array}{l}\text { Species ID from } \\
\text { GenBank database }\end{array}$ & Mountain range & Collection date & Analysis date \\
\hline 43 & 11041401 & NA & Santa Rita Mtns, AZ & $4 / 11 / 2014$ & $6 / 28 / 2014$ \\
\hline 44 & 11041401 & Panthera onca & Santa Rita Mtns, AZ & $4 / 11 / 2014$ & $1 / 15 / 2015$ \\
\hline 45 & 08051401 & Panthera onca & Santa Rita Mtns, AZ & $5 / 8 / 2014$ & $6 / 28 / 2014$ \\
\hline 46 & 08051402 & Panthera onca & Santa Rita Mtns, AZ & $5 / 8 / 2014$ & $6 / 28 / 2014$ \\
\hline 47 & 08051403 & Panthera onca & Santa Rita Mtns, AZ & $5 / 8 / 2014$ & $6 / 28 / 2014$ \\
\hline 48 & 22061402 & Pecari tajacu & Santa Rita Mtns, AZ & $6 / 22 / 2014$ & $6 / 28 / 2014$ \\
\hline 49 & 22061403 & NA & Santa Rita Mtns, AZ & $6 / 22 / 2014$ & $6 / 28 / 2014$ \\
\hline 50 & 22101402 & NA & Santa Rita Mtns, AZ & $10 / 22 / 2014$ & $1 / 15 / 2015$ \\
\hline 51 & 07011501 & Panthera onca & Santa Rita Mtns, AZ & $1 / 7 / 2015$ & $1 / 15 / 2015$ \\
\hline 52 & 07011502 & Panthera onca & Santa Rita Mtns, AZ & $1 / 7 / 2015$ & $1 / 15 / 2015$ \\
\hline 53 & 07011503 & Panthera onca & Santa Rita Mtns, AZ & 1/7/2015 & $1 / 15 / 2015$ \\
\hline 54 & 07011504 & Panthera onca & Santa Rita Mtns, AZ & $1 / 7 / 2015$ & $1 / 15 / 2015$ \\
\hline 55 & 07011505 & NA & Santa Rita Mtns, AZ & $1 / 7 / 2015$ & $1 / 15 / 2015$ \\
\hline 56 & 07011506 & Panthera onca & Santa Rita Mtns, AZ & $1 / 7 / 2015$ & $1 / 15 / 2015$ \\
\hline 57 & Small cat scat 59 & Urocyon & Santa Rita Mtns, AZ & $5 / 27 / 2014$ & 7/9/2014 \\
\hline 58 & Small cat scat 60 & Lynx rufus & Santa Rita Mtns, AZ & $5 / 27 / 2014$ & 7/9/2014 \\
\hline 59 & Small cat scat 61 & Lynx rufus & Santa Rita Mtns, AZ & $5 / 27 / 2014$ & 7/9/2014 \\
\hline 60 & 63 & Lynx rufus & Patagonia Mtns, AZ & $5 / 27 / 2014$ & 7/9/2014 \\
\hline
\end{tabular}


Table 11. A list of mountain ranges showing 43 semi-randomly selected camera locations where iButtons were deployed.

[UTM zone, GPS location, and elevation in meters are also shown. iButton locations with jaguar detections are bold (n=9). iButton locations with ocelot detections are shown in italics. iButton locations recording jaguar and ocelot detections are bold and italicized ( $\mathrm{n}=2)$. UTM, Universal Transverse Mercator; GPS, Global Positioning System]

\begin{tabular}{|c|c|c|c|c|}
\hline Mountain range & Location & UTM zone & $\begin{array}{l}\text { GPS location (UTM East \& North, } \\
\text { rounded) }\end{array}$ & $\begin{array}{l}\text { Elevation } \\
\text { (meters) }\end{array}$ \\
\hline Atascosa & ATA03 & $12 \mathrm{R}$ & 480000,3470000 & 1,450 \\
\hline Atascosa & ATA05 & $12 \mathrm{R}$ & 480000, 3480000 & 1,210 \\
\hline Baboquivari & BAB02 & $12 \mathrm{R}$ & 440000,3510000 & 1,337 \\
\hline Baboquivari & ВАВ07 & $12 \mathrm{R}$ & 440000,3500000 & 1,353 \\
\hline Baboquivari & BAB10 & $12 \mathrm{R}$ & 440000, 3490000 & 1,237 \\
\hline Baboquivari & BAB14 & $12 \mathrm{R}$ & 440000, 3510000 & 1,501 \\
\hline Canelo Hills & CAN06 & $12 \mathrm{R}$ & 520000, 3490000 & 1,270 \\
\hline Canelo Hills & CAN07 & $12 \mathrm{R}$ & 530000, 3490000 & 1,380 \\
\hline Cerro Colorado & CER03 & $12 \mathrm{R}$ & 470000,3500000 & 1,190 \\
\hline Chiricahua & CHI02 & $12 \mathrm{R}$ & 650000,3530000 & 1,910 \\
\hline Chiricahua & CHI11 & $12 \mathrm{R}$ & 650000,3520000 & 1,854 \\
\hline Dos Cabezas & DOS02 & $12 \mathrm{R}$ & 640000,3560000 & 1,684 \\
\hline Dos Cabezas & DOS03 & $12 \mathrm{R}$ & 640000,3550000 & 1,527 \\
\hline Huachuca & HUA03 & $12 \mathrm{R}$ & 550000,3480000 & 1,680 \\
\hline Ниасhиса & HUA04 & $12 \mathrm{R}$ & 560000,3470000 & 1,910 \\
\hline Huachuca & HUA09 & $12 \mathrm{R}$ & 550000,3470000 & 1,740 \\
\hline Huachuca & HUA15 & $12 \mathrm{R}$ & 560000,3470000 & 2,160 \\
\hline Huachuca & HUA16 & $12 \mathrm{R}$ & 550000,3480000 & 1,800 \\
\hline Pajarito & PAJ01 & $12 \mathrm{R}$ & 480000, 3470000 & 1,210 \\
\hline Pajarito & PAJ07 & $12 \mathrm{R}$ & 480000,3470000 & 1,300 \\
\hline Pajarito & PAJ18 & $12 \mathrm{R}$ & 490000,3470000 & 1,250 \\
\hline Patagonia & PAT02 & $12 \mathrm{R}$ & 520000,3470000 & 1,430 \\
\hline Santa Rita & SAN01 & $12 \mathrm{R}$ & 520000,3510000 & 1,440 \\
\hline Santa Rita & SAN04 & $12 \mathrm{R}$ & 520000, 3520000 & 1,680 \\
\hline Santa Rita & SAN08 & $12 \mathrm{R}$ & 520000,3520000 & 1,810 \\
\hline Santa Rita & SAN11 & $12 \mathrm{R}$ & 510000,3500000 & 1,585 \\
\hline Santa Rita & SAN15 & $12 \mathrm{R}$ & 520000,3510000 & 1,540 \\
\hline Santa Rita & SAN16 & $12 \mathrm{R}$ & 5200003510000 & 1,520 \\
\hline Santa Rita & SAN19 & $12 \mathrm{R}$ & 510000,3490000 & 1,770 \\
\hline Santa Rita & SAN20 & $12 \mathrm{R}$ & 510000,3500000 & 1,885 \\
\hline Santa Rita & SAN22 & $12 \mathrm{R}$ & 510000,3500000 & 1,775 \\
\hline Santa Rita & SAN23 & $12 \mathrm{R}$ & 510000,3500000 & 1,720 \\
\hline Santa Rita & SAN24 & $12 \mathrm{R}$ & 510000,3500000 & 1,625 \\
\hline
\end{tabular}




\begin{tabular}{lcccc}
\hline Mountain range & Location & UTM zone & $\begin{array}{c}\text { GPS location (UTM East \& North, } \\
\text { rounded) }\end{array}$ & $\begin{array}{c}\text { Elevation } \\
\text { (meters) }\end{array}$ \\
\hline Santa Rita & SAN30 & $12 \mathrm{R}$ & $\mathbf{5 1 0 0 0 0 , 3 4 9 0 0 0 0}$ & $\mathbf{1 , 4 9 5}$ \\
Santa Rita & SAN34 & $12 \mathrm{R}$ & 500000,3500000 & 1,710 \\
Santa Rita & SAN51 & $12 \mathrm{R}$ & 500000,3500000 & 1,620 \\
Santa Rita & SAN56 & $12 \mathrm{R}$ & 510000,3510000 & 1,795 \\
San Luis & SLU03 & $12 \mathrm{R}$ & 450000,3490000 & 1,125 \\
San Luis & SLU07 & $12 \mathrm{R}$ & 460000,3480000 & 1,250 \\
Tumacacori & TUM01 & $12 \mathrm{R}$ & 480000,3490000 & 1,100 \\
Tumacacori & TUM05 & $12 \mathrm{R}$ & 480000,3490000 & 1,300 \\
Whetstone & WHE03 & $12 \mathrm{R}$ & 550000,3520000 & 1,817 \\
Whetstone & WHE08 & $12 \mathrm{R}$ & 550000,3510000 & 1,729 \\
\hline
\end{tabular}

Table 12. WorldClim bioclimatic variables evaluated for jaguar habitat suitability model performance (Hijmans and others, 2005; http://www.worldclim.org).

[Bioclimatic variable name and a description of the bioclimatic variable are included, also whether that variable was included in the final model]

\begin{tabular}{llc}
\hline \multicolumn{1}{c}{ Variable name } & \multicolumn{1}{c}{ Description } & $\begin{array}{c}\text { Included in final } \\
\text { model }\end{array}$ \\
\hline BIO1 & Annual mean temperature & Yes \\
BIO6 & Minimum temperature of coldest month & No \\
BIO12 & Annual precipitation & Yes \\
BIO19 & Precipitation of coldest quarter & No \\
\hline
\end{tabular}

Table 13. Evaluation statistics for top performing jaguar habitat suitability models for southeastern Arizona and southwestern New Mexico.

[Which model used, which beta test version used, how many parameters were used, $\mathrm{AIC}_{\mathrm{C}}, \Delta$ (delta) $\mathrm{AIC}_{\mathrm{C}}$, Likelihood of that model, weight, $\mathrm{AUC}_{\text {test }}$, and $\mathrm{AUC}_{\text {test }} \mathrm{SD}$ are included. $\mathrm{AIC}_{\mathrm{C}}$, Akaike Information Criterion; $\Delta$, change; SD, standard deviation]

\begin{tabular}{|c|c|c|c|c|c|c|c|c|}
\hline Model & Beta & Parameters & $\mathrm{AIC}_{\mathrm{c}}$ & $\Delta \mathrm{AIC}_{\mathrm{c}}$ & Likelihood & Weight & $A U C_{\text {test }}$ & $A U C_{\text {test }} S D$ \\
\hline 1 & 3 & 16 & 1283.25 & 0.00 & 1.0000 & 0.9982 & 0.873 & 0.024 \\
\hline 2 & 3 & 12 & 1296.47 & 13.22 & 0.0013 & 0.0013 & 0.875 & 0.014 \\
\hline 3 & 3 & 18 & 1299.19 & 15.94 & 0.0003 & 0.0003 & 0.878 & 0.015 \\
\hline 4 & 5 & 13 & 1301.39 & 18.15 & 0.0001 & 0.0001 & 0.872 & 0.031 \\
\hline
\end{tabular}


Table 14. Bioclimatic and environmental variables included in the four top performing jaguar habitat suitability models (Models 1, 2, 3, and 4) for southeastern Arizona and southwestern New Mexico.

[NDVI, Normalized Difference Vegetation Index]

\begin{tabular}{lccc}
\hline \multicolumn{1}{c}{ Variables } & Models 1, 4 & Model 2 & Model 3 \\
\hline Elevation & & & $\mathrm{x}$ \\
Human Influence Index & $\mathrm{x}$ & & $\mathrm{x}$ \\
NDVI (August, 2014) & $\mathrm{x}$ & & $\mathrm{x}$ \\
NDVI (March 2015) & $\mathrm{x}$ & & $\mathrm{x}$ \\
Tree cover & $\mathrm{x}$ & $\mathrm{x}$ & $\mathrm{x}$ \\
Terrain Ruggedness Index & $\mathrm{x}$ & $\mathrm{x}$ & $\mathrm{x}$ \\
Bio1 (Annual Mean & $\mathrm{x}$ & $\mathrm{x}$ & $\mathrm{x}$ \\
Temperature) & $\mathrm{x}$ & $\mathrm{x}$ & $\mathrm{x}$ \\
Bio12 (Annual Precipitation) & & & \\
\hline
\end{tabular}

Table 15. Jaguar habitat suitability statistics for current photo locations (2012-2015), all historical locations (19652009), and these locations combined.

[Location type, number, minimum, maximum, mean, and median habitat suitability are included. $\mathrm{N}$, number of locations; HS, habitat suitability]

\begin{tabular}{lccccc}
\hline \multicolumn{1}{c}{ Location type } & N & HS min & HS max & HS mean & $\begin{array}{c}\text { HS } \\
\text { median }\end{array}$ \\
\hline All & 58 & 0.086 & 0.847 & 0.591 & 0.656 \\
Historical & 35 & 0.086 & 0.843 & 0.539 & 0.611 \\
Current & 23 & 0.327 & 0.847 & 0.679 & 0.715 \\
\hline
\end{tabular}




\title{
Appendixes
}

\section{Appendix 1. Document Provided to Landowners and Grazing Permittees about the Jaguar Monitoring Project to Solicit Cooperation in Access to Land That They Own or Use}

\author{
Jaguar Survey and Monitoring Project \\ Ranching Community (Landowner/Grazing Permittee) Coordination Protocol
}

February 2013

The University of Arizona Jaguar Survey and Monitoring Project has identified your area for potential placement of wildlife cameras to track the potential movement of jaguars. Your area is one of over 120 sites that are being considered as possible locations where jaguar might occur in the United States-Mexico Border region from the east side of the Baboquivaris in Arizona to the "Boot-heel" area of southwestern New Mexico. At each site a pair of cameras will be located and periodically checked to download photos and replace batteries as needed. The cameras may then be moved to a new location, or will remain fixed for the duration of the project (2-3 years). Large carnivore scats will also be collected for genetic analysis.

Field cameras will be set and monitored in accordance with the following protocol.

1. Project personnel will coordinate with landowners/grazing permittees before cameras are set.

2. If it is necessary to access public lands through private property, project personnel will request written permission of landowners for access. (In some cases, where rural roads crisscross many parcels and/or landowners are out of state or not available to be notified, field teams may drive/ride through on existing, non-posted roads). When permission is granted for access, field teams or the project manager will coordinate with landowners as to when they are likely to be on the property (ideally within a $2-5$ day window) to set cameras or return to download photos, and we will provide names and vehicle descriptions. Notifications can be by phone or email. Field teams will sign in and out at headquarters or other access points when requested and open/close any gates as appropriate.

3. If it is desirable to place cameras on private land, project personnel will seek landowners written permission. Field team members will work with landowners to arrange a convenient schedule for camera placement. Landowners may participate in monitoring with the field team. Notification, sign-in, and gate use will be conducted as above. Field team members will be carrying a letter or ID card identifying them as a project team member.

4. If a jaguar is detected on this site by either the cameras or genetically verified scat ID, we will notify you within 24 hours of this detection confirmation, along with the U.S. Fish and Wildlife Service (USFWS), the state wildlife management agency (AZ or NM), and appropriate public land management agencies if applicable. Project managers and USFWS may prepare a press release to communicate the jaguar detection, and landowners/permittees will receive a courtesy call or email about the press release when it is announced as well. In keeping with past jaguar detection efforts, we and the agencies will not voluntarily make the specific location public. Simply the county or mountain range may be used. We hope that you agree with and follow this convention to assure only appropriate disclosure of related information to protect both the jaguar and your privacy. We are aware that in some cases it may be important for you to contact your neighbors about this detection. Please use your own discretion when contacting others that need to know of these findings.

If a jaguar is detected, the team will deploy additional cameras in the area, and, with the aid of a specially trained scat dog, collect scat for analyses in the genetics lab. Field teams will coordinate with landowners regarding the schedule for these activities as above.

5. Periodic updates of camera findings, based on six month reports prepared for the USFWS, will be provided at meetings, or through a UA or USFWS website if the information is appropriate for public release, to all participating

landowners/permittees. Annual updates for the public will also be prepared by the project team.

For further information, please contact Lisa Haynes, (520)977-8249 lynx@email.arizona.edu / Jaguar Survey Project Manager, School of Natural Resources and the Environment, BioSciences East 325, University of Arizona, Tucson, AZ 85721 (FAX 520.621.8801) Or, if Lisa is not available, contact Kirk Emerson (520) 621-3315, kemerson@email.arizona.edu If you are a public lands grazing permittee or a landowner whom we've contacted to set cameras and conduct field activities, please fill out the appropriate section below (A or B or both) and either mail/scan \& email/or FAX to above address. 
A. PUBLIC OR STATE TRUST LANDS GRAZING PERMITTEE:

Name

- $\quad$ Please coordinate with me about your field activities (Please select as many as apply):

i. By phone Phone number:

ii. By email Email address:

iii. In person Description (e.g. stop by ranch headquarters on way in, etc.):

- $\quad$ Ongoing coordination not necessary

B. PRIVATE LANDOWNER: Name

- $\quad$ Please coordinate with me about your field activities (Please select as many as apply):

i. By phone Phone number:

ii. By email Email address:

iii. In person Description (e.g. stop by ranch headquarters on way in, etc.):

- $\quad$ Ongoing coordination not necessary

I give my permission for you to conduct field activities (check one or both) cross my private land to access other project areas:

on my private land and/or Date: 


\section{Appendix 2. All Camera Sites with Rounded Coordinates (UTM 12N - NAD83) and Environmental Characteristics from Arizona and New Mexico, 2012-2015}

[yellow, ocelot photo; blue, jaguar photo; orange, ocelot and jaguar photos). TRI, terrain ruggedness index; NDVI-eModis, normalized difference vegetation index for March, 2015; NDVI-AVHRR, normalized difference vegetation index for August, 2014. Human influence is an index ranging from 0 to 64 with human influence increasing from 0 (none) to 64 (maximum)]

\begin{tabular}{|c|c|c|c|c|c|c|c|c|c|c|c|c|}
\hline Mountain range & Site code & $\begin{array}{l}\text { UTM } \\
\text { East }\end{array}$ & $\begin{array}{l}\text { UTM } \\
\text { North }\end{array}$ & Elev (m) & TRI & $\begin{array}{l}\text { NDVI- } \\
\text { eMODIS }\end{array}$ & $\begin{array}{l}\text { NDVI- } \\
\text { AVHRR }\end{array}$ & $\begin{array}{l}\text { Distance } \\
\text { to water } \\
\text { (m) }\end{array}$ & $\begin{array}{l}\text { Distance } \\
\text { to roads } \\
(\mathrm{m})\end{array}$ & $\begin{array}{l}\text { Human } \\
\text { Influence }\end{array}$ & $\begin{array}{c}\text { Tree } \\
\text { cover (\%) }\end{array}$ & $\begin{array}{c}\text { Biotic } \\
\text { communitya }\end{array}$ \\
\hline Atascosa & ATA01 & 490000 & 3480000 & 1,362 & 797 & 0.31 & 0.5 & 741 & 3,584 & 13 & 15 & MEW \\
\hline Atascosa & ATA02 & 490000 & 3470000 & 1,346 & 754 & 0.28 & 0.53 & 612 & 4,355 & 18 & 12 & MEW \\
\hline Atascosa & ATA03 & 490000 & 3480000 & 1,375 & 1,101 & 0.29 & 0.5 & 291 & 5,647 & 18 & 11 & MEW \\
\hline Atascosa & ATA04 & 490000 & 3480000 & 1,330 & 855 & 0.31 & 0.51 & 1,769 & 8,872 & 13 & 19 & MEW \\
\hline Atascosa & ATA05 & 490000 & 3480000 & 1,226 & 624 & 0.24 & 0.5 & 1,993 & 11,683 & 13 & 13 & MEW \\
\hline Baboquivari & BAB01 & 450000 & 3510000 & 1,733 & 1,181 & 0.35 & 0.53 & 1,975 & 9,599 & 8 & 14 & MEW \\
\hline Baboquivari & BAB02 & 440000 & 3510000 & 1,595 & 1,275 & 0.34 & 0.54 & 2,770 & 9,684 & 8 & 17 & MEW \\
\hline Baboquivari & ВАВ03 & 450000 & 3520000 & 1,369 & 1,290 & 0.39 & 0.47 & 3,319 & 10,972 & 10 & 15 & MEW \\
\hline Baboquivari & BAB04 & 450000 & 3520000 & 1,378 & 1,153 & 0.48 & 0.51 & 2,781 & 10,109 & 10 & 29 & MEW \\
\hline Baboquivari & BAB05a & 450000 & 3520000 & 1,761 & 1,519 & 0.42 & 0.52 & 1,518 & 9,517 & 10 & 12 & MEW \\
\hline Baboquivari & BAB05b & 450000 & 3520000 & 1,761 & 1,519 & 0.42 & 0.52 & 1,518 & 9,517 & 10 & 12 & MEW \\
\hline Baboquivari & BAB06 & 450000 & 3520000 & 1,618 & 1,036 & 0.33 & 0.41 & 1,014 & 7,515 & 10 & 9 & MEW \\
\hline Baboquivari & BAB07 & 440000 & 3510000 & 1,347 & 867 & 0.29 & 0.39 & 384 & 12,346 & 7 & 7 & SG \\
\hline Baboquivari & BAB08 & 440000 & 3500000 & 1,150 & 878 & 0.34 & 0.37 & 1,422 & 12,148 & 7 & 12 & SG \\
\hline Baboquivari & BAB09 & 440000 & 3500000 & 1,269 & 605 & 0.34 & 0.47 & 136 & 10,791 & 8 & 4 & SG \\
\hline Baboquivari & BAB10 & 440000 & 3490000 & 1,131 & 538 & 0.32 & 0.49 & 1,305 & 4,848 & 10 & 2 & MEW \\
\hline Baboquivari & BAB11 & 440000 & 3500000 & 1,270 & 852 & 0.3 & 0.51 & 232 & 10,197 & 6 & 5 & SG \\
\hline Baboquivari & BAB12 & 440000 & 3490000 & 1,323 & 610 & 0.31 & 0.52 & 413 & 8,447 & 6 & 5 & SG \\
\hline Baboquivari & BAB13 & 450000 & 3520000 & 1,443 & 1,222 & 0.39 & 0.47 & 3,223 & 10,828 & 10 & 15 & MEW \\
\hline Baboquivari & BAB14 & 450000 & 3520000 & 1,378 & 1,153 & 0.48 & 0.51 & 2,651 & 10,110 & 10 & 28 & MEW \\
\hline Baboquivari & BAB15 & 450000 & 3520000 & 1,761 & 1,519 & 0.42 & 0.52 & 1,518 & 9,517 & 10 & 12 & MEW \\
\hline Baboquivari & BAB16 & 440000 & 3490000 & 1,314 & 623 & 0.33 & 0.51 & 75 & 9,303 & 6 & 4 & SG \\
\hline Baboquivari & BAB17 & 450000 & 3520000 & 1,295 & 1,120 & 0.4 & 0.5 & 1,997 & 9,238 & 10 & 18 & MEW \\
\hline
\end{tabular}




\begin{tabular}{|c|c|c|c|c|c|c|c|c|c|c|c|c|}
\hline Mountain range & Site code & $\begin{array}{l}\text { UTM } \\
\text { East }\end{array}$ & $\begin{array}{l}\text { UTM } \\
\text { North }\end{array}$ & $\operatorname{Elev}(\mathrm{m})$ & TRI & $\begin{array}{l}\text { NDVI- } \\
\text { eMODIS }\end{array}$ & $\begin{array}{l}\text { NDVI- } \\
\text { AVHRR }\end{array}$ & $\begin{array}{l}\text { Distance } \\
\text { to water } \\
(\mathrm{m})\end{array}$ & $\begin{array}{l}\text { Distance } \\
\text { to roads } \\
\text { (m) }\end{array}$ & $\begin{array}{l}\text { Human } \\
\text { Influence }\end{array}$ & $\begin{array}{c}\text { Tree } \\
\text { cover (\%) }\end{array}$ & $\begin{array}{c}\text { Biotic } \\
\text { communitya }\end{array}$ \\
\hline Canelo Hills & CAN01 & 550000 & 3470000 & 1,581 & 627 & 0.31 & 0.44 & 1,545 & 3,654 & 11 & 14 & PGBG \\
\hline Canelo Hills & CAN02 & 550000 & 3480000 & 1,635 & 522 & 0.4 & 0.48 & 838 & 1,756 & 10 & 24 & MEW \\
\hline Canelo Hills & CAN03 & 540000 & 3490000 & 1,588 & 778 & 0.26 & 0.54 & 1,168 & 5,402 & 10 & 13 & MEW \\
\hline Canelo Hills & CAN04 & 540000 & 3490000 & 1,518 & 668 & 0.28 & 0.55 & 1,752 & 5,603 & 6 & 12 & MEW \\
\hline Canelo Hills & CAN05 & 540000 & 3490000 & 1,783 & 640 & 0.33 & 0.5 & 411 & 3,493 & 10 & 11 & MEW \\
\hline Canelo Hills & CAN06 & 530000 & 3490000 & 1,340 & 635 & 0.26 & 0.55 & 1,931 & 4,362 & 6 & 14 & SG \\
\hline Canelo Hills & CAN07 & 530000 & 3490000 & 1,391 & 761 & 0.27 & 0.52 & 818 & 6,220 & 6 & 13 & MEW \\
\hline Canelo Hills & CAN08 & 540000 & 3490000 & 1,454 & 850 & 0.26 & 0.38 & 1,283 & 7,769 & 6 & 16 & MEW \\
\hline Canelo Hills & CAN09 & 540000 & 3490000 & 1,630 & 590 & 0.31 & 0.51 & 550 & 4,557 & 10 & 15 & MEW \\
\hline Canelo Hills & CAN10 & 540000 & 3490000 & 1,677 & 781 & 0.3 & 0.5 & 1,227 & 4,953 & 10 & 14 & MEW \\
\hline Canelo Hills & CAN11 & 540000 & 3480000 & 1,765 & 982 & 0.35 & 0.49 & 1,936 & 3,899 & 6 & 15 & MEW \\
\hline Canelo Hills & CAN12 & 550000 & 3480000 & 1,695 & 941 & 0.33 & 0.51 & 1,103 & 3,414 & 6 & 16 & MEW \\
\hline Canelo Hills & CAN13 & 550000 & 3480000 & 1,711 & 759 & 0.29 & 0.33 & 1,024 & 3,204 & 6 & 14 & MEW \\
\hline Canelo Hills & CAN14 & 550000 & 3480000 & 1,712 & 929 & 0.38 & 0.36 & 55 & 3,002 & 6 & 11 & PGBG \\
\hline Canelo Hills & CAN15 & 550000 & 3480000 & 1,644 & 604 & 0.28 & 0.4 & 1,362 & 3,222 & 6 & 13 & PGBG \\
\hline Cerro Colorado & CER01 & 480000 & 3510000 & 1,280 & 670 & 0.25 & 0.27 & 557 & 17,428 & 10 & 10 & SG \\
\hline Cerro Colorado & CER02 & 470000 & 3510000 & 1,092 & 520 & 0.2 & 0.28 & 1,786 & 16,371 & 10 & 8 & SG \\
\hline Cerro Colorado & CER03 & 470000 & 3510000 & 1,162 & 942 & 0.25 & 0.29 & 1,841 & 17,772 & 10 & 6 & SG \\
\hline Chiricauhua & CHI01 & 660000 & 3540000 & 2,003 & 1,198 & 0.39 & 0.42 & 5,015 & 6,655 & 5 & 32 & MEW \\
\hline Chiricauhua & CHIO2 & 660000 & 3540000 & 1,884 & 1,140 & 0.45 & 0.49 & 2,298 & 8,890 & 9 & 31 & MEW \\
\hline Chiricauhua & CHI03 & 670000 & 3530000 & 1,883 & 1,170 & 0.45 & 0.37 & 2,985 & 17,173 & 5 & 28 & MEW \\
\hline Chiricauhua & CHI04 & 660000 & 3530000 & 1,989 & 1,093 & 0.48 & 0.51 & 3,372 & 15,121 & 5 & 39 & PMCF \\
\hline Chiricauhua & CHI05 & 660000 & 3520000 & 1,928 & 1,058 & 0.4 & 0.23 & 911 & 19,037 & 5 & 32 & MEW \\
\hline Chiricauhua & CHIO6 & 660000 & 3520000 & 1,828 & 922 & 0.39 & 0.44 & 2,687 & 18,410 & 9 & 26 & MEW \\
\hline Chiricauhua & CHI07 & 660000 & 3520000 & 1,966 & 884 & 0.34 & 0.37 & 368 & 19,635 & 5 & 32 & MEW \\
\hline Chiricauhua & CHI08 & 680000 & 3530000 & 1,466 & 1,247 & 0.28 & 0.48 & 1,334 & 9,911 & 9 & 16 & CD \\
\hline Chiricauhua & CHI09 & 680000 & 3530000 & 1,487 & 816 & 0.23 & 0.48 & 1,008 & 9,981 & 9 & 13 & CD \\
\hline Chiricauhua & CHI10 & 660000 & 3540000 & 2,082 & 1,099 & 0.26 & 0.43 & 1,001 & 7,905 & 5 & 35 & MEW \\
\hline Chiricauhua & CHI11 & 660000 & 3530000 & 1,924 & 1,169 & 0.36 & 0.55 & 2,938 & 14,689 & 5 & 29 & PMCF \\
\hline Chiricauhua & CHI12 & 660000 & 3540000 & 1,858 & 1,121 & 0.42 & 0.45 & 4,040 & 6,778 & 9 & 43 & MEW \\
\hline
\end{tabular}




\begin{tabular}{|c|c|c|c|c|c|c|c|c|c|c|c|c|}
\hline Mountain range & Site code & $\begin{array}{l}\text { UTM } \\
\text { East }\end{array}$ & $\begin{array}{l}\text { UTM } \\
\text { North }\end{array}$ & Elev (m) & TRI & $\begin{array}{l}\text { NDVI- } \\
\text { eMODIS }\end{array}$ & $\begin{array}{l}\text { NDVI- } \\
\text { AVHRR }\end{array}$ & $\begin{array}{c}\text { Distance } \\
\text { to water } \\
\text { (m) }\end{array}$ & $\begin{array}{l}\text { Distance } \\
\text { to roads } \\
\text { (m) }\end{array}$ & $\begin{array}{l}\text { Human } \\
\text { Influence }\end{array}$ & $\begin{array}{c}\text { Tree } \\
\text { cover (\%) }\end{array}$ & $\begin{array}{c}\text { Biotic } \\
\text { communitya }\end{array}$ \\
\hline Chiricauhua & CHI13 & 660000 & 3540000 & 2,005 & 1,369 & 0.43 & 0.53 & 2,765 & 11,114 & 9 & 43 & MEW \\
\hline Chiricauhua & CHI14 & 670000 & 3530000 & 1,737 & 1,339 & 0.33 & 0.62 & 1,979 & 13,148 & 9 & 22 & MEW \\
\hline Coyote & COY01 & 450000 & 3540000 & 1,049 & 936 & 0.26 & 0.28 & 3,634 & 3,329 & 10 & 3 & SG \\
\hline Coyote & COY02 & 450000 & 3540000 & 1,347 & 1,340 & 0.31 & 0.31 & 3,150 & 3,105 & 10 & 7 & SG \\
\hline Coyote & COY03 & 450000 & 3540000 & 1,677 & 1,388 & 0.33 & 0.34 & 2,333 & 3,686 & 10 & 9 & SG \\
\hline Coyote & COY04 & 450000 & 3540000 & 1,347 & 1,340 & 0.31 & 0.31 & 2,866 & 3,280 & 10 & 9 & SG \\
\hline Coyote & COY05 & 450000 & 3540000 & 1,347 & 1,340 & 0.31 & 0.3 & 2,998 & 3,269 & 10 & 8 & SG \\
\hline Dos Cabezas & DOS01 & 640000 & 3560000 & 1,829 & 1,110 & 0.34 & 0.42 & 2,707 & 5,997 & 5 & 10 & MEW \\
\hline Dos Cabezas & DOS02 & 640000 & 3560000 & 1,847 & 1,003 & 0.24 & 0.43 & 624 & 6,239 & 5 & 9 & SG \\
\hline Dos Cabezas & DOS03 & 640000 & 3560000 & 1,561 & 643 & 0.21 & 0.42 & 84 & 8,042 & 9 & 2 & SG \\
\hline Huachuca & HUA01 & 550000 & 3490000 & 1,658 & 900 & 0.37 & 0.43 & 3,319 & 4,323 & 11 & 20 & MEW \\
\hline Huachuca & HUA02 & 550000 & 3480000 & 1,769 & 575 & 0.36 & 0.47 & 702 & 1,439 & 12 & 15 & MEW \\
\hline Huachuca & HUA03 & 550000 & 3480000 & 1,716 & 578 & 0.39 & 0.36 & 1,190 & 194 & 15 & 18 & MEW \\
\hline Huachuca & HUA04 & 570000 & 3470000 & 2,001 & 1,656 & 0.32 & 0.42 & 1,049 & 6,579 & 15 & 18 & MEW \\
\hline Huachuca & HUA05 & 560000 & 3470000 & 2,242 & 1,543 & 0.59 & 0.35 & 3,819 & 6,843 & 15 & 35 & MEW \\
\hline Huachuca & HUA06 & 560000 & 3480000 & 2,203 & 1,317 & 0.57 & 0.53 & 856 & 5,225 & 12 & 32 & MEW \\
\hline Huachuca & HUA07 & 560000 & 3470000 & 1,708 & 838 & 0.4 & 0.47 & 36 & 2,044 & 15 & 11 & PGBG \\
\hline Huachuca & HUA08 & 560000 & 3470000 & 1,988 & 1,095 & 0.48 & 0.45 & 3,125 & 7,913 & 15 & 27 & MEW \\
\hline Huachuca & HUA09 & 560000 & 3480000 & 1,743 & 506 & 0.36 & 0.48 & 538 & 1,288 & 15 & 17 & PGBG \\
\hline Huachuca & HUA10 & 560000 & 3480000 & 1,827 & 878 & 0.36 & 0.55 & 1,258 & 2,915 & 11 & 20 & MEW \\
\hline Huachuca & HUA11 & 560000 & 3480000 & 2,073 & 1,080 & 0.64 & 0.53 & 3,484 & 5,248 & 11 & 44 & MEW \\
\hline Huachuca & HUA12 & 550000 & 3480000 & 1,681 & 784 & 0.44 & 0.5 & 2,330 & 3,325 & 14 & 27 & MEW \\
\hline Huachuca & HUA13 & 570000 & 3480000 & 1,651 & 722 & 0.52 & 0.58 & 2,868 & 2,952 & 21 & 20 & MEW \\
\hline Huachuca & HUA14 & 570000 & 3480000 & 1,632 & 890 & 0.37 & 0.57 & 4,062 & 2,040 & 11 & 20 & MEW \\
\hline Huachuca & HUA15 & 560000 & 3480000 & 2,143 & 1,436 & 0.65 & 0.57 & 4,509 & 7,039 & 11 & 50 & PMCF \\
\hline Huachuca & HUA16 & 560000 & 3480000 & 2,234 & 1,809 & 0.52 & 0.52 & 2,963 & 7,387 & 14 & 24 & PMCF \\
\hline Huachuca & HUA17 & 570000 & 3480000 & 2,262 & 1,587 & 0.43 & 0.58 & 5,787 & 4,623 & 11 & 27 & MEW \\
\hline Huachuca & HUA18 & 550000 & 3480000 & 1,751 & 1,080 & 0.45 & 0.5 & 2,577 & 3,700 & 14 & 27 & MEW \\
\hline Huachuca & HUA19 & 560000 & 3480000 & 2,055 & 1,080 & 0.64 & 0.54 & 3,338 & 5,287 & 11 & 43 & MEW \\
\hline Huachuca & HUA20 & 560000 & 3470000 & 1,854 & 795 & 0.32 & 0.51 & 2,071 & 3,674 & 15 & 20 & MEW \\
\hline
\end{tabular}




\begin{tabular}{|c|c|c|c|c|c|c|c|c|c|c|c|c|}
\hline Mountain range & Site code & $\begin{array}{l}\text { UTM } \\
\text { East }\end{array}$ & $\begin{array}{l}\text { UTM } \\
\text { North }\end{array}$ & Elev (m) & TRI & $\begin{array}{l}\text { NDVI- } \\
\text { eMODIS }\end{array}$ & $\begin{array}{l}\text { NDVI- } \\
\text { AVHRR }\end{array}$ & $\begin{array}{c}\text { Distance } \\
\text { to water } \\
(\mathrm{m})\end{array}$ & $\begin{array}{c}\text { Distance } \\
\text { to roads } \\
(\mathrm{m})\end{array}$ & $\begin{array}{l}\text { Human } \\
\text { Influence }\end{array}$ & $\begin{array}{c}\text { Tree } \\
\text { cover (\%) }\end{array}$ & $\begin{array}{c}\text { Biotic } \\
\text { community }^{a}\end{array}$ \\
\hline Huachuca & HUA21 & 560000 & 3480000 & 2,323 & 1,383 & 0.55 & 0.6 & 5,054 & 7,686 & 11 & 42 & PMCF \\
\hline Huachuca & HUA22 & 570000 & 3470000 & 1,956 & 1,748 & 0.6 & 0.44 & 2,969 & 6,854 & 11 & 44 & MEW \\
\hline Huachuca & HUA23 & 570000 & 3470000 & 2,229 & 1,627 & 0.39 & 0.5 & 1,437 & 6,246 & 15 & 31 & MEW \\
\hline Pajarito & PAJ01 & 480000 & 3470000 & 1,180 & 631 & 0.33 & 0.49 & 863 & 8,760 & 18 & 16 & MEW \\
\hline Pajarito & PAJ02 & 480000 & 3480000 & 1,292 & 480 & 0.27 & 0.52 & 368 & 7,773 & 17 & 15 & MEW \\
\hline Pajarito & PAJ03a & 480000 & 3470000 & 1,315 & 631 & 0.33 & 0.48 & 1,114 & 9,591 & 14 & 17 & MEW \\
\hline Pajarito & PAJ03b & 480000 & 3470000 & 1,315 & 631 & 0.33 & 0.48 & 999 & 9,455 & 14 & 16 & MEW \\
\hline Pajarito & PAJ04 & 480000 & 3470000 & 1,237 & 763 & 0.34 & 0.43 & 1,932 & 10,825 & 14 & 16 & MEW \\
\hline Pajarito & PAJ05 & 480000 & 3470000 & 1,306 & 680 & 0.28 & 0.45 & 213 & 8,854 & 14 & 17 & MEW \\
\hline Pajarito & PAJ06 & 490000 & 3470000 & 1,455 & 904 & 0.28 & 0.54 & 1,858 & 3,278 & 14 & 18 & MEW \\
\hline Pajarito & PAJ07 & 490000 & 3470000 & 1,317 & 745 & 0.26 & 0.6 & 1,375 & 4,640 & 14 & 15 & MEW \\
\hline Pajarito & PAJ08a & 490000 & 3470000 & 1,403 & 805 & 0.29 & 0.51 & 855 & 2,629 & 13 & 19 & MEW \\
\hline Pajarito & PAJ08b & 490000 & 3470000 & 1,403 & 805 & 0.29 & 0.51 & 862 & 2,643 & 13 & 18 & MEW \\
\hline Pajarito & PAJ09a & 490000 & 3470000 & 1,457 & 716 & 0.29 & 0.51 & 633 & 2,865 & 13 & 18 & MEW \\
\hline Pajarito & PAJ09b & 490000 & 3470000 & 1,457 & 716 & 0.29 & 0.51 & 570 & 3,049 & 13 & 17 & MEW \\
\hline Pajarito & PAJ10 & 490000 & 3470000 & 1,472 & 719 & 0.31 & 0.55 & 2,740 & 4,883 & 14 & 21 & MEW \\
\hline Pajarito & PAJ11 & 490000 & 3470000 & 1,415 & 736 & 0.29 & 0.51 & 878 & 3,951 & 14 & 18 & MEW \\
\hline Pajarito & PAJ12 & 490000 & 3470000 & 1,229 & 796 & 0.28 & 0.54 & 491 & 3,239 & 14 & 12 & MEW \\
\hline Pajarito & PAJ13 & 490000 & 3470000 & 1,234 & 706 & 0.27 & 0.5 & 1,285 & 678 & 17 & 17 & MEW \\
\hline Pajarito & PAJ14 & 490000 & 3470000 & 1,523 & 825 & 0.27 & 0.44 & 162 & 5,736 & 13 & 14 & MEW \\
\hline Pajarito & PAJ15 & 490000 & 3470000 & 1,470 & 744 & 0.29 & 0.52 & 467 & 3,729 & 13 & 13 & MEW \\
\hline Pajarito & PAJ16a & 490000 & 3470000 & 1,448 & 692 & 0.28 & 0.61 & 659 & 6,308 & 14 & 15 & MEW \\
\hline Pajarito & PAJ16b & 490000 & 3470000 & 1,448 & 692 & 0.28 & 0.61 & 659 & 6,308 & 14 & 15 & MEW \\
\hline Pajarito & PAJ17 & 490000 & 3470000 & 1,204 & 446 & 0.26 & 0.47 & 1,110 & 596 & 17 & 10 & MEW \\
\hline Pajarito & PAJ18 & 490000 & 3470000 & 1,362 & 904 & 0.29 & 0.54 & 1,760 & 2,695 & 14 & 17 & MEW \\
\hline Pajarito & PAJ19 & 490000 & 3470000 & 1,457 & 716 & 0.29 & 0.51 & 570 & 3,049 & 13 & 17 & MEW \\
\hline Pajarito & PAJ20 & 490000 & 3470000 & 1,362 & 904 & 0.29 & 0.54 & 1,760 & 2,695 & 14 & 17 & MEW \\
\hline Patagonia & PAT01 & 530000 & 3470000 & 1,537 & 1,090 & 0.31 & 0.52 & 2,208 & 11,773 & 17 & 28 & MEW \\
\hline Patagonia & PAT02 & 520000 & 3480000 & 1,439 & 746 & 0.27 & 0.57 & 2,068 & 6,738 & 13 & 14 & MEW \\
\hline Patagonia & PAT03 & 520000 & 3480000 & 1,895 & 1,111 & 0.37 & 0.53 & 3,804 & 7,040 & 15 & 27 & MEW \\
\hline
\end{tabular}




\begin{tabular}{|c|c|c|c|c|c|c|c|c|c|c|c|c|}
\hline Mountain range & Site code & $\begin{array}{l}\text { UTM } \\
\text { East }\end{array}$ & $\begin{array}{l}\text { UTM } \\
\text { North }\end{array}$ & Elev (m) & TRI & $\begin{array}{l}\text { NDVI- } \\
\text { eMODIS }\end{array}$ & $\begin{array}{l}\text { NDVI- } \\
\text { AVHRR }\end{array}$ & $\begin{array}{c}\text { Distance } \\
\text { to water } \\
\text { (m) }\end{array}$ & $\begin{array}{l}\text { Distance } \\
\text { to roads } \\
\text { (m) }\end{array}$ & $\begin{array}{l}\text { Human } \\
\text { Influence }\end{array}$ & $\begin{array}{c}\text { Tree } \\
\text { cover (\%) }\end{array}$ & $\begin{array}{c}\text { Biotic } \\
\text { communitya }\end{array}$ \\
\hline Patagonia & PAT04 & 520000 & 3480000 & 1,765 & 1,055 & 0.38 & 0.53 & 3,765 & 6,780 & 15 & 26 & MEW \\
\hline Patagonia & PAT05 & 520000 & 3480000 & 1,814 & 985 & 0.38 & 0.58 & 3,892 & 7,503 & 13 & 26 & MEW \\
\hline Patagonia & PAT06 & 530000 & 3470000 & 1,593 & 611 & 0.38 & 0.56 & 1,214 & 18,694 & 9 & 20 & MEW \\
\hline Patagonia & РAT07 & 530000 & 3470000 & 1,429 & 944 & 0.27 & 0.55 & 2,491 & 10,707 & 17 & 17 & MEW \\
\hline Patagonia & РАТ08 & 520000 & 3480000 & 1,557 & 1,061 & 0.36 & 0.5 & 1,910 & 8,689 & 13 & 28 & MEW \\
\hline Patagonia & PAT09a & 530000 & 3480000 & 1,856 & 1,070 & 0.38 & 0.51 & 2,777 & 9,372 & 17 & 31 & MEW \\
\hline Patagonia & PAT09b & 530000 & 3480000 & 1,856 & 1,070 & 0.38 & 0.51 & 2,781 & 9,363 & 17 & 31 & MEW \\
\hline Patagonia & PAT10 & 530000 & 3470000 & 1,837 & 1,194 & 0.54 & 0.55 & 3,339 & 13,505 & 13 & 33 & MEW \\
\hline Patagonia & PAT11a & 530000 & 3470000 & 1,733 & 757 & 0.46 & 0.53 & 1,569 & 14,031 & 17 & 33 & MEW \\
\hline Patagonia & PAT11b & 530000 & 3470000 & 1,733 & 757 & 0.46 & 0.53 & 1,425 & 14,203 & 17 & 33 & MEW \\
\hline Patagonia & PAT13 & 530000 & 3480000 & 1,826 & 809 & 0.39 & 0.52 & 4,075 & 8,016 & 13 & 36 & MEW \\
\hline Patagonia & PAT14 & 530000 & 3480000 & 1,889 & 977 & 0.4 & 0.5 & 3,105 & 8,872 & 17 & 29 & MEW \\
\hline Patagonia & PAT15 & 530000 & 3470000 & 1,674 & 1,047 & 0.45 & 0.54 & 1,611 & 11,602 & 17 & 29 & MEW \\
\hline Patagonia & PAT16 & 530000 & 3480000 & 1,684 & 611 & 0.43 & 0.52 & 2,546 & 12,664 & 13 & 33 & MEW \\
\hline Patagonia & PAT17 & 520000 & 3470000 & 1,471 & 745 & 0.26 & 0.56 & 2,363 & 11,105 & 17 & 13 & MEW \\
\hline Patagonia & PAT18 & 520000 & 3480000 & 1,794 & 948 & 0.26 & 0.59 & 2,962 & 7,066 & 13 & 15 & MEW \\
\hline Patagonia & PAT19 & 530000 & 3470000 & 1,860 & 853 & 0.43 & 0.51 & 2,428 & 12,610 & 17 & 33 & MEW \\
\hline Patagonia & PAT20 & 520000 & 3480000 & 1,801 & 1,154 & 0.41 & 0.55 & 3,381 & 5,962 & 15 & 26 & MEW \\
\hline Patagonia & РАT21 & 530000 & 3470000 & 1,825 & 853 & 0.48 & 0.53 & 3,107 & 13,480 & 17 & 30 & MEW \\
\hline Patagonia & PAT22 & 530000 & 3480000 & 1,717 & 811 & 0.48 & 0.5 & 2,198 & 12,170 & 17 & 32 & MEW \\
\hline Patagonia & PAT23 & 520000 & 3480000 & 1,842 & 1,028 & 0.38 & 0.53 & 3,508 & 6,716 & 11 & 31 & MEW \\
\hline Patagonia & РAT24 & 530000 & 3480000 & 1,725 & 1,007 & 0.39 & 0.53 & 4,096 & 7,710 & 15 & 22 & MEW \\
\hline Patagonia & PAT25 & 520000 & 3480000 & 1,895 & 1,111 & 0.37 & 0.53 & 3,780 & 7,018 & 15 & 27 & MEW \\
\hline Patagonia & PAT26 & 530000 & 3490000 & 1,438 & 730 & 0.29 & 0.49 & 1,338 & 6,321 & 10 & 15 & MEW \\
\hline Patagonia & PAT27 & 530000 & 3480000 & 1,706 & 1,117 & 0.38 & 0.48 & 2,645 & 8,704 & 17 & 22 & MEW \\
\hline Peloncillo & PEL01 & 690000 & 3490000 & 1,746 & 557 & 0.36 & 0.39 & 485 & 13,221 & 8 & 14 & MEW \\
\hline Peloncillo & PEL02 & 690000 & 3490000 & 1,727 & 557 & 0.41 & 0.39 & 1,838 & 12,888 & 8 & 14 & MEW \\
\hline Peloncillo & PEL03 & 690000 & 3490000 & 1,743 & 711 & 0.33 & 0.4 & 1,454 & 13,953 & 4 & 13 & MEW \\
\hline Peloncillo & PEL04 & 690000 & 3500000 & 1,703 & 982 & 0.3 & 0.45 & 717 & 14,635 & 4 & 11 & MEW \\
\hline Peloncillo & PEL05 & 690000 & 3490000 & 1,802 & 753 & 0.35 & 0.45 & 2,334 & 14,957 & 4 & 19 & MEW \\
\hline
\end{tabular}




\begin{tabular}{|c|c|c|c|c|c|c|c|c|c|c|c|c|}
\hline Mountain range & Site code & $\begin{array}{l}\text { UTM } \\
\text { East }\end{array}$ & $\begin{array}{l}\text { UTM } \\
\text { North }\end{array}$ & Elev (m) & TRI & $\begin{array}{l}\text { NDVI- } \\
\text { eMODIS }\end{array}$ & $\begin{array}{l}\text { NDVI- } \\
\text { AVHRR }\end{array}$ & $\begin{array}{c}\text { Distance } \\
\text { to water } \\
\text { (m) }\end{array}$ & $\begin{array}{c}\text { Distance } \\
\text { to roads } \\
(\mathrm{m})\end{array}$ & $\begin{array}{l}\text { Human } \\
\text { Influence }\end{array}$ & $\begin{array}{c}\text { Tree } \\
\text { cover (\%) }\end{array}$ & $\begin{array}{c}\text { Biotic } \\
\text { communitya }\end{array}$ \\
\hline Peloncillo & PEL06 & 690000 & 3490000 & 1,697 & 953 & 0.32 & 0.53 & 1,640 & 15,895 & 5 & 20 & MEW \\
\hline Peloncillo & PEL07 & 680000 & 3490000 & 1,691 & 798 & 0.36 & 0.55 & 540 & 16,369 & 5 & 21 & MEW \\
\hline Peloncillo & PEL08 & 690000 & 3490000 & 1,725 & 662 & 0.32 & 0.34 & 1,169 & 12,441 & 8 & 14 & MEW \\
\hline Peloncillo & PEL09 & 690000 & 3490000 & 1,816 & 746 & 0.31 & 0.4 & 362 & 13,356 & 4 & 12 & MEW \\
\hline Peloncillo & PEL10 & 690000 & 3490000 & 1,850 & 714 & 0.3 & 0.51 & 632 & 11,662 & 4 & 10 & MEW \\
\hline Peloncillo & PEL11 & 690000 & 3490000 & 1,677 & 609 & 0.29 & 0.4 & 1,975 & 10,541 & 8 & 12 & MEW \\
\hline Peloncillo & PEL12 & 680000 & 3480000 & 1,367 & 448 & 0.24 & 0.61 & 2,470 & 16,675 & 5 & 7 & SG \\
\hline Peloncillo & PEL13 & 690000 & 3490000 & 1,614 & 1,032 & 0.26 & 0.51 & 1,281 & 14,158 & 9 & 16 & MEW \\
\hline Peloncillo & PEL14 & 690000 & 3490000 & 1,710 & 912 & 0.35 & 0.53 & 1,089 & 13,627 & 4 & 14 & MEW \\
\hline Peloncillo & PEL15 & 690000 & 3490000 & 1,763 & 636 & 0.4 & 0.4 & 51 & 11,557 & 8 & 7 & MEW \\
\hline Peloncillo & PEL16 & 680000 & 3490000 & 1,569 & 808 & 0.3 & 0.62 & 1,038 & 18,127 & 9 & 7 & $\mathrm{SG}$ \\
\hline Peloncillo & PEL17 & 690000 & 3520000 & 1,520 & 1,026 & 0.21 & 0.33 & 480 & 6,318 & 4 & 6 & MEW \\
\hline Peloncillo & PEL18 & 690000 & 3490000 & 1,614 & 1,032 & 0.26 & 0.51 & 787 & 14,750 & 9 & 17 & MEW \\
\hline Peloncillo & PEL19 & 690000 & 3490000 & 1,889 & 1,075 & 0.33 & 0.5 & 937 & 15,314 & 9 & 11 & MEW \\
\hline Peloncillo & PEL20 & 680000 & 3480000 & 1,358 & 550 & 0.27 & 0.6 & 2,267 & 16,927 & 5 & 7 & SG \\
\hline Peloncillo & PEL21 & 690000 & 3490000 & 1,756 & 670 & 0.41 & 0.32 & 1,270 & 12,747 & 8 & 15 & MEW \\
\hline Peloncillo & PEL22 & 680000 & 3480000 & 1,358 & 550 & 0.27 & 0.6 & 2,267 & 16,927 & 5 & 7 & SG \\
\hline Peloncillo & PEL23a & 680000 & 3490000 & 1,636 & 1,078 & 0.37 & 0.57 & 601 & 16,026 & 9 & 17 & MEW \\
\hline Peloncillo & PEL23b & 680000 & 3490000 & 1,590 & 1,051 & 0.37 & 0.57 & 417 & 15,846 & 9 & 18 & MEW \\
\hline Santa Rita & SAN01 & 520000 & 3520000 & 1,509 & 970 & 0.26 & 0.6 & 2,276 & 6,659 & 18 & 15 & SG \\
\hline Santa Rita & SAN02 & 520000 & 3520000 & 1,713 & 1,006 & 0.29 & 0.59 & 1,666 & 5,566 & 18 & 12 & SG \\
\hline Santa Rita & SAN03 & 520000 & 3520000 & 1,726 & 877 & 0.35 & 0.5 & 1,694 & 4,575 & 18 & 12 & SG \\
\hline Santa Rita & SAN04 & 520000 & 3520000 & 1,802 & 792 & 0.34 & 0.56 & 2,499 & 5,529 & 18 & 17 & $\mathrm{SG}$ \\
\hline Santa Rita & SAN05 & 520000 & 3520000 & 1,663 & 912 & 0.32 & 0.5 & 872 & 3,376 & 18 & 10 & MEW \\
\hline Santa Rita & SAN06 & 520000 & 3520000 & 1,305 & 984 & 0.27 & 0.56 & 2,275 & 7,872 & 14 & 12 & $\mathrm{SG}$ \\
\hline Santa Rita & SAN07 & 520000 & 3520000 & 1,509 & 970 & 0.26 & 0.6 & 1,597 & 6,333 & 18 & 17 & MEW \\
\hline Santa Rita & SAN08 & 520000 & 3520000 & 1,648 & 1,108 & 0.26 & 0.58 & 2,389 & 5,716 & 14 & 14 & $\mathrm{SG}$ \\
\hline Santa Rita & SAN09 & 520000 & 3510000 & 1,544 & 1,373 & 0.3 & 0.56 & 374 & 14,079 & 11 & 14 & MEW \\
\hline Santa Rita & SAN10 & 520000 & 3510000 & 1,410 & 1,221 & 0.34 & 0.58 & 2,471 & 12,065 & 11 & 25 & MEW \\
\hline Santa Rita & SAN11 & 520000 & 3500000 & 1,716 & 1,036 & 0.38 & 0.59 & 672 & 8,177 & 6 & 19 & MEW \\
\hline
\end{tabular}




\begin{tabular}{|c|c|c|c|c|c|c|c|c|c|c|c|c|}
\hline Mountain range & Site code & $\begin{array}{l}\text { UTM } \\
\text { East }\end{array}$ & $\begin{array}{l}\text { UTM } \\
\text { North }\end{array}$ & Elev (m) & TRI & $\begin{array}{l}\text { NDVI- } \\
\text { eMODIS }\end{array}$ & $\begin{array}{l}\text { NDVI- } \\
\text { AVHRR }\end{array}$ & $\begin{array}{c}\text { Distance } \\
\text { to water } \\
\text { (m) }\end{array}$ & $\begin{array}{c}\text { Distance } \\
\text { to roads } \\
(\mathrm{m})\end{array}$ & $\begin{array}{l}\text { Human } \\
\text { Influence }\end{array}$ & $\begin{array}{c}\text { Tree } \\
\text { cover (\%) }\end{array}$ & $\begin{array}{c}\text { Biotic } \\
\text { community }\end{array}$ \\
\hline Santa Rita & SAN12 & 520000 & 3500000 & 1,764 & 1,176 & 0.32 & 0.55 & 1,083 & 8,926 & 6 & 17 & MEW \\
\hline Santa Rita & SAN13 & 520000 & 3500000 & 1,746 & 1,000 & 0.43 & 0.57 & 722 & 10,096 & 6 & 24 & MEW \\
\hline Santa Rita & SAN14 & 520000 & 3500000 & 1,658 & 879 & 0.34 & 0.56 & 1,364 & 8,349 & 6 & 22 & MEW \\
\hline Santa Rita & SAN15 & 520000 & 3520000 & 1,713 & 1,006 & 0.29 & 0.58 & 2,121 & 5,336 & 18 & 10 & SG \\
\hline Santa Rita & SAN16 & 520000 & 3520000 & 1,584 & 1,103 & 0.25 & 0.62 & 2,168 & 6,731 & 18 & 11 & SG \\
\hline Santa Rita & SAN17 & 520000 & 3520000 & 1,509 & 970 & 0.26 & 0.6 & 1,891 & 6,181 & 18 & 15 & SG \\
\hline Santa Rita & SAN18 & 520000 & 3500000 & 1,730 & 849 & 0.47 & 0.58 & 1,068 & 11,180 & 6 & 30 & MEW \\
\hline Santa Rita & SAN19 & 520000 & 3500000 & 1,807 & 1,032 & 0.35 & 0.54 & 1,112 & 11,692 & 6 & 25 & MEW \\
\hline Santa Rita & SAN20 & 520000 & 3510000 & 2,089 & 1,725 & 0.49 & 0.39 & 2,405 & 12,296 & 6 & 28 & PMCF \\
\hline Santa Rita & SAN21 & 520000 & 3510000 & 1,969 & 1,305 & 0.48 & 0.37 & 2,567 & 11,318 & 6 & 27 & PMCF \\
\hline Santa Rita & SAN22 & 520000 & 3510000 & 1,836 & 1,124 & 0.44 & 0.52 & 1,387 & 9,725 & 6 & 34 & PMCF \\
\hline Santa Rita & SAN23 & 520000 & 3500000 & 1,901 & 1,269 & 0.52 & 0.56 & 585 & 9,802 & 6 & 35 & MEW \\
\hline Santa Rita & SAN24 & 520000 & 3500000 & 1,734 & 982 & 0.46 & 0.59 & 611 & 8,517 & 6 & 22 & MEW \\
\hline Santa Rita & SAN25 & 520000 & 3500000 & 1,495 & 933 & 0.35 & 0.58 & 364 & 6,528 & 6 & 16 & MEW \\
\hline Santa Rita & SAN26 & 520000 & 3510000 & 1,765 & 799 & 0.37 & 0.4 & 370 & 9,585 & 11 & 25 & MEW \\
\hline Santa Rita & SAN27 & 520000 & 3510000 & 1,678 & 1,140 & 0.42 & 0.51 & 2,281 & 7,889 & 6 & 23 & MEW \\
\hline Santa Rita & SAN28 & 520000 & 3510000 & 1,678 & 1,140 & 0.42 & 0.5 & 2,478 & 8,100 & 6 & 23 & MEW \\
\hline Santa Rita & SAN29 & 520000 & 3500000 & 1,949 & 924 & 0.61 & 0.59 & 2,225 & 11,042 & 6 & 38 & MEW \\
\hline Santa Rita & SAN30 & 520000 & 3500000 & 1,658 & 879 & 0.34 & 0.56 & 1,304 & 8,794 & 6 & 29 & MEW \\
\hline Santa Rita & SAN31 & 520000 & 3500000 & 2,149 & 1,534 & 0.46 & 0.54 & 742 & 10,660 & 6 & 26 & MEW \\
\hline Santa Rita & SAN32 & 520000 & 3500000 & 1,793 & 1,176 & 0.42 & 0.55 & 27 & 8,385 & 6 & 20 & MEW \\
\hline Santa Rita & SAN33 & 520000 & 3500000 & 1,900 & 865 & 0.5 & 0.55 & 2,246 & 10,263 & 6 & 34 & MEW \\
\hline Santa Rita & SAN34 & 510000 & 3510000 & 1,912 & 1,362 & 0.48 & 0.39 & 3,933 & 14,668 & 13 & 17 & MEW \\
\hline Santa Rita & SAN35 & 510000 & 3510000 & 1,855 & 1,309 & 0.48 & 0.42 & 4,782 & 15,309 & 7 & 37 & MEW \\
\hline Santa Rita & SAN36 & 520000 & 3510000 & 2,332 & 1,462 & 0.52 & 0.43 & 3,785 & 14,054 & 6 & 33 & MEW \\
\hline Santa Rita & SAN37 & 520000 & 3500000 & 1,731 & 1,115 & 0.39 & 0.59 & 455 & 9,230 & 6 & 26 & MEW \\
\hline Santa Rita & SAN38 & 510000 & 3500000 & 1,971 & 1,468 & 0.38 & 0.57 & 4,852 & 15,890 & 6 & 16 & MEW \\
\hline Santa Rita & SAN39 & 510000 & 3500000 & 1,794 & 1,098 & 0.57 & 0.57 & 2,203 & 13,953 & 6 & 46 & MEW \\
\hline Santa Rita & SAN40 & 510000 & 3500000 & 1,909 & 1,116 & 0.57 & 0.59 & 3,260 & 14,420 & 6 & 43 & MEW \\
\hline Santa Rita & SAN41 & 510000 & 3510000 & 2,052 & 1,246 & 0.58 & 0.57 & 4,130 & 14,288 & 6 & 48 & MEW \\
\hline
\end{tabular}




\begin{tabular}{|c|c|c|c|c|c|c|c|c|c|c|c|c|}
\hline Mountain range & Site code & $\begin{array}{l}\text { UTM } \\
\text { East }\end{array}$ & $\begin{array}{l}\text { UTM } \\
\text { North }\end{array}$ & Elev (m) & TRI & $\begin{array}{l}\text { NDVI- } \\
\text { eMODIS }\end{array}$ & $\begin{array}{l}\text { NDVI- } \\
\text { AVHRR }\end{array}$ & $\begin{array}{l}\text { Distance } \\
\text { to water } \\
\text { (m) }\end{array}$ & $\begin{array}{l}\text { Distance } \\
\text { to roads } \\
(\mathrm{m})\end{array}$ & $\begin{array}{l}\text { Human } \\
\text { Influence }\end{array}$ & $\begin{array}{c}\text { Tree } \\
\text { cover (\%) }\end{array}$ & $\begin{array}{c}\text { Biotic } \\
\text { communitya }\end{array}$ \\
\hline Santa Rita & SAN42 & 510000 & 3510000 & 1,446 & 1,402 & 0.34 & 0.49 & 2,994 & 11,996 & 7 & 17 & SG \\
\hline Santa Rita & SAN43a & 510000 & 3510000 & 1,677 & 1,052 & 0.37 & 0.48 & 3,078 & 14,161 & 13 & 12 & SG \\
\hline Santa Rita & SAN43b & 510000 & 3510000 & 1,677 & 1,052 & 0.37 & 0.48 & 3,079 & 14,185 & 13 & 12 & SG \\
\hline Santa Rita & SAN44 & 520000 & 3500000 & 1,901 & 1,401 & 0.51 & 0.54 & 55 & 9,976 & 6 & 31 & MEW \\
\hline Santa Rita & SAN45 & 520000 & 3510000 & 2,512 & 1,648 & 0.48 & 0.41 & 3,302 & 13,177 & 6 & 35 & MEW \\
\hline Santa Rita & SAN46 & 520000 & 3510000 & 1,658 & 692 & 0.24 & 0.55 & 1,371 & 6,449 & 11 & 13 & MEW \\
\hline Santa Rita & SAN47 & 520000 & 3510000 & 1,410 & 1,221 & 0.34 & 0.58 & 2,421 & 12,065 & 11 & 23 & MEW \\
\hline Santa Rita & SAN48 & 520000 & 3500000 & 1,803 & 1,030 & 0.4 & 0.54 & 1,281 & 9,178 & 6 & 24 & MEW \\
\hline Santa Rita & SAN49 & 520000 & 3510000 & 2,229 & 1,534 & 0.48 & 0.53 & 1,156 & 10,749 & 6 & 26 & PMCF \\
\hline Santa Rita & SAN50 & 510000 & 3510000 & 1,623 & 1,052 & 0.38 & 0.5 & 3,672 & 14,771 & 13 & 11 & MEW \\
\hline Santa Rita & SAN50 & 510000 & 3510000 & 1,623 & 1,052 & 0.38 & 0.5 & 3,655 & 14,736 & 13 & 12 & MEW \\
\hline Santa Rita & SAN51 & 510000 & 3510000 & 1,677 & 1,052 & 0.37 & 0.48 & 3,295 & 14,436 & 13 & 12 & SG \\
\hline Santa Rita & SAN52a & 510000 & 3510000 & 1,298 & 963 & 0.28 & 0.56 & 1,195 & 14,878 & 11 & 20 & SG \\
\hline Santa Rita & SAN52b & 510000 & 3510000 & 1,298 & 963 & 0.28 & 0.56 & 1,232 & 14,912 & 11 & 20 & $\mathrm{SG}$ \\
\hline Santa Rita & SAN54 & 520000 & 3500000 & 1,750 & 949 & 0.4 & 0.56 & 1,517 & 8,976 & 6 & 33 & MEW \\
\hline Santa Rita & SAN55 & 510000 & 3500000 & 1,453 & 1,107 & 0.28 & 0.57 & 443 & 13,114 & 10 & 12 & SG \\
\hline Santa Rita & SAN56 & 520000 & 3510000 & 1,781 & 1,494 & 0.42 & 0.5 & 2,263 & 11,679 & 11 & 25 & PMCF \\
\hline Santa Rita & SAN57 & 520000 & 3510000 & 1,859 & 1,444 & 0.4 & 0.44 & 1,181 & 10,787 & 11 & 27 & MEW \\
\hline Santa Rita & SAN58 & 520000 & 3510000 & 1,836 & 1,124 & 0.44 & 0.52 & 1,638 & 8,922 & 6 & 26 & MEW \\
\hline Santa Rita & SAN59 & 520000 & 3500000 & 1,642 & 774 & 0.29 & 0.52 & 197 & 6,790 & 6 & 9 & MEW \\
\hline Santa Rita & SAN60 & 510000 & 3510000 & 1,715 & 1,307 & 0.33 & 0.44 & 2,833 & 13,883 & 13 & 19 & SG \\
\hline Santa Rita & SAN61 & 510000 & 3500000 & 1,554 & 1,344 & 0.38 & 0.53 & 3,915 & 15,026 & 13 & 21 & SG \\
\hline Sierrita & SIE01 & 480000 & 3520000 & 1,272 & 609 & 0.25 & 0.43 & 26 & 15,843 & 10 & 3 & SG \\
\hline Sierrita & SIE02 & 480000 & 3520000 & 1,168 & 457 & 0.25 & 0.38 & 3 & 13,861 & 10 & 2 & SG \\
\hline Sierrita & SIE03 & 480000 & 3520000 & 1,188 & 597 & 0.28 & 0.33 & 1,293 & 12,711 & 10 & 7 & SG \\
\hline San Luis & SLU01 & 460000 & 3490000 & 1,150 & 296 & 0.24 & 0.44 & 39 & 8,233 & 10 & 5 & SG \\
\hline San Luis & SLU02 & 460000 & 3490000 & 1,136 & 293 & 0.28 & 0.43 & 943 & 7,396 & 10 & 8 & SG \\
\hline San Luis & SLU03 & 460000 & 3500000 & 1,088 & 450 & 0.25 & 0.48 & 427 & 8,831 & 10 & 5 & SG \\
\hline San Luis & SLU04a & 460000 & 3480000 & 1,213 & 601 & 0.33 & 0.5 & 1,460 & 10,044 & 7 & 9 & SG \\
\hline San Luis & SLU04b & 460000 & 3480000 & 1,213 & 601 & 0.33 & 0.5 & 1,587 & 10,166 & 7 & 8 & SG \\
\hline
\end{tabular}




\begin{tabular}{|c|c|c|c|c|c|c|c|c|c|c|c|c|}
\hline Mountain range & Site code & $\begin{array}{l}\text { UTM } \\
\text { East }\end{array}$ & $\begin{array}{l}\text { UTM } \\
\text { North }\end{array}$ & Elev (m) & TRI & $\begin{array}{l}\text { NDVI- } \\
\text { eMODIS }\end{array}$ & $\begin{array}{l}\text { NDVI- } \\
\text { AVHRR }\end{array}$ & $\begin{array}{c}\text { Distance } \\
\text { to water } \\
\text { (m) }\end{array}$ & $\begin{array}{l}\text { Distance } \\
\text { to roads } \\
\text { (m) }\end{array}$ & $\begin{array}{l}\text { Human } \\
\text { Influence }\end{array}$ & $\begin{array}{c}\text { Tree } \\
\text { cover (\%) }\end{array}$ & $\begin{array}{c}\text { Biotic } \\
\text { communitya }\end{array}$ \\
\hline San Luis & SLU05 & 460000 & 3480000 & 1,165 & 592 & 0.29 & 0.47 & 317 & 13,635 & 5 & 10 & SG \\
\hline San Luis & SLU06 & 460000 & 3480000 & 1,246 & 573 & 0.32 & 0.44 & 2,228 & 13,404 & 7 & 9 & MEW \\
\hline San Luis & SLU07 & 460000 & 3490000 & 1,274 & 641 & 0.3 & 0.46 & 0 & 14,505 & 10 & 6 & MEW \\
\hline Tumacacori & TUM01 & 490000 & 3500000 & 1,112 & 695 & 0.27 & 0.3 & 1,866 & 6,185 & 13 & 11 & SG \\
\hline Tumacacori & TUM02 & 490000 & 3500000 & 1,245 & 498 & 0.24 & 0.43 & 140 & 8,674 & 13 & 6 & SG \\
\hline Tumacacori & TUM03 & 490000 & 3500000 & 1,386 & 958 & 0.25 & 0.53 & 1,088 & 7,237 & 13 & 6 & SG \\
\hline Tumacacori & TUM04 & 490000 & 3500000 & 1,211 & 470 & 0.25 & 0.45 & 1,847 & 10,165 & 13 & 8 & SG \\
\hline Tumacacori & TUM05 & 490000 & 3490000 & 1,291 & 823 & 0.23 & 0.53 & 1,670 & 7,612 & 13 & 8 & SG \\
\hline Whetstone & WHE01 & 560000 & 3530000 & 1,795 & 1,325 & 0.47 & 0.53 & 2,697 & 5,758 & 9 & 25 & MEW \\
\hline Whetstone & WHE02 & 560000 & 3530000 & 1,819 & 1,385 & 0.47 & 0.49 & 1,994 & 7,362 & 9 & 26 & MEW \\
\hline Whetstone & WHE03 & 560000 & 3520000 & 2,139 & 1,132 & 0.47 & 0.53 & 3,887 & 5,763 & 9 & 21 & MEW \\
\hline Whetstone & WHE04 & 560000 & 3520000 & 1,834 & 1,212 & 0.42 & 0.46 & 5,545 & 4,919 & 9 & 19 & MEW \\
\hline Whetstone & WHE05 & 560000 & 3520000 & 1,675 & 1,361 & 0.43 & 0.42 & 3,820 & 4,722 & 9 & 19 & MEW \\
\hline Whetstone & WHE06 & 550000 & 3520000 & 1,963 & 1,188 & 0.51 & 0.43 & 3,076 & 7,302 & 10 & 30 & MEW \\
\hline Whetstone & WHE07 & 560000 & 3520000 & 1,875 & 1,332 & 0.48 & 0.42 & 2,023 & 5,472 & 9 & 21 & MEW \\
\hline Whetstone & WHE08 & 550000 & 3520000 & 1,651 & 964 & 0.26 & 0.36 & 2,292 & 6,143 & 10 & 11 & MEW \\
\hline Whetstone & WHE09 & 550000 & 3520000 & 1,636 & 690 & 0.29 & 0.39 & 3,256 & 8,093 & 14 & 11 & MEW \\
\hline
\end{tabular}

${ }^{\mathrm{a}}$ MEW, Madrean evergreen woodland; SG, semidesert grassland; PGBG, Plains and Great Basin grassland; PMCF, Petran montane conifer forest; CD, Chihuahuan desertscrub (Brown 1994). 
Appendix 3. Survey Sites (UTM coordinates) by Mountain Range and Number of Days Camera(s) Were Active at Each Site

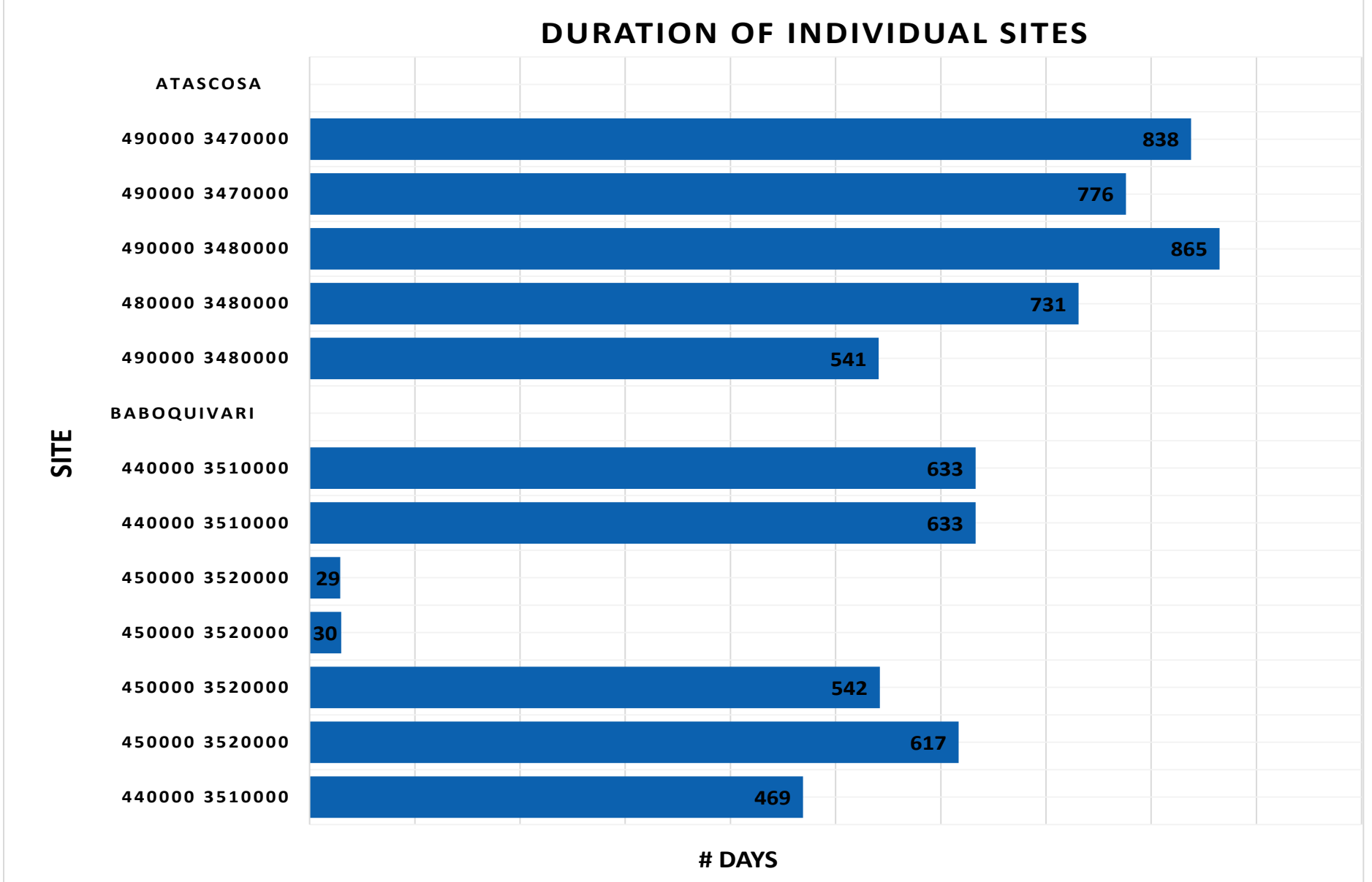




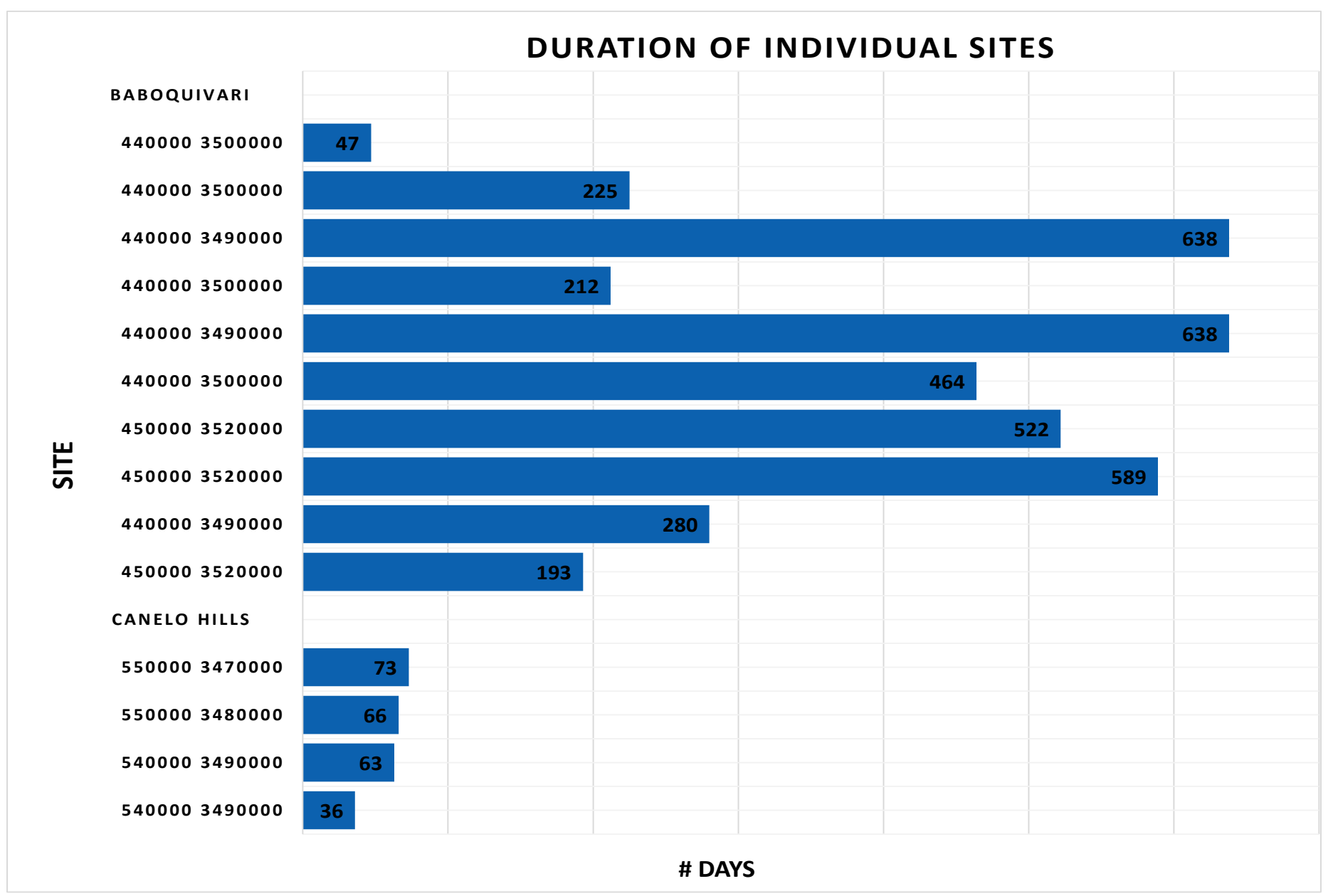




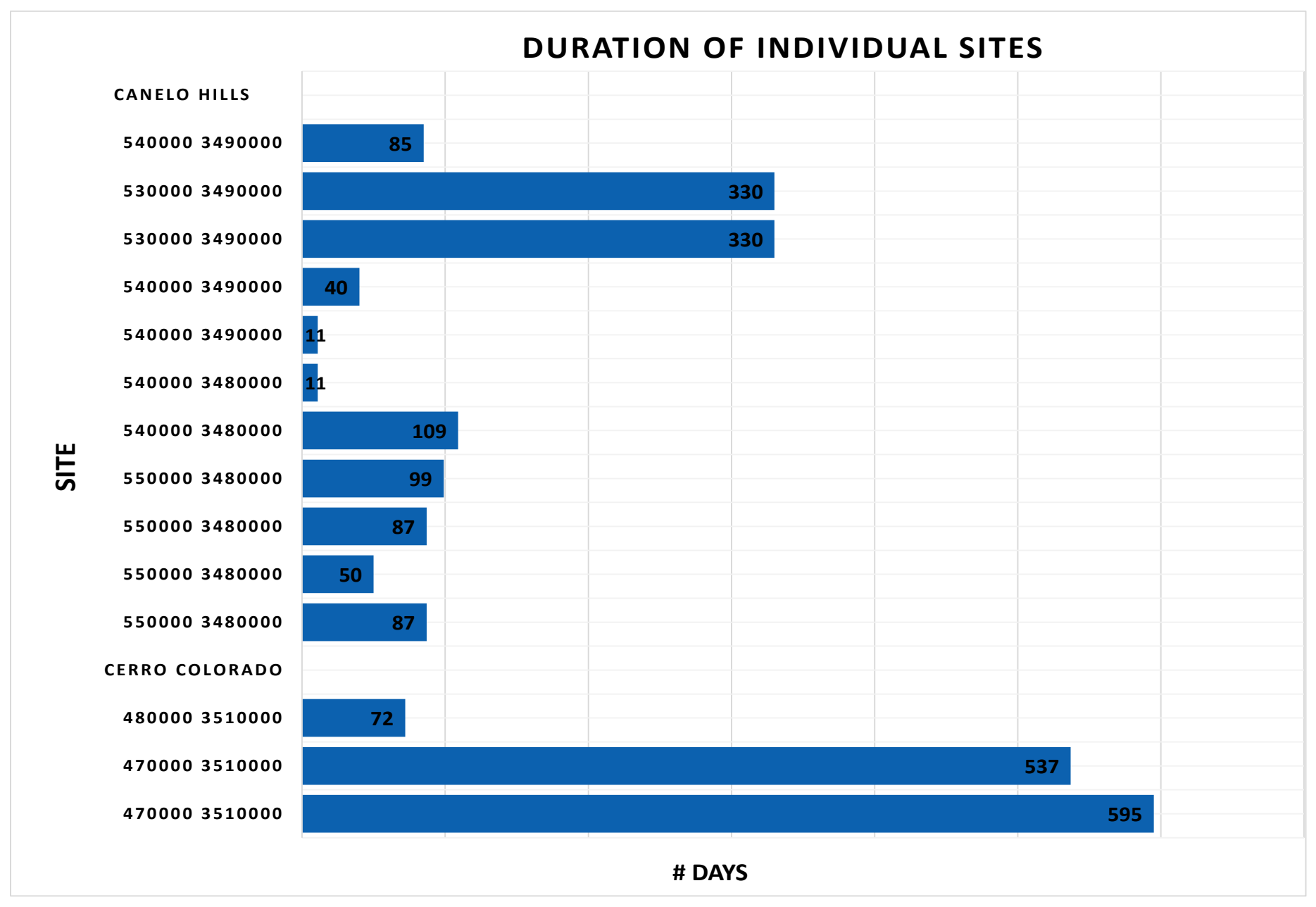




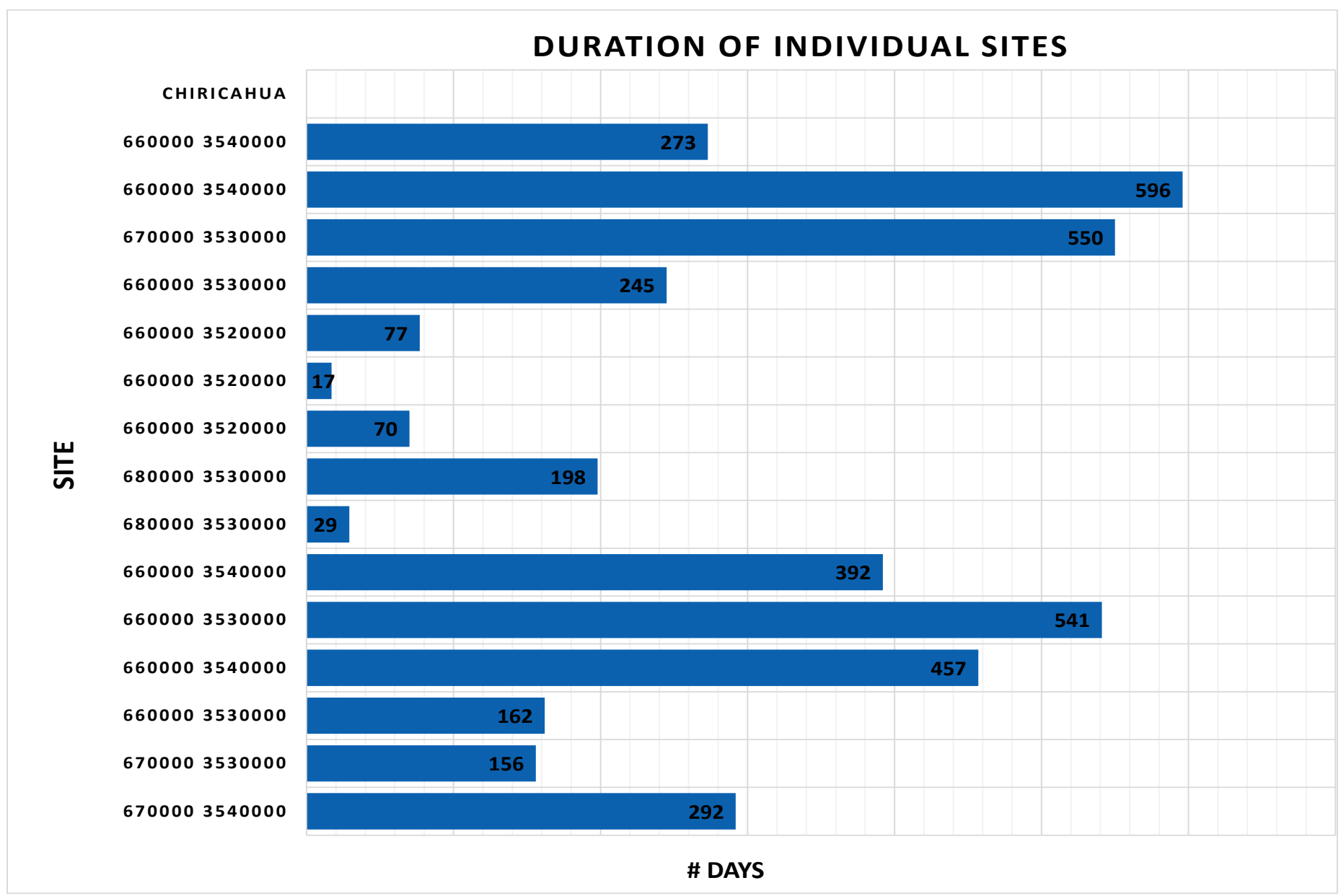




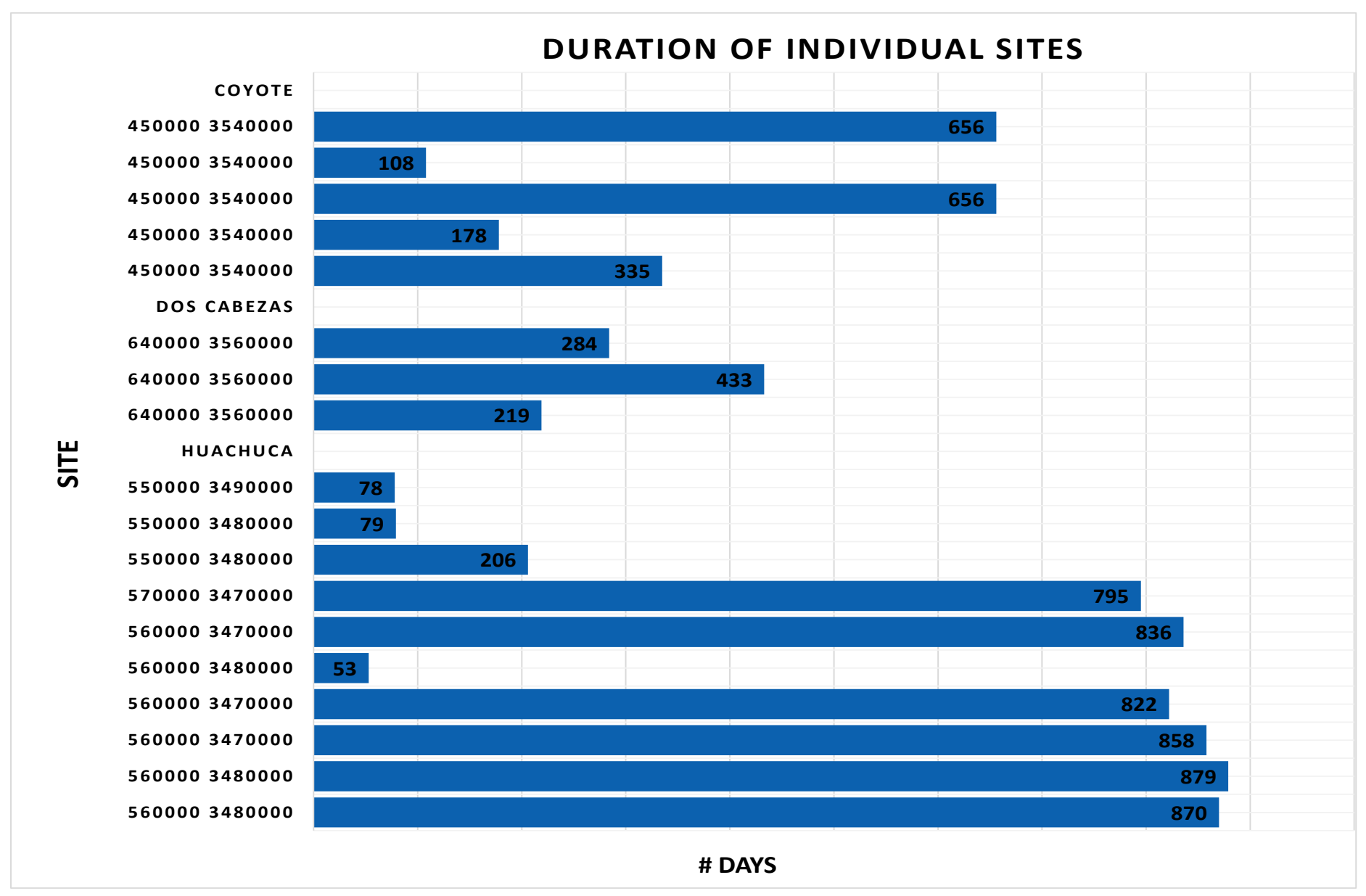




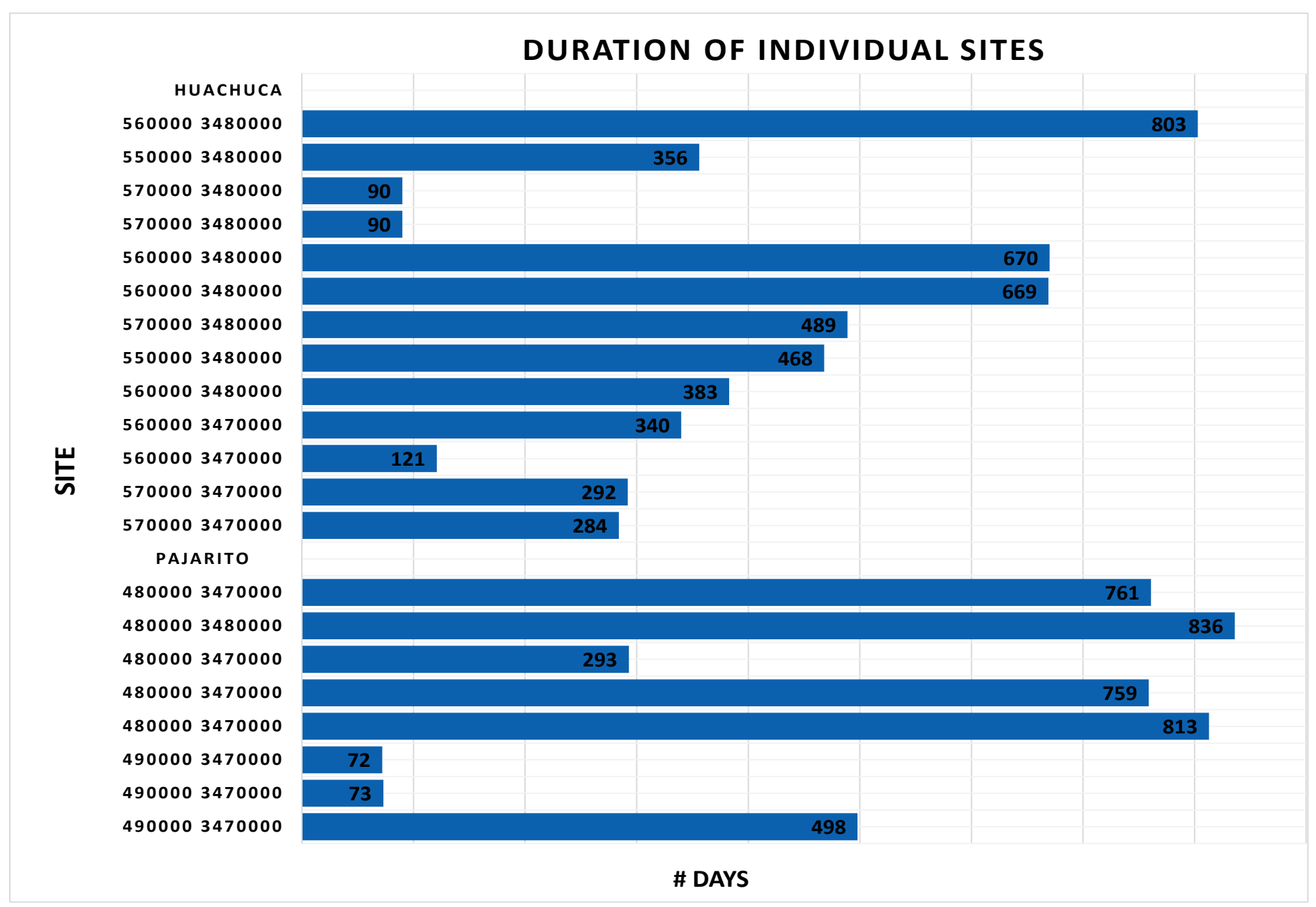




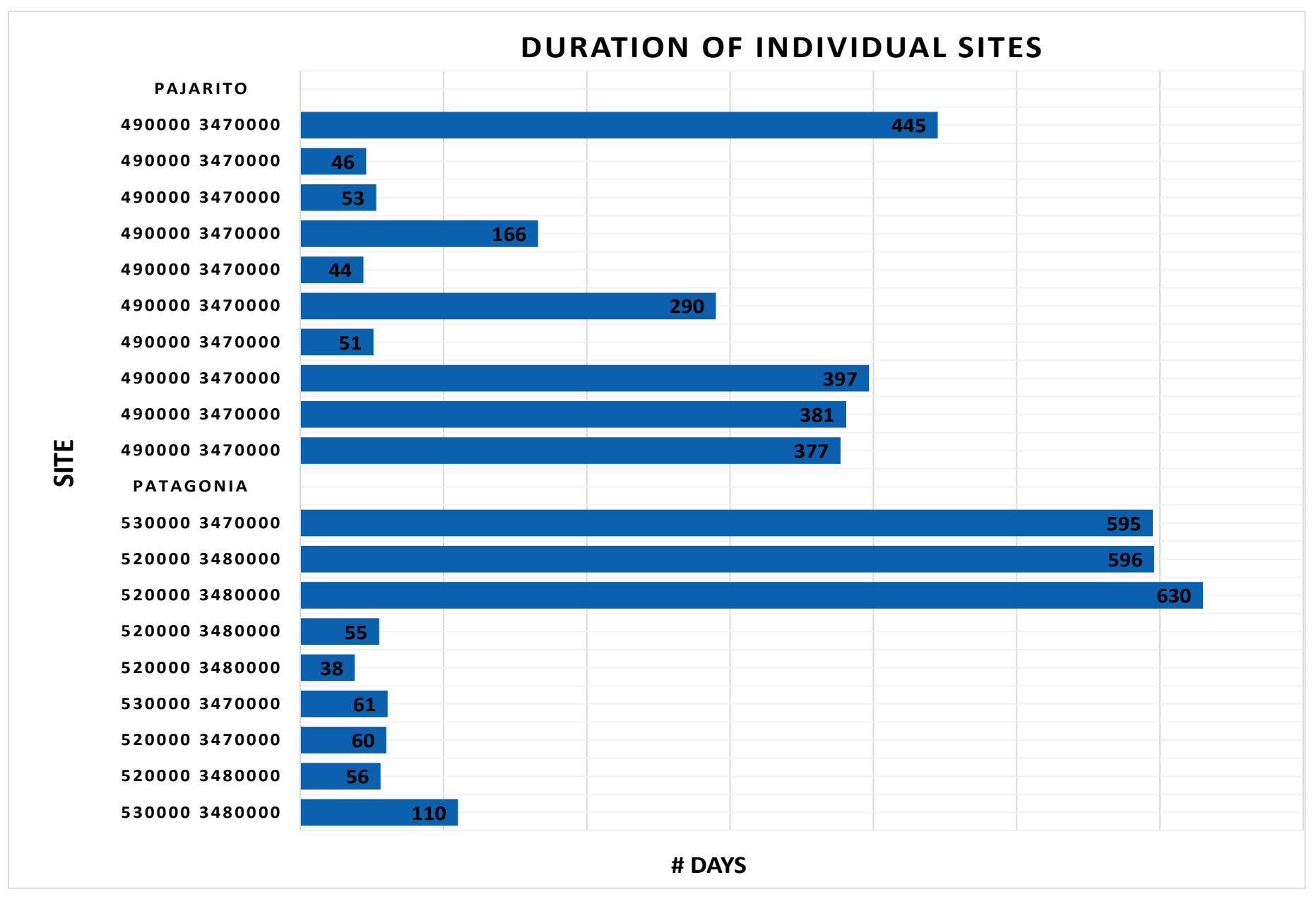




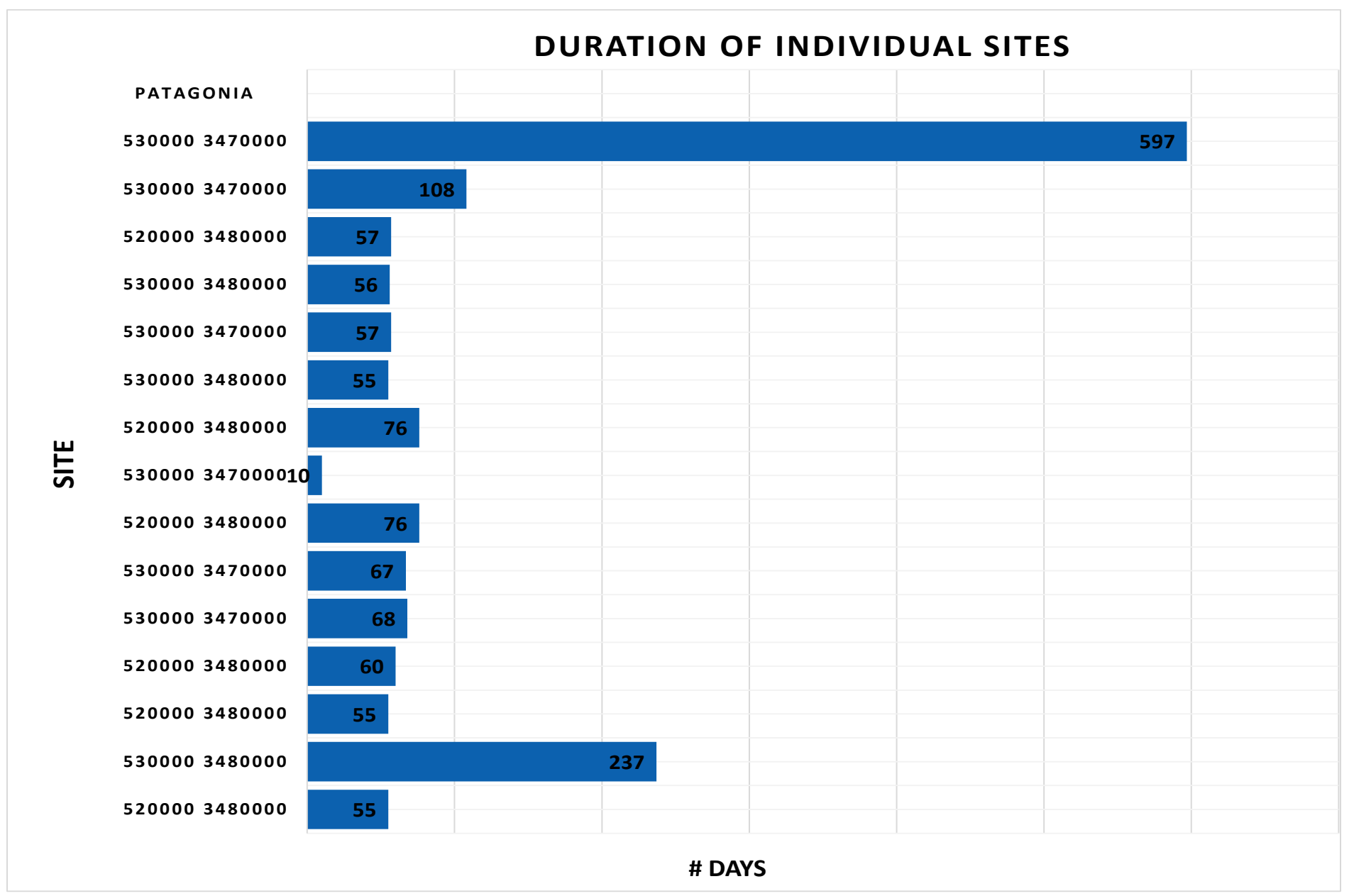




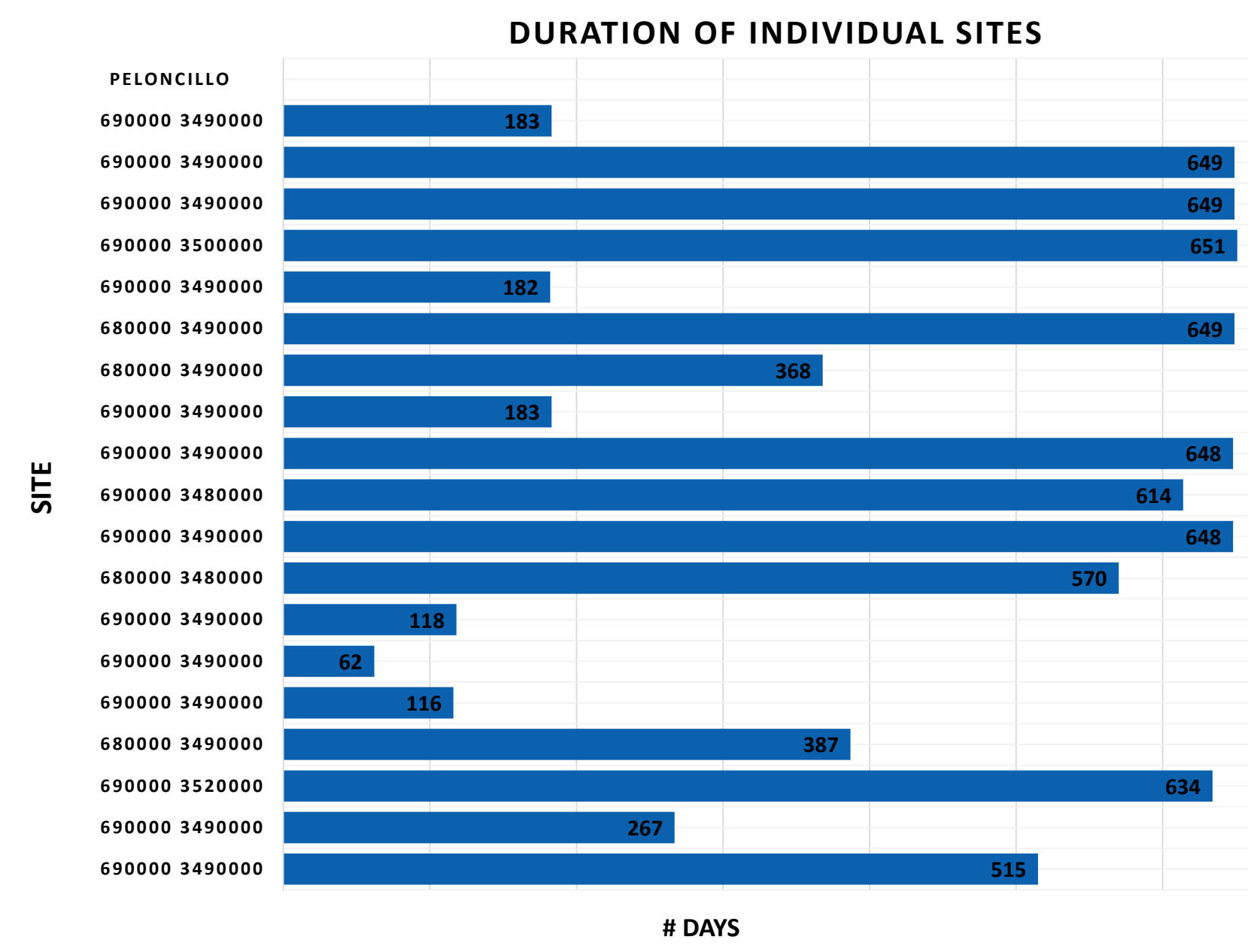




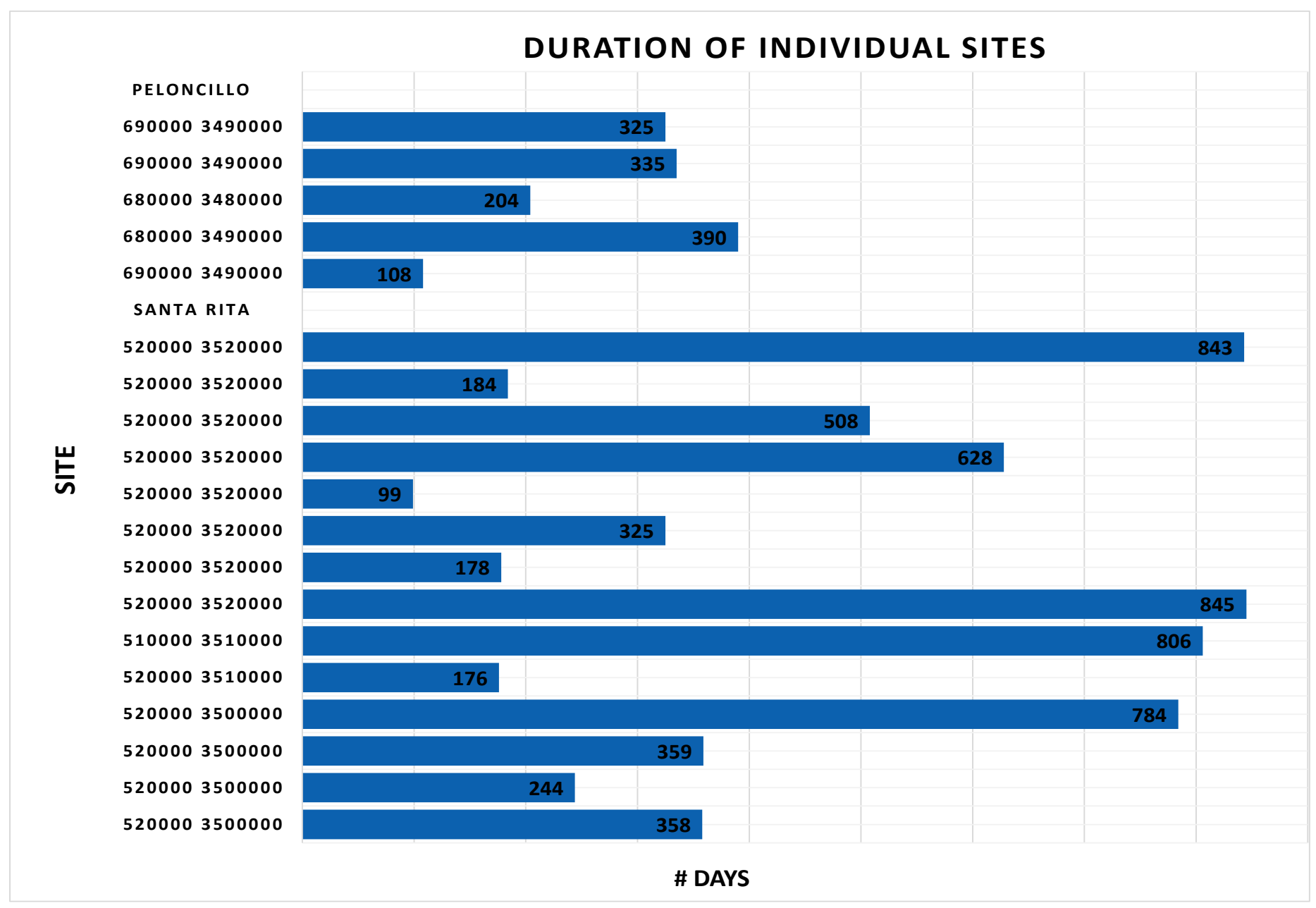




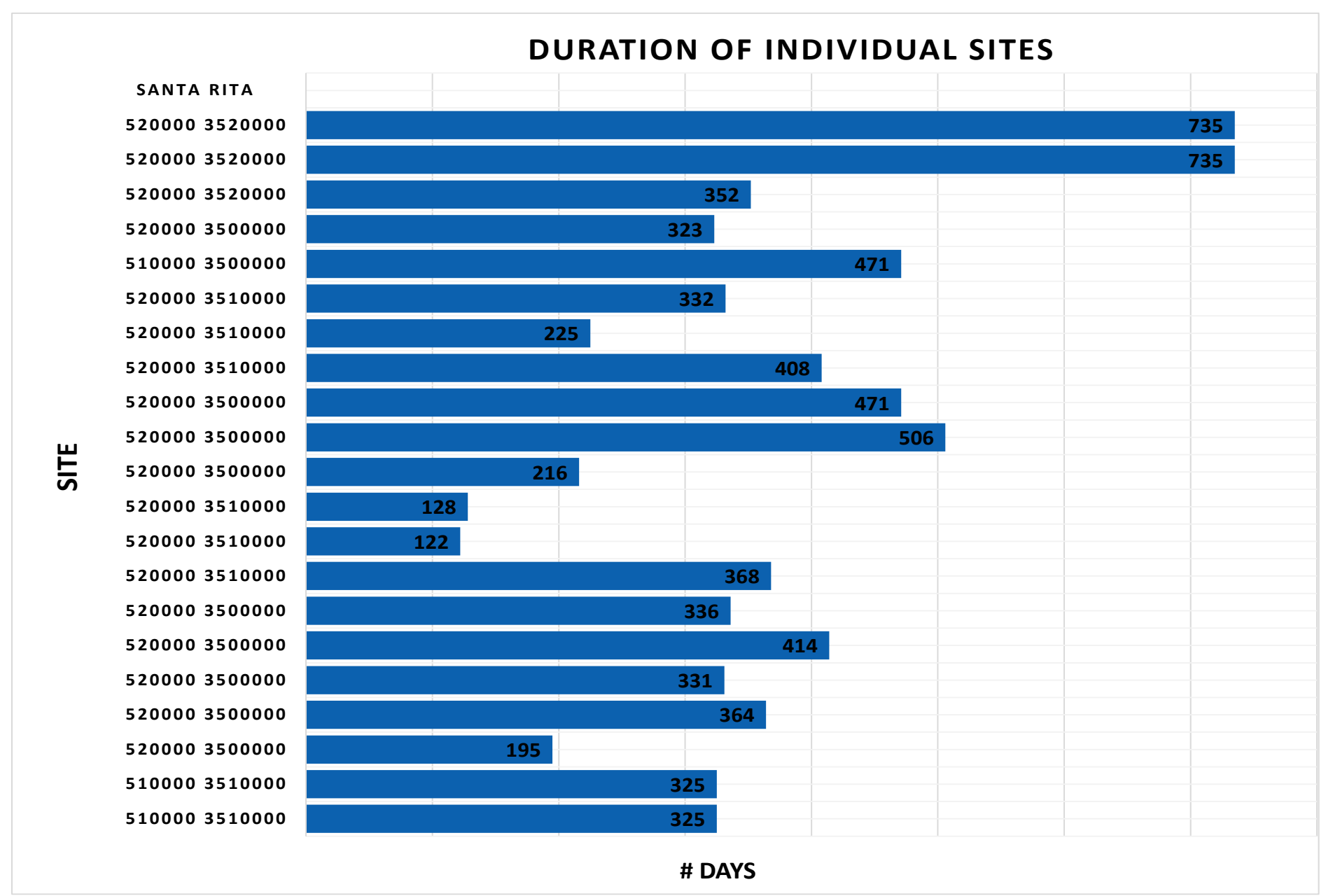




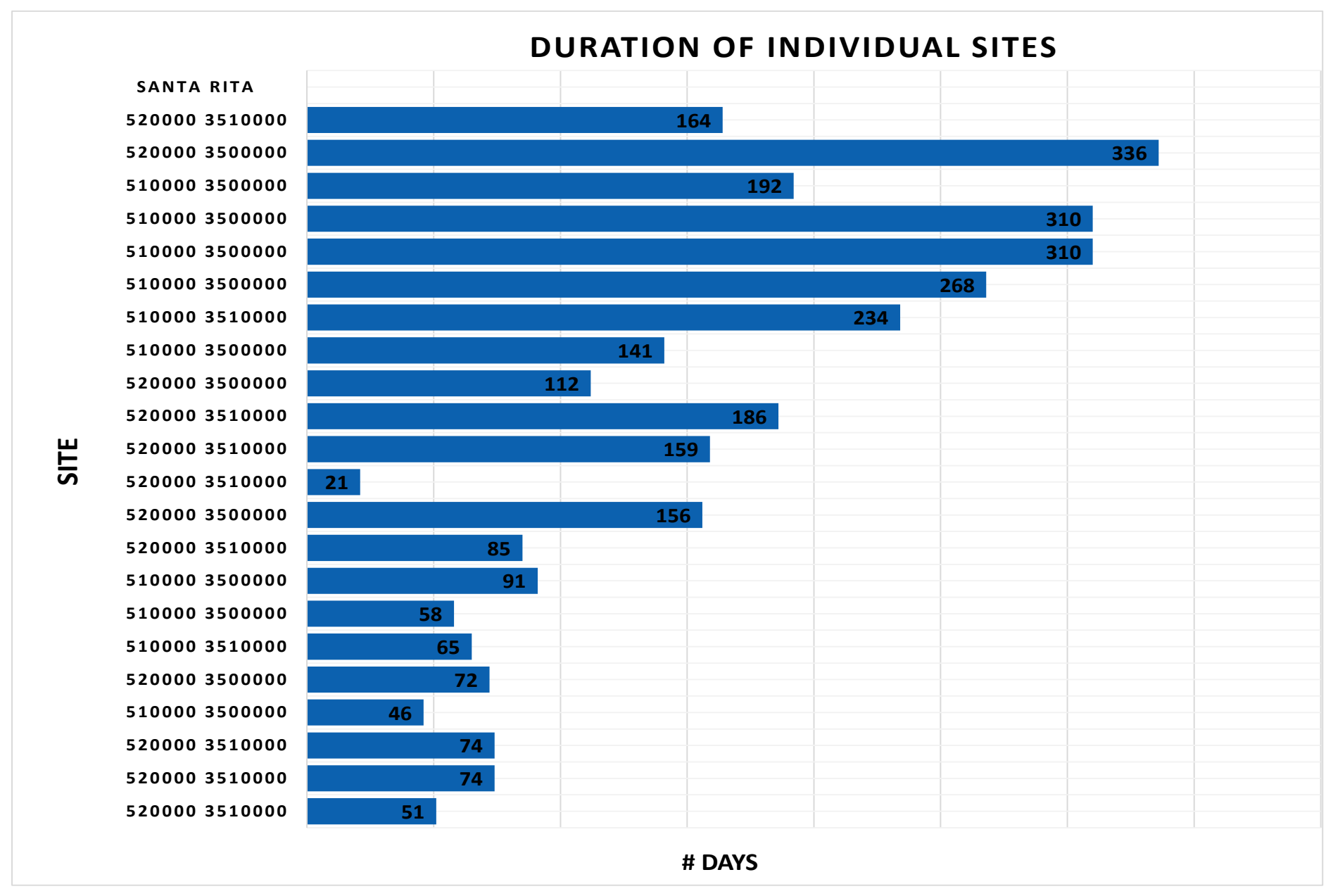




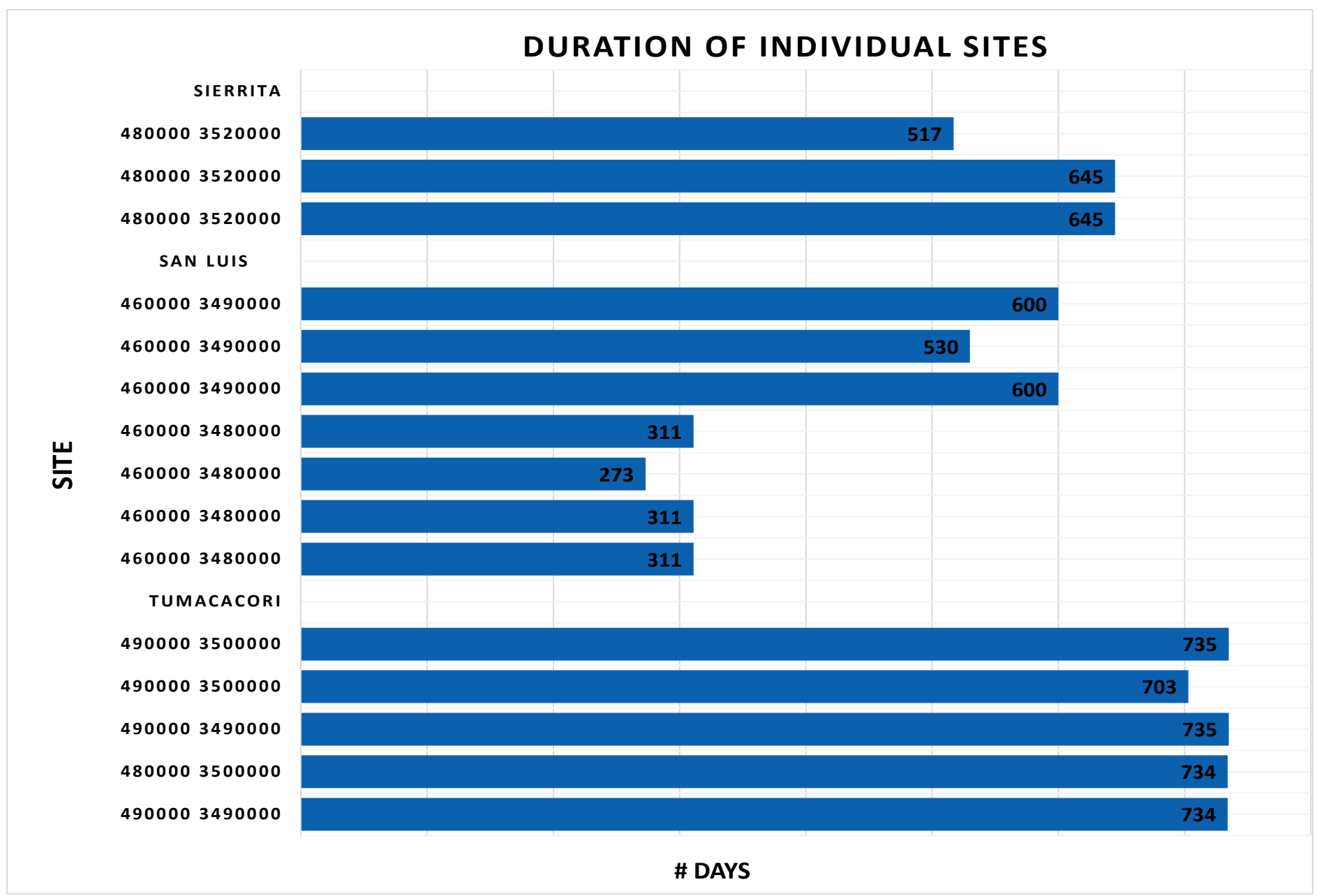




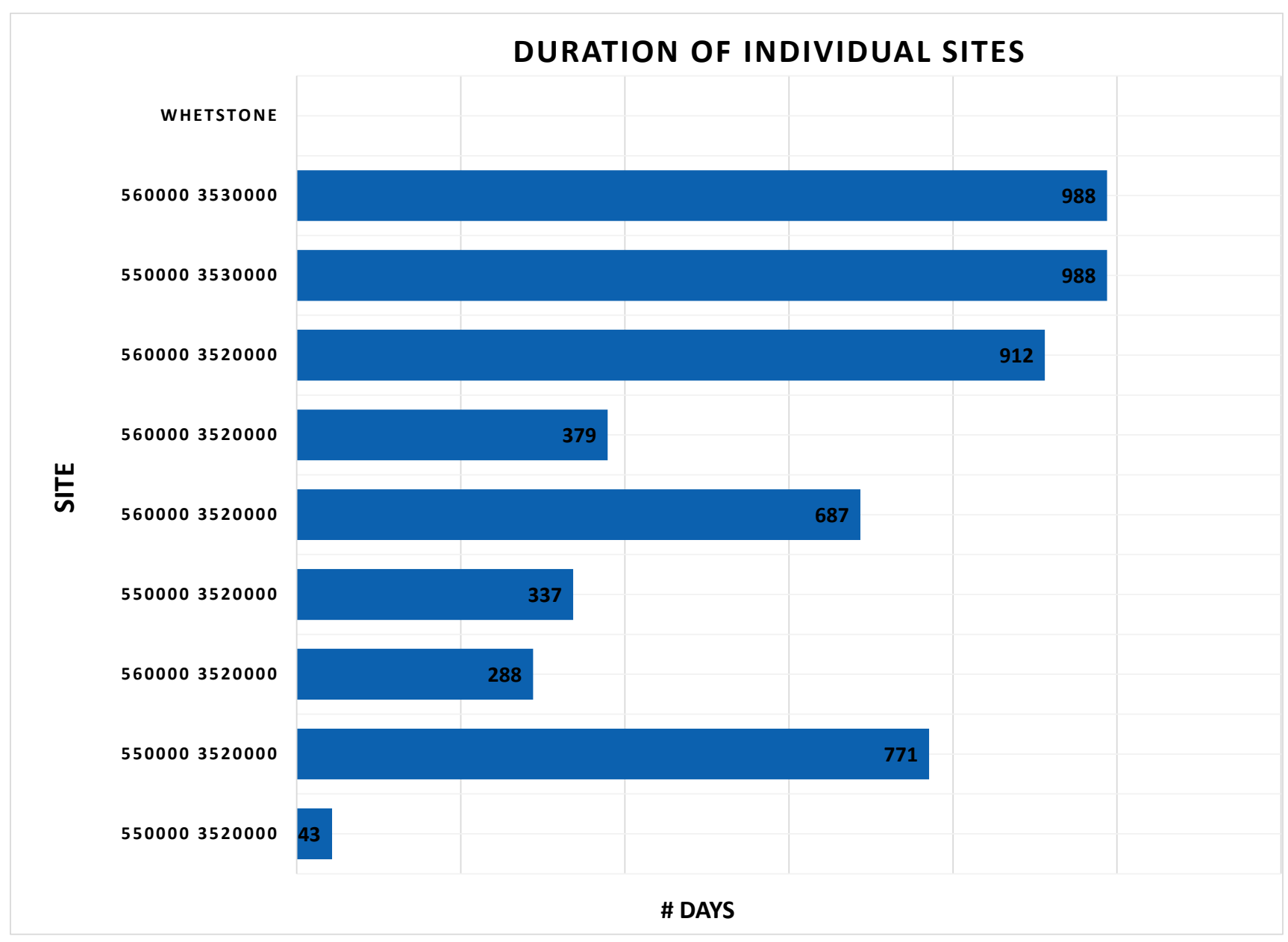


Appendix 4. Spatial Representation of Camera Theft and Vandalism from April 2012 to February 2015 on Eastern Side of Study Area

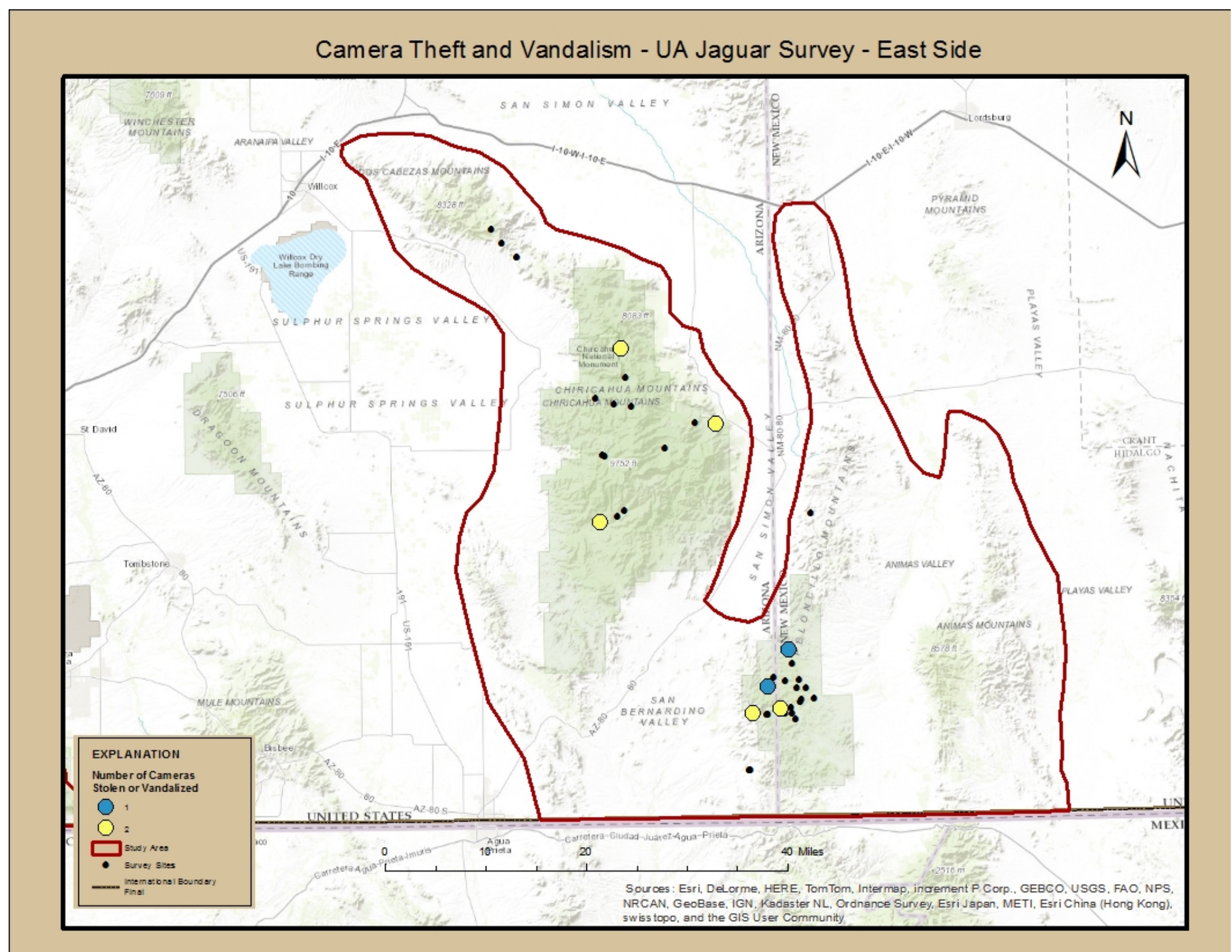


Appendix 5. Spatial Representation of Camera Theft and Vandalism from April 2012 to February 2015 on Western Side of Study Area

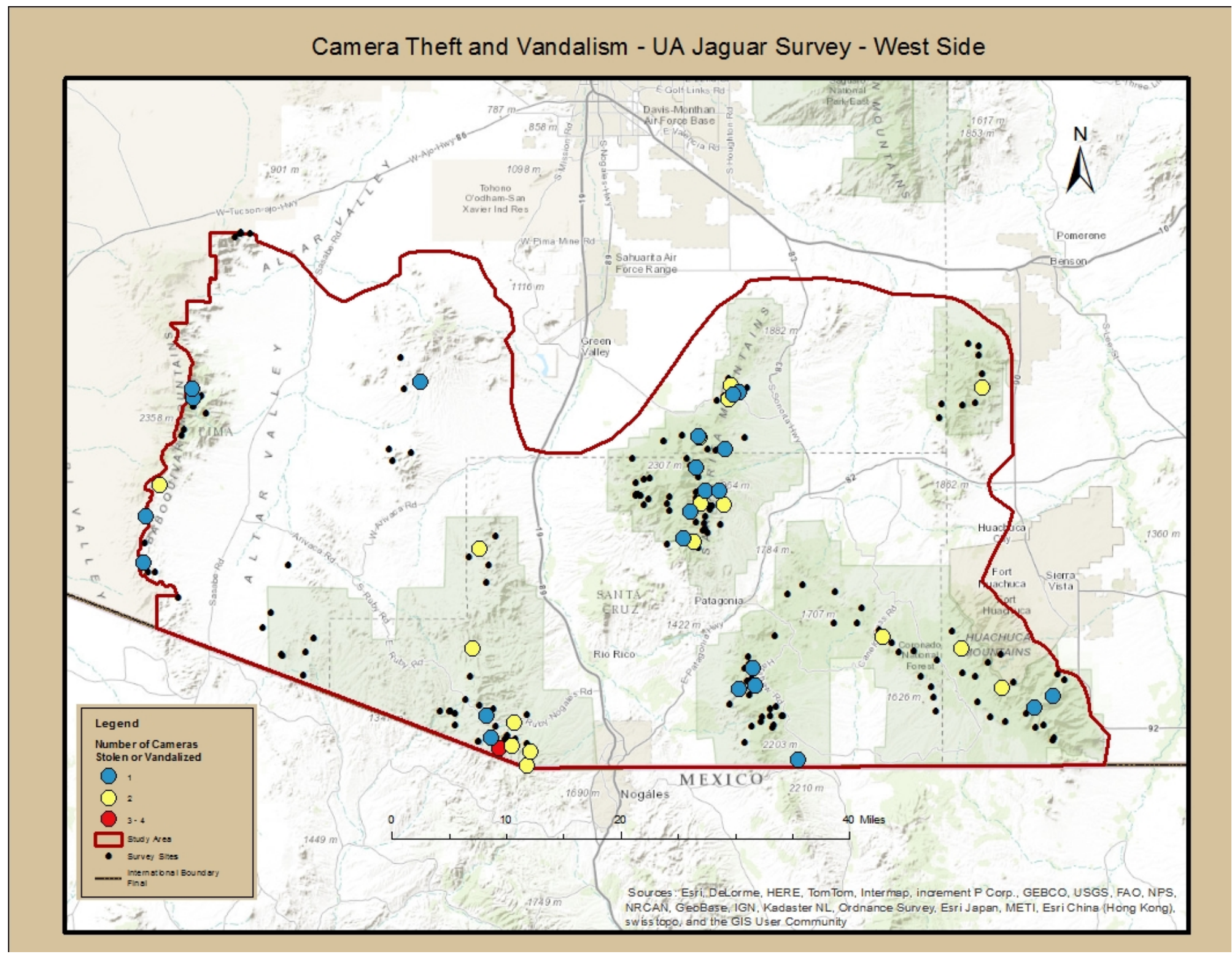


Appendix 6. Quantity of Cameras Stolen/Vandalized per Quarter of Study. Data Represents April 2012 through February 2015

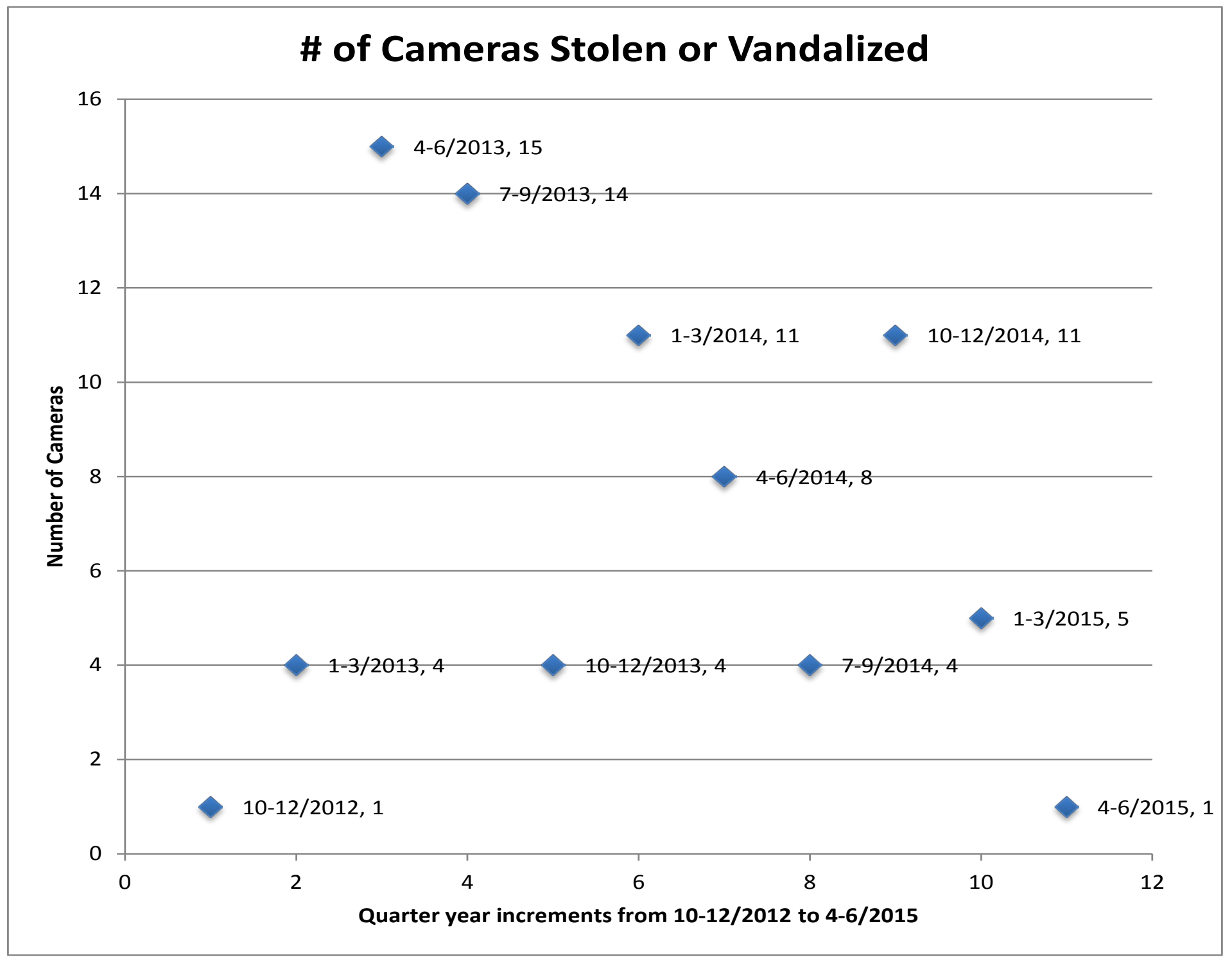


Publishing support provided by the U.S. Geological Survey Science Publishing Network, Menlo and Tacoma Publishing Service Centers

For more information concerning the research in this report, contact the Leader, Arizona Cooperative Fish and Wildlife Research Unit U.S. Geological Survey

325 Biosciences East

Tucson, Arizona 85721

http://www.coopunits.org/Arizona/ 
